G C A T

T A C G

G C A T
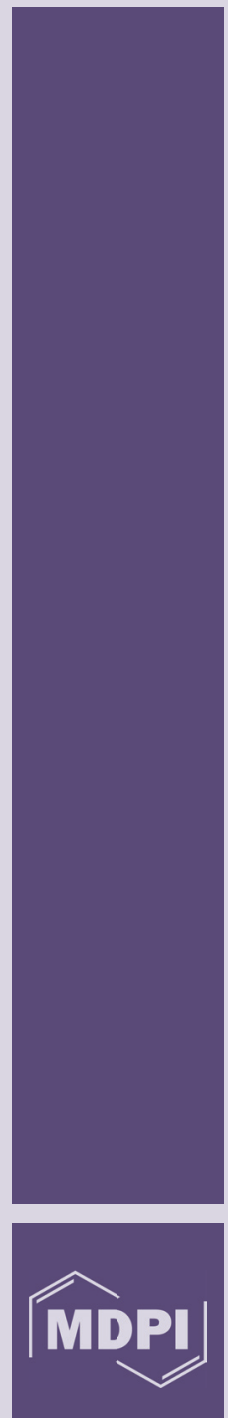

\title{
Grand Celebration: 10th Anniversary of the Human Genome Project
}

\section{Volume 1}

Edited by

John Burn, James R. Lupski, Karen E. Nelson and Pabulo H. Rampelotto

Printed Edition of the Special Issue Published in Genes 
John Burn, James R. Lupski, Karen E. Nelson and

Pabulo H. Rampelotto (Eds.)

\section{Grand Celebration: 10th Anniversary of the Human Genome Project}

\section{Volume 1}


This book is a reprint of the special issue that appeared in the online open access journal Genes (ISSN 2073-4425) in 2014 (available at:

http://www.mdpi.com/journal/genes/special_issues/Human_Genome).

\author{
Guest Editors \\ John Burn \\ University of Newcastle \\ UK \\ James R. Lupski \\ Baylor College of Medicine \\ USA \\ Karen E. Nelson \\ J. Craig Venter Institute (JCVI) \\ USA \\ Pabulo H. Rampelotto \\ Federal University of Rio Grande do Sul \\ Brazil
}

Editorial Office

MDPI AG

Klybeckstrasse 64

Basel, Switzerland
Publisher

Shu-Kun Lin
Assistant Editor

Rongrong Leng

\title{
1. Edition 2016
}

MDPI • Basel • Beijing • Wuhan

ISBN 978-3-03842-123-8 complete edition (Hbk)

ISBN 978-3-03842-169-6 complete edition (PDF)

ISBN 978-3-03842-124-5 Volume 1 (Hbk) ISBN 978-3-03842-170-2 Volume 1 (PDF)

ISBN 978-3-03842-125-2 Volume 2 (Hbk) ISBN 978-3-03842-171-9 Volume 2 (PDF)

ISBN 978-3-03842-126-9 Volume 3 (Hbk) ISBN 978-3-03842-172-6 Volume 3 (PDF)

(C) 2016 by the authors; licensee MDPI, Basel, Switzerland. All articles in this volume are Open Access distributed under the Creative Commons License (CC-BY), which allows users to download, copy and build upon published articles even for commercial purposes, as long as the author and publisher are properly credited, which ensures maximum dissemination and a wider impact of our publications. However, the dissemination and distribution of physical copies of this book as a whole is restricted to MDPI, Basel, Switzerland. 


\section{Table of Contents}

List of Contributors ................................................................................................ VII

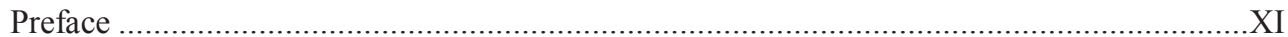

\section{Debra J. H. Mathews and Leila Jamal}

Revisiting Respect for Persons in Genomic Research

Reprinted from: Genes 2014, 5(1), 1-12

http://www.mdpi.com/2073-4425/5/1/1

Vincent Timmerman, Alleene V. Strickland and Stephan Züchner

Genetics of Charcot-Marie-Tooth (CMT) Disease within the Frame of the Human Genome

Project Success

Reprinted from: Genes 2014, 5(1), 13-32

http://www.mdpi.com/2073-4425/5/1/13

\section{Megan E. Aldrup-MacDonald and Beth A. Sullivan}

The Past, Present, and Future of Human Centromere Genomics

Reprinted from: Genes 2014, 5(1), 33-50

http://www.mdpi.com/2073-4425/5/1/33

\section{Nathalie Chami and Guillaume Lettre}

Lessons and Implications from Genome-Wide Association Studies (GWAS) Findings of Blood Cell Phenotypes

Reprinted from: Genes 2014, 5(1), 51-64

http://www.mdpi.com/2073-4425/5/1/51

Jose Russo, Julia Santucci-Pereira and Irma H. Russo

The Genomic Signature of Breast Cancer Prevention

Reprinted from: Genes 2014, 5(1), 65-83

http://www.mdpi.com/2073-4425/5/1/65 


\section{Katsushi Tokunaga}

Lessons from Genome-Wide Search for Disease-Related Genes with Special Reference to HLA-Disease Associations

Reprinted from: Genes 2014, 5(1), 84-96

http://www.mdpi.com/2073-4425/5/1/84

\section{Hannelore Ehrenreich and Klaus-Armin Nave}

Phenotype-Based Genetic Association Studies (PGAS) — Towards Understanding the

Contribution of Common Genetic Variants to Schizophrenia Subphenotypes

Reprinted from: Genes 2014, 5(1), 97-105

http://www.mdpi.com/2073-4425/5/1/97

Albino Bacolla, David N. Cooper and Karen M. Vasquez

Mechanisms of Base Substitution Mutagenesis in Cancer Genomes

Reprinted from: Genes 2014, 5(1), 108-146

http://www.mdpi.com/2073-4425/5/1/108 104

Muhammad Imran Khan, Maleeha Azam, Muhammad Ajmal, Rob W. J. Collin, Anneke I. den Hollander, Frans P. M. Cremers and Raheel Qamar

The Molecular Basis of Retinal Dystrophies in Pakistan

Reprinted from: Genes 2014, 5(1), 176-195

http://www.mdpi.com/2073-4425/5/1/176

Sara L. Pulit, Maarten Leusink, Androniki Menelaou and Paul I. W. de Bakker

Association Claims in the Sequencing Era

Reprinted from: Genes 2014, 5(1), 196-213

http://www.mdpi.com/2073-4425/5/1/196 163

\section{Megan J. Puckelwartz and Elizabeth M. McNally}

Genetic Profiling for Risk Reduction in Human Cardiovascular Disease

Reprinted from: Genes 2014, 5(1), 214-234

http://www.mdpi.com/2073-4425/5/1/214 181

\section{David J. Elliott}

Illuminating the Transcriptome through the Genome

Reprinted from: Genes 2014, 5(1), 235-253

http://www.mdpi.com/2073-4425/5/1/235 203 
Nicola Whiffin and Richard S. Houlston

Architecture of Inherited Susceptibility to Colorectal Cancer: A Voyage of Discovery

Reprinted from: Genes 2014, 5(2), 270-284

http://www.mdpi.com/2073-4425/5/2/270 .

Dianne F. Newbury, Anthony P. Monaco and Silvia Paracchini

Reading and Language Disorders: The Importance of Both Quantity and Quality

Reprinted from: Genes 2014, 5(2), 285-309

http://www.mdpi.com/2073-4425/5/2/285 



\section{List of Contributors}

Muhammad Ajmal: Department of Biosciences, Faculty of Science, COMSATS Institute of Information Technology, Islamabad 45600, Pakistan; Department of Human Genetics, Radboud University Medical Center, Nijmegen 6500 HB, The Netherlands.

Megan E. Aldrup-MacDonald: Department of Molecular Genetics and Microbiology, Duke University Medical Center, Durham, NC 27710, USA; Division of Human Genetics, Duke University, Durham, NC 27710, USA.

Maleeha Azam: Department of Biosciences, Faculty of Science, COMSATS Institute of Information Technology, Islamabad 45600, Pakistan; Department of Human Genetics, Radboud University Medical Center, Nijmegen 6500 HB, The Netherlands.

Albino Bacolla: Dell Pediatric Research Institute, Division of Pharmacology and Toxicology, College of Pharmacy, The University of Texas at Austin, 1400 Barbara Jordan Blvd., Austin, TX 78723, USA.

Nathalie Chami: Montreal Heart Institute, Faculté de Médecine, Université de Montréal, 5000 Bélanger Street, Montréal, QC H1T 1C8, Canada.

Rob W. J. Collin: Radboud Institute for Molecular Life Sciences/Department of Human Genetics, Radboud University Medical Center, Nijmegen 6500 HB, The Netherlands.

David N. Cooper: Institute of Medical Genetics, School of Medicine, Cardiff University, Cardiff CF14 4XN, UK.

Frans P. M. Cremers: Department of Biosciences, Faculty of Science, COMSATS Institute of Information Technology, Islamabad 45600, Pakistan; Radboud Institute for Molecular Life Sciences/Department of Human Genetics, Radboud University Medical Center, Nijmegen 6500 HB, The Netherlands.

Paul I. W. de Bakker: Department of Medical Genetics, Institute for Molecular Medicine / Julius Center for Health Sciences and Primary Care, University Medical Center Utrecht, Universiteitsweg 100, 3584 CG, Utrecht, The Netherlands.

Anneke I. den Hollander: Department of Ophthalmology/Department of Human Genetics, Radboud University Medical Center, Nijmegen 6500 HB, The Netherlands.

Hannelore Ehrenreich: Max Planck Institute of Experimental Medicine, Hermann-ReinStr.3, 37075 Göttingen, Germany; DFG Center for Nanoscale Microscopy and Molecular Physiology of the Brain (CNMPB), Hermann-Rein-Str.3, 37075 Göttingen, Germany.

David J. Elliott: Institute of Genetic Medicine, Newcastle University, Newcastle, NE1 3BZ, UK.

Richard S. Houlston: Division of Genetics and Epidemiology / Molecular and Population Genetics Team, Genetics and Epidemiology, The Institute of Cancer Research, Sutton, SM2 $5 \mathrm{NG}, \mathrm{UK}$. 
Leila Jamal: Department of Health Policy and Management, Johns Hopkins Bloomberg School of Health, 615 North Wolfe St., Baltimore, MD 21205, USA; Division of Neurogenetics, Kennedy Krieger Institute, 801 N. Broadway, Rm. 564, Baltimore, MD 21205, USA.

Muhammad Imran Khan: Department of Biosciences, Faculty of Science, COMSATS Institute of Information Technology, Islamabad 45600, Pakistan; Department of Human Genetics, Radboud University Medical Center, Nijmegen 6500 HB, The Netherlands.

Guillaume Lettre: Montreal Heart Institute, Faculté de Médecine, Université de Montréal, 5000 Bélanger Street, Montréal, QC H1T 1C8, Canada.

Maarten Leusink: Julius Center for Health Sciences and Primary Care / Department of Medical Genetics, Institute for Molecular Medicine, University Medical Center Utrecht, Universiteitsweg 100, 3584 CG, Utrecht, The Netherlands; Division of Pharmacoepidemiology \& Clinical Pharmacology, Utrecht Institute for Pharmaceutical Sciences, Utrecht University, Universiteitsweg 99, 3584 CG, Utrecht, The Netherlands.

Debra J. H. Mathews: Johns Hopkins Berman Institute of Bioethics, 1809 Ashland Avenue, Baltimore, MD 21205, USA.

Elizabeth M. McNally: Department of Human Genetics/Department of Medicine, University of Chicago, Chicago, IL 60637, USA.

Androniki Menelaou: Department of Medical Genetics, Institute for Molecular Medicine, University Medical Center Utrecht, Universiteitsweg 100, 3584 CG, Utrecht, The Netherlands.

Anthony P. Monaco: Tufts University, Ballou Hall, Medford, MA 02155, USA.

Klaus-Armin Nave: Max Planck Institute of Experimental Medicine, Hermann-Rein-Str.3, 37075 Göttingen, Germany; DFG Center for Nanoscale Microscopy and Molecular Physiology of the Brain (CNMPB), Hermann-Rein-Str.3, 37075 Göttingen, Germany.

Dianne F. Newbury: Wellcome Trust Centre for Human Genetics, University of Oxford, Oxford OX3 7BN, UK.

Silvia Paracchini: School of Medicine, University of St. Andrews, St. Andrews, KY16 9TF, UK. Megan J. Puckelwartz: Department of Medicine, University of Chicago, Chicago, IL 60637, USA.

Sara L. Pulit: Department of Medical Genetics, Institute for Molecular Medicine, University Medical Center Utrecht, Universiteitsweg 100, 3584 CG, Utrecht, The Netherlands.

Raheel Qamar: Department of Biosciences, Faculty of Science, COMSATS Institute of Information Technology, Islamabad 45600, Pakistan; Al-Nafees Medical College \& Hospital, Isra University, Islamabad 45600, Pakistan.

Irma H. Russo: The Irma H. Russo MD Breast Cancer Research Laboratory, Fox Chase Cancer Center, Temple University Health System, 333 Cottman Avenue, Philadelphia, PA 19111, USA.

Jose Russo: The Irma H. Russo MD Breast Cancer Research Laboratory, Fox Chase Cancer Center, Temple University Health System, 333 Cottman Avenue, Philadelphia, PA 19111, USA. 
Julia Santucci-Pereira: The Irma H. Russo MD Breast Cancer Research Laboratory, Fox Chase Cancer Center, Temple University Health System, 333 Cottman Avenue, Philadelphia, PA 19111, USA.

Alleene V. Strickland: Department of Human Genetics, Hussman Institute for Human Genomics, University of Miami Miller School of Medicine, Biomedical Research Building, Room 523, LC: M-860, 1501 NW 10 Ave., Miami, FL 33136, USA.

Beth A. Sullivan: Division of Human Genetics/Department of Molecular Genetics and Microbiology, Duke University Medical Center, Durham, NC 27710, USA.

Vincent Timmerman: Peripheral Neuropathy Group, Molecular Genetics Department, VIB, University of Antwerp, Universiteitsplein 1, Antwerpen B2610, Belgium; Neurogenetics Group, Institute Born Bunge, University of Antwerp, Antwerpen B2610, Belgium.

Katsushi Tokunaga: Department of Human Genetics, Graduate School of Medicine, University of Tokyo, Tokyo 113-0013, Japan.

Karen M. Vasquez: Dell Pediatric Research Institute, Division of Pharmacology and Toxicology, College of Pharmacy, The University of Texas at Austin, 1400 Barbara Jordan Blvd., Austin, TX 78723, USA.

Nicola Whiffin: Molecular and Population Genetics Team, Genetics and Epidemiology, The Institute of Cancer Research, Sutton, SM2 5NG, UK.

Stephan Züchner: Department of Human Genetics, Hussman Institute for Human Genomics, University of Miami Miller School of Medicine, Biomedical Research Building, Room 523, LC: M-860, 1501 NW 10 Ave., Miami, FL 33136, USA. 



\section{Preface}

In 1990, scientists began working together on one of the largest biological research projects ever proposed. The project proposed to sequence the three billion nucleotides in the human genome. The Human Genome Project took 13 years and was completed in April 2003, at a cost of approximately three billion dollars. It was a major scientific achievement that forever changed the understanding of our own nature. The sequencing of the human genome was in many ways a triumph for technology as much as it was for science. From the Human Genome Project, powerful technologies have been developed (e.g., microarrays and next generation sequencing) and new branches of science have emerged (e.g., functional genomics and pharmacogenomics), paving new ways for advancing genomic research and medical applications of genomics in the 21 st century. The investigations have provided new tests and drug targets, as well as insights into the basis of human development and diagnosis/treatment of cancer and several mysterious humans diseases. This genomic revolution is prompting a new era in medicine, which brings both challenges and opportunities. Parallel to the promising advances over the last decade, the study of the human genome has also revealed how complicated human biology is, and how much remains to be understood. The legacy of the understanding of our genome has just begun. To celebrate the 10th anniversary of the essential completion of the Human Genome Project, in April 2013 Genes launched this Special Issue, which highlights the recent scientific breakthroughs in human genomics, with a collection of papers written by authors who are leading experts in the field.

John Burn, James R. Lupski,

Karen E. Nelson and Pabulo H. Rampelotto

Guest Editors 



\title{
Revisiting Respect for Persons in Genomic Research
}

\section{Debra J. H. Mathews and Leila Jamal}

\begin{abstract}
The risks and benefits of research using large databases of personal information are evolving in an era of ubiquitous, internet-based data exchange. In addition, information technology has facilitated a shift in the relationship between individuals and their personal data, enabling increased individual control over how (and how much) personal data are used in research, and by whom. This shift in control has created new opportunities to engage members of the public as partners in the research enterprise on more equal and transparent terms. Here, we consider how some of the technological advances driving and paralleling developments in genomics can also be used to supplement the practice of informed consent with other strategies to ensure that the research process as a whole honors the notion of respect for persons upon which human research subjects protections are premised. Further, we suggest that technological advances can help the research enterprise achieve a more thoroughgoing respect for persons than was possible when current policies governing human subject research were developed. Questions remain about the best way to revise policy to accommodate these changes.
\end{abstract}

Reprinted from Genes. Cite as: Mathews, D.J.H.; Jamal, L. Revisiting Respect for Persons in Genomic Research. Genes 2014, 5, 1-12.

\section{Introduction}

The risks and benefits of research using large databases of personal information are evolving in an era of ubiquitous, internet-based data exchange. Here, we consider some of the technological advances driving and paralleling developments in genomics, and how they can be used to supplement the practice of informed consent to ensure that the research process as a whole honors the notion of respect for persons upon which human research subjects protections are premised.

The cost of next-generation sequencing has declined precipitously in recent years, increasing the potential of genomic research to expand knowledge of human biology and disease [1]. To render human genome data meaningful for individuals, investigators must collect and analyze information contributed by many individuals from diverse populations over long periods of time. To build large datasets, people are asked to donate biospecimens and personal data, including genomic data, to repositories of de-identified tissue and data used by many researchers [2]. Indeed, in an effort to harness the scientific potential of such large datasets, many of the world's leading research institutions recently announced ambitious plans to build a global, interoperable framework for sharing genomic and other research data more broadly in the future [3], and the NIH is currently developing a revised data-sharing policy [4]. As this new era of genomic research progresses, it is critical that we attend not only to the benefits that such broad sharing will have for science and medicine, but also to the proportionality of risks and benefits borne by contributors to biorepositories and genome databases. 
The structures and norms guiding the development and use of such repositories were established at a time when the re-identification of individual data contributors was thought to be unlikely, and the anonymization of personal data was a reasonable strategy for mitigating risks to research subjects from loss of confidentiality and subsequent discrimination. As we have learned over the past five years, it is no longer possible to credibly guarantee that anonymized or de-identified samples and data will remain de-identified in large data repositories [5-7]. The increased technical capacity to reidentify individuals in databases can be addressed in a number of ways: (1) we can clamp down on sharing; (2) we can merely be transparent about the risks during the informed consent process and allow those individuals willing to assume the risks to do so [8]; or (3) we can shift our attention to increasing penalties for re-identification and misuse of identifiable data [9]. Limiting use would be an unfortunate and ill-considered outcome, reducing research and medical benefits to society and foiling the intentions of many individual contributors who are, after all, providing samples and data to further science and clinical innovation. Transparency and penalties for misuse may be necessary to address the increased risk of re-identification, but they are not sufficient. Here, we suggest that, where technological capacity exists, technological advances can help the research enterprise achieve a more thoroughgoing respect for persons than was possible when current policies governing human subject research were developed. Further, by restricting access to data and failing to recognize that some individuals may exercise their autonomy by enabling use of their genomic and personal data, researchers and regulators hobble science and fail to truly honor the notion of respect for persons that underlies the entire enterprise. That said, questions remain about the best way to revise policy to accommodate the changed landscape.

\section{Background}

Concerns about the ethical use of human genomic and other personal data in prospective cohort studies are longstanding [10]. However, the increased use of next-generation sequencing in research reanimates three challenges on an unprecedented scale. First, next-generation sequencing can generate data from every known disease-associated gene or DNA sample. As more is learned about the contribution of genomic factors to disease risk, an individual genome sequence will acquire new meaning to the person from whom it originated and will contribute to the interpretation of others' genomes.

Second, next-generation sequencing has co-evolved with powerful computing infrastructures for analyzing and exchanging enormous volumes of personal data. To facilitate the efficient use of resources, there has been a growing tendency to establish large databases and open-access policies to store and share human genomic and other research data. This trend favors the "emergence" of many hypotheses from large datasets long after a participant's initial informed consent to research, and facilitates the re-use and combining of datasets by multiple researchers. As a result, secondary and tertiary data users may be far removed from the original context in which research data were obtained, blurring the lines of accountability for responsible data use.

Third, it has become easier to re-identify individual contributors to databases based on publicly-available internet data, as the latter has grown more abundant [5-7]. Consequently, the 
privacy risks associated with contributing biospecimens and genomic data to research must now be assessed broadly, rather than in relation to the activities of any one project.

A current challenge facing policymakers is to develop standards for using not only archived tissues samples and data, but also newly generated genomic information in research to benefit society while respecting heterogeneous beliefs about privacy [11-14] and while safeguarding research participants from uncertain risks. This dilemma is often framed as a tension between serving individual autonomy interests by keeping data confidential on the one hand, and advancing public beneficence by sharing data liberally on the other. However, this polarized view may be oversimplified. Internet users have increasingly come to use social media-blogs, Facebook, Twitter, wikis, forums - to become content creators and sharers in their own right. While norms are still evolving, information technology (IT) has facilitated a shift in the relationship between individuals and their personal data, enabling increased individual control over how (and how much) personal data are used in research, and by whom. This shift in control has created new opportunities to engage members of the public as partners in the research enterprise on more equal and transparent terms. Conceptions of privacy-including what should remain private and what privacy means in various online spaces - and risks of breaching confidentiality are changing even as genomic data are accumulating rapidly.

\section{The Rationale for Informed Consent}

An ethical duty to secure the autonomous and voluntary informed consent of human research subjects emerged in response to specific and grave concerns - about physical harm, discrimination, stigma - that arose from inhumane and coercive research practices in the U.S., Europe and elsewhere during the 20th century $[15,16]$. Today, to uphold the bioethical principle of respect for persons, the United States Federal Policy for the Protection of Human Subjects ("The Common Rule") requires investigators to obtain informed consent from prospective research subjects before collecting or using their individually identifiable biological materials or data in research studies [17]. The doctrine of informed consent was conceived to ensure respect for persons as autonomous agents in clinical care and research. Motivated to prevent further unethical research practices, the U.S. National Research Act of 1974 both mandated Institutional Review Board (IRB) review for research and convened a National Commission for the Protection of Human Subjects of Biomedical and Behavioral Research, which produced The Belmont Report, the foundation of much of the Common Rule.

The Belmont Report identifies three ethical principles: respect for persons, beneficence, and justice, which are paired with three corresponding means of translating principle into action: informed consent, assessing risks and benefits, and fair selection of subjects. The original Belmont concept of "autonomy" embedded in respect for persons is elaborated as follows:

An autonomous person is an individual capable of deliberation about personal goals and of acting under the direction of such deliberation. To respect autonomy is to give weight to an autonomous person's considered opinions and choices while refraining from obstructing their actions unless they are clearly detrimental to others. To show lack of respect for an autonomous 
agent is to repudiate that person's considered judgments or to withhold information necessary to make a considered judgment, when there are no compelling reasons to do so [18] [underlining added].

The Belmont Report formed the basis of the first formal research regulations adopted by the Department of Health and Human Services (HHS) in 1981, only slightly modified in the currently prevailing Common Rule.

\section{The Changing Research Landscape}

It is widely agreed that since the adoption of the Common Rule, the advent of genomic research has changed the research landscape, as have its risks and benefits, as a result of technological advances that make it cheaper and easier to generate, analyze, and share large volumes of data [19,20]. Just as significant, many technological advances in the same period have diversified the tools available to mitigate or offset the risks facing contributors to genomic research.

\subsection{The Shifting Relationship between Identifiability and Ethics Review}

Historically, the risks of genetic and genomic research have been mitigated by nondisclosure (e.g., of non-paternity), and sample and data anonymization or de-identification. Stripping identifiers or severing links between tissues and tissue donors were, justifiably, seen as effective measures to mitigate risks to individuals' privacy interests, by restricting access to their personal information. Yet privacy is a complex, variably defined concept encompassing a plurality of related issues; informational secrecy is merely one of its dimensions. Further, the practice of respecting privacy by restricting access to individual information undermines the pursuit of public benefit through aggregation of large amounts of personal data in research databases, and may not actually align with research subjects' values [21,22].

The concerns addressed by restricting access to personal information include threats to valued social and economic opportunities as a result of privacy breaches and threats to individual autonomy, including risk of social stigma and unwanted scrutiny, making it harder to exercise basic liberties in the course of daily life [23]. Further, some individuals simply do not want others (e.g., researchers) to know information about them that they do not know themselves, or that they do not wish to know about themselves.

The moral case for gaining access to personal information also varies. In science, the argument is often made that such access will advance scientific knowledge, leading to improved healthcare and other societal benefits [24,25]. Justifying the use of personal information to achieve ends like these is difficult when the contribution of individual information to these outcomes is unclear, and even harder when not all parties involved are in agreement about the desirability of the ends. The various interests protected and hindered by confidentiality provisions make it impossible to arrive at a consensus risk-benefit profile for a pool of research subjects that can be assessed each time personal information is transferred from one holder to another.

Given the choice, some individuals might decline to make their personally identifiable health information available to researchers; others might elect to share their data to enable scientists to develop new treatments, to help advance biomedical science, or to forge connections to other 
individuals with common diagnoses or health concerns; still others might choose to share with academic but not commercial researchers, or with breast cancer researchers, but not those who study psychiatric disease. Whether a person is motivated to enroll in research by personal history of illness, intellectual curiosity, or feelings of altruism or social responsibility, the tradeoffs involved in contributing personal information to a biorepository are dynamic and variable over time, and contributors' values and goals are diverse. Current policy that uniformly restricts access to data as a form of privacy protection both fails to respect those participants who would wish to have and share their data freely and limits the potential benefits to science and society that may accrue from the use of those data.

In recent years, it has become increasingly possible to re-identify individual data contributors to large electronic datasets [5-7]. This is significant because under the regulatory status quo, full ethics review is primarily reserved for projects using personal data considered "identifiable" under the Common Rule, meaning that the identity of the subject can be "readily ascertained" by the investigator from the information. Informed consent is not typically sought from individuals before their "de-identified" data are used in research. In human genomics, this policy is problematic due to the inherent identifiability of human sequence data and the need sometimes to interpret these data in the context of detailed phenotypic information.

The prevailing notion that investigators can balance the risk-benefit profile of genomic research by divorcing data from individual identifiers is also problematic because de-identification may actually impoverish the quality of research data to an extent that undermines scientific progress. De-identification might also preclude the return of individual research results to participants in instances when such results have implications for their well-being. Further, de-identification denies participants the opportunity to exercise their autonomy by managing the use of their data over time, as their circumstances and views change. From an individual's perspective, the foreclosure of these benefits and limitations on their autonomy might actually worsen the risk-benefit profile of participating in research.

\subsection{Growth of Online Data-Sharing}

Simultaneous with the emergence of next-gen sequencing technologies, there has been a profound shift in the nature of online information sharing in the course of daily life. Today's Internet contains vast quantities of user-volunteered, identifiable data disclosed for purposes as varied as commercial exchange, social networking, recreational gaming, and health support and promotion. Facebook, Pinterest, patient discussion boards, posted Fitbit reports and myriad other forms of Internet sharing have changed what, how and with whom we share. In many online healthrelated communities, members develop and test their own hypotheses, assuming roles typically reserved for "experts", and operating outside traditional human subjects protections frameworks (see Section 5.4 below). Further, some have begun to advocate not for the ability to keep one's data private, but rather for the ability to have and to share one's data freely [26]. Such calls for the freedom to share reflect the oft-ignored feature of autonomy as defined in the Belmont Report, respect for individuals' ability to pursue their interests so long as they do not harm others (see underling above). 
Norms of information exchange are also changing. When investigators and institutions are trusted, research participants tend not to mind contributing identifiable data to multiple research projects provided that they are kept informed about the nature of the research to which they are contributing [27,28]. Furthermore, several studies have shown that individual concerns about privacy are highly variable and seem to be affected by the tradeoffs that individuals make among three considerations: their privacy concerns, their perceptions of the utility of study participation, and the degree of reciprocity they perceive from investigators using their data $[29,30]$.

Taken together - the limitations of informed consent, the growing ease of re-identifying donors and the value of donor-associated data, the proliferation of new IT platforms, and evidence for a so-called "privacy-utility tradeoff" made by research participants - these new realities suggest it is time to revise how we configure an ethical relationship between donors and users of genomic research data. If we wish to uphold the notion of respect for persons on which we base human research subject protections, we must both "give weight to an autonomous person's considered opinions and choices" and refrain "from obstructing their actions unless they are clearly detrimental to others." Limiting autonomy by restricting individuals' access to and sharing of their own data, or ability to modify their preferences regarding data use over time fails to uphold the second requirement of respect for persons.

\section{Application of IT to Both Research and Research Subject Protections}

The importance of trust and reciprocity to research participation suggests that revising the relationship between donors and users toward a more collaborative model might also encourage and support participation in genomic research, to the potential benefit of both parties and society as a whole. Many argue that research subjects must become more active partners in the research process itself: true participants, rather than mere subjects [10-12]. To realize this aim, and achieve the hoped for trust and reciprocity, new digital systems for collecting and curating research data (including genomic data) have been developed by innovators in both the for-profit and non-profit sectors. Below, we describe a heterogeneous group of evolving new approaches to collecting and using biospecimens and genomic data in research. Given their novelty and continuing evolution, it is not our aim to classify them prematurely or draw a false equivalence among them. Our goals are to draw attention to the innovative ways these approaches re-imagine the relationship between research participants and researchers, and to highlight some of the empirical questions that must be addressed, as we attempt to evaluate the ethical implications of the new research models.

\subsection{The Personal Genome Project and Open Consent}

The Harvard-based Personal Genome Project (PGP) [31] has abandoned the notion that de-identification of genomic research data and samples is plausible or even desirable, privileging the values of "veracity" and reciprocity in the conduct of research [32]. The PGP is a longitudinal genome research study enrolling participants through a detailed, web-based informed consent process (including a mandatory genetics exam) that secures "open consent" from participants for ongoing research use of their individual genomic and phenotypic data. PGP participants are free to 
upload as little or as much personal information as they wish to their online PGP profiles, within its defined parameters. Although these profiles do not display names, the PGP makes no promises that data contributed to the project will remain de-identified or anonymous. In return for assuming the risks of re-identification, the PGP offers participants individual research data and hosts an annual research meeting to which participants are invited, demonstrating the PGP's belief that reciprocity may play an important role in earning and securing the trust of their study participants.

\subsection{Portable Legal Consent}

The Portable Legal Consent (PLC), developed by the Consent to Research project, is designed to address the challenges of broad data sharing. The PLC gives participants who wish to donate data to research the opportunity to attach a single research consent to their health and genetic data, which they then upload to a secure website. These data can then be used for research purposes by any researcher who agrees to specific terms of data use including: an intent to publish research results in an open-access forum, a promise not to attempt to re-identify individual research participants, and a promise not to distribute data among third parties who do not agree to the PLC conditions. While participants may withdraw their data from the database at any time, they are clearly advised that once data are uploaded, it may not be possible to remove them from all sources (for example, from researchers who have already downloaded, shared, or used the data).

\subsection{Registry for All Disease ("Reg4All”)}

In 2012, the umbrella disease advocacy organization Genetic Alliance created Reg4All [33] to collect information relevant to many health conditions. Using a "dynamic consent" platform, Reg4All participants select fine-grained consent rules to determine how their personal data are viewed, by whom, and for what purposes. The system's privacy settings include "deny the use of my data in any form for any purpose"; "allow discovery and retrieval of all of my data in the registry", and "make my data available to ONLY this research project". Preferences also allow varying degrees of contact between registry participants and investigators interested in using their data. Participants may make their data available to specific clinical trials and research studies, or they may allow their data to be used openly by all. For each decision about data use, a participant may choose to give consent, deny consent, or postpone the decision until later. A participant may choose to enter their preferences once and retain them, or they may choose to change their choices at a later date. The overall vision of Reg4All is to re-imagine the researcher-participant relationship as a reciprocal collaboration over time.

\section{4. "Apomediated", Peer-Produced Research}

The term "apomediation" describes the relatively non-hierarchical nature of information-sharing in some research communities [34,35]. Apomediated initiatives create virtual spaces in which individuals are encouraged to propose and carry out their own research studies using self-reported data. Examples include PatientsLikeMe (PLM), which provides self-tracking and social networking tools to its over 220,000 users in exchange for permission to share their data with researchers listed 
on the PLM website. Since 2012, PLM's peer-reviewed publications have covered measures of functional disability in multiple sclerosis, epilepsy care quality, and Parkinson's disease progression [36-38]. Other initiatives include DIYGenomics, which has hosted a crowd-sourced study of the relationship between polymorphisms in the Methylenetetrahydrofolate reductase (MTHFR) gene, homocysteine levels, and vitamin B deficiency, and Genomera, which in beta version allows members of online communities to initiate studies related to nutrition, sleep patterns, exercise, and genome variation [39].

\section{Open Questions}

The ability of IT and social media to change how genomic and other health data are shared and interpreted has generated excitement among health-oriented constituencies. Advocacy organizations have embraced social media's role in helping patients become more engaged in their own healthcare and in research [40-42]. That said, using social media to share personal information raises its own ethical issues, and robust, longitudinal studies examining the effectiveness or safety of using social media to manage health information are needed. Some question whether existing initiatives are as "participant-centric" as they claim, given that commercial incentives may generate conflicts of interest in some cases [43]. One obvious concern is that personal information may be acquired surreptitiously or abused [44]. Another concern is that "gamified" survey data may not always be contributed voluntarily by users, given the compulsive nature of some forms of internet gaming [45]. Yet other concerns focus on financial motivations of the entities controlling the data - will participant and researcher incentives always stay in alignment [43]?

Thus far, we have few data on basic questions about these new models for doing research, such as: do granular data-sharing choices unduly hinder or bias the collection of research data? Who, if anyone, is alienated or excluded by systems like those we have described above? It is important to acknowledge that many participants in genomics research will not have ready access to or experience with the kinds of technologies we discuss here-will variation in access to technology lead to or exacerbate existing disparities between different research populations? Which, and how many, data-sharing options are necessary to secure autonomous and respectful research participation? What happens when study participants assume roles traditionally held by researchers?

Interactive websites have been demonstrated to be effective at educating the public about genomics, and individual data-sharing attitudes have been found to be highly nuanced and variable. We believe that the approaches highlighted above are promising strategies for managing many of the challenges of modern genomic research, while fostering autonomy. However, to realize their full potential, they must be developed in parallel with empirical studies of their benefits and harms, both intended and unintended.

\section{Conclusions}

Current informed consent practices are unequal to the task of upholding authentic respect for persons in contemporary genomic research. New models that take advantage of advances in both genomic research and IT promise to address this shortfall, but require further study of their associated 
benefits and harms. Careful study will be necessary to guide the evolution of these new models, and to ensure that research both adequately balances protections and benefits against the burdens and uncertainties borne by participants in genomic studies, and does not unnecessarily limit participants' actions.

Prior work in bioethics has addressed privacy concerns narrowly, by focusing on privacy as a strict function of identifiability or a form of informational secrecy [46-48]. This focus misses other broad interests individuals may have in sharing their own health and genomic data and information. The conception of privacy as informational secrecy lends itself to a view of genomic information-sharing as a false dichotomy, in which information is either wholly private or wholly public. By restricting access to data and failing to recognize that some individuals may exercise their autonomy by enabling use of their genomic and personal data, researchers and regulators hobble science and fail to truly honor the notion of respect for persons that underlies the entire enterprise.

The scientific, bioethics, and research oversight communities frequently frame the debate as privacy versus public beneficence and equate respect for persons with informed consent. Such norms and practices impede meaningful reform of human subjects protections. Further, we lack the empirical evidence necessary to evaluate emerging models of engaging with research subjects and participants that more fully embody the original concept of respect for persons. The research enterprise as a whole must accommodate the cultural shift that is taking place in the relationship between individuals and their health information. Appreciating and understanding this transformation will be an indispensible step in adapting ethical guidelines to the realities of modern information use and patients who want and expect to be true participants in research.

\section{Acknowledgments}

The authors would like to thank Robert Cook-Deegan for his helpful comments on an earlier version of this manuscript.

\section{Author Contributions}

Conception and design: DJHM, LJ. Wrote the paper: DJHM, LJ.

\section{Conflicts of Interest}

The authors declare no conflict of interest.

\section{References}

1. Pasche, B.; Absher, D. Whole-genome sequencing: A step closer to personalized medicine. JAMA 2011, 305, 1596-1597.

2. Greenbaum, D.; Sboner, A.; Mu, X.J.; Gerstein, M. Genomics and privacy: Implications of the new reality of closed data for the field. PLoS Comput. Biol. 2011, 7, E1002278.

3. Hayden, E.C. Geneticists push for global data-sharing. Nature 2013, 498, 16-17. 
4. Draft NIH Genomic Data Sharing Policy Request for Public Comments; 2013. Available online: http://www.bioethics.net/2013/10/nih-requests-comment-on-genomic-data-sharingpolicy-draft/ (accessed on 29 December 2013).

5. Gymrek, M.; McGuire, A.L.; Golan, D.; Halperin, E.; Erlich, Y. Identifying personal genomes by surname inference. Science 2013, 339, 321-324.

6. Homer, N.; Szelinger, S.; Redman, M.; Duggan, D.; Tembe, W.; Muehling, J.; Pearson, J.V.; Stephan, D.A.; Nelson, S.F.; Craig, D.W. Resolving individuals contributing trace amounts of DNA to highly complex mixtures using high-density SNP genotyping microarrays. PLoS Genet. 2008, 4, E1000167.

7. Sweeney, L.A.A.; Winn, J. Identifying Participants in the Personal Genome Project by Name; Data Privacy Lab, Harvard University: Cambridge, MA, USA, 2013.

8. Lunshof, J.E.; Chadwick, R.; Vorhaus, D.B.; Church, G.M. From genetic privacy to open consent. Nat. Rev. Genet. 2008, 9, 406-411.

9. Bambauer, J.Y. Tragedy of the data commons. Harv. J. Law Technol. 2011, 25, doi:10.2139/ ssrn.1789749.

10. Geller, L.N.; Alper, J.S.; Billings, P.R.; Barash, C.I.; Beckwith, J.; Natowicz, M.R. Individual, family, and societal dimensions of genetic discrimination: A case study analysis. Sci. Eng. Ethics 1996, 2, 71-88.

11. McGuire, A.L.; Caulfield, T.; Cho, M.K. Research ethics and the challenge of whole-genome sequencing. Nat. Rev. Genet. 2008, 9, 152-156.

12. Kaye, J. The tension between data sharing and the protection of privacy in genomics research. Annu. Rev. Genomics Hum. Genet. 2012, 13, 415-431.

13. Privacy and Progress in Whole Genome Sequencing; President's Commission for the Study of Bioethical Issues: Washington, DC, USA, 2012.

14. Knoppers, B.M.; Dove, E.S.; Litton, J.E.; Nietfeld, J.J. Questioning the limits of genomic privacy. Am. J. Hum. Genet. 2012, 91, 577-578.

15. Rice, T.W. The historical, ethical, and legal background of human-subjects research. Respir. Care 2008, 53, 1325-1329.

16. Menikoff, J. Research involving human subjects: Ethical and regulatory issues. Handb. Clin. Neurol. 2013, 118, 289-299.

17. Code of Federal Regulations Title 45, Section 46. In Federal Policy for the Protection of Human Subjects; U.S. Department of Health \& Human Services: Washington, DC, USA, 2008.

18. The National Commission for the Protection of Human Subjects of Biomedical and Behavioral Research. The Belmont Report: Ethical Principles and Guidelines for the Protection of Human Subjects of Research; Department of Health, Education, and Welfare: Washington, DC, USA, 1979.

19. Meslin, E.M.; Cho, M.K. Research ethics in the era of personalized medicine: Updating science's contract with society. Public Health Genomics 2010, 13, 378-384.

20. Bunnik, E.M.; de Jong, A.; Nijsingh, N.; de Wert, G.M. The new genetics and informed consent: Differentiating choice to preserve autonomy. Bioethics 2013, 27, 348-355. 
21. Mello, M.M.; Wolf, L.E. The havasupai Indian tribe case-Lessons for research involving stored biologic samples. NEJM 2010, 363, 204-207.

22. McGuire, A.L.; Oliver, J.M.; Slashinski, M.J.; Graves, J.L.; Wang, T.; Kelly, P.A.; Fisher, W.; Lau, C.C.; Goss, J.; Okcu, M.; et al. To share or not to share: A randomized trial of consent for data sharing in genome research. Genet. Med. 2011, 13, 948-955.

23. Powers, M. Justice and Genetics: Privacy protection and the Moral Basis of Public Policy. In Genetic Secrets: Protecting Privacy and Confidentiality in the Genetic Era; Rothstein, M.A., Ed.; Yale University Press: New Haven, CT, USA, 1997; pp. 355-368.

24. Sharing Clinical Research Data: Workshop Summary; The National Academy of Sciences: Washington, DC, USA, 2013.

25. Terry, S.F.; Shelton, R.; Biggers, G.; Baker, D.; Edwards, K. The haystack is made of needles. Genet. Test. Mol. Biomark. 2013, 17, 175-177.

26. Terry, S.F.; Terry, P.F. Power to the people: Participant ownership of clinical trial data. Sci. Transl. Med. 2011, doi:10.1126/scitranslmed.3001857.

27. Ludman, E.J.; Fullerton, S.M.; Spangler, L.; Trinidad, S.B.; Fujii, M.M.; Jarvik, G.P.; Larson, E.B.; Burke, W. Glad you asked: Participants' opinions of re-consent for dbGaP data submission. JERHRE 2010, 5, 9-16.

28. Lemke, A.A.; Wolf, W.A.; Hebert-Beirne, J.; Smith, M.E. Public and biobank participant attitudes toward genetic research participation and data sharing. Public Health Genomics 2010, $13,368-377$.

29. Oliver, J.M.; Slashinski, M.J.; Wang, T.; Kelly, P.A.; Hilsenbeck, S.G.; McGuire, A.L. Balancing the risks and benefits of genomic data sharing: Genome research participants' perspectives. Public Health Genomics 2012, 15, 106-114.

30. Hobbs, A.; Starkbaum, J.; Gottweis, U.; Wichmann, H.E.; Gottweis, H. The privacy-reciprocity connection in biobanking: Comparing german with UK Strategies. Public Health Genomics 2012, 15, 272-284.

31. Personal Genome Project. Available online: http://www.personalgenomes.org/ (accessed on 17 January 2014).

32. Angrist, M. Eyes wide open: The personal genome project, citizen science and veracity in informed consent. Per. Med. 2009, 6, 691-699.

33. Reg4All. Available online: https://reg4all.org/ (accessed on 17 January 2014).

34. Eysenbach, G. Medicine 2.0: Social networking, collaboration, participation, apomediation, and openness. J. Med. Int. Res. 2008, 10, E22.

35. O'Connor, D. The apomediated world: Regulating research when social media has changed research. J. Law Med. Ethics 2013, 41, 470-483.

36. Wicks, P.; Fountain, N.B. Patient assessment of physician performance of epilepsy quality-of-care measures. Neurol. Clin. Prac. 2012, 2, 335-342.

37. Wicks, P.; Vaughan, T.E.; Massagli, M.P. The multiple sclerosis rating scale, revised (Msrs-R): Development, refinement, and psychometric validation using an online community. Health Qual. Life Outcomes 2012, doi:10.1186/1477-7525-10-70. 
38. Little, M.; Wicks, P.; Vaughan, T.; Pentland, A. Quantifying short-term dynamics of parkinson's disease using self-reported symptom data from an internet social network. J. Med. Int. Res. 2013, 15, E20.

39. Swan, M. Crowdsourced health research studies: An important emerging complement to clinical trials in the public health research ecosystem. J. Med. Int. Res. 2012, 14, E46.

40. Inspire. Available online: http://www.inspire.com/ (accessed on 31 October 2013).

41. Trialx. Available online: http://trialx.com/ (accessed on 5 November 2013).

42. Army of women. Available online: http://www.armyofwomen.org/ (accessed on 5 November 2013).

43. Vayena, E.; Tasioulas, J. Adapting standards: Ethical oversight of participant-led health research. PLoS Med. 2013, 10, E1001402.

44. Vayena, E.; Mastroianni, A.; Kahn, J. Caught in the web: Informed consent for online health research. Sci. Transl. Med. 2013, 5, $173 \mathrm{fs} 176$.

45. Foddy, B. The Ethics of Gamification: Little Rewards for Everything. Available online: http://blog.practicalethics.ox.ac.uk/2011/02/the-ethics-of-gamification-little-rewards-for-everything/ (accessed on 29 December 2013).

46. Gutmann, A.; Wagner, J.W. Found your DNA on the web: Reconciling privacy and progress. Hastings Center Rep. 2013, 43, 15-18.

47. Roche, P.; Glantz, L.H.; Annas, G.J. The genetic privacy act: A proposal for national legislation. Am. Bar Assoc. 1996, 37, 1-11.

48. Rothstein, M.A. Is deidentification sufficient to protect health privacy in research? Am. J. Bioeth. 2010, 10, 3-11. 


\title{
Genetics of Charcot-Marie-Tooth (CMT) Disease within the Frame of the Human Genome Project Success
}

\author{
Vincent Timmerman, Alleene V. Strickland and Stephan Züchner
}

\begin{abstract}
Charcot-Marie-Tooth (CMT) neuropathies comprise a group of monogenic disorders affecting the peripheral nervous system. CMT is characterized by a clinically and genetically heterogeneous group of neuropathies, involving all types of Mendelian inheritance patterns. Over 1000 different mutations have been discovered in 80 disease-associated genes. Genetic research of CMT has pioneered the discovery of genomic disorders and aided in understanding the effects of copy number variation and the mechanisms of genomic rearrangements. CMT genetic study also unraveled common pathomechanisms for peripheral nerve degeneration, elucidated gene networks, and initiated the development of therapeutic approaches. The reference genome, which became available thanks to the Human Genome Project, and the development of next generation sequencing tools, considerably accelerated gene and mutation discoveries. In fact, the first clinical whole genome sequence was reported in a patient with CMT. Here we review the history of CMT gene discoveries, starting with technologies from the early days in human genetics through the high-throughput application of modern DNA analyses. We highlight the most relevant examples of CMT genes and mutation mechanisms, some of which provide promising treatment strategies. Finally, we propose future initiatives to accelerate diagnosis of CMT patients through new ways of sharing large datasets and genetic variants, and at ever diminishing costs.
\end{abstract}

Reprinted from Genes. Cite as: Timmerman, V.; Strickland, A.V.; Züchner, S. Genetics of Charcot-Marie-Tooth (CMT) Disease within the Frame of the Human Genome Project Success. Genes 2014, 5, 13-32.

\section{Introduction}

Charcot-Marie-Tooth (CMT) disease was so named to acknowledge J.M. Charcot, P. Marie, and H.H. Tooth, who originally described this inherited peripheral neuropathy in the 19th century $[1,2]$. CMT occurs worldwide with an estimated prevalence of $1 / 2,500$. CMT is a neuromuscular disorder characterized by progressive and length-dependent degeneration of peripheral nerves resulting in muscle weakness and wasting in distal limbs, feet and hands. Onset varies from childhood to late adulthood and clinical severity ranges from mild to severe between patients. The neurophysiological and neuropathological defects in the motor and/or sensory nerves create foot deformities, walking disabilities, wheelchair dependence, and sensory deficits. Over the years, clinical and genetic studies have demonstrated that CMT is extremely heterogeneous. A classification was proposed in the 1970s aiming to group the most common CMT variants as hereditary motor and sensory neuropathies (HMSN). In CMT type 1 the myelinating Schwann cells are affected, while axons are degenerated in CMT2. Besides these two autosomal dominant inherited CMT types, recessive and X-linked demyelinating and axonal CMT subtypes have been described and also included in the HMSN classification [3]. Depending on the severity of motor or sensory deficiency, other CMT 
variants were grouped into predominantly distal hereditary motor neuropathies (distal HMN) and hereditary sensory and autonomic neuropathies (HSAN) [4,5]. More recently, clinical and genetic overlaps have been reported between CMT neuropathies and hereditary spastic paraplegias. In addition, there have been cases with more complex clinical phenotypes involving other tissues, such as skin and bone (reviews by [6-8]), further complicating the original CMT classification.

Figure 1. Genes and loci for Charcot-Marie-Tooth (CMT) and related inherited peripheral neuropathies.

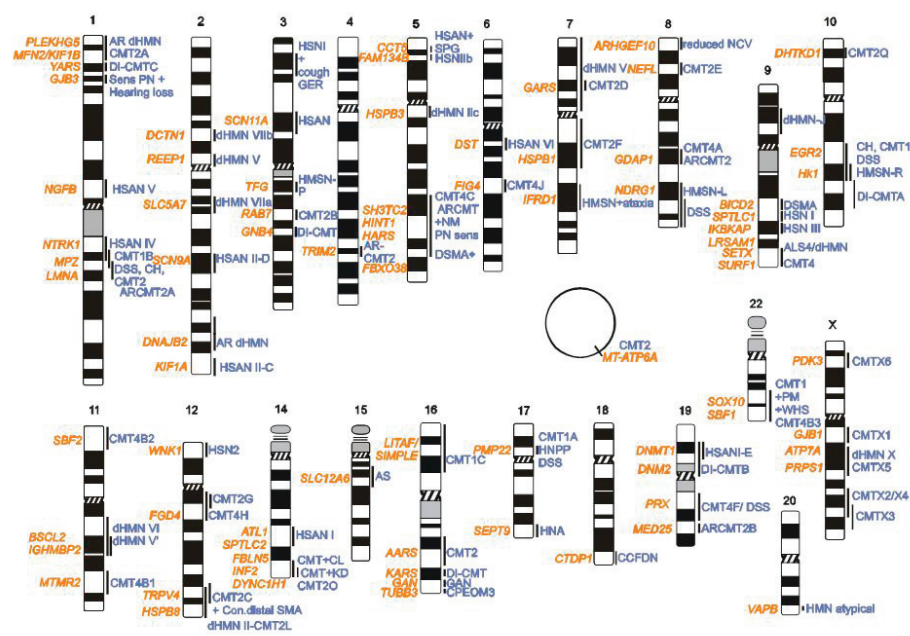

The figure shows 80 currently known genes (orange symbols) and their corresponding chromosomal loci (vertical bars). The corresponding phenotypes are indicated by blue symbols and are according to the disease nomenclature. Note that the disease names may not always correspond to information available in OMIM, GeneReviews, or in other publicly available databases. The full names of the gene symbols and year of gene identification are provided in Supplementary Table S1.

The first CMT locus was mapped in 1982 [9], and 30 years of genetic research has not only allowed the successful identification of 80 disease-causing genes, but also pioneered the discovery of novel genomic mechanisms (Figure 1). Loci and genes for CMT and related peripheral neuropathies were initially identified using genetic linkage studies, positional cloning, or candidate gene approaches. Since the publication of the Human Genome in 2001 [10,11], the development of high-throughput technologies, such as whole genome mapping (WGM), whole genome sequencing (WGS), and whole exome sequencing (WES) $[12,13]$ accelerated the gene and mutation discovery in CMT research. Genetic research in CMT has shown that all Mendelian inheritance patterns are possible. However, besides dominant, recessive, and X-linked inherited CMT types, mutations also occur de novo in isolated patients. More recently, a CMT phenotype was associated with a defect in MT-ATP6A, a gene encoded by the mitochondrial DNA [14]. Different CMT phenotypes can be caused by mutations in the same gene, and conversely mutations in different genes may result in the same phenotype. This is further complicated by the fact that some mutations are extremely rare and occur in specific subtypes. Mutations in more than 20 genes cause primary alterations of the 
myelin sheath; well-known examples include $M P Z, P M P 22$, and GJB1. Mutations in genes with axonal functions, however, result in axonal CMT and associated phenotypes (e.g., NEFL, GAN). Their gene products have cell-type specific functions, allowing underlying disease mechanisms to be logically inferred. Other mutations have been reported to cause intermediate CMT, with both myelin and axonal phenotypes. The availability of the Human Genome also contributed to the identification of mutations in genes that were not the primary functional candidates for CMT neuropathies. Examples include mutations found in ubiquitously expressed genes coding for amino-acyl tRNA synthetases (GARS, YARS, HARS, MARS, AARS), small heat shock proteins (HSPB1, HSPB3, HSPB8) and enzymes involved in membrane and transport metabolism (SPTLC1, $S P T L C 2, M T M R 2, S B F 1, S B F 2$ ), whose resulting gene products have housekeeping functions and pleiotropic activities. In addition, CMT disease-associated genes are expressed in different cellular compartments of the developing and myelinating Schwann cells and/or the neuronal axons [15]. Some of these genes have been shown to function in the nucleus as transcription factors (EGR2, $S O X 10, D N M T 1)$, others in vesicle transport (RAB7A), in the Golgi (FAM134B), endoplasmic reticulum (SPTLC1, REEP1, ATL1), or the mitochondria (MFN2, GDAP1). For most of these genes, it still remains an enigma why the mutant proteins cause such specific, length-dependent degeneration of peripheral nerves in CMT patients (Figure 2).

Figure 2. Functional categories containing enriched molecular and cellular functions of genes involved in CMT and related neuropathies.



Ingenuity Pathway Analysis (IPA version 17199142, Ingenuity Systems [16]) was used to summarize the molecular and cellular functions that were most strongly associated with genes linked to inherited peripheral neuropathies. Detailed gene functions are provided as Supplementary Tables S2. Corresponding $p$-values in the supplementary files were calculated using Fisher's exact test and corrected for multiple testing using the Benjamini-Hochberg method. Note that the same gene can be present in various clusters. 
The tremendous success of molecular genetics between 1990 and 2004 can be attributed to multiplex studies of large CMT families suitable for positional cloning and candidate gene screening. Because such families have become rare, the pace of gene discovery slowed down soon after the publication of the Human Genome (Figure 3). Reflecting the clinical reality, the majority of patients with peripheral neuropathies derive from nuclear families or represent isolated patients with severe phenotypes. Despite their huge potential, these patients and nuclear families were beyond the reach of classical gene discovery approaches. Fortunately, this situation has changed spectacularly with the introduction of novel, affordable sequencing technologies which allow massive, genome-wide analysis of entire exomes (all protein coding regions) or even genomes. We will discuss the history of CMT gene discoveries by providing a few highlights where the Human Genome Project (HGP) contributed to the gene finding. As not all discoveries can be discussed, we provide a comprehensive table listing all currently known disease-causing genes for CMT, as well as the original technologies used to find the associated genes and mutations (Supplementary Table S1). Further details can be obtained from corresponding references to the literature, via the OMIM database [17], IPNMDB database [18], or LOVD database [19], which in part provide a list of mutations and genetic variants.

Figure 3. Historical overview of gene identification in CMT and related inherited peripheral neuropathies.

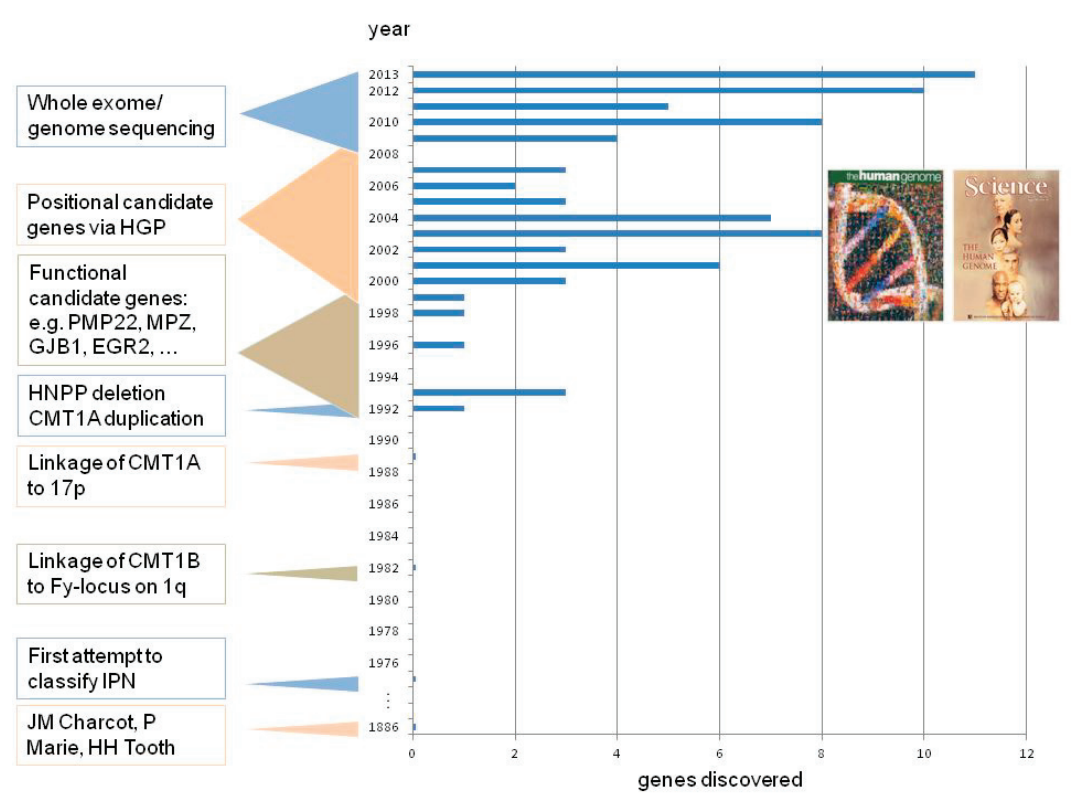

This figure shows the number of genes found per year since the identification of the CMT1A duplication in 1991. Note a peak in gene discovery soon after the publication of the HGP in 2001. A second peak occurs when next generation sequencing (NGS) tools became available from 2009. We also highlighted the introduction of NGS methods (WES and WGS), as well as the major functional and positional CMT genes identified before and after the publication of the HGP. All genes identified per year are listed in Supplementary Table S1. 


\section{CMT Genetics as a Pioneer for Genomic Mechanisms and Emerging Genome Technologies}

\subsection{Early Linkage Studies}

In 1982, T. Bird assigned the first locus for autosomal dominant CMT by screening the Duffy-blood group marker in a family with demyelinating HMSN. He obtained genetic linkage between CMT and the Duffy locus on chromosome 1 [9], which was soon confirmed in other CMT families and defined as the CMT1B subtype (reviewed in [20]). However, the Duffy-blood group marker did not segregate in several other large CMT families which were grouped within the CMT1A subtype of HMSN [21-23]. It took as long as 10 years to find that the $M P Z$ gene coding for the major peripheral myelin protein (P0) was mutated in CMT1B patients [24]. The MPZ gene was assigned to chromosome 1q22-q23 and was the perfect candidate gene for a demyelinating peripheral neuropathy. Further genetic research, based on sequencing the coding region of the $M P Z$ gene, demonstrated that mainly heterozygous, missense mutations occur in CMT1B. Other rare mutations in $M P Z$ were associated with severe and early onset peripheral neuropathies, such as Roussy-Levy syndrome [25], Dejerine-Sottas syndrome [26] and congenital hypomyelination [27]. At least 117 mutations have been described in MPZ [18], and genotype/phenotype correlations associated a few MPZ mutations with axonal HMSN or CMT2 [28,29]. Although CMT patients can be routinely screened for $M P Z$ mutations in DNA diagnostic labs, the CMT1B subtype is less frequent than CMT1A [30,31].

\subsection{CMT1A-The First 'Genomic Disorder'}

The CMT1A subtype is the most common HMSN and one of the first genetic examples of a submicroscopic genomic disorder. The CMT1A locus was assigned, in 1989, to chromosome 17 through genetic linkage studies in large HMSN families using restriction fragment length polymorphic (RFLP) markers [21-23,32]. These families were excluded for linkage to the CMT1B locus on chromosome 1q22-q23. A multipoint linkage study allowed refinement of the CMT1A locus to a $30 \mathrm{cM}$ region on 17p11.2-p12 [33]. In 1991 a tandem-duplication of 1.4 megabases (Mb) on chromosome band $17 \mathrm{p} 12$ was identified as a frequent cause for CMT1A and represented $70 \%$ of CMT1 in many populations [30,31,34,35]. Several molecular methods revealed the CMT1A duplication: the presence of three informative alleles by RFLP analysis and polymorphic dinucleotide $(\mathrm{CA})_{\mathrm{n}}$ repeats in affected individuals, the identification of a patient-specific junction fragment by pulsed-field gel electrophoresis (PFGE), and the duplication of probes detected by fluorescence in situ hybridization (FISH) (reviewed in [36]). A deletion of the same chromosomal region in $17 \mathrm{p} 12$ resulted in a distinct form of inherited peripheral neuropathy, known as hereditary neuropathy with liability to pressure palsy (HNPP) [37]. Through the availability of large insert clones, such as yeast artificial chromosomes (YAC), P1 artificial chromosomes (PAC) and bacterial artificial chromosomes (BAC), it became possible to build clone contigs of chromosome 17. These clones could be further subcloned into smaller fragments, which could then undergo DNA sequencing analysis. This procedure allowed sequencing of $1,421,129 \mathrm{bp}$ of DNA at the CMT1A duplication and HNPP deletion region. Furthermore, this $1.4 \mathrm{Mb}$ chromosomal region was 
found to be flanked by two $24 \mathrm{~kb}$ homologous low copy repeats (LCRs) called the proximal and distal CMT1A-REPs. This unique genomic architecture creates a non-allelic homologous recombination (NAHR) which cause the CMT1A duplication or HNPP deletion. Further analysis of the genomic region nearby the CMT1A-REPs demonstrated an evolutionary mechanism for the formation of the CMT1A-REP and the creation of novel genes by DNA rearrangement [38-40]. The PMP22 gene, encoding the peripheral myelin protein 22 , was physically assigned in the middle of the 1.4-Mb CMT1A region using somatic chromosomal hybrid cell lines, PFGE restriction, and YAC maps [41-44]. As a consequence, one additional copy of PMP22 is responsible for CMT1A, whereas loss of one copy of PMP22 results in HNPP, highlighting a gene dosage effect as the mechanism for these disorders [45]. In addition, some CMT1A and HNPP neuropathy patients have apparent rare copy number variations (CNVs) of an atypical size in the $17 \mathrm{p} 12$ region [46]. More recent, detailed analysis of these genomic rearrangements by high-density, oligonucleotide-based array comparative genomic hybridization $(\mathrm{aCGH})$ and subsequent sequencing of the CMT1A/HNPP breakpoint revealed non-recurrent rearrangements including: non-homologous end joining (NHEJ), Alu-Alu-mediated recombination, and DNA replication-based mechanisms such as fork stalling and template switching (FoSTeS) and microhomology-mediated break-induced replication (MMBIR) [47]. All these studies confirmed that PMP22, either altered by dosage or dysregulation, is the major gene responsible for CMT1A and HNPP. The identification of the CMT1A duplication and reciprocal HNPP deletion on $17 \mathrm{p} 12$ has also shown that rare CNVs involving both coding and non-coding sequences can cause human disorders (reviewed in $[48,49]$ ). Further genetic research in CMT and HNPP resulted in the identification of 61 different point mutations in PMP22.

Some of these PMP22 mutations have been described in naturally occurring mouse mutants (trembler mouse) or have been modeled in transgenic animals [50,51] (reviewed in [52]). Rodent models expressing multiple copies of the PMP22 gene mimic the human CMT1A duplication and have been instrumental in understanding the disease mechanism and developing therapeutic approaches. Anti-progesterone or ascorbic acid (Vitamin C) has been used to alleviate the typical demyelinating neuropathy in CMT1A rat and mouse models respectively [53,54]. Based on this data, multicenter clinical trials with the aim to treat CMT1A duplication patients have been developed for adults and children, but did not reveal significant improvement of the disease symptoms [55-60]. Recently, clinicians and researchers have focused on the natural history of peripheral neuropathies and developed reliable clinical and DNA diagnostic guidelines [61,62]. These internationally accepted guidelines will be important to support other treatment strategies for CMT1A currently under investigation.

\subsection{Genetic and Physical Mapping, and the Contribution of the Human Genome Reference to Gene Finding in CMT}

The HGP has significantly contributed to the identification of genes that were not considered likely candidates for peripheral neuropathies. Here we provide a research example for CMT-related neuropathies where the motor neurons are predominantly affected. The clinical characteristics of this entity, also known as distal hereditary motor neuropathy (HMN), have been defined by A. Harding and P.K. Thomas in 1980 [63]. The identification of the distal HMN genes also started 
through genetic linkage studies in extended families in which the disease was dominantly transmitted (reviewed in [64]). These studies were labor intensive and time consuming due to the limited availability of genetic markers, which were mainly RFLPs analyzed by Southern blotting and hybridization with radioactive labeled probes. Linkage excluded the CMT1A and CMT1B loci on chromosomes 17 and 1 respectively [65]. However, thanks to the discovery of highly polymorphic short tandem repeat (STR) markers and their detection through PCR methods, genome-wide scans (GWS) allowed the identification of one of the first distal HMN loci on chromosome 12. The GWS was performed with a multiplex procedure for genotyping microsatellite markers (referred to as afm-markers) combined with a hybridization-based detection technology [66]. A total of $187(\mathrm{CA})_{\mathrm{n}}$ repeat polymorphisms located on chromosomes 1 to 12 were genotyped at a mean distance of 15 $\mathrm{cM}$. Based on the segregation analysis of STR alleles, the presence of informative recombinants, and multipoint linkage analysis, a candidate region for the distal HMN gene was delineated to a region of $13 \mathrm{cM}$ [67]. Although a large part of chromosome 12 was then assembled into integrated physical, genetic, and cytogenetic maps, the distal part of 12q, including the critical region of the distal HMN locus, was not yet converted into a high-density contig. Attempts to construct a contiguous YAC-based map of the chromosomal region were not successful due to the presence of gaps or chimeric YAC clones. However, the use of PAC and BAC clones, which contained few deletions and were rarely chimeric, allowed the generation of a complete PAC/BAC contig. The PAC and BAC libraries were screened with known STR markers as well as with markers derived from cloned end-fragments of PACs and BACs using STS content mapping, PFGE analysis, Southern blotting, and fiber fluorescence in situ hybridizations (FISH). This final clone contig of 12 q24 allowed mapping candidate genes or expressed sequenced tags (ESTs) within the critical distal HMN region [68]. The combination of genetic linkage studies (including haplotype analysis of polymorphic markers and the identification of recombinants) and the availability of the clone contig allowed further reduction of the locus from 5 to $1.7 \mathrm{Mb}$. From this refined region, known genes were selected from the HGP data $[10,11]$ for Sanger sequencing using one of the first ABI automated DNA sequencing machines. In two large distal HMN families, a missense mutation $(\mathrm{K} 141 \mathrm{~N})$ was found in the HSPB8 gene coding for the $22-\mathrm{kDa}$ small heat shock protein B8 (HSP22/HSPB8). In two other distal HMN families, another mutation targeted the same lysine residue (K141E) in the HSPB8 protein [69]. Interestingly, a very similar strategy resulted in the identification of another CMT locus on chromosome 7q11-q21 (CMT2F) in a large family with autosomal dominant axonal CMT [70]. A missense mutation (S135F) in the HSPB1 gene encoding the 27-kDa small heat-shock protein B1 (HSP27/HSPB1) segregated in this CMT2F family. Screening for HSPB1 mutations in additional CMT and HMN families confirmed the previously observed mutation and identified several additional missense mutations [71]. Both small heat shock proteins act as molecular chaperones but are also involved in many essential cellular processes such as apoptosis, autophagy, splicing, cytoskeleton dynamics, and neuronal survival (reviewed by [72]). Transgenic mouse models for mutant $H S P B 1$ have been created, which develop neurological symptoms similar to the human condition. Alpha-tubulin is less acetylated in sciatic nerves of mutant HSPBI mice when compared to wild type animals, and treatment with HDAC inhibitors (which avoids deacetylation of tubulin), ameliorated the axonal degeneration in the HSPBI mutant 
mouse [73]. Studies aimed at developing better treatment strategies for this group of axonal CMT are being tested in cell and animal models.

\subsection{CMT2A-The Importance of a Finished Human Genome Reference}

The chromosomal locus for the first axonal form of CMT was mapped to chromosome 1p36 in 1993 [74]. Despite a sizable number of mapped, large families and efforts to identify the underlying gene at multiple laboratories, no gene was discovered for over 10 years. What complicated the search was an incomplete map for chromosome 1. Within the established linkage region existed a gap of unknown size and content. In 2001, an elegant study involving cell and mouse models of Kiflb showed mitochondrial transport deficiencies due to loss-of-function mutations [75]. A single small CMT2 family from Japan with suggestive linkage to 1p35-36 [74] was reported to carry a specific Q98L missense mutation that showed functional deficits in a cell culture-based assay. The fact that a mutation in the motor protein Kiflb can underlie a peripheral neuropathy led to the conclusion that KIFIB is the long sought after CMT2A gene [75]. Subsequent mutation screening studies of linked CMT2A families, however, came back empty-handed. To our knowledge, no additional KIFIB mutations have been reported in the literature. This raised the possibility of a second gene in the region. With steadily improving genomic maps of chromosome 1 , an international collaboration eventually identified mutations in the gene coding for MFN2 in all previously linked CMT2A families [76]. MFN2 is now established as the most common CMT2 gene accounting for $\sim 20 \%$ of all axonal cases. Amongst the most severe and early onset forms of CMT2, MFN2 carries a mutation in $\sim 90 \%$ of cases [77]. The MFN2 screening has also revealed a broader phenotypic spectrum that includes early- and late-onset cases of HMSN [78], severe and mild manifestation of symptoms [79,80] accompanying optic atrophy (HMSN VI) [81], and involvement of upper motor neurons (HMSN V) [82]. Rarely, a recessive/co-dominant trait is possible in MFN2 [77,83].

\section{Next Generation Sequencing Boosted the Identification of CMT Associated Genes}

\subsection{Targeted Next-Generation Sequencing and Its Limitation in CMT Gene Finding}

As next generation sequencing (NGS) platforms become more and more accessible and affordable, many CMT laboratories are shifting their research towards smaller families and isolated patients who still represent a large group of genetically unsolved patients. These revolutionary technologies will allow studying isolated patients with severe phenotypes that, until recently, were beyond our reach.

Multiple studies have successfully combined whole-genome SNP genotyping, subsequent target capturing, and parallel sequencing. This approach revealed a single novel missense variant in FBLN5 causing autosomal dominant CMT with hyperelastic skin and age-related macular degeneration [84]. A similar approach, combining whole-genome SNP genotyping, homozygosity mapping and NGS, allowed the identification of mutations in HINT1 causing an autosomal recessive axonal neuropathy with neuromyotonia [85]. Other examples of NGS of CMT genes, with or without additional clinical features, are provided in Supplementary Table S1. As whole 
exome and whole genome studies become more affordable, such targeted studies will not be competitive in the near future. Furthermore, the current limitation of targeted NGS is the lack of complete coverage of some genes and the inability to detect non-exonic mutations and copy number variations (CNVs) [86,87].

Importantly, current diagnostic sequencing of disease genes heavily relies on targeted NGS-based methods. By creating gene panels that includes all known CMT genes, the cost of sequence production can be radically reduced, and for the first time, clinicians will have a comprehensive view of the mutational load in all CMT genes. This technique allows for a much better characterization of genotype/phenotype correlations. It will likely also uncover digenic and other unusual mutational mechanisms. The phenotypic spectra of each CMT gene will be comprehensively defined over the coming decade. At the moment a technical drawback for this approach is the less-than-100\% coverage of a sequence of interest. It is expected, however, that the technology will soon match and outperform traditional Sanger sequencing in sensitivity and specificity.

\subsection{Whole Exome Sequencing as a Successful Approach in CMT Gene Finding}

Whole exome sequencing (WES), aiming to sequence an abbreviated version of the entire genome, has become a powerful and cost-efficient method. CMT research was one of the earliest adaptors of this new technology. Montenegro et al. reported a study of a large CMT pedigree and the identification of a known GJB1 mutation for the X-linked variant of CMT [88]. This was somewhat unexpected, as the family pedigree initially appeared to exclude an X-linked trait. After mutation identification, it appeared that a distant branch of the pedigree with male-to-male transmission was never clinically evaluated and likely carried a different phenotype. This study further detailed the challenges of data interpretation and incompleteness of sequence coverage of coding sequences, as well as possible strategies to resolve these shortcomings of WES. As discussed below, this approach is now well established in many clinical laboratories, only two years later.

One can now analyse whole exomes in trios (patients and their parents) for de novo dominant mutations. Examples of novel genes for CMT and related inherited peripheral neuropathies discovered solely based on WES include MARS [89], BICD2 [90-92], PDK3 [93], SCN11A [94], SLC5A7 [95], and TUBB3 [96] (Supplementary Table S1). The gene discovery rate will increase to as much as one new gene per month in the CMT field alone, until the majority of rare genes have been identified. However, WES may still be hindered by the lack of complete coverage of some genes [86,87]. Regardless, it is widely expected that these new genes will allow for a precise delineation of pathways that are key to the pathogenesis of CMT and related axonopathies.

\subsection{First Whole-Genome Sequencing of a CMT Patient}

The first whole-genome sequence (WGS) of a CMT patient was published in 2010 [97]. This study demonstrated for the first time in all of medicine that WGS can identify clinically relevant variants and provide diagnostic information [98]. The DNA sample of the index patient belonged to a family with recessive CMT and was sequenced on the SOLiD (Sequencing by Oligonucleotide Ligation and Detection) next-generation-sequencing platform developed by Applied Biosystems. 
The accuracy in sequencing of 50-base reads on the SOLiD system exceeded $99 \%$ and 12 multiple sequences were read simultaneously. Overlapping reads increased the overall sequence accuracy and reduced the risk of obtaining false positive sequence variants. In the patient sample a compound heterozygous mutation was identified in $S H 3 T C 2$, a previously known gene for recessive CMT [97,99]. The two mutations in SH3TC2 co-segregated with the CMT disease phenotype in the pedigree. All four affected individuals had slowed nerve conduction velocities, which is indicative of a demyelinating CMT phenotype. Interestingly, the Y169H mutation also seemed to co-segregate with an electrophysiologically defined axonal neuropathy phenotype that was evident in the four affected siblings as well as the proband's father and grandmother. By contrast, the first R954X variant in SH3TC2 was associated with subclinical electrophysiological evidence of carpal tunnel syndrome, regardless of the presence of the second R169H mutation. Although some of the proband's family were shown to have one or the other of these mutations, only the proband and his three siblings, who were also diagnosed with CMT, had both mutations [97]. These observations underline the importance of careful phenotyping for the valid interpretation of genomic variant data. In their study, J.R. Lupski and colleagues [97] identified over 9,000 non-synonymous single nucleotide variants (SNVs), 148 of which involved stop codons, and 112 of which were located in conserved exon splice-sites, which presumably had severe consequences for the affected proteins. Moreover, 21 of these changes were previously described as causing a Mendelian disease other than CMT. Thus, the identification of phenotypically relevant variations by means of WGS can be difficult. At the time, the authors estimated the cost of their study at $\sim \$ 50,000$ - the same study today, three years later, would amount to less than $\$ 10,000$.

\section{Future Perspectives and the Need to Share Large Datasets and Genetic Variants}

In the early days of molecular genetics, access to rare, large families was a prerequisite for linkage studies and positional cloning strategies. With the introduction of WGS and WES, this hurdle has largely been cleared. Today these novel high-throughput technologies allow the simultaneous analysis of approximately 20,000 genes in the human genome in an unbiased way. Besides the tremendous generation of DNA sequence data from complete genomes or exomes, these emerging NGS technologies also permit geneticists to tackle phenotypes that were previously largely inaccessible via Sanger sequencing. Because these methods are so powerful, NGS projects are shifting towards nuclear families and isolated patients, representing a very large group of genetically undefined patients. For each nuclear family, one can sequence two affected individuals and search for variants in genes that are shared between patients. Different strategies can be applied to study isolated patients; when the individuals are severely affected, this can be the consequence of a de novo mutation, and by sequencing both parents and the patient, de novo variations can be identified. Another approach involves sequencing unrelated index patients and detecting variations in the same gene in different individuals across families, which is a very strong and independent argument in favor of a pathogenic link between a certain gene and the CMT neuropathy. To this end, novel, innovative genome data analysis platforms have emerged, such as Genomes Management Application (GEM.app) [100]. GEM.app allows laboratories around the world to analyze their data jointly, collaborate ad hoc on specific novel genes, or establish networks of 
collaboration. This is possible via a strictly web-based system with secure access to data [101]. Every user has full control over their own data, but also sees counts of variants by gene, phenotype and variant type for all exomes/genomes in this system. The majority of novel CMT and HSP (hereditary spastic paraplegia) genes are currently discovered via GEM.app by over 200 users from 24 different countries. A variation of this approach is the Genome Variant Database for Human Diseases [101], which is heavily biased towards axonopathies. This system contains more than 500 exomes that can be freely queried to search for a "second family" to support a new gene.

Large scale screening of patients allows determination of CMT mutation frequencies, establishment of phenotypic borders of these heterogeneous neuropathies, and at the same time, exploration of phenotypic overlaps between CMT and other neuropathies. As such, NGS will be an important tool for personalized and preventive medicine. Several database initiatives aim at capturing more complete lists of clinically relevant mutations in human diseases. These include the Leiden Open Variant Database [19], the Human Gene Mutation Database [102], and a specific CMT database currently constructed by the Inherited Neuropathy Consortium [103] However, CMT genetics have already identified more than 1,000 mutations in 80 disease associated genes, and novel NGS tools will unravel at least an equal amount of CMT associated genes, making it more appropriate to study the common disease mechanisms. Importantly, the development of NGS technologies also led to the discovery of novel mutations in known genes, uncovering their phenotypic spectrum and highlighting pleiotropic effects.

Finally, we cannot forget the important role of functional studies in unraveling gene and protein functions, and in particular the study of mutations in cell and animal models [104]. In general, these studies were designed to understand the complex pathomechanisms of axonal degeneration and myelination defects in the peripheral nervous system. Cell and animal models that have been developed for a large group of peripheral neuropathy associated genes will be instrumental for treatment of CMT and related disorders [105]. However, focusing on treatment strategies for axonal degeneration or demyelination, or aiming at treating motor and sensory defects, might be more relevant than aiming at rescuing all CMT mutations individually.

\section{Acknowledgments}

Research in Vincent Timmerman's lab is supported in part by research grants from the University of Antwerp, the Flanders Fund for Scientific Research (FWO), the Medical Foundation Queen Elisabeth (GSKE), and the Association Belge contre les maladies Neuromusculaires (ABMM), Belgium. VT is a partner within the FP7 NEUROMICS EU project. We appreciate the help of Bart Smets, VIB-DMG Centralized Service Facility, for running the Ingenuity Pathway Analysis. Research in Stephan Züchner's lab is currently supported by the Muscular Dystrophy Association, the Charcot-Marie-Tooth Association, and NIH (5R01NS075764, 5U54NS065712).

\section{Author Contributions}

Wrote the paper: VT, AS and SZ. 


\section{Conflicts of Interest}

The authors declare no conflict of interest.

\section{References}

1. Charcot, J.-M.; Marie, P. Sur une forme particulière d'atrophie musculaire progressive, souvent familiale, debutant par les pieds et les jambes et atteignant plus tard les mains (in French). Rev. Med. 1886, 6, 97-138.

2. Tooth, H.H. The Peroneal Type of Progressive Muscular Atrophy; H.K. Lewis and Co.: London, UK, 1886.

3. Dyck, P.J. Definition and basis of classification of hereditary neuropathy with neuronal atrophy and degeneration. In Peripheral Neuropathy, 1st ed.; Dyck, P.J., Thomas, P.K., Lambert, E.H., Eds.; W.B. Saunders Company: Philadelphia, PA, USA, 1975; pp. 825-867.

4. Dyck, P.J.; Thomas, P.K.; Griffin, J.W.; Low, P.A.; Poduslo, J.F. Peripheral Neuropathy, 4th ed.; WB Saunders: Philadelphia, PA, USA, 2005.

5. Harding, A.E.; Thomas, P.K. The clinical features of hereditary motor and sensory neuropathy types I and II. Brain 1980, 103, 259-280.

6. Timmerman, V.; Clowes, V.E.; Reid, E. Overlapping molecular pathological themes link Charcot-Marie-Tooth neuropathies and hereditary spastic paraplegias. Exp. Neurol. 2013, 246, 14-25.

7. Reilly, M.M.; Murphy, S.M.; Laura, M. Charcot-Marie-Tooth disease. J. Peripher. Nerv. Syst. 2011, 16, 1-14.

8. Saporta, M.A.; Shy, M.E. Inherited peripheral neuropathies. Neurol. Clin. 2013, 31, 597-619.

9. Bird, T.D.; Ott, J.; Giblett, E.R. Evidence for linkage of Charcot-Marie-Tooth neuropathy to the Duffy locus on chromosome 1. Am. J. Hum. Genet. 1982, 34, 388-394.

10. Venter, J.C.; Adams, M.D.; Myers, E.W.; Li, P.W.; Mural, R.J.; Sutton, G.G.; Smith, H.O.; Yandell, M.; Evans, C.A.; Holt, R.A.; et al. The sequence of the human genome. Science 2001, 291, 1304-1351.

11. Lander, E.S.; Linton, L.M.; Birren, B.; Nusbaum, C.; Zody, M.C.; Baldwin, J.; Devon, K.; Dewar, K.; Doyle, M.; FitzHugh, W.; et al. Initial sequencing and analysis of the human genome. Nature 2001, 409, 860-921.

12. Ng, S.B.; Turner, E.H.; Robertson, P.D.; Flygare, S.D.; Bigham, A.W.; Lee, C.; Shaffer, T.; Wong, M.; Bhattacharjee, A.; Eichler, E.E.; et al. Targeted capture and massively parallel sequencing of 12 human exomes. Nature 2009, 461, 272-276.

13. Ng, S.B.; Buckingham, K.J.; Lee, C.; Bigham, A.W.; Tabor, H.K.; Dent, K.M.; Huff, C.D.; Shannon, P.T.; Jabs, E.W.; Nickerson, D.A.; et al. Exome sequencing identifies the cause of a mendelian disorder. Nat. Genet. 2010, 42, 30-35.

14. Pitceathly, R.D.; Murphy, S.M.; Cottenie, E.; Chalasani, A.; Sweeney, M.G.; Woodward, C.; Mudanohwo, E.E.; Hargreaves, I.; Heales, S.; Land, J.; et al. Genetic dysfunction of MT-ATP6 causes axonal Charcot-Marie-Tooth disease. Neurology 2012, 79, 1145-1154. 
15. Suter, U.; Scherer, S.S. Disease mechanisms in inherited neuropathies. Nat. Rev. Neurosci. 2003, 4, 714-726.

16. Ingenuity Systems. Available online: http://www.ingenuity.com/ (accessed on 18 October 2013).

17. Online Mendelian Inheritance in Man Database (OMIM). Available online: http://ncbi. nlm.nih.gov/omim/ (accessed on 20 November 2013).

18. Inherited Peripheral Neuropathy Mutation Database (IPNMDB). Available online: http:// molgen.vib-ua.be/CMTMutations/ (accessed on 20 November 2013).

19. Leiden Open (Source) Variation Database (LOVD). Available online: http://lovd.nl/ (accessed on 20 November 2013).

20. Bird, T.D. Historical perspective of defining Charcot-Marie-Tooth type 1B. Ann. N. Y. Acad. Sci. 1999, 883, 6-13.

21. Vance, J.M.; Nicholson, G.A.; Yamaoka, L.H.; Stajich, J.; Stewart, J.S.; Speer, M.C.; Hung, W.-J.; Roses, A.D.; Barker, D.; Pericak-Vance, M.A. Linkage of Charcot-Marie-Tooth neuropathy type 1a to chromosome 17. Exp. Neurol. 1989, 104, 186-189.

22. Raeymaekers, P.; Timmerman, V.; de Jonghe, P.; Swerts, L.; Gheuens, J.; Martin, J.-J.; Muylle, L.; de Winter, G.; Vandenberghe, A.; van Broeckhoven, C. Localization of the mutation in an extended family with Charcot- Marie-Tooth neuropathy (HMSN I). Am. J. Hum. Genet. 1989, 45, 953-958.

23. Middleton-Price, H.R.; Harding, A.E.; Monteiro, C.; Berciano, J.; Malcolm, S. Linkage of hereditary motor and sensory neuropathy type I to the pericentromeric region of chromosome 17. Am. J. Hum. Genet. 1990, 46, 92-94.

24. Hayasaka, K.; Himoro, M.; Sato, W.; Takada, G.; Uyemura, K.; Shimizu, N.; Bird, T.; Conneally, P.M.; Chance, P.F. Charcot-Marie-Tooth neuropathy type $1 \mathrm{~B}$ is associated with mutations of the myelin P0 gene. Nat. Genet. 1993, 5, 31-34.

25. Planté-Bordeneuve, V.; Guiochon-Mantel, A.; Lacroix, C.; Lapresle, J.; Said, G. The Roussy-Levy family: From the original description to the gene. Ann. Neurol. 1999, 46, 770-773.

26. Pareyson, D.; Menichella, D.; Botti, S.; Sghirlanzoni, A.; Fallica, E.; Mora, M.; Ciano, C.; Shy, M.E.; Taroni, F. Heterozygous null mutation in the P0 gene associated with mild Charcot-Marie-Tooth disease. Ann. N. Y. Acad. Sci. 1999, 883, 477-480.

27. Warner, L.E.; Hilz, M.J.; Appel, S.H.; Killian, J.M.; Kolodny, E.H.; Karpati, G.; Watters, G.V.; Nelis, E.; van Broeckhoven, C.; Lupski, J.R. Clinical phenotypes of different $M P Z\left(\mathrm{P}_{0}\right)$ mutations may include Charcot-Marie-Tooth 1B, Dejerine-Sottas and congenital hypomyelination. Neuron 1996, 17, 451-460.

28. Schiavon, F.; Rampazzo, A.; Merlini, L.; Angelini, C.; Mostacciuolo, M.L. Mutations of the same sequence of the myelin P0 gene causing two different phenotypes. Hum. Mutat. 1998, $11, \mathrm{~S} 217-\mathrm{S} 219$.

29. De Jonghe, P.; Timmerman, V.; Ceuterick, C.; Nelis, E.; de Vriendt, E.; Löfgren, A.; Vercruyssen, A.; Verellen, C.; van Maldergem, L.; Martin, J.-J.; et al. The Thr124Met mutation in the peripheral myelin protein zero (MPZ) gene is associated with a clinically distinct Charcot-Marie-Tooth phenotype. Brain 1999, 122, 281-290. 
30. Nelis, E.; van Broeckhoven, C.; de Jonghe, P.; Löfgren, A.; Vandenberghe, A.; Latour, P.; Le Guern, E.; Brice, A.; Mostacciuolo, M.L.; Schiavon, F.; et al. Estimation of the mutation frequencies in Charcot-Marie-Tooth disease type 1 and hereditary neuropathy with liability to pressure palsies: A European collaborative study. Eur. J. Hum. Genet. 1996, 4, 25-33.

31. Szigeti, K.; Garcia, C.; Lupski, J.R. Charcot-Marie-Tooth disease and related hereditary polyneuropathies: Molecular diagnostics determine aspects of medical management. Genet. Med. 2006, 8, 86-92.

32. Patel, P.I.; Franco, B.; Garcia, C.; Slaugenhaupt, S.A.; Nakamura, Y.; Ledbetter, D.H.; Chakravarti, A.; Lupski, J.R. Genetic mapping of autosomal dominant Charcot-Marie-Tooth disease in a large French-Acadian kindred: Identification of new linked markers on chromosome 17. Am. J. Hum. Genet. 1990, 46, 801-809.

33. Timmerman, V.; Raeymaekers, P.; de Jonghe, P.; de Winter, G.; Swerts, L.; Jacobs, K.; Gheuens, J.; Martin, J.-J.; Vandenberghe, A.; van Broeckhoven, C. Assignment of the Charcot-Marie-Tooth neuropathy type 1 (CMT 1a) gene to 17p11.2-p12. Am. J. Hum. Genet. 1990, 47, 680-685.

34. Raeymaekers, P.; Timmerman, V.; Nelis, E.; de Jonghe, P.; Hoogendijk, J.E.; Baas, F.; Barker, D.F.; Martin, J.-J.; de Visser, M.; Bolhuis, P.A.; et al. HMSN Collaborative Research Group Duplication in chromosome 17p11.2 in Charcot-Marie-Tooth neuropathy type 1a (CMT 1a). Neuromuscul. Disord. 1991, 1, 93-97.

35. Lupski, J.R.; Montes de Oca-Luna, R.; Slaugenhaupt, S.; Pentao, L.; Guzzetta, V.; Trask, B.J.; Saucedo-Cardenas, O.; Barker, D.F.; Killian, J.M.; Garcia, C.A.; et al. DNA duplication associated with Charcot-Marie-Tooth disease type 1A. Cell 1991, 66, 219-239.

36. Timmerman, V.; Lupski, J.R. The CMT1A duplication and HNPP deletion. In Genomic Disorders: The Genomic Basis of Disease, 1st ed.; Lupski, J.R., Stankiewicz, P., Eds.; Humana Press: Totowa, NJ, USA, 2006.

37. Chance, P.F.; Alderson, M.K.; Leppig, K.A.; Lensch, M.W.; Matsunami, N.; Smith, B.; Swanson, P.D.; Odelberg, S.J.; Distsche, C.M.; Bird, T.D. DNA deletion associated with hereditary neuropathy with liability to pressure palsies. Cell 1993, 72, 143-151.

38. Reiter, L.T.; Murakami, T.; Koeuth, T.; Pentao, L.; Muzny, D.M.; Gibbs, R.A.; Lupski, J.R. A recombination hotspot responsible for two inherited peripheral neuropathies is located near a mariner transposon-like element. Nat. Genet. 1996, 12, 288-297.

39. Kennerson, M.L.; Nassif, N.T.; Dawkins, J.L.; DeKroon, R.M.; Yang, J.G.; Nicholson, G.A. The Charcot-Marie-Tooth binary repeat contains a gene transcribed from the opposite strand of a partially duplicated region of the COX10 gene. Genomics 1997, 46, 61-69.

40. Inoue, K.; Dewar, K.; Katsanis, N.; Reiter, L.T.; Lander, E.S.; Devon, K.L.; Wyman, D.W.; Lupski, J.R.; Birren, B. The 1.4 Mb CMT1A duplication/HNPP deletion genomic region reveals unique genome architectural features and provides insights into the recent evolution of new genes. Genome Res. 2001, 11, 1018-1033. 
41. Matsunami, N.; Smith, B.; Ballard, L.; Lensch, M.W.; Robertson, M.; Albertsen, H.; Hanemann, C.O.; Müller, H.W.; Bird, T.D.; White, R.; et al. Peripheral myelin protein-22 gene maps in the duplication in chromosome $17 \mathrm{p} 11.2$ associated with Charcot-Marie-Tooth 1A. Nat. Genet. 1992, 1, 176-179.

42. Patel, P.I.; Roa, B.B.; Welcher, A.A.; Schoener-Scott, R.; Trask, B.J.; Pentao, L.; Snipes, G.J.; Garcia, C.A.; Francke, U.; Shooter, E.M.; et al. The gene for the peripheral myelin protein PMP-22 is a candidate for Charcot-Marie-Tooth disease type 1A. Nat. Genet. 1992, 1, 159-165.

43. Timmerman, V.; Nelis, E.; van Hul, W.; Nieuwenhuijsen, B.W.; Chen, K.L.; Wang, S.; Ben Othman, K.; Cullen, B.; Leach, R.J.; Hanemann, C.O.; et al. The peripheral myelin protein gene PMP-22 is contained within the Charcot-Marie-Tooth disease type 1A duplication. Nat. Genet. 1992, 1, 171-175.

44. Valentijn, L.J.; Bolhuis, P.A.; Zorn, I.; Hoogendijk, J.E.; van den Bosch, N.; Hensels, G.W.; Stanton, V., Jr.; Housman, D.E.; Fischbeck, K.H.; Ross, D.A.; et al. The peripheral myelin gene PMP-22/GAS-3 is duplicated in Charcot- Marie-Tooth disease type 1A. Nat. Genet. 1992, 1, $166-170$.

45. Lupski, J.R.; Wise, C.A.; Kuwano, A.; Pentao, L.; Parker, J.; Glaze, D.; Ledbetter, D.; Greenberg, F.; Patel, P.I. Gene dosage is a mechanism for Charcot-Marie-Tooth disease type 1A. Nat. Genet. 1992, 1, 29-33.

46. Palau, F.; Löfgren, A.; de Jonghe, P.; Bort, S.; Nelis, E.; Sevilla, T.; Martin, J.-J.; Vílchez, J.; Prieto, F.; van Broeckhoven, C. Origin of the de novo duplication in Charcot-Marie-Tooth disease type 1A: Unequal nonsister chromatid exchange during spermatogenesis. Hum. Mol. Genet. 1993, 2, 2031-2035.

47. Zhang, F.; Seeman, P.; Liu, P.; Weterman, M.A.; Gonzaga-Jauregui, C.; Towne, C.F.; Batish, S.D.; de Vriendt, E.; de Jonghe, P.; Rautenstrauss, B.; et al. Mechanisms for nonrecurrent genomic rearrangements associated with CMT1A or HNPP: Rare CNVs as a cause for missing heritability. Am. J. Hum. Genet. 2010, 86, 892-903.

48. Boone, P.M.; Wiszniewski, W.; Lupski, J.R. Genomic medicine and neurological disease. Hum. Genet. 2011, 130, 103-121.

49. Stankiewicz, P.; Lupski, J.R. Structural variation in the human genome and its role in disease. Annu. Rev. Med. 2010, 61, 437-455.

50. Suter, U.; Welcher, A.A.; Özcelik, T.; Snipes, G.; Kosaras, B.; Francke, U.; Billings-Gagliardi, S. Trembler mouse carries a point mutation in a myelin gene. Nature 1992, 356, 241-244.

51. Suter, U.; Moskow, J.J.; Welcher, A.A.; Snipes, G.; Kosaras, B.; Sidman, R.; Buchberg, A.; Shooter, E. A leucine-to-proline mutation in the putative first transmembrane domain of the 22-kDa peripheral myelin protein in the trembler-J mouse. Proc. Natl. Acad. Sci. USA 1992, 89, 4382-4386.

52. Fledrich, R.; Stassart, R.M.; Sereda, M.W. Murine therapeutic models for Charcot-Marie-Tooth (CMT) disease. Br. Med. Bull. 2012, 102, 89-113.

53. Sereda, M.W.; zu Horste, G.M.; Suter, U.; Uzma, N.; Nave, K.-A. Therapeutic administration of progesterone antagonist in a model of Charcot-Marie-Tooth disease (CMT-1A). Nat. Med. 2003, 9, 1533-1537. 
54. Passage, E.; Norreel, J.C.; Noack-Fraissignes, P.; Sanguedolce, V.; Pizant, J.; Thirion, X.; Robaglia-Schlupp, A.; Pellissier, J.F.; Fontes, M. Ascorbic acid treatment corrects the phenotype of a mouse model of Charcot-Marie-Tooth disease. Nat. Med. 2004, 10, 396-401.

55. Verhamme, C.; de Haan, R.J.; Vermeulen, M.; Baas, F.; de Visser, M.; van Schaik, I.N. Oral high dose ascorbic acid treatment for one year in young CMT1A patients: A randomised, double-blind, placebo-controlled phase II trial. BMC Med. 2009, 7, 70.

56. Pareyson, D.; Schenone, A.; Rizzuto, N.; Fabrizi, G.M.; Santoro, L.; Vita, G.; Quattrone, A.; Padua, L.; Gemignani, F.; Visioli, F.; et al. Clinical and electrophysiological evaluation of 222 patients with Charcot-Marie-Tooth disease type 1A recruited in the CMT-TRIAAL (ascorbic acid therapy for Charcot-Marie-Tooth 1A disease). J. Neurol. 2008, 255, 104-105.

57. Pareyson, D.; Schenone, A.; Fabrizi, G.M.; Santoro, L.; Padua, L.; Quattrone, A.; Vita, G.; Gemignani, F.; Visioli, F.; Solari, A.; et al. A multicenter, randomized, double-blind, placebo-controlled trial of long-term ascorbic acid treatment in Charcot-Marie-Tooth disease type 1A (CMT-TRIAAL): The study protocol [EudraCT no.: 2006-000032-27]. Pharmacol. Res. 2006, 54, 436-441.

58. Burns, J.; Ouvrier, R.A.; Yiu, E.M.; Joseph, P.D.; Kornberg, A.J.; Fahey, M.C.; Ryan, M.M. Ascorbic acid for Charcot-Marie-Tooth disease type 1A in children: A randomised, double-blind, placebo-controlled, safety and efficacy trial. Lancet Neurol. 2009, 8, 537-544.

59. Lewis, R.A.; McDermott, M.P.; Herrmann, D.N.; Hoke, A.; Clawson, L.L.; Siskind, C.; Feely, S.M.; Miller, L.J.; Barohn, R.J.; Smith, P.; et al. High-dosage ascorbic acid treatment in Charcot-Marie-Tooth disease type 1A: Results of a randomized, double-masked, controlled trial. JAMA Neurol. 2013, 70, 981-987.

60. Micallef, J.; Attarian, S.; Dubourg, O.; Gonnaud, P.M.; Hogrel, J.Y.; Stojkovic, T.; Bernard, R.; Jouve, E.; Pitel, S.; Vacherot, F.; et al. Effect of ascorbic acid in patients with Charcot-Marie-Tooth disease type 1A: A multicentre, randomised, double-blind, placebo-controlled trial. Lancet Neurol. 2009, 8, 1103-1110.

61. Saporta, A.S.; Sottile, S.L.; Miller, L.J.; Feely, S.M.; Siskind, C.E.; Shy, M.E. Charcot-Marie-Tooth disease subtypes and genetic testing strategies. Ann. Neurol. 2011, 69, 22-33.

62. Aretz, S.; Rautenstrauss, B.; Timmerman, V. Clinical utility gene card for: HMSN/HNPP HMSN types 1, 2, 3, 6 (CMT1,2,4, DSN, CHN, GAN, CCFDN, HNA); HNPP. Eur. J. Hum. Genet. 2010, 18, doi:10.1038/ejhg.2010.75.

63. Harding, A.E.; Thomas, P.K. Hereditary distal spinal muscular atrophy. A report on 34 cases and a review of the literature. J. Neurol. Sci. 1980, 45, 337-348.

64. Irobi, J.; Dierick, I.; Jordanova, A.; Claeys, K.; de Jonghe, P.; Timmerman, V. Unravelling the genetics of distal hereditary motor neuronopathies. NeuroMol. Med. 2006, 8, 131-146.

65. Timmerman, V.; Raeymaekers, P.; Nelis, E.; de Jonghe, P.; Muylle, L.; Ceuterick, C.; Martin, J.-J.; van Broeckhoven, C. Linkage analysis of distal hereditary motor neuropathy type II (distal HMN II) in a single pedigree. J. Neurol. Sci. 1992, 109, 41-48.

66. Gyapay, G.; Morissette, J.; Vignal, A.; Dib, C.; Fizames, C.; Millasseau, P.; Marc, S.; Bernardi, G.; Lathrop, M.; Weissenbach, J. The 1993-94 Genethon human genetic linkage map [see comments]. Nat. Genet. 1994, 7, 246-339. 
67. Timmerman, V.; de Jonghe, P.; Simokovic, S.; Löfgren, A.; Beuten, J.; Nelis, E.; Ceuterick, C.; Martin, J.-J.; van Broeckhoven, C. Distal hereditary motor neuropathy type II (distal HMN II): Mapping of a locus to chromosome 12q24. Hum. Mol. Genet. 1996, 5, 1065-1069.

68. Irobi, J.; Tissir, F.; de Jonghe, P.; de Vriendt, E.; van Broeckhoven, C.; Timmerman, V.; Beuten, J. A clone contig of 12q24.3 encompassing the distal hereditary motor neuropathy type II gene. Genomics 2000, 65, 34-43.

69. Irobi, J.; van Impe, K.; Seeman, P.; Jordanova, A.; Dierick, I.; Verpoorten, N.; Michalik, A.; de Vriendt, E.; Jacobs, A.; van Gerwen, V.; et al. Hot-spot residue in small heat-shock protein 22 causes distal motor neuropathy. Nat. Genet. 2004, 36, 597-601.

70. Ismailov, S.M.; Fedotov, V.P.; Dadali, E.L.; Polyakov, A.V.; van Broeckhoven, C.; Ivanov, V.I.; de Jonghe, P.; Timmerman, V.; Evgrafov, O.V. A new locus for autosomal dominant Charcot-Marie-Tooth disease type 2 (CMT2F) maps to chromosome 7q11-q21. Eur. J. Hum. Genet. 2001, 9, 646-650.

71. Evgrafov, O.V.; Mersiyanova, I.V.; Irobi, J.; van den Bosch, L.; Dierick, I.; Schagina, O.; Verpoorten, N.; van Impe, K.; Fedotov, V.P.; Dadali, E.L.; et al. Mutant small heat-shock protein 27 causes axonal Charcot-Marie-Tooth disease and distal hereditary motor neuropathy. Nat. Genet. 2004, 36, 602-606.

72. Holmgren, A.; Bouhy, D.; Timmerman, V. Molecular Biology of small HSPs associated with Peripheral Neuropathies. eLS 2012, doi:10.1002/9780470015902.a0024294.

73. D’Ydewalle, C.; Krishnan, J.; Chiheb, D.M.; van Damme, P.; Irobi, J.; Kozikowski, A.P.; Vanden Berghe, P.; Timmerman, V.; Robberecht, W.; van den Bosch, L. HDAC6 inhibitors reverse axonal loss in a mouse model of mutant HSPB1-induced Charcot-Marie-Tooth disease. Nat. Med. 2011, 17, 968-974.

74. Saito, M.; Hayashi, Y.; Suzuki, T.; Tanaka, H.; Hozumi, I.; Tsuji, S. Linkage mapping of the gene for Charcot-Marie-Tooth disease type 2 to chromosome 1p (CMT2A) and the clinical features of CMT2A. Neurology 1997, 49, 1630-1635.

75. Zhao, C.; Takita, J.; Tanaka, Y.; Setou, M.; Nakagawa, T.; Takeda, S.; Wei Yang, H.; Terada, S.; Nakata, T.; Takei, Y.; et al. Charcot-Marie-Tooth disease type 2A caused by mutation in a microtubule motor KIF1Bbeta. Cell 2001, 105, 587-597.

76. Zuchner, S.; Mersiyanova, I.V.; Muglia, M.; Bissar-Tadmouri, N.; Rochelle, J.; Dadali, E.L.; Zappia, M.; Nelis, E.; Patitucci, A.; Senderek, J.; et al. Mutations in the mitochondrial GTPase mitofusin 2 cause Charcot-Marie-Tooth neuropathy type 2A. Nat. Genet. 2004, 36, 449-451.

77. Verhoeven, K.; Claeys, K.; Züchner, S.; Schröder, J.M.; Weis, J.; Ceuterick, C.; Jordanova, A.; Nelis, E.; de Vriendt, E.; van Hul, M.; et al. Mitofusin 2 mutation distribution and genotype/phenotype correlation in Charcot-Marie-Tooth type 2. Brain 2006, 129, 2093-2102.

78. Lv, H.; Wang, L.; Li, W.; Qiao, X.; Li, Y.; Wang, Z.; Yuan, Y. Mitofusin 2 gene mutation causing early-onset CMT2A with different progressive courses. Clin. Neuropathol. 2013, 32, $16-23$. 
79. Chung, K.W.; Kim, S.B.; Park, K.D.; Choi, K.G.; Lee, J.H.; Eun, H.W.; Suh, J.S.; Hwang, J.H.; Kim, W.K.; Seo, B.C.; et al. Early onset severe and late-onset mild Charcot-Marie-Tooth disease with mitofusin 2 (MFN2) mutations. Brain 2006, 129, 2103-2118.

80. Feely, S.M.; Laura, M.; Siskind, C.E.; Sottile, S.; Davis, M.; Gibbons, V.S.; Reilly, M.M.; Shy, M.E. MFN2 mutations cause severe phenotypes in most patients with CMT2A. Neurology 2011, 76, 1690-1696.

81. Züchner, S.; de Jonghe, P.; Jordanova, A.; Claeys, K.; Guergelcheva, V.; Cherninkova, S.; Hamilton, S.R.; van Stavern, G.; Krajewski, K.; Stajich, J.; et al. Axonal neuropathy with optic atrophy (HMSN VI) is caused by mutations in mitofusin 2. Ann. Neurol. 2006, 59, 276-281.

82. Zhu, D.; Kennerson, M.L.; Walizada, G.; Züchner, S.; Vance, J.M.; Nicholson, G.A. Charcot-Marie-Tooth with pyramidal signs is genetically heterogeneous: Families with and without MFN2 mutations. Neurology 2005, 65, 496-497.

83. McCorquodale, D.S.; Montenegro, G.; Peguero, A.; Carlson, N.; Speziani, F.; Price, J.; Taylor, S.W.; Melanson, M.; Vance, J.M.; Zuchner, S. Mutation screening of mitofusin 2 in Charcot-Marie-Tooth disease type 2. J. Neurol. 2011, 258, 1234-1239.

84. Auer-Grumbach, M.; Weger, M.; Fink-Puches, R.; Papic, L.; Frohlich, E.; Auer-Grumbach, P.; El Shabrawi-Caelen, L.; Schabhuttl, M.; Windpassinger, C.; Senderek, J.; et al. Fibulin-5 mutations link inherited neuropathies, age-related macular degeneration and hyperelastic skin. Brain 2011, 134, 1839-1852.

85. Zimon, M.; Baets, J.; Almeida-Souza, L.; de Vriendt, E.; Nikodinovic, J.; Parman, Y.; Battalo Gcaron, L.E.; Matur, Z.; Guergueltcheva, V.; Tournev, I.; et al. Loss-of-function mutations in HINT1 cause axonal neuropathy with neuromyotonia. Nat. Genet. 2012, 44, 1080-1083.

86. Azzedine, H.; Senderek, J.; Rivolta, C.; Chrast, R. Molecular genetics of charcot-marie-tooth disease: From genes to genomes. Mol. Syndromol. 2012, 3, 204-214.

87. Rossor, A.M.; Polke, J.M.; Houlden, H.; Reilly, M.M. Clinical implications of genetic advances in Charcot-Marie-Tooth disease. Nat. Rev. Neurol. 2013, 9, 562-571.

88. Montenegro, G.; Powell, E.; Huang, J.; Speziani, F.; Edwards, Y.J.; Beecham, G.; Hulme, W.; Siskind, C.; Vance, J.; Shy, M.; et al. Exome sequencing allows for rapid gene identification in a Charcot-Marie-Tooth family. Ann. Neurol. 2011, 69, 464-470.

89. Gonzales, M.A.; McLaughlin, H.M.; Houlden, H.; Guo, M. Exome sequencing identifies a significant variant in methionyl-tRNA synthetase (MARS) in a family with late-onset CMT2. J. Neurol. Neurosurg. Psychiatry 2013, 84, 1247-1249.

90. Peeters, K.; Litvinenko, I.; Asselbergh, B.; Almeida-Souza, L.; Chamova, T.; Geuens, T.; Ydens, E.; Zimon, M.; Irobi, J.; de Vriendt, E.; et al. Molecular defects in the motor adaptor BICD2 Cause proximal spinal muscular atrophy with autosomal-dominant inheritance. Am. J. Hum. Genet. 2013, 92, 955-964.

91. Neveling, K.; Martinez-Carrera, L.A.; Holker, I.; Heister, A.; Verrips, A.; Hosseini-Barkooie, S.M.; Gilissen, C.; Vermeer, S.; Pennings, M.; Meijer, R.; et al. Mutations in BICD2, which encodes a golgin and important motor adaptor, cause congenital autosomal-dominant spinal muscular atrophy. Am. J. Hum. Genet. 2013, 92, 946-954. 
92. Oates, E.C.; Rossor, A.M.; Hafezparast, M.; Gonzalez, M.; Speziani, F.; Macarthur, D.G.; Lek, M.; Cottenie, E.; Scoto, M.; Foley, A.R.; et al. Mutations in BICD2 cause dominant congenital spinal muscular atrophy and hereditary spastic paraplegia. Am. J. Hum. Genet. 2013, 92, 965-973.

93. Kennerson, M.L.; Yiu, E.M.; Chuang, D.T.; Kidambi, A.; Tso, S.C.; Ly, C.; Chaudhry, R.; Drew, A.P.; Rance, G.; Delatycki, M.B.; et al. A new locus for X-linked dominant Charcot-Marie-Tooth disease (CMTX6) is caused by mutations in the pyruvate dehydrogenase kinase isoenzyme 3 (PDK3) gene. Hum. Mol. Genet. 2013, 22, 1404-1416.

94. Leipold, E.; Liebmann, L.; Korenke, G.C.; Heinrich, T.; Giesselmann, S.; Baets, J.; Ebbinghaus, M.; Goral, R.O.; Stodberg, T.; Hennings, J.C.; et al. A de novo gain-of-function mutation in SCN11A causes loss of pain perception. Nat. Genet. 2013, 45, 1399-1404.

95. Barwick, K.E.; Wright, J.; Al-Turki, S.; McEntagart, M.M.; Nair, A.; Chioza, B.; Al-Memar, A.; Modarres, H.; Reilly, M.M.; Dick, K.J.; et al. Defective presynaptic choline transport underlies hereditary motor neuropathy. Am. J. Hum. Genet. 2012, 91, 1103-1107.

96. Tischfield, M.A.; Baris, H.N.; Wu, C.; Rudolph, G.; van Maldergem, L.; He, W.; Chan, W.M.; Andrews, C.; Demer, J.L.; Robertson, R.L.; et al. Human TUBB3 mutations perturb microtubule dynamics, kinesin interactions, and axon guidance. Cell 2010, 140, 74-87.

97. Lupski, J.R.; Reid, J.G.; Gonzaga-Jauregui, C.; Rio, D.D.; Chen, D.C.; Nazareth, L.; Bainbridge, M.; Dinh, H.; Jing, C.; Wheeler, D.A.; et al. Whole-genome sequencing in a patient with Charcot-Marie-Tooth neuropathy. N. Engl. J. Med. 2010, 362, 1181-1191.

98. Züchner, S. Peripheral neuropathies: Whole genome sequencing identifies causal variants in CMT. Nat. Rev. Neurol. 2010, 6, 424-425.

99. Senderek, J.; Bergmann, C.; Stendel, C.; Kirfel, J.; Verpoorten, N.; de Jonghe, P.; Timmerman, V.; Chrast, R.; Verheijen, M.H.G.; Lemke, G.; et al. Mutations in a gene encoding a novel SH3/TPR domain protein cause autosomal recessive Charcot-Marie-Tooth type 4C neuropathy. Am. J. Hum. Genet. 2003, 73, 1106-1119.

100. Gonzalez, M.A.; Lebrigio, R.F.; van Booven, D.; Ulloa, R.H.; Powell, E.; Speziani, F.; Tekin, M.; Schule, R.; Zuchner, S. GEnomes Management Application (GEM.app): A new software tool for large-scale collaborative genome analysis. Hum. Mutat. 2013, 34, 842-846.

101. Genome Variant Database for Human Diseases. Available online: http://www.genomics.med. miami.edu/ (accessed on 20 November 2013).

102. Human Gene Mutation Database. Available online: http://www.biobase-international.com/ product/hgmd/ (accessed on 20 November 2013).

103. Inherited Neuropathy Consortium. Available online: http://rarediseasesnetwork.epi. usf.edu/INC/ (accessed on 20 November 2013).

104. Niemann, A.; Berger, P.; Suter, U. Pathornechanisms of mutant proteins in Charcot-Marie-Tooth disease. NeuroMol. Med. 2006, 8, 217-241.

105. Bouhy, D.; Timmerman, V. Animal models and therapeutic prospects for Charcot-Marie-Tooth disease. Ann. Neurol. 2013, 74, 391-396. 


\title{
The Past, Present, and Future of Human Centromere Genomics
}

\section{Megan E. Aldrup-MacDonald and Beth A. Sullivan}

\begin{abstract}
The centromere is the chromosomal locus essential for chromosome inheritance and genome stability. Human centromeres are located at repetitive alpha satellite DNA arrays that compose approximately $5 \%$ of the genome. Contiguous alpha satellite DNA sequence is absent from the assembled reference genome, limiting current understanding of centromere organization and function. Here, we review the progress in centromere genomics spanning the discovery of the sequence to its molecular characterization and the work done during the Human Genome Project era to elucidate alpha satellite structure and sequence variation. We discuss exciting recent advances in alpha satellite sequence assembly that have provided important insight into the abundance and complex organization of this sequence on human chromosomes. In light of these new findings, we offer perspectives for future studies of human centromere assembly and function.
\end{abstract}

Reprinted from Genes. Cite as: Aldrup-MacDonald, M.E.; Sullivan, B.A. The Past, Present, and Future of Human Centromere Genomics. Genes 2014, 5, 33-50.

\section{Introduction}

The centromere is the chromosomal locus that controls chromosome segregation during cell division. Visually, the centromere appears on metaphase chromosomes, at least in metazoans that have excellent cytology, as a primary constriction. This is also the site of kinetochore assembly, the multi-protein structure that forms to coordinate attachment to and movement of chromosomes along microtubules. The proteins associated with centromeres are conserved among species, consistent with the functional significance of the locus. A surprising feature of centromeres is that the DNA sequences present at the locus are dissimilar, not only among organisms but often within the same organism. However, protein components of centromeres are shared among species, suggesting an epigenetic basis for centromere assembly. Such centromere proteins (CENPs) include CENP-A, CENP-C, and CENP-T that are important for structural and functional aspects of the centromere and kinetochore. CENP-A is of particular significance since it is a histone H3 variant that contributes to specialized chromatin at centromeres. The Holliday Junction Recognition Protein HJURP and its fungal homolog Scm3 are chaperones that direct the loading of CENP-A into chromatin primed by the Mis18 complex and ensure propagation of epigenetically marked centromeric nucleosomes (reviewed by [1,2]).

Despite the lack of sequence identity, many centromeres are located in regions of repetitive DNA or satellites. In humans, repetitive alpha satellite DNA defines the centromere. The sequence basis of centromere identity is widely debated, since variant centromeres have been identified in humans and other organisms. These unusual centromeres include neocentromeres, new centromeres that are formed on unique or non-centromeric DNA sequences [3,4]. Dicentric human chromosomes, those chromosomes that are formed by fusion or translocation, have two regions of centromeric DNA, but often only one is the site of kinetochore formation. In these instances, the alpha satellite 
DNA appears to be neither necessary nor sufficient for centromere function. Nevertheless, other evidence exists that supports the importance of DNA sequence in centromere formation in humans, particularly de novo centromere assembly. In this review, we will discuss advances in our understanding of human centromeric DNA, from the discovery of human centromeric sequences through integration of physical and genetic maps of centromeres during the Human Genome Project era to the first centromeric genome assemblies that are only now emerging.

\section{Alpha Satellite DNA: Discovery, Organization, and Variation}

Human centromeres, and in fact most primate centromeres, are composed of alpha satellite DNA [5]. This sequence is thought to be important for centromere function since it is present at the primary constriction of all human chromosomes. It comprises up to $5 \%$ of the genome. Alpha satellite is based on a $171 \mathrm{bp}$ monomer arranged in a tandem, head-to-tail fashion. Individual monomers share $50 \%-70 \%$ sequence identity. An integral number of monomers give rise to a higher order repeat (HOR) unit that is itself repeated in a largely uninterrupted fashion so that within a given centromeric locus, the alpha satellite array can span from 250 to $5,000 \mathrm{~kb}$. Such re-iteration of the HOR gives rise to a homogenized alpha satellite array in which the HORs differ in sequence by only a few percent (Figure 1), even though the constituent monomers show much less sequence similarity [6,7]. Some monomers within the HORs contain a 17 bp sequence called the CENP-B box, a motif that is recognized by the DNA-binding centromere protein CENP-B [8]. Outside of the higher order arrays, monomers are randomly arranged and span the region between the homogeneous array and the chromosome arm [9].

Figure 1. The genomic organization of human centromeres. The primary sequence at human centromeres is alpha satellite DNA that is based on $171 \mathrm{bp}$ monomers (colored arrows) organized in a tandem head-to tail fashion. The monomeric sequences differ by as much as $40 \%$. A set number of monomers give rise to a higher order repeat (colored bars with black arrowhead) and confer chromosome-specificity. Higher order repeats are themselves reiterated hundreds to thousands of times, so that the alpha satellite arrays are highly homogenous and span several hundred kilobases to several megabases. Unordered monomeric alpha satellite DNA flanks the higher order arrays, becoming progressively more divergent farther away from centromeric core.

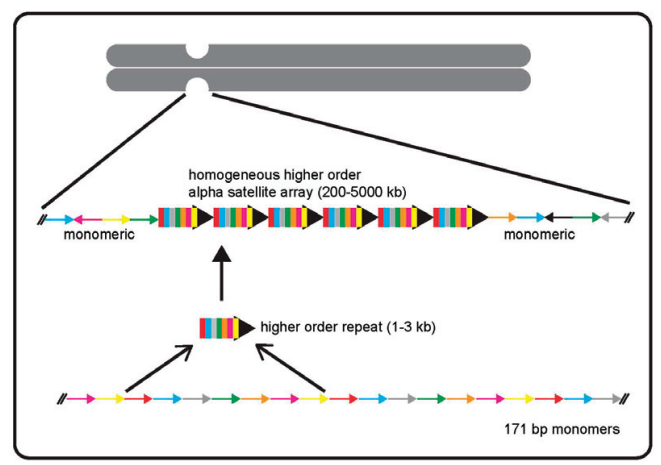


Figure 2. Heterogeneity of alpha satellite DNA. The alpha satellite DNA at centromeres exhibits several types of polymorphism. (A) Total array size, defined by the number of higher order repeats (HOR; gray arrows), varies between homologues and among individuals; (B) The same alpha satellite array from a given chromosome type can contain HORs of different sizes. In addition, the number of each HOR variant can vary. For example, an alpha satellite array can contain a mixture of 10-mers and 6-mers, with a greater number of 10-mers. Another array from the same chromosome in a different individual might have an equal number of 10-mers and 6-mers or, alternatively, more 6-mers than 10-mers; (C) Alpha satellite DNA can also vary at the level of monomer (black arrowheads) type and arrangement. Some monomers (gray arrowheads) contain a specific sequence element called the CENP-B box. Others monomers can contain identical nucleotide changes or SNPs (yellow arrowheads) within the same array. Multiple SNPs (hot pink, orange, gray, yellow arrowheads) can be present in the same HOR or distributed across an alpha satellite array. Each type of variation (array size, HOR size, SNPs) is not mutually exclusive and all contribute to the heterogeneity of alpha satellite DNA in the human population.

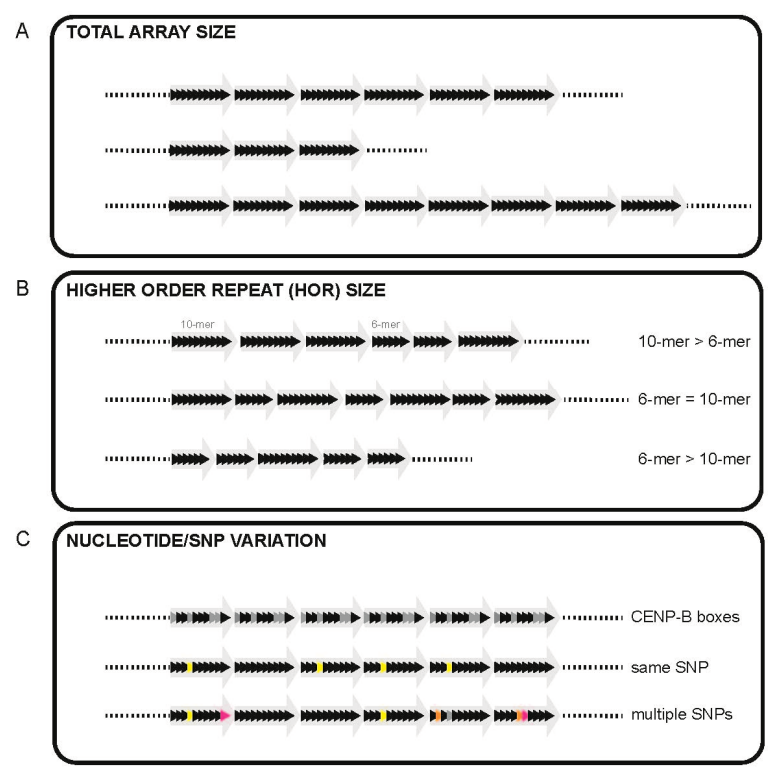

Variation within the alpha satellite is common and complex. Each chromosome type is defined by an alpha satellite array in which the multimers of the HOR contain a particular number of tandem monomers $[7,10,11]$. The homogeneity of HORs of the same monomer number makes the alpha satellite array chromosome-specific and distinguishable from related sequences at other centromeres. Certain chromosomes share greater homology of HORs based on monomer subtypes and organization, allowing them to be classified into one of three suprachromosomal families [12]. Diverged monomeric alpha satellite falls into two additional suprachromosomal families [13]. On a given chromosome type, the number of times the HOR is re-iterated is heterogeneous, spanning 
hundreds to thousands of copies. Consequently, total array size on a given chromosome varies between homologs and among individuals (Figure 2) [14-18]. Although array sizes can be as small as a few hundred kilobases or as large as five megabases $[16,19,20]$, the range appears to be less extensive on a particular chromosome type [21]. Array size polymorphisms are largely stable through meiosis since they can be efficiently tracked through multigenerational families [17]. These polymorphisms make alpha satellite a useful centromeric marker for tracking inheritance of individual chromosomes.

Additional alpha satellite variation exists at the level of the HOR. On a given chromosome, the primary HOR unit can exhibit size polymorphisms that are most likely the result of deletions caused by unequal exchange $[22,23]$. Human chromosome 17 is a good example of HOR polymorphism within the D17Z1 alpha satellite array. The predominant HOR on D17Z1 is a 16-monomer (16-mer) [18,22]. However, less prevalent 15-mer and 14-mer HORs are present on many D17Z1 arrays, as well as 13-mers and 12-mers [22,24]. Within this group, the 13-mer is the most abundant after the 16-mer. These size polymorphisms create centromeric haplotypes, with the 16-/15-/14-mer comprising a haplotype found on $65 \%$ of chromosome $17 \mathrm{~s}$ and the additional 13 -mer present on $35 \%$ of chromosome $17 \mathrm{~s}[22,25]$. A recent study that evaluated centromere assembly on multiple chromosome $17 \mathrm{~s}$ suggested that HOR variants might have different functional capacities [26]. This possibility, however, remains to be formally tested in an independent functional assay.

\section{Functional Studies that Have Defined Genomic Centromeres}

The strongest evidence for implicating alpha satellite DNA in human centromere function came from two lines of chromosome engineering experiments that took "bottom up" and "top down" approaches (Figure 3). In the "top down" strategy, telomere-mediated chromosomal truncation was used to modify the $\mathrm{X}$ chromosome or $\mathrm{Y}$ chromosome, some of which had been transferred to either rodent somatic cell hybrids or DT40 chicken cells (Figure 3B) [27,28]. Because DT40 cells are proficient in homologous recombination, targeted seeding of the telomere truncation constructs accelerated the deletion process. Multiple rounds of telomere truncation generated a series of deleted chromosomes, each containing less X or Y chromosome material (Figure 3B). The stability of the minichromosomes was monitored and those that maintained the least amount of the original chromosome but were still mitotically stable were concluded to contain the minimal sequence(s) necessary for centromere function. In both truncated $\mathrm{X}$ and $\mathrm{Y}$ chromosomes, minichromosomes containing alpha satellite DNA arrays DXZ1 and DYZ3, respectively, equated with the most stable linear minichromosomes. These studies strongly implicated alpha satellite DNA as the sequence that corresponds to centromere function and chromosome stability.

However, it could be argued that in the top-down studies the centromere, once established on any sequence, stays at that sequence, and does not shift with truncation. At the same time, pioneering experiments were being developed by two groups to take a "bottom up" approach to define the sequences required for centromere function. In these studies, alpha satellite sequences were introduced into linear yeast artificial chromosome (YAC) or circular bacterial artificial chromosome (BAC) vectors (Figure 3A). Hunt Willard's group created first generation artificial chromosomes from synthetic alpha satellite arrays [29]. One higher order repeat from D17Z1 
(chromosome 17) or DYZ3 (chromosome Y) was amplified through successive rounds of directional cloning to yield a $1 \mathrm{Mb}$ array that was inserted into a BAC vector. Introduction of these artificial chromosome assembly constructs by liposome-mediated transfection into the HT1080 cell line yielded clones that contained a microchromosome or human artificial chromosome (HAC). The HACs recruited centromere proteins and were stable in mitosis for at least 6 months. Careful analysis of the HACs showed that the D17Z1 HACs were completely derived from the input construct. However, the DYZ3-derived HAC had acquired additional alpha satellite sequences from host chromosomes. The functional significance of the inability of DYZ3 to form a functional HAC containing only Y centromere sequences was not fully appreciated at the time. Subsequent studies shed light on the correlation between DYZ3 sequence and its competence for de novo centromere assembly (see below).

At the same time that the Willard group was creating HACs from synthetic alpha satellite DNA, Howard Cooke's and Hiroshi Masumoto's groups were collaborating to clone large alpha satellite arrays from human chromosome 21 into linear YAC vectors. In their studies, the higher order array $\alpha 21-\mathrm{I}$ and the unordered monomeric array $\alpha 21$-II were introduced into HT1080 cells and compared for de novo centromere competency [30]. Only YACs containing the $\alpha 21-\mathrm{I}$ HOR array were capable of forming mitotically stable HACs that properly assembled centromere proteins. These innovative studies complemented those of the Willard group, and contributed important structure-function information that implicated HOR alpha satellite as a preferred substrate for de novo centromere assembly. In the time that has elapsed since these groundbreaking experiments, additional studies have established HACs as models for testing the genomic (and epigenetic) requirements for de novo centromere assembly and function. Circular BAC and PAC vectors, rather than linear YACs, are the most useful assembly vectors and are associated with high rates of HAC formation [31,32]. Not all alpha satellite arrays translate to HAC formation. Y chromosome alpha satellite DNA (DYZ3) lacks CENP-B boxes and is unable to efficiently form de novo centromeres on HACs [29,32]. Furthermore, arrays containing mutated CENP-B boxes cannot form de novo HACs [33]. Thus, the presence of CENP-B binding sites is required for centromere assembly. This has been a perplexing finding, given that the $\mathrm{Y}$ chromosome clearly assembles a functional centromere and recruits essential centromere proteins. These findings hint at key differences between de novo versus established centromere function that are not well understood.

Initial studies that tested the ability of alpha satellite to nucleate functional centromeres introduced cosmids containing human alpha satellite DNA from chromosome 17 into African green monkey (AGM) cells [34]. These experiments did not result in supernumerary chromosomes or HACs, but instead, integration of the alpha satellite construct into AGM chromosomes (Figure 3A). Indeed, up to $60 \%$ of HAC constructs introduced into human cells integrate into the genome rather than forming an independent chromosome. While some might point out that this argues against the case for sequence-dependent centromere assembly, another interpretation is that de novo chromosome assembly and de novo centromere formation are two different processes. Indeed, some integrated alpha satellite arrays recruit centromere proteins [34,35], although they may not retain some or all of the proteins long-term. At the very least, both integrated and free-lying HAC studies suggest that alpha satellite provides sequence information for some aspects of centromere function. 
Figure 3. Minichromosome-based assays defining alpha satellite as the functional human centromere. (A) In the late 1990s, human artificial chromosome (HAC) assays (bottom up approach) were developed to test the ability of alpha satellite DNA to form de novo centromeres. Synthetic or clone arrays of alpha satellite DNA, such as D17Z1 from human chromosome 17 (green), were cloned into bacterial or plasmid (P1) artificial chromosome (BAC/PAC) vectors containing selectable marker genes (SM). The chromosome assembly constructs were introduced by transfection into human cells. In approximately half of the cells, an autonomous de novo chromosome (arrowhead) was produced, consisting of the same alpha satellite DNA (D17Z1, green, as shown) as the parental chromosome (arrow). Inset shows DAPI (DNA) staining of HAC. In the other proportion of transfected clones, the alpha satellite assembly $\mathrm{BAC} / \mathrm{PAC}$ vector does not make a HAC, but integrates once or multiple times (as shown) into one or more chromosomes. In these instances, the alpha satellite DNA does not recruit any, or all, centromere proteins and is not a functional centromere. Inset shows DAPI (DNA) stained chromosome that contains multiple insertions of D17Z1. (B) In a complementary top-down approach, existing chromosomes (X and Y) were systematically deleted using plasmid constructs containing mammalian telomeric sequence (yellow arrowheads). These experiments yielded partially deleted chromosomes with integrated telomeres (red-orange-yellow rectangles) that were progressively deleted. Mitotic chromosome segregation of these minichromosomes was used as a measure of chromosome stability. Based on the molecular composition of the stable minichromosomes that were recovered, alpha satellite DNA (pink oval) was defined as the minimal sequence required for centromere function.

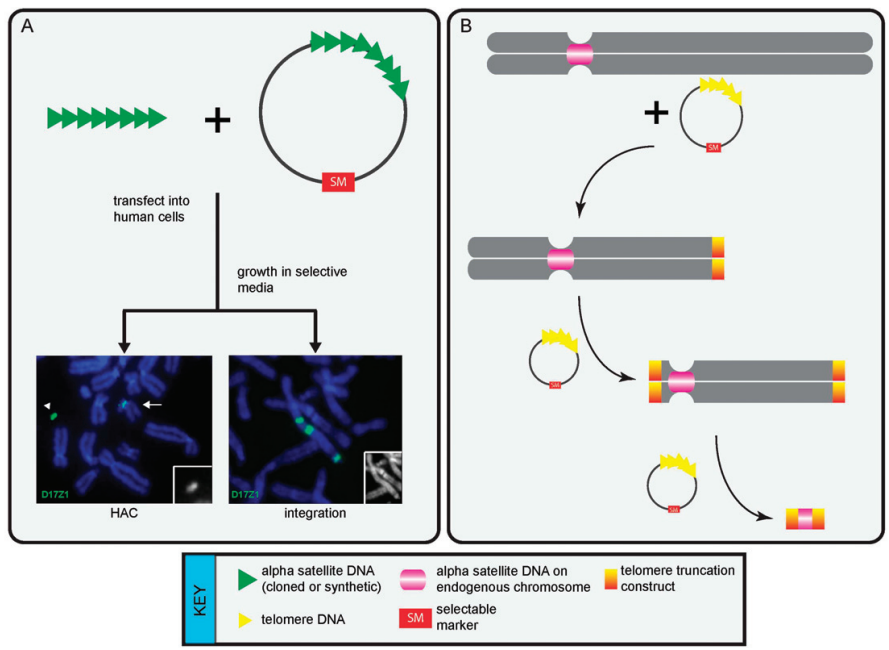

Contemporary studies are now using centromere-based chromosome engineering to create a new generation of HACs that contain alpha satellite in addition to tetracycline operator (tetO) sequences [36]. The tetO sequences are bound with high affinity by the tet repressor (tetR) that can 
be fused to different proteins in order to manipulate the chromatin or protein composition of the HAC $[37,38]$. In this way, centromere assembly on the alpha satellite can be enhanced or inhibited, the long-term stability of the HAC can be monitored by tethering tetR fluorescent protein fusions, and expression of genes included on the HAC can be tested [39].

\section{Centromere Regions in the Human Genome Project Era}

As the understanding of the relationship between alpha satellite DNA and centromere function emerged at the end of the 20th century, it led to a call for the identification and mapping of functional centromere sequences [40]. However, the nature of alpha satellite, with its megabase-scale regions of higher-order repetitive structure, made it highly refractory to sequencing and assembly [41]. As the Human Genome Project (HGP) rapidly increased the sequence information available for testing human genome function, gains were largely not seen at the pericentromeres and centromeres of most human chromosomes. A 1998 plan for the project that outlined the HGP's goal for a 2001 working draft and a 2003 final draft acknowledged that "the small proportion of highly repeated sequence represented by the centromeres and other constitutive heterochromatic regions of the genome" might not be included in the final reference assembly [42]. A contemporary perspective on the plan warned of the possibility that potentially important duplications and tandem repeats would be "swept under the carpet". There was a repeated call for at least some centromeric regions to be characterized in order to confirm that their structure was as homogenous as originally claimed [43]. But again, due to the computational complexity required to accurately assemble such highly repetitive regions, few labs attempted to close these sequence gaps [44-46]. A decade later, multi-megabase-sized gaps remain at the centromeres of most chromosome assemblies. This problem is not exclusive to the human genome, since centromere and pericentromere sequence gaps in other organisms such as mouse and Drosophila remain unclosed [47-50]. Only in the past year have advances in sequencing technologies and innovative computational efforts focused on elucidating alpha satellite structure helped to make a full understanding of the genome and some of its most critical elements a real possibility [51,52].

\section{Linking Physical and Genetic Maps of Human Centromeres}

By the late 1990s and early 2000s, several groups had pushed forward the centromere field by producing integrated physical and genetic maps of centromere regions including chromosomes X, 5, and 12 [53-55]. These studies used pulsed-field gel electrophoresis to estimate physical alpha satellite array sizes and either radiation hybrid or linkage analyses to estimate genetic distance across the centromere. In addition to confirming the repression of recombination across centromeres, the integrated maps that resulted allowed for the anchoring of alpha satellite regions to existing genomic maps, and sometimes identified unique pericentric sequences that had not been represented in the human genome drafts [55].

Of the sequence assemblies around the centromere that do exist, the pericentric regions are the best characterized. Within these regions, a high proportion of segmental duplications have accumulated $[44,56]$. Many pericentric duplications corresponding to unmapped regions of the 
genome were identified using monochromosomal somatic cell hybrids and PCR or FISH with known pericentric sequences and genomic BACs that recognized paralogous sequences across the genome [56]. Genome-wide analysis of the January 2001 draft assembly further revealed pericentromeric and subtelomeric enrichment for duplicated sequences, and showed that such sequences were frequently present in unmapped or misassembled segments [57]. The discordance between FISH and BLAST results in these analyses was much higher than the genome-wide rate reported in the same year [58]. Together, these studies demonstrated the importance of elucidating highly duplicated pericentric regions in order to accurately understand the Human Genome Project's results. More recent progress was made in assembling "inaccessible" regions by using linkage disequilibrium analysis of genetically distinct (admixed) genomes to map almost $20 \mathrm{Mb}$ of sequence near centromeres [59]. As the number of admixed genomes available for analysis increases, this powerful technique is expected to reduce the gaps in the current reference assembly.

Figure 4. The detailed genomic organization of the human $X$ centromere. The first contiguous genomic map of a human centromere (CEN) on the $\mathrm{X}$ chromosome was completed in 2001 and showed that the higher order array (large light gray arrays containing black monomer arrowheads) is flanked by unordered, monomeric alpha satellite DNA (multi-colored arrows). The regions between monomeric alpha satellite and the chromosome short $(\mathrm{Xp})$ and long $(\mathrm{Xq})$ arms contain other types of satellite DNA, such as gamma satellite and HSAT4. LINEs (red lollipops) and SINEs (purple lollipops) punctuate the repetitive DNA between the centromere and chromosome arms. The Xq pericentromere contains monomeric alpha satellite and a LINE element at the pericentromere-arm junction. Some of the monomers within the unordered Xq satellite contain CENP-B boxes (black asterisks). The functional significance of these monomers remains unclear.



\section{Correlating the Genetic and Functional Centromere}

In 2001, a major breakthrough in reaching beyond the boundaries of alpha satellite occurred when chromosome $\mathrm{X}$ short arm (Xp) genomic clones were mapped into the homogenous higher order DXZ1 array [60]. This tour-de-force used combined in silico and high-stringency BAC clone screening to demonstrate that even in higher order alpha satellite, enough sequence variation existed to assemble a contig extending almost half a megabase from the satellite boundary towards the centromere core that is the location of the functional centromere (Figure 4). This study revealed that heterogeneity of alpha satellite DNA increased with more distance from the DXZ1 core. These 
studies permitted the definition of transitions between the higher order alpha satellite and flanking regions. Monomers of alpha satellite DNA that are not ordered into multi-monomer repeat units are located directly outside of the homogenous HOR domain [9]. These monomers exhibit enough sequence variation that they can be more easily assembled and in fact represent most of the alpha satellite that exists in the human reference assembly $[60,61]$. The monomeric alpha satellite regions show greater sequence dissimilarity and more interspersed elements, such as L1 sequences, as they approach the chromosome arms. Currently, HSAX and HSA8 are the only human chromosomes represented in the genome assembly with contiguous sequence from higher-order alpha satellite to both arms $[62,63]$.

Subsequent to these findings, several groups began analyzing alpha satellite at increasing sequence depth, discovering new alpha satellite polymorphisms and repeat organization. Building on the work of previous decades, targeted sequencing of several well-characterized arrays was performed. The high copy number of alpha satellite HORs on each chromosome permitted analysis of intra-homolog SNPs in addition to inter-individual variation that was paired with restriction digestion for haplotype analysis [64]. These studies revisited the molecular basis for variation within alpha satellite by pinpointing where unequal exchange occurred to produce array homogenization.

\section{The Computational Challenge of Alpha Satellite Genome Assemblies}

The bottleneck in generating alpha satellite assemblies has undoubtedly been the sophistication of assembly tools that are required to order distinguishable monomeric sequences within highly homogenous arrays. Several groups have developed in silico tools for analyzing higher order alpha satellite sequence available in genome assemblies [65,66]. These computational and in silico approaches are most effective when combined with experimental approaches that mapped clones by FISH to verify their location in or near the higher order array. Indeed, such dry/wet approaches were used to map the region spanning the Xp centromere-arm junction and to characterize the centromere of human chromosome 17 [60,61,67]. In the latter instance, a novel higher-order array (D17Z1-B) was discovered on chromosome 17 [67], emphasizing the power of this integrative approach. Another novel HOR array, localized by BLAST to HSA22 and verified by FISH to hybridize to HSA14 and 22, was found by "rescuing" unassembled alpha satellite sequence information from whole genome sequencing (WGS) repositories [68]. These studies revealed that while challenging to assemble, repetitive satellite regions, particularly in the centromere, hold a wealth of complex genomic structure and potentially functional information.

\section{Assembling Centromeres in the Present Day}

Previous studies utilized traditional sequencing technologies that have the potential to contain several 171-bp monomers per read. Next generation short-read sequencing technology has enabled the recent increase in whole-genome sequencing and the amount of human sequence information available overall. Nevertheless, short reads present a particular challenge for assembling alpha satellite sequence. It appears that this obstacle of aligning short-read alpha satellite sequences can be overcome to utilize functional information gleaned from chromatin immunoprecipitation with centromeric protein antibodies and Illumina sequencing of the DNA that is captured [51]. This 
ChIP-sequencing (ChIP-seq) approach utilized the reference assembly as well as the HuRef genome, first by aligning the HuRef alpha satellite reads to the reference assembly. After this alignment, the reference alpha satellite was broken into sliding windows, and the alignment checked back onto the HuRef reads to determine the "mappability" of each window. This mappability information was then used for alignment of the short Illumina reads generated by ChIP-seq. It should be noted, however, that this study did not have the means to extend beyond the edges of the reference assembly into the homogenous centromere cores (see Future Perspectives). Another major discovery from the assembly annotation of this work was that many more chromosomes than previously thought contain two or more higher order alpha satellite arrays [51,61,69-71]. This finding has raised the complexity of centromeres to a new level and introduced the possibility that the location of centromere assembly may be quite variable in humans. This is indeed the case for human chromosome 17 on which the centromere can be assembled at either of the two higher order repeat arrays [26]. This new information suggests that in addition to alpha satellite haplotypes, there may a number of functional centromeric genotypes. How a functional genotype might affect long-term chromosome stability is an open question.

\section{Future Perspectives}

It is now 2014, so what can we expect from the centromere field in the next decade? Based on the foundation laid by the Human Genome Project era, the most exciting areas of centromere research are in some of the following areas.

\subsection{Centromere Assemblies}

Clearly, the most significant frontier that remains to be explored in centromere biology is complete genomic centromere assemblies. With the recent advances in the past two years alone using long-template sequencing and advanced computational approaches that have sampled, annotated, and assembled centromere sequences in multiple genomes, centromere reference sequences are a real possibility. Just recently, ordering of monomer sequences from whole-genome shotgun reads has produced the first linear characterization of centromeric assemblies for alpha satellite arrays from chromosomes X and Y [72]. Increasing read lengths offered by multiple platforms offer the potential to contain several multi-kilobase HORs in one read. In fact, long PacBio reads have already accelerated the discovery and mapping of centromeric tandem repeats in a variety of species [52]. These third generation sequencing techniques should enable longer alpha satellite sequence assemblies and better understanding of centromere structure and neighboring variant HORs. Completion of even a few centromere assemblies will undoubtedly be important, but given the amount of variation in alpha satellite organization and size, the ultimate goal would be to produce centromere assemblies for each individual. These personalized maps would be useful for defining the spectrum of sequences that correlate with functional competency. In addition, they will allow identification of other features - such as genes or non-coding elements - that are present within current centromere/pericentromere gaps. These sequences may require centromeric locations for proper function, similar to heterochromatic genes in Drosophila [48,73]. Indeed, a human 
muscle disorder has been mapped to the gene KCNJ18 that is located in an assembly gap on $17 \mathrm{p} 11.2$ [74]. It is possible that other genes or elements within centromere regions may be associated with diseases for which the molecular basis remains undefined.

\subsection{Centromeric Variation and Functional Capacity}

The ability to confidently assemble centromeric contigs should permit identification of the full range of variability in alpha satellite, including sequence and size variants [72]. Such variation will shed light on the molecular mechanisms that regulate alpha satellite homogenization, but also effects of fundamental processes such as DNA replication and DNA repair on alpha satellite stability. Ultimately, characterization of alpha satellite variation would reveal the range of sequences that are capable of supporting centromere function. HAC studies have taught us that not all alpha satellite sequences have the capacity to support de novo centromere assembly [29,32]. The reasons for this have been largely unexplored, and mostly attributed to the presence or absence of CENP-B boxes in alpha satellite [33,75]. One would expect that like a given complex human disease that is often associated with various SNPs, many types of sequence variation would be associated with diminished centromere function. Complete, personalized centromeric assemblies linked to functional centromere status would expedite experiments to compare efficiencies of various sequence variants in de novo centromere assembly and/or centromere maintenance.

\subsection{Maps of Functional Centromeric Domains}

The consensus in the centromere field is that centromere identity is specified by epigenetic mechanisms. However, without detailed genomic information, this theory is not irrefutable. Centromere proteins, such as the histone H3-like protein CENP-A, are assembled onto alpha satellite DNA to create a specialized type of nucleosome within unique chromatin that distinguishes the centromere from the rest of the genome [76,77]. CENP-A and other proteins create a complicated network of protein sub-complexes that link the chromatin to the structural kinetochore that interacts with spindle microtubules [78]. However, chromatin that contains CENP-A nucleosomes is only assembled on a portion of alpha satellite DNA [79,80]. How and why CENP-A is recruited to only a subset of alpha satellite HOR and/or monomers is unclear. Recent studies have revealed that CENP-A nucleosomes on the human $\mathrm{X}$ chromosome are positioned at monomers that do not contain CENP-B boxes [81]. One could speculate that distribution of CENP-B boxes within an alpha satellite array and sequence variation that interrupts the CENP-B box motif or makes the motif non-functional (not bound by CENP-B) might impact CENP-A chromatin assembly and centromere function. Complete centromeric assemblies of many human chromosomes will be important for addressing this possibility experimentally.

\section{Conclusions}

Since the discovery of alpha satellite DNA in the late 1970s, the field has moved from identification of centromeric sequences at every human centromere to a basic molecular understanding of the organization and structure of alpha satellite monomers into homogeneous 
higher order repetitive arrays (Figure 5). The Human Genome Project was essential in providing a rough and limited reference assembly for centromeres of three chromosomes $(X, 8,17)$. These fundamental studies of alpha satellite DNA paved the way for pioneering functional assays in which the sequence was tested in de novo centromere assembly in human artificial chromosome assays. HACs have been the gold standard for testing centromere assembly, but are now being used to explore chromosome stability and gene expression. The next challenge will be to complete genomic assemblies for all human centromeres in multiple individuals and populations and to develop the next generation of functional assays to test the role of alpha satellite variation in centromere function, chromosome stability, and disease association.

Figure 5. Timeline of major discoveries in human centromere genomics. Since the discovery of alpha satellite DNA in 1979, the understanding of the sequence, organization, and functional aspects of this sequence flourished during the Human Genome Project era. Recent years have shown the use of human artificial chromosomes (HACs) and the creation of the first database of alpha satellite sequences linked to their functional capacity.

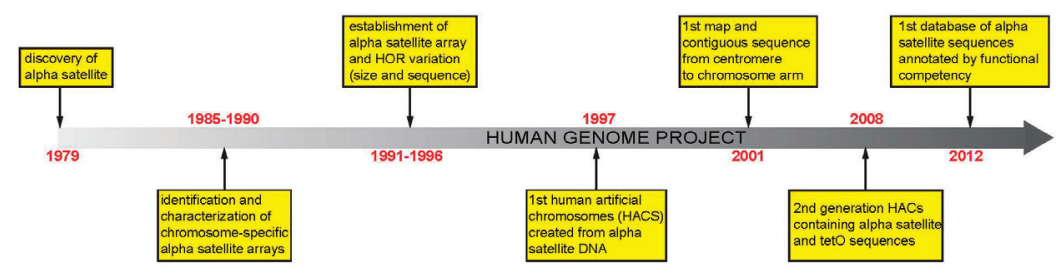

\section{Acknowledgments}

We apologize to our colleagues whose work on alpha satellite DNA and human centromeres could not be cited due to space constraints. Research in the Sullivan lab is supported in part by R01 GM098500 (NIH) and Gene Discovery and Translational Research Grant \#1-FY13-517 (March of Dimes Foundation).

\section{Author Contributions}

Wrote the paper: MEAM, BAS.

\section{Conflicts of interest}

The authors declare no conflict of interest.

\section{References}

1. Panchenko, T.; Black, B.E. The epigenetic basis for centromere identity. Prog. Mol. Subcell. Biol. 2009, 48, 1-32.

2. Valente, L.P.; Silva, M.C.; Jansen, L.E. Temporal control of epigenetic centromere specification. Chromosome Res. 2012, 20, 481-492.

3. Choo, K.H. Domain organization at the centromere and neocentromere. Dev. Cell 2001, 1, 165-177. 
4. Warburton, P.E. Chromosomal dynamics of human neocentromere formation. Chromosome Res. 2004, 12, 617-626.

5. Manuelidis, L.; Wu, J.C. Homology between human and simian repeated DNA. Nature 1978, 276, 92-94.

6. Waye, J.S.; Willard, H.F. Nucleotide sequence heterogeneity of alpha satellite repetitive DNA: A survey of alphoid sequences from different human chromosomes. Nucleic Acids Res. 1987, $15,7549-7569$.

7. Willard, H.F. Chromosome-specific organization of human alpha satellite DNA. Am. J. Hum. Genet. 1985, 37, 524-532.

8. Muro, Y.; Masumoto, H.; Yoda, K.; Nozaki, N.; Ohashi, M.; Okazaki, T. Centromere protein $\mathrm{B}$ assembles human centromeric alpha-satellite DNA at the 17-bp sequence, CENP-B box. J. Cell Biol. 1992, 116, 585-596.

9. Schueler, M.G.; Sullivan, B.A. Structural and functional dynamics of human centromeric chromatin. Annu. Rev. Genomics Hum. Genet. 2006, 7, 301-313.

10. Choo, K.H.; Vissel, B.; Nagy, A.; Earle, E.; Kalitsis, P. A survey of the genomic distribution of alpha satellite DNA on all the human chromosomes, and derivation of a new consensus sequence. Nucleic Acids Res. 1991, 19, 1179-1182.

11. Vissel, B.; Choo, K.H. Human alpha satellite DNA - Consensus sequence and conserved regions. Nucleic Acids Res. 1987, 15, 6751-6752.

12. Alexandrov, I.A.; Mitkevich, S.P.; Yurov, Y.B. The phylogeny of human chromosome specific alpha satellites. Chromosoma 1988, 96, 443-453.

13. Alexandrov, I.; Kazakov, A.; Tumeneva, I.; Shepelev, V.; Yurov, Y. Alpha-satellite DNA of primates: Old and new families. Chromosoma 2001, 110, 253-266.

14. Devilee, P.; Kievits, T.; Waye, J.S.; Pearson, P.L.; Willard, H.F. Chromosome-specific alpha satellite DNA: Isolation and mapping of a polymorphic alphoid repeat from human chromosome 10. Genomics 1988, 3, 1-7.

15. Mahtani, M.M.; Willard, H.F. Pulsed-field gel analysis of alpha-satellite DNA at the human X chromosome centromere: High-frequency polymorphisms and array size estimate. Genomics 1990, 7, 607-613.

16. Greig, G.M.; Parikh, S.; George, J.; Powers, V.E.; Willard, H.F. Molecular cytogenetics of alpha satellite DNA from chromosome 12: Fluorescence in situ hybridization and description of DNA and array length polymorphisms. Cytogenet. Cell Genet. 1991, 56, 144-148.

17. Wevrick, R.; Willard, H.F. Long-range organization of tandem arrays of alpha satellite DNA at the centromeres of human chromosomes: High-frequency array-length polymorphism and meiotic stability. Proc. Natl. Acad. Sci. USA 1989, 86, 9394-9398.

18. Willard, H.F.; Waye, J.S.; Skolnick, M.H.; Schwartz, C.E.; Powers, V.E.; England, S.B. Detection of restriction fragment length polymorphisms at the centromeres of human chromosomes by using chromosome-specific alpha satellite DNA probes: Implications for development of centromere-based genetic linkage maps. Proc. Natl. Acad. Sci. USA 1986, 83, 5611-5615.

19. Abruzzo, M.A.; Griffin, D.K.; Millie, E.A.; Sheean, L.A.; Hassold, T.J. The effect of Y-chromosome alpha-satellite array length on the rate of sex chromosome disomy in human sperm. Hum. Genet. 1996, 97, 819-823. 
20. Oakey, R.; Tyler-Smith, C. Y chromosome DNA haplotyping suggests that most European and Asian men are descended from one of two males. Genomics 1990, 7, 325-330.

21. Willard, H.F. Evolution of alpha satellite. Curr. Opin. Genet. Dev. 1991, 1, 509-514.

22. Waye, J.S.; Willard, H.F. Molecular analysis of a deletion polymorphism in alpha satellite of human chromosome 17: Evidence for homologous unequal crossing-over and subsequent fixation. Nucleic Acids Res. 1986, 14, 6915-6927.

23. Waye, J.S.; Willard, H.F. Structure, organization, and sequence of alpha satellite DNA from human chromosome 17: Evidence for evolution by unequal crossing-over and an ancestral pentamer repeat shared with the human X chromosome. Mol. Cell. Biol. 1986, 6, 3156-3165.

24. Willard, H.F.; Greig, G.M.; Powers, V.E.; Waye, J.S. Molecular organization and haplotype analysis of centromeric DNA from human chromosome 17: Implications for linkage in neurofibromatosis. Genomics 1987, 1, 368-373.

25. Warburton, P.E.; Willard, H.F. Interhomologue sequence variation of alpha satellite DNA from human chromosome 17: Evidence for concerted evolution along haplotypic lineages. J. Mol. Evol. 1995, 41, 1006-1015.

26. Maloney, K.A.; Sullivan, L.L.; Matheny, J.E.; Strome, E.D.; Merrett, S.L.; Ferris, A.; Sullivan, B.A. Functional epialleles at an endogenous human centromere. Proc. Natl. Acad. Sci. USA 2012, 109, 13704-13709.

27. Brown, K.E.; Barnett, M.A.; Burgtorf, C.; Shaw, P.; Buckle, V.J.; Brown, W.R. Dissecting the centromere of the human Y chromosome with cloned telomeric DNA. Hum. Mol. Genet. 1994, 3, 1227-1237.

28. Farr, C.J.; Bayne, R.A.; Kipling, D.; Mills, W.; Critcher, R.; Cooke, H.J. Generation of a human X-derived minichromosome using telomere-associated chromosome fragmentation. EMBO J. 1995, 14, 5444-5454.

29. Harrington, J.J.; van Bokkelen, G.; Mays, R.W.; Gustashaw, K.; Willard, H.F. Formation of de novo centromeres and construction of first-generation human artificial microchromosomes. Nat. Genet. 1997, 15, 345-355.

30. Ikeno, M.; Grimes, B.R.; Okazaki, T.; Nakano, M.; Saitoh, K.; Hoshino, H.; McGill, N.I.; Cooke, H.; Masumoto, H. Construction of YAC-based mammalian artificial chromosomes. Nat. Biotechnol. 1998, 16, 431-439.

31. Grimes, B.R.; Babcock, J.; Rudd, M.K.; Chadwick, B.; Willard, H.F. Assembly and characterization of heterochromatin and euchromatin on human artificial chromosomes. Genome Biol. 2004, 5, R89.

32. Grimes, B.R.; Rhoades, A.A.; Willard, H.F. Alpha-satellite DNA and vector composition influence rates of human artificial chromosome formation. Mol. Ther. 2002, 5, 798-805.

33. Ohzeki, J.; Nakano, M.; Okada, T.; Masumoto, H. CENP-B box is required for de novo centromere chromatin assembly on human alphoid DNA. J. Cell Biol. 2002, 159, 765-775.

34. Haaf, T.; Warburton, P.E.; Willard, H.F. Integration of human alpha-satellite DNA into simian chromosomes: Centromere protein binding and disruption of normal chromosome segregation. Cell 1992, 70, 681-696.

35. Nakashima, H.; Nakano, M.; Ohnishi, R.; Hiraoka, Y.; Kaneda, Y.; Sugino, A.; Masumoto, H. Assembly of additional heterochromatin distinct from centromere-kinetochore chromatin is required for de novo formation of human artificial chromosome. J. Cell Sci. 2005, 118, 5885-5898. 
36. Nakano, M.; Cardinale, S.; Noskov, V.N.; Gassmann, R.; Vagnarelli, P.; Kandels-Lewis, S.; Larionov, V.; Earnshaw, W.C.; Masumoto, H. Inactivation of a human kinetochore by specific targeting of chromatin modifiers. Dev. Cell 2008, 14, 507-522.

37. Bergmann, J.H.; Rodriguez, M.G.; Martins, N.M.; Kimura, H.; Kelly, D.A.; Masumoto, H.; Larionov, V.; Jansen, L.E.; Earnshaw, W.C. Epigenetic engineering shows H3K4me2 is required for HJURP targeting and CENP-A assembly on a synthetic human kinetochore. EMBO J. 2011, 30, 328-340.

38. Cardinale, S.; Bergmann, J.H.; Kelly, D.; Nakano, M.; Valdivia, M.M.; Kimura, H.; Masumoto, H.; Larionov, V.; Earnshaw, W.C. Hierarchical inactivation of a synthetic human kinetochore by a chromatin modifier. Mol. Biol. Cell 2009, 20, 4194-4204.

39. Kononenko, A.V.; Lee, N.C.; Earnshaw, W.C.; Kouprina, N.; Larionov, V. Re-engineering an alphoid(tetO)-HAC-based vector to enable high-throughput analyses of gene function. Nucleic Acids Res. 2013, 41, e107.

40. Murphy, T.D.; Karpen, G.H. Centromeres take flight: Alpha satellite and the quest for the human centromere. Cell 1998, 93, 317-320.

41. Henikoff, S. Near the edge of a chromosome's "black hole". Trends Genet. 2002, 18, 165-167.

42. Collins, F.S.; Patrinos, A.; Jordan, E.; Chakravarti, A.; Gesteland, R.; Walters, L. New goals for the U.S. Human Genome Project: 1998-2003. Science 1998, 282, 682-689.

43. Eichler, E.E. Repetitive conundrums of centromere structure and function. Hum. Mol. Genet. 1999, 8, 151-155.

44. Horvath, J.E.; Bailey, J.A.; Locke, D.P.; Eichler, E.E. Lessons from the human genome: Transitions between euchromatin and heterochromatin. Hum. Mol. Genet. 2001, 10, 2215-2223.

45. Horvath, J.E.; Viggiano, L.; Loftus, B.J.; Adams, M.D.; Archidiacono, N.; Rocchi, M.; Eichler, E.E. Molecular structure and evolution of an alpha satellite/non-alpha satellite junction at 16p11. Hum. Mol. Genet. 2000, 9, 113-123.

46. She, X.; Horvath, J.E.; Jiang, Z.; Liu, G.; Furey, T.S.; Christ, L.; Clark, R.; Graves, T.; Gulden, C.L.; Alkan, C.; et al. The structure and evolution of centromeric transition regions within the human genome. Nature 2004, 430, 857-864.

47. Hoskins, R.A.; Carlson, J.W.; Kennedy, C.; Acevedo, D.; Evans-Holm, M.; Frise, E.; Wan, K.H.; Park, S.; Mendez-Lago, M.; Rossi, F.; et al. Sequence finishing and mapping of Drosophila melanogaster heterochromatin. Science 2007, 316, 1625-1628.

48. Smith, C.D.; Shu, S.; Mungall, C.J.; Karpen, G.H. The Release 5.1 annotation of Drosophila melanogaster heterochromatin. Science 2007, 316, 1586-1591.

49. Kalitsis, P.; Griffiths, B.; Choo, K.H. Mouse telocentric sequences reveal a high rate of homogenization and possible role in Robertsonian translocation. Proc. Natl. Acad. Sci. USA 2006, 103, 8786-8791.

50. Mouse Genome Sequencing Consortium; Waterston, R.H.; Lindblad-Toh, K.; Birney, E.; Rogers, J.; Abril, J.F.; Agarwal, P.; Agarwala, R.; Ainscough, R.; Alexandersson, M.; et al. Initial sequencing and comparative analysis of the mouse genome. Nature 2002, 420, 520-562.

51. Hayden, K.E.; Strome, E.D.; Merrett, S.E.; Lee, H.R.; Rudd, M.K.; Willard, H.F. Sequences associated with centromere competency in the human genome. Mol. Cell. Biol. 2012, 33, 763-772. 
52. Melters, D.P.; Bradnam, K.R.; Young, H.A.; Telis, N.; May, M.R.; Ruby, J.G.; Sebra, R.; Peluso, P.; Eid, J.; Rank, D.; et al. Comparative analysis of tandem repeats from hundreds of species reveals unique insights into centromere evolution. Genome Biol. 2013, 14, R10.

53. Mahtani, M.M.; Willard, H.F. Physical and genetic mapping of the human X chromosome centromere: Repression of recombination. Genome Res. 1998, 8, 100-110.

54. Puechberty, J.; Laurent, A.M.; Gimenez, S.; Billault, A.; Brun-Laurent, M.E.; Calenda, A.; Marçais, B.; Prades, C.; Ioannou, P.; Yurov, Y.; et al. Genetic and physical analyses of the centromeric and pericentromeric regions of human chromosome 5: Recombination across 5cen. Genomics 1999, 56, 274-287.

55. Vermeesch, J.R.; Duhamel, H.; Raeymaekers, P.; van Zand, K.; Verhasselt, P.; Fryns, J.P.; Marynen, P. A physical map of the chromosome 12 centromere. Cytogenet. Genome Res. 2003, 103, 63-73.

56. Horvath, J.E.; Schwartz, S.; Eichler, E.E. The mosaic structure of human pericentromeric DNA: A strategy for characterizing complex regions of the human genome. Genome Res. 2000, 10, 839-852.

57. Bailey, J.A.; Yavor, A.M.; Massa, H.F.; Trask, B.J.; Eichler, E.E. Segmental duplications: Organization and impact within the current human genome project assembly. Genome Res. 2001, 11, 1005-1017.

58. Cheung, V.G.; Nowak, N.; Jang, W.; Kirsch, I.R.; Zhao, S.; Chen, X.N.; Furey, T.S.; Kim, U.J.; Kuo, W.L.; Olivier, M.; et al. Integration of cytogenetic landmarks into the draft sequence of the human genome. Nature 2001, 409, 953-958.

59. Genovese, G.; Handsaker, R.E.; Li, H.; Kenny, E.E.; McCarroll, S.A. Mapping the human reference genome's missing sequence by three-way admixture in Latino genomes. Am. J. Hum. Genet. 2013, 93, 411-421.

60. Schueler, M.G.; Higgins, A.W.; Rudd, M.K.; Gustashaw, K.; Willard, H.F. Genomic and genetic definition of a functional human centromere. Science 2001, 294, 109-115.

61. Rudd, M.K.; Willard, H.F. Analysis of the centromeric regions of the human genome assembly. Trends Genet. 2004, 20, 529-533.

62. Nusbaum, C.; Mikkelsen, T.S.; Zody, M.C.; Asakawa, S.; Taudien, S.; Garber, M.; Kodira, C.D.; Schueler, M.G.; Shimizu, A.; Whittaker, C.A.; et al. DNA sequence and analysis of human chromosome 8. Nature 2006, 439, 331-335.

63. Ross, M.T.; Grafham, D.V.; Coffey, A.J.; Scherer, S.; McLay, K.; Muzny, D.; Platzer, M.; Howell, G.R.; Burrows, C.; Bird, C.P.; et al. The DNA sequence of the human X chromosome. Nature 2005, 434, 325-337.

64. Roizes, G. Human centromeric alphoid domains are periodically homogenized so that they vary substantially between homologues. Mechanism and implications for centromere functioning. Nucleic Acids Res. 2006, 34, 1912-1924.

65. Paar, V.; Pavin, N.; Rosandic, M.; Gluncic, M.; Basar, I.; Pezer, R.; Zinic, S.D. ColorHOR - Novel graphical algorithm for fast scan of alpha satellite higher-order repeats and HOR annotation for GenBank sequence of human genome. Bioinformatics 2005, 21, 846-852.

66. Rosandic, M.; Paar, V.; Gluncic, M.; Basar, I.; Pavin, N. Key-string algorithm-Novel approach to computational analysis of repetitive sequences in human centromeric DNA. Croat. Med. J. 2003, 44, 386-406. 
67. Rudd, M.K.; Schueler, M.G.; Willard, H.F. Sequence organization and functional annotation of human centromeres. Cold Spring Harb. Symp. Quant. Biol. 2003, 68, 141-149.

68. Alkan, C.; Ventura, M.; Archidiacono, N.; Rocchi, M.; Sahinalp, S.C.; Eichler, E.E. Organization and evolution of primate centromeric DNA from whole-genome shotgun sequence data. PLoS Comput. Biol. 2007, 3, 1807-1818.

69. Alexandrov, I.A.; Mashkova, T.D.; Akopian, T.A.; Medvedev, L.I.; Kisselev, L.L.; Mitkevich, S.P.; Yurov, Y.B. Chromosome-specific alpha satellites: Two distinct families on human chromosome 18. Genomics 1991, 11, 15-23.

70. Choo, K.H.; Earle, E.; Vissel, B.; Filby, R.G. Identification of two distinct subfamilies of alpha satellite DNA that are highly specific for human chromosome 15. Genomics 1990, 7, 143-151.

71. Wevrick, R.; Willard, H.F. Physical map of the centromeric region of human chromosome 7: Relationship between two distinct alpha satellite arrays. Nucleic Acids Res. 1991, 19, 2295-2301.

72. Miga, K.H.; Newton, Y.; Jain, M.; Altemose, N.; Willard, H.F.; Kent, W.J. Centromere reference models for human chromosomes X and Y satellite arrays. arXiv 2013, arXiv:1307.0035v3[q-bio.GN].

73. Schulze, S.; Sinclair, D.A.; Silva, E.; Fitzpatrick, K.A.; Singh, M.; Lloyd, V.K.; Morin, K.A.; Kim, J.; Holm, D.G.; Kennison, J.A.; et al. Essential genes in proximal 3L heterochromatin of Drosophila melanogaster. Mol. Gen. Genet. 2001, 264, 782-789.

74. Ryan, D.P.; da Silva, M.R.; Soong, T.W.; Fontaine, B.; Donaldson, M.R.; Kung, A.W.; Jongjaroenprasert, W.; Liang, M.C.; Khoo, D.H.; Cheah, J.S.; et al. Mutations in potassium channel Kir2.6 cause susceptibility to thyrotoxic hypokalemic periodic paralysis. Cell 2010, 140, 88-98.

75. Masumoto, H.; Nakano, M.; Ohzeki, J. The role of CENP-B and alpha-satellite DNA: De novo assembly and epigenetic maintenance of human centromeres. Chromosome Res. 2004, 12, 543-556.

76. Blower, M.D.; Sullivan, B.A.; Karpen, G.H. Conserved organization of centromeric chromatin in flies and humans. Dev. Cell 2002, 2, 319-330.

77. Sullivan, B.A.; Karpen, G.H. Centromeric chromatin exhibits a histone modification pattern that is distinct from both euchromatin and heterochromatin. Nat. Struct. Mol. Biol. 2004, 11, 1076-1083.

78. Hori, T.; Fukagawa, T. Establishment of the vertebrate kinetochores. Chromosome Res. 2012, 20, 547-561.

79. Spence, J.M.; Critcher, R.; Ebersole, T.A.; Valdivia, M.M.; Earnshaw, W.C.; Fukagawa, T.; Farr, C.J. Co-localization of centromere activity, proteins and topoisomerase II within a subdomain of the major human X alpha-satellite array. EMBO J. 2002, 21, 5269-5280.

80. Sullivan, L.L.; Boivin, C.D.; Mravinac, B.; Song, I.Y.; Sullivan, B.A. Genomic size of CENP-A domain is proportional to total alpha satellite array size at human centromeres and expands in cancer cells. Chromosome Res. 2011, 19, 457-470.

81. Hasson, D.; Panchenko, T.; Salimian, K.J.; Salman, M.U.; Sekulic, N.; Alonso, A.; Warburton, P.E.; Black, B.E. The octamer is the major form of CENP-A nucleosomes at human centromeres. Nat. Struct. Mol. Biol. 2013, 20, 687-695. 


\title{
Lessons and Implications from Genome-Wide Association Studies (GWAS) Findings of Blood Cell Phenotypes
}

\author{
Nathalie Chami and Guillaume Lettre
}

Abstract: Genome-wide association studies (GWAS) have identified reproducible genetic associations with hundreds of human diseases and traits. The vast majority of these associated single nucleotide polymorphisms (SNPs) are non-coding, highlighting the challenge in moving from genetic findings to mechanistic and functional insights. Nevertheless, large-scale (epi)genomic studies and bioinformatic analyses strongly suggest that GWAS hits are not randomly distributed in the genome but rather pinpoint specific biological pathways important for disease development or phenotypic variation. In this review, we focus on GWAS discoveries for the three main blood cell types: red blood cells, white blood cells and platelets. We summarize the knowledge gained from GWAS of these phenotypes and discuss their possible clinical implications for common (e.g., anemia) and rare (e.g., myeloproliferative neoplasms) human blood-related diseases. Finally, we argue that blood phenotypes are ideal to study the genetics of complex human traits because they are fully amenable to experimental testing.

Reprinted from Genes. Cite as: Chami, N.; Lettre, G. Lessons and Implications from Genome-Wide Association Studies (GWAS) Findings of Blood Cell Phenotypes. Genes 2014, 5, 51-64.

\section{Genetics of Red Blood Cells, White Blood Cells and Platelets}

Blood is mostly composed of plasma and blood cells and plays a major role in a variety of functions involved in general human homeostasis: it transports oxygen, nutrients and hormones to tissues, removes waste, performs immunological functions and contributes tissue damage repair through coagulation. The main three blood cell types carry out most of these activities: red blood cells (RBC, or erythrocytes) transport oxygen, white blood cells (WBC, or leukocytes) coordinate some of the immune responses, and platelets are the bricks that form blood clots to prevent excessive bleeding. All of these cell types originate through proliferation and differentiation from common precursors (hematopoietic stem cells) [1].

An aberrant number, size or feature of the three main blood cell types characterizes multiple human diseases (Table 1). In many cases, the triggering factor is of environmental origin, often poor nutrition or infections (e.g., malaria, HIV). Germline and somatic mutations can also cause severe blood disorders, such as mutations in glucose-6 phosphate dehydrogenase $(G 6 P D)$ which is responsible for chronic hemolytic anemia or mutations in oncogenes or tumor suppressor genes that result in leukemia. It is also known that blood cell phenotypes vary between healthy individuals, and that some of this inter-individual variation is controlled by genetics. In a large study of healthy Sardinians $(N=6,148)$, the heritability estimates for RBC, WBC and platelet counts were, respectively, $0.67,0.38$ and 0.53 [2]. Similar heritability estimates were obtained when analyzing phenotype concordance in healthy monozygotic and dizygotic twins from the United Kingdom [3]. 
These results indicate that a large fraction of the phenotypic variation in these blood traits is controlled by DNA sequence variants segregating in healthy individuals.

Table 1. Main blood cell traits routinely measured in standard complete blood count (CBC).

\begin{tabular}{ccc}
\hline Trait & Description & Unit \\
\hline Red blood cell (RBC) count & Count of RBC per microliter & Million cells per microliter $\left(\times 10^{6} / \mu \mathrm{L}\right)$ \\
Hemoglobin (HGB) & Hemoglobin concentration & Gram per deciliter $(\mathrm{g} / \mathrm{dL})$ \\
Hematocrit (HCT) & Fraction of blood that contains hemoglobin & Percentage $(\%)$ \\
Mean corpuscular hemoglobin (MCH) & Amount of hemoglobin per RBC & Picogram $(\mathrm{pg})$ \\
Mean corpuscular volume (MCV) & Average volume of RBC & Femtoliter $(\mathrm{fL})$ \\
MCH concentration (MCHC) & Hemoglobin divided by hematocrit & Gram per deciliter $(\mathrm{g} / \mathrm{dL})$ \\
RBC distribution width (RDW) & Distribution of RBC volume & Percentage $(\%)$ \\
White blood cell (WBC) count & Number of WBC per liter (include all main subtypes) & Billion cells per liter $\left(\times 10^{9} / \mathrm{L}\right)$ \\
Platelet (PLT) count & Number of PLT per liter & Billion cells per liter $\left(\times 10^{9} / \mathrm{L}\right)$ \\
Mean platelet volume (MPV) & Average platelet volume & Femtoliter $(\mathrm{fL})$ \\
\hline
\end{tabular}

The clinical importance of this heritable variation in blood cell phenotypes is unclear. However, it is interesting that epidemiological studies have detected links between WBC or platelet counts and the risk to suffer from cardio- and cerebrovascular diseases [4-6]. As for most epidemiological observations, however, it is difficult to determine if changes in hematological parameters are pathological or reflect consequences of disease manifestation. Using Mendelian randomization methodologies, in which inherited genetic variants associated with hematological traits are used as instruments to test the causal effect of the traits on diseases, may provide an answer to this question [7]. Such an approach was successfully used to determine that LDL-cholesterol and triglyceride levels, but unlikely HDL-cholesterol levels, are causes of coronary artery diseases [8,9]. Understanding how DNA polymorphisms modulate blood cell phenotypes in health (and diseases) could provide new opportunities to study hematopoiesis, improve their use in medicine as biomarkers and maybe even help in the development of new drugs. To this list, we would also add that hematological traits are ideal phenotypes to further our understanding of the genetics of human complex diseases and traits because experimental systems exist to functionally validate genetic findings.

\section{Genome-Wide Association Studies (GWAS) for Blood Cell Phenotypes}

Before GWAS, little was known about the role of SNPs and other common DNA sequence variants on normal variation in blood cell phenotypes. Candidate gene DNA sequencing experiments have identified mutations in the globin loci, but also in the erythropoietin receptor $(E P O R)$ and hemochromatosis (HFE) genes [10,11]. Genome-wide linkage studies also found a few reproducible signals, most notably a linkage peak on chromosome $6 \mathrm{q} 23$ that encompasses the MYB transcription factor [12,13]. These findings could not, however, explain the heritability of these blood cell phenotypes in normal individuals.

As for many other complex human traits and diseases, the capacity to test associations with genotypes across the genome by GWAS opened a new world. Prior to the GWAS era, genetic association studies often had sample sizes that were too small and were limited to testing only 
known genes [14]. With GWAS, it became possible to genotype all genes independently of previous knowledge. Blood cell traits are particularly amenable to the GWAS approach because they are routinely and accurately measured in large cohorts, and initial findings can be tested for replication in other cohorts because it is easy to harmonize these phenotypes (Figure 1) [15]. In general, one of the main challenges for GWAS has been to pinpoint functional genes and variants associated with a given trait. Although this remains a challenge, blood cell traits are particularly well-suited for genetic and functional follow-up. As mentioned earlier, fine-mapping by dense genotyping and DNA re-sequencing is possible because the traits are usually available in most cohorts or biobanks, including participants of different ethnicities (see below). There is also the possibility to test the functions of new genes in cell culture systems or model organisms because the phenotypes are often cell autonomous and the assays already well-developed. Using this approach, investigators showed that SNPs at 6p21.1 modulate erythrocyte traits through a regulatory effect on the cyclin D3 (CCND3) gene [16]. Large-scale gene silencing and other functional experiments in fruit flies, zebrafish and mice were also used to validate several new genes involved in platelet and RBC development within loci identified by GWAS $[17,18]$.

All the steps described in Figure 1 now take advantage of powerful bioinformatic tools and other resources freely available on the web. For instance, comparative genomics has identified DNA bases that are conserved through evolution and therefore more likely to be functionally important [19]. There are also software that can predict based on conservation and physicochemical properties whether a DNA polymorphism that changes an amino acid is likely detrimental or not [20,21]. We can also quickly query large gene expression datasets to determine if the genes near an associated SNP are expressed in the relevant tissue(s) for the phenotypes of interest (as an example, see reference [22]). And when genotypes are available, it is possible to test in silico if the GWAS SNPs (or SNPs in linkage disequilibrium) control gene expression through regulatory mechanisms; that is, if the variants are expression quantitative trait loci (eQTL) [23]. The ENCODE and Roadmap Epigenomics Projects have used next-generation DNA sequencing applications, including DNAse I hypersensitive sites mapping and chromatin immunoprecipitation with antibodies against several histone tail modifications (ChIP-seq), to define regulatory sequences in human cell lines and tissues [24-26]. Using a complementary approach (FAIRE-seq), Paul et al. identified regions of open chromatin in primary human blood cells and showed that SNPs associated with RBC and platelet phenotypes are enriched in these regions [27]. All this vast genomic information is useful in prioritizing causal genes and variants at GWAS loci, and investigators are developing algorithms to facilitate its integration $[28,29]$.

Several GWAS for hematological traits have already been published [17,18,30-46]. The largest studies, carried out in Europeans or individuals of European ancestry, have so far identified at genome-wide significance ( $p$-value $<5 \times 10^{-8}$ ) 75, 10 and 68 SNPs associated with RBC, WBC and platelet traits respectively $[17,18,45]$. The lower number of SNPs associated with WBC count could be explained by a lower heritability (see above), but also because the sample size for the WBC GWAS was smaller $(N=11,823)$ in comparison with the GWAS for RBC $(N=135,367)$ and platelet $(N=66,867)$ traits. Despite their large number, these variants only explain a small fraction of the heritable variation in these phenotypes $(<10 \%)$. They are, however, not random but clustered 
near genes involved in relevant biological pathways and enriched for regulatory functions by expression quantitative trait loci (eQTL) and epigenomic analyses. Most loci are associated with a single blood cell type but by comparing the different studies, we found seven loci that are associated with at least two different cell types (Table 2). These include SH2B3, a gene that encodes the adapter protein LNK that interacts with JAK2 and modulates JAK-STAT signaling in hematopoietic cells, and $M Y B$, that encodes a transcription factor essential for definitive hematopoiesis. Both $S H 2 B 3$ and $M Y B$ SNPs are associated with the three main blood cell types. The other loci presented in Table 2 include genes associated with a combination of two phenotypes, maybe suggesting different functions in different hematopoietic lineages.

Figure 1. Ideal study design to identify single nucleotide polymorphisms (SNPs) associated with human complex traits and diseases using genome-wide association studies (GWAS). For blood cell phenotypes, GWAS were particularly successful because sample sizes are large, phenotypes are easy to measure and are accurate, and well-characterized experimental models already exist.

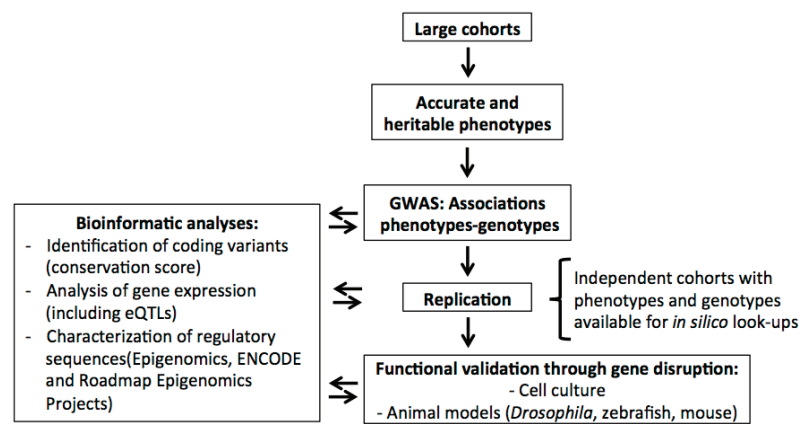

Table 2. Loci identified by GWAS that carry SNPs associated with at least two of the three main blood cell types. For each association, we report the ethnic group in which the genetic associations were found. We also listed only one gene per locus, although for many loci, the causal gene is unknown. RBC: red blood cell; WBC: white blood cell.

\begin{tabular}{|c|c|c|c|c|c|}
\hline Locus & Location & RBC & WBC & Platelet & References \\
\hline TMCC2 & $1 \mathrm{q} 32.1$ & Caucasian & & Caucasian & {$[17,18]$} \\
\hline ARHGEF3 & $3 \mathrm{p} 14.3$ & African American & & Caucasian & {$[17,30,36,38]$} \\
\hline$L R R C 16 A$ & $6 \mathrm{p} 22.2$ & African American & & African American & {$[31,37]$} \\
\hline $\begin{array}{c}H B S 1 L- \\
M Y B\end{array}$ & $6 \mathrm{q} 22-\mathrm{q} 23.3$ & $\begin{array}{l}\text { African American/ } \\
\text { Caucasian/Japanese }\end{array}$ & Caucasian & $\begin{array}{c}\text { African American/ } \\
\text { Caucasian }\end{array}$ & {$[17,18,31,32,34,35,37]$} \\
\hline$I L-6$ & $7 \mathrm{p} 21$ & & Japanese & Japanese & [47] \\
\hline$R C L 1$ & $9 \mathrm{p} 24.1-\mathrm{p} 23$ & Caucasian & & Caucasian/Japanese & {$[17,18,32,34]$} \\
\hline SH2B3 & $12 \mathrm{q} 24$ & Caucasian & Caucasian & Caucasian/Japanese & {$[17,32-35,38]$} \\
\hline
\end{tabular}

\section{Some Loci Associated with Blood Cell Traits Are Population-Specific}

It is difficult to compare association results for hematological traits across different populations because the sample size of the respective GWAS, and thus the statistical power to discover associations, is very different. For instance for RBC phenotypes, the largest studies in Caucasians 
and African Americans included, respectively, 135,367 and 16,496 participants [18,31]. Despite this caveat, many of the loci found in African Americans or Asians were also present in Caucasians; this general transferability of results across ethnic groups has been observed for other complex human traits $[48,49]$. For blood cell traits, however, there are notable exceptions. A SNP upstream of the Duffy antigen/receptor for chemokines $(D A R C)$ gene explains a large fraction of the variation in WBC and neutrophil counts, and is responsible for benign neutropenia [50]. This variant, which is monomorphic in Caucasians, is under positive selection in persons of African ancestry because it provides protection against Plasmodium vivax malaria infections. Similarly, genetic variation near the $\alpha$-globin, the $\beta$-globin and the G6PD genes are associated with RBC indices in Africa-derived populations and are relatively common in frequency because they provide a selective advantage against malaria infections. These observations suggest that as we continue to query the human genome for associations with blood cell phenotypes, integrating evidence of natural selection would be a powerful approach.

\section{Genetic Modifiers of Disease Severity}

Several human diseases, which afflict a large fraction of the human population, are characterized by abnormally low or high counts of the three main blood cell types, or some unusual values for their features or contents. Anemia is a decrease of RBC count and hemoglobin levels $(<11 \mathrm{~g} / \mathrm{dL}$ in women or $<13 \mathrm{~g} / \mathrm{dL}$ in men) and is characterized by a wide spectrum of symptoms from simple fatigue to heart failure [51]. The World Health Organization estimates that anemia affects 1.62 billion people in the World [52]. The main causes of anemia are poor nutrition and iron deficiency, infections (e.g., malaria) and RBC diseases such as the hemoglobinopathies. Although the effect size of an individual SNP associated with RBC count or hemoglobin levels is not sufficient to cause anemia, a combination of hemoglobin-reducing alleles at many SNPs could have an impact on the risk to develop this disorder. Maybe more importantly, without causing anemia itself, this genetic score could influence clinical severity in at-risk populations (e.g., children with a small number of hemoglobin-increasing alleles that live in a region where malaria is endemic). Since anemia is mostly frequent in Africa and South-East Asia, it is critical to continue to search for genetic associations with hemoglobin levels in these populations [52].

There are many other human diseases that are diagnosed, like anemia, through abnormal counts of the main blood cell types (e.g., cancers). One example is myeloproliferative neoplasms (MPNs), diseases of the bone marrow characterized by excess cell production [53]. By far the main cause of MPNs is a somatic gain-of-function mutation in the kinase gene JAK2 (Val617Phe), which activates cell proliferation in the myeloid lineage [54,55], and changes platelet formation and reactivity [56]. It has never been tested whether SNPs associated with blood cell counts could modify complication risk in MPN patients with a JAK2 (Val617Phe) mutation. For instance, MPN patients are at high risk of stroke, but it is unknown if such patients that also carry a large number of platelet-increasing alleles are at even higher stroke risk. Such analyses, on MPNs but also all other diseases characterized by a blood phenotype, are simple and could test the role that SNPs associated with normal variation in hematological traits may have on our risk to develop more severe disorders and related complications [18]. 


\section{BCL11A Modifies Clinical Severity in Hemoglobinopathies}

In adults, hemoglobin ( $\mathrm{HbA})$ is composed of two $\alpha$ - and two $\beta$-globin subunits that form a tetramer with the heme moiety to transport oxygen from the lungs to the different organs. Prior to birth, the $\beta$-globin gene is silent and the $\beta$-globin subunits are encoded by the $\gamma$-globin genes to form fetal hemoglobin $(\mathrm{HbF})$. The switch from $\mathrm{HbF}$ to $\mathrm{HbA}$ production is a transcriptionally and epigenetically tightly regulated process [57]. For most healthy individuals, the switch itself has no clinical impact. However, for $\beta$-thalassemia and sickle cell disease patients with mutations in the $\beta$-globin gene, understanding and modulating the globin switch is currently the most promising therapeutic strategy. Conceptually, this is easy to appreciate: if the disease-causing mutations are in the $\beta$-globin gene, then re-activating $\gamma$-globin gene expression to form "normal" $\beta$-globin subunits would bypass the problem. This approach is supported by an extensive literature on the natural history of hemoglobinopathies and epidemiological studies [58]. For instance, it has been shown that sickle cell disease patients that normally produce more $\mathrm{HbF}$ have better survival prognostic and less severe disease complications than patients with low HbF levels [59-61].

Although as adults we mostly produce $\mathrm{HbA}$, we continue to make residual levels of $\mathrm{HbF}$. Inter-individual variation in $\mathrm{HbF}$ levels is highly heritable $\left(h^{2} \sim 0.6-0.9\right)$ [2,62]. Genetic investigations, including GWAS, have identified common genetic variation at three loci $(B C L 11 A$, $H B S 1 L-M Y B$ and $\beta$-globin) that have strong phenotypic effects and that together explain almost half of the heritable variation in $\mathrm{HbF}$ levels [63-66]. These HbF-associated SNPs are also associated with clinical severity in $\beta$-hemoglobinopathy patients: transfusion-dependency in $\beta$-thalassemia and painful crises in sickle cell disease $[65,67,68]$. This again emphasizes the importance of $\mathrm{HbF}$ as a strong modifier of severity for these diseases.

$B C L 11 \mathrm{~A}$ encodes a transcription factor that had no known function in the globin switch before its discovery in two GWAS for HbF levels $[63,65]$. Since then, we have learned that BCL11A is a potent transcriptional repressor of $\gamma$-globin gene expression and that its inactivation in the erythroid lineage can treat a sickle cell disease mouse model through re-activation of $\mathrm{HbF}$ production $[69,70]$. More recently, both genetic and molecular fine-mapping work has determined that HbF-associated SNPs located in a BCL11A intron disrupt en erythroid enhancer that controls BCL11A expression [71]. This model was confirmed by targeted deletion of the enhancer through genome engineering that blocked BCL11A expression and re-activated $\gamma$-globin gene expression and HbF production [16]. As genome editing methods are rapidly improving, this proof-of-concept experiment suggests a new therapeutic strategy in which the $B C L 11 A$ enhancer would be deleted ex vivo in a hemoglobinopathy patient's cells to re-activate $\mathrm{HbF}$ production, and the cells would then be transplanted back to the patient [72]. The characterization of BCL11A and its role in HbF production serves as a powerful example to illustrate the success of GWAS from new biology to potentially innovative therapy.

\section{Orphan Blood Cell Diseases}

Although we did not assess the statistical significance of the enrichment, we observed that many of the SNPs associated with blood cell traits are located near genes that are mutated in severe hematological disorders and inherited in a Mendelian fashion. These include SNPs near HK1 
(hemolytic anemia), TMPRSS6, HFE and TFR2 (iron deficiency) or TUBB1 (thrombocytopenia). This observation is similar to the situation of many other complex human phenotypes (e.g., lipids, height, diabetes) where GWAS have identified hypomorphic alleles near human syndrome genes for related phenotypes. As such, the long list of loci found by GWAS provides a framework to investigate human syndromes characterized by aberrant blood features, mapped to a chromosome arm by linkage studies, but where the gene culprit has not been identified yet.

Table 3. Orphan human syndromes mapped to a chromosomal band and characterized by a blood cell phenotype. Only such syndromes that overlap with a locus identified by GWAS for the corresponding blood cell trait are included in this table. We generated this list by querying the Online Mendelian Inheritance in Man (OMIM) database with the following keywords: anemia, blood, hemoglobin, leukopenia, neutropenia, platelet, thrombocytopenia.

\begin{tabular}{|c|c|c|c|c|c|c|c|c|}
\hline \multicolumn{4}{|c|}{ Mendelian genetics: orphan syndromes } & \multicolumn{5}{|c|}{ Genome-wide association studies } \\
\hline Locus & Disease & OMIM\# & Description & SNP & Position & Phenotype & $\begin{array}{l}\text { Candidate- } \\
\text { gene(s) }\end{array}$ & Ref. \\
\hline $5 \mathrm{q} 31$ & Familial eosinophilia & 131400 & $\begin{array}{l}\text { Characterized by peripheral } \\
\text { hypereosinophilia with or } \\
\text { without other organ } \\
\text { involvement }\end{array}$ & rs4143832 & $\begin{array}{l}\text { chr5: } \\
131,862,977\end{array}$ & $\begin{array}{l}\text { Eosinophil } \\
\text { count }\end{array}$ & IL5 & {$[33]$} \\
\hline $6 \mathrm{p} 21$ & $\begin{array}{l}\text { Macroblobulinemia, } \\
\text { susceptibility to } \\
\text { Waldenstrom }\end{array}$ & 153600 & $\begin{array}{l}\text { Malignant B-cell neoplasm } \\
\text { characterized by } \\
\text { lymphoplasmacytic infiltration } \\
\text { of the bone marrow and } \\
\text { hypersecretion of monoclonal } \\
\text { immunoglobulin M (IgM) } \\
\text { protein }\end{array}$ & rs2517524 & $\begin{array}{l}\text { chr6: } \\
31,025,713\end{array}$ & $\begin{array}{l}\text { White blood } \\
\text { cell }\end{array}$ & $H L A$ region & {$[45]$} \\
\hline $15 \mathrm{q} 21$ & $\begin{array}{l}\text { Dyserythropoietic } \\
\text { anemia, congenital } \\
\text { type III }\end{array}$ & 105600 & $\begin{array}{l}\text { Characterized by } \\
\text { nonprogressive mild to } \\
\text { moderate hemolytic anemia, } \\
\text { macrocytosis in the peripheral } \\
\text { blood, and giant multinucleated } \\
\text { erythroblasts in the bone } \\
\text { marrow }\end{array}$ & rs 1532085 & $\begin{array}{l}\text { chr15: } \\
58,683,366\end{array}$ & Hemoglobin & $L I P C$ & {$[18]$} \\
\hline $19 q 13$ & $\begin{array}{l}\text { Transient } \\
\text { erythroblastopenia of } \\
\text { childhood }\end{array}$ & 227050 & Red blood cell aplasia & rs3892630 & $\begin{array}{l}\text { chr19: } \\
33,181484\end{array}$ & $\begin{array}{l}\text { Mean } \\
\text { corpuscular } \\
\text { volume }\end{array}$ & NUDTI9 & {$[18]$} \\
\hline
\end{tabular}

To investigate this hypothesis, we queried the Online Mendelian Inheritance in Man (OMIM) database [73]. In a non-exhaustive search, we identified four such orphan diseases where the genomic locations overlap with SNPs identified by GWAS (Table 3). For three of the diseases, GWAS findings suggest a strong candidate gene (IL5, LIPC, NUDT19) for re-sequencing in affected individuals. As we continue to map these rare blood disorders, cross-referencing with GWAS hits may provide a strong filter to prioritize genes for genetic testing. 


\section{Conclusions}

GWAS have identified hundreds of loci that carry common genetic variants associated with RBC, WBC and platelet phenotypes. Many of these genetic associations still need to be linked to causal genes and genetic variants, yet because tractable cellular and animal models are available, this might be simpler for blood cell traits than it is for most complex human phenotypes. By design, GWAS interrogate common DNA variants, leaving untested low-frequency and rare sequence variation. The development of next-generation DNA sequencing platforms and exome genotyping arrays now provides the tools to test the role of this rarer genetic variation on blood cell phenotypes. Much criticism has been raised against GWAS because identified SNPs have poor predictive value; this is also true for SNPs associated with blood cell traits. However, this observation needs to be counter-balanced by the potential gain in improving our understanding of human biology in health and disease. GWAS blood cell trait loci provide new opportunities to study hematopoiesis, natural selection and the various ways common segregating DNA sequence variants can modify disease severity, paving the way for the development of more specific therapies.

\section{Acknowledgments}

This work was funded by grants from the Doris Duke Charitable Foundation (2012126), the Canadian Institute of Health Research (123382) and the Canada Research Chair Program.

\section{Author Contributions}

Wrote the paper: NC, GL.

\section{Conflicts of Interest}

The authors declare no conflict of interest.

\section{References}

1. Orkin, S.H.; Zon, L.I. Hematopoiesis: An evolving paradigm for stem cell biology. Cell 2008, 132, 631-644.

2. Pilia, G.; Chen, W.M.; Scuteri, A.; Orru, M.; Albai, G.; Dei, M.; Lai, S.; Usala, G.; Lai, M.; Loi, P.; et al. Heritability of cardiovascular and personality traits in 6,148 sardinians. PLoS Genet. 2006, 2, e132.

3. Garner, C.; Tatu, T.; Reittie, J.E.; Littlewood, T.; Darley, J.; Cervino, S.; Farrall, M.; Kelly, P.; Spector, T.D.; Thein, S.L. Genetic influences on F cells and other hematologic variables: A twin heritability study. Blood 2000, 95, 342-346.

4. Hoffman, M.; Blum, A.; Baruch, R.; Kaplan, E.; Benjamin, M. Leukocytes and coronary heart disease. Atherosclerosis 2004, 172, 1-6.

5. Boos, C.J.; Lip, G.Y. Assessment of mean platelet volume in coronary artery disease-What does it mean? Thromb. Res. 2007, 120, 11-13. 
6. Nieswandt, B.; Kleinschnitz, C.; Stoll, G. Ischaemic stroke: A thrombo-inflammatory disease? J. Physiol. 2011, 589, 4115-4123.

7. Ebrahim, S.; Davey Smith, G. Mendelian randomization: Can genetic epidemiology help redress the failures of observational epidemiology? Hum. Genet. 2008, 123, 15-33.

8. Voight, B.F.; Peloso, G.M.; Orho-Melander, M.; Frikke-Schmidt, R.; Barbalic, M.; Jensen, M.K.; Hindy, G.; Holm, H.; Ding, E.L.; Johnson, T.; et al. Plasma HDL cholesterol and risk of myocardial infarction: A mendelian randomisation study. Lancet 2012, 380, 572-580.

9. Do, R.; Willer, C.J.; Schmidt, E.M.; Sengupta, S.; Gao, C.; Peloso, G.M.; Gustafsson, S.; Kanoni, S.; Ganna, A.; Chen, J.; et al. Common variants associated with plasma triglycerides and risk for coronary artery disease. Nat. Genet. 2013, 45, 1345-1352.

10. Zeng, S.M.; Yankowitz, J.; Widness, J.A.; Strauss, R.G. Etiology of differences in hematocrit between males and females: Sequence-based polymorphisms in erythropoietin and its receptor. J. Gend. Specif. Med.: JGSM: 2001, 4, 35-40.

11. McLaren, C.E.; Barton, J.C.; Gordeuk, V.R.; Wu, L.; Adams, P.C.; Reboussin, D.M.; Speechley, M.; Chang, H.; Acton, R.T.; Harris, E.L.; et al. Determinants and characteristics of mean corpuscular volume and hemoglobin concentration in white HFE C282Y homozygotes in the hemochromatosis and iron overload screening study. Am. J. Hematol. 2007, 82, 898-905.

12. Lin, J.P.; O’Donnell, C.J.; Jin, L.; Fox, C.; Yang, Q.; Cupples, L.A. Evidence for linkage of red blood cell size and count: Genome-wide scans in the framingham heart study. Am. J. Hematol. 2007, 82, 605-610.

13. Menzel, S.; Jiang, J.; Silver, N.; Gallagher, J.; Cunningham, J.; Surdulescu, G.; Lathrop, M.; Farrall, M.; Spector, T.D.; Thein, S.L. The HBS1L-MYB intergenic region on chromosome 6q23.3 influences erythrocyte, platelet, and monocyte counts in humans. Blood 2007, 110, 3624-3626.

14. Lohmueller, K.E.; Pearce, C.L.; Pike, M.; Lander, E.S.; Hirschhorn, J.N. Meta-analysis of genetic association studies supports a contribution of common variants to susceptibility to common disease. Nat. Genet. 2003, 33, 177-182.

15. Lettre, G. The search for genetic modifiers of disease severity in the beta-hemoglobinopathies. Cold Spring Harbor Perspect. Med. 2012, 2, doi:10.1101/cshperspect.a015032.

16. Sankaran, V.G.; Ludwig, L.S.; Sicinska, E.; Xu, J.; Bauer, D.E.; Eng, J.C.; Patterson, H.C.; Metcalf, R.A.; Natkunam, Y.; Orkin, S.H.; et al. Cyclin D3 coordinates the cell cycle during differentiation to regulate erythrocyte size and number. Genes Dev. 2012, 26, 2075-2087.

17. Gieger, C.; Radhakrishnan, A.; Cvejic, A.; Tang, W.; Porcu, E.; Pistis, G.; Serbanovic-Canic, J.; Elling, U.; Goodall, A.H.; Labrune, Y.; et al. New gene functions in megakaryopoiesis and platelet formation. Nature 2011, 480, 201-208.

18. Van der Harst, P.; Zhang, W.; Mateo Leach, I.; Rendon, A.; Verweij, N.; Sehmi, J.; Paul, D.S.; Elling, U.; Allayee, H.; Li, X.; et al. Seventy-five genetic loci influencing the human red blood cell. Nature 2012, 492, 369-375.

19. Lindblad-Toh, K.; Garber, M.; Zuk, O.; Lin, M.F.; Parker, B.J.; Washietl, S.; Kheradpour, P.; Ernst, J.; Jordan, G.; Mauceli, E.; et al. A high-resolution map of human evolutionary constraint using 29 mammals. Nature 2011, 478, 476-482. 
20. Adzhubei, I.A.; Schmidt, S.; Peshkin, L.; Ramensky, V.E.; Gerasimova, A.; Bork, P.; Kondrashov, A.S.; Sunyaev, S.R. A method and server for predicting damaging missense mutations. Nat. Methods 2010, 7, 248-249.

21. Kumar, P.; Henikoff, S.; Ng, P.C. Predicting the effects of coding non-synonymous variants on protein function using the sift algorithm. Nat. Protoc. 2009, 4, 1073-1081.

22. Wu, C.; Orozco, C.; Boyer, J.; Leglise, M.; Goodale, J.; Batalov, S.; Hodge, C.L.; Haase, J.; Janes, J.; Huss, J.W., 3rd; et al. BioGPS: An extensible and customizable portal for querying and organizing gene annotation resources. Genome Biol. 2009, 10, R130.

23. Cookson, W.; Liang, L.; Abecasis, G.; Moffatt, M.; Lathrop, M. Mapping complex disease traits with global gene expression. Nat. Rev. Genet. 2009, 10, 184-194.

24. Bernstein, B.E.; Birney, E.; Dunham, I.; Green, E.D.; Gunter, C.; Snyder, M. An integrated encyclopedia of DNA elements in the human genome. Nature 2012, 489, 57-74.

25. Bernstein, B.E.; Stamatoyannopoulos, J.A.; Costello, J.F.; Ren, B.; Milosavljevic, A.; Meissner, A.; Kellis, M.; Marra, M.A.; Beaudet, A.L.; Ecker, J.R.; et al. The NIH roadmap epigenomics mapping consortium. Nat. Biotechnol. 2010, 28, 1045-1048.

26. Maurano, M.T.; Humbert, R.; Rynes, E.; Thurman, R.E.; Haugen, E.; Wang, H.; Reynolds, A.P.; Sandstrom, R.; Qu, H.; Brody, J.; et al. Systematic localization of common disease-associated variation in regulatory DNA. Science 2012, 337, 1190-1195.

27. Paul, D.S.; Albers, C.A.; Rendon, A.; Voss, K.; Stephens, J.; van der Harst, P.; Chambers, J.C.; Soranzo, N.; Ouwehand, W.H.; Deloukas, P. Maps of open chromatin highlight cell type-restricted patterns of regulatory sequence variation at hematological trait loci. Genome Res. 2013, 23, 1130-1141.

28. Ward, L.D.; Kellis, M. Haploreg: A resource for exploring chromatin states, conservation, and regulatory motif alterations within sets of genetically linked variants. Nucleic Acids Res. 2012, 40, D930-D934.

29. Schaub, M.A.; Boyle, A.P.; Kundaje, A.; Batzoglou, S.; Snyder, M. Linking disease associations with regulatory information in the human genome. Genome Res. 2012, 22, 1748-1759.

30. Auer, P.L.; Johnsen, J.M.; Johnson, A.D.; Logsdon, B.A.; Lange, L.A.; Nalls, M.A.; Zhang, G.; Franceschini, N.; Fox, K.; Lange, E.M.; et al. Imputation of exome sequence variants into population- based samples and blood-cell-trait-associated loci in African Americans: NHLBI go exome sequencing project. Am. J. Hum. Genet. 2012, 91, 794-808.

31. Chen, Z.; Tang, H.; Qayyum, R.; Schick, U.M.; Nalls, M.A.; Handsaker, R.; Li, J.; Lu, Y.; Yanek, L.R.; Keating, B.; et al. Genome-wide association analysis of red blood cell traits in African Americans: The cogent network. Hum. Mol. Genet. 2013, 22, 2529-2538.

32. Ganesh, S.K.; Zakai, N.A.; van Rooij, F.J.; Soranzo, N.; Smith, A.V.; Nalls, M.A.; Chen, M.H.; Kottgen, A.; Glazer, N.L.; Dehghan, A.; et al. Multiple loci influence erythrocyte phenotypes in the charge consortium. Nat. Genet. 2009, 41, 1191-1198.

33. Gudbjartsson, D.F.; Bjornsdottir, U.S.; Halapi, E.; Helgadottir, A.; Sulem, P.; Jonsdottir, G.M.; Thorleifsson, G.; Helgadottir, H.; Steinthorsdottir, V.; Stefansson, H.; et al. Sequence variants affecting eosinophil numbers associate with asthma and myocardial infarction. Nat. Genet. 2009, 41, 342-347. 
34. Kamatani, Y.; Matsuda, K.; Okada, Y.; Kubo, M.; Hosono, N.; Daigo, Y.; Nakamura, Y.; Kamatani, N. Genome-wide association study of hematological and biochemical traits in a Japanese population. Nat. Genet. 2010, 42, 210-215.

35. Lo, K.S.; Wilson, J.G.; Lange, L.A.; Folsom, A.R.; Galarneau, G.; Ganesh, S.K.; Grant, S.F.; Keating, B.J.; McCarroll, S.A.; Mohler, E.R., 3rd; et al. Genetic association analysis highlights new loci that modulate hematological trait variation in caucasians and African Americans. Hum. Genet. 2011, 129, 307-317.

36. Meisinger, C.; Prokisch, H.; Gieger, C.; Soranzo, N.; Mehta, D.; Rosskopf, D.; Lichtner, P.; Klopp, N.; Stephens, J.; Watkins, N.A.; et al. A genome-wide association study identifies three loci associated with mean platelet volume. Am. J. Hum. Genet. 2009, 84, 66-71.

37. Qayyum, R.; Snively, B.M.; Ziv, E.; Nalls, M.A.; Liu, Y.; Tang, W.; Yanek, L.R.; Lange, L.; Evans, M.K.; Ganesh, S.; et al. A meta-analysis and genome-wide association study of platelet count and mean platelet volume in African Americans. PLoS Genet. 2012, 8, e1002491.

38. Soranzo, N.; Spector, T.D.; Mangino, M.; Kuhnel, B.; Rendon, A.; Teumer, A.; Willenborg, C.; Wright, B.; Chen, L.; Li, M.; et al. A genome-wide meta-analysis identifies 22 loci associated with eight hematological parameters in the haemgen consortium. Nat. Genet. 2009, 41, 1182-1190.

39. Soranzo, N.; Rendon, A.; Gieger, C.; Jones, C.I.; Watkins, N.A.; Menzel, S.; Doring, A.; Stephens, J.; Prokisch, H.; Erber, W.; et al. A novel variant on chromosome 7q22.3 associated with mean platelet volume, counts, and function. Blood 2009, 113, 3831-3837.

40. Chambers, J.C.; Zhang, W.; Li, Y.; Sehmi, J.; Wass, M.N.; Zabaneh, D.; Hoggart, C.; Bayele, H.; McCarthy, M.I.; Peltonen, L.; et al. Genome-wide association study identifies variants in TMPRSS6 associated with hemoglobin levels. Nat. Genet. 2009, 41, 1170-1172.

41. Reiner, A.P.; Lettre, G.; Nalls, M.A.; Ganesh, S.K.; Mathias, R.; Austin, M.A.; Dean, E.; Arepalli, S.; Britton, A.; Chen, Z.; et al. Genome-wide association study of white blood cell count in 16,388 African Americans: The continental origins and genetic epidemiology network (cogent). PLoS Genet. 2011, 7, e1002108.

42. Crosslin, D.R.; McDavid, A.; Weston, N.; Nelson, S.C.; Zheng, X.; Hart, E.; de Andrade, M.; Kullo, I.J.; McCarty, C.A.; Doheny, K.F.; et al. Genetic variants associated with the white blood cell count in 13,923 subjects in the emerge network. Hum. Genet. 2012, 131, 639-652.

43. Okada, Y.; Hirota, T.; Kamatani, Y.; Takahashi, A.; Ohmiya, H.; Kumasaka, N.; Higasa, K.; Yamaguchi-Kabata, Y.; Hosono, N.; Nalls, M.A.; et al. Identification of nine novel loci associated with white blood cell subtypes in a Japanese population. PLoS Genet. 2011, 7, e1002067.

44. Li, J.; Glessner, J.T.; Zhang, H.; Hou, C.; Wei, Z.; Bradfield, J.P.; Mentch, F.D.; Guo, Y.; Kim, C.; Xia, Q.; et al. GWAS of blood cell traits identifies novel associated loci and epistatic interactions in Caucasian and African-American children. Hum. Mole. Genet. 2013, 22, 1457-1464.

45. Nalls, M.A.; Couper, D.J.; Tanaka, T.; van Rooij, F.J.; Chen, M.H.; Smith, A.V.; Toniolo, D.; Zakai, N.A.; Yang, Q.; Greinacher, A.; et al. Multiple loci are associated with white blood cell phenotypes. PLoS Genet. 2011, 7, e1002113. 
46. Shameer, K.; Denny, J.C.; Ding, K.; Jouni, H.; Crosslin, D.R.; de Andrade, M.; Chute, C.G.; Peissig, P.; Pacheco, J.A.; Li, R.; et al. A genome- and phenome-wide association study to identify genetic variants influencing platelet count and volume and their pleiotropic effects. Hum. Genet. 2014, 133, 95-109.

47. Okada, Y.; Takahashi, A.; Ohmiya, H.; Kumasaka, N.; Kamatani, Y.; Hosono, N.; Tsunoda, T.; Matsuda, K.; Tanaka, T.; Kubo, M.; et al. Genome-wide association study for C-reactive protein levels identified pleiotropic associations in the IL6 locus. Hum. Mol. Genet. 2011, 20, 1224-1231.

48. Monda, K.L.; Chen, G.K.; Taylor, K.C.; Palmer, C.; Edwards, T.L.; Lange, L.A.; Ng, M.C.; Adeyemo, A.A.; Allison, M.A.; Bielak, L.F.; et al. A meta-analysis identifies new loci associated with body mass index in individuals of African ancestry. Nat. Genet. 2013, 45, 690-696.

49. N’Diaye, A.; Chen, G.K.; Palmer, C.D.; Ge, B.; Tayo, B.; Mathias, R.A.; Ding, J.; Nalls, M.A.; Adeyemo, A.; Adoue, V.; et al. Identification, replication, and fine-mapping of loci associated with adult height in individuals of African ancestry. PLoS Genet. 2011, 7, e1002298.

50. Reich, D.; Nalls, M.A.; Kao, W.H.; Akylbekova, E.L.; Tandon, A.; Patterson, N.; Mullikin, J.; Hsueh, W.C.; Cheng, C.Y.; Coresh, J.; et al. Reduced neutrophil count in people of African descent is due to a regulatory variant in the duffy antigen receptor for chemokines gene. PLoS Genet. 2009, 5, e1000360.

51. Greenburg, A.G. Pathophysiology of anemia. Am. J. Med. 1996, 101, 7S-11S.

52. Worldwide Prevalence of Anaemia 1993-2005 (WHO Global Database on Anaemia). Available online:http://whqlibdoc.Who.Int/publications/2008/9789241596657_eng.pdf (accessed on 19 November 2013).

53. Skoda, R. The genetic basis of myeloproliferative disorders. Am. Soc. Hematol. Educ. Program 2007, 2007, 1-10.

54. Oh, S.T.; Gotlib, J. JAK2 V617F and beyond: Role of genetics and aberrant signaling in the pathogenesis of myeloproliferative neoplasms. Expert Rev. Hematol. 2010, 3, 323-337.

55. Vannucchi, A.M.; Pieri, L.; Guglielmelli, P. JAK2 allele burden in the myeloproliferative neoplasms: Effects on phenotype, prognosis and change with treatment. Ther. Adv. Hematol. 2011, 2, 21-32.

56. Hobbs, C.M.; Manning, H.; Bennett, C.; Vasquez, L.; Severin, S.; Brain, L.; Mazharian, A.; Guerrero, J.A.; Li, J.; Soranzo, N.; et al. JAK2V617F leads to intrinsic changes in platelet formation and reactivity in a knock-in mouse model of essential thrombocythemia. Blood 2013, 122, 3787-3797.

57. Sankaran, V.G.; Xu, J.; Orkin, S.H. Advances in the understanding of haemoglobin switching. Br. J. Haematol. 2010, 149, 181-194.

58. Sankaran, V.G.; Lettre, G.; Orkin, S.H.; Hirschhorn, J.N. Modifier genes in mendelian disorders: The example of hemoglobin disorders. Ann. N. Y. Acad. Sci. 2010, 1214, 47-56. 
59. Platt, O.S.; Brambilla, D.J.; Rosse, W.F.; Milner, P.F.; Castro, O.; Steinberg, M.H.; Klug, P.P. Mortality in sickle cell disease. Life expectancy and risk factors for early death. N. Engl. J. Med. 1994, 330, 1639-1644.

60. Platt, O.S.; Thorington, B.D.; Brambilla, D.J.; Milner, P.F.; Rosse, W.F.; Vichinsky, E.; Kinney, T.R. Pain in sickle cell disease. Rates and risk factors. N. Engl. J. Med. 1991, 325, 11-16.

61. Castro, O.; Brambilla, D.J.; Thorington, B.; Reindorf, C.A.; Scott, R.B.; Gillette, P.; Vera, J.C.; Levy, P.S. The acute chest syndrome in sickle cell disease: Incidence and risk factors. The cooperative study of sickle cell disease. Blood 1994, 84, 643-649.

62. Thein, S.L.; Craig, J.E. Genetics of HB F/F cell variance in adults and heterocellular hereditary persistence of fetal hemoglobin. Hemoglobin 1998, 22, 401-414.

63. Menzel, S.; Garner, C.; Gut, I.; Matsuda, F.; Yamaguchi, M.; Heath, S.; Foglio, M.; Zelenika, D.; Boland, A.; Rooks, H.; et al. A QTL influencing F cell production maps to a gene encoding a zinc-finger protein on chromosome 2p15. Nat. Genet. 2007, 39, 1197-1199.

64. Thein, S.L.; Menzel, S.; Peng, X.; Best, S.; Jiang, J.; Close, J.; Silver, N.; Gerovasilli, A.; Ping, C.; Yamaguchi, M.; et al. Intergenic variants of HBS1L-MYB are responsible for a major quantitative trait locus on chromosome 6q23 influencing fetal hemoglobin levels in adults. Proc. Natl. Acad. Sci. USA 2007, 104, 11346-11351.

65. Uda, M.; Galanello, R.; Sanna, S.; Lettre, G.; Sankaran, V.G.; Chen, W.; Usala, G.; Busonero, F.; Maschio, A.; Albai, G.; et al. Genome-wide association study shows BCL11A associated with persistent fetal hemoglobin and amelioration of the phenotype of beta-thalassemia. Proc. Natl. Acad. Sci. USA 2008, 105, 1620-1625.

66. Galarneau, G.; Palmer, C.D.; Sankaran, V.G.; Orkin, S.H.; Hirschhorn, J.N.; Lettre, G. Fine-mapping at three loci known to affect fetal hemoglobin levels explains additional genetic variation. Nat. Genet. 2010, 42, 1049-1051.

67. Lettre, G.; Sankaran, V.G.; Bezerra, M.A.; Araujo, A.S.; Uda, M.; Sanna, S.; Cao, A.; Schlessinger, D.; Costa, F.F.; Hirschhorn, J.N.; et al. DNA polymorphisms at the BCL11A, HBS1L-MYB, and beta-globin loci associate with fetal hemoglobin levels and pain crises in sickle cell disease. Proc. Natl. Acad. Sci. USA 2008, 105, 11869-11874.

68. Nuinoon, M.; Makarasara, W.; Mushiroda, T.; Setianingsih, I.; Wahidiyat, P.A.; Sripichai, O.; Kumasaka, N.; Takahashi, A.; Svasti, S.; Munkongdee, T.; et al. A genome-wide association identified the common genetic variants influence disease severity in beta(0)-thalassemia/hemoglobin E. Hum. Genet. 2010, 127, 303-314.

69. Sankaran, V.G.; Menne, T.F.; Xu, J.; Akie, T.E.; Lettre, G.; van Handel, B.; Mikkola, H.K.; Hirschhorn, J.N.; Cantor, A.B.; Orkin, S.H. Human fetal hemoglobin expression is regulated by the developmental stage-specific repressor BCL11A. Science 2008, 322, 1839-1842.

70. Xu, J.; Peng, C.; Sankaran, V.G.; Shao, Z.; Esrick, E.B.; Chong, B.G.; Ippolito, G.C.; Fujiwara, Y.; Ebert, B.L.; Tucker, P.W.; et al. Correction of sickle cell disease in adult mice by interference with fetal hemoglobin silencing. Science 2011, 334, 993-996.

71. Bauer, D.E.; Kamran, S.C.; Lessard, S.; Xu, J.; Fujiwara, Y.; Lin, C.; Shao, Z.; Canver, M.C.; Smith, E.C.; Pinello, L.; et al. An erythroid enhancer of BCL11A subject to genetic variation determines fetal hemoglobin level. Science 2013, 342, 253-257. 
72. Hardison, R.C.; Blobel, G.A. Genetics. Gwas to therapy by genome edits? Science 2013, 342, 206-207.

73. Online Mendelian Inheritance in Man. Available online: http://omim.org/ (accessed on 18 November 2013). 


\title{
The Genomic Signature of Breast Cancer Prevention
}

\author{
Jose Russo, Julia Santucci-Pereira and Irma H. Russo
}

\begin{abstract}
The breast of parous postmenopausal women exhibits a specific signature that has been induced by a full term pregnancy. This signature is centered in chromatin remodeling and the epigenetic changes induced by methylation of specific genes which are important regulatory pathways induced by pregnancy. Through the analysis of the genes found to be differentially methylated between women of varying parity, multiple positions at which beta-catenin production and use is inhibited were recognized. The biological importance of the pathways identified in this specific population cannot be sufficiently emphasized because they could represent a safeguard mechanism mediating the protection of the breast conferred by full term pregnancy.
\end{abstract}

Reprinted from Genes. Cite as: Russo, J.; Santucci-Pereira, J.; Russo, I.H. The Genomic Signature of Breast Cancer Prevention. Genes 2014, 5, 65-83.

\section{Introduction}

More than 300 years ago, an excess in breast cancer mortality in nuns was reported, in whom the increased risk was attributed to their childlessness [1] until MacMahon et al. [2] found an almost linear relationship between a woman's risk and the age at which she bore her first child. This work confirmed that pregnancy had a protective effect that was evident from the early teen years and persisted until the middle twenties [1]. Other studies have reported that additional pregnancies and breastfeeding confer greater protection to young women, including a statistically significantly reduced risk of breast cancer in women with deleterious BRCA1 mutations who breast-fed for a cumulative total of more than one year [3,4]. Our studies, designed to unravel what specific changes occurred in the breast during pregnancy that confer a lifetime protection from developing cancer, led us to the discovery that endogenous endocrinological or environmental influences affecting breast development before the first full term pregnancy were important modulators of the susceptibility of the breast to undergo neoplastic transformation. The fact that exposure of the breast of young nulliparous females to environmental physical agents [5] or chemical toxicants $[6,7]$ results in a greater rate of cell transformation suggests that the immature breast possesses a greater number of susceptible cells that can become the site of the origin of cancer, similarly to what has been reported in experimental animal models [8-11]. In these models, the initiation of cancer is prevented by the differentiation of the mammary gland induced by pregnancy [11,12]. The molecular changes involved in this phenomenon are just starting to be unraveled [13-18]. The protection conferred by pregnancy is age-specific since a delay in childbearing after age 24 progressively increases the risk of cancer development. Eventually, this risk becomes greater than that of nulliparous women when the first full term pregnancy (FFTP) occurs after 35 years of age [2]. The higher breast cancer risk which has been associated with early menarche further emphasizes the importance of the length of the susceptibility "window" that encompasses the period of breast development occurring between menarche and the first 
pregnancy, when the organ is more susceptible to undergo complete differentiation under physiological hormonal stimuli. Differentiation is a hallmark that protects the breast from developing cancer by lessening the risk of suffering genetic or epigenetic damages. This postulate is supported by our observations that the architectural pattern of lobular development in parous women with cancer differs from that of parous women without cancer; the former being similar to the architectural pattern of lobular development of nulliparous women with or without cancer. Thus, the higher breast cancer risk in parous women might have resulted from either a failure of the breast to fully differentiate under the influence of the hormones of pregnancy and/or proliferation of transformed cells initiated by early damage or genetic predisposition [18].

Numerous studies have been performed to understand how the dramatic modifications that occur during pregnancy in the pattern of lobular development and differentiation, cell proliferation, and steroid hormone receptor content of the breast influence cancer risk [18]. Studies at the molecular level using different platforms for global genome analysis have confirmed the universality of this phenomenon in various strains of rats and mice [13-21]. Studies in experimental animal models have been useful for uncovering the sequential genomic changes occurring in the mammary gland in response to multiple hormonal stimuli of pregnancy that lead to the imprinting of a permanent genomic signature. Our results support our hypothesis that post-menopausal parous women exhibit a genomic "signature" that differs from the expression present in the breast of nulliparous women, who traditionally represent a high breast cancer risk group.

\section{Phenotypic Changes Induced by Pregnancy in the Human Breast}

Our study has been done using core biopsies of nulliparous (NP) and parous (P) postmenopausal women $[22,23]$. The nulliparous group included both nulligravida nulliparous $(\mathrm{NN})$ and gravida nulliparous (GN); both NN and GN women were considered within the NP as a single group for most analyses, unless indicated otherwise. Our previous studies have in great part clarified the role of pregnancy-induced breast differentiation in the reduction in breast cancer risk, as well as the identification of lobules type 1 (Lob 1) or the terminal ductal lobular unit (TDLU) as the site of origin of breast cancer [4,7,24]. The morphological, physiological and genomic changes resulting from pregnancy and hormonally-induced differentiation of the breast and their influence on breast cancer risk have been addressed in previous publications $[4,7,24,25]$. Our observations that during the post-menopausal years the breast of both parous and nulliparous women contains preponderantly Lob 1, and the fact that nulliparous women are at higher risk of developing breast cancer than parous women, indicate that Lob 1 in these two groups of women either differ biologically, or exhibit different susceptibility to carcinogenesis [25]. The breast tissues of the P and NP women contained ducts and Lob 1 [4,12,26].

The microscopic analysis of the breast tissue revealed that the population of luminal cells lining ducts and Lob 1 was composed of cells that were characterized by their nuclear appearance into two types: one that contained large and palely stained nuclei with prominent nucleoli and another consisting of small hyper chromatic nuclei [27]. The pale staining of the large former nuclei is a feature indicative of a high content of non-condensed euchromatin; these nuclei were called euchromatin-rich nuclei (EUN). The hyperchromasia observed in the latter nuclei was indicative of 
chromatin condensation and high content of heterochromatin; these nuclei were identified as heterochromatin-rich nucleus (HTN). The analysis of the distribution of HTN and EUN cells in histological sections of the breast core biopsies revealed that EUN were more abundant in the NP than in the P breast tissues, whereas the inverse was true for the HTN; these differences were statistically significant [27]. We have confirmed the differences between the HTN and EUN using a quantitative image analysis system [27]. The nuclear size (diameter, area and perimeter) of the EUN as a whole was significantly higher $(p<0.05)$ than that of the HTN in both nulliparous and parous women. Differences were also found to be statistically significant $(p<0.05)$ regarding the nuclear shape (nuclear feret ratio) in the breast of nulliparous women, indicating that in these breasts the nuclei of the HTN had a more elongated ellipsoidal shape than the EUN. The light absorbance (mean gray values/nucleus) was always greater for EUN than for HTN of both NP and $\mathrm{P}$ breasts, either considered as two groups or individually, an indication that under densitometric terms HTN were always more densely stained than EUN. Comparison of the EUN of nulliparous vs. parous breasts revealed significant differences in nuclear size, stainability and densitometric energy, leading us to conclude that epithelial cell nuclei were larger, less stainable and with smaller regions with uniform densitometric intensity in nulliparous breasts. Comparison of the HTN of nulliparous $v s$. parous breasts revealed significant differences in nuclear diameter, perimeter, shape and stainability; cell nuclei showed larger contours and more elongated ellipsoidal shape and they were more stainable in nulliparous breasts. These observations indicated that a shift of the EUN cell population to a more densely packed chromatin cell (HTN) had occurred in association with the history of pregnancy as a distinctive pattern of the postmenopausal parous breast [27].

Since chromatin condensation is part of the process of chromatin remodeling towards gene silencing that is highly regulated by methylation of histones, we verified this phenomenon by immunohistochemistry (IHC) incubating NP and P breast tissues with antibodies against histone 3 dimethylated at lysine 9 (H3K9me2) and trimethylated at lysine 27 (H3K27me3) [27]. The IHC stain revealed that methylation of $\mathrm{H} 3$ at both lysine 9 and 27 was increased in the heterochromatin condensed nuclei of epithelial cells of the parous breast when compared to the euchromatin rich nuclei of the nulliparous breast. In the nulliparous breast, the reactivity in individual cells was less intense and the number of positive cells was significantly lower. These variations in chromatin reorganization were supported by the upregulation of CBX3, CHD2, L3MBTL, and EZH2 genes controlling this process (Table 1) [27].

Table 1. Genes upregulated in the parous breast.

\begin{tabular}{cccc}
\hline Symbol & Log Ratio & P value & Gene Name \\
\hline & \multicolumn{2}{c}{ Apoptosis $(G O: 0006915 ;$ GO:0006917; GO:0008624; GO:0042981) } \\
CASP4 & 0.37 & 0.0003 & caspase 4, apoptosis-related cysteine peptidase \\
RUNX3 & 0.36 & 0.0000 & runt-related transcription factor 3 \\
LUC7L3 & 0.34 & 0.0002 & LUC7-like 3 (S. cerevisiae) \\
ELMO3 & 0.30 & 0.0003 & engulfment and cell motility 3 \\
& & DNA repair (GO:0006281; GO:0006284) \\
SFPQ & 0.46 & 0.0002 & splicing factor proline/glutamine-rich \\
MBD4 & 0.36 & 0.0003 & methyl-CpG binding domain protein 4 \\
RBBP8 & 0.32 & 0.0000 & retinoblastoma binding protein 8 \\
\hline
\end{tabular}


Table 1. Cont.

\begin{tabular}{|c|c|c|c|}
\hline Symbol & Log Ratio & $P$ value & Gene Name \\
\hline \multicolumn{4}{|c|}{ Cell adhesion (GO:0007155; GO:0030155) } \\
\hline NRXN1 & 0.60 & 0.0001 & neurexin 1 \\
\hline DSC3 & 0.51 & 0.0000 & desmocollin 3 \\
\hline COL27A1 & 0.44 & 0.0002 & collagen, type XXVII, alpha 1 \\
\hline PNN & 0.37 & 0.0001 & pinin, desmosome associated protein \\
\hline COL4A6 & 0.36 & 0.0008 & collagen, type IV, alpha 6 \\
\hline LAMC2 & 0.34 & 0.0008 & laminin, gamma 2 \\
\hline COL7A1 & 0.33 & 0.0002 & collagen, type VII, alpha 1 \\
\hline COL16A1 & 0.31 & 0.0000 & collagen, type XVI, alpha 1 \\
\hline LAMA3 & 0.30 & 0.0008 & laminin, alpha 3 \\
\hline \multicolumn{4}{|c|}{ Cell cycle (GO:0000075; GO:0007049; GO:0045786) } \\
\hline SYCP2 & 0.45 & 0.0000 & synaptonemal complex protein 2 \\
\hline PNN & 0.37 & 0.0001 & pinin, desmosome associated protein \\
\hline RUNX3 & 0.36 & 0.0000 & runt-related transcription factor 3 \\
\hline RBBP8 & 0.32 & 0.0000 & retinoblastoma binding protein 8 \\
\hline \multicolumn{4}{|c|}{ Cell differentiation (GO:0001709; GO:0030154; GO:0030216) } \\
\hline MGP & 0.53 & 0.0003 & matrix Gla protein \\
\hline KRT5 & 0.41 & 0.0002 & keratin 5 \\
\hline GATA3 & 0.35 & 0.0009 & GATA binding protein 3 \\
\hline LAMA3 & 0.30 & 0.0008 & laminin, alpha 3 \\
\hline \multicolumn{4}{|c|}{ Cell proliferation (GO:0008283; GO:0008284; GO:0008285; GO:0042127; GO:0050679; GO:0050680) } \\
\hline PTN & 0.67 & 0.0002 & Pleiotrophin \\
\hline KRT5 & 0.41 & 0.0002 & keratin 5 \\
\hline RUNX3 & 0.36 & 0.0000 & runt-related transcription factor 3 \\
\hline IL28RA & 0.34 & 0.0003 & interleukin 28 receptor, alpha (interferon, lambda receptor) \\
\hline CDCA7 & 0.31 & 0.0005 & cell division cycle associated 7 \\
\hline \multicolumn{4}{|c|}{ Cell motility (GO:0006928; GO:0030334) } \\
\hline DNALI1 & 0.37 & 0.0001 & dynein, axonemal, light intermediate chain 1 \\
\hline LAMA3 & 0.30 & 0.0008 & laminin, alpha 3 \\
\hline \multicolumn{4}{|c|}{ G-protein coupled receptor pathway (GO:0007186) } \\
\hline OXTR & 0.54 & 0.0006 & oxytocin receptor \\
\hline \multicolumn{4}{|c|}{$\begin{array}{l}\text { RNA metabolic process (GO:0000398; GO:0001510; GO:0006376; GO:0006396; GO:0006397; GO:0006401; } \\
\text { GO:0008380) }\end{array}$} \\
\hline METTL3 & 0.69 & 0.0000 & methyltransferase like 3 \\
\hline HNRPDL & 0.65 & 0.0001 & heterogeneous nuclear ribonucleoprotein D-like \\
\hline HNRNPD & 0.59 & 0.0003 & $\begin{array}{c}\text { heterogeneous nuclear ribonucleoprotein } \mathrm{D}(\mathrm{AU} \text {-rich element RNA } \\
\text { binding protein } 1,37 \mathrm{kDa})\end{array}$ \\
\hline HNRNPA2B1 & 0.56 & 0.0003 & heterogeneous nuclear ribonucleoprotein A2/B1 \\
\hline SFPQ & 0.47 & 0.0006 & splicing factor proline/glutamine-rich \\
\hline RBM25 & 0.38 & 0.0009 & RNA binding motif protein 25 \\
\hline RBMX & 0.38 & 0.0000 & RNA binding motif protein, $\mathrm{X}$-linked \\
\hline LUC7L3 & 0.34 & 0.0002 & LUC7-like 3 (S. cerevisiae) \\
\hline SFRS1 & 0.30 & 0.0001 & splicing factor, arginine/serine-rich 1 \\
\hline \multicolumn{4}{|c|}{ RNA transport (GO:0050658) } \\
\hline HNRNPA2B1 & 0.56 & 0.0003 & heterogeneous nuclear ribonucleoprotein $\mathrm{A} 2 / \mathrm{B} 1$ \\
\hline
\end{tabular}


Table 1. Cont.

\begin{tabular}{|c|c|c|c|}
\hline Symbol & Log Ratio & $P$ value & Gene Name \\
\hline \multicolumn{4}{|c|}{$\begin{array}{c}\text { Transcription (GO:0006350; GO:0006355; GO:0006357; GO:0006366; GO:0016481; GO:0045449; GO:0045893; } \\
\text { GO:0045941) }\end{array}$} \\
\hline HNRPDL & 0.65 & 0.0001 & heterogeneous nuclear ribonucleoprotein D-like \\
\hline HNRNPD & 0.59 & 0.0003 & $\begin{array}{l}\text { heterogeneous nuclear ribonucleoprotein } \mathrm{D}(\mathrm{AU} \text {-rich element } \\
\text { RNA binding protein } 1,37 \mathrm{kDa})\end{array}$ \\
\hline CBX3 & 0.53 & 0.0003 & chromobox homolog 3 (HP1 gamma homolog, Drosophila) \\
\hline NFKBIZ & 0.48 & 0.0001 & $\begin{array}{l}\text { nuclear factor of kappa light polypeptide gene enhancer } \\
\text { in B-cells inhibitor, zeta }\end{array}$ \\
\hline FUBP1 & 0.47 & 0.0002 & far upstream element (FUSE) binding protein 1 \\
\hline SFPQ & 0.47 & 0.0006 & splicing factor proline/glutamine-rich \\
\hline $\mathrm{EZH} 2$ & 0.44 & 0.0000 & enhancer of zeste homolog 2 (Drosophila) \\
\hline ZNF207 & 0.41 & 0.0007 & zinc finger protein 207 \\
\hline ZNF711 & 0.41 & 0.0003 & zinc finger protein 711 \\
\hline GATA3 & 0.38 & 0.0009 & GATA binding protein 3 \\
\hline PNN & 0.37 & 0.0003 & pinin, desmosome associated protein \\
\hline ZNF107 & 0.37 & 0.0001 & zinc finger protein 107 \\
\hline RUNX3 & 0.36 & 0.0000 & runt-related transcription factor 3 \\
\hline CCNL1 & 0.35 & 0.0009 & cyclin L1 \\
\hline ZNF692 & 0.34 & 0.0000 & zinc finger protein 692 \\
\hline CHD2 & 0.33 & 0.0001 & chromodomain helicase DNA binding protein 2 \\
\hline RBBP8 & 0.32 & 0.0000 & retinoblastoma binding protein 8 \\
\hline ZNF789 & 0.32 & 0.0005 & zinc finger protein 789 \\
\hline CDCA7 & 0.31 & 0.0005 & cell division cycle associated 7 \\
\hline \multicolumn{4}{|c|}{ Chromatin organization (GO:0006333; GO:0006338) } \\
\hline $\mathrm{CBX} 3$ & 0.53 & 0.0003 & chromobox homolog 3 (HP1 gamma homolog, Drosophila) \\
\hline CHD2 & 0.33 & 0.0001 & chromodomain helicase DNA binding protein 2 \\
\hline \multicolumn{4}{|c|}{ Cell division (GO:0051301) } \\
\hline SYCP2 & 0.45 & 0.0000 & synaptonemal complex protein 2 \\
\hline \multicolumn{4}{|c|}{ DNA metabolic process (GO:0006139; GO:0006260; GO:0006310; GO:0015074) } \\
\hline METTL3 & 0.69 & 0.0000 & methyltransferase like 3 \\
\hline SFPQ & 0.46 & 0.0002 & splicing factor proline/glutamine-rich \\
\hline GOLGA2B & 0.32 & 0.0001 & golgin A2 family, member B \\
\hline \multicolumn{4}{|c|}{ Lactation (GO:0007595) } \\
\hline OXTR & 0.54 & 0.0006 & oxytocin receptor \\
\hline
\end{tabular}

\section{Transcriptomic Differences Induced by Pregnancy}

Analysis of P and NP gene expression microarrays revealed that there were 305 probe sets, corresponding to 208 distinct genes, differentially expressed between these two groups. Of the 305 probe sets, 267 were up- and 38 were down-regulated [22,23]. From these 267 up-regulated genes, we described biological processes that were representative of the transcriptomic differences between the parous and the nulliparous breasts. Using bioinformatics based analysis of microarray data, we found that the biological processes involving the splicing machinery and mRNA processing were prevalent in the parous breast and were represented by the following upregulated genes: LUC7L3, SFRS1, HNRNPA2B1, HNRNPD, RBM25, SFRS5, METTL3, HNRNPDL, and 
SFPQ (Table 1). Transcription regulation and chromatin organization were also highly represented in the parous breast by the upregulation of CBX3, EBF1, GATA3, RBBP8, CCNL1, CCNL2, CDCA7, EZH2, FUBP1, NFKBIZ, RUNX3, ZNF107, ZNF207, ZNF692, ZNF711, ZNF789, CDCA7, and ZNF692 (Table 1). The parous breast also expressed upregulation of six non-coding regions that included XIST, MALAT-1 (or NEAT2) and NEAT1 [27].

Genes that were down-regulated in the parous breast represented transcription regulation, encompassing CBL, FHL5, NFATC3, NCR3C1, TCF7L2, and a set of genes that were involved in IGF-like growth factor signaling, somatic stem cell maintenance, muscle cell differentiation and apoptosis, such as IGF1, RASD1, EBF1, SOX 1,SOX6, SOX 17, RALGAPA2 and ABHD5. In rodents, also was observed the reduction of expression of genes related to growth factors, such as Igf1 [15]. The level of expression was confirmed to be differentially expressed between nulliparous and parous breast tissues by real time RT-PCR for the following genes: CREBZF, XIST, MALAT1, NEAT1, CCNL2, GATA3, DDX17, HNRPDL, SOX6, SNHG12, SOX 17 and C1orf168 [23]. In addition to the level of expression, the localization of the alternative splicing regulator cyclin L2 protein (CCNL2) [28], was verified by IHC. CCNL2 protein was expressed in the nucleus of epithelial cells in breast tissues from NP and P women, although the level of expression was significantly higher in Lob 1 in the parous breast when compared with similar structures found in the breast of nulliparous women. These observations confirmed the localization of this gene product in the splicing factor compartment (nuclear speckles) [29].

\section{Shifting of the Cell Population in the Human Breast}

We found a shift in the cell population of the postmenopausal breast as a manifestation of the reprogramming of the organ after pregnancy. These observations are in agreement with what is observed in the rat mammary gland, which also contains two types of luminal epithelial cells, designated dark (DC) and intermediate (IC) cells, in addition to the myoepithelial cells [30]. The DC and IC are equivalent to the HTN and EUN cells described in the present work. DCs increase after pregnancy and lactational involution; whereas the ICs significantly outnumber the DC in ductal hyperplasias and ductal carcinomas [30,31]. Our analysis of nuclear ultrastructural and morphometric parameters of rodent IC have allowed us to differentiate the mammary progenitor stem cell from the cancer stem cells [25,30,31]. Nuclear morphometric analysis of breast and ovarian carcinomas has confirmed the predictive value of nuclear grade on the progression of premalignant lesions to invasiveness [32-34]. Our findings of a significant decrease in the number of EUN with a subsequent increase in the number of HTN cells expressing specific biomarkers identified at the chromatin and transcriptional levels support the value of morphometric analysis as an adjuvant to molecular studies [27]. Our data clearly indicate that there are morphological indications of chromatin remodeling in the parous breast, such as the increase in the number of epithelial cells with condensed chromatin and increased reactivity with anti-H3K9me2 and H3K27me3 antibodies. Histone methylation is a major determinant for the formation of active and inactive regions of the genome and is crucial for the proper programming of the genome during development [35]. In the parous breast, there is upregulation of transcription factors and chromatin remodeling genes such as CHD2 or chromodomain helicase DNA binding protein 2 and the CBX3 
or Chromobox homolog 3, whose products are required for controlling recruitment of protein/protein or DNA/protein interactions. CBX3 is involved in transcriptional silencing in heterochromatin-like complexes, and recognizes and binds $\mathrm{H} 3$ tails methylated at lysine 9, leading to epigenetic repression. Two other important genes related to the polycomb group (PcG) protein that are upregulated in the parous breast are the L3MBTL gene or 1(3)mbt-like and the histone-lysine $\mathrm{N}$-methyltransferase or EZH2. Members of the PcG form multimeric protein complexes that maintain the transcriptional repressive state of genes over successive cell generations (Table 1). EZH2 is an enzyme that acts mainly as a gene silencer, performing this role by the addition of three methyl groups to lysine 27 of histone 3 , a modification that leads to chromatin condensation [30,36,37].

\section{Methylation Changes in the DNA of Parous Women are Part of Chromatin Remodeling and the Genomic Signature of Pregnancy}

The chromatin remodeling process is demonstrated not only by the shifting of the EUN to the HTN cells, but also confirmed by the increase in methylation of histones H3K9me2 and H3K27me3. This is an indication that methylation of other genes could also be involved in the process. Using the DNA from five nulliparous and five parous breast core biopsies and applying the MBD-cap sequencing methodology [38], we have identified 583 genes showing different levels of methylation between the parous and nulliparous breasts. From the 583 genes, 455 were hypermethylated in the parous while 128 were hypermethylated in the nulliparous breast, confirming the reprogramming of the chromatin to a more silenced or resting stage. To get a better understanding of the methylation profile of the 583 genes, we used Integrative Genomics Viewer (IGV) software [39,40]. IGV was utilized to identify the distinct areas, throughout the entire gene, where the methylation levels differed between the sample groups. The identification of these areas, known as differentially methylated regions (DMRs), is important because they are more likely to affect gene expression [41]. We performed the comparison between the nulliparous and parous methylation profiles against the human reference genome "hg 18" and against each other. For example, the gene COBRA 1, which is the cofactor of BRCA1 and has been shown to work in its regulatory pathway [42], was hypermethylated in the nulliparous breast. It is shown in Figure 1 that the methylation levels for each sample at each base pair that an area of higher methylation occurring in at least four of the samples of one group as compared to all members of the opposing group, that area was defined as a (DMR) (Figures 1 and 2). COBRA1 had a DMR near the end of the gene, which was marked in Figure 1 using the IGV's marking tool. When a differentially methylated area is found and marked, hovering over the red marker at the top of the sample area gives the exact chromosomal location. Every gene within the 583 gene list was closely examined for DMRs. The chromosomal locations at which these DMRs were found and marked were recorded in Tables 2 and 3. 
Figure 1. Overview of how the DNA methylation levels appear in the Integrative Genomics Viewer (IGV). At the top of the figure is the ideogram of the chromosome given by IGV, with the area currently being examined marked in red. At the bottom is the overall shape of the gene containing exons and introns. Exons are shown as thicker blue sections on the overall gene. The gray bars represent the methylation levels of each volunteer at each base pair. They are created by combining each read resulting from the sequencing done on the samples. The higher they are, the higher the percentage of methylation is at any given base pair. When there was an area of higher methylation occurring in at least four of the members of one parity group as compared to all members of the opposing group, that area was defined as a differentially methylated region (DMR).

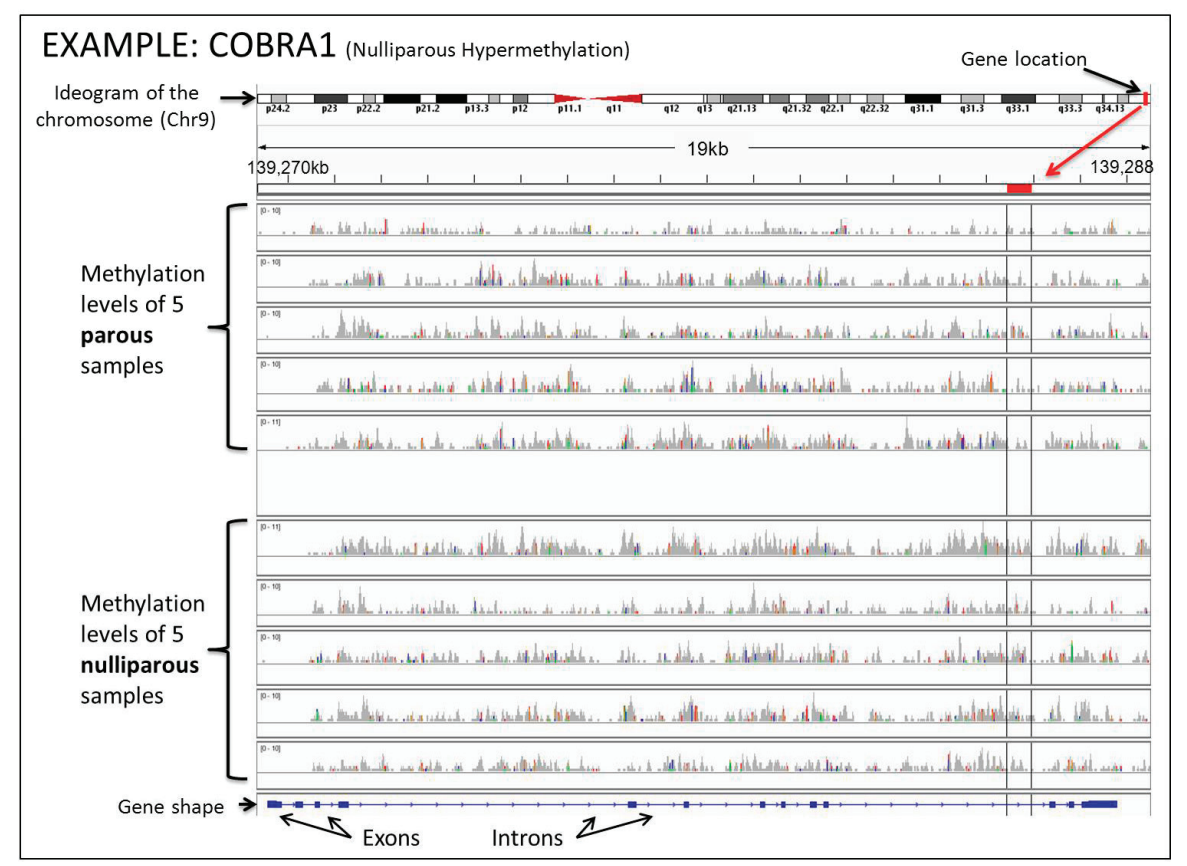

After analysis of the 583 genes using the IGV, we have identified the DMRs of 53 genes. Of the 455 parous hypermethylated genes, 41 had DMRs. These were NEGR1, NUF2, SYT14, POU4F1, FLRT2, ASAP2, DNAJC13, IFITM4P, ZNF292, SDK1, ELAVL4, DACT1, SPATA5L1, DYNC112, NLGN1, MAN1A1, AK5, DPYD, PROX1, PDE3A, NOVA1, SKAP1, ANKRD12, B4GALT5, CNTN4, ROBO1, GSK3B, INPP4B, FNIP2, IL6ST, TICAM2, PPP2CA, C6orf138, PRKAR2B, TTLL7, MAN1A2, CDC42BPA, OSBP, STIM2, NR3C2, and REV3L. The exact locations of these DMRs are recorded in Table 2. A point of interest within these genes is that DNAJC13 and GSK3B, while statistically given to be hypermethylated within parous women, had DMRs which suggested nulliparous hypermethylation. Because of this and for the scope of this experiment, those genes are treated as nulliparous hypermethylated. Of the 128 nulliparous hypermethylated genes, 12 had DMRs. These were NHSL2, PTX4, LRRC37A3, C20orf166-AS1, 
TPPP, NELF, SAMD10, CELSR1, FZD1, TNFRSF18, SRMS, and COBRA1. The chromosomal locations of these DMRs can be seen in Table 3. Within this list only C20orf166-AS1 was found to have a DMR in the direction opposite to what the statistics showed. Visual examples of these differentially methylated areas are seen in Figure 2 and Supplementary Figures S1-S4.

Figure 2. DMRs for PRKAR2B. At the top we see the gene shape, with the red marked DMRs. Any colored locations within the gray bars indicate a nucleotide read which is different from the reference genome.

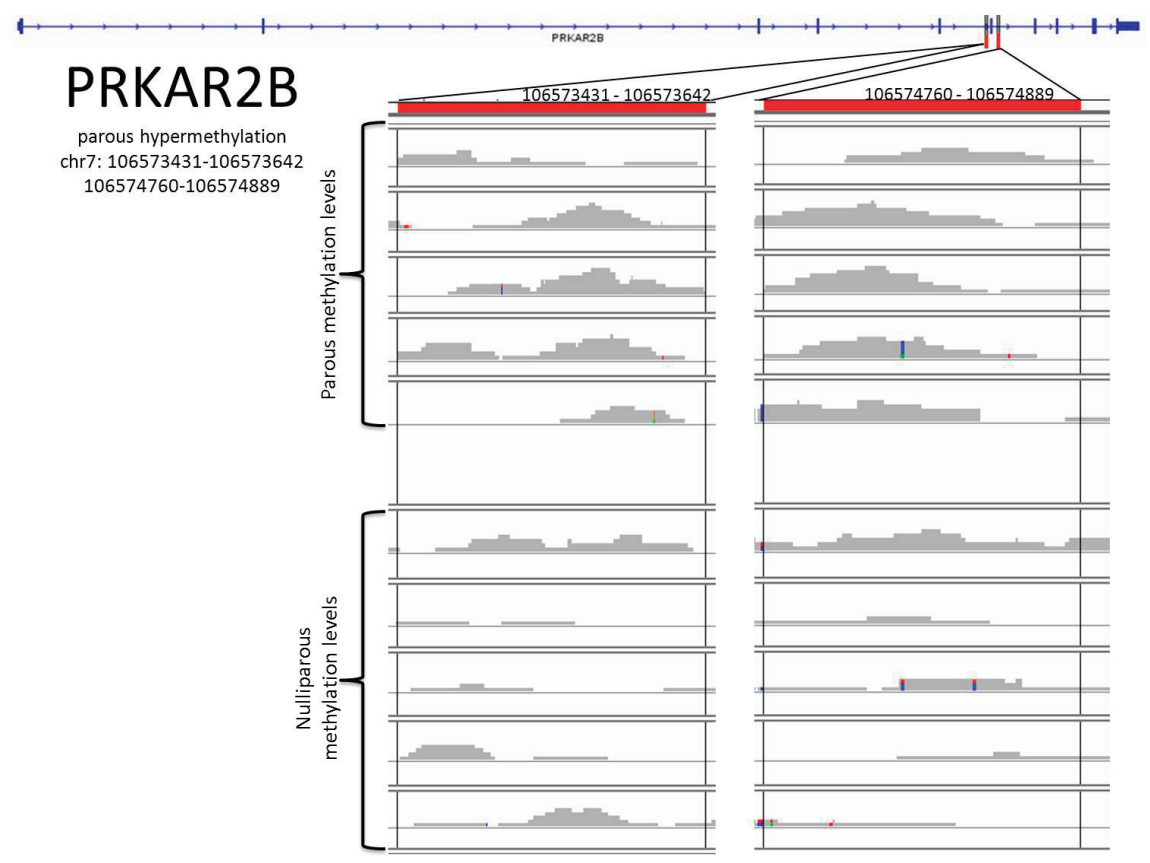

Table 2. DMRs within parous hypermethylated genes.

\begin{tabular}{ccc}
\hline & & Parous Hypermethylated Genes \\
\hline \multirow{2}{*}{ NEGR1 } & \multirow{2}{*}{ chr1 } & $71702567-71703327$ \\
& $72142369-72142934$ \\
\hline \multirow{2}{*}{ NUF2 } & chr1 & $161576182-161576653$ \\
\hline \multirow{2}{*}{ SYT14 } & \multirow{2}{*}{ chr1 } & $208309959-208310406$ \\
& & $208206495-208206910$ \\
\hline POU4F1 & chr13 & $78072725-78073146$ \\
FLRT2 & chr14 & $85155301-85155789$ \\
\hline \multirow{2}{*}{ ASAP2 } & \multirow{2}{*}{ chr2 } & $9266977-9267464$ \\
& Chr3 & $9432659-9433115$ \\
\hline DNAJC13 & Chr6 & $133712540-133712930$ \\
\hline IFITM4P & Chr6 & $29826792-29827266$ \\
\hline ZNF292 & \multirow{2}{*}{ Chr7 } & $88022117-88022631$ \\
\hline \multirow{2}{*}{ SDK1 } & Chr1 & $4121961-4122279$ \\
& Chr14 & $4230104-4230384$ \\
\hline ELAVL4 & Chr15 & $50387715-50388146$ \\
\hline DACT1 & & $58182547-58182717$ \\
\hline SPATA5L1 & $43494615-43495210$ \\
\hline
\end{tabular}


Table 2. Cont.

\begin{tabular}{|c|c|c|}
\hline \multicolumn{3}{|c|}{ Parous Hypermethylated Genes } \\
\hline DYNC1I2 & Chr2 & $172279940-172280462$ \\
\hline \multirow{3}{*}{ NLGN1 } & \multirow{3}{*}{$\mathrm{Chr} 3$} & $175147546-175148159$ \\
\hline & & $175156296-175156626$ \\
\hline & & $175277928-175278476$ \\
\hline MAN1A1 & Chr6 & $119623891-119624320$ \\
\hline \multirow{2}{*}{ AK5 } & \multirow{2}{*}{ Chr1 } & $77616541-77616886$ \\
\hline & & $77655265-77655548$ \\
\hline DPYD & Chr1 & $98153997-98154252$ \\
\hline PROX1 & Chr1 & $212267523-212267905$ \\
\hline PDE3A & Chr12 & $20432463-20432808$ \\
\hline NOVA1 & Chr14 & $26015695-26016215$ \\
\hline SKAP1 & Chr17 & $43591761-43592022$ \\
\hline ANKRD12 & Chr18 & $9168269-9168654$ \\
\hline B4GALT5 & Chr20 & $47704095-47704520$ \\
\hline CNTN4 & Chr3 & $2572819-2573349$ \\
\hline ROBO1 & Chr3 & $79026030-79023709$ \\
\hline GSK3B & Chr3 & $121258375-121258501$ \\
\hline \multirow{3}{*}{ INPP4B } & \multirow{3}{*}{ Chr4 } & $143292977-143293319$ \\
\hline & & $143347212-143347585$ \\
\hline & & $143966478-143966985$ \\
\hline \multirow{2}{*}{ FNIP2 } & \multirow{2}{*}{ Chr4 } & $159911129-159911596$ \\
\hline & & $160015288-160015809$ \\
\hline IL6ST & Chr5 & $55271135-55271466$ \\
\hline \multirow{2}{*}{ TICAM2 } & \multirow{2}{*}{ Chr5 } & $114955685-114955992$ \\
\hline & & $114956473-114956938$ \\
\hline PPP2CA & Chr5 & $133567556-133567871$ \\
\hline \multirow{2}{*}{ C6orf138 } & \multirow{2}{*}{ Chr6 } & $48025616-48025836$ \\
\hline & & $48067151-48067418$ \\
\hline \multirow{2}{*}{ PRKAR2B } & \multirow{2}{*}{ Chr7 } & $106573431-106573642$ \\
\hline & & $106574760-106574889$ \\
\hline TTLL7 & Chr1 & $84185339-84185660$ \\
\hline MAN1A2 & Chr1 & $117816180-117816444$ \\
\hline CDC42BPA & Chr1 & $225520202-225520399$ \\
\hline \multirow{2}{*}{ OSBP } & \multirow{2}{*}{ Chr11 } & $59121100-59121437$ \\
\hline & & $59121927-59122155$ \\
\hline STIM2 & $\mathrm{Chr} 4$ & $26572404-26572775$ \\
\hline NR3C2 & Chr4 & $149367631-149368052$ \\
\hline REV3L & Chr6 & $111804054-111804285$ \\
\hline
\end{tabular}

Table 3. DMRs within nulliparous hypermethylated genes.

\begin{tabular}{ccc}
\hline & Nulliparous Hypermethylated Genes \\
\hline NHSL2 & chrX & $71270541-71271527$ \\
C16orf38 (PTX4) & Chr16 & $1476600-1476773$ \\
LRRC37A3 & Chr17 & $60311872-60311982$ \\
C20orf200 (C20orf166-AS1) & Chr20 & $60557111-60557421$ \\
TPPP & Chr5 & $742334-742618$ \\
NELF & Chr9 & $139471353-139471653$ (HYPO) \\
SAMD10 & Chr20 & $139471653-139471895$ \\
CELSR1 & Chr22 & $62077471-62077661$ \\
FZD1 & Chr7 & $45272965-45273071$ \\
TNFRSF18 & Chr1 & $90733372-90733621$ \\
SRMS & Chr20 & $1130349-1130634$ \\
COBRA1 & Chr9 & $61646714-61647041$ \\
& & $139285424-139285977$ \\
\hline
\end{tabular}


Analysis and research into the functions of these 53 genes identified seven which interacted with each other in either the Wnt signaling pathway or its controlling PI3K/AKT/mTOR pathways. The DMRs of these genes (DACT1, PPP2CA, GSK3B, ROBO1, INPP4B, IL6ST, FZD1) are shown in Supplementary Figures S1-S4. An overview of the involvement in the canonical Wnt pathway is shown in Figure 3. The interworking of these genes with each other and with other genes within the statistically methylated 583 can be seen in Figure 4.

Figure 3. Canonical WNT/ $\beta$-catenin signaling genes marked in green are hypermethylated in parous women (suggesting down-regulation of the gene in parous women). Genes in red are hypermethylated within nulliparous women. Genes marked with $(*)$ were observed differentially expressed the microarray data. This canonical pathway was generated through the use of IPA (Ingenuity ${ }^{\circledR}$ Systems) [43].



Of the seven genes with DMRs which we have shown to work together in the Wnt pathway or its controllers, three worked directly in canonical Wnt signaling. Interestingly, when we analyzed the genes differentially expressed between parous and nulliparous [23], we found genes that also participate in the Wnt pathway, such as CSNK1A1 and SOX family (Figure 3). FZD1, which is the hypermethylated in the nulliparous breast, codes for the Frizzled receptor. When activated, this 
receptor directly activates Disheveled (Dsh) in the cytosol to begin the Wnt signaling cascade [44]. GSK3B, which also contains DMRs hypermethylated in the nulliparous women, has as main rule to decrease beta-catenin levels in the Wnt signaling pathway [45]. PPP2CA (PP2A) is suggested to work both upstream and downstream of beta-catenin to assist in its stabilization [46]. DACT1 assists in Wnt signaling by up-regulating GSK3B [47]. ROBO1, INPP4B and IL6ST genes are active in PI3K dependent AKT signaling [48-50].

Figure 4. Interaction of target genes in $W n t / \beta$-catenin signaling. The green genes are statistically parous hypermethylated, while the ones colored red are statistically nulliparous hypermethylated. The darker genes have recorded DMRs, and this is to the exception of GSK3B, which was first found statistically significant hypermethylated in the parous breast, but its DMR is hypermethylated in the nulliparous samples. This network was generated through the use of IPA (Ingenuity ${ }^{\circledR}$ Systems) [43].



The potential significance of the Wnt signaling pathway is rooted in an experiment performed in 1982 to find which genes would mutate in mice injected with mouse mammary tumor virus locating int1, a proto-oncogene [51]. Int1 was soon found to be highly conserved across multiple species, including drosophila and humans. Int1 was discovered to be the mammalian homologue of the drosophila Wingless $(\mathrm{Wg})$, a gene previously found to be a segment polarity gene in embryonic development. The Wnt signaling pathway was given its name from the combination of $\mathrm{Wg}$ and int1, and has always had a close relationship to both differentiation and breast cancer.

Mammary development requires complex, reciprocal epithelial mesenchymal interactions. During embryonic development, Wnt signaling is involved in the initiation and early formation of mammary buds [52]. Then, during pregnancy, the pathway is activated to help the differentiation of mammary ducts in preparation for lactation. It does this by increasing beta-catenin levels in the cytosol and the nucleus, which in turn increases epithelial-mesenchymal transition and aids in 
transcription. After weaning, the mammary glands go through involution and the E-cadherin binding domain for beta-catenin is truncated [53]. This decreases cellular adhesion and signal epithelial apoptosis. The result is a lessened need for beta-catenin. In fact, overexpression of betacatenin during involution results in a lack of complete involution [54]. This suggests that lowered beta-catenin expression is essential for proper mammary involution. Studies in mouse model systems clearly demonstrate that activated Wnt signaling leads to mammary tumorigenesis [55]. Misra et al. observed alteration in Fzd4 and Wnt2 expression in rats after full term pregnancy [20]. Other studies have shown an increase in cytosolic/nuclear beta-catenin in about $60 \%$ of breast cancers. This is usually explained by the pathway's ability to aid in epithelial-mesenchymal transition and cell proliferation, two things incredibly important in the progression of cancer. Recently, the Wnt signaling pathway has been directly implicated in the parity induced protective effect against breast cancer [56]. It was revealed that parity induces differentiation and down-regulates the Wnt/Notch signaling ratio of basal stem/progenitor cells in mice. The down-regulation was attributed to a reduced expression of Wnt4, a necessary ligand in the activation stages of the Wnt pathway, in the mammary cells of parous mice [56].

The nulliparous hypermethylation of FZD1 suggests an up-regulation of the Frizzled family receptors and through this an up-regulation of all three types of Wnt signaling, indeed, we observed a slight overexpression of this gene in the parous women (not statistically significant). Increased Wnt signaling is associated with an increase in EMT in both development and cancer [57,58]. However, despite the Wnt signaling pathways being seemingly up-regulated, key genes within the pathways appear within our data to be down-regulated, thus changing the outcome of the signals sent through the Frizzled receptors. Signals sent through the Fz receptors activate the phosphoprotein Disheveled (Dsh). Dsh has three highly conserved protein domains, which interact differently depending on which Wnt pathway it is interacting with [44]. An up-regulation of FZD1 assumes an overall up-regulation of Dsh activation, and thus an increase in all three Wnt pathways. The three pathways are the canonical Wnt/beta-catenin pathway, the noncanonical planar cell polarity (PCP) pathway, and the noncanonical Wnt/calcium pathway.

The canonical pathway is the only one to involve beta-catenin, which is the TCF/LEF binding protein responsible for increased transcription and EMT [57,58]. Intracellular beta-catenin levels are maintained through constant creation and destruction, the processes of which are suggested to be regulated differently between our parity groups.

The canonical Wnt pathway contains the beta-catenin destruction complex, which is usually down-regulated or disrupted after the activation of Wnt signaling. The most effective way this occurs is through the binding of Fz to LRP5/6, which will disrupt the destruction complex before it can begin [59]. Our analysis showed an increased methylation of LRP5 within parous women, which suggests a decreased expression of LRP5/6 and a decreased cellular capability to stop the beta-catenin destruction complex in this way. The beta-catenin destruction complex begins with the binding of GSK3 to Axin, which leaves GSK3's active site open to phosphorylate beta-catenin. Once phosphorylated, beta-catenin is ubiquitinated and sent to the proteasome for removal [59]. It is suggested that initial tumor development requires rapid and effective repression of GSK3B [58]. In our analysis through IGV, GSK3B was found to have a DMR hypermethylated in the nulliparous 
samples. This suggests an increase in expression of GSK3 within parous women and subsequently an increase in the activity of the beta-catenin destruction complex.

PPP2CA, found to be hypermethylated within parous women, is also closely involved in canonical Wnt signaling. While the effect of PPP2CA in this context is still unclear, research leans toward a positive ability to stabilize beta-catenin [59]. The parous hypermethylation of PPP2CA, which suggests a lower expression in parous women, supports the idea of decreased beta-catenin.

The noncanonical Wnt/calcium pathway, which is also found to be up-regulated in parous women as a result of increased FZD1 expression, occurs independently of beta-catenin. However, the noncanonical Wnt/calcium pathway is an inhibitor of canonical Wnt/beta-catenin signaling further along the line by stopping the transcriptional efforts of beta-catenin in the nucleus [60]. This inhibition occurs in one of two ways. The first uses the CaMKII-TAK1-NLK pathway, which inhibits beta-catenin-TCF-dependent transcription through the phosphorylation of TCF. The second uses NFAT-mediated transcriptional regulation to suppress beta-catenin-dependent-transcription.

Whereas more mechanistic studies need to be done in human breast cells, the data analyzed thus far indicate that the methylation of genes involved in Wnt signaling pathway could be another path involved in the protective effect of pregnancy in the human breast.

\section{Conclusions}

Our work [22,23,27] clearly demonstrates that the breast of parous postmenopausal women exhibits a specific signature that has been induced by a full term pregnancy. This signature reveals for the first time that the differentiation process is centered in chromatin remodeling and the epigenetic changes induced by methylation of specific genes, that are important regulatory pathways induced by pregnancy. Through the analysis of the genes found to be differentially methylated between women of varying parity, multiple positions at which beta-catenin production and use is inhibited were recognized. First, the ability of the Fz receptor to bind to LRP5/6 and disrupt the beta-catenin destruction complex was down-regulated by a decrease in LRP5. Then, an increase in GSK3B suggests a strong up-regulation of the beta-catenin destruction complex, wherein GSK3B is responsible for marking beta-catenin for deletion. Third, a decrease in PPP2CA lowers its ability to stabilize beta-catenin. All of these transpire to decrease the amount of beta-catenin able to make it through the cytosol and into the nucleus. Once in the nucleus, however, the increased expression of the noncanonical Wnt/calcium signaling pathway interferes with the ability to beta-catenin to bind to TCF and help in transcription and EMT. The added effect of all of these differential methylations leans toward the conclusion that beta-catenin, especially as it pertains to the Wnt signaling pathway, is regulated differently between parous and nulliparous women. The decrease in beta-catenin production and accumulation may be a leftover effect from mammary involution, which would have been the last process of remodeling the mammary glands had undergone. This suggests that the decreased capacity for beta-catenin accumulation caused by involution is what causes the protective effect of pregnancy against breast cancer. The biological importance of the pathways identified in this specific population cannot be sufficiently emphasized due to the fact that they could represent another safeguard mechanism besides the ones discussed earlier [27], mediating the protection of the breast conferred by full term pregnancy. 


\section{Acknowledgments}

This work was supported by grant 02-2008-034 from the Avon Foundation for Women Breast Cancer Research Program, NIH core grant CA06927 to Fox Chase Cancer Center and an appropriation from the Commonwealth of Pennsylvania. The authors thank the women of Norrbotten County, Sweden, for their willing contribution to the project and the staff of the Mammography Department, Sunderby Hospital, Luleå, Sweden.

\section{Author Contributions}

Designed the experiments: JR, IHR, JS-P. Analyzed the data: JR, JS-P. Wrote the paper: JR, JS-P, IHR.

\section{Conflicts of Interest}

The authors declare no conflict of interest.

\section{References}

1. Clarke, C.A.; Purdie, D.M.; Glaser, S.L. Population attributable risk of breast cancer in white women associated with immediately modifiable risk factors. BMC Cancer 2006, 6, 170-181.

2. MacMahon, B.; Cole, P.; Lin, T.M.; Lowe, C.R.; Mirra, A.P.; Ravnihar, B.; Salber, E.J.; Valaoras, V.G.; Yuasa, S. Age at first birth and breast cancer risk. Bull. World Health Organ. 1970, 43, 209-221.

3. Jemal, A.; Siegel, R.; Ward, E.; Murray, T.; Xu, J.; Thun, M.J. Cancer statistics, 2007. CA Cancer J. Clin. 2007, 57, 43-66.

4. Russo, J.; Balogh, G.A.; Russo, I.H. Full-Term pregnancy induces a specific genomic signature in the human breast. Cancer Epidemiol. Biomark. Prev. 2008, 17, 51-66.

5. Russo, J.; Russo, I.H. Influence of differentiation and cell kinetics on the susceptibility of the rat mammary gland to carcinogenesis. Cancer Res. 1980, 40, 2677-2687.

6. Tay, L.K.; Russo, J. Formation and removal of 7,12-dimethylbenz[a]anthracene-nucleic acid adducts in rat mammary epithelial cells with different susceptibility to carcinogenesis. Carcinogenesis 1981, 2, 1327-1333.

7. Russo, I.H.; Koszalka, M.; Russo, J. Comparative study of the influence of pregnancy and hormonal treatment on mammary carcinogenesis. Br. J. Cancer 1991, 64, 481-484.

8. Sinha, D.K.; Pazik, J.E.; Dao, T.L. Prevention of mammary carcinogenesis in rats by pregnancy: Effect of full-term and interrupted pregnancy. Br. J. Cancer 1988, 57, 390-394.

9. Srivastava, P.; Russo, J.; Mgbonyebi, O.P.; Russo, I.H. Growth inhibition and activation of apoptotic gene expression by human chorionic gonadotropin in human breast epithelial cells. Anticancer Res. 1998, 18, 4003-4010.

10. Thomas, D.B.; Rosenblatt, K.A.; Ray, R.M. Re: "Breastfeeding and reduced risk of breast cancer in an Icelandic cohort study". Am. J. Epidemiol. 2001, 154, 975-977.

11. Russo, J.; Moral, R.; Balogh, G.A.; Mailo, D.; Russo, I.H. The protective role of pregnancy in breast cancer. Breast Cancer Res. 2005, 7, 131-142. 
12. Russo, J.; Russo, I.H. Role of differentiation in the pathogenesis and prevention of breast cancer. Endocr. Relat. Cancer 1997, 4, 7-21.

13. Srivastava, P.; Russo, J.; Russo, I.H. Chorionic gonadotropin inhibits rat mammary carcinogenesis through activation of programmed cell death. Carcinogenesis 1997, 18, 1799-1808.

14. Ginger, M.R.; Gonzalez-Rimbau, M.F.; Gay, J.P.; Rosen, J.M. Persistent changes in gene expression induced by estrogen and progesterone in the rat mammary gland. Mol. Endocrinol. 2001, 15, 1993-2009.

15. D'Cruz, C.M.; Moody, S.E.; Master, S.R.; Hartman, J.L.; Keiper, E.A.; Imielinski, M.B.; Cox, J.D.; Wang, J.Y.; Ha, S.I.; Keister, B.A.; et al. Persistent parity-induced changes in growth factors, TGF-beta3, and differentiation in the rodent mammary gland. Mol. Endocrinol. 2002, 16, 2034-2051.

16. Henry, M.D.; Triplett, A.A.; Oh, K.B.; Smith, G.H.; Wagner, K.U. Parity-induced mammary epithelial cells facilitate tumorigenesis in MMTV-neu transgenic mice. Oncogene 2004, 23, 6980-6985.

17. Medina, D. Breast cancer: The protective effect of pregnancy. Clin. Cancer Res. 2004, 10, 380S-384S.

18. Endocrine control of breast development. In Molecular Basis of Breast Cancer: Prevention and Treatment; Russo, J., Russo, I.H., Eds.; Springer: Berlin, Germany, 2004; pp. 64-67.

19. Ginger, M.R.; Rosen, J.M. Pregnancy-Induced changes in cell-fate in the mammary gland. Breast Cancer Res. 2003, 5, 192-197.

20. Misra, Y.; Bentley, P.A.; Bond, J.P.; Tighe, S.; Hunter, T.; Zhao, F.Q. Mammary gland morphological and gene expression changes underlying pregnancy protection of breast cancer tumorigenesis. Physiol. Genomics 2012, 44, 76-88.

21. Santucci-Pereira, J.; George, C.; Armiss, D.; Russo, I.; Vanegas, J.; Sheriff, F.; Lopez de Cicco, R.; Su, Y.; Russo, R.; Bidinotto, L.; et al. Mimicking pregnancy as a strategy for breast cancer prevention. Breast Cancer Manag. 2013, 2, 283-294.

22. Belitskaya-Levy, I.; Zeleniuch-Jacquotte, A.; Russo, J.; Russo, I.H.; Bordas, P.; Ahman, J.; Afanasyeva, Y.; Johansson, R.; Lenner, P.; Li, X.; et al. Characterization of a genomic signature of pregnancy identified in the breast. Cancer Prev. Res. 2011, 4, 1457-1464.

23. Peri, S.; de Cicco, R.L.; Santucci-Pereira, J.; Slifker, M.; Ross, E.A.; Russo, I.H.; Russo, P.A.; Arslan, A.A.; Belitskaya-Levy, I.; Zeleniuch-Jacquotte, A.; et al. Defining the genomic signature of the parous breast. BMC Med. Genomics 2012, 5, 46-57.

24. Russo, J.; Rivera, R.; Russo, I.H. Influence of age and parity on the development of the human breast. Breast Cancer Res. Treat. 1992, 23, 211-218.

25. Russo, I.H.; Russo, J. Pregnancy-induced changes in breast cancer risk. J. Mammary Gland Biol. Neoplasia 2011, 16, 221-233.

26. Molecular Basis of Breast Cancer: Prevention and Treatment; Russo, J., Russo, I.H., Eds.; Springer-Verlag: Berlin, Germany, 2004; p. 447.

27. Russo, J.; Santucci-Pereira, J.; de Cicco, R.L.; Sheriff, F.; Russo, P.A.; Peri, S.; Slifker, M.; Ross, E.; Mello, M.L.; Vidal, B.C.; et al. Pregnancy-induced chromatin remodeling in the breast of postmenopausal women. Int. J. Cancer 2012, 131, 1059-1070.

28. Long, J.C.; Caceres, J.F. The SR protein family of splicing factors: Master regulators of gene expression. Biochem. J. 2009, 417, 15-27. 
29. Herrmann, A.; Fleischer, K.; Czajkowska, H.; Muller-Newen, G.; Becker, W. Characterization of cyclin L1 as an immobile component of the splicing factor compartment. FASEB J. 2007, $21,3142-3152$.

30. Russo, I.H.; Russo, J. Mammary gland neoplasia in long-term rodent studies. Environ. Health Perspect. 1996, 104, 938-967.

31. Russo, J.; Tait, L.; Russo, I.H. Susceptibility of the mammary gland to carcinogenesis. III. The cell of origin of rat mammary carcinoma. Am. J. Pathol. 1983, 113, 50-66.

32. Tan, P.H.; Goh, B.B.; Chiang, G.; Bay, B.H. Correlation of nuclear morphometry with pathologic parameters in ductal carcinoma in situ of the breast. Mod. Pathol. 2001, 14, 937-941.

33. Bussolati, G.; Marchio, C.; Gaetano, L.; Lupo, R.; Sapino, A. Pleomorphism of the nuclear envelope in breast cancer: A new approach to an old problem. J. Cell Mol. Med. 2008, 12, 209-218.

34. Palmer, J.E.; Sant Cassia, L.J.; Irwin, C.J.; Morris, A.G.; Rollason, T.P. The prognostic value of nuclear morphometric analysis in serous ovarian carcinoma. Int. J. Gynecol. Cancer 2008, 18, 692-701.

35. Cao, R.; Wang, L.; Wang, H.; Xia, L.; Erdjument-Bromage, H.; Tempst, P.; Jones, R.S.; Zhang, Y. Role of histone H3 lysine 27 methylation in Polycomb-group silencing. Science 2002, 298, 1039-1043.

36. Kubicek, S.; Schotta, G.; Lachner, M.; Sengupta, R.; Kohlmaier, A.; Perez-Burgos, L.; Linderson, Y.; Martens, J.H.; O'Sullivan, R.J.; Fodor, B.D.; et al. The role of histone modifications in epigenetic transitions during normal and perturbed development. Ernst. Schering Res. Found. Workshop 2006, 57, 1-27.

37. Lin, W.; Dent, S.Y. Functions of histone-modifying enzymes in development. Curr. Opin. Genet. Dev. 2006, 16, 137-142.

38. Zuo, T.; Tycko, B.; Liu, T.M.; Lin, H.J.; Huang, T.H. Methods in DNA methylation profiling. Epigenomics 2009, 1, 331-345.

39. Robinson, J.T.; Thorvaldsdottir, H.; Winckler, W.; Guttman, M.; Lander, E.S.; Getz, G.; Mesirov, J.P. Integrative genomics viewer. Nat. Biotechnol. 2011, 29, $24-26$.

40. Thorvaldsdottir, H.; Robinson, J.T.; Mesirov, J.P. Integrative Genomics Viewer (IGV): High-performance genomics data visualization and exploration. Brief Bioinform. 2013, 14, 178-192.

41. Rakyan, V.K.; Down, T.A.; Thorne, N.P.; Flicek, P.; Kulesha, E.; Graf, S.; Tomazou, E.M.; Backdahl, L.; Johnson, N.; Herberth, M.; et al. An integrated resource for genome-wide identification and analysis of human tissue-specific differentially methylated regions (tDMRs). Genome Res. 2008, 18, 1518-1529.

42. Aiyar, S.E.; Cho, H.; Lee, J.; Li, R. Concerted transcriptional regulation by BRCA1 and COBRA1 in breast cancer cells. Int. J. Biol. Sci. 2007, 3, 486-492.

43. Ingenuity ${ }^{\circledR}$ Pathway Analysis. v.18030641. Available online: http://www.ingenuity.com/ (accessed on 24 January 2014).

44. Habas, R.; Dawid, I.B. Dishevelled and Wnt signaling: Is the nucleus the final frontier? J. Biol. 2005, 4, 2. 
45. Wang, X.; Goode, E.L.; Fredericksen, Z.S.; Vierkant, R.A.; Pankratz, V.S.; Liu-Mares, W.; Rider, D.N.; Vachon, C.M.; Cerhan, J.R.; Olson, J.E.; et al. Association of genetic variation in genes implicated in the beta-catenin destruction complex with risk of breast cancer. Cancer Epidemiol. Biomarkers Prev. 2008, 17, 2101-2108.

46. Ratcliffe, M.J.; Itoh, K.; Sokol, S.Y. A positive role for the PP2A catalytic subunit in Wnt signal transduction. J. Biol. Chem. 2000, 275, 35680-35683.

47. Cheyette, B.N.; Waxman, J.S.; Miller, J.R.; Takemaru, K.; Sheldahl, L.C.; Khlebtsova, N.; Fox, E.P.; Earnest, T.; Moon, R.T. Dapper, a Dishevelled-associated antagonist of beta-catenin and JNK signaling, is required for notochord formation. Dev. Cell 2002, 2, 449-461.

48. Zhao, L.; Hart, S.; Cheng, J.; Melenhorst, J.J.; Bierie, B.; Ernst, M.; Stewart, C.; Schaper, F.; Heinrich, P.C.; Ullrich, A.; et al. Mammary gland remodeling depends on gp130 signaling through Stat3 and MAPK. J. Biol. Chem. 2004, 279, 44093-44100.

49. Chang, P.H.; Hwang-Verslues, W.W.; Chang, Y.C.; Chen, C.C.; Hsiao, M.; Jeng, Y.M.; Chang, K.J.; Lee, E.Y.; Shew, J.Y.; Lee, W.H. Activation of Robo1 signaling of breast cancer cells by Slit2 from stromal fibroblast restrains tumorigenesis via blocking PI3K/Akt/beta-catenin pathway. Cancer Res. 2012, 72, 4652-4661.

50. Bertucci, M.C.; Mitchell, C.A. Phosphoinositide 3-kinase and INPP4B in human breast cancer. Ann. N. Y. Acad. Sci. 2013, 1280, 1-5.

51. Nusse, R.; Varmus, H. Three decades of Wnts: A personal perspective on how a scientific field developed. EMBO J. 2012, 31, 2670-2684.

52. Turashvili, G.; Bouchal, J.; Burkadze, G.; Kolar, Z. Wnt signaling pathway in mammary gland development and carcinogenesis. Pathobiology 2006, 73, 213-223.

53. Vallorosi, C.J.; Day, K.C.; Zhao, X.; Rashid, M.G.; Rubin, M.A.; Johnson, K.R.; Wheelock, M.J.; Day, M.L. Truncation of the beta-catenin binding domain of E-cadherin precedes epithelial apoptosis during prostate and mammary involution. J. Biol. Chem. 2000, 275, 3328-3334.

54. Imbert, A.; Eelkema, R.; Jordan, S.; Feiner, H.; Cowin, P. Delta N89 beta-catenin induces precocious development, differentiation, and neoplasia in mammary gland. J. Cell Biol. 2001, $153,555-568$.

55. Howe, L.R.; Brown, A.M. Wnt signaling and breast cancer. Cancer Biol. Ther. 2004, 3, 36-41.

56. Meier-Abt, F.; Milani, E.; Roloff, T.; Brinkhaus, H.; Duss, S.; Meyer, D.S.; Klebba, I.; Balwierz, P.J.; van Nimwegen, E.; Bentires-Alj, M. Parity induces differentiation and reduces Wnt/Notch signaling ratio and proliferation potential of basal stem/progenitor cells isolated from mouse mammary epithelium. Breast Cancer Res. 2013, 15, R36.

57. Prasad, C.P.; Rath, G.; Mathur, S.; Bhatnagar, D.; Parshad, R.; Ralhan, R. Expression analysis of E-cadherin, Slug and GSK3beta in invasive ductal carcinoma of breast. BMC Cancer 2009, 9, 325-335.

58. Logullo, A.F.; Nonogaki, S.; Pasini, F.S.; Osorio, C.A.; Soares, F.A.; Brentani, M.M. Concomitant expression of epithelial-mesenchymal transition biomarkers in breast ductal carcinoma: Association with progression. Oncol. Rep. 2010, 23, 313-320. 
59. Kimelman, D.; Xu, W. Beta-catenin destruction complex: Insights and questions from a structural perspective. Oncogene 2006, 25, 7482-7491.

60. Sugimura, R.; Li, L. Noncanonical Wnt signaling in vertebrate development, stem cells, and diseases. Birth Defects Res. C Embryo Today 2010, 90, 243-256. 


\section{Lessons from Genome-Wide Search for Disease-Related Genes with Special Reference to HLA-Disease Associations}

\section{Katsushi Tokunaga}

Abstract: The relationships between diseases and genetic factors are by no means uniform. Single-gene diseases are caused primarily by rare mutations of specific genes. Although each single-gene disease has a low prevalence, there are an estimated 5000 or more such diseases in the world. In contrast, multifactorial diseases are diseases in which both genetic and environmental factors are involved in onset. These include a variety of diseases, such as diabetes and autoimmune diseases, and onset is caused by a range of various environmental factors together with a number of genetic factors. With the astonishing advances in genome analysis technology in recent years and the accumulation of data on human genome variation, there has been a rapid progress in research involving genome-wide searches for genes related to diseases. Many of these studies have led to the recognition of the importance of the human leucocyte antigen (HLA) gene complex. Here, the current state and future challenges of genome-wide exploratory research into variations that are associated with disease susceptibilities and drug/therapy responses are described, mainly with reference to our own experience in this field.

Reprinted from Genes. Cite as: Tokunaga, K. Lessons from Genome-Wide Search for Disease-Related Genes with Special Reference to HLA-Disease Associations. Genes 2014, 5, 84-96.

\section{Development of Genome-Wide Searches}

The greatest attraction of the strategy of genome-wide searches for genes related to diseases is the potential for the discovery of the involvement of completely new genes that could not have been predicted using existing knowledge or data. The previous method for genome-wide search of multifactorial disease-susceptibility genes was non-parametric linkage analysis, which does not presuppose any specific inheritance mode. One such method is the affected sib-pair method. However, it is not easy to collect a large number of samples with affected sib-pairs, so the detection power of this method is inevitably low [1]. Consequently, only limited results have been obtained so far.

The genome-wide association study (GWAS), however, makes use of the high statistical power of association analysis traditionally used for investigating the possible involvement of specific candidate genes, and applies it genome-wide [1]. Two pioneering GWAS studies were carried out in Japan. One was the first single nucleotide polymorphism (SNP)-based GWAS for myocardial infarction, which utilized an approximately 90,000 SNPs [2]. The other was the first microsatellite-based GWAS for rheumatoid arthritis, which used approximately 30,000 microsatellite polymorphisms [3]. However, only a few research groups adopted either of these platforms, due to the labor and cost they involved.

GWAS advanced to a new stage from 2006 onward, mainly as a result of two developments in infrastructure. The first was information infrastructure, typified by the Database of Single Nucleotide Polymorphisms (dbSNP) [4], the International HapMap Project [5] and the 1000 Genomes Project [6], which gathered together a vast range of information of genome variation that spanned the 
entire human genome. The other development was in technology infrastructure; this was the commercial release of platforms that allowed the analysis of several thousands of samples performed on several hundreds of thousands of SNPs and could be carried out relatively easily. The application of these developments meant that SNP-based GWAS became a broad-based, practical strategy, and in 2007, several studies were published from large-scale collaborations between multiple institutions. The subsequent rush to discover gene polymorphisms associated with different diseases or traits using GWAS was dramatic, and over 1600 types of significant associations with 250 diseases or traits have been reported [7]. Nevertheless, attention should be paid for GWAS in ethnically diverse populations, since the genome-wide SNP typing chips have been designed based on mainly European data, these chips may have limited utility in certain populations.

\section{Identified Susceptibility Genes to Multifactorial Diseases}

\subsection{Population Differences in Disease Susceptibility Genes}

A disease for which GWAS have shown striking results is type II diabetes. In 2007, several groups from Europe and North America reported results from different GWAS on several thousand patients and controls [8-11]. Over 11 susceptibility loci were identified, and over half of these were newly discovered. The following year, two independent groups from Japan reported a new susceptibility gene, $K C N Q 1[12,13]$. Table 1 shows a comparison between European and Japanese populations of the allele frequency, odds ratio and $p$-value of TCF7L2, the most important susceptibility gene found in European populations, and $K C N Q 1$, which was discovered in Japanese. $T C F 7 L 2$ showed a $p$-value of $10^{-48}$ in European populations, indicating a definite association with type II diabetes [8]. Among Japanese, however, the $p$-value is at a level of no more than $10^{-4}$ [14]. The main reason for this is the difference in minor allele frequency, which is lower in Japanese by an order of magnitude. Consequently, although the odds ratio is similar to European populations, no clear association was observed in an analysis of 2000 patients and 2000 healthy controls. A contrasting relationship can be seen with KCNQ1 [12]. The $p$-value for Japanese samples was $10^{-29}$, indicating a definite association with type II diabetes, and the same clear association was found for Korean and Chinese samples. However, although European samples showed the same tendency of the odds ratio, the $p$-value was at a level of no more than $10^{-4}$.

Table 1. Population differences of susceptibility genes to type II diabetes.

\begin{tabular}{ccccc}
\hline Gen (SNP) & Population & Odds Ratio & $\boldsymbol{p}$ & Minor Allele Frequency \\
\hline TCF7L2 (rs7903146) & European [8-11] & 1.37 & $1.0 \times 10^{-48}$ & $0.31 / 0.25$ \\
TCF7L2 (rs7903146) & Japanese [14] & 1.70 & $7.0 \times 10^{-4}$ & $0.05 / 0.02$ \\
KCNQ1 (rs2237892) & European [12] & 1.29 & $7.8 \times 10^{-4}$ & $0.03 / 0.05$ \\
KCNQ1 (rs2237892) & Japanese [12] & 1.43 & $3.0 \times 10^{-29}$ & $0.31 / 0.40$ \\
\hline
\end{tabular}

In other words, the main type II diabetes-susceptibility genes for European and East Asian populations, respectively, are, in fact, shared susceptibility genes by both populations, but because they differ greatly in frequency, their contribution in each respective population is different. 
Several genetic factors, in addition to environmental factors, such as stress, are involved in the onset of narcolepsy, one of the hypersomnia. In the past, the only gene well established as a genetic factor for narcolepsy was $H L A-D R / D Q[15]$; then, we carried out a GWAS to search for new genetic factors [16]. As a result, an SNP located between $C P T 1 B$ and $C H K B$ on Chromosome 22 was found to be associated with narcolepsy. Japanese and Koreans were found to have similar allele frequency and both showed a significant association. However, although the odds ratio showed similar trends in European Americans and African Americans, we could not find a significant difference association, because of the low frequency of the susceptibility allele. We have also experienced significant population differences in other diseases, including tuberculosis [17], rheumatoid arthritis [18], glaucoma [19] and primary biliary cirrhosis [20].

The above diseases serve as examples of different contributions of multiple genetic factors in each population. Consequently, the study of each individual population would be essential to build a complete picture of the important genetic factors to complex diseases in the various human populations.

\subsection{Susceptibility Genes Common to Different Diseases}

There has been an increase in the number of reports of genetic factors that are common to different diseases. GPC5 (glypican-5) has been found to be a susceptibility gene common to nephrotic syndrome diseases, such as membranous nephropathy, immunoglobulin A nephropathy and diabetic nephropathy (Table 2) [21]. We further confirmed the expression of the GPC5 protein in the glomerular podocytes and showed that the risk allele is associated with a high level of GPC5 expression.

Table 2. Common susceptibility gene GPC5 (glypican 5) for acquired nephrotic syndrome [21].

\begin{tabular}{ccccc}
\hline Panel & Case: Minor Allele Frequency & Control: Minor Allele Frequency & $\boldsymbol{p}^{*}$ & Odds Ratio \\
\hline 1 & 0.237 & 0.167 & $5.8 \times 10^{-3}$ & $2.33(1.25-4.35)$ \\
2 & 0.195 & 0.159 & $2.0 \times 10^{-5}$ & $3.44(1.89-6.25)$ \\
3 & 0.224 & 0.174 & $8.7 \times 10^{-6}$ & $2.39(1.61-3.55)$ \\
Combined & 0.219 & 0.168 & $6.0 \times 10^{-11}$ & $2.54(1.91-3.40)$ \\
\hline
\end{tabular}

* Based on the recessive model of the minor allele ( $\mathrm{GG}+\mathrm{GA} v s$. AA).

Meta-analysis of the largest-scale GWAS in Japan on rheumatoid arthritis (RA) led to the discovery of susceptibility genes that are common to various different autoimmune disorders [18]. The GWAS was performed on approximately 4000 patients and 17,000 controls, and a replication study was carried out with 5000 patients and 22,000 controls. In addition to previously reported susceptibility genes, nine new susceptibility genes were discovered. Among these are several susceptibility genes that have been also reported for systemic lupus erythematosus (SLE) and Graves' disease.

Another example in our recent experience was primary biliary cirrhosis [20]. We performed a GWAS by a nation-wide collaboration; as a result, we discovered two new susceptibility genes. Interestingly, one of these, TNFSF15, has also been reported as a susceptibility gene for inflammatory bowel disease, including Crohn's disease and ulcerative colitis. There are numerous other reports of genetic factors that are found to be common to various autoimmune and inflammatory diseases [22,23]. 
The presence of common susceptibility genes for different diseases suggests that at least part of the pathogenic mechanism of these diseases is shared. These results may contribute to the elucidation of the pathogenic mechanism of these diseases and to the development of new therapies.

\subsection{Towards the Understanding of Pathogenic Mechanisms}

As mentioned earlier, the new narcolepsy-susceptibility region, $C P T 1 B / C H K B$, was discovered through a GWAS performed to search for genetic factors other than the established factor, HLA [16]. Subjects possessing the risk allele of the susceptibility SNP showed significantly lower levels of mRNA expression of both $C P T 1 B$ and $C H K B$. We also observed that narcolepsy patients show abnormally low levels of carnitine [24], on which $C P T 1 B$ (carnitine palmitoyltransferase 1B) is relevant, and that carnitine improves the sleep of the patients [25]. Carnitine is known as the transporter of long-chain fatty acids into mitochondria, thus playing a crucial role in energy production.

Moreover, the new susceptibility gene, TRA (T cell receptor $\alpha$ ), was discovered through a GWAS performed by a joint international research group [26]. SNPs located in the J region of TRA showed significant associations with narcolepsy in European and Asian populations. TRA and HLA are key molecules in the regulation of immune response in the acquired immunity. The same joint international research group also found that a polymorphism of $P 2 R Y 11$, which is also involved in the regulation of the immune system, is associated with narcolepsy [27]. From these results, it may be assumed that narcolepsy onset has at least two mechanisms: both autoimmunity to orexin (hypocretin)-producing cells and a disorder of fatty acid $\beta$-oxidation.

If we appreciate that multiple susceptibility genes that have been discovered belong to specific pathways or networks, they will provide useful hints toward clarifying the mechanism of disease onset or disease formation and also developing new drugs.

\section{Identified Response Genes to Drugs/Therapies}

\subsection{Development of New Gene Tests}

GWAS studies are extremely useful in the search for drug-response genes. We performed a GWAS as part of a multi-institutional joint research group investigating hepatitis $C$ virus related diseases. As a result of this GWAS, we discovered that $I L 28 B$ on Chromosome 19 was strongly associated with non-responder patients to the combined therapy of PEGylated interferon-alpha and ribavirin for chronic hepatitis C [28]. This was a completely unexpected result. The GWAS was performed on only 78 non-responders and 64 responders to this therapy; nevertheless a $p$-value at the level of $10^{-12}$ was obtained, reaching the genome-wide significance level (Figure 1). About $70 \%-80 \%$ of the non-responding patients possessed the minor alleles of several SNPs in the $I L 28 B$ region, and combining the replication study data, the $p$-value was $10^{-27}-10^{-32}$ and the odds ratio was $17-30$ (Figure 2).

Response to the interferon-alpha therapy had been considered to be determined mainly by the virus genotype and concentration. However, the discovery that response is, in fact, mostly determined by a human genetic factor had a major impact. IL28B SNP typing has already been 
introduced into the routine clinical testing in Japan and is used as important reference data in the determination of therapeutic strategies.

Figure 1. A genome-wide association study (GWAS) on the response to the combined therapy of PEGylated interferon-alpha and ribavirin for chronic hepatitis $\mathrm{C}$ identified two SNPs on Chromosome 19 [28].



Figure 2. The strong association of $I L 28 B$ with therapy response for chronic hepatitis C: $80 \%$ of non-responders possess the minor allele [28].

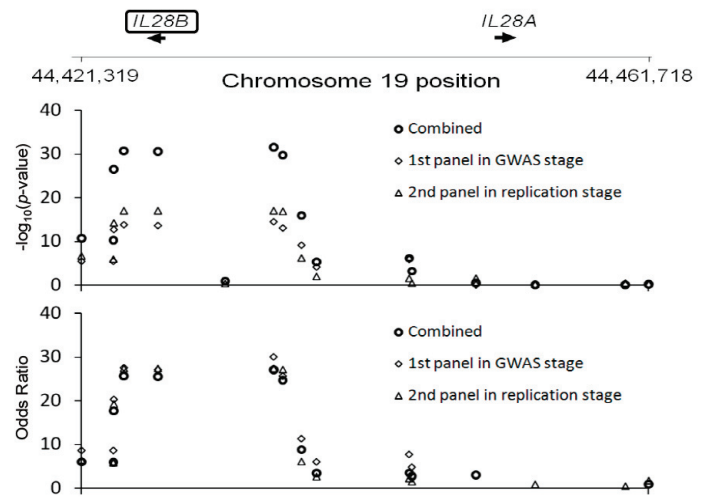

\subsection{Identification of New Therapeutic Targets}

The discovery of $I L 28 B$, which is strongly associated with response to treatment for hepatitis C, indicated another highly interesting possibility. $I L 28 B$ is a member of the interferon $\lambda$ family and is assumed to exhibit its defensive activity against viral infection mediated by similar receptors and intracellular signal transduction pathway as interferon $\alpha$, which was used in the treatment of hepatitis C. $I L 28 B$ itself is therefore expected to be a powerful contender for the development of new hepatitis $\mathrm{C}$ drugs. In fact, $I L-29$, a member of the same family, has already been subjected to clinical trial for a new drug. 
In addition to the above, genes involved in response to many drugs have been reported, and an increasing number of genetic factors are being identified for the first time as a result of GWAS. Drug-response genes generally tend to show greater odds ratios than disease-susceptibility genes, so that even with a relatively small sample size, there is a high likelihood of being able to identify the relevant gene. Ever greater results may therefore be expected in the future.

\section{Particular Importance of HLA}

\subsection{Immune-Mediated Diseases and HLA}

GWAS studies have been conducted for a number of diseases to date, and many of these have reported $H L A$ as a susceptibility gene. In our own experience, narcolepsy [16], hepatitis B [29], rheumatoid arthritis [18], primary biliary cirrhosis [20], Stevens-Johnson syndrome, insulin autoimmune syndrome and type I diabetes have all shown strong association with certain $H L A$ gene(s). Of these, narcolepsy, rheumatoid arthritis, primary biliary cirrhosis, type I diabetes and insulin autoimmune syndrome were associated most strongly with the $H L A-D R$ and $H L A-D Q$ regions, while hepatitis B and Stevens-Johnson syndrome were associated most strongly with the HLA-DP and $H L A-A$ genes, respectively.

With regard to narcolepsy, Juji et al. [30] first reported in 1984 an extremely strong association with $H L A-D R 2$ (HLA-DRB1*1501-DQB1*0602 haplotype according to the recent sequence-level nomenclature). We also found an extremely strong association between narcolepsy and the $H L A-D R / D Q$ region with an SNP-based GWAS (Figure 3) [16]. If the results of HLA analysis in European and African populations are considered together, the primary susceptibility allele is assumed to be $H L A-D Q B 1 * 0602$.

Figure 3. GWAS confirmed the most strong association of the $H L A-D R / D Q$ region with narcolepsy [16].

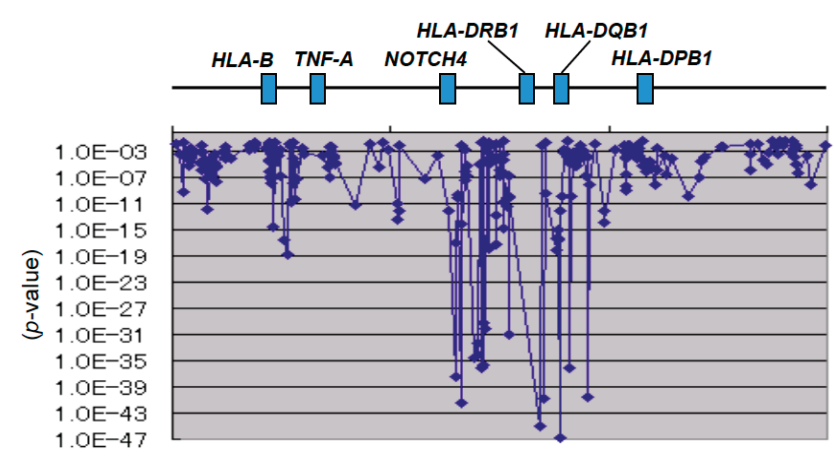

Numerous GWAS have also been carried out for rheumatoid arthritis in Japan and elsewhere, and the $H L A-D R / D Q$ region has been shown to have stronger association than any other region of the genome [18]. HLA-DR4 has been known to be strongly associated with rheumatoid arthritis since the latter half of the 1970s; recent analysis at the sequence level has shown that $D R B 1 * 0401$ is most strongly associated in European populations and $D R B 1 * 0405$ among Japanese. However, there are 
several other $D R B 1$ alleles that also exhibit susceptibility or resistance, and a hierarchy may be seen in their odds ratios.

With primary biliary cirrhosis, also, the $H L A-D R / D Q$ region showed the strongest association in the GWAS of European populations [31] and in the first GWAS of an Asian population [20]. From the analysis of HLA itself, HLA-DRB1*0803-DQB1*0602 and HLA-DRB1*0405-DQB1*0401 have been reported as susceptible haplotypes in the Japanese population [32], while $H L A-D R B 1 * 0801-D Q B 1 * 04$ was reported in European descendants [33].

\subsection{Drug Hypersensitivity and HLA}

There has also been great interest in $H L A$ in its association with drug hypersensitivity. In 2002, it was reported that nearly $80 \%$ of patients who showed a hypersensitivity against the HIV drug, abacavir, possessed $H L A-B * 5701$, with an odds ratio of 117 [34]. In 2004, a group from Taiwan found that of 44 patients with Stevens-Johnson syndrome induced by carbamazepine used for epilepsy seizures or as a psychotropic drug, all had $H L A-B^{*} 1502$ [35]. However, less than $0.1 \%$ of Japanese possess $H L A-B * 5701$, while $H L A-B * 1502$ is extremely rare. Consequently, it was predicted that the associations observed in the previous reports are hardly seen at all among Japanese.

In fact, Ozeki et al. [36] reported that adverse reactions in the skin as a result of carbamazepine are associated with $H L A-A * 3101$. We reported independently that Stevens-Johnson syndrome/toxic epidermal necrolysis accompanied by eye manifestations caused by certain types of cold remedies is associated with $H L A-A * 0206$ [37]. Now, GWAS for this type of Stevens-Johnson syndrome has identified new susceptibility gene(s). Accordingly, GWAS can be powerful tool to investigate hypersensitivity to different kinds of drugs, and there is particular interest in associations with the $H L A$ gene complex.

\subsection{Characteristics of HLA and the Importance of HLA Typing}

There are a number of unique characteristics of $H L A$ genes and their polymorphisms, which indicates the limitation of SNP-based analysis and the importance of typing $H L A$ genes themselves. First, the $H L A$ genes are broadly classified into the Class I and Class II genes. Genes that exhibit high degrees of polymorphisms include $H L A-A,-B$ and $-C$ in Class I and $H L A-D R B 1,-D Q A 1,-D Q B 1$, $-D P A 1$, and -DPB1 in Class II. Including HLA and non-HLA genes, a total of some 130 genes encoding proteins are densely located within a physical distance of about $4 \mathrm{Mbp}$ on the short arm of Chromosome 6. They also show stronger linkage disequilibria than any other region of human genome. For these reasons, specifying a gene locus that is primarily associated with a disease is no easy task.

Second, commercially available genome-wide SNP typing arrays are unable to analyze the SNPs of the $H L A-D R$ region. This is because there is copy number polymorphism of the $D R B$ genes in the region: there are four functional $D R B$ genes $(D R B 1, B 3, B 4$ and $B 5)$ and five pseudogenes (DRB2, $B 6, B 7, B 8$ and $B 9$ ), and the gene composition differs depending upon the $D R B$ haplotype. The SNPs of this region therefore do not conform to the Hardy-Weinberg equilibrium and, so, are not included on the arrays. Consequently, even though the $H L A-D Q$ region may appear to show primary 
association from the results of an SNP-based GWAS, the adjacent $H L A$-DR region with extremely strong linkage disequilibrium must also be considered as a candidate region.

Third, genes in the Class II region are each adjacent on the genome as a pair, comprising an A gene and a B gene, and are linked to each other with a strong linkage disequilibrium. It is therefore very difficult to specify which gene of the pair is the primary one.

Fourth, as mentioned above, the HLA gene exhibits a high degree of polymorphism, and there are a huge number of alleles. There are almost no SNPs or SNP haplotypes that correspond one-on-one to individual $H L A$ alleles. For example, more than 1300 alleles of $H L A-D R B 1$ have been admitted worldwide to date; for example, around 20 alleles with relatively high frequency and a great number of rare alleles have been found in the Japanese population; however, this sort of subclassification is not possible from SNP haplotypes.

Furthermore, a major feature is that a striking diversity between different populations can be observed. In other words, many $H L A$ alleles are distributed only in certain regional populations.

Imputation of $H L A$ alleles using $H L A$ region SNP data is reported to have an accuracy of over 94\% in European populations [38-40]. However, it is not perfect, especially for infrequent alleles, and the imputation is not yet fully available in Japanese or other Asian populations. The typing of the $H L A$ genes is preferable for specifying $H L A$ alleles directly involved in susceptibility, because there are multiple susceptibility alleles and resistance alleles, as well as 'neutral' alleles, and for many of these, the odds ratios are not consistent.

With regard to the $H L A$-associated diseases, therefore, detailed analysis, including the typing of the $H L A$ genes themselves, are necessary to identify the primary $H L A$ genes and alleles for each individual disease. These data will prove invaluable in clarifying the molecular mechanism through which HLA is associated with disease.

\section{Conclusions and Issues for the Future}

There are two hypotheses regarding the involvement of genome variation in common diseases: the common disease (common variants hypothesis and the common disease) and the rare variants hypothesis. In this regard, there is the argument that the common variants identified by GWAS as causing susceptibility to multifactorial diseases can only account for a small proportion of the genetic factors of disease, so that rare variants must also be important. This was symbolized by the term "missing heritability" [41], when only around 20 susceptibility loci for type II diabetes had been identified. Even in total, these could only explain about $5 \%$ of heritability. To date, over 60 common susceptibility loci have been identified, and this number is increasing all the time as a result of GWAS and meta-analyses carried out on greater scales. Further, it has been shown by the latest statistical analysis using all the GWAS data that around $40 \%-60 \%$ of all genetic factors can be explained. Therefore, it is assumed that there are still a great many relatively weak common susceptibility variants that have yet to be discovered.

To put it differently, we have not yet utilized the data obtained from GWAS to the fullest extent. For example, susceptibility genes that are not discovered by gathering samples from patients with the same disease name may be discovered by collecting detailed clinical data for each patient and then carrying out an analysis focused on clinical subsets. Considering a common disease from the 
viewpoint of its genetic architecture, the disease could be a collection of the many diseases that resemble each other, but also exhibit heterogeneity. Furthermore, it is likely that many susceptibility gene polymorphisms do not reach the so-called genome-wide significance level and, instead, exhibit moderate $p$-values. Establishing a method to identify the real susceptibility loci from this gray area is an issue that will need to be resolved in the future. It will be necessary to develop new methods that synthesize data from genetic ontology, pathway/network informatics and other fields and to establish statistical methods that can detect both intra-gene and inter-gene interactions. Our collaborators developed one such method that greatly improves the detection power of susceptibility loci [42].

Other than investigation by means of SNPs, there is also a need to clarify the degree to which variation, such as copy number variation $(\mathrm{CNV})$ and short insertion/deletion variation, account for genetic factors in disease. Massive sequencing using next-generation sequencers is leading to astounding developments; to date, it has been very useful in identifying single genes responsible for hereditary diseases, and it has recently started to be applied to the search for susceptibility genes of multifactorial diseases. Until now, exome analysis has not turned up major results with respect to multifactorial disease. Considering that the majority of susceptibility SNPs identified by GWAS have been discovered in regions that regulate gene expression rather than in regions that code proteins, large-scale whole genome sequencing with a large number of patient and control samples may be needed. Then, the major challenge for the future is to establish a system to extract valuable data from the huge data produced by this new technology and to detect variants associated with certain multifactorial diseases.

$H L A$ is already essential in clinical testing, such as organ and bone marrow transplantation and platelet transfusion. In addition, its association with over 100 types of diseases, including various autoimmune and inflammatory disorders, as well as infectious diseases, has been reported since the 1970s. Research aimed at understanding the mechanism of HLA-disease association commenced in the 1980s, but even now, the mechanism is not clearly known. In the 1990s, also, researchers carried out many analyses of antigenic peptides eluted from $H L A$ molecules prepared from mass cultured cells and analyses of T-cell clones created from patient samples, but were unable to gain a complete understanding of pathogenic peptides or the mechanisms of disease onset. It is hoped that there will be breakthroughs in the search for solutions to the huge riddle of disease mechanisms through advances, such as the diversity analysis of each $H L A$ haplotype using next-generation sequencers, expression analysis of each $H L A$ molecule using the latest protein chemistry and high-order structure analysis of the $H L A$-antigenic peptide-T-cell receptor complex.

Finally, the sharing of a huge amount of data produced by genome-wide variation analyses on various diseases through public databases, such as the Database of Genotypes and Phenotypes (dbGaP) [43], European Genome-Phenome Archive (EGA) [44] and GWAS Central [45], is crucial for the promotion of the complete identification of disease susceptibility genes and the understanding of the molecular mechanism of disease onset. We have also developed a public database for studies on the Japanese population [46-48]. 


\section{Acknowledgments}

This work was supported by grants-in-aid for scientific research from the Japanese Ministry of Education, Culture, Sports, Science and Technology, the Japanese Ministry of Health, Labor and Welfare and the Japan Science and Technology Agency.

\section{Conflicts of Interest}

The author declares no conflict of interest.

\section{References}

1. Risch, N. Searching for genetic determinants in the millennium. Nature 2000, 405, 847-856.

2. Ozaki, K.; Ohnishi, Y.; Iida, A.; Sekine, A.; Yamada, R.; Tsunoda, T.; Sato, H.; Hori, M.; Nakamura, Y.; Tanaka, T. Functional SNPs in the lymphotoxin-alpha gene that are associated with susceptibility to myocardial infarction. Nat. Genet. 2002, 32, 650-654.

3. Tamiya, G.; Shinya, M.; Imanishi, T.; Ikuta, T.; Makino, S.; Okamoto, K.; Furugaki, K.; Matsumoto, T.; Mano, S.; Ando, S.; et al. Whole genome association study of rheumatoid arthritis using 27039 microsatellites. Hum. Mol. Genet. 2005, 14, 2305-2321.

4. Database of Single Nucleotide Polymorphisms (dbSNP). Available online: http://www.ncbi. nlm.nih.gov/snp/ (accessed on 11 February 2014).

5. Database of HapMap Project. Available online: http://hapmap.ncbi.nlm.nih.gov/ (accessed on 11 February 2014).

6. Database of 1000 Genomes Project. Available online: http://www.1000genomes.org/ (accessed on 11 February 2014).

7. A Catalog of Published Genome-Wide Association Studies. Available online: http://www. genome.gov/gwastudies/ (accessed on 11 February 2014).

8. Sladek, R.; Rocheleau, G.; Rung, J.; Dina, C.; Shen, L.; Serre, D.; Boutin, P.; Vincent, D.; Belisle, A.; Hadjadj, S.; et al. Genome-wide association study identifies novel risk loci for type 2 diabetes. Nature 2007, 445, 881-885.

9. Saxena, R.; Voight, B.F.; Lyssenko, V.; Burtt, N.P.; de Bakker, P.I.; Chen, H.; Roix, J.J.; Kathiresan, S.; Hirschhorn, J.N.; Daly, M.J.; et al. Genome-wide association analysis identifies loci for type 2 diabetes and triglyceride levels. Science 2007, 316, 1331-1336.

10. Scott, L.J.; Mohlke, K.L.; Bonnycastle, L.L.; Willer, C.J.; Li, Y.; Duren, W.L.; Erdos, M.R.; Stringham, H.M; Chines, P.S.; Jackson, A.U.; et al. A genome-wide association study of type 2 diabetes in Finns detects multiple susceptibility variants. Science 2007, 316, 1341-1345.

11. Wellcome Trust Case Control Consortium. Genome-wide association study of 14,000 cases of seven common diseases and 3000 shared controls. Nature 2007, 447, 661-678.

12. Yasuda, K.; Miyake, K.; Horikawa, Y.; Hara, K.; Osawa, H.; Furuta, H.; Hirota, Y.; Mori, H.; Jonsson, A.; Sato, Y.; et al. Variants in KCNQ1 are associated with susceptibility to type 2 diabetes mellitus. Nat. Genet. 2008, 40, 1092-1097. 
13. Unoki, H.; Takahashi, A.; Kawaguchi, T.; Hara, K.; Horikoshi, M.; Andersen, G.; Ng, D.P.; Holmkvist, J.; Borch-Johnsen, K.; Jørgensen, T.; et al. SNPs in KCNQ1 are associated with susceptibility to type 2 diabetes in East Asian and European populations. Nat. Genet. 2008, 40, 1098-1102.

14. Miyake, K.; Horikawa, Y.; Hara, K.; Yasuda, K.; Osawa, H.; Furuta, H.; Hirota, Y.; Yamagata, K.; Hinokio, Y.; Oka, Y.; et al. Association of TCF7L2 polymorphisms with susceptibility to type 2 diabetes in 4,087 Japanese subjects. J. Hum. Genet. 2008, 53, 174-180.

15. Matsuki, K.; Juji, T.; Tokunaga, K.; Naohara, T.; Satake, M.; Honda, Y. Human histocompatibility leukocyte antigen (HLA) haplotype frequencies estimated from the data on HLA class I, II, and III antigens in 111 Japanese narcoleptics. J. Clin. Invest. 1985, 76, 2078-2083.

16. Miyagawa, T.; Kawashima, M.; Nishida, N.; Ohashi, J.; Kimura, R.; Fujimoto, A.; Shimada, M.; Morishita, S.; Shigeta, T.; Lin, L.; et al. Variant between CPT1B and CHKB associated with susceptibility to narcolepsy. Nat. Genet. 2008, 40, 1324-1328.

17. Mahasirimongkol, S.; Yanai, H.; Mushiroda, T.; Promphittayarat, W.; Wattanapokayakit, S.; Phromjai, J.; Yuliwulandari, R.; Wichukchinda, N.; Yowang, A.; Yamada, N.; et al. Genome-wide association studies of tuberculosis in Asians identify distinct at-risk locus foe young tuberculosis. J. Hum. Genet. 2012, 57, 363-367.

18. Okada, Y.; Terao, C.; Ikari, K.; Kochi, Y.; Ohmura, K.; Suzuki, A.; Kawaguchi, T.; Stahl, E.A.; Kurreeman, F.A.; Nishida, N.; et al. Meta-analysis of genome-wide association studies identifies multiple novel loci associated with rheumatoid arthritis in the Japanese population. Nat. Genet. 2012, 44, 511-516.

19. Takamoto, M.; Kaburaki, T.; Mabuchi, A.; Araie, M.; Amano, S.; Aihara, M.; Tomidokoro, A.; Iwase, A.; Mabuchi, F.; Kashiwagi, K.; et al. Common variants on chromosome 9q21 are associated with normal tension glaucoma. PLoS One 2012, 7, e40107.

20. Nakamura, M.; Nishida, N.; Kawashima, M.; Aiba, Y.; Tanaka, A.; Yasunami, M.; Nakamura, H.; Komori, A.; Nakamuta, M.; Zeniya, M.; et al. Genome-wide association study identifies TNFSF15 and POU2AF1 as susceptibility loci for primary biliary cirrhosis in the Japanese population. Am. J. Hum. Genet. 2012, 91, 721-728.

21. Okamoto, K.; Tokunaga, K.; Doi, K.; Fujita, T.; Suzuki, H.; Katoh, T.; Watanabe, T.; Nishida, N.; Mabuchi, A.; Takahashi, A.; et al. Common variation in GPC5 is associated with acquired nephrotic syndrome. Nat. Genet. 2011, 43, 459-463.

22. Cotsapas, C.; Voight, B.F.; Rossin, E.; Lage, K.; Neale, B.M.; Wallace, C.; Abecasis, G.R.; Barrett, J.C.; Behrens, T.; Cho, J.; et al. Pervasive sharing of genetic effects in autoimmune disease. PLoS Genet. 2011, 7, e1002254.

23. Parkes, M.; Cortes, A.; van Heel, D.A.; Brown, M.A. Genetic insights into common pathways and complex relationships among immune-mediated diseases. Nat. Rev. Genet. 2013, 14, 661-673.

24. Miyagawa, T.; Miyadera, H.; Tanaka, S.; Kawashima, M.; Shimada, M.; Honda, Y.; Tokunaga, K.; Honda, M. Abnormally low serum acylcarnitine level in narcolepsy. Sleep 2011, 34, 349-353. 
25. Miyagawa, T.; Kawamura, H.; Obuchi, M.; Ikesaki, A.; Ozaki, A.; Tokunaga, K.; Inoue, Y.; Honda, M. Effects of oral L-carnitine administration in narcolepsy patients: A randomized, double-blind, cross-over and placebo-controlled trial. PLoS One 2013, 8, e53707.

26. Hallmayer, J.; Faraco, J.; Lin, L.; Hesselson, S.; Winkelmann, J.; Kawashima, M.; Mayer, G.; Plazzi, G.; Nevsimalova, S.; Bourgin, P.; et al. Narcolepsy is strongly associated with the TCR alpha locus. Nat. Genet. 2009, 41, 708-711.

27. Kornum, B.R.; Kawashima, M.; Faraco, J.; Lin, L.; Rico, T.J.; Hesselson, S.; Axtell, R.C.; Kuipers, H.; Weiner, K.; Hamacher, A.; et al. Common variants in P2RY11 are associated with narcolepsy. Nat. Genet. 2011, 43, 66-71.

28. Tanaka, Y.; Nishida, N.; Sugiyama, M.; Kurosaki, M.; Matsuura, K.; Sakamoto, N.; Nakagawa, M.; Korenaga, M.; Hino, K.; Hige, S.; et al. Genome-wide association of IL28B with response to pegylated interferon-alpha and ribavirin therapy for chronic hepatitis $\mathrm{C}$. Nat. Genet. 2009, 41, 1105-1109.

29. Nishida, N.; Sawai, H.; Matsuura, K.; Sugiyama, M.; Ahn, S.H.; Park, J.Y.; Hige, S.; Kang, J.H.; Suzuki, K.; Kurosaki, M.; et al. Genome-wide association study confirming association of $H L A-D P$ with protection against chronic hepatitis $\mathrm{B}$ and viral clearance in Japanese and Korean. PLoS One 2012, 7, e39175.

30. Juji, T.; Satake, M.; Honda, Y.; Doi, Y. HLA antigens in Japanese patients with narcolepsy. All patients were DR2 positive. Tissue Antigens 1984, 24, 316-319.

31. Hirschfield, G.M.; Liu, X.; Xu, C.; Lu, Y.; Xie, G.; Lu, Y.; Gu, X.; Walker, E.J.; Jing, K.; Juran, B.D.; et al. Primary biliary cirrhosis associated with HLA, IL12A, and IL12RB2 variants. N. Engl. J. Med. 2009, 360, 2544-2555.

32. Nakamura, M.; Yasunami, M.; Kondo, H.; Horie, H.; Aiba, Y.; Komori, A.; Migita, K.; Yatsuhashi, H.; Ito, M.; Shimoda, S.; et al. Analysis of $H L A-D R B 1$ polymorphisms in Japanese patients with primary biliary cirrhosis (PBC): The $H L A-D R B 1$ polymorphism determines the relative risk of antinuclear antibodies for disease progression in PBC. Hepatol. Res. 2010, 40, 494-504.

33. Begovich, A.B.; Klitz, W.; Moonsamy, P.V.; van de Water, J.; Peltz, G.; Gershwin, M.E. Genes within the HLA class II region confer both predisposition and resistance to primary biliary cirrhosis. Tissue Antigens 1994, 43, 71-77.

34. Mallal, S.; Nolan, D.; Witt, C.; Masel, G.; Martin, A.M.; Moore, C.; Sayer, D.; Castley, A.; Mamotte, C.; Maxwell, D.; et al. Association between presence of HLA-B*5701, HLA-DR7 and $H L A-D Q 3$ and hypersensitivity to HIV-1 reverse-transcriptase inhibitor abacavir. Lancet 2002, $359,727-732$.

35. Chung, W.H.; Hung, S.I.; Hong, H.S.; Hsih, M.S.; Yang, L.C.; Ho, H.C.; Wu, J.Y.; Chen, Y.T. Medical genetics: A marker for Stevens-Johnson syndrome. Nature 2004, 428, 486.

36. Ozeki, T.; Mushiroda, T.; Yowang, A.; Takahashi, A.; Kubo, M.; Shirakata, Y.; Ikezawa, Z.; Iijima, M.; Shiohara, T.; Hashimoto, K.; et al. Genome-wide association study identifies $H L A-A * 3101$ allele as a genetic risk factor for carbamazepine-induced cutaneous adverse drug reactions in Japanese population. Hum. Mol. Genet. 2011, 20, 1034-1041. 
37. Ueta, M.; Tokunaga, K.; Sotozono, C.; Inatomi, T.; Yabe, T.; Matsushita, M.; Mitsuishi, Y.; Kinoshita, S. HLA class I and II gene polymorphisms in Stevens-Johnson syndrome with ocular complications in Japanese. Mol. Vis. 2008, 14, 550-555.

38. Dilthey, A.; Leslie, S.; Moutsianas, L.; Shen, J.; Cox, C.; Nelson, M.R.; McVean, G. Multi-population classical HLA type imputation. PLoS Comput. Biol. 2013, 9, e1002877.

39. Zheng, X.; Shen, J.; Cox, C.; Wakefield, J.C.; Ehm, M.G.; Nelson, M.R.; Weir, B.S. HIBAG-HLA genotype imputation with attribute bagging. Pharmacogenomics J. 2013, doi:10.1038/tpj.2013.18.

40. Jia, X.; Han, B.; Onengut-Gumuscu, S.; Chen, W.M.; Concannon, P.J.; Rich, S.S.; Raychaudhuri, S.; de Bakker, P.I. Imputing amino acid polymorphisms in human leucocyte antigens. PLoS One 2013, 8, e64683.

41. Maher, B. Personal genomes: The case of the missing heritability. Nature 2008, 456, 18-21.

42. Dinu, I.; Mahasirimongkol, S.; Liu, Q.; Yanai, H.; El-Din, N.S.; Kreiter, E.; Wu, X.; Jabbari, S.; Tokunaga, K.; Yasui, Y. SNP-SNP interactions discovered by logic regression explain Crohn's disease genetics. PLoS One 2012, 7, e43035.

43. Database of Genotypes and Phenotypes (dbGaP). Available online: http://www.ncbi.nlm. nih.gov/gap/ (accessed on 11 February 2014).

44. European Genome-Phenome Archive (EGA). Available online: https://www.ebi.ac.uk/ega/ (accessed on 11 February 2014).

45. GWAS Central. Available online: https://www.gwascentral.org/ (accessed on 11 February 2014).

46. Koike, A.; Nishida, N.; Inoue, I.; Tsuji, S.; Tokunaga, K. Genome-wide association database developed in the Japanese Integrated Database Project. J. Hum. Genet. 2009, 54, 543-546.

47. Koike, A.; Nishida, N.; Yamashita, D.; Tokunaga, K. Comparative analysis of copy number variation detection methods and database construction. BMC Genet. 2011, 12, e29.

48. Human Genome Variation Database. Available online: https://gwas.biosciencedbc.jp/index.html/ (accessed on 11 February 2014). 


\title{
Phenotype-Based Genetic Association Studies (PGAS)-Towards Understanding the Contribution of Common Genetic Variants to Schizophrenia Subphenotypes
}

\author{
Hannelore Ehrenreich and Klaus-Armin Nave
}

\begin{abstract}
Neuropsychiatric diseases ranging from schizophrenia to affective disorders and autism are heritable, highly complex and heterogeneous conditions, diagnosed purely clinically, with no supporting biomarkers or neuroimaging criteria. Relying on these "umbrella diagnoses", genetic analyses, including genome-wide association studies (GWAS), were undertaken but failed to provide insight into the biological basis of these disorders. "Risk genotypes" of unknown significance with low odds ratios of mostly $<1.2$ were extracted and confirmed by including ever increasing numbers of individuals in large multicenter efforts. Facing these results, we have to hypothesize that thousands of genetic constellations in highly variable combinations with environmental co-factors can cause the individual disorder in the sense of a final common pathway. This would explain why the prevalence of mental diseases is so high and why mutations, including copy number variations, with a higher effect size than SNPs, constitute only a small part of variance. Elucidating the contribution of normal genetic variation to (disease) phenotypes, and so re-defining disease entities, will be extremely labor-intense but crucial. We have termed this approach PGAS ("phenotype-based genetic association studies"). Ultimate goal is the definition of biological subgroups of mental diseases. For that purpose, the GRAS (Göttingen Research Association for Schizophrenia) data collection was initiated in 2005. With $>3000$ phenotypical data points per patient, it comprises the world-wide largest currently available schizophrenia database ( $>$ 1200), combining genome-wide SNP coverage and deep phenotyping under highly standardized conditions. First PGAS results on normal genetic variants, relevant for e.g., cognition or catatonia, demonstrated proof-of-concept. Presently, an autistic subphenotype of schizophrenia is being defined where an unfortunate accumulation of normal genotypes, so-called pro-autistic variants of synaptic genes, explains part of the phenotypical variance. Deep phenotyping and comprehensive clinical data sets, however, are expensive and it may take years before PGAS will complement conventional GWAS approaches in psychiatric genetics.
\end{abstract}

Reprinted from Genes. Cite as: Ehrenreich, H.; Nave, K. Phenotype-Based Genetic Association Studies (PGAS) - Towards Understanding the Contribution of Common Genetic Variants to Schizophrenia Subphenotypes. Genes 2014, 5, 97-105.

\section{Schizophrenia Is a Heterogeneous Group of Diseases Diagnosed Purely Clinically}

The diagnosis of schizophrenia (as of most mental diseases) is to this day an exclusively clinical one which, based on the leading classification systems, DSM and ICD, demands the simultaneous presence of a number of symptoms that are labeled "positive" or "negative". In addition, persistence of these symptoms for at least 6 months is requested. As such, schizophrenia is the unifying term of a highly complex and heterogeneous group of multigenetic disorders, with an 
array of environmental hazards having influenced onset and course. Common to this group of disorders are merely some gross phenotypical traits. Biomarkers in unequivocal support of the diagnosis are missing and also modern imaging technologies, despite revealing brain matter loss or various functional alterations, have not yet assisted in better understanding disease etiology or pathogenesis, or in defining objective diagnostic criteria. This conglomerate of uncertainties, however, is the groundwork on which genetic studies have been and are being built on.

\section{Despite High Heritability of Schizophrenia No "Disease Genes" Have Been Uncovered}

There is no doubt that schizophrenia has a major genetic root. Family studies yielded heritability estimates of up to $80 \%$ for schizophrenia [1,2]. Monozygotic twins manifest concordance rates of $\sim 50 \%$, thus - on top of a clear genetic origin - pointing to a considerable influence of non-genetic causes, i.e., environmental and/or epigenetic factors [3-6]. Searching for the genetic part of schizophrenia etiology, a considerable number of explorations have been undertaken, ranging from segregation or linkage analyses to association studies based on candidate genes [7,8]. Most of the so identified "disease genes", e.g., DISC1 [9], turned out not to respect disease borders. In fact, the DISC1 translocation was found associated as much with mental health as with affective diseases, schizophrenia, autism, or personality disorders [10]. DISC1 and others may thus at best deserve the label of a global risk gene for mental disorders.

So far, no definite "schizophrenia genes" of general significance could be identified. Recently reported genome-wide association studies (GWAS) of schizophrenia identified a number of genetic risk markers significantly associated with the disease, but unfortunately could not extract any universally convincing "disease genes". The initially limited reproducibility over studies and ethnicities has improved with increasing numbers to $\sim 60,000$ individuals (PGC, Psychiatric Genetic Consortium) [11]. Associations, however, remain hampered by very low odds ratios (OR distinctly <1.2) [12,13]. Altogether, it appears highly unlikely that even larger GWAS, based on the umbrella term "schizophrenia", will succeed in unraveling the genetic basis of these conditions or in identifying relevant disease genes.

\section{3. “GWAS Hits" Do Not Predict Disease Severity or Other Relevant Schizophrenia Phenotypes}

Which information do we then gain from the GWAS-identified risk genotypes? In fact, even an accumulation of the GWAS-identified "top 10" risk genotypes [14] does not lead to a more severe disease phenotype. In other words: If an individual possessed all "top 10" risk markers at the same time ("accumulated risk"), his disease will not be more grave than if he carried none of the risk genotypes.

Another attempt to make use of the GWAS-derived information has been the definition of polygenic schizophrenia risk scores (PSS). Besides the genome-wide significant risk loci, a substantial proportion of schizophrenia risk has been hypothesized to lie in markers not achieving genome-wide significance. Thus, quantitative PSS were calculated based on nominal associations of each SNP from the PGC GWAS. These PSS explained up to $6 \%$ of variance regarding the 
diagnosis schizophrenia in an independent sample [11]. Subsequently, PSS effects on various disease-relevant phenotypes, e.g., brain matter dimensions, were explored with variable success $[15,16]$. Employing the GRAS data collection of $>1200$ well characterized schizophrenic subjects (see below), we could not uncover the slightest association of PSS with any lead features of schizophrenia. In contrast, we found in the same schizophrenic population dramatic effects of accumulated environmental risk on age at onset of the disease [17].

\section{Definition of Biological Subgroups of Schizophrenia: The Essential Next Step}

In summary, we note that all genetic "schizophrenia markers" discovered by GWAS up to date do not contribute more to the entirety of this disease than a minimally heightened probability (OR < 1.2) of receiving the "umbrella diagnosis schizophrenia". This whole picture could, however, look quite different when considering biological subgroups of schizophrenia as illustrated in Figure 1. GWAS on well-defined disease-relevant subphenotypes may ultimately not only lead to the identification of entirely new risk genotypes but, importantly, also to a re-distribution of some of the identified GWAS risk markers to certain subgroups, resulting then in much higher odds ratios.

Figure 1. Schematic presentation of three hypothetical subphenotypes of schizophrenia embedded in a sample Manhattan plot. The non-overlapping red, blue or yellow dots comprise genetic constellations ("assemblies") suggested to account for disease subforms with respective subphenotypes. Note that typical genome-wide association studies (GWAS) "top hits", as defined by the highest significance levels for the clinical endpoint diagnosis ("schizophrenia"), most likely fall into different assemblies. This can explain their low odd ratios in a large and diverse patient group and the apparent lack of interactions. The depicted schema is purely hypothetical and shall illustrate the difficulties to define disease genes by conventional GWAS approaches based on endpoint diagnoses.

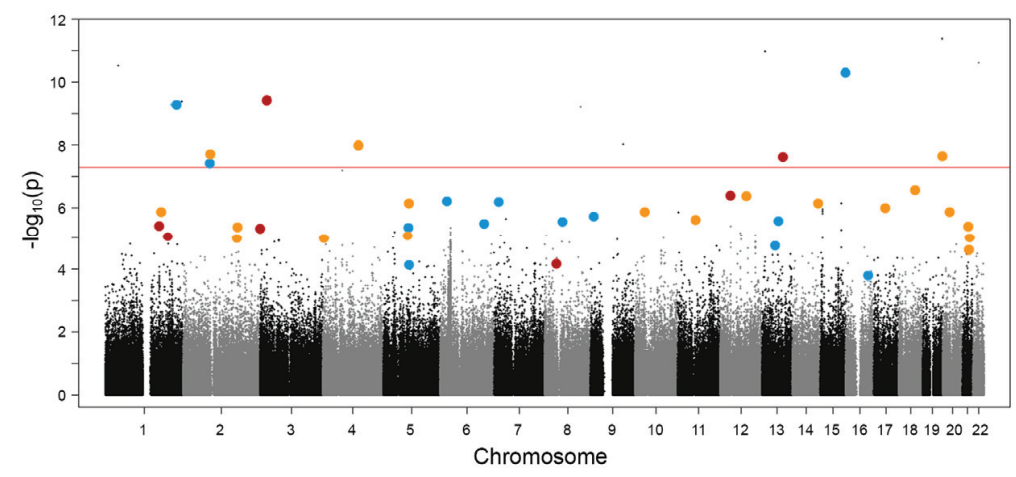

In order to define biological subgroups of schizophrenia, the GRAS (Göttingen Research Association for Schizophrenia) data collection was initiated in 2004 [18,19]. The lead hypothesis of GRAS states that schizophrenia is caused by thousands of possible combinations of genetic markers interacting with a large array of different environmental risk factors. There may be rare 
cases where a 'per se' critical genetic load exists and the disease onset is independent of additional external factors. In the vast majority of cases, however, only the interaction between certain genetic predispositions and environmental factors will lead to disease onset. The overlap of genetic risk factors between individual schizophrenic patients/families probably is fairly low. This in turn might explain why it is obviously impossible to obtain common risk genes of schizophrenia with convincing odds ratios. According to the primary GRAS hypothesis, schizophrenia is the result of a combination of 'unfortunate' normal genetic variants exposed to unfavorable environmental influence. Apart from its scientific significance, undoubtedly, this hypothesis strongly supports any anti-stigma campaign.

We hypothesize that a considerable proportion of the population across cultures (likely 50\%) may harbor a principal genetic make-up for developing mental diseases whereas the remaining $50 \%$ of individuals could never acquire them due to absence of respective genetic prerequisites. Only in a small fraction of risk carriers the disease will break out $(\sim 0.5 \%-1 \%$ of the population across cultures) [20], co-induced by a multitude of potential external inputs, while the bulk of carriers will stay healthy and forward their predisposition to their offspring.

\section{Deep Phenotyping Is a Prerequisite for PGAS: The GRAS Data Collection}

If all human genetic approaches to schizophrenia as a "classic" genetic disease apparently failed, how will it be possible to learn more about the contribution of genes/genotypes to relevant disease subphenotypes? Motivated by this central question we started an alternative and at the same time complementary approach to conventional GWAS. We call this approach PGAS, which stands for phenotype-based genetic association study. PGAS enables us to explore the contribution of genes/genetic markers to schizophrenic subphenotypes. To start PGAS, we had to establish a comprehensive standardized phenotypical characterization of schizophrenic patients, the above introduced GRAS data collection. Within a few years we compiled the presently world-wide largest phenotypical database of schizophrenic patients comprising presently $\sim 1200$ subjects, with slow steady-state recruitment ongoing. Although much larger data bases of schizophrenic subjects exist, unfortunately, they do not even come close to the deep phenotyping information of GRAS. A total of 23 psychiatric hospitals all over Germany were involved as hosting centers in this effort $[18,19]$. Inclusion criteria for the patients were confirmed diagnosis of schizophrenia or schizoaffective disorder, and the ability to cooperate at least to a minimal degree. The GRAS population is representative for those schizophrenic patients in central Europe/Germany who are in contact with the healthcare system. Unrivalled in the GRAS data collection is the fact that all cross-sectional examinations of all patients were performed by one and the same travelling team. This fact contributes in a highly significant manner to the homogeneity of the resulting database, containing at present $82 \%$ schizophrenic and $18 \%$ schizoaffective subjects $[18,19]$.

The GRAS data collection does not only encompass sociodemographic and basic clinical parameters but also, for example, a very comprehensive cognitive test battery, an extensive investigation of neurological signs and symptoms, a thorough medication history, several tests of extrapyramidal side effects of antipsychotic medication, complete information on drug abuse/addiction or other comorbidities, to name just a few of the covered data modules. Apart from 
the comprehensive cross-sectional analyses, we collected nearly all psychiatric medical reports and discharge letters of all GRAS patients, allowing also for fairly solid longitudinal data analysis, e.g., information on disease course and outcome. Altogether, we have about 3000 data points per patient, which allow us to perform PGAS $[18,19]$. Importantly, most GRAS patients agreed to be re-contacted for follow-up studies, thereby enabling targeted analyses in the future, e.g., detailed morphometrical or functional magnetic resonance imaging studies, or inducible pluripotent stem cell approaches to study cellular consequences of complex genotypes.

\section{Phenotype-Based Genetic Association Studies (PGAS): Proof-of-Principle}

First proof-of-principle studies for the PGAS approach were performed over the last years. We could show that genetic variants within the complexin2 gene (6 single nucleotide polymorphisms) influence the cognitive capability of schizophrenic patients [18]. This gene encodes a synaptic protein that is crucial for the regulation of neurotransmitter release. Similarly, the calcium-activated potassium channel SK3 is involved in the modulation of neuroplasticity and influences cognition of schizophrenic individuals genotype-dependently (as a function of the length of a CAG repeat in the $N$-terminal region) [21]. Moreover, a distinct combination of erythropoietin (EPO) and EPO receptor (EPOR) genotypes leads to a remarkable cognitive benefit in schizophrenic subjects as compared to all other possible EPO/EPOR genotype combinations [22]. Of note, the association of certain genetic variants with cognition in schizophrenia may likewise hold true for healthy individuals and other disease populations [22]. This clearly emphasizes the importance of investigating the genotype contribution to phenotypes in general, rather than immediately focusing on risk genes in complex diseases.

Among the discussed candidate risk genes of schizophrenia is NRG1 [23-25]. Analyzing its disease-relevance in the GRAS population yielded for the first time an association with age at disease onset and severity of positive symptoms [26]. A myelin-associated gene, CNP, turned out to co-determine the occurrence of a catatonia-depression syndrome upon aging [27]. Importantly, in most of these PGAS approaches, either replication in an independent human cohort and/or behavioral phenotypes in corresponding mouse mutants were demonstrated. In fact, the translation to mouse models, and even to cultured cells, plays an important part in the search for mechanistic comprehension of the observed phenomena.

\section{PGAS Will Be Instrumental for Definition of Biological Schizophrenia Subgroups}

Even though the above mentioned first proof-of-principle studies were important to set the stage for PGAS, it is now mandatory to work on the "bigger picture". The availability of genome-wide genetic data on the GRAS sample enables us to better understand the genetic basis of neuropsychiatric phenotypes. Using the Axiom ${ }^{\mathrm{TM}}$ Caucasian European array as a backbone, we have enriched it by including additional markers of putative functional importance [28]. At present, we are in the process of defining biological subphenotypes of schizophrenia which are less complex as compared to the 'umbrella diagnosis schizophrenia' and can be described and well quantified by respective phenotype scores. Prerequisite for applying these scores to genetic studies is a high internal consistency of the selected score items, reflected by a demanded Cronbach's alpha of $>80 \%$. As a 
first example, we have operationalized an autistic subphenotype of schizophrenia. This subphenotype, which comprises lead features of high-functioning autism (deficits in social interaction and communication, as well as repetitive behaviors/stereotypies), shows a normal distribution in the GRAS population, thus allowing for extreme group comparisons with respect to the associated genotypical information. Our primary target genes for the autistic subphenotype are synaptic genes where we find an unfortunate accumulation of normal genotypes, that we consider as "pro-autistic" variants of these genes, to explain an appreciable part of the phenotypical variance. A sample of Asperger autists is presently recruited with the aim to replicate the genotype-phenotype associations found in the autistic subgroup of schizophrenic individuals. Similarly, a psychomotor and a schizoaffective subphenotype of schizophrenia will be phenotypically as well as-subsequently-genetically characterized, targeting myelin- and ion channel-associated genes, respectively [29].

\section{Conclusions}

To conclude, the lack of objective diagnoses and the non-existence of classical disease genes have forced us to re-consider the genetic approach to mental diseases. Deep clinical phenotyping as prerequisite for PGAS, combined with genome-wide SNP coverage, makes it possible to improve our understanding of the molecular-genetic architecture of schizophrenia and likely other mental diseases via systematic phenotype-based approaches. These in turn necessitate highly labor-intense groundwork since they depend on the availability of comprehensive phenotypical databases comparable to the GRAS data collection. Some efforts in a similar direction are launched in other disciplines, for instance in a first trial to integrate available electronic medical record data and GWAS information [30]. Regarding the depth of phenotyping, however, this approach certainly needs to be substantially improved. Even if "deep phenotyping" is much more tedious than collecting thousands of subjects with merely a certain diagnostic label versus healthy individuals, it will be the only way to reach the goal, i.e., novel definitions of biologically sound disease subgroups. It can only be hoped that big consortia and sponsors will follow this rationale such that the number of deeply phenotyped individuals will grow. At the end of the road, PGAS will permit better insights into the complex genotype-phenotype interactions of schizophrenia (and other neuropsychiatric diseases) and in this way open up more targeted therapeutic strategies for biological subphenotypes of the disease.

\section{Acknowledgments}

This work has received continuous support by the Max Planck Society, the Max Planck Förderstiftung, and the DFG (CNMPB).

\section{Author Contributions}

Both authors discussed the contents, designed the paper outline, and jointly wrote the manuscript.

\section{Conflicts of Interest}

The authors declare no conflict of interest. 


\section{References}

1. Cardno, A.G.; Gottesman, I.I. Twin studies of schizophrenia: From bow-and-arrow concordances to star wars mx and functional genomics. Am. J. Med. Genet. 2000, 97, 12-17.

2. Lichtenstein, P.; Yip, B.H.; Bjork, C.; Pawitan, Y.; Cannon, T.D.; Sullivan, P.F.; Hultman, C.M. Common genetic determinants of schizophrenia and bipolar disorder in swedish families: A population-based study. Lancet 2009, 373, 234-239.

3. Cardno, A.G.; Marshall, E.J.; Coid, B.; Macdonald, A.M.; Ribchester, T.R.; Davies, N.J.; Venturi, P.; Jones, L.A.; Lewis, S.W.; Sham, P.C.; et al. Heritability estimates for psychotic disorders: The maudsley twin psychosis series. Arch. Gen. Psychiatry 1999, 56, 162-168.

4. Franzek, E.; Beckmann, H. Different genetic background of schizophrenia spectrum psychoses: A twin study. Am. J. Psychiatry 1998, 155, 76-83.

5. Svrakic, D.M.; Zorumski, C.F.; Svrakic, N.M.; Zwir, I.; Cloninger, C.R. Risk architecture of schizophrenia: The role of epigenetics. Curr. Opin. Psychiatry 2013, 26, 188-195.

6. Van Os, J.; Kenis, G.; Rutten, B.P. The environment and schizophrenia. Nature 2010, 468, 203-212.

7. Giusti-Rodriguez, P.; Sullivan, P.F. The genomics of schizophrenia: Update and implications. J. Clin. Invest. 2013, 123, 4557-4563.

8. Sullivan, P.F. The genetics of schizophrenia. PLoS Med. 2005, 2, e212.

9. St. Clair, D.; Blackwood, D.; Muir, W.; Carothers, A.; Walker, M.; Spowart, G.; Gosden, C.; Evans, H.J. Association within a family of a balanced autosomal translocation with major mental illness. Lancet 1990, 336, 13-16.

10. Brandon, N.J.; Millar, J.K.; Korth, C.; Sive, H.; Singh, K.K.; Sawa, A. Understanding the role of disc1 in psychiatric disease and during normal development. J. Neurosci. 2009, 29, 12768-12775.

11. Ripke, S.; O’Dushlaine, C.; Chambert, K.; Moran, J.L.; Kahler, A.K.; Akterin, S.; Bergen, S.E.; Collins, A.L.; Crowley, J.J.; Fromer, M.; et al. Genome-wide association analysis identifies 13 new risk loci for schizophrenia. Nat. Genet. 2013, 45, 1150-1159.

12. Consortium, I.S.; Purcell, S.M.; Wray, N.R.; Stone, J.L.; Visscher, P.M.; O’Donovan, M.C.; Sullivan, P.F.; Sklar, P. Common polygenic variation contributes to risk of schizophrenia and bipolar disorder. Nature 2009, 460, 748-752.

13. O’Donovan, M.C.; Craddock, N.; Norton, N.; Williams, H.; Peirce, T.; Moskvina, V.; Nikolov, I.; Hamshere, M.; Carroll, L.; Georgieva, L.; et al. Identification of loci associated with schizophrenia by genome-wide association and follow-up. Nat. Genet. 2008, 40, 1053-1055.

14. Papiol, S.; Malzahn, D.; Kästner, A.; Sperling, S.; Begemann, M.; Stefansson, H.; Bickeboller, H.; Nave, K.A.; Ehrenreich, H. Dissociation of accumulated genetic risk and disease severity in patients with schizophrenia. Transl. Psychiatry 2011, 1, e45.

15. Papiol, S.; Mitjans, M.; Assogna, F.; Piras, F.; Hammer, C.; Caltagirone, C.; Arias, B.; Ehrenreich, H.; Spalletta, G. Polygenic determinants of white matter volume derived from gwas lack reproducibility in a replicate sample. Transl. Psychiatry 2014, doi:10.1038/tp.2013.126.

16. Terwisscha van Scheltinga, A.F.; Bakker, S.C.; van Haren, N.E.; Derks, E.M.; Buizer-Voskamp, J.E.; Boos, H.B.; Cahn, W.; Hulshoff Pol, H.E.; Ripke, S.; Ophoff, R.A.; et al. Genetic schizophrenia risk variants jointly modulate total brain and white matter volume. Biol. Psychiatry 2013, 73, 525-531. 
17. Stepniak, B.; Papiol, S.; Hammer, C.; Ramin, A.; Everts, S.; Hennig, L.; Begemann, M.; Ehrenreich, H. Accumulated environmental risk determining the onset of schizophrenia. Submitted for publication, 2014.

18. Begemann, M.; Grube, S.; Papiol, S.; Malzahn, D.; Krampe, H.; Ribbe, K.; Friedrichs, H.; Radyushkin, K.A.; El-Kordi, A.; Benseler, F.; et al. Modification of cognitive performance in schizophrenia by complexin 2 gene polymorphisms. Arch. Gen. Psychiatry 2010, 67, 879-888.

19. Ribbe, K.; Friedrichs, H.; Begemann, M.; Grube, S.; Papiol, S.; Kästner, A.; Gerchen, M.F.; Ackermann, V.; Tarami, A.; Treitz, A.; et al. The cross-sectional gras sample: A comprehensive phenotypical data collection of schizophrenic patients. BMC Psychiatry 2010, 10, 91.

20. Van Os, J.; Kapur, S. Schizophrenia. Lancet 2009, 374, 635-645.

21. Grube, S.; Gerchen, M.F.; Adamcio, B.; Pardo, L.A.; Martin, S.; Malzahn, D.; Papiol, S.; Begemann, M.; Ribbe, K.; Friedrichs, H.; et al. A cag repeat polymorphism of kcnn3 predicts sk3 channel function and cognitive performance in schizophrenia. EMBO Mol. Med. 2011, 3, 309-319.

22. Kästner, A.; Grube, S.; El-Kordi, A.; Stepniak, B.; Friedrichs, H.; Sargin, D.; Schwitulla, J.; Begemann, M.; Giegling, I.; Miskowiak, K.W.; et al. Common variants of the genes encoding erythropoietin and its receptor modulate cognitive performance in schizophrenia. Mol. Med. 2012, 18, 1029-1040.

23. Gong, Y.G.; Wu, C.N.; Xing, Q.H.; Zhao, X.Z.; Zhu, J.; He, L. A two-method meta-analysis of neuregulin 1(nrg1) association and heterogeneity in schizophrenia. Schizophr. Res. 2009, $111,109-114$.

24. Li, D.; Collier, D.A.; He, L. Meta-analysis shows strong positive association of the neuregulin 1 (nrg1) gene with schizophrenia. Hum. Mol. Genet. 2006, 15, 1995-2002.

25. Munafo, M.R.; Thiselton, D.L.; Clark, T.G.; Flint, J. Association of the nrg1 gene and schizophrenia: A meta-analysis. Mol. Psychiatry 2006, 11, 539-546.

26. Papiol, S.; Begemann, M.; Rosenberger, A.; Friedrichs, H.; Ribbe, K.; Grube, S.; Schwab, M.H.; Jahn, H.; Gunkel, S.; Benseler, F.; et al. A phenotype-based genetic association study reveals the contribution of neuregulin1 gene variants to age of onset and positive symptom severity in schizophrenia. Am. J. Med. Genet. Part B Neuropsychiatry Genet. 2011, 156, 340-345.

27. Hagemeyer, N.; Goebbels, S.; Papiol, S.; Kästner, A.; Hofer, S.; Begemann, M.; Gerwig, U.C.; Boretius, S.; Wieser, G.L.; Ronnenberg, A.; et al. A myelin gene causative of a catatonia-depression syndrome upon aging. EMBO Mol. Med. 2012, 4, 528-539.

28. Hammer, C.; Stepniak, B.; Schneider, A.; Papiol, S.; Tantra, M.; Begemann, M.; Siren, A.L.; Pardo, L.A.; Sperling, S.; Mohd Jofrry, S.; et al. Neuropsychiatric disease relevance of circulating anti-nmda receptor autoantibodies depends on blood-brain barrier integrity. Mol. Psychiatry 2013, doi:10.1038/mp.2013.110.

29. Ehrenreich, H.; Nave, K.-A. Max Planck Institute of Experimental Medicine, and DFG Center for Nanoscale Microscopy and Molecular Physiology of the Brain (CNMPB), Hermann-Rein-Str.3, 37075 Göttingen, Germany. Unpublished work, 2014. 
30. Denny, J.C.; Bastarache, L.; Ritchie, M.D.; Carroll, R.J.; Zink, R.; Mosley, J.D.; Field, J.R.; Pulley, J.M.; Ramirez, A.H.; Bowton, E.; et al. Systematic comparison of phenome-wide association study of electronic medical record data and genome-wide association study data. Nat. Biotechnol. 2013, 31, 1102-1111. 


\title{
Mechanisms of Base Substitution Mutagenesis in Cancer Genomes
}

\author{
Albino Bacolla, David N. Cooper and Karen M. Vasquez
}

Abstract: Cancer genome sequence data provide an invaluable resource for inferring the key mechanisms by which mutations arise in cancer cells, favoring their survival, proliferation and invasiveness. Here we examine recent advances in understanding the molecular mechanisms responsible for the predominant type of genetic alteration found in cancer cells, somatic single base substitutions (SBSs). Cytosine methylation, demethylation and deamination, charge transfer reactions in DNA, DNA replication timing, chromatin status and altered DNA proofreading activities are all now known to contribute to the mechanisms leading to base substitution mutagenesis. We review current hypotheses as to the major processes that give rise to SBSs and evaluate their relative relevance in the light of knowledge acquired from cancer genome sequencing projects and the study of base modifications, DNA repair and lesion bypass. Although gene expression data on APOBEC3B enzymes provide support for a role in cancer mutagenesis through $\mathrm{U}: \mathrm{G}$ mismatch intermediates, the enzyme preference for single-stranded DNA may limit its activity genome-wide. For SBSs at both CG:CG and YC:GR sites, we outline evidence for a prominent role of damage by charge transfer reactions that follow interactions of the DNA with reactive oxygen species (ROS) and other endogenous or exogenous electron-abstracting molecules.

Reprinted from Genes. Cite as: Bacolla, A.; Cooper, D.N.; Vasquez, K.M. Mechanisms of Base Substitution Mutagenesis in Cancer Genomes. Genes 2014, 5, 108-146.

\section{Introduction}

The explosion of cancer genome sequencing projects over the past few years has revealed the complexity of the processes whose alterations are associated with, and are often causative of, various types of cancer [1,2]. These include mutational mechanisms that give rise to tissue-specific mutation rates, variations in mutation frequencies in distinct compartments of chromosomes, sequence context-dependent mutation spectra, and regional hypermutation, often termed kataegis (thunderstorm) [3]. Cancer genome studies have also served to catalogue the extent to which translocations, chromosomal gains and losses and focal copy-number alterations take place, often mediated by catastrophic chromosomal shattering events, as in the case of some bone and pediatric cancers [4-8]. From a mechanistic and therapeutic perspective, the arsenal of gene classes and pathways that are frequently altered, such as signal transduction, metabolism, DNA repair, transcription, epigenetics, RNA splicing and protein homeostasis, has also greatly expanded [1,2,9-11]. Attempts to address key questions concerning the causes leading to the mutational events that characterize and contribute to driving a normal cell towards tumorigenesis have also burgeoned [12-14]. These attempts are, however, necessarily indirect since the only material available for analysis are the catalogues of mutations that survived and accumulated, in most cases, over long periods of time. With few exceptions, such as mutation patterns observed in the lung 
cancers of heavy smokers and in skin cancers following UV exposure, most mutational patterns have remained enigmatic.

The goal of this review is to examine two prominent single base substitution (SBS) patterns observed in cancer genomes, both of which display sequence context-dependent signatures: $\mathrm{C} \rightarrow \mathrm{T}$ transitions at $\mathrm{CG}: \mathrm{CG}$ (the colon separates complementary bases written in a $5^{\prime} \rightarrow 3^{\prime}$ direction) dinucleotide sequences and substitutions at $\mathrm{C}: \mathrm{G}$ base-pairs in the context of $\mathrm{Y} \underline{\mathrm{C}}: \underline{\mathrm{GR}}$ (Y, pyrimidine; R, purine) motifs. Whereas spontaneous deamination of 5-methylcytosine has been proposed to account for the first pattern, two mechanisms have been recently suggested for the latter pattern: over-activity by the APOBEC family of cytosine deaminases and electron transfer following oxidative damage. After considering several factors associated with SBSs, such as regional variations in mutation frequencies, mechanisms leading to base modification, and DNA repair systems, we conclude that for both SBS patterns, oxidative base damage from ROS and other electron-abstracting molecules appears to play a more significant role than previously anticipated.

\section{Meta-Analyses of Cancer Genomes}

\subsection{Mutational Signatures in Cancer Genomes}

The large number of sequenced cancer genomes now available has made it possible to address the issue of mutational spectra and relative mutation frequencies, both exome-wide and genome-wide across different cancer types. Some of the largest meta-analyses have included 4,938,362 somatic substitutions and small insertions/deletions (indels) from 7,042 primary cancers of 30 different classes [13], 1,000,000 somatic exome mutations from 4,800 tumors representing 19 different cancers [15], 617,354 somatic mutations in 3,281 tumors from 12 cancer types [16], 533,482 somatic SBSs from 1,149 cancer samples and 2 cell lines representing 14 different tissues [17], and 373,909 non-silent coding mutations in 3,083 tumor-normal pairs across 27 tumor types [18]. The prevalence of SBSs was highly variable, both between and within cancer classes, ranging from $\sim 0.001$ per megabase $(\mathrm{Mb})$ to $>400$ per $\mathrm{Mb}$, with childhood cancers generally carrying the fewest mutations, acute myeloid leukemia exhibiting a very low median mutation frequency $(\sim 0.28 / \mathrm{Mb})$, and cancers associated with chronic mutagen exposure, such as lung (tobacco smoking) and malignant melanoma (UV light) displaying the highest mutation frequencies $(8.15 / \mathrm{Mb}$ for lung squamous cell carcinoma) $[13,15,16]$.

With regard to mutational spectra, the most consistent and frequent mutational signature across cancer types has been noted at CG:CG (we identify both nucleosides and nucleotides by their base) dinucleotides, with 25/30 cancer types in [13] and 13/14 in [17], and gastrointestinal tumors displaying $\underline{\mathrm{CG}}: \underline{\mathrm{CG}} \rightarrow \mathrm{TG}: \mathrm{CA}$ (target base underlined) among the highest fractions $[13,16,17]$ (Table 1A). The preponderance of $\mathrm{C} \rightarrow \mathrm{T}$ transitions at $\mathrm{CG}: \mathrm{CG}$ sequences has been attributed to high rates of spontaneous deamination of 5-methylcytosine $(5 \mathrm{mC})$ as compared to unmethylated cytosine $[13,16,17,19]$; such deamination events yield $\mathrm{T}: \mathrm{G}$ mismatches and, subsequently, $\mathrm{G} \rightarrow \mathrm{A}$ transitions at the next round of DNA replication. A second prominent mutational signature found across several cancer types, including breast, ovary, bladder, head and neck, cervix, liver and lung $[13,15-17,20,21]$, has been noted at $\mathrm{C}: \mathrm{G}$ base-pairs in the context of T$: \underline{\mathrm{G}} \mathrm{A}$ dinucleotides 
$(\mathrm{C} \rightarrow \mathrm{T}, \mathrm{C} \rightarrow \mathrm{G}$ and $\mathrm{C} \rightarrow \mathrm{A})$ (Table $1 \mathrm{~A})$; this has been attributed either to over-activity of members of the apolipoprotein B mRNA-editing catalytic polypeptide (APOBEC) cytosine deaminases $[13,15,18,20]$, or to electron transfer reactions following oxidative damage [17].

Table 1. Mutational signatures in cancer genomes.

\begin{tabular}{|c|c|c|c|c|}
\hline \multicolumn{5}{|c|}{ A. Main mutational signatures revealed from meta-analyses of cancer genomes } \\
\hline $\begin{array}{c}\text { Total number of } \\
\text { mutations }\end{array}$ & $\begin{array}{c}\text { Total number of } \\
\text { cancer types }\end{array}$ & $\begin{array}{l}\text { Major SBS signature } \\
\text { (\% cancer types) }\end{array}$ & Sequence context & References \\
\hline $4,938,362$ & 30 & $\begin{array}{c}\mathrm{C}: \mathrm{G} \rightarrow \mathrm{T}: \mathrm{A}(80 \%) \\
\mathrm{C}: \mathrm{G} \rightarrow \mathrm{T}: \mathrm{A} \text { or } \mathrm{G}: \mathrm{C}(50 \%)\end{array}$ & 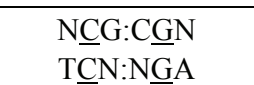 & [13] \\
\hline $1,000,000$ & 19 & $\mathrm{C}: \mathrm{G} \rightarrow$ any subst. $(32 \%)$ & TCEN:NGA & [15] \\
\hline 617,354 & 12 & $\begin{array}{l}\mathrm{C}: \mathrm{G} \rightarrow \mathrm{T}: \mathrm{A}(33 \%) \\
\mathrm{C}: \mathrm{G} \rightarrow \mathrm{G}: \mathrm{C}(25 \%)\end{array}$ & $\begin{array}{l}\overline{\mathrm{C}} \mathrm{G}: \overline{\mathrm{C}} \overline{\mathrm{G}} \\
\mathrm{T} \underline{\mathrm{C}}: \underline{\mathrm{GA}}\end{array}$ & {$[16]$} \\
\hline 533,482 & 14 & $\begin{array}{l}\mathrm{C}: \mathrm{G} \rightarrow \text { any subst. }(93 \%) \\
\mathrm{C}: \mathrm{G} \rightarrow \text { any subst. }(36 \%)\end{array}$ & 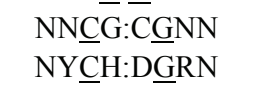 & [17] \\
\hline 373,909 & 27 & $\begin{array}{c}\mathrm{C}: \mathrm{G} \rightarrow \mathrm{T}: \mathrm{A}(30 \%) \\
\mathrm{C}: \mathrm{G} \rightarrow \text { any subst. }(11 \%)\end{array}$ & $\begin{array}{l}\overline{\mathrm{C}} \mathrm{G}: \overline{\mathrm{C}} \overline{\mathrm{G}} \\
\mathrm{T} \underline{\mathrm{C}}: \underline{\mathrm{GA}}\end{array}$ & {$[18]$} \\
\hline \multicolumn{5}{|c|}{ B. Main cancer type-specific mutational signatures } \\
\hline Cancer type & Putative cause & SBS signature & Sequence context & References \\
\hline Lung cancer & $\begin{array}{l}\text { tobacco smoke } \\
\text { arsenic exposure }\end{array}$ & $\begin{array}{l}\mathrm{C}: \mathrm{G} \rightarrow \mathrm{A}: \mathrm{T} \\
\mathrm{T}: \mathrm{A} \rightarrow \mathrm{G}: \mathrm{C}\end{array}$ & $\begin{array}{l}\text { none } \\
\text { none }\end{array}$ & $\begin{array}{c}{[22,27-30]} \\
{[35]}\end{array}$ \\
\hline Melanoma & $\begin{array}{l}\mathrm{UV}, \text { APOBEC3A } \\
\text { unknown }\end{array}$ & $\begin{array}{c}\mathrm{C}: \mathrm{G} \rightarrow \mathrm{T}: \mathrm{A} \\
\mathrm{G}: \mathrm{C} \rightarrow \text { any subst. }\end{array}$ & $\begin{array}{l}\text { pyrimidine dimers } \\
\text { NGRA:TY } \underline{\mathrm{CN}}\end{array}$ & $\begin{array}{c}{[36-42,44]} \\
{[17]}\end{array}$ \\
\hline Liver carcinoma & carcinogens & $\mathrm{T}: \mathrm{A} \rightarrow \mathrm{C}: \mathrm{G}$ & none & {$[13,18]$} \\
\hline Leukemia & unknown & $\mathrm{A}: \mathrm{T} \rightarrow \mathrm{T}: \mathrm{A}$ & TA $\underline{\text { TA }}$ & {$[13,18]$} \\
\hline Endometrial cancer & POLE $^{\mathrm{P} 286 \mathrm{R}}$ & $\mathrm{G}: \mathrm{C} \rightarrow \mathrm{T}: \mathrm{A}$ & A $\underline{\overline{G A}} \bar{T} \underline{T} \underline{T}$ & \\
\hline
\end{tabular}

$N$, any nucleotide; $Y, \mathrm{C}$ or $\mathrm{T} ; R, \mathrm{~A}$ or $\mathrm{G} ; D, \mathrm{~A}$ or $\mathrm{G}$ or $\mathrm{T} ; H, \mathrm{~A}$ or $\mathrm{C}$ or $\mathrm{T}$.

Cancer type-specific mutational signatures have also been identified, particularly in cancers of the lung and skin. For example, in a cohort of 17 non-small cell lung cancer patients, the total number of somatic mutations was $\sim 10$-fold higher in smokers (median 15,659, range 7,424-26,202) than in never-smokers (median 888, range 842-1,268) [22], consistent with other reports that smoking-associated lung cancer is distinguished by a significantly high number of mutations per $\mathrm{Mb}$ [23-28]. Tumors from smokers were also characterized by high fractions (up to $46 \%$ ) of $\mathrm{C}: \mathrm{G} \rightarrow \mathrm{A}: \mathrm{T}$ transversions $[22,27-30]$ (Table 1B), a signature of exposure to alkylating nitrosamines and polycyclic aromatic hydrocarbons (PAHs) present in tobacco smoke, which yield miscoding $\mathrm{G}$ adducts [31-34]. This conclusion is further supported by a recent whole-genome sequencing analysis of an arsenic exposure-related lung squamous cell carcinoma, which was instead characterized by a high fraction $(16.3 \%)$ of $\mathrm{T}: \mathrm{A} \rightarrow \mathrm{G}: \mathrm{C}$ transversions but a low fraction $(\sim 6.1 \%)$ of $\mathrm{C}: \mathrm{G} \rightarrow \mathrm{A}: \mathrm{T}$ transversions [35] (Table 1B). In melanomas, up to $87 \%$ of all mutations were represented by $\mathrm{C}: \mathrm{G} \rightarrow \mathrm{T}: \mathrm{A}$ transitions, mostly at pyrimidine dimers [36-42], consistent with DNA translesion synthesis across UV-induced covalently linked pyrimidine dimers [43]. Mutations in skin cancer at pyrimidine dimers, particularly $\mathrm{CC} \rightarrow \mathrm{TT}$ transitions on the non-transcribed strand of expressed genes, have also been attributed to APOBEC3A, a member of the APOBEC family of cytosine deaminases which are active mostly on single-stranded DNA, and expressed in skin keratinocytes [44] 
(Table 1B). Other prominent patterns included $\mathrm{T} \rightarrow \mathrm{C}$ transitions in hepatocellular carcinomas,

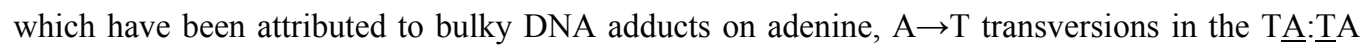
context, particularly in leukemia samples $[13,18]$, a 2 -fold increase in mutations at NGRA relative to NGRB (B = C or $\mathrm{G}$ or $\mathrm{T}$ ) in melanomas [17] (Table 1B) and several others, for which the underlying mechanisms remain unknown.

\subsection{Mismatch Repair and DNA Replicative Polymerase Proofreading Genes}

The high fidelity of human DNA replication achieves nucleotide incorporation error rates of $\sim 10^{-9}-10^{-10}$ in part through the proofreading $\left(3^{\prime} \rightarrow 5^{\prime}\right.$ exonuclease) activities of replicative polymerases, Pol $\varepsilon$ and $\mathrm{Pol} \delta$, and postreplicative mismatch repair (MMR), which decrease the rates of misincorporation on the newly synthesized daughter strands by 100-1,000-fold each [45-48]. The proofreading domains reside in the large 261 and $125 \mathrm{kDa}$ POLE and POLD1 catalytic subunits of the Pol $\varepsilon$ and Pol $\delta$ holoenzymes, respectively, which perform their specific function predominantly during leading-strand (Pol $\varepsilon$ ) and lagging-strand (Pol $\delta$ ) DNA synthesis in $\mathrm{S}$ phase [49]. The MMR pathway comprises 6 genes (MSH2, MSH3, MSH6, MLH1, PMS1 and PMS2) whose products yield 4 types of heterodimeric complexes [MutS $\alpha$ (MSH2/6), MutS $\beta$ (MSH2/3), MutL $\alpha$ (MLH1/PMS2) and MutL $\beta$ (MLH1/PMS1)], active on mismatches, bulges, small loops, and a number of DNA lesions [50,51].

More than 1,000 constitutional gene variants in MLH1, MSH2, MSH6 and PMS2 have been classified as pathogenic or likely pathogenic in patients affected by Lynch syndrome [52], an autosomal dominant condition also known as hereditary non-polyposis colorectal cancer (HNPCC) and characterized by increased susceptibility to colorectal $(25 \%-70 \%)$, endometrial $(30 \%-70 \%)$, and other types of cancer [53]. Such MMR defects have also been known to lead to an accumulation of mutations, mostly in the form of microsatellite length changes (microsatellite instability, MSI) [53]. The patterns of SBSs in cancers showing MSI have recently been addressed from the exome and whole-genome sequencing data of two large cohorts of colorectal [54] and endometrial [55] cancer patients (224 and 373 tumors, respectively), and the reconstructed whole-genomes from two gastric cancer patients [56]. In general, there was no relationship between MSI status (high/low) and SBS mutation rates. By contrast, most samples with elevated SBS mutation rates also displayed somatic mutations in the proofreading POLE domain [57]. In addition, POLE-mutated samples could be classified into two distinct groups based on $M L H 1$ status: group 1, with low SBS mutations rates, $M L H 1$ inactivation and MSI-high; and group 2, with high SBS mutation rates, functional $M L H 1$ and MSI-low. Thus, paradoxically, concomitant POLE and MLHI mutations do not appear to act synergistically on SBS mutation rates in most patients [57].

Mutations in the proofreading domains of POLE and POLD1 were also reported in two human colorectal cancer cell lines (DLD-1 and LoVo) and 1/76 colorectal cancer patients, all three samples exhibiting MMR deficiency [58]. More recently, two recurrent germline mutations in the proofreading domains of POLE (L424V) and POLD1 $(\mathrm{S} 478 \mathrm{~N})$ have been found in a cohort of 3,805 colorectal cancer patients selected for family history of colorectal tumors and multiple adenomas [59]. In the 62 tumors analyzed, no MSI was found; rather, loss of heterozygosity, chromosomal instability and driver mutations in known cancer genes, including $K R A S, B R A F$, 
$A P C, P I K 3 C A, F B X W 7$, but not $C T N N B 1$, were revealed, suggesting that mutant POLE ${ }^{\mathrm{L} 424 \mathrm{~V}}$ and POLD $1^{\mathrm{S} 478 \mathrm{~N}}$ may promote tumor formation by increasing the rates of SBS, without any apparent bias for a predominant type of base substitution [59]. Twelve missense somatic mutations predicted to affect the proofreading domain of POLE, and all associated with microsatellite stability, have also been identified in a study of 173 endometrial cancers, P286R being the most commonly represented (6 times) POLE mutation [60]. Using a panel of 75 cancer genes, POLE-mutated tumors exhibited an $\sim 6$-fold increase in mutations relative to non- $P O L E$-mutated tumors, with a prevalence of $\mathrm{G}: \mathrm{C} \rightarrow \mathrm{T}: \mathrm{A}$ transversions, particularly at $\mathrm{G}: \mathrm{C}$ base-pairs flanked $5^{\prime}$ and $3^{\prime}$ by an A:T base-pair [60]. Further validation of the putative role for POLE, and to a lesser extent POLD1, mutations in endometrial cancer has been obtained from an analysis of unpublished TCGA genomic data, where $21(8.5 \%)$ and $1(0.4 \%)$ tumors out of 248 samples were found to harbor POLE and POLD1 mutations, respectively, including 8 cases of P286R and 5 cases of V411L changes in POLE [60]. POLE/POLD1-mutated cancers displayed high SBS rates, ranging from 227 to 14,695 exonic events, compared to a range of 22 to 2,014 in cancers lacking POLE/POLD1 mutations. Likewise, cancers carrying the $\mathrm{POLE}^{\mathrm{P} 286 \mathrm{R}}$ allele exhibited an overrepresentation of $\mathrm{G}: \mathrm{C} \rightarrow \mathrm{T}: \mathrm{A}$ substitutions at A $\underline{G A}$ :TECT motifs (Table 1B), supporting a DNA sequence-specific proofreading defect for this particular POLE mutation [60]. In summary, germline and somatic mutations in the POLE and POLD1 genes appear to predispose to, or promote, colorectal and endometrial cancers in part by increasing the rates of SBSs [61]. Less direct investigations from cell culture nuclear extracts suggest that defects in DNA replication fidelity might also be associated with ovarian cancers [62].

\subsection{The APOBEC Family of Cytosine Deaminases}

The family of APOBEC cytosine deaminases comprises eleven members with distinct functions: activation-induced deaminase (AID), a B cell-specific enzyme required for both somatic hypermutation (SHM) and class-switch recombination (CSR); APOBEC1, which is expressed primarily in the gastrointestinal compartment and is active in the transcript sequence editing of the apolipoprotein B mRNA; APOBEC2, which is expressed in heart and skeletal muscles and which appears to be essential for muscle development; APOBEC3s (A, B, C, D, F, G and H), which are active against exogenous viruses and endogenous retroelements and hence important for innate immunity; and APOBEC4, which is mostly expressed in the testes but whose function remains unknown [63]. APOBECs have in common a zinc-dependent cytidine deaminase domain (ZDD), which catalyzes the conversion of cytosine and deoxycytidine to uracil and deoxyuracil [64] in single-stranded RNA and DNA, often in a sequence-dependent context.

Editing activities of APOBEC3s play a critical role in restricting viral infectivity, and have also been postulated to have counteracted the actions against genome stability, mostly in terms of integration, exerted by both non-LTR (long terminal repeats) and LTR retrotransposons during evolutionary time $[63,65]$. For example, in $\Delta$ vif (virion infectivity factor) HIV-1 particles, APOBEC3G proteins interact with the nucleocapsid domain of viral Gag to form nucleoprotein complexes with several Pol-II and Pol-III transcribed RNAs, which are then encapsulated into virions. During HIV-1 reverse transcription, up to $10 \%$ of cytosines can be deaminated to uracil on the minus strand of the viral complementary DNA (cDNA), thereby promoting loss of genetic 
information and the production of large populations of defective virions [63]. Vif antagonizes the activity of APOBEC3G (and other APOBEC3s) by binding and targeting the enzyme for polyubiquitination and subsequent degradation $[63,66,67]$. Other viruses targeted by APOBEC3 enzymes include human T-cell lymphotropic virus (HTLV), hepatitis B virus (HBV), hepatitis C virus (HCV), human papillomavirus (HPV) and human herpesviruses (HHV). In addition to deaminase activity, APOBEC enzymes restrict exogeneous viruses and endogenous retroelements through editing-independent activities. These include inhibition of viral replication, for example by interfering with tRNA priming and the initiation of DNA replication during HIV-1 reverse transcription, binding with positive regulators of viral gene expression, as in the case of heterogeneous nuclear ribonucleoprotein K (hnRNP) for HBV [63], and other less well-characterized mechanisms aimed at restricting non-LTR retrotransposon transcription, DNA synthesis and integration [65].

At least three recent reports have suggested the involvement of aberrant APOBEC3B deaminase activity as a frequent cause of SBSs in cancer. For example, APOBEC3B mRNA was found to be upregulated relative to controls in 28/38 established breast cancer cell lines and to be expressed in the nucleus [68]. In selected nuclear extracts, $\mathrm{C} \rightarrow \mathrm{U}$ editing activity was detected on synthetic DNA substrates specifically at $\underline{T} \underline{\text { : }} \underline{\mathrm{G}} \mathrm{A}$ dinucleotides when treated with a control shRNA, whereas no activity was evident upon treatment with short hairpin RNA (shRNA) targeting APOBEC3B mRNA. In these cell lines, treatment with anti-APOBEC3B shRNA also led to a decrease in genomic uracil loads from $\sim 100,000$ to $\sim 60,000$ per haploid genome by HPLC-ESI-MS/MS and, as expected, test amplicons displayed a decrease in $\mathrm{C} \rightarrow \mathrm{T}$ transition mutation frequency. The involvement of APOBEC3B activity in cancer was further supported by data on Ref-seq APOBEC3B expression, which was shown to be high in several tumor types, including breast, uterus, bladder, head and neck and lung (both adenocarcinoma and squamous cell carcinoma) [15,20]. The top five cancer types with the majority of mutations at $\mathrm{C}$ : G base-pairs were also among the top six datasets in terms of APOBEC3B mRNA expression, and a positive correlation between the proportion of mutations at $\mathrm{C}: \mathrm{G}$ base-pairs and median APOBEC3B levels was observed. Bladder, cervical, lung squamous cell carcinoma, lung adenocarcinoma, head and neck, and breast cancers shared a strong bias for $\mathrm{T} \underline{\mathrm{CN}}(\mathrm{N}=\mathrm{A}$ or $\mathrm{C}$ or $\mathrm{G}$ or $\mathrm{T})$ mutation signatures, as observed for the recombinant APOBEC3B protein. Interestingly, a significant enrichment of strand-coordinated and clustered ( 2 or more per $10 \mathrm{~kb}$ ) $\mathrm{C} \rightarrow \mathrm{T}$ and $\mathrm{C} \rightarrow \mathrm{G}$ mutations at $\mathrm{T} \underline{\mathrm{CW}}(\mathrm{W}=\mathrm{A}$ or $\mathrm{T}$ ) motifs were discovered, a number of which were in close proximity to chromosomal rearrangement breakpoints, particularly in bladder, cervical, head and neck, breast and lung tumors [15,20], a phenomenon termed kataegis [3]. In summary, these analyses are consistent with the possibility that aberrant APOBEC deaminase


to several different types of human cancer.

\subsection{Electron Transfer in DNA Oxidation}

Mutation spectra analyses of SBSs arising spontaneously, both in cell culture and in whole animals, have indicated the frequent occurrence of sequence context-dependent mutations. For example, an analysis of 837 spontaneous SBSs in the supF tRNA gene in 18 cell lines and 2 transgenic mouse models indicated that the most mutable regions involved guanine and cytosine 
tracts [69]. In human osteosarcoma cells, shRNA knock-down of the WRN helicase gene, mutations in which are associated with the progeroid Werner syndrome, led to a doubling in genomic 8-oxo-7,8-dihydro-2'-deoxyguanosine (8-oxoG) content and an increase in SBSs in the supF reporter gene, consisting largely of $\mathrm{G} \rightarrow \mathrm{C}(49 \%), \mathrm{G} \rightarrow \mathrm{A}(28 \%)$ and $\mathrm{G} \rightarrow \mathrm{T}(23 \%)$ substitutions at $\underline{\mathrm{GA}} \mathrm{T} \underline{\mathrm{C}}$ dinucleotides within the supF reporter gene [70]. Because 8-oxoG is a well-recognized marker of oxidative damage and guanine oxidation depends upon flanking sequence (i.e., GR $>\mathrm{GY}$; R $=\mathrm{A}$ or $\mathrm{G} ; \mathrm{Y}=\mathrm{T}$ or $\mathrm{C}$ ) as a result of stacking-induced electron transfer [71,72], these data suggested a role for oxidative damage in sequence context-dependent mutagenesis.

A recent study addressed the question as to whether electron transfer might also cause sequence context-dependent SBSs in cancer [17]. The analysis compared the fractions of mutations occurring

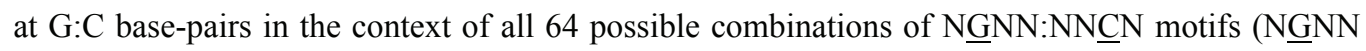
for simplicity), and included 21 cancer datasets representing 14 tissues comprising 1,149 patient samples and 2 cell lines for a total of 533,482 SBSs. With the exception of two melanoma datasets, C $\underline{G N N}$ sequences were more frequently mutated than $\mathrm{DGNN}(\mathrm{D}=\mathrm{A}$ or $\mathrm{G}$ or $\mathrm{T})$, consistent with the CG:CG dinucleotide, the most prominent substrate for cytosine methylation, being a common mutation hotspot (Table 1A). In 7 cancer datasets, including lung, head and neck and melanoma, for which association with exposure to either cigarette smoke or sunlight was documented, G followed by a 3' purine was associated with increased mutations as compared to a 3' pyrimidine, i.e., DGRN > D $\underline{G} Y N$ (Table 1A). Notably, significant correlations were observed between the fractions of mutated DGNN motifs and the sequence-dependent free energies of base stacking along the DGNN motifs for 5 of the 7 cancer datasets. Significant correlations were also observed between the fractions of mutated DGNN motifs and the energies required to abstract an electron from the target guanines, as assessed from the values of vertical ionization energies computed for all G-centered trimer motifs. These results are consistent with the conclusion that DNA oxidation may be a source of sequence context-dependent SBSs in cancer as a result of electron transfer, as postulated from model sequences in vitro [73,74].

\subsection{DNA Replication Timing}

During eukaryotic DNA replication, more than 20,000 pre-replicative complexes comprising the origin recognition complex (ORC), Cdc6 and Cdt1 load inactive minichromosome maintenance (MCM) helicase complexes to generate "licensed" replication origins. Initiation of DNA synthesis is controlled by Dbf4-dependent kinase (DDK), which recruits Cdc45 and Sld3, and cyclindependent kinase (CDK), which by phosphorylating Sld3 and Sld2 recruit GINS and additional factors, including DNA polymerases. Origin firing is controlled both temporally and spatially. Chromatin correlating positively with gene expression, $\mathrm{G}+\mathrm{C}$-richness and active chromatin marks is replicated in early $\mathrm{S}$ phase in the nuclear interior, whereas chromatin associated with gene-poor regions, A + T-richness and repressive chromatin marks is preferentially replicated during late $\mathrm{S}$ phase at the nuclear periphery $[75,76]$.

In cancer, late replicating chromatin has been shown to harbor higher relative fractions of SBSs than early replicating chromatin. For example, the fractions of SBSs from several completely sequenced cancer genomes (melanoma, prostate cancer, small cell lung cancer, chronic lymphocytic 
leukemia and colorectal cancer) were found to be significantly more extensive in the constant late than in the constant early replicating zones of neutrally evolving regions [77]. Such genomic regions were those remaining after sequences from centromeres, telomeres, the Y chromosome, genes, promoters, repetitive elements and ultra-conserved regions had been excluded. Analyses of the mutation spectra indicated that, with the exception of $\mathrm{A} \rightarrow \mathrm{T}(\mathrm{T} \rightarrow \mathrm{A})$ transversions, which occurred more often in the constant late replicating regions in all five cancer types, the relative proportions of substitutions were very similar between constant early and constant late replicating regions, even though mutation frequencies were higher in the latter. Similarly, a large study of SBSs in exomes from 3,083 tumor-normal pairs representing 27 different cancer types also found that the average mutation fraction was higher $(\sim 2.9$-fold $)$ in the latest- as compared to the earliestreplicating percentiles [18].

The increased mutation frequency in late as compared to early replicating regions does not appear to be a unique property of cancer cells. For example, a comparison of 1-Mb non-overlapping regions containing SBSs between human and chimpanzee and the pooled cancer data [77] indicated that most regions harboring human-chimpanzee SBSs also harbored SBSs in cancer. Similarly, in human populations, late-replicating regions of the human genome have been shown to be characterized by a greater density of single nucleotide polymorphisms (SNPs) than early replicating regions [78]. Finally, deep-sequencing of human lymphoblastoid cell lines from father-mother-offspring trios revealed that transition mutations were $>2$-fold more abundant in late-replicating than in early-replicating regions of the genome, whereas transversion mutations were increased $>6$-fold [79]. In summary, these analyses suggest that the increased mutation rate in late- versus early-DNA replicating regions, which has been noted in both population and cancer genome studies, are potentially caused by mechanisms that share some commonalities between the germline and the soma.

\subsection{Chromatin Organization}

Chromatin structure has been found to strongly correlate with regional SBS rates along chromosomes in cancer genomes. Chromatin organization is regulated by many factors, including epigenetics, i.e., reversible changes both at the level of DNA and involving histone tail amino acids. Chromatin condensation, or heterochromatin, is associated with reduced gene expression and involves the accumulation of specific histone marks, including methylation of lysines 9 and 27 on histone 3 (H3K9me2 and H3K27me3) by histone methyltransferases (HMT) such as G9a, GLP and SETBD1, and DNA methylation at C5 of cytosine, mainly at CG:CG dinucleotides, by DNA (cytosine-5)-methyltransferases (DNMT1, DNMT3a and DNMT3b). Among other critical interactions, H3K9me2 serves as a high-affinity binding site for the recruitment of heterochromatin protein 1 (HP1), a platform protein that collapses chromatin into higher-order fibers as a result of dimerization between nucleosomes. By contrast, promoter DNA demethylation and acetylation of H3K9 maintains open chromatin structure, or euchromatin, and supports active gene transcription [80]. These represent only a few epigenetic marks that are known to influence chromatin structure.

A total of 84,879 unique SBS positions from leukemia, melanoma, small cell lung cancer and prostate cancer genomes were used to identify potential sources of mutation rate variation across the genome, by correlating site variation with a set of 46 diverse genetic and epigenetic features 
that had been mapped genome-wide in human cells [81]. On a megabase scale, cancer SBS density correlated with many features of somatic cell chromatin organization, with the highest positive correlations being represented by the repressive histone modification $\mathrm{H} 3 \mathrm{~K} 9 \mathrm{me}$, followed by $\mathrm{H} 3 \mathrm{~K} 9 \mathrm{me} 2$ and $\mathrm{H} 4 \mathrm{~K} 20 \mathrm{me} 3$. In fact, $>55 \%$ of the variance in cancer SBS regional variation could be accounted for by combining features, with $\mathrm{H} 3 \mathrm{~K} 9 \mathrm{me} 3$ alone associating with more than $40 \%$ of the observed variance in SBS density. Interestingly, the associations remained strong when only non-genic or only genic regions of the genome were considered, suggesting that transcription or transcription-coupled nucleotide excision repair may have played a comparatively minor role as compared to epigenetic modifications. We should note that, in a separate study, levels of gene transcription were found to correlate inversely with mutation rates [18], although the process of transcription is known to be a source of genetic instability $[82,83]$. The use of a metric that employed data on physical contacts between regions through three-dimensional folding of chromosomes, thereby distinguishing between densely packed chromatin with strong short-range interactions and accessible euchromatin with more diverse interactions, also revealed an anti-correlation pattern with somatic SBS density [81]. These findings led the authors to propose that chromatin organization is a major determinant of variation in regional mutation rates in cancer. Specifically, SBS rates in cancer cells appear to be highest in inaccessible, heterochromatin-like regions and lowest in accessible euchromatin-like domains. Reversible histone acetylation and deacetylation events in the cell are also critical for mutation avoidance. For example, failure to


increased the rates of SBS $\sim 10$-fold and the rates of gross chromosomal rearrangements 15,600-fold, whereas lack of H3K59 acetylation in rtt109A cells led to a $\sim 10$-fold increase in complex mutations [84]. These effects were synergistic with mutations in MMR and the proofreading activities of replicative Pols $\delta$ and $\varepsilon$, suggesting a model in which cyclic acetylation and deacetylation of chromatin is critical for replication fidelity.

\section{Mechanisms of Base Modification}

\subsection{Cytosine Methylation and Demethylation}

The ability of site-specific DNA (cytosine-5)-methyltransferases to transfer the methyl group from $S$-adenosylmethionine to the C5 position of cytosine in the context (mainly) of CG:CG dinucleotides in mammals and the role of DNA methylation in transcriptional regulation, genomic imprinting and silencing of repetitive DNA have been well reviewed $[85,86]$. During the past few years, it has become evident that DNA methylation can be reversed by a group of enzymes belonging to the ten-eleven translocation (TET1, 2, and 3) family of iron and $\alpha$-ketoglutarate $(\alpha-K G)$-dependent dioxygenases, which utilize molecular oxygen to transfer a hydroxyl group to $5 \mathrm{mC}$ to form 5 -hydroxymethylcytosine $(5 \mathrm{hmC}$ ) (Figure 1 , top). Whereas approximately $4 \%$ of all cytosines ( $70 \%-80 \%$ of CG:CGs) are estimated to be methylated, only $\sim 0.1 \%-0.7 \%$ total appear to be marked by hydroxymethylation [87]. TET enzymes can further oxidize $5 \mathrm{hmC}$ sequentially to yield 5-formylcytosine $(5 \mathrm{fC})$ and 5-carboxycytosine $(5 \mathrm{caC})$, and evidence is increasing for a role of thymine DNA glycosylase (TDG), a member of the base excision repair pathway, in cleaving $5 \mathrm{fC}$ 
and $5 \mathrm{caC}$ thereby yielding abasic sites which are then replaced with unmodified cytosines $[88,89]$ (Figure 1, top).

A critical step in the TET-dependent oxidation reactions is the role played by $\alpha-\mathrm{KG}$, which binds to a His-His-Asp-coordinated Fe(II) cluster in the enzymatic active site to effect the transfer of an oxygen to the substrate $5 \mathrm{mC}$ and release $5 \mathrm{hmC}$. $\alpha-\mathrm{KG}$ is synthesized from isocitrate in a fully reversible reaction by different isoforms of $\mathrm{NADP}^{+}$-dependent isocitrate dehydrogenases (IDH), with IDH2 and IDH3 acting in the mitochondria as part of the tricarboxylic acid (TCA) cycle (Krebs cycle), and IDH1 providing a source of NADPH in the cytoplasm for lipid biosynthesis and protection from oxidative stress [90].

Recurrent gain-of-function mutations in IDH1 and/or IDH2 typify $\sim 70 \%$ of sporadic high-grade gliomas and secondary glioblastomas, $\sim 10 \%$ of acute myeloid leukemias [16] and colangiocarcinomas [91], and have been reported in patients with acute lymphoblastic leukemia, chondrosarcomas, angioimmunoblastic T-cell lymphoma, cholangiocarcinoma and pancreatic cancers $[90,92]$, where they have been found to induce DNA hypermethylation at CG:CG islands and shores in a tissue-specific manner [91]. The mutations, which occur at the active site of IDHs, alter the reaction order of the enzymes such that high concentrations of D-2-hydroxyglutarate (2-HG), rather than $\alpha-\mathrm{KG}$, are released [92] (Figure 2). Thus, 2-HG binding to both TET enzymes as well as other cellular dioxygenases, including histone demethylases and propyl hydroxylases, effectively inhibits their activities.

Figure 1. (Top) Cytosine methylation and demethylation pathways; (Bottom) Products of cytosine and C5-substituted cytosine deamination.

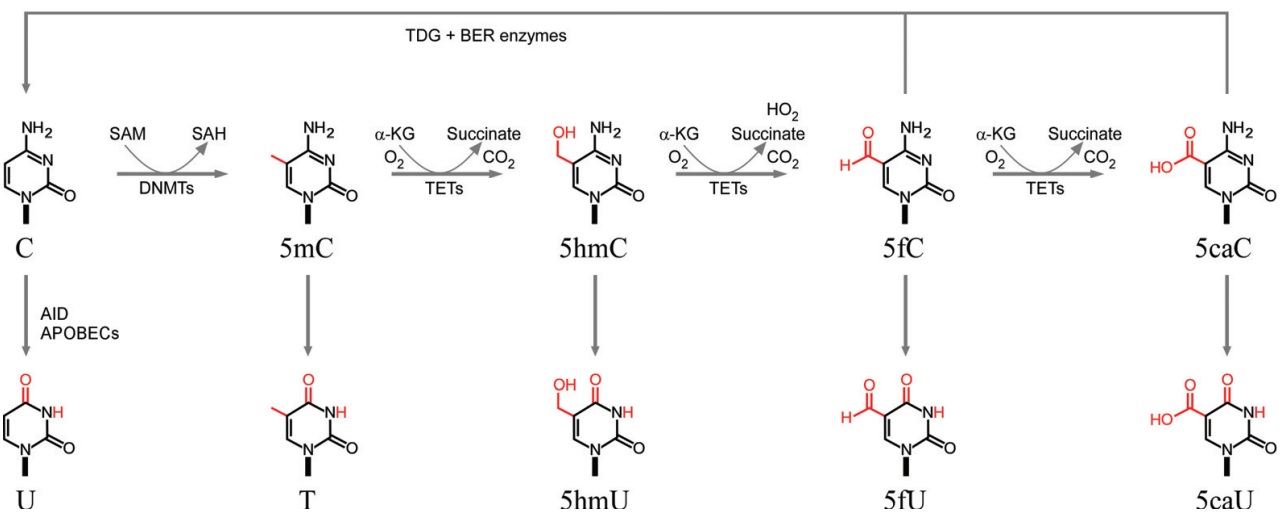

Figure 2. Conversion of isocitrate to $\alpha$-ketoglutarate $(\alpha-K G)$ by isocitrate dehydrogenases (IDH) enzymes and conversion of $\alpha-K G$ to D-2-hydroxyglutarate (2$\mathrm{HG}$ ) by gain-of-function mutations in $\mathrm{IDH} 1$ or $\mathrm{IDH} 2$.

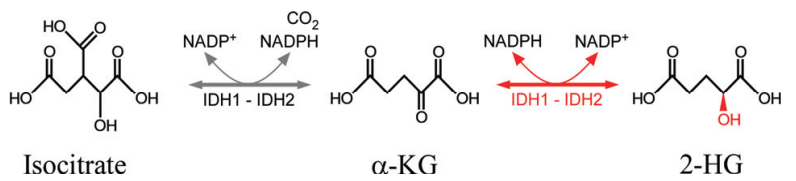


In melanoma, loss of $5 \mathrm{mC}$ through oxidation to $5 \mathrm{hmC}$ has been observed both as a result of IDH1 or IDH2 neomorphic mutations as well as the downregulation of TET and IDH2 genes [93]. A comparison between benign nevi and melanoma further supported the selective loss of $5 \mathrm{hmC}$ in melanoma; in addition, the extent of $5 \mathrm{hmC}$ loss in melanoma correlated directly with Breslow depth, a predictor of prognosis, pathological stage and, most significantly, Kaplan-Meier survival curves [93]. Large losses of 5hmC peaks and higher levels of $5 \mathrm{mC}$ in melanoma versus nevi were detected within gene coding and flanking regions, including genes associated with adherens junctions, Wnt signaling, additional pathways in cancer, and melanogenesis pathways, implying a role for $5 \mathrm{hmC}$ in pathways that are fundamental to cellular differentiation and dedifferentiation. In mouse embryonic stem cells, $5 \mathrm{fC}$ and $5 \mathrm{hmC}$ were found to be enriched in intragenic regions, especially within exons and enhancers, where they colocalized with histone acetyltransferase p300 sites, DNaseI hypersensitive sites and CTCF-bound regions, specifically at poised enhancers, which are marked by $\mathrm{H} 3 \mathrm{~K} 4 \mathrm{me} 1[+]$ and $\mathrm{H} 3 \mathrm{~K} 27 \mathrm{ac}[-]$, in comparison to active enhancers $(\mathrm{H} 3 \mathrm{~K} 4 \mathrm{me} 1[+] \mathrm{H} 3 \mathrm{~K} 27 \mathrm{ac}[+])$, concomitantly with a decrease in $5 \mathrm{mC}$ [88]. Accordingly, in the absence of TDG, accumulation of $5 \mathrm{fC}$ correlated with increased binding of the transcriptional activator p300 at poised enhancers. These data support a role for $5 \mathrm{mC}$ and $5 \mathrm{hmC}$ oxidation in the regulation of the epigenetic state of functional enhancer elements in mammalian genomes. CG:CG hypermethylation at specific genes was also found to represent a marker for relapse-free survival time after surgery in a cohort of 444 patients with non-small cell lung cancer. Specifically, patients with zero to one methylated markers in the HIST1H4F, PCDHGB6, NPBWR1, ALX1 and HOXA9 genes were characterized by a longer relapse-free survival time than those with two or more hypermethylated markers; $48 \%$ from the enriched methylated group relapsed, as compared with only $18 \%$ of those in the less methylated group [94].

\subsection{Deamination of Cytosine Bases}

\subsubsection{Spontaneous Deamination}

The rate constants for the spontaneous deamination of cytosine $(\mathrm{C})$ and protonated $\mathrm{C}$ to uracil (U), $5 \mathrm{mC}$ to $\mathrm{T}, 5 \mathrm{hmC}$ to $5 \mathrm{hmU}, 5 \mathrm{fC}$ to $5 \mathrm{fU}$ and $5 \mathrm{caC}$ to $5 \mathrm{caU}$ (Figure 1 , bottom) have been determined from both the extrapolations of Arrhenius plots and genetic assays [95-100]. In doublestranded DNA, deamination rates for $\mathrm{C}$ and $5 \mathrm{mC}$ are extremely slow, of the order of $10^{-13} \mathrm{~s}^{-1}$ (Table 2), which translates into half-lives ranging between $\sim 30,000$ to $\sim 85,000$ years. Rates increase approximately 3-orders of magnitude in both single-stranded DNA and in isolated deoxyribonucleotides, supporting the view that hydrogen bonding plays a major role in shielding cytosines from spontaneous deamination. Comparison of the data given in Table 2 indicates that rates of deamination for $5 \mathrm{mC}$ are only marginally higher than for cytosine. Rates increase by an additional 3-orders of magnitude upon protonation; however, since cytosine protonation occurs at acidic $\mathrm{pH}(\mathrm{pKa}=2.4)$ within a $\mathrm{C}: \mathrm{G}$ Watson-Crick base-pair [101], it is unlikely to play a significant role in vivo. That DNA melting is rate-limiting for cytosine deamination is further suggested by evolutionary studies, which indicate that CG:CGs embedded within $\mathrm{G}+\mathrm{C}$-rich areas (H isochores), and thus characterized by increased melting temperatures, have been depleted to a 
lesser extent than $\mathrm{CG}$ :CGs embedded in $\mathrm{G}+\mathrm{C}$-poor ( $\mathrm{L}$ isochores) regions, which melt at lower temperatures [102]. Further support is provided by the finding that $\mathrm{C} \rightarrow \mathrm{T}$ transitions decrease gradually with increasing nucleosome occupancy score in comparative studies of $S$. cerevisiae, medaka (Oryzias lapites) and C. elegans genomes [103]. Analysis of the hydrolytic deamination reaction using density functional theory shed considerable light on the requirements for base unpairing and the effect of protonation [104]. Two pathways have been identified (Figure 3). In pathway A, upon protonation of N3 (Figure 3a), nucleophilic addition of a first water molecule to carbon $\mathrm{C} 4$, leads to the formation of a tetrahedral intermediate with the assistance of a second water molecule (Figure $3 \mathrm{~b}$ ). The $\mathrm{C} 4-\mathrm{N} 4$ bond is then broken and a proton transfer takes place from the hydroxyl group at $\mathrm{C} 4$ to $\mathrm{NH}_{3}$, thereby forming thymine and an ammonium cation. In pathway $\mathrm{B}$, nucleophilic addition of the first water molecule to $\mathrm{C} 4$ occurs on the neutral $5 \mathrm{mC}$ (Figure $3 \mathrm{~b}$ ), again with the assistance of a second water molecule, yielding a neutral tetrahedral intermediate (not shown). The exocyclic amino group is then protonated through an intermolecular proton transfer, after which the reaction proceeds as in pathway A. For both pathways, the nucleophilic addition is the rate-determining step; however, whereas nucleophilic addition to carbon $\mathrm{C} 4$ of $5 \mathrm{mC}$ is easier than to the $\mathrm{N} 3$-protonated form, the trend is reversed in the case of $\mathrm{C}$ and $\mathrm{N} 3$-protonated C. Thus, deamination of $5 \mathrm{mC}$ is more difficult than that of $\mathrm{C}$ in pathway $\mathrm{A}$, whereas the opposite is seen in pathway B.

This study has several implications. First, nucleophilic attack and formation of the tetrahedral intermediate cannot occur on duplex DNA; second, only pathway B is compatible with the greater susceptibility of $5 \mathrm{mC}$ to deamination, compared with $\mathrm{C}$; third, a protonated base is not required for pathway B; and fourth, for pathway B, the activation free energy for $5 \mathrm{mC}(134.1 \mathrm{~kJ} / \mathrm{mol}$ in aqueous solution) is only $4.4 \mathrm{~kJ} / \mathrm{mol}$ less than that associated with $\mathrm{C}(138.5 \mathrm{~kJ} / \mathrm{mol}$ in aqueous solution), implying that the susceptibility to deamination of $5 \mathrm{mC}$ relative to $\mathrm{C}$ is no more than $4-5$-fold. In summary, these investigations show that (1) spontaneous deamination of cytosine bases only occurs in single-stranded DNA; (2) deamination of $5 \mathrm{mC}$ is only marginally more efficient than for C; and (3) all $\mathrm{C} 5$ substituted bases, with the exception of $5 \mathrm{caC}$, display detectable (and rather similar) rates of deamination.

\subsubsection{ROS-Induced Deamination}

ROS, such as the non-radical hydrogen peroxide $\left(\mathrm{H}_{2} \mathrm{O}_{2}\right)$ and free superoxide radicals $\left(\mathrm{O}_{2}{ }^{-}\right)$, are generated as a result of mitochondrial respiration, from the activation of growth factor receptors through NADPH oxidase, the arachidonic acid cascade and others, and play crucial roles as signal transduction molecules and neuroregulators [105,106]. $\mathrm{H}_{2} \mathrm{O}_{2}$ may also generate free radicals, including the hydroxyl radical $(\mathrm{OH})$, the most potent oxidizing radical generated by the cell, through routes including ionizing radiation, interactions with $\mathrm{O}_{2}{ }^{-}$through the Haber-Weiss reaction and by interactions with transition metal ions $[\mathrm{Fe}(\mathrm{II})$ and $\mathrm{Cu}(\mathrm{I}) / \mathrm{Cu}(\mathrm{II})]$ through Fenton chemistry, as exemplified in reaction 1.

$$
\mathrm{Cu}(\mathrm{II})+\mathrm{H}_{2} \mathrm{O}_{2} \rightarrow \mathrm{Cu}(\mathrm{I})+\mathrm{H}_{2} \mathrm{O}+\mathrm{H}^{+} ; \mathrm{Cu}(\mathrm{I})+\mathrm{H}_{2} \mathrm{O}_{2} \rightarrow \mathrm{Cu}(\mathrm{II})+\mathrm{OH}+\mathrm{OH}^{-}
$$


Free radicals constitute an important endogenous source of damage to DNA and other molecules, and therefore strict homeostatic controls exist in the cell between the generation and neutralization of ROS species by catalase, superoxide dismutase 1 and 2 (SOD1 and 2), the glutathione peroxidase (GPX) and peroxiredoxin (PRX) families of detoxifying enzymes and other antioxidants, such as vitamins $\mathrm{C}$ and $\mathrm{E}[106,107]$. A number of studies in leukemia, breast cancer, ovarian cancer, benign and malignant prostate cancer, non-small cell lung carcinoma, cervical squamous cell carcinoma, stomach cancer, and Hodgkin's disease patients all concur with the conclusion that levels of ROS detoxifying enzymes are generally lower in cancer than in surrounding normal tissue, leading to oxidative stress, i.e., altered ROS homeostasis in favor of increased steady-state levels of ROS [107]. In addition, as in the case of $B C R-A B L 1$ translocations in myeloid leukemia, Rac2 activation has been shown to reduce the mitochondrial membrane potential $\left(\Delta \Psi_{m}\right)$, thereby inducing electron leakage from the mitochondrial respiratory chain complexes I-III and II-III (MRC-cIII) and, as a consequence, a 2- to 6-fold increase in cellular ROS [108].

Treatment of duplex oligonucleotides containing methylated and unmethylated CG:CGs with $\mathrm{Cu}(\mathrm{II}) / \mathrm{H}_{2} \mathrm{O}_{2} /$ ascorbate to effect Fenton-type reactions led to much more frequent modifications of $5 \mathrm{mC}$ than $\mathrm{C}$ [109]. One of the main products involved the saturation of the C5-C6 double-bond of $5 \mathrm{mC}$, to yield 5-methyl-5,6-dihydroxy-5,6-dihydro-2'-deoxycytidine (5-methyl-2'-deoxycytidine glycol, $5 \mathrm{mCg}$ ). Kinetic determinations of the spontaneous deamination of two stereoisomers of $5 \mathrm{mCg}$, i.e., $5 \mathrm{mCg}(5 S, 6 S)$ and $5 \mathrm{mCg}(5 R, 6 R)$ in duplex DNA, which affords 5,6-dihydroxy-5,6-dihydrothymidine (thymidine glycol, Tg) [110], indicated rates in the range of $\sim 10^{-6} \mathrm{~s}^{-1}$, similar to the values determined for isolated nucleotides. Thus, ROS-induced saturation of the C5-C6 double-bond in $5 \mathrm{mC}$ increases rates of deamination by $\sim 4$ orders of magnitude with respect to single-stranded DNA, and $\sim 7$ orders of magnitude with respect to unmodified $5 \mathrm{mC}$ in double-stranded DNA. The susceptibility of duplex DNA to damage by Fenton-type reactions has been assessed using 5S rDNA, either alone or upon reconstitution on nucleosome particles. The number of single base lesions was found to be 8-fold higher on nucleosomal DNA than on isolated DNA, implying that Fenton chemistry is not only unrestricted by chromatin compaction but actually appears to be facilitated [111]. Although the roles of histone octamers in permitting DNA damage are not fully understood, X-ray crystal structure studies revealed the presence of many divalent metal binding sites in nucleosome particles [112], and several peptide models of histones H2A, H2B, H3 and H4 have been shown to coordinate $\mathrm{Cu}(\mathrm{II})$, mostly through macrochelate rings involving histidine and carboxylate groups [113]. The extremely short $(<1 \mathrm{~ns})$ half-life of $\mathrm{OH}$ likely restricts Fenton chemistry at sites of $\mathrm{OH}$ generation. Thus, given that $\mathrm{H}_{2} \mathrm{O}_{2}$ is relatively stable and able to diffuse across cells, copper coordination within nucleosome particles might provide a suitable environment for oxidative DNA damage in chromatin. Determinations of copper concentrations by both atomic absorption spectroscopy and X-ray fluorescence in plasma and tumor samples from several types of cancer indicate that levels are usually higher (up to 2- to 3-fold) in cancer patients than in normal controls [107]. Indeed, high levels of copper appear to be required for tumor growth [114]. 
Table 2. Rates of spontaneous deamination for cytosines.

\begin{tabular}{|c|c|c|c|c|}
\hline Deaminating base & Sequence context & Deaminated base product & Deamination rate at $37^{\circ} \mathrm{C}\left(s^{-1}\right)$ & References \\
\hline $\mathrm{C}$ & Free nucleoside & $\mathrm{U}$ & $9.4 \pm 0.5 \times 10^{-10}$ & [95] \\
\hline $3 \mathrm{mC}^{+}$ & Free nucleoside & $3 \mathrm{mU}$ & $5 \times 10^{-7}$ & {$[100]$} \\
\hline $3 \mathrm{mC}^{+}$ & Free nucleotide & $3 \mathrm{mU}$ & $13 \times 10^{-7}$ & {$[100]$} \\
\hline $5 \mathrm{mC}$ & Free nucleoside & $\mathrm{T}$ & $7.8 \pm 0.3 \times 10^{-10}$ & {$[95]$} \\
\hline $5 \mathrm{hmC}$ & Free nucleoside & $5 \mathrm{hmU}$ & $5.8 \pm 0.8 \times 10^{-10}$ & [95] \\
\hline $5 \mathrm{fC}$ & Free nucleoside & $5 \mathrm{fU}$ & $1.2 \pm 0.2 \times 10^{-9}$ & [95] \\
\hline $5 \mathrm{caC}$ & Free nucleoside & $5 \mathrm{caU}$ & not detected & {$[95]$} \\
\hline $5 \mathrm{mCg}(5 S, 6 S)$ & Free nucleoside & $\operatorname{Tg}$ & $1.1 \times 10^{-5}$ & [115] \\
\hline $5 \mathrm{mCg}(5 R, 6 R)$ & Free nucleoside & $\operatorname{Tg}$ & $8.6 \times 10^{-6}$ & [115] \\
\hline $\mathrm{C}$ & ssDNA & $\mathrm{U}$ & $2.1 \times 10^{-10}$ & [96] \\
\hline $\mathrm{C}$ & ssDNA & $\mathrm{U}$ & $\sim 1 \times 10^{-10}$ & [98] \\
\hline $5 \mathrm{mC}$ & ssDNA & $\mathrm{T}$ & $9.5 \times 10^{-10}$ & [96] \\
\hline $\mathrm{C}$ & dsDNA & $\mathrm{U}$ & $2.6 \times 10^{-13}$ & [97] \\
\hline $\mathrm{C}$ & dsDNA & U & $4 \times 10^{-13}$ & [99] \\
\hline $\mathrm{C}$ & dsDNA & $\mathrm{U}$ & $\sim 7 \times 10^{-13}$ & [98] \\
\hline $5 \mathrm{mC}$ & dsDNA & $\mathrm{T}$ & $5.8 \times 10^{-13}$ & [97] \\
\hline $5 \mathrm{mC}$ & dsDNA & $\mathrm{T}$ & $1.5 \times 10^{-11}$ & [99] \\
\hline $5 \mathrm{mCg}(5 S, 6 S)$ & dsDNA & $\mathrm{Tg}$ & $5.2 \times 10^{-6}$ & [109] \\
\hline $5 \mathrm{mCg}(5 R, 6 R)$ & dsDNA & $\mathrm{Tg}$ & $7.0 \times 10^{-6}$ & [109] \\
\hline
\end{tabular}

C, cytosine; 5mC, 5-methylcytosine; $3 \mathrm{mC}^{+}, N^{3}$-methylcytosine; 5hmC, 5-hydroxymethylcytosine; $5 \mathrm{fC}$, 5-formylcytosine; $5 \mathrm{caC}, 5$-carboxycytosine; $5 \mathrm{mCg}(5 \mathrm{~S}, 6 \mathrm{~S})$ and $5 \mathrm{mCg}(5 R, 6 R), 5$-methylcytosine glycol stereoisomers; $\mathrm{U}$, uracil; $3 \mathrm{mU}$, $N^{3}$-methyluracil; T, thymine; 5hmU, 5-hydroxymethyluracil; 5fU, 5-formyluracil; Tg, thymine glycols.

Figure 3. (a) Numbering scheme for cytosines; (b) Pathways for the spontaneous deamination of $\mathrm{C}$ and $5 \mathrm{mC}$ (only $5 \mathrm{mC}$ is shown).

(a)<smiles>Cc1cn(C)c(=O)nc1[NH]</smiles>

Numbering scheme (b)

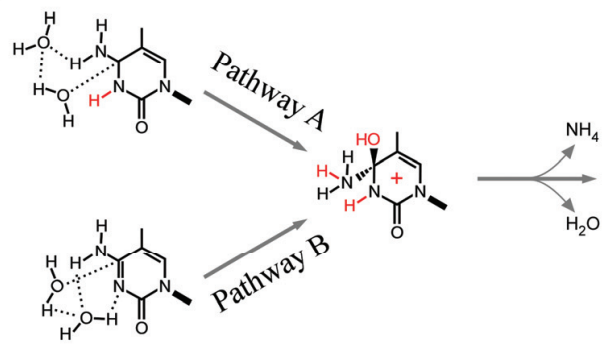



$\mathrm{T}$

In summary, oxidation of $5 \mathrm{mC}$ by copper ions and ROS generate cytosine glycol intermediates, which deaminate at high rates to yield Tg:G mispairs. DNA oxidation by Fenton chemistry is enhanced in nucleosomal DNA, in which several coordination sites for copper and other metal ions have been identified. Both ROS and copper concentrations have been found to be enhanced in tumors, raising the possibility that ROS-dependent deamination at methylated CG:CG sites may contribute to mutation in cancer. 


\subsubsection{Enzymatic Deamination by Single-Stranded DNA-Specific AID/APOBECs}

AID/APOBEC enzymes catalyze the deamination of cytosine to uracil on single-stranded DNA (Figure 1, bottom). The active site of APOBEC enzymes is recognized by a conserved motif (H-X-E-X $23-28-\mathrm{P}-\mathrm{C}-\mathrm{X}-\mathrm{C}$ ), in which a coordinated zinc ion carries out the nucleophilic attack during the deamination reaction. In the high-resolution crystal structures of human APOBEC 2 and the catalytic domain of APOBEC3G (aa 197-380), a water molecule serves as a hydrogen donor, whereas a conserved glutamate residue (E100 in APOBEC2 and E259 in APOBEC3G) functions as a proton shuffler during the hydrolytic cycle [116]. As with other DNA metabolizing enzymes, the target cytosine is flipped-out and inserted into the active site; because the flipping step appears to involve passive DNA breathing, it probably accounts for the greater enzymatic activities observed for deamination of single-stranded, as opposed to double-stranded, nucleic acids [117,118]. Whether AID/APOBEC enzymes deaminate cytosines modified at $\mathrm{C} 5$, including $5 \mathrm{mC}, 5 \mathrm{hmC}, 5 \mathrm{fC}$ and $5 \mathrm{caC}$ has been controversial, with recent studies reporting generally weak or no activities towards C5-substituted cytosines [118-120], and earlier work reporting strong activities on $5 \mathrm{mC}$ by human AID and rat APOBEC1 [121]. Experiments performed by scoring mutations either in viral DNA or in vitro with model sequences indicate strong effects on deamination rates by nucleotides flanking the target cytosine: optimal substrates include $(\mathrm{C} / \mathrm{G}) \mathrm{T} \underline{\mathrm{C}}(\mathrm{A} / \mathrm{G})$ for APOBEC3B [122,123], T(T/C)ㅡㅡ for APOBEC3C [124], CCㅡA for APOBEC3G [122,124-127], TTCET for APOBEC3F [124-126], WRCY ( $\mathrm{W}=\mathrm{A}$ or $\mathrm{T} ; \mathrm{R}=\mathrm{A}$ or $\mathrm{G} ; \mathrm{Y}=\mathrm{C}$ or $\mathrm{T}$ ) for AID [128], although comparative analyses using enzymatic kinetic constants awaits further work. In summary, APOBEC enzymes favor deamination of unmodified cytosine residues, to yield C-to-U modifications, in single-stranded DNA and in a sequence-dependent manner that is specific to each family member.

\subsection{Sequence Context-Dependent Guanine Oxidation Products}

\subsubsection{Guanine}

DNA oxidation plays a significant role in the pathophysiology of cancer, with epidemiological studies demonstrating a strong association between the generation of ROS and reactive nitrogen species (RNS) from chronic inflammation and increased cancer risk [129]. Guanine has the lowest redox potential of all DNA bases, and it has consistently been found to be a highly susceptible site for reactions with a variety of agents, including singlet oxygen, $\mathrm{OH}$ radicals, peroxynitrite, UV radiation with riboflavin and many others [130]. For example, in a study in which duplex DNA was subjected to $266 \mathrm{~nm}$ wavelength laser pulses as a source of photonic ionization, the quantum yield for the formation of 8 -oxoG was much higher than that of oxidized nucleosides arising from the degradation of the other bases [131]. In addition, extensive experimental evidence supports a role for sequence context in terms of the chemistry and extent of DNA damage at guanine residues. Pioneering work in which DNA cleavage was induced by riboflavin as an electron-accepting photosensitizer in double-stranded 30-mers containing a target $\mathrm{G}$ in different sequence contexts (5'-TXGYT-3'), showed that the extent of cleavage at the target $G$ depended upon DNA flanking sequence composition [73], implying that the ease of losing an electron by the target $\mathrm{G}$ was also 
dependent on flanking sequence. Indeed, computations of the ionization potentials (i.e., the energy required to abstract an electron) (IPs) for the target $\mathrm{G}$ were found not only to differ with varying $\mathrm{X}$ and $\mathrm{Y}$ in a $5^{\prime}-\underline{\mathrm{XGY}}-3^{\prime}$ context, but also to correlate inversely with the extent of cleavage [73]. Specifically, the ionization potentials at a $\mathrm{G}$ followed by a $3^{\prime} \mathrm{A}$ or $\mathrm{G}$ were found to be up to $0.44 \mathrm{eV}$ lower than when followed by a $3^{\prime} \mathrm{T}$ or $\mathrm{C}$, whereas the base composition $5^{\prime}$ of a $\mathrm{G}$ made little $(<0.1 \mathrm{eV})$ contribution [74]. Hence, these and other investigations have together laid the foundation for the concept that a positive charge (a hole) inserted into DNA by abstracting an electron migrates through base stacking, either by hopping from one base to the next over long distances or by a tunneling mechanism over short distances (1-3 bases) [132], from the original location to sites of lowest IPs, i.e., 5' G in $\underline{\mathrm{G}} \mathrm{A}$ and $\underline{\mathrm{GG}}$ sequences.

\subsubsection{8-oxoG}

In addition to unmodified bases, sequence context-dependent reactivity has also been observed for 8-oxoG, one of the main products of DNA exposed to ROS and RNS. 8-oxoG is several orders-of-magnitude more susceptible to further oxidation than $G$ itself due to a lower ionization potential (6.93 versus $7.31 \mathrm{eV}$ for unstacked 8-oxoG and $\mathrm{G}$, respectively) [133], yielding more stable secondary oxidation products, including dehydroguanidinohydantoin (DGh), $\mathrm{N}$-nitro-dehydroguanidinohydantoin $\quad\left(\mathrm{NO}_{2}\right.$-DGh), 5-guanidinohydantoin $\quad(\mathrm{Gh}), \quad 2$-imino-5, 5'-spirodihydantoin (Sp), 2,5-diamino-4H-imidazol-4-one (imidazolone, Iz), its hydrolysis product 2,2,4-triamino-5(2H)-oxazolone (oxazolone, Oz) [134] and guanidinoformimine (Gf), the decarboxylated product of $\mathrm{Oz}$ [135] (Figure 4).

Earlier studies demonstrated that 8 -oxoG reactivity to a variety of oxidants, including $\mathrm{NiCR} / \mathrm{KHSO}_{5}, \mathrm{IrCl}_{6}{ }^{2-}, \mathrm{IrBr}_{6}{ }^{2-}, \mathrm{Fe}(\mathrm{CN}) 6^{3-}, \mathrm{SO}_{4}^{-}$and ${ }^{1} \mathrm{O}_{2}$, increased when located $5^{\prime}$ to a $\mathrm{G}$ (8-oxoGG) compared to $3^{\prime}$ to a $\mathrm{G}$ (G8-oxoG) [136], a trend that followed the computed sequence-dependent ionization potentials (6.38 eV for 8-oxoGG and $6.51 \mathrm{eV}$ for G8-oxoG) [133]. More recent studies further established the sequence-dependent reactivity of 8-oxoG in duplex oligonucleotides to UVA-irradiated riboflavin to follow: C8-oxoGA $\approx$ A8-oxoGG $>$ G8-oxoGG $>$ C8-oxoGT $>$ T8-oxoGC $>$ A8-oxoGC, supporting a model whereby indiscriminate removal of electrons from all four nucleobases by riboflavin creates holes that migrate to sites of lower IPs (8-oxoG), with 8-oxoG reactivity modulated by sequence-dependent variations in the IPs by neighboring bases [137]. In addition to the extent of reactivity, also the types of products formed by riboflavin-oxidized 8-oxoG varied with flanking sequence composition. For example, although three main products were generally observed $(\mathrm{Sp}>\mathrm{Gh}>\mathrm{Iz})$, at low riboflavin concentrations $(<15 \mu \mathrm{M})$ oligonucleotides containing G8-oxoGG, C8-oxoGT and T8-oxoGC yielded relatively high levels of $\mathrm{Sp}$ that decreased as a function of increasing riboflavin concentration. By contrast, at riboflavin doses $>30 \mu \mathrm{M}$, DGh was the most abundant species in some sequence contexts (G8-oxoGG and C8-oxoGA), with Iz matching DGh in the A8-oxoGG and G8-oxoGG sequence contexts. In contrast to riboflavin, nitrosoperoxycarbonate $\left(\mathrm{ONOOCO}_{2}{ }^{-}\right)$, generated from macrophage-derived nitric oxide (NO) and superoxide $\left(\mathrm{O}_{2}{ }^{-}\right)$, failed to yield sequence-dependent 8-oxoG reactivity and displayed a rather uniform spectrum of oxidation products, which were dominated by DGh $>$ Oxaluric acid $>$ $\mathrm{NO}_{2}$-DGh [138]. Likewise, $\mathrm{Gh}$ and $\mathrm{Sp}$ have been established as the main products of $\mathrm{G}$ and 
8-oxoG oxidation by peroxynitrite, peroxyl radicals, and hypochlorous acid, reactive species also released by macrophages during an inflammatory response [139]. Thus, as noted by Lim et al. [138], "the observation of strong sequence context effects on the final chemistry of DNA oxidation complicates our understanding of the mechanistic basis for both mutation frequency and mutational spectra caused by DNA damage in vivo", a task that is further complicated by sequence-dependent variations in the rates of DNA repair of individual DNA lesions. The analyses reported above clearly point to a critical role being played by charge (electron) transfer in the sequence context-dependent oxidation of DNA and the migration of the original sites of damage to

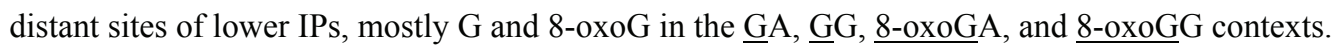

Figure 4. Sequence context-dependent reaction products of 8-oxoG.

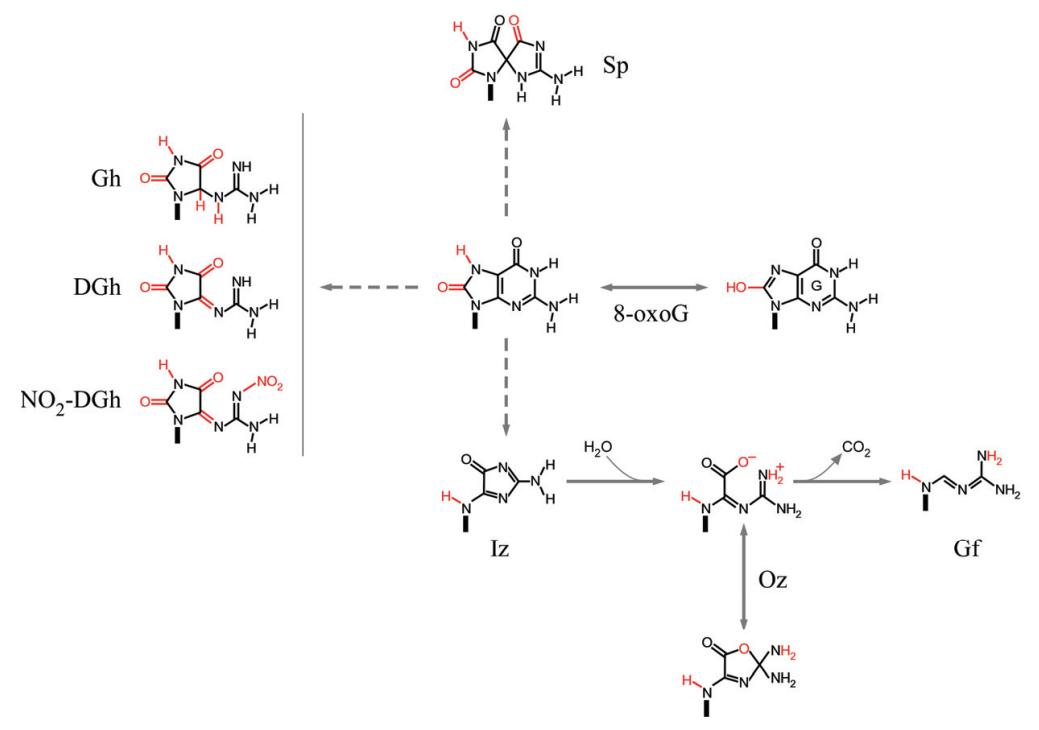

\subsubsection{Charge Transfer in Nucleosomal DNA}

Whereas bases in single-stranded DNA are generally more easily oxidized than in double-stranded DNA [138], the stability of stacking interactions in duplex DNA enables the charge transfer process towards guanine bases to take place more efficiently in duplex DNA than in single-stranded DNA. For example, increasing the ionic strength, which results in a more stable duplex, also enhanced the yield of 8-oxoG and, concomitantly, decreased the yield of thymine and adenine oxidation products upon $266 \mathrm{~nm}$ laser pulses in isolated DNA [131]. Charge transfer was also shown to occur more efficiently in chromatinized DNA than in naked DNA. A detailed study of the location and types of guanine oxidation products generated by UVA photodamage along a duplex DNA fragment wrapped around a nucleosome core particle (NCP) indicated that, whereas in naked DNA lesions were mostly localized at the distal sites, in nucleosomal DNA there was substantial enhancement of internally (i.e., in contact with the NCP) damaged guanine sites [140]. Surprisingly, removing the histone tails from nucleosomes, most of which were in molecular contact with the packaged DNA, was sufficient to abrogate the effects of nucleosomal packing on 
long-range charge transfer, implying that weakening histone-DNA interactions also dampened the efficiency of charge transfer along DNA. In addition, a shift in the nature of guanine lesions was observed, from $\mathrm{Oz}$ mostly in the linker regions and in naked DNA to 8-oxoG for those sites in closest contact with the NCP. Because the guanine radical cation $\left(\mathrm{G}^{+}\right)$, a key intermediate in guanine oxidation, may react with either oxygen or water to yield $\mathrm{Oz}$ and 8-oxoG, respectively, these results clearly show that the NCP shields $\mathrm{G}^{+}$from reacting with molecular oxygen. As the authors pointed out, the implications of this study are two-fold: first, "the enhancement of damage in the most tightly packaged nucleosomes could result in enhanced guanine oxidation in heterochromatin versus euchromatin"; and second "the distribution of guanine oxidation products is modulated by nucleosomal packaging. Therefore, the spectrum of guanine lesions generated by DNA oxidation could vary in different regions of chromatin".

In summary, sites of oxidation in DNA may migrate from their original location to sites of lower ionization potentials, e.g., predominantly $G$ in the context of $\underline{G A}$ and $\underline{G}$ sequences (charge/electron transfer); charge transfer also occurs towards 8-oxoG, which yields different oxidation products depending on the nature of the oxidizing agent and flanking sequence composition. Finally, charge transfer in DNA is favored in chromatin, where guanine oxidation products are modulated by their position along the NCP.

\section{DNA Repair Pathways and Synthesis across Modified Bases and Mismatches}

\subsection{Base Excision Repair}

Base excision repair (BER) is initiated by the activity of a DNA glycosylase that recognizes small perturbations in the DNA helical structure caused by base modifications or a mismatched base-pair [141]. The basic steps of BER, the distinction between short and long patch repair, the nature of monofunctional versus bifunctional enzymes and the involvement of Pol $\beta$ in cancer have been thoroughly reviewed [141-143]. Herein, we shall focus on those BER enzymes that have been shown to process the modified bases and mismatches described above.

\subsubsection{Modified CG:CG Sites}

Two monofunctional DNA glycosylases display a preference for correcting T:G and $U: G$ mismatches in the CG:CG sequence context in double-stranded DNA, methyl-CpG binding domain protein 4 (MBD4) and thymine DNA glycosylase (TDG). MBD4 contains an N-terminal methylCpG binding domain and a C-terminal DNA glycosylase domain that acts on T:G, 5hmU:G and $\mathrm{U}: \mathrm{G}$ mismatches with relative rate constants of $0.5,1.0$ and $1.7 \mathrm{~min}^{-1}$, respectively [144], and on Tg:G with half the efficiency observed for $\mathrm{T}: \mathrm{G}$. Thus, the enzyme is poised to recognize deamination products of $5 \mathrm{mC}: \mathrm{G}, 5 \mathrm{hmC}: \mathrm{G}$ and $\mathrm{C}: \mathrm{G}$ within $\mathrm{CG}: \mathrm{CG}$ sequences [145]. Consistent with these activities in vitro, $M b d 4^{-/-}$mice display a $\sim 3$-fold increase in $\mathrm{C} \rightarrow \mathrm{T}$ transitions at $\mathrm{CG}$ :CG sites relative to wild-type littermates [146,147]; however, a direct role for $\mathrm{Mbd}^{-/-}$in accelerating tumorigenesis has not been confirmed [148]. Competition experiments indicate that CG:CG methylation enhances MBD4 binding and that, whereas glycosylase activity is observed on reconstituted chromatin, activity is enhanced upon histone tail acetylation, consistent with 
increased accessibility of the target sites on a less compact chromatin environment [149]. TDG actively processes a number of lesions resulting from oxidation, alkylation and deamination of $\mathrm{C}$, $5 \mathrm{mC}, 5 \mathrm{hmC}, \mathrm{T}$ and A, with the strongest activities observed on $\mathrm{U}: \mathrm{G}>\mathrm{T}: \mathrm{G}>\mathrm{Tg}: \mathrm{G}$ mismatches. The enzyme also cleaves the products of $5 \mathrm{hmC}$ oxidation, $5 \mathrm{fC}$ and $5 \mathrm{caC}$. The TDG gene is expressed at high levels in the G2-M and G1 phases of the cell cycle, and then rapidly declines at the onset of S-phase. Loss of TDG expression is embryonic lethal in mice and, indeed, a number of investigations support a role for TDG in demethylation during embyogenesis, whereas interactions with transcription factors, transcriptional coregulators, DNMT3a, DNMT3b and others, suggest a scenario in which coordinated CG:CG methylation/demethylation and chromatin organization serve to regulate gene expression [145]. A striking preponderance (86\%) of $\mathrm{C} \rightarrow \mathrm{T}$ transitions at mutated CG:CG sites, which are normally methylated, was recently reported in a mismatch-repair (PMS2) deficient 13-year-old colorectal cancer patient with a heterozygous germline missense mutation in $T D G$ [150], in line with a potential role for TDG in repairing C5-substituted C deaminated products at $\mathrm{CG}: \mathrm{CG}$ sites.

Because thymidine glycol may exist in four different configurations, 5Rcistrans (Figure 5a) and $5 S_{\text {cis/trans }}$ (Figure $5 b$ ) pairs, the efficiency of repair by DNA glycosylases will vary depending upon whether Tg isomers oppose $\mathrm{G}(\mathrm{Tg}: \mathrm{G})$, which results from $5 \mathrm{mC}$ oxidation and deamination, or A ( $\mathrm{Tg}: \mathrm{A}$ ), which results from $\mathrm{T}$ oxidation. Under conditions of single turnover, the stereoselectivity of nth endonuclease III-like 1 (NTHL1, a bifunctional enzyme with $\beta$-lyase activity) was similar for $\operatorname{Tg}(5 R)$ : $\mathrm{A}$ and $\operatorname{Tg}(5 R): \mathrm{G}$, but the amount of $\operatorname{Tg}(5 R)$ cleaved was $\sim 13$-fold higher than for the $\operatorname{Tg}(5 S)$ due to stronger product inhibition by the latter. By contrast, for nei endonuclease VIII-like 1 (NEIL1, a bifunctional enzyme with $\beta, \delta$-lyase activity), no stereoselectivity was detected; however, Tg:G was excised much more rapidly than Tg:A, suggesting that NEIL1 may be primarily involved

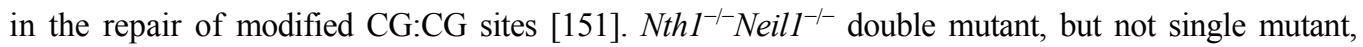
mice developed a high incidence of lung and liver tumors after the first year, implying overlapping roles in DNA repair [152].

Figure 5. (a) cis-trans stereoisomer pair of $5 R$ thymine glycol; (b) cis-trans stereoisomer pair of $5 S$ thymine glycol.

(a)



cis- $5 R, 6 S$

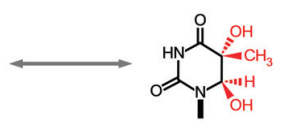

trans- $5 R, 6 R$ (b)

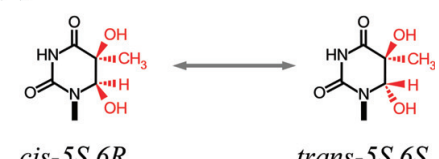

NEIL1 synthesis is activated during S-phase, and NEIL1 has been proposed to act at the replication fork to remove oxidative DNA lesions in a scheme involving: (1) damage recognition on the single-stranded template but cleavage inhibition by replication protein A (RPA); (2) fork reversal, which places the lesion back into duplex DNA; (3) base cleavage; and (4) resumption of the collapsed replication fork and DNA synthesis. Although NEIL2, which displays substrate specificities similar to NEIL1, was able to partially complement NEIL1 at the replication fork, its activity is believed to be more relevant during transcription [153]. A study on promoter 
methylation for 160 DNA repair genes in $\sim 40$ head and neck squamous cell carcinoma samples and controls identified NEIL1 as the most prominently hypermethylated gene, with $81 \%$ samples (35/43) displaying significant hypermethylation relative to controls, suggesting a role for diminished DNA repair activity in cancer onset or progression [154].

\subsubsection{Uracil}

Two additional monofunctional DNA glycosylases, uracil-DNA glycosylase 2 (UNG2) and single-stranded selective monofunctional uracil-DNA glycosylase 1 (SMUG1), serve to remove uracil from nuclear DNA [141]. UNG2 is a single-stranded DNA-specific enzyme that plays an indispensable role in somatic hypermutation (SHM), which is part of the antigen-driven high-affinity antibody diversification program in follicular B cells, by removing uracil generated by AID at WRCYY sequence hotspots. In vitro, SMUG1 is active either on single-stranded or double-stranded DNA, depending on salt concentrations. However, at physiological mono and divalent metal ion concentrations, SMUG1 is active only on double-stranded DNA, and therefore it is considered to be a double-stranded-specific DNA glycosylase. This distinction is crucial, since it places UNG2 as the sole enzyme acting on uracil in single-stranded DNA in vivo. Thus, in addition to sequence-specificity, the ability of RPA, a single-stranded DNA binding protein, to recruit UNG2 to single-stranded DNA has been proposed as a key feature that restricts UNG2 (rather than SMUG1, TDG or MBD4) activity to SHM [155]. In mice, during SHM, UNG2-generated AP sites are "copied" by error-prone translesion synthesis (TLS) polymerases during DNA replication, including Rev1 and Pol $\eta$, yielding $\mathrm{C} \rightarrow \mathrm{T}, \mathrm{C} \rightarrow \mathrm{G}$ and $\mathrm{C} \rightarrow \mathrm{A}$ mutations [128]. In chronic myeloid leukemia in chronic phase (CML-CP) hematopoietic stem cells, the kinase activity associated with the $B C L-A B L 1$ translocation was found to inhibit UNG2 activity, thereby promoting mutations arising from increased ROS-mediated oxidative base lesions [156].

\subsubsection{Guanine Lesions}

Two bifunctional double-strand-specific DNA glycosylases, 8-oxoguanine DNA glycosylase 1 (OGG1) and mutY homologue (MUTYH), act upon 8-oxoG, a highly miscoding lesion that instructs A incorporation (8-oxoG:A base-pairs) by the replicative DNA Pol $\delta / \varepsilon$. Knockout mice for both enzymes are strongly prone to lung, ovarian cancers and lymphomas, and have shortened life spans [157], whereas human germline biallelic MUTYH mutations have been implicated in MUTYH-associated polyposis, a condition associated with increased risk of colorectal cancer [158]. OGG1 cleaves 8-oxoG only when paired with C, owing to specific contacts made with both bases, which trigger catalysis [159]. By contrast, MUTYH specifically cleaves the A base in 8oxoG:A base-pairs by using a central interconnector domain (ICD) to coordinate the action between the N-terminal catalytic domain and the C-terminal 8-oxoG recognition domain. In addition, the ICD serves as a structural scaffold to direct MUTYH activity to replication foci through specific interactions, including PCNA and Rad9-Rad1-Hus1 (the 9-1-1 complex) [160]. After induction of oxidative stress, the co-localization of OGG1-containing BER patches with $\mathrm{H} 3 \mathrm{meK} 4$ or acetylated 
histone $\mathrm{H} 4$ in euchromatic regions and the exclusion from heterochromatic regions suggests that chromatin compaction hinders BER [161]. These conclusions are in line with dinucleosome reconstitution experiments in vitro, in which 8-oxoG cleavage in the linker region separating the two nucleosomes was unhindered in the absence of H1, but was decreased $\sim 10$-fold upon H1 binding to the linker [162].

In vitro, $\mathrm{Oz}$ in $\mathrm{Oz}: \mathrm{C}$ and $\mathrm{Gh}$ in $\mathrm{Gh}: \mathrm{C}$ mispairs in duplex oligonucleotides were cleaved by NEIL1 as efficiently as the well-recognized pyrimidine lesion 5hU:G, implying that guanine oxidation lesions, in addition to pyrimidine lesions, are good substrates for the enzyme. Similar results were obtained with NTHL1, although Gh was cleaved slightly more efficiently than Oz [163]. NEIL1 and NHTL1 also cleaved Oz:G as efficiently as Oz:C, which would give rise to $\mathrm{G} \rightarrow \mathrm{C}$ transversions; Oz:G mispairs are predicted to arise from Pol $\alpha$ or $\varepsilon$ replication across $\mathrm{Oz}$, as mentioned below.

Although the ability of NEIL1 and NEIL2 to remove Sp and Gh lesions has been well documented [164-166], a critical role for NEIL3 has recently emerged [167]. Neil3 ${ }^{-/-}$mice exhibited learning and memory deficits, impaired proliferation of neural stem/progenitor cells [168], and tissue extracts from $\mathrm{Neil}^{-/-}$mice, but not from wild-type littermates, displayed defective nicking activities on hydantoin lesions only when present on single-stranded DNA [169]. The purified human catalytic domain of NEIL3 was also found to display strong preference for Sp and Gh when compared to several other lesions, including 5-OHC and 5-OHU, with the greatest turnover number $\left(0.035 \mathrm{~s}^{-1}\right)$ on $\mathrm{Gh}$ in single-stranded DNA, as assessed from single turnover experiments, $\sim 2$-fold higher than on double-stranded Gh and single-/double-stranded Sp. Thus, removal of $\mathrm{Sp}$ and $\mathrm{Gh}$ lesions appears to depend critically on NEIL3. Interestingly, the enzyme elicited uncoordinated cleavage and $\beta$-lyase activities, suggesting that it can act both as a monofunctional and as a bifunctional glycosylase [170].

\subsection{Lesion Bypass}

Base lesions that remain unrepaired can serve as templates during DNA synthesis, and are either copied by the normal replicative DNA polymerases ( $\mathrm{Pol} \alpha, \delta$ and $\varepsilon$ ) or alternatively may block replication, in which case they are bypassed by one of 10 specialized polymerases lacking $3^{\prime} \rightarrow 5^{\prime}$ proofreading exonuclease activity during TLS, which limits DNA synthesis to a few bases across the blocking lesion $[43,142]$. Of the modified bases described above, C5 modified cytosines direct mostly guanine incorporation, and hence are not mutagenic; by contrast, their deaminated counterparts (thymine and uracil derivatives) enable the incorporation of adenine by replicative polymerases [171], which yields $\mathrm{C} \rightarrow \mathrm{T}(\mathrm{G} \rightarrow \mathrm{A})$ transitions. Tg stereroisomers block replication; investigations in vitro with DNA Polymerase gp43 from bacteriophage RB69 (a polymerase of the B-family, which in humans includes $\operatorname{Pol} \alpha, \delta, \varepsilon$ and $\zeta$ ) revealed that whereas Tg is weakly bypassed and correctly paired with A, extension is inhibited by the enzymatic exonuclease activity [172] and, thus, extended through TLS by Pol $\alpha, \zeta, v, \eta$ or $\theta$, either alone or in combination [43,173-179]. Thus, TLS across Tg:G base-pairs originating from $5 \mathrm{mC}$ oxidation and subsequent deamination are expected to lead almost quantitatively to $\mathrm{C} \rightarrow \mathrm{T}(\mathrm{G} \rightarrow \mathrm{A})$ transitions. 8-oxoG is able to functionally mimic thymine in the syn conformation, and DNA synthesis by replicative polymerases has been 
shown to yield both the correct 8-oxoG:C base-pair and the incorrect 8-oxoG(syn):A(anti) Hoogsteen base-pair [180-182] (Figure 6a-c) in $~ 3: 2$ ratios and to be extended, mostly by Pol $\lambda$ [182-187]. The 8-oxoG:A base-pair would therefore give rise to $\mathrm{G} \rightarrow \mathrm{T}(\mathrm{C} \rightarrow \mathrm{A})$ transversions.

The crystal structure of bacteriophage B-family Polymerase RB69 bound to the templating $R$-stereoisomer of $\mathrm{Gh}$ revealed that the base was flipped in a non-templating position. However, the results also suggested that either slow rotation by the $R$-isomer or, more effectively, the $S$-isomer would present the pyrimidine-like hydantoin side to the enzyme, thereby instructing incorporation of a purine (A or G) [188]. Indeed, a Y567A mutant of RB69 was found to insert both bases with $>100$-fold increased efficiency, whereas extension was blocked [189]. These data suggest that, as in the case with Tg stereoisomers, Gh:A and Gh:G are switched to the enzymatic exonucleolytic domain, thereby triggering a futile incorporation/degradation cycle that effectively blocks DNA replication and renders Gh an obligate mutagen. Thus, a TLS extender polymerase may assist in lesion bypass across Gh in vivo; in addition, Pol $\eta$ was found to bypass Gh efficiently and to incorporate either A or G in primer extension assays [190]. Although Sp was also found to yield $\mathrm{G} \rightarrow \mathrm{C}$ and $\mathrm{G} \rightarrow \mathrm{T}$ transversions [191], no structural data are currently available for polymerease:Sp complexes.

In primer extension assays, Pol $\eta$ was found to partially extend past the Oz, Iz and Gf lesions $(\mathrm{Oz}>\mathrm{Iz}>\mathrm{Gf})$, whereas Pol $\kappa$ was almost completely blocked [135]. Sequence analyses of the extended products indicated that Pol $\eta$ incorporated either $\mathrm{C}(55 \%-65 \%)$ or $\mathrm{G}(35 \%-45 \%)$, whereas Pol $\kappa$ incorporated C (41\%-58\%), G (25\%-37\%) and A (16\%-33\%), across all lesions. Thus, $\mathrm{T}$ incorporation seems to be limited with both TLS polymerases. How these lesions are processed by replicative DNA polymerases and the extent to which each leads to SBSs remains to be determined. The stabilization energies $(\Delta E)$ for a number of isomers of the oxidative guanine products $\mathrm{Gh}, \mathrm{Sp}, \mathrm{Iz}$ and $\mathrm{Oz}$ base-paired with $\mathrm{G}$ (Figure $6 \mathrm{~d}-\mathrm{g}$ ) have been computed by $a b$ initio molecular orbital calculations; using density functional theory, $\Delta E$ values varied from $28.2 \mathrm{kcal} / \mathrm{mol}$ for $\mathrm{Sp}: \mathrm{G}$ to $20.7 \mathrm{kcal} / \mathrm{mol}$ for $\mathrm{Oz}: \mathrm{G}(30.9 \mathrm{kcal} / \mathrm{mol}$ for the canonical G:C base-pair) $[130,192]$.

Figure 6. (a) Canonical G:C base-pair; (b) Canonical A:T base-pair; (c) 8-oxoG(syn):A(anti) base-pair; (d) Gh:G base-pair; (e) Sp:G base-pair; (f) Iz:G base-pair; (g) Oz:G base-pair. For noncanonical base-pairs, the templating base is shown in blue.

(a)

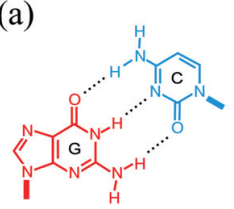

(e)



(b)

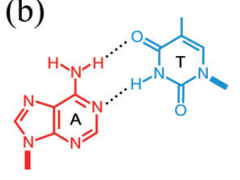

(f)



(c)



(g)

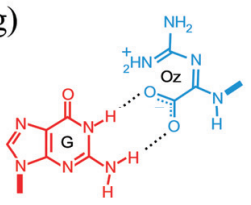

(d)






\subsection{Transcription-Coupled Repair}

Given that cancer genome analyses have indicated that the number of SBSs is often lower at sites of putative base lesions on the transcribed strand than on the non-transcribed strand [13,30,193], we will briefly discuss some key aspects of transcription-coupled repair. Nucleotide excision repair (NER) is considered to be the main pathway for the repair of UV photo-induced DNA lesions; CPDs and 6-4 pyrimidine-pyrimidone photo products (6-4PP) [194], and defects in NER components are associated with inherited DNA repair syndromes such as xeroderma pigmentosum [195] and Cockayne syndrome [194], that display severe hypersensitivity to sunlight. NER also actively repairs many types of DNA adducts that cause helical distortions, including environmental mutagens such as benzo[ $a]$ pyrene and other PAHs, aromatic amines, oxidative endogenous lesions such as cyclopurines, and adducts caused by cancer chemotherapeutic agents, including cisplatin [196]. NER comprises two distinctive subpathways, global genome NER (GG-NER) and transcription-coupled NER (TC-NER), which acts specifically on the transcribed strand of actively transcribed genes [194]. TC-NER is activated by the physical blockage imposed by bulky DNA adducts and abasic sites on RNA Polymerase II (RNAPII), but not by small lesions such as thymine glycols and 8-oxoG [197,198]. RNAPII is found in transient association with Cockayne syndrome type B protein (CSB, the product of the ERCC6 gene) and the UV-stimulated scaffold protein A (UVSSA)-ubiquitin-specific peptidase 7 (USP7) complex. RNAPII stalling is believed to stabilize RNAPII/CSB interactions and activate the UVSSA/USP7 complex, thus protecting CSB from degradation through deubiquitination. Stabilized CSB recruits a complex that includes Cockayne syndrome WD repeat protein (CSA, product of the ERCC 8 gene), damage-specific DNA binding protein 1 (DDB1), cullin 4A (CUL4A) and others, which mediates downstream events, including chromatin remodeling, permitting backtracking of the RNAPII complex and exposing the lesion for repair by the GG-NER complex. Specific DNA adducts have been shown to be repaired exclusively by TC-NER but not by GG-NER. For example, exome sequencing of urothelial carcinomas of the upper urinary tract associated with chronic exposure to aristolochic acid, a natural compound from traditional herb medicine, revealed a characteristic $A \rightarrow T(T \rightarrow A)$ mutational signature on non-transcribed strands leading to splicing defects [199,200], which was attributed to a failure of GG-NER, but not TC-NER, to recognize aristolactam-DNA adducts [201]. In addition to its role in TC-NER, CSB has been reported to facilitate lesion bypass by the RNAPII complex [202], to associate with components of BER, including OGG1, NEIL1 and AP1, and to elicit critical functions in mitochondria related to DNA repair, ROS homeostasis and others [203-205].

More recently, immunoprecipitation experiments in human gastric epithelial AGS cells revealed a direct association between the DNA glycosylase NEIL2, RNAPII and heterogeneous nuclear ribonucleoprotein U (hnRNP-U) [206]. On a 51-mer oligonucleotide, NEIL2 activity on a single 5-OHU lesion was stimulated 5- to 6-fold by hnRNP-U and likewise, on a plasmid system, a reconstituted transcription-repair complex comprising RNAPII, NEIL2, hnRNP-U and the BER components Pol $\beta$, Lig III $\alpha$, PNK was proficient in repairing a 5-OHU lesion on the transcribed, but not on the non-transcribed, strand under conditions of active transcription. Co-localizations of NEIL2 with hnRNP-U with actively transcribed genes were confirmed by pulling down 
NEIL2-FLAG expressing AGS and neuroblastoma SK-N-BE2-(C) cells, followed by chromatin immunoprecipitation with hnRNP-U antibodies. Finally, a rise in mutations was inferred on selected, transcribed, genes in NEIL2 knock-down compared to control cells. These composite data extend previous investigations [207], and document the preferential removal of oxidized DNA bases in actively transcribed genes by a system linking BER to the transcriptional apparatus. As already pointed out [206], it remains to be seen whether components of NER, such as CSB, also take part in BER related to transcription. In summary, whereas common oxidative DNA lesions may not be recognized by TC-NER, they are likely to represent a substrate for BER on the transcribed strand in association with transcription.

\section{Proposed Mutational Mechanisms}

Herein we have provided information on the most prominent SBS mutation patterns found in cancer genomes, i.e., $\mathrm{C} \rightarrow \mathrm{T}$ transitions at $\mathrm{CG}: \mathrm{CG}$ sites and substitutions at $\mathrm{C}: \mathrm{G}$ base-pairs in the context of $\underline{Y} \underline{G}: \underline{G R}$ dinucleotides, in an attempt to examine the validity of currently proposed models of mutagenesis. The main points may be summarized as follows: (i) higher fractions of SBSs have been found in cancer genomes within gene-poor regions, which are associated with heterochromatin and replicate late during the cell cycle, than in euchromatin, a pattern that mimics the one observed in population analyses; (ii) there are several established mechanisms for base substitutions at $\mathrm{C}$ residues, and their relative importance is only now being recognized. These involve deamination, oxidation, BER, TC-NER, TLS, and APOBEC activities; (iii) both guanine and 8-oxoG undergo sequence context- and ROS-dependent oxidation reactions that are consistent with an electron transfer (charge or hole migration) mechanism, whose efficiency is enhanced in the context of nucleosomal DNA; (iv) BER displays widely overlapping substrate specificity; however, NEIL enzymes appear to play a more prominent role in repairing guanine lesions, such as Gh and Sp, which are obligate mutagens during TLS; (v) most endogenous DNA lesions do not activate TC-NER; however, there are hints for a functional link between BER and transcription, leading to preferential repair on the transcribed strand.

$\mathrm{C} \rightarrow \mathrm{T}$ transitions at $\mathrm{CG}: \mathrm{CG}$ sites have generally been attributed to faster spontaneous deamination of $5 \mathrm{mC}$ relative to $\mathrm{C}[13,16,17,19]$. Our current analysis suggests that this explanation may be somewhat too restrictive. First, the fact that a family of C5-substituted Cs exists at CG:CG sites implies that $\mathrm{C} \rightarrow \mathrm{T}$ transitions are not limited to $5 \mathrm{mC}$ but, rather, to any of the $\mathrm{C} 5$-substituted species, i.e., $5 \mathrm{hmC}, 5 \mathrm{fC}$ and $5 \mathrm{caC}$. Second, as noted [208], the modest (5-fold at the most) increase in spontaneous deamination rates for $\mathrm{C} 5$-substituted $\mathrm{Cs}$ relative to $\mathrm{Cs}$ contrasts with the larger fractions of mutated Cs at CG:CG sites relative to non-CG:CG sites (up to 10-50 times), both in cancer and the germline [17,209]. Third, there does not seem to be a rational barrier to the possibility that $5 \mathrm{mC}$ oxidation and further deamination may yield $\mathrm{Tg}: \mathrm{G}$ mismatches at $\mathrm{CG}: \mathrm{CG}$ sites, which would then lead to $\mathrm{C} \rightarrow \mathrm{T}$ transitions during TLS. In fact, the observation that such a type of oxidation is facilitated by nucleosome occupancy raises the prospect for thymine glycols in Tg:G mispairs being a more prominent source of mutation at CG:CG sites than T:G mismatches, as previously pointed out [208]. The finding that $\mathrm{C}: \mathrm{G} \rightarrow \mathrm{T}: \mathrm{A}$ substitutions at $\mathrm{CG}: \mathrm{CG}$ dinucleotides showed a strong positive correlation with the age at cancer diagnosis in ER-cancers, but not in 
ER+ cancers [193], further supports the conclusion that spontaneous deamination of $5 \mathrm{mC}$ may not be the only mechanism leading to mutations at $\mathrm{CG}$ :CG.

With respect to mutations at $\mathrm{Y} \underline{\mathrm{C}}$ : $\underline{\mathrm{GR}}$ sites, the gene expression data on APOBEC3B enzymes provide strong support for a role in cancer mutagenesis through $\mathrm{U}: \mathrm{G}$ mismatch intermediates, as in the case of SHM. However, the extent to which the enzyme preference for single-stranded DNA may limit their activity genome-wide, possibly at sites of clustered and strand-coordinated mutations (kataegis) [13,210], remains to be determined. For example, whole-genome sequencing of gastric cancers, in which a prominent $\mathrm{C} \rightarrow \mathrm{T}$ signature at $\mathrm{GC}$ dinucleotides in coding-regions did not overlap with a preponderance of $\mathrm{C} \rightarrow \mathrm{A}$ transversions at CCT or TCA motifs genome-wide, were attributed to AID activation (on single-stranded DNA during transcription) and ROS/NOS, respectively, following $H$. pylori infection [56]. The findings that: (i) oxidative DNA damage occurs at Y $\underline{\mathrm{C}}: \underline{G R}$ sites, which overlap with APOBEC3B specificity; (ii) numerous oxidation products can form at the target $\underline{G R}$; (iii) efficient charge transfer and high mutation rates co-localize with heterochromatin; and (iv) some oxidation products of guanine are obligate mutagens during TLS, make it tempting to attribute a significant role for oxidative damage in mutations at $\mathrm{YC}$ : $\underline{G R}$ sites genome-wide in cancer mutagenesis. Thus, for SBSs at both $\mathrm{CG}: \mathrm{CG}$ and $\mathrm{Y} \underline{\mathrm{C}} \underline{\mathrm{GR}}$ sites, we suggest a prominent role for oxidative damage by ROS and other electron-abstracting species.

One severe limitation in elucidating mechanisms of SBS is obviously the lack of information on the steady-state levels of modified bases and mismatches in cancer cells. Current efforts to address this critical issue [211-215] should at least temper these limitations, not only for the limited number of base modifications discussed here, but also for the much larger repertoire that may be formed by both endogenous and environmental agents, and which have not been addressed herein.

\section{Acknowledgments}

We thank Richard D. Wood and Robert D. Wells for comments and suggestions. This work was supported by grants from the National Institutes of Health (CA097175 and CA093729) to K.M.V.

\section{Author Contributions}

Wrote the paper: AB, DNC and KMV.

\section{Conflict of Interest}

The authors declare no conflict of interest.

\section{References}

1. Garraway, L.A.; Lander, E.S. Lessons from the cancer genome. Cell 2013, 153, 17-37.

2. Vogelstein, B.; Papadopoulos, N.; Velculescu, V.E.; Zhou, S.; Diaz, L.A., Jr.; Kinzler, K.W. Cancer genome landscapes. Science 2013, 339, 1546-1558. 
3. Nik-Zainal, S.; Alexandrov, L.B.; Wedge, D.C.; van Loo, P.; Greenman, C.D.; Raine, K.; Jones, D.; Hinton, J.; Marshall, J.; Stebbings, L.A.; et al. Mutational processes molding the genomes of 21 breast cancers. Cell 2012, 149, 979-993.

4. Forment, J.V.; Kaidi, A.; Jackson, S.P. Chromothripsis and cancer: Causes and consequences of chromosome shattering. Nat. Rev. Cancer 2012, 12, 663-670.

5. Yang, L.; Luquette, L.J.; Gehlenborg, N.; Xi, R.; Haseley, P.S.; Hsieh, C.H.; Zhang, C.; Ren, X.; Protopopov, A.; Chin, L.; et al. Diverse mechanisms of somatic structural variations in human cancer genomes. Cell 2013, 153, 919-929.

6. Drier, Y.; Lawrence, M.S.; Carter, S.L.; Stewart, C.; Gabriel, S.B.; Lander, E.S.; Meyerson, M.; Beroukhim, R.; Getz, G. Somatic rearrangements across cancer reveal classes of samples with distinct patterns of DNA breakage and rearrangement-induced hypermutability. Genome Res. 2013, 23, 228-235.

7. Bunting, S.F.; Nussenzweig, A. End-joining, translocations and cancer. Nat. Rev. Cancer 2013, 13, 443-454.

8. Zack, T.I.; Schumacher, S.E.; Carter, S.L.; Cherniack, A.D.; Saksena, G.; Tabak, B.; Lawrence, M.S.; Zhang, C.Z.; Wala, J.; Mermel, C.H.; et al. Pan-cancer patterns of somatic copy number alteration. Nat. Genet. 2013, 45, 1134-1140.

9. Watson, I.R.; Takahashi, K.; Futreal, P.A.; Chin, L. Emerging patterns of somatic mutations in cancer. Nat. Rev. Genet. 2013, 14, 703-718.

10. Ciriello, G.; Miller, M.L.; Aksoy, B.A.; Senbabaoglu, Y.; Schultz, N.; Sander, C. Emerging landscape of oncogenic signatures across human cancers. Nat. Genet. 2013, 45, 1127-1133.

11. Lange, S.S.; Takata, K.; Wood, R.D. DNA polymerases and cancer. Nat. Rev. Cancer 2011, 11, 96-110.

12. Alexandrov, L.B.; Nik-Zainal, S.; Wedge, D.C.; Campbell, P.J.; Stratton, M.R. Deciphering signatures of mutational processes operative in human cancer. Cell. Rep. 2013, 3, 246-259.

13. Alexandrov, L.B.; Nik-Zainal, S.; Wedge, D.C.; Aparicio, S.A.; Behjati, S.; Biankin, A.V.; Bignell, G.R.; Bolli, N.; Borg, A.; Borresen-Dale, A.L.; et al. Signatures of mutational processes in human cancer. Nature 2013, 500, 415-421.

14. Fischer, A.; Illingworth, C.J.; Campbell, P.J.; Mustonen, V. EMu: Probabilistic inference of mutational processes and their localization in the cancer genome. Genome Biol. 2013, 14, R39.

15. Burns, M.B.; Temiz, N.A.; Harris, R.S. Evidence for APOBEC3B mutagenesis in multiple human cancers. Nat. Genet. 2013, 45, 977-983.

16. Kandoth, C.; McLellan, M.D.; Vandin, F.; Ye, K.; Niu, B.; Lu, C.; Xie, M.; Zhang, Q.; McMichael, J.F.; Wyczalkowski, M.A.; et al. Mutational landscape and significance across 12 major cancer types. Nature 2013, 502, 333-339.

17. Bacolla, A.; Temiz, N.A.; Yi, M.; Ivanic, J.; Cer, R.Z.; Donohue, D.E.; Ball, E.V.; Mudunuri, U.S.; Wang, G.; Jain, A.; et al. Guanine holes are prominent targets for mutation in cancer and inherited disease. PLoS Genet. 2013, 9, e1003816.

18. Lawrence, M.S.; Stojanov, P.; Polak, P.; Kryukov, G.V.; Cibulskis, K.; Sivachenko, A.; Carter, S.L.; Stewart, C.; Mermel, C.H.; Roberts, S.A.; et al. Mutational heterogeneity in cancer and the search for new cancer-associated genes. Nature 2013, 499, 214-218. 
19. Ivanov, D.; Hamby, S.E.; Stenson, P.D.; Phillips, A.D.; Kehrer-Sawatzki, H.; Cooper, D.N.; Chuzhanova, N. Comparative analysis of germline and somatic microlesion mutational spectra in 17 human tumor suppressor genes. Hum. Mutat. 2011, 32, 620-632.

20. Roberts, S.A.; Lawrence, M.S.; Klimczak, L.J.; Grimm, S.A.; Fargo, D.; Stojanov, P.; Kiezun, A.; Kryukov, G.V.; Carter, S.L.; Saksena, G.; et al. An APOBEC cytidine deaminase mutagenesis pattern is widespread in human cancers. Nat. Genet. 2013, 45, 970-976.

21. Greenman, C.; Stephens, P.; Smith, R.; Dalgliesh, G.L.; Hunter, C.; Bignell, G.; Davies, H.; Teague, J.; Butler, A.; Stevens, C.; et al. Patterns of somatic mutation in human cancer genomes. Nature 2007, 446, 153-158.

22. Govindan, R.; Ding, L.; Griffith, M.; Subramanian, J.; Dees, N.D.; Kanchi, K.L.; Maher, C.A.; Fulton, R.; Fulton, L.; Wallis, J.; et al. Genomic landscape of non-small cell lung cancer in smokers and never-smokers. Cell 2012, 150, 1121-1134.

23. Imielinski, M.; Berger, A.H.; Hammerman, P.S.; Hernandez, B.; Pugh, T.J.; Hodis, E.; Cho, J.; Suh, J.; Capelletti, M.; Sivachenko, A.; et al. Mapping the hallmarks of lung adenocarcinoma with massively parallel sequencing. Cell 2012, 150, 1107-1120.

24. Seo, J.S.; Ju, Y.S.; Lee, W.C.; Shin, J.Y.; Lee, J.K.; Bleazard, T.; Lee, J.; Jung, Y.J.; Kim, J.O.; Shin, J.Y.; et al. The transcriptional landscape and mutational profile of lung adenocarcinoma. Genome Res. 2012, 22, 2109-2119.

25. Ding, L.; Getz, G.; Wheeler, D.A.; Mardis, E.R.; McLellan, M.D.; Cibulskis, K.; Sougnez, C.; Greulich, H.; Muzny, D.M.; Morgan, M.B.; et al. Somatic mutations affect key pathways in lung adenocarcinoma. Nature 2008, 455, 1069-1075.

26. TCGARN. Comprehensive genomic characterization of squamous cell lung cancers. Nature 2012, 489, 519-525.

27. Xiong, D.; Li, G.; Li, K.; Xu, Q.; Pan, Z.; Ding, F.; Vedell, P.; Liu, P.; Cui, P.; Hua, X.; et al. Exome sequencing identifies MXRA5 as a novel cancer gene frequently mutated in non-small cell lung carcinoma from Chinese patients. Carcinogenesis 2012, 33, 1797-1805.

28. Liu, P.; Morrison, C.; Wang, L.; Xiong, D.; Vedell, P.; Cui, P.; Hua, X.; Ding, F.; Lu, Y.; James, M.; et al. Identification of somatic mutations in non-small cell lung carcinomas using whole-exome sequencing. Carcinogenesis 2012, 33, 1270-1276.

29. Lee, W.; Jiang, Z.; Liu, J.; Haverty, P.M.; Guan, Y.; Stinson, J.; Yue, P.; Zhang, Y.; Pant, K.P.; Bhatt, D.; et al. The mutation spectrum revealed by paired genome sequences from a lung cancer patient. Nature 2010, 465, 473-477.

30. Pleasance, E.D.; Stephens, P.J.; O’Meara, S.; McBride, D.J.; Meynert, A.; Jones, D.; Lin, M.L.; Beare, D.; Lau, K.W.; Greenman, C.; et al. A small-cell lung cancer genome with complex signatures of tobacco exposure. Nature 2010, 463, 184-190.

31. Drablos, F.; Feyzi, E.; Aas, P.A.; Vaagbo, C.B.; Kavli, B.; Bratlie, M.S.; Pena-Diaz, J.; Otterlei, M.; Slupphaug, G.; Krokan, H.E. Alkylation damage in DNA and RNA-Repair mechanisms and medical significance. DNA Repair 2004, 3, 1389-1407.

32. Matter, B.; Wang, G.; Jones, R.; Tretyakova, N. Formation of diastereomeric benzo[a]pyrene diol epoxide-guanine adducts in p53 gene-derived DNA sequences. Chem. Res. Toxicol. 2004, 17, 731-741. 
33. Ziegel, R.; Shallop, A.; Jones, R.; Tretyakova, N. K-ras gene sequence effects on the formation of 4-(methylnitrosamino)-1-(3-pyridyl)-1-butanone (NNK)-DNA adducts. Chem. Res. Toxicol. 2003, 16, 541-550.

34. Anna, L.; Holmila, R.; Kovacs, K.; Gyorffy, E.; Gyori, Z.; Segesdi, J.; Minarovits, J.; Soltesz, I.; Kostic, S.; Csekeo, A.; et al. Relationship between TP53 tumour suppressor gene mutations and smoking-related bulky DNA adducts in a lung cancer study population from Hungary. Mutagenesis 2009, 24, 475-480.

35. Martinez, V.D.; Thu, K.L.; Vucic, E.A.; Hubaux, R.; Adonis, M.; Gil, L.; MacAulay, C.; Lam, S.; Lam, W.L. Whole-genome sequencing analysis identifies a distinctive mutational spectrum in an arsenic-related lung tumor. J. Thorac. Oncol. 2013, 8, 1451-1455.

36. Pleasance, E.D.; Cheetham, R.K.; Stephens, P.J.; McBride, D.J.; Humphray, S.J.; Greenman, C.D.; Varela, I.; Lin, M.L.; Ordonez, G.R.; Bignell, G.R.; et al. A comprehensive catalogue of somatic mutations from a human cancer genome. Nature 2010, 463, 191-196.

37. Berger, M.F.; Hodis, E.; Heffernan, T.P.; Deribe, Y.L.; Lawrence, M.S.; Protopopov, A.; Ivanova, E.; Watson, I.R.; Nickerson, E.; Ghosh, P.; et al. Melanoma genome sequencing reveals frequent PREX2 mutations. Nature 2012, 485, 502-506.

38. Nikolaev, S.I.; Rimoldi, D.; Iseli, C.; Valsesia, A.; Robyr, D.; Gehrig, C.; Harshman, K.; Guipponi, M.; Bukach, O.; Zoete, V.; et al. Exome sequencing identifies recurrent somatic MAP2K1 and MAP2K2 mutations in melanoma. Nat. Genet. 2012, 44, 133-139.

39. Stark, M.S.; Woods, S.L.; Gartside, M.G.; Bonazzi, V.F.; Dutton-Regester, K.; Aoude, L.G.; Chow, D.; Sereduk, C.; Niemi, N.M.; Tang, N.; et al. Frequent somatic mutations in MAP3K5 and MAP3K9 in metastatic melanoma identified by exome sequencing. Nat. Genet. 2012, 44, 165-169.

40. Krauthammer, M.; Kong, Y.; Ha, B.H.; Evans, P.; Bacchiocchi, A.; McCusker, J.P.; Cheng, E.; Davis, M.J.; Goh, G.; Choi, M.; et al. Exome sequencing identifies recurrent somatic RAC1 mutations in melanoma. Nat. Genet. 2012, 44, 1006-1014.

41. Wei, X.; Walia, V.; Lin, J.C.; Teer, J.K.; Prickett, T.D.; Gartner, J.; Davis, S.; Program, N.C.S.; Stemke-Hale, K.; Davies, M.A.; et al. Exome sequencing identifies GRIN2A as frequently mutated in melanoma. Nat. Genet. 2011, 43, 442-446.

42. Dutton-Regester, K.; Kakavand, H.; Aoude, L.G.; Stark, M.S.; Gartside, M.G.; Johansson, P.; O’Connor, L.; Lanagan, C.; Tembe, V.; Pupo, G.M.; et al. Melanomas of unknown primary have a mutation profile consistent with cutaneous sun-exposed melanoma. Pigment Cell Melanoma Res. 2013, 26, 852-860.

43. Sale, J.E. Translesion DNA synthesis and mutagenesis in eukaryotes. Cold Spring Harb. Perspect. Biol. 2013, 5, a012708.

44. Pham, P.; Landolph, A.; Mendez, C.; Li, N.; Goodman, M.F. A biochemical analysis linking APOBEC3A to disparate HIV-1 restriction and skin cancer. J. Biol. Chem. 2013, 288, 29294-29304.

45. Korona, D.A.; Lecompte, K.G.; Pursell, Z.F. The high fidelity and unique error signature of human DNA polymerase epsilon. Nucleic Acids Res. 2011, 39, 1763-1773. 
46. Agbor, A.A.; Goksenin, A.Y.; LeCompte, K.G.; Hans, S.H.; Pursell, Z.F. Human Pol epsilon-dependent replication errors and the influence of mismatch repair on their correction. DNA Repair 2013, 12, 954-963.

47. Jiricny, J. Postreplicative mismatch repair. Cold Spring Harb. Perspect. Biol. 2013, 5, a012633.

48. Pluciennik, A.; Dzantiev, L.; Iyer, R.R.; Constantin, N.; Kadyrov, F.A.; Modrich, P. PCNA function in the activation and strand direction of MutLalpha endonuclease in mismatch repair. Proc. Natl. Acad. Sci. USA 2010, 107, 16066-16071.

49. Arana, M.E.; Kunkel, T.A. Mutator phenotypes due to DNA replication infidelity. Semin. Cancer Biol. 2010, 20, 304-311.

50. Preston, B.D.; Albertson, T.M.; Herr, A.J. DNA replication fidelity and cancer. Semin. Cancer Biol. 2010, 20, 281-293.

51. Aquilina, G.; Bignami, M. Mismatch repair in correction of replication errors and processing of DNA damage. J. Cell. Physiol. 2001, 187, 145-154.

52. Thompson, B.A.; Spurdle, A.B.; Plazzer, J.P.; Greenblatt, M.S.; Akagi, K.; Al-Mulla, F.; Bapat, B.; Bernstein, I.; Capella, G.; den Dunnen, J.T.; et al. Application of a 5-tiered scheme for standardized classification of 2,360 unique mismatch repair gene variants in the InSiGHT locus-specific database. Nat. Genet. 2014, 46, 107-115.

53. Vasen, H.F.; Blanco, I.; Aktan-Collan, K.; Gopie, J.P.; Alonso, A.; Aretz, S.; Bernstein, I.; Bertario, L.; Burn, J.; Capella, G.; et al. Revised guidelines for the clinical management of Lynch syndrome (HNPCC): Recommendations by a group of European experts. Gut 2013, 62, 812-823.

54. TCGAN. Comprehensive molecular characterization of human colon and rectal cancer. Nature 2012, 487, 330-337.

55. TCGARN; Kandoth, C.; Schultz, N.; Cherniack, A.D.; Akbani, R.; Liu, Y.; Shen, H.; Robertson, A.G.; Pashtan, I.; Shen, R.; et al. Integrated genomic characterization of endometrial carcinoma. Nature 2013, 497, 67-73.

56. Nagarajan, N.; Bertrand, D.; Hillmer, A.M.; Zang, Z.J.; Yao, F.; Jacques, P.E.; Teo, A.S.; Cutcutache, I.; Zhang, Z.; Lee, W.H.; et al. Whole-genome reconstruction and mutational signatures in gastric cancer. Genome Biol. 2012, 13, R115.

57. Kim, T.M.; Laird, P.W.; Park, P.J. The landscape of microsatellite instability in colorectal and endometrial cancer genomes. Cell 2013, 155, 858-868.

58. Yoshida, R.; Miyashita, K.; Inoue, M.; Shimamoto, A.; Yan, Z.; Egashira, A.; Oki, E.; Kakeji, Y.; Oda, S.; Maehara, Y. Concurrent genetic alterations in DNA polymerase proofreading and mismatch repair in human colorectal cancer. Eur. J. Hum. Genet. 2011, 19, 320-325.

59. Palles, C.; Cazier, J.B.; Howarth, K.M.; Domingo, E.; Jones, A.M.; Broderick, P.; Kemp, Z.; Spain, S.L.; Guarino, E.; Salguero, I.; et al. Germline mutations affecting the proofreading domains of POLE and POLD1 predispose to colorectal adenomas and carcinomas. Nat. Genet. 2013, 45, 136-144. 
60. Church, D.N.; Briggs, S.E.; Palles, C.; Domingo, E.; Kearsey, S.J.; Grimes, J.M.; Gorman, M.; Martin, L.; Howarth, K.M.; Hodgson, S.V.; et al. DNA polymerase epsilon and delta exonuclease domain mutations in endometrial cancer. Hum. Mol. Genet. 2013, 22, 2820-2828.

61. Briggs, S.; Tomlinson, I. Germline and somatic polymerase epsilon and delta mutations define a new class of hypermutated colorectal and endometrial cancers. J. Pathol. 2013, 230, $148-153$.

62. Dai, H.; Hickey, R.J.; Liu, J.; Bigsby, R.M.; Lanner, C.; Malkas, L.H. Error-promoting DNA synthesis in ovarian cancer cells. Gynecol. Oncol. 2013, 131, 198-206.

63. Vieira, V.C.; Soares, M.A. The role of cytidine deaminases on innate immune responses against human viral infections. Biomed Res. Int. 2013, 2013, 683095.

64. Smith, H.C.; Bennett, R.P.; Kizilyer, A.; McDougall, W.M.; Prohaska, K.M. Functions and regulation of the APOBEC family of proteins. Semin. Cell Dev. Biol. 2012, 23, 258-268.

65. Koito, A.; Ikeda, T. Intrinsic immunity against retrotransposons by APOBEC cytidine deaminases. Front. Microbiol. 2013, 4, 28.

66. Bohn, M.F.; Shandilya, S.M.; Albin, J.S.; Kouno, T.; Anderson, B.D.; McDougle, R.M.; Carpenter, M.A.; Rathore, A.; Evans, L.; Davis, A.N.; et al. Crystal structure of the DNA cytosine deaminase APOBEC3F: The catalytically active and HIV-1 Vif-binding domain. Structure 2013, 21, 1042-1050.

67. Kitamura, S.; Ode, H.; Nakashima, M.; Imahashi, M.; Naganawa, Y.; Kurosawa, T.; Yokomaku, Y.; Yamane, T.; Watanabe, N.; Suzuki, A.; et al. The APOBEC3C crystal structure and the interface for HIV-1 Vif binding. Nat. Struct. Mol. Biol. 2012, 19, 1005-1010.

68. Burns, M.B.; Lackey, L.; Carpenter, M.A.; Rathore, A.; Land, A.M.; Leonard, B.; Refsland, E.W.; Kotandeniya, D.; Tretyakova, N.; Nikas, J.B.; et al. APOBEC3B is an enzymatic source of mutation in breast cancer. Nature 2013, 494, 366-370.

69. Lewis, P.D.; Harvey, J.S.; Waters, E.M.; Skibinski, D.O.; Parry, J.M. Spontaneous mutation spectra in supF: Comparative analysis of mammalian cell line base substitution spectra. Mutagenesis 2001, 16, 503-515.

70. Bacolla, A.; Wang, G.; Jain, A.; Chuzhanova, N.A.; Cer, R.Z.; Collins, J.R.; Cooper, D.N.; Bohr, V.A.; Vasquez, K.M. Non-B DNA-forming sequences and WRN deficiency independently increase the frequency of base substitution in human cells. J. Biol. Chem. 2011, 286, 10017-10026.

71. Giese, B. Long-distance electron transfer through DNA. Annu. Rev. Biochem. 2002, 71, 51-70.

72. Sugiyama, H.; Saito, I. Theoretical studies of GC-specific photocleavage of DNA via electron transfer: Significant lowering of ionization potential and 5'-localization of HOMO of stacked GG bases in B-form DNA. J. Am. Chem. Soc. 1996, 118, 7063-7068.

73. Saito, I.; Nakamura, T.; Nakatani, K.; Yoshioka, Y.; Yamaguchi, K.; Sugiyama, H. Mapping of the hot spots for DNA damage by one-electron oxidation: Efficacy of GG doublets and GGG triplets as a trap in long-range hole migration. J. Am. Chem. Soc. 1998, 120, $12686-12687$. 
74. Senthilkumar, K.; Grozema, F.C.; Guerra, C.F.; Bickelhaupt, F.M.; Siebbeles, L.D. Mapping the sites for selective oxidation of guanines in DNA. J. Am. Chem. Soc. 2003, 125, 13658-13659.

75. Yamazaki, S.; Hayano, M.; Masai, H. Replication timing regulation of eukaryotic replicons: Rif1 as a global regulator of replication timing. Trends Genet. 2013, 29, 449-460.

76. Aparicio, O.M. Location, location, location: It's all in the timing for replication origins. Genes Dev. 2013, 27, 117-128.

77. Liu, L.; De, S.; Michor, F. DNA replication timing and higher-order nuclear organization determine single-nucleotide substitution patterns in cancer genomes. Nat. Commun. 2013, 4, 1502.

78. Stamatoyannopoulos, J.A.; Adzhubei, I.; Thurman, R.E.; Kryukov, G.V.; Mirkin, S.M.; Sunyaev, S.R. Human mutation rate associated with DNA replication timing. Nat. Genet. 2009, 41, 393-395.

79. Koren, A.; Polak, P.; Nemesh, J.; Michaelson, J.J.; Sebat, J.; Sunyaev, S.R.; McCarroll, S.A. Differential relationship of DNA replication timing to different forms of human mutation and variation. Am. J. Hum. Genet. 2012, 91, 1033-1040.

80. Gavin, D.P.; Chase, K.A.; Sharma, R.P. Active DNA demethylation in post-mitotic neurons: A reason for optimism. Neuropharmacology 2013, 75, 233-245.

81. Schuster-Bockler, B.; Lehner, B. Chromatin organization is a major influence on regional mutation rates in human cancer cells. Nature 2012, 488, 504-507.

82. Kim, N.; Jinks-Robertson, S. Transcription as a source of genome instability. Nat. Rev. Genet. 2012, 13, 204-214.

83. Wang, G.; Christensen, L.A.; Vasquez, K.M. Z-DNA-forming sequences generate large-scale deletions in mammalian cells. Proc. Natl. Acad. Sci. USA 2006, 103, 2677-2682.

84. Kadyrova, L.Y.; Mertz, T.M.; Zhang, Y.; Northam, M.R.; Sheng, Z.; Lobachev, K.S.; Shcherbakova, P.V.; Kadyrov, F.A. A reversible histone H3 acetylation cooperates with mismatch repair and replicative polymerases in maintaining genome stability. PLOS Genet. 2013, 9, e1003899.

85. Kinney, S.R.; Pradhan, S. Regulation of expression and activity of DNA (cytosine-5) methyltransferases in mammalian cells. Prog. Mol. Biol. Transl. Sci. 2011, 101, 311-333.

86. Smith, Z.D.; Meissner, A. DNA methylation: Roles in mammalian development. Nat. Rev. Genet. 2013, 14, 204-220.

87. Thomson, J.P.; Moggs, J.G.; Wolf, C.R.; Meehan, R.R. Epigenetic profiles as defined signatures of xenobiotic exposure. Mutat. Res. 2013, doi:10.1016/j.mrgentox.2013.08.007.

88. Song, C.X.; Szulwach, K.E.; Dai, Q.; Fu, Y.; Mao, S.Q.; Lin, L.; Street, C.; Li, Y.; Poidevin, M.; Wu, H.; et al. Genome-wide profiling of 5-formylcytosine reveals its roles in epigenetic priming. Cell 2013, 153, 678-691.

89. Kohli, R.M.; Zhang, Y. TET enzymes, TDG and the dynamics of DNA demethylation. Nature 2013, 502, 472-479.

90. Losman, J.A.; Kaelin, W.G., Jr. What a difference a hydroxyl makes: Mutant IDH, (R)-2-hydroxyglutarate, and cancer. Genes Dev. 2013, 27, 836-852. 
91. Guilhamon, P.; Eskandarpour, M.; Halai, D.; Wilson, G.A.; Feber, A.; Teschendorff, A.E.; Gomez, V.; Hergovich, A.; Tirabosco, R.; Fernanda Amary, M.; et al. Meta-analysis of IDH-mutant cancers identifies EBF1 as an interaction partner for TET2. Nat. Commun. 2013, 4, 2166.

92. Rendina, A.R.; Pietrak, B.; Smallwood, A.; Zhao, H.; Qi, H.; Quinn, C.; Adams, N.D.; Concha, N.; Duraiswami, C.; Thrall, S.H.; et al. Mutant IDH1 enhances the production of 2-hydroxyglutarate due to its kinetic mechanism. Biochemistry 2013, 52, 4563-4577.

93. Lian, C.G.; Xu, Y.; Ceol, C.; Wu, F.; Larson, A.; Dresser, K.; Xu, W.; Tan, L.; Hu, Y.; Zhan, Q.; et al. Loss of 5-hydroxymethylcytosine is an epigenetic hallmark of melanoma. Cell 2012, 150, 1135-1146.

94. Sandoval, J.; Mendez-Gonzalez, J.; Nadal, E.; Chen, G.; Carmona, F.J.; Sayols, S.; Moran, S.; Heyn, H.; Vizoso, M.; Gomez, A.; et al. A prognostic DNA methylation signature for stage I non-small-cell lung cancer. J. Clin. Oncol. 2013, 31, 4140-4147.

95. Schiesser, S.; Pfaffeneder, T.; Sadeghian, K.; Hackner, B.; Steigenberger, B.; Schroder, A.S.; Steinbacher, J.; Kashiwazaki, G.; Hofner, G.; Wanner, K.T.; et al. Deamination, oxidation, and C-C bond cleavage reactivity of 5-hydroxymethylcytosine, 5-formylcytosine, and 5-carboxycytosine. J. Am. Chem. Soc. 2013, 135, 14593-14599.

96. Ehrlich, M.; Norris, K.F.; Wang, R.Y.; Kuo, K.C.; Gehrke, C.W. DNA cytosine methylation and heat-induced deamination. Biosci. Rep. 1986, 6, 387-393.

97. Shen, J.C.; Rideout, W.M., 3rd; Jones, P.A. The rate of hydrolytic deamination of 5-methylcytosine in double-stranded DNA. Nucleic Acids Res. 1994, 22, 972-976.

98. Frederico, L.A.; Kunkel, T.A.; Shaw, B.R. A sensitive genetic assay for the detection of cytosine deamination: Determination of rate constants and the activation energy. Biochemistry 1990, 29, 2532-2537.

99. Zhang, X.; Mathews, C.K. Effect of DNA cytosine methylation upon deamination-induced mutagenesis in a natural target sequence in duplex DNA. J. Biol. Chem. 1994, 269, 7066-7069.

100. Sowers, L.C.; Sedwick, W.D.; Shaw, B.R. Hydrolysis of N3-methyl-2'-deoxycytidine: Model compound for reactivity of protonated cytosine residues in DNA. Mutat. Res. 1989, 215, $131-138$.

101. Nikolova, E.N.; Goh, G.B.; Brooks, C.L., 3rd; Al-Hashimi, H.M. Characterizing the protonation state of cytosine in transient G.C Hoogsteen base pairs in duplex DNA. J. Am. Chem. Soc. 2013, 135, 6766-6769.

102. Fryxell, K.J.; Zuckerkandl, E. Cytosine deamination plays a primary role in the evolution of mammalian isochores. Mol. Biol. Evol. 2000, 17, 1371-1383.

103. Chen, X.; Chen, Z.; Chen, H.; Su, Z.; Yang, J.; Lin, F.; Shi, S.; He, X. Nucleosomes suppress spontaneous mutations base-specifically in eukaryotes. Science 2012, 335, 1235-1238.

104. Labet, V.; Morell, C.; Cadet, J.; Eriksson, L.A.; Grand, A. Hydrolytic deamination of 5-methylcytosine in protic medium-A theoretical study. J. Phys. Chem. A 2009, 113, $2524-2533$. 
105. Patel, J.C.; Rice, M.E. Classification of $\mathrm{H}(2) \mathrm{O}(2)$ as a neuromodulator that regulates striatal dopamine release on a subsecond time scale. ACS Chem. Neurosci. 2012, 3, 991-1001.

106. Nogueira, V.; Hay, N. Molecular pathways: Reactive oxygen species homeostasis in cancer cells and implications for cancer therapy. Clin. Cancer Res. 2013, 19, 4309-4314.

107. Gupte, A.; Mumper, R.J. Elevated copper and oxidative stress in cancer cells as a target for cancer treatment. Cancer Treat. Rev. 2009, 35, 32-46.

108. Nieborowska-Skorska, M.; Kopinski, P.K.; Ray, R.; Hoser, G.; Ngaba, D.; Flis, S.; Cramer, K.; Reddy, M.M.; Koptyra, M.; Penserga, T.; et al. Rac2-MRC-cIII-generated ROS cause genomic instability in chronic myeloid leukemia stem cells and primitive progenitors. Blood 2012, 119, 4253-4263.

109. Cao, H.; Jiang, Y.; Wang, Y. Kinetics of deamination and $\mathrm{Cu}(\mathrm{II}) / \mathrm{H}_{2} \mathrm{O}_{2} /$ ascorbate-induced formation of 5-methylcytosine glycol at CpG sites in duplex DNA. Nucleic Acids Res. 2009, 37, 6635-6643.

110. Wagner, J.R.; Cadet, J. Oxidation reactions of cytosine DNA components by hydroxyl radical and one-electron oxidants in aerated aqueous solutions. Acc. Chem. Res. 2010, 43, 564-571.

111. Liang, Q.; Dedon, P.C. $\mathrm{Cu}(\mathrm{II}) / \mathrm{H}_{2} \mathrm{O}_{2}$-induced DNA damage is enhanced by packaging of DNA as a nucleosome. Chem. Res. Toxicol. 2001, 14, 416-422.

112. Tan, S.; Davey, C.A. Nucleosome structural studies. Curr. Opin. Struct. Biol. 2011, 21, 128-136.

113. Zavitsanos, K.; Nunes, A.M.; Malandrinos, G.; Hadjiliadis, N. Copper effective binding with 32-62 and 94-125 peptide fragments of histone H2B. J. Inorg. Biochem. 2011, 105, 102-110.

114. Ishida, S.; Andreux, P.; Poitry-Yamate, C.; Auwerx, J.; Hanahan, D. Bioavailable copper modulates oxidative phosphorylation and growth of tumors. Proc. Natl. Acad. Sci. USA 2013, 110, 19507-19512.

115. Bienvenu, C.; Cadet, J. Synthesis and kinetic study of the deamination of the cis diastereomers of 5,6-dihydroxy-5,6-dihydro-5-methyl-2'-deoxycytidine. J. Org. Chem. 1996, 61, 2632-2637.

116. Bransteitter, R.; Prochnow, C.; Chen, X.S. The current structural and functional understanding of APOBEC deaminases. Cell. Mol. Life Sci. 2009, 66, 3137-3147.

117. Larijani, M.; Martin, A. The biochemistry of activation-induced deaminase and its physiological functions. Semin. Immunol. 2012, 24, 255-263.

118. Iwatani, Y.; Takeuchi, H.; Strebel, K.; Levin, J.G. Biochemical activities of highly purified, catalytically active human APOBEC3G: Correlation with antiviral effect. J. Virol. 2006, 80, 5992-6002.

119. Nabel, C.S.; Jia, H.; Ye, Y.; Shen, L.; Goldschmidt, H.L.; Stivers, J.T.; Zhang, Y.; Kohli, R.M. AID/APOBEC deaminases disfavor modified cytosines implicated in DNA demethylation. Nat. Chem. Biol. 2012, 8, 751-758.

120. Wijesinghe, P.; Bhagwat, A.S. Efficient deamination of 5-methylcytosines in DNA by human APOBEC3A, but not by AID or APOBEC3G. Nucleic Acids Res. 2012, 40, 9206-9217. 
121. Morgan, H.D.; Dean, W.; Coker, H.A.; Reik, W.; Petersen-Mahrt, S.K. Activation-induced cytidine deaminase deaminates 5-methylcytosine in DNA and is expressed in pluripotent tissues: Implications for epigenetic reprogramming. J. Biol. Chem. 2004, 279, 52353-52360.

122. Bishop, K.N.; Holmes, R.K.; Sheehy, A.M.; Davidson, N.O.; Cho, S.J.; Malim, M.H. Cytidine deamination of retroviral DNA by diverse APOBEC proteins. Curr. Biol. 2004, 14, $1392-1396$.

123. Leonard, B.; Hart, S.N.; Burns, M.B.; Carpenter, M.A.; Temiz, N.A.; Rathore, A.; Isaksson Vogel, R.; Nikas, J.B.; Law, E.K.; Brown, W.L.; et al. APOBEC3B upregulation and genomic mutation patterns in serous ovarian carcinoma. Cancer Res. 2013, 73, 7222-7231.

124. Langlois, M.A.; Beale, R.C.; Conticello, S.G.; Neuberger, M.S. Mutational comparison of the single-domained APOBEC3C and double-domained APOBEC3F/G anti-retroviral cytidine deaminases provides insight into their DNA target site specificities. Nucleic Acids Res. 2005, 33, 1913-1923.

125. Wiegand, H.L.; Doehle, B.P.; Bogerd, H.P.; Cullen, B.R. A second human antiretroviral factor, APOBEC3F, is suppressed by the HIV-1 and HIV-2 Vif proteins. EMBO J. 2004, 23, 2451-2458.

126. Liddament, M.T.; Brown, W.L.; Schumacher, A.J.; Harris, R.S. APOBEC3F properties and hypermutation preferences indicate activity against HIV-1 in vivo. Curr. Biol. 2004, 14, 1385-1391.

127. Kohli, R.M.; Maul, R.W.; Guminski, A.F.; McClure, R.L.; Gajula, K.S.; Saribasak, H.; McMahon, M.A.; Siliciano, R.F.; Gearhart, P.J.; Stivers, J.T. Local sequence targeting in the AID/APOBEC family differentially impacts retroviral restriction and antibody diversification. J. Biol. Chem. 2010, 285, 40956-40964.

128. Krijger, P.H.; Tsaalbi-Shtylik, A.; Wit, N.; van den Berk, P.C.; de Wind, N.; Jacobs, H. Rev1 is essential in generating $\mathrm{G}$ to $\mathrm{C}$ transversions downstream of the Ung2 pathway but not the Msh2+Ung2 hybrid pathway. Eur. J. Immunol. 2013, 43, 2765-2770.

129. Cui, L.; Ye, W.; Prestwich, E.G.; Wishnok, J.S.; Taghizadeh, K.; Dedon, P.C.; Tannenbaum, S.R. Comparative analysis of four oxidized guanine lesions from reactions of DNA with peroxynitrite, singlet oxygen, and gamma-radiation. Chem. Res. Toxicol. 2013, 26, 195-202.

130. Kino, K.; Sugiyama, H. UVR-induced G-C to C-G transversions from oxidative DNA damage. Mutat. Res. 2005, 571, 33-42.

131. Douki, T.; Angelov, D.; Cadet, J. UV laser photolysis of DNA: Effect of duplex stability on charge-transfer efficiency. J. Am. Chem. Soc. 2001, 123, 11360-11366.

132. Giese, B.; Amaudrut, J.; Kohler, A.K.; Spormann, M.; Wessely, S. Direct observation of hole transfer through DNA by hopping between adenine bases and by tunnelling. Nature 2001, 412, 318-320.

133. Prat, F.; Houk, K.N.; Foote, C.S. Effect of guanine stacking on the oxidation of 8-oxoguanine in B-DNA. J. Am. Chem. Soc. 1998, 120, 845-846.

134. Cadet, J.; Wagner, J.R. DNA base damage by reactive oxygen species, oxidizing agents, and UV radiation. Cold Spring Harb. Perspect. Biol. 2013, 5, a012559. 
135. Stathis, D.; Lischke, U.; Koch, S.C.; Deiml, C.A.; Carell, T. Discovery and mutagenicity of a guanidinoformimine lesion as a new intermediate of the oxidative deoxyguanosine degradation pathway. J. Am. Chem. Soc. 2012, 134, 4925-4930.

136. Hickerson, R.P.; Prat, F.; Muller, J.G.; Foote, C.S.; Burrows, C.J. Sequence and stacking dependence of 8-oxoguanine oxidation: Comparison of one-electron vs. singlet oxygen mechanisms. J. Am. Chem. Soc. 1999, 121, 9423-9428.

137. Lim, K.S.; Taghizadeh, K.; Wishnok, J.S.; Babu, I.R.; Shafirovich, V.; Geacintov, N.E.; Dedon, P.C. Sequence-dependent variation in the reactivity of 8-Oxo-7,8-dihydro-2'deoxyguanosine toward oxidation. Chem. Res. Toxicol. 2012, 25, 366-373.

138. Lim, K.S.; Cui, L.; Taghizadeh, K.; Wishnok, J.S.; Chan, W.; DeMott, M.S.; Babu, I.R.; Tannenbaum, S.R.; Dedon, P.C. In situ analysis of 8-oxo-7,8-dihydro-2'-deoxyguanosine oxidation reveals sequence- and agent-specific damage spectra. J. Am. Chem. Soc. 2012, 134, 18053-18064.

139. McKibbin, P.L.; Fleming, A.M.; Towheed, M.A.; Van Houten, B.; Burrows, C.J.; David, S.S. Repair of hydantoin lesions and their amine adducts in DNA by base and nucleotide excision repair. J. Am. Chem. Soc. 2013, 135, 13851-13861.

140. Davis, W.B.; Bjorklund, C.C.; Deline, M. Probing the effects of DNA-protein interactions on DNA hole transport: The N-terminal histone tails modulate the distribution of oxidative damage and chemical lesions in the nucleosome core particle. Biochemistry 2012, 51, 3129-3142.

141. Krokan, H.E.; Bjoras, M. Base excision repair. Cold Spring Harb. Perspect. Biol. 2013, $5, \mathrm{a} 012583$.

142. Sarasin, A. An overview of the mechanisms of mutagenesis and carcinogenesis. Mutat. Res. 2003, 544, 99-106.

143. Ray, S.; Menezes, M.R.; Senejani, A.; Sweasy, J.B. Cellular roles of DNA polymerase beta. Yale J. Biol. Med. 2013, 86, 463-469.

144. Hashimoto, H.; Zhang, X.; Cheng, X. Excision of thymine and 5-hydroxymethyluracil by the MBD4 DNA glycosylase domain: Structural basis and implications for active DNA demethylation. Nucleic Acids Res. 2012, 40, 8276-8284.

145. Sjolund, A.B.; Senejani, A.G.; Sweasy, J.B. MBD4 and TDG: Multifaceted DNA glycosylases with ever expanding biological roles. Mutat. Res. 2013, 743-744, 12-25.

146. Millar, C.B.; Guy, J.; Sansom, O.J.; Selfridge, J.; MacDougall, E.; Hendrich, B.; Keightley, P.D.; Bishop, S.M.; Clarke, A.R.; Bird, A. Enhanced CpG mutability and tumorigenesis in MBD4-deficient mice. Science 2002, 297, 403-405.

147. Wong, E.; Yang, K.; Kuraguchi, M.; Werling, U.; Avdievich, E.; Fan, K.; Fazzari, M.; Jin, B.; Brown, A.M.; Lipkin, M.; et al. Mbd4 inactivation increases $\mathrm{C} \rightarrow \mathrm{T}$ transition mutations and promotes gastrointestinal tumor formation. Proc. Natl. Acad. Sci. USA 2002, 99, $14937-$ 14942. 
148. Sansom, O.J.; Bishop, S.M.; Bird, A.; Clarke, A.R. MBD4 deficiency does not increase mutation or accelerate tumorigenesis in mice lacking MMR. Oncogene 2004, 23, 5693-5696.

149. Ishibashi, T.; So, K.; Cupples, C.G.; Ausio, J. MBD4-mediated glycosylase activity on a chromatin template is enhanced by acetylation. Mol. Cell. Biol. 2008, 28, 4734-4744.

150. Vasovcak, P.; Krepelova, A.; Menigatti, M.; Puchmajerova, A.; Skapa, P.; Augustinakova, A.; Amann, G.; Wernstedt, A.; Jiricny, J.; Marra, G.; et al. Unique mutational profile associated with a loss of TDG expression in the rectal cancer of a patient with a constitutional PMS2 deficiency. DNA Repair 2012, 11, 616-623.

151. Ocampo-Hafalla, M.T.; Altamirano, A.; Basu, A.K.; Chan, M.K.; Ocampo, J.E.; Cummings, A., Jr.; Boorstein, R.J.; Cunningham, R.P.; Teebor, G.W. Repair of thymine glycol by hNth1 and hNeill is modulated by base pairing and cis-trans epimerization. DNA Repair 2006, 5, 444-454.

152. Chan, M.K.; Ocampo-Hafalla, M.T.; Vartanian, V.; Jaruga, P.; Kirkali, G.; Koenig, K.L.; Brown, S.; Lloyd, R.S.; Dizdaroglu, M.; Teebor, G.W. Targeted deletion of the genes encoding NTH1 and NEIL1 DNA N-glycosylases reveals the existence of novel carcinogenic oxidative damage to DNA. DNA Repair 2009, 8, 786-794.

153. Hegde, M.L.; Hegde, P.M.; Bellot, L.J.; Mandal, S.M.; Hazra, T.K.; Li, G.M.; Boldogh, I.; Tomkinson, A.E.; Mitra, S. Prereplicative repair of oxidized bases in the human genome is mediated by NEIL1 DNA glycosylase together with replication proteins. Proc. Natl. Acad. Sci. USA 2013, 110, E3090-E3099.

154. Chaisaingmongkol, J.; Popanda, O.; Warta, R.; Dyckhoff, G.; Herpel, E.; Geiselhart, L.; Claus, R.; Lasitschka, F.; Campos, B.; Oakes, C.C.; et al. Epigenetic screen of human DNA repair genes identifies aberrant promoter methylation of NEIL1 in head and neck squamous cell carcinoma. Oncogene 2012, 31, 5108-5116.

155. Doseth, B.; Ekre, C.; Slupphaug, G.; Krokan, H.E.; Kavli, B. Strikingly different properties of uracil-DNA glycosylases UNG2 and SMUG1 may explain divergent roles in processing of genomic uracil. DNA Repair 2012, 11, 587-593.

156. Slupianek, A.; Falinski, R.; Znojek, P.; Stoklosa, T.; Flis, S.; Doneddu, V.; Pytel, D.; Synowiec, E.; Blasiak, J.; Bellacosa, A.; et al. BCR-ABL1 kinase inhibits uracil DNA glycosylase UNG2 to enhance oxidative DNA damage and stimulate genomic instability. Leukemia 2013, 27, 629-634.

157. Xie, Y.; Yang, H.; Cunanan, C.; Okamoto, K.; Shibata, D.; Pan, J.; Barnes, D.E.; Lindahl, T.; McIlhatton, M.; Fishel, R.; et al. Deficiencies in mouse Myh and Oggl result in tumor predisposition and $\mathrm{G}$ to $\mathrm{T}$ mutations in codon 12 of the $K$-ras oncogene in lung tumors. Cancer Res. 2004, 64, 3096-3102.

158. Ruggieri, V.; Pin, E.; Russo, M.T.; Barone, F.; Degan, P.; Sanchez, M.; Quaia, M.; Minoprio, A.; Turco, E.; Mazzei, F.; et al. Loss of MUTYH function in human cells leads to accumulation of oxidative damage and genetic instability. Oncogene 2013, 32, 4500-4508.

159. Bjoras, M.; Seeberg, E.; Luna, L.; Pearl, L.H.; Barrett, T.E. Reciprocal "flipping" underlies substrate recognition and catalytic activation by the human 8-oxo-guanine DNA glycosylase. J. Mol. Biol. 2002, 317, 171-177. 
160. Luncsford, P.J.; Chang, D.Y.; Shi, G.; Bernstein, J.; Madabushi, A.; Patterson, D.N.; Lu, A.L.; Toth, E.A. A structural hinge in eukaryotic MutY homologues mediates catalytic activity and Rad9-Rad1-Hus1 checkpoint complex interactions. J. Mol. Biol. 2010, 403, 351-370.

161. Amouroux, R.; Campalans, A.; Epe, B.; Radicella, J.P. Oxidative stress triggers the preferential assembly of base excision repair complexes on open chromatin regions. Nucleic Acids Res. 2010, 38, 2878-2890.

162. Menoni, H.; Shukla, M.S.; Gerson, V.; Dimitrov, S.; Angelov, D. Base excision repair of 8-oxoG in dinucleosomes. Nucleic Acids Res. 2012, 40, 692-700.

163. Kino, K.; Takao, M.; Miyazawa, H.; Hanaoka, F. A DNA oligomer containing 2,2,4-triamino-5(2H)-oxazolone is incised by human NEIL1 and NTH1. Mutat Res. 2012, 734, 73-77.

164. Dou, H.; Mitra, S.; Hazra, T.K. Repair of oxidized bases in DNA bubble structures by human DNA glycosylases NEIL1 and NEIL2. J. Biol. Chem. 2003, 278, 49679-49684.

165. Hailer, M.K.; Slade, P.G.; Martin, B.D.; Rosenquist, T.A.; Sugden, K.D. Recognition of the oxidized lesions spiroiminodihydantoin and guanidinohydantoin in DNA by the mammalian base excision repair glycosylases NEIL1 and NEIL2. DNA Repair 2005, 4, 41-50.

166. Krishnamurthy, N.; Zhao, X.; Burrows, C.J.; David, S.S. Superior removal of hydantoin lesions relative to other oxidized bases by the human DNA glycosylase hNEIL1. Biochemistry 2008, 47, 7137-7146.

167. Liu, M.; Doublie, S.; Wallace, S.S. Neil3, the final frontier for the DNA glycosylases that recognize oxidative damage. Mutat. Res. 2013, 743-744, 4-11.

168. Regnell, C.E.; Hildrestrand, G.A.; Sejersted, Y.; Medin, T.; Moldestad, O.; Rolseth, V.; Krokeide, S.Z.; Suganthan, R.; Luna, L.; Bjoras, M.; et al. Hippocampal adult neurogenesis is maintained by Neil3-dependent repair of oxidative DNA lesions in neural progenitor cells. Cell Rep. 2012, 2, 503-510.

169. Rolseth, V.; Krokeide, S.Z.; Kunke, D.; Neurauter, C.G.; Suganthan, R.; Sejersted, Y.; Hildrestrand, G.A.; Bjoras, M.; Luna, L. Loss of Neil3, the major DNA glycosylase activity for removal of hydantoins in single stranded DNA, reduces cellular proliferation and sensitizes cells to genotoxic stress. Biochim. Biophys. Acta 2013, 1833, 1157-1164.

170. Krokeide, S.Z.; Laerdahl, J.K.; Salah, M.; Luna, L.; Cederkvist, F.H.; Fleming, A.M.; Burrows, C.J.; Dalhus, B.; Bjoras, M. Human NEIL3 is mainly a monofunctional DNA glycosylase removing spiroimindiohydantoin and guanidinohydantoin. DNA Repair 2013, 12, 1159-1164.

171. Wardle, J.; Burgers, P.M.; Cann, I.K.; Darley, K.; Heslop, P.; Johansson, E.; Lin, L.J.; McGlynn, P.; Sanvoisin, J.; Stith, C.M.; et al. Uracil recognition by replicative DNA polymerases is limited to the archaea, not occurring with bacteria and eukarya. Nucleic Acids Res. 2008, 36, 705-711.

172. Aller, P.; Duclos, S.; Wallace, S.S.; Doublie, S. A crystallographic study of the role of sequence context in thymine glycol bypass by a replicative DNA polymerase serendipitously sheds light on the exonuclease complex. J. Mol. Biol. 2011, 412, 22-34. 
173. Fischhaber, P.L.; Gerlach, V.L.; Feaver, W.J.; Hatahet, Z.; Wallace, S.S.; Friedberg, E.C. Human DNA polymerase kappa bypasses and extends beyond thymine glycols during translesion synthesis in vitro, preferentially incorporating correct nucleotides. J. Biol. Chem. 2002, 277, 37604-37611.

174. Yoon, J.H.; Bhatia, G.; Prakash, S.; Prakash, L. Error-free replicative bypass of thymine glycol by the combined action of DNA polymerases kappa and zeta in human cells. Proc. Natl. Acad. Sci. USA 2010, 107, 14116-14121.

175. Takata, K.; Shimizu, T.; Iwai, S.; Wood, R.D. Human DNA polymerase N (POLN) is a low fidelity enzyme capable of error-free bypass of 5S-thymine glycol. J. Biol. Chem. 2006, 281, 23445-23455.

176. Takata, K.; Arana, M.E.; Seki, M.; Kunkel, T.A.; Wood, R.D. Evolutionary conservation of residues in vertebrate DNA polymerase $\mathrm{N}$ conferring low fidelity and bypass activity. Nucleic Acids Res. 2010, 38, 3233-3244.

177. Kusumoto, R.; Masutani, C.; Iwai, S.; Hanaoka, F. Translesion synthesis by human DNA polymerase eta across thymine glycol lesions. Biochemistry 2002, 41, 6090-6099.

178. Hogg, M.; Seki, M.; Wood, R.D.; Doublie, S.; Wallace, S.S. Lesion bypass activity of DNA polymerase theta (POLQ) is an intrinsic property of the pol domain and depends on unique sequence inserts. J. Mol. Biol. 2011, 405, 642-652.

179. Seki, M.; Masutani, C.; Yang, L.W.; Schuffert, A.; Iwai, S.; Bahar, I.; Wood, R.D. High-efficiency bypass of DNA damage by human DNA polymerase Q. EMBO J. 2004, 23, 4484-4494.

180. Shibutani, S.; Takeshita, M.; Grollman, A.P. Insertion of specific bases during DNA synthesis past the oxidation-damaged base 8-oxodG. Nature 1991, 349, 431-434.

181. Fazlieva, R.; Spittle, C.S.; Morrissey, D.; Hayashi, H.; Yan, H.; Matsumoto, Y. Proofreading exonuclease activity of human DNA polymerase delta and its effects on lesion-bypass DNA synthesis. Nucleic Acids Res. 2009, 37, 2854-2866.

182. Markkanen, E.; Castrec, B.; Villani, G.; Hubscher, U. A switch between DNA polymerases delta and lambda promotes error-free bypass of 8-oxo-G lesions. Proc. Natl. Acad. Sci. USA 2012, 109, 20401-20406.

183. Markkanen, E.; Hubscher, U.; van Loon, B. Regulation of oxidative DNA damage repair: The adenine:8-oxo-guanine problem. Cell Cycle 2012, 11, 1070-1075.

184. Brown, J.A.; Duym, W.W.; Fowler, J.D.; Suo, Z. Single-turnover kinetic analysis of the mutagenic potential of 8-oxo-7,8-dihydro-2'-deoxyguanosine during gap-filling synthesis catalyzed by human DNA polymerases lambda and beta. J. Mol. Biol. 2007, 367, 1258-1269.

185. Freudenthal, B.D.; Beard, W.A.; Wilson, S.H. DNA polymerase minor groove interactions modulate mutagenic bypass of a templating 8-oxoguanine lesion. Nucleic Acids Res. 2013, 41, 1848-1858.

186. Avkin, S.; Livneh, Z. Efficiency, specificity and DNA polymerase-dependence of translesion replication across the oxidative DNA lesion 8-oxoguanine in human cells. Mutat. Res. 2002, 510, 81-90. 
187. Maga, G.; Crespan, E.; Markkanen, E.; Imhof, R.; Furrer, A.; Villani, G.; Hubscher, U.; van Loon, B. DNA polymerase delta-interacting protein 2 is a processivity factor for DNA polymerase lambda during 8-oxo-7,8-dihydroguanine bypass. Proc. Natl. Acad. Sci. USA 2013, 110, 18850-18855.

188. Aller, P.; Ye, Y.; Wallace, S.S.; Burrows, C.J.; Doublie, S. Crystal structure of a replicative DNA polymerase bound to the oxidized guanine lesion guanidinohydantoin. Biochemistry 2010, 49, 2502-2509.

189. Beckman, J.; Wang, M.; Blaha, G.; Wang, J.; Konigsberg, W.H. Substitution of Ala for Tyr567 in RB69 DNA polymerase allows dAMP and dGMP to be inserted opposite Guanidinohydantoin. Biochemistry 2010, 49, 8554-8563.

190. Kino, K.; Ito, N.; Sugasawa, K.; Sugiyama, H.; Hanaoka, F. Translesion synthesis by human DNA polymerase eta across oxidative products of guanine. Nucleic Acids Symp. Ser. (Oxf.) 2004, 48, 171-172.

191. Henderson, P.T.; Delaney, J.C.; Muller, J.G.; Neeley, W.L.; Tannenbaum, S.R.; Burrows, C.J.; Essigmann, J.M. The hydantoin lesions formed from oxidation of 7,8-dihydro-8-oxoguanine are potent sources of replication errors in vivo. Biochemistry 2003, 42, 9257-9262.

192. Suzuki, M.; Kino, K.; Morikawa, M.; Kobayashi, T.; Komori, R.; Miyazawa, H. Calculation of the stabilization energies of oxidatively damaged guanine base pairs with guanine. Molecules 2012, 17, 6705-6715.

193. Stephens, P.J.; Tarpey, P.S.; Davies, H.; van Loo, P.; Greenman, C.; Wedge, D.C.; Nik-Zainal, S.; Martin, S.; Varela, I.; Bignell, G.R.; et al. The landscape of cancer genes and mutational processes in breast cancer. Nature 2012, 486, 400-404.

194. Vermeulen, W.; Fousteri, M. Mammalian transcription-coupled excision repair. Cold Spring Harb. Perspect. Biol. 2013, 5, a012625.

195. Lehmann, A.R.; McGibbon, D.; Stefanini, M. Xeroderma pigmentosum. Orphanet J. Rare Dis. 2011, 6, 70 .

196. Scharer, O.D. Nucleotide excision repair in eukaryotes. Cold Spring Harb. Perspect. Biol. 2013, 5, a012609.

197. Tornaletti, S.; Maeda, L.S.; Lloyd, D.R.; Reines, D.; Hanawalt, P.C. Effect of thymine glycol on transcription elongation by T7 RNA polymerase and mammalian RNA polymerase II. J. Biol. Chem. 2001, 276, 45367-45371.

198. Tornaletti, S.; Maeda, L.S.; Kolodner, R.D.; Hanawalt, P.C. Effect of 8-oxoguanine on transcription elongation by $\mathrm{T} 7$ RNA polymerase and mammalian RNA polymerase II. DNA Repair 2004, 3, 483-494.

199. Hoang, M.L.; Chen, C.H.; Sidorenko, V.S.; He, J.; Dickman, K.G.; Yun, B.H.; Moriya, M.; Niknafs, N.; Douville, C.; Karchin, R.; et al. Mutational signature of aristolochic acid exposure as revealed by whole-exome sequencing. Sci. Transl. Med. 2013, 5, 197ra102.

200. Poon, S.L.; Pang, S.T.; McPherson, J.R.; Yu, W.; Huang, K.K.; Guan, P.; Weng, W.H.; Siew, E.Y.; Liu, Y.; Heng, H.L.; et al. Genome-wide mutational signatures of aristolochic acid and its application as a screening tool. Sci. Transl. Med. 2013, 5, $197 \mathrm{ra101.}$ 
201. Sidorenko, V.S.; Yeo, J.E.; Bonala, R.R.; Johnson, F.; Scharer, O.D.; Grollman, A.P. Lack of recognition by global-genome nucleotide excision repair accounts for the high mutagenicity and persistence of aristolactam-DNA adducts. Nucleic Acids Res. 2012, 40, 2494-2505.

202. Charlet-Berguerand, N.; Feuerhahn, S.; Kong, S.E.; Ziserman, H.; Conaway, J.W.; Conaway, R.; Egly, J.M. RNA polymerase II bypass of oxidative DNA damage is regulated by transcription elongation factors. EMBO J. 2006, 25, 5481-5491.

203. Khobta, A.; Epe, B. Repair of oxidatively generated DNA damage in Cockayne syndrome. Mech. Ageing Dev. 2013, 134, 253-260.

204. D'Errico, M.; Pascucci, B.; Iorio, E.; van Houten, B.; Dogliotti, E. The role of CSA and CSB protein in the oxidative stress response. Mech. Ageing Dev. 2013, 134, 261-269.

205. Aamann, M.D.; Muftuoglu, M.; Bohr, V.A.; Stevnsner, T. Multiple interaction partners for Cockayne syndrome proteins: Implications for genome and transcriptome maintenance. Mech. Ageing Dev. 2013, 134, 212-224.

206. Banerjee, D.; Mandal, S.M.; Das, A.; Hegde, M.L.; Das, S.; Bhakat, K.K.; Boldogh, I.; Sarkar, P.S.; Mitra, S.; Hazra, T.K. Preferential repair of oxidized base damage in the transcribed genes of mammalian cells. J. Biol. Chem. 2011, 286, 6006-6016.

207. Inukai, N.; Yamaguchi, Y.; Kuraoka, I.; Yamada, T.; Kamijo, S.; Kato, J.; Tanaka, K.; Handa, H. A novel hydrogen peroxide-induced phosphorylation and ubiquitination pathway leading to RNA polymerase II proteolysis. J. Biol. Chem. 2004, 279, 8190-8195.

208. Pfeifer, G.P.; Besaratinia, A. Mutational spectra of human cancer. Hum. Genet. 2009, 125, 493-506.

209. Walser, J.C.; Furano, A.V. The mutational spectrum of non-CpG DNA varies with CpG content. Genome Res. 2010, 20, 875-882.

210. Roberts, S.A.; Sterling, J.; Thompson, C.; Harris, S.; Mav, D.; Shah, R.; Klimczak, L.J.; Kryukov, G.V.; Malc, E.; Mieczkowski, P.A.; et al. Clustered mutations in yeast and in human cancers can arise from damaged long single-strand DNA regions. Mol. Cell 2012, 46, 424-435.

211. Galashevskaya, A.; Sarno, A.; Vagbo, C.B.; Aas, P.A.; Hagen, L.; Slupphaug, G.; Krokan, H.E. A robust, sensitive assay for genomic uracil determination by LC/MS/MS reveals lower levels than previously reported. DNA Repair 2013, 12, 699-706.

212. Mangerich, A.; Knutson, C.G.; Parry, N.M.; Muthupalani, S.; Ye, W.; Prestwich, E.; Cui, L.; McFaline, J.L.; Mobley, M.; Ge, Z.; et al. Infection-induced colitis in mice causes dynamic and tissue-specific changes in stress response and DNA damage leading to colon cancer. Proc. Natl. Acad. Sci. USA 2012, 109, E1820-E1829.

213. Chan, S.W.; Dedon, P.C. The biological and metabolic fates of endogenous DNA damage products. J. Nucleic Acids 2010, $2010,929047$.

214. Gaikwad, N.W. Metabolomic profiling unravels DNA adducts in human breast that are formed from peroxidase mediated activation of estrogens to quinone methides. PLoS One 2013, 8, e65826.

215. Tretyakova, N.; Goggin, M.; Sangaraju, D.; Janis, G. Quantitation of DNA adducts by stable isotope dilution mass spectrometry. Chem. Res. Toxicol. 2012, 25, 2007-2035. 


\title{
The Molecular Basis of Retinal Dystrophies in Pakistan
}

\author{
Muhammad Imran Khan, Maleeha Azam, Muhammad Ajmal, Rob W. J. Collin, \\ Anneke I. den Hollander, Frans P. M. Cremers and Raheel Qamar
}

\begin{abstract}
The customary consanguineous nuptials in Pakistan underlie the frequent occurrence of autosomal recessive inherited disorders, including retinal dystrophy (RD). In many studies, homozygosity mapping has been shown to be successful in mapping susceptibility loci for autosomal recessive inherited disease. RDs are the most frequent cause of inherited blindness worldwide. To date there is no comprehensive genetic overview of different RDs in Pakistan. In this review, genetic data of syndromic and non-syndromic RD families from Pakistan has been collected. Out of the 132 genes known to be involved in non-syndromic RD, 35 different genes have been reported to be mutated in families of Pakistani origin. In the Pakistani RD families 90\% of the mutations causing non-syndromic $\mathrm{RD}$ and all mutations causing syndromic forms of the disease have not been reported in other populations. Based on the current inventory of all Pakistani $\mathrm{RD}$-associated gene defects, a cost-efficient allele-specific analysis of $11 \mathrm{RD}$-associated variants is proposed, which may capture up to $35 \%$ of the genetic causes of retinal dystrophy in Pakistan.
\end{abstract}

Reprinted from Genes. Cite as: Khan, M.I.; Azam, M.; Ajmal, M.; Collin, R.W.J.; den Hollander, A.I.; Cremers, F.P.M.; Qamar, R. The Molecular Basis of Retinal Dystrophies in Pakistan. Genes 2014, 5, 176-195.

\section{Introduction}

Inherited retinal dystrophies (RD) belong to a group of clinically and genetically heterogeneous disorders [1]. The clinical sub-classification of this group of diseases is based on the nature of the disease (stationary or progressive), the inheritance pattern, and the dysfunctional part of the retina [2]. The disease is either congenital, occurring early in life, such as Leber congenital amaurosis (LCA; MIM\# 204000), and congenital stationary night blindness (CSNB; MIM\# 310500), or might have a later onset, such as in retinitis pigmentosa (RP; MIM\# 268000), cone-rod dystrophy (CRD; MIM\# 604116), and cone dystrophy (CD; MIM\# 602093) [3]. In addition to disorders confined to the eye, there are syndromic forms of the disease in which retinal dystrophy is either among the primary clinical symptoms or might manifest at an advanced stage. The most common syndromic form of RD is Usher syndrome (USH; MIM\# 276900), in which RP is associated with variable degrees of hearing loss and vestibular dysfunction [4]. Other types of syndromic RD include Bardet-Biedl syndrome (BBS; MIM\# 209900), Senior-Loken syndrome (SLSN; MIM\# 266900), Joubert syndrome (JBTS; MIM\# 213300), and Meckel syndrome (MKS; MIM\# 249000). All these syndromes exhibit severe clinical features in addition to retinal degeneration $[5,6]$.

The estimated worldwide prevalence of RD is 1 in 3000 individuals [7]. RP is the most frequent phenotype among the RDs, affecting 1 in 4000 individuals [8,9]. In Pakistan the frequency of RD is not very well defined, but a hospital-based study estimated autosomal recessive RP to be the most prevalent [10]. In several developing countries, as opposed to Western countries, consanguinity has 
always been a major contributing factor in the high prevalence of autosomal recessive disorders [11]. In Pakistan more than $60 \%$ of marriages are consanguineous and among them about $80 \%$ are between first cousins [12]. Such consanguineous families are ideal for homozygosity based genetic mapping studies aimed at the identification of the underlying genetic defect $[13,14]$.

As a result of several technological advances, 201 genes implicated in different forms of RD have been identified to date [15]. Among these genes, 132 are linked to non-syndromic forms of the disease with some genetic overlap between different classes $[1,3,16]$. In the developed countries, genetic testing using medium-to-high throughput genotyping methods are now being routinely used for proper disease diagnosis [17]. This has resulted in the establishment of many genotype-phenotype correlations [17-19]. In the last two decades, several studies have described the genetic causes of different retinal dystrophies in consanguineous Pakistani families. However, to date, there has been no comprehensive ophthalmogenetic overview of all forms of RD that have been identified in Pakistan. Therefore, this literature review provides an overview of all published genetic data of syndromic and non-syndromic RD that have been described for Pakistani families.

\section{Experimental}

A comprehensive literature review was performed for mutations and loci, which have been described previously for Pakistani individuals with syndromic and non-syndromic retinal diseases. The Retinal Network (RetNet) [15], National Centre for Biotechnology Information (NCBI) [20], Online Mendelian Inheritance in Man (OMIM) [21], The Human Gene Mutation Database (HGMD) [22], and published literature were used to search for the causative genes. In order to predict the pathogenicity of the reported missense mutations, in silico analysis including, polymorphism phenotyping (PolyPhen-2) [23], and sorting tolerant from intolerant (SIFT) [24] were performed. The frequency of these variants in the healthy population was checked via the exome variant server (EVS) [25].

\section{Results}

\subsection{Overview of Molecular Genetic Studies in Non-Syndromic RD in Pakistan}

Thus far, fifty-six studies have reported on the genetic causes of non-syndromic RD including arCRD, arCSNB, arLCA, and arRP in Pakistani persons, most of which belong to consanguineous families. The genetic data of a total of 466 Pakistani RD patients from 103 families (Tables 1 and 2), have been described in the current review. Among these retinal phenotypes, arRP was found to be the most frequently occurring RD (59\%), followed by arLCA (19\%), arCRD (10\%), and arCSNB (9\%) (Tables 1 and 2; Figure 1). Autosomal recessive inheritance seems to predominate in the RD families (96\%) and only two autosomal dominant RP (adRP) families have been described (Tables 1 and 2). Of these, one adRP family carries a mutation in RHO (MIM\# 180380) [26], while in one family a frequent variant (c.2138G $>$ A) in SEMA4A (MIM\# 607292) has been described to cause adRP, however in silico prediction and exome variant server (EVS) frequency do not support the pathogenicity of the latter variant (Table 2) [27]. The compiled data demonstrate that out of the 132 genes known to be involved in non-syndromic $\mathrm{RD}$, mutations in 36 different genes are causing 
disease in patients of Pakistani origin (Table 1; Figure 2), reflecting the genetic heterogeneity of the disease in this population. The most frequently mutated genes were AIPL1 (MIM\# 604392), CRB1 (MIM\# 604210), TULP1 (MIM\# 602280), RPGRIP1 (MIM\# 605446), RP1 (MIM\# 180100), SEMA4A, LCA5 (MIM\# 611408), and PDE6A (MIM\# 180071) (Figure 2). Most of the reported mutations, and those identified in the current cohort, were novel to this population except for mutations in ABCA4 (MIM\# 601691), CRB1, CERKL (MIM\# 608381), RPE65 (MIM\# 180069), RPGR (MIM\# 312610), and SPATA7 (MIM\# 609868), which were initially identified in persons of different ethnicity (Table 1). As expected, all the reported disease associated alleles are rare variants and in silico analysis predicted these variants to have a deleterious effect on protein function (Table S1).

Table 1. Mutations identified in Pakistani patients with non-syndromic retinal dystrophies.

\begin{tabular}{|c|c|c|c|c|c|c|c|}
\hline Gene & RefSeq Id & Nucleotide variant & Protein variant & Phenotype & \# Families & \# Patients & References \\
\hline$A B C A 4$ & NM_000350.2 & c. $6658 \mathrm{C}>\mathrm{T}$ & p. $\left(\mathrm{G} \ln 2220^{*}\right)$ & $\operatorname{arRP}$ & 1 & 6 & {$[28,29]$} \\
\hline ADAM9 & NM_003816.2 & c. $766 \mathrm{C}>\mathrm{T}$ & p. $\left(\operatorname{Arg} 256^{*}\right)$ & $\operatorname{arCRD}$ & 1 & 4 & {$[30]$} \\
\hline AIPL1 & NM_201253.2 & c. $116 \mathrm{C}>\mathrm{A}$ & p.(Thr39Asp) & $\operatorname{arLCA}$ & 1 & 6 & {$[31]$} \\
\hline AIPLI & NM_014336.3 & c. $834 \mathrm{G}>\mathrm{A}$ & p. $\left(\operatorname{Trp} 278^{*}\right)$ & EORP & 11 & 25 & {$[29,31-34]$} \\
\hline BEST1 & NM_001139443.1 & c. $418 \mathrm{C}>\mathrm{G}$ & p.(Leu140Val) & $\operatorname{arRP}$ & 1 & 4 & {$[35]$} \\
\hline CERKL & NM_001030311.2 & c. $316 \mathrm{C}>\mathrm{A}$ & p.(Arg106Ser) & $\operatorname{arRP}$ & 1 & 3 & {$[36]$} \\
\hline CERKL & NM_001030311.2 & c. $847 \mathrm{C}>\mathrm{T}$ & p. $\left(\operatorname{Arg} 283^{*}\right)$ & $\operatorname{arRP}$ & 1 & 6 & {$[29,37,38]$} \\
\hline$C L R N I^{\dagger}$ & NM_001195794.1 & c. $92 \mathrm{C}>\mathrm{T}$ & p.(Pro31Leu) & $\operatorname{arRP}$ & 1 & 6 & [39] \\
\hline$C L R N I^{\dagger}$ & NM_001195794.1 & c. $461 \mathrm{~T}>\mathrm{G}$ & p.(Leu154Trp) & $\operatorname{arRP}$ & 1 & 6 & [39] \\
\hline$C N G A I$ & NM_00142564.1 & c.626_627del & p.(Ile209Serfs*26) & $\operatorname{arRP}$ & 1 & 7 & [40] \\
\hline$C N G A I$ & NM_00142564.1 & c. $1298 \mathrm{G}>\mathrm{A}$ & P.(Gly433Asp) & $\operatorname{arRP}$ & 1 & 3 & {$[41]$} \\
\hline$C N G A 3$ & NM_001298.2 & c. $822 \mathrm{G}>\mathrm{T}$ & p.(Arg274Ser) & $\operatorname{arCRD}(\mathrm{ACHM})$ & 1 & 4 & {$[42]$} \\
\hline$C N G A 3$ & NM_001298.2 & c. $827 \mathrm{~A}>\mathrm{G}$ & p.(Asn276Ser) & $\operatorname{arCRD}(\mathrm{ACHM})$ & 1 & 6 & {$[43]$} \\
\hline$C N G B I$ & NM_001297.4 & c. $412-1 \mathrm{G}>\mathrm{A}$ & p.(?) & $\operatorname{arRP}$ & 1 & 10 & [44] \\
\hline$C N G B I$ & NM_001297.4 & c. $2284 \mathrm{C}>\mathrm{T}$ & p.(Arg762Cys) & $\operatorname{arRP}$ & 1 & 5 & {$[44]$} \\
\hline$C N G B I$ & NM_001297.4 & c. $2493-2 \mathrm{~A}>\mathrm{G}$ & p.(?) & $\operatorname{arRP}$ & 1 & 10 & [41] \\
\hline$C N G B 3$ & NM_019098.4 & c. $1825 \mathrm{del}$ & p.(Val609Trpfs*9) & $\operatorname{arCRD}(\mathrm{ACHM})$ & 1 & 2 & {$[42]$} \\
\hline$C R B 1$ & NM_201253.2 & c. $107 \mathrm{C}>\mathrm{G}$ & p. $\left(\operatorname{Ser} 36^{*}\right)$ & $\operatorname{arLCA}$ & 1 & 10 & {$[33]$} \\
\hline$C R B I$ & NM_201253.2 & c. $2234 \mathrm{C}>\mathrm{T}$ & p.(Thr745Met) & $\operatorname{arRP}$ & 1 & 2 & {$[41,45]$} \\
\hline$C R B I$ & NM_201253.2 & c. $2536 \mathrm{G}>\mathrm{A}$ & p.(Gly846Arg) & $\operatorname{arRP}$ & 1 & 6 & {$[31]$} \\
\hline$C R B 1$ & NM_201253.2 & c. $3101 \mathrm{~T}>\mathrm{C}$ & p.(Leu989Thr) & $\operatorname{arLCA}$ & 1 & 8 & {$[31]$} \\
\hline$C R B 1$ & NM_201253.2 & c. $3296 \mathrm{C}>\mathrm{A}$ & p.(Thr1099Lys) & $\operatorname{arRP}$ & 1 & 9 & {$[44]$} \\
\hline$C R B I$ & NM_201253.2 & c. $3343 \_3352 \mathrm{del}$ & p.(Gly1115Ilefs*23) & $\operatorname{arRP}$ & 1 & 9 & {$[46]$} \\
\hline$C R B 1$ & NM_201253.2 & c. $3347 \mathrm{~T}>\mathrm{C}$ & p.(Leu1071Pro) & $\operatorname{arRP}$ & 1 & 7 & {$[31]$} \\
\hline$C R B 1$ & NM_201253.2 & c. $3962 \mathrm{G}>\mathrm{C}$ & p.(Cys1321Ser) & $\operatorname{arRP}$ & 1 & 5 & [46] \\
\hline$E Y S$ & NM_001142800.1 & c. $8299 \mathrm{G}>\mathrm{T}$ & p.(Asp2767Tyr) & $\operatorname{arRP}$ & 1 & 7 & {$[47]$} \\
\hline GNATI & NM_144499.2 & c. $386 \mathrm{~A}>\mathrm{G}$ & p.(Asp129Gly) & $\operatorname{arCSNB}$ & 1 & 1 & {$[48]$} \\
\hline$G R K 1$ & NM_002929 & c. $614 \mathrm{C}>\mathrm{A}$ & p. $\left(\right.$ Ser $\left.205^{*}\right)$ & arCSNB (Oguchi) & 1 & 9 & [49] \\
\hline GRK1 & NM_002929 & c. $827+623 \_883 \mathrm{del}$ & p.(?) & arCSNB (Oguchi) & 1 & 3 & {$[50]$} \\
\hline$I M P G 2$ * & NM_016247.3 & c. $1680 \mathrm{~T}>\mathrm{A}$ & p. $($ Tyr560*) & $\operatorname{arRP}$ & 1 & 2 & {$[51]$} \\
\hline$L C A 5^{*}$ & NM_181714.3 & c. $643 \mathrm{del}$ & p.(Leu215Tyrfs*11) & $\operatorname{arLCA}$ & 1 & 4 & {$[52]$} \\
\hline$L C A 5^{*}$ & NM_181714.3 & c.1151del & p.(Pro384Glnfs*17) & $\operatorname{arLCA}$ & 3 & 13 & {$[33,53]$} \\
\hline MERTK & NM_00634.2 & c. $718 \mathrm{G}>\mathrm{T}$ & p.(Glu240*) & $\operatorname{arRP}$ & 1 & 4 & {$[54]$} \\
\hline$N M N A T 1 \ddagger$ & NM_022787.3 & c. $25 \mathrm{G}>\mathrm{A}$ & p.(Val9Met) & $\operatorname{arLCA}$ & 1 & 5 & {$[55]$} \\
\hline$N M N A T 1^{\ddagger}$ & NM_022787.3 & c. $838 \mathrm{~T}>\mathrm{C}$ & p. $* 280 \mathrm{Glnext} * 16$ & $\operatorname{arLCA}$ & 1 & 8 & {$[56]$} \\
\hline PDE6A & NM_000440.2 & c. $889 \mathrm{C}>\mathrm{T}$ & p.(Gly297Ser) & $\operatorname{arRP}$ & 1 & 4 & {$[57]$} \\
\hline PDE6A & NM_000440.2 & c. $1264-2 A>G$ & p.(?) & $\operatorname{arRP}$ & 1 & 5 & {$[57]$} \\
\hline PDE6A & NM_000440.2 & c. $1630 \mathrm{C}>\mathrm{T}$ & p.(Arg544Trp) & arRP & 1 & 3 & [29] \\
\hline PDE6A & NM_000440.2 & c.2218_2219insT & p.(Ala740Valfs*2) & $\operatorname{arRP}$ & 1 & 3 & {$[57]$} \\
\hline PDE6B & NM_000283.3 & c. $1160 \mathrm{C}>\mathrm{T}$ & p.(Pro387Leu) & $\operatorname{arRP}$ & 1 & 6 & {$[58]$} \\
\hline$P D E 6 B$ & NM_000283.3 & c. $1655 \mathrm{G}>\mathrm{A}$ & p. (Arg552Gln) & $\operatorname{arRP}$ & 1 & 9 & {$[58]$} \\
\hline PDE6B & NM_000283.3 & c. $1722+1 \mathrm{G}>\mathrm{A}$ & p.(?) & $\operatorname{arRP}$ & 1 & 4 & [44] \\
\hline
\end{tabular}


Table 1. Cont.

\begin{tabular}{|c|c|c|c|c|c|c|c|}
\hline Gene & RefSeq Id & Nucleotide variant & Protein variant & Phenotype & \# Families & \# Patients & References \\
\hline PROMI & NM_006017.2 & c. $1726 \mathrm{C}>\mathrm{T}$ & p. $\left(\mathrm{G} \ln 576^{*}\right)$ & $\operatorname{arRP}$ & 1 & 6 & [59] \\
\hline RDH12 & NM_152443.2 & c. $506 \mathrm{G}>\mathrm{A}$ & p. $(\operatorname{Arg} 169 \mathrm{Gln})$ & $\operatorname{arLCA/EORD~}$ & 2 & 2 & [60] \\
\hline RDHI2 & NM_152443.2 & c. $619 \mathrm{~A}>\mathrm{G}$ & p.(Asn207Asp) & $\operatorname{arLCA} / \mathrm{EORD}$ & 1 & 1 & [60] \\
\hline RDH5 & NM_001199771.1 & c. $758 \mathrm{~T}>\mathrm{G}$ & p.(Met253Arg) & $\operatorname{arCSNB}(\mathrm{FA})$ & 1 & 6 & [61] \\
\hline RDH5 & NM_001199771.1 & c.913_917del & p.(Val305Hisfs*29) & $\operatorname{arCSNB}(\mathrm{FA})$ & 1 & 2 & [61] \\
\hline RHO & NM_000539.3 & c. $448 \mathrm{G}>\mathrm{A}$ & p.(Glu150Lys) & $\operatorname{arRP}$ & 2 & 6 & [62] \\
\hline RHO & NM_000539.3 & c. $1045 \mathrm{~T}>\mathrm{G}$ & p. $(* 349$ Gluext*52) & $\operatorname{adRP}$ & 1 & 8 & [26] \\
\hline$R L B P I$ & NM_000326.4 & c. $346 \mathrm{G}>\mathrm{C}$ & p.(Gly116Arg) & FA & 1 & 4 & [63] \\
\hline$R L B P I$ & NM_000326.4 & c. $466 \mathrm{C}>\mathrm{T}$ & p. $\left(\operatorname{Arg} 156^{*}\right)$ & FA & 1 & 6 & [63] \\
\hline$R P I$ & NM_006269.1 & c.1458_1461dup & p.(Glu488*) & $\operatorname{arRP}$ & 2 & 9 & {$[64,65]$} \\
\hline$R P I$ & NM_006269.1 & c. $4555 \mathrm{del}$ & p.(Arg1519Glufs*2) & $\operatorname{arRP}$ & 1 & 5 & [65] \\
\hline$R P I$ & NM_006269.1 & c. $5252 \mathrm{del}$ & p.(Asn1751Ilefs*4) & $\operatorname{arRP}$ & 1 & 4 & [65] \\
\hline RPE65 & NM_000329.2 & c. $131 \mathrm{G}>\mathrm{A}$ & p.(Arg44Gln) & EORP & 1 & 3 & {$[41,66,67]$} \\
\hline RPE65 & NM_000329.2 & c.361del & p.(Ser121Leufs*6) & EORP & 1 & 4 & {$[41,67]$} \\
\hline RPE65 & NM_000329.2 & c. $751 \mathrm{G}>\mathrm{T}$ & p.(Val251Phe) & $\operatorname{arLCA}$ & 1 & 6 & [33] \\
\hline$R P G R$ & NM_001034853.1 & c. $2426 \_2427 \mathrm{del}$ & p.(Glu809Glyfs*25) & $\mathrm{xlRP}$ & 1 & 8 & {$[41,68]$} \\
\hline RPGRIPI & NM_020366.3 & c. $587+1 \mathrm{G}>\mathrm{C}$ & p.(?) & $\operatorname{arLCA}$ & 1 & 1 & [33] \\
\hline RPGRIPI & NM_020366.3 & c. $1180 \mathrm{C}>\mathrm{T}$ & p. $(\mathrm{G} \ln 394 *)$ & $\operatorname{arLCA}$ & 1 & 1 & [33] \\
\hline$R P G R I P 1$ & NM_020366.3 & c. $2480 \mathrm{G}>\mathrm{T}$ & p.(Arg827Leu) & $\operatorname{arCRD}, \operatorname{arLCA}$ & 2 & 9 & {$[33,69]$} \\
\hline RPGRIP1 & NM_020366.3 & c. $3620 \mathrm{~T}>\mathrm{G}$ & p. $\left(\right.$ Leu $\left.1207^{*}\right)$ & $\operatorname{arLCA}$ & 1 & 1 & [33] \\
\hline$S A G$ & NM_000541.4 & c. $916 \mathrm{G}>\mathrm{T}$ & p.(Glu306*) & $\operatorname{arCSNB}$ & 1 & 1 & [70] \\
\hline$S E M A 4 A^{\text {* }}$ & NM_022367.3 & c. $1033 \mathrm{G}>\mathrm{C}$ & p.(Asp345His) & & 4 & 4 & \\
\hline$S E M A 4 A^{\ddagger}$ & NM_022367.3 & c. $1049 \mathrm{~T}>\mathrm{G}$ & p.(Phe350Cys) & arCRD, arRP & 4 & 4 & [27] \\
\hline$S L C 24 A 1^{\ddagger}$ & NM_004727.2 & c.1613_1614del & p.(Phe538Cysfs*23) & $\operatorname{arCSNB}$ & 1 & 5 & [71] \\
\hline SPATA7 & NM_018418.4 & c. $253 \mathrm{C}>\mathrm{T}$ & p. $\left(\operatorname{Arg} 85^{*}\right)$ & $\operatorname{arLCA} / \operatorname{arRD}$ & 2 & 3 & [72] \\
\hline SPATA7 & NM_018418.4 & c.960dup & p.(Pro321Thrfs*6) & $\operatorname{arLCA} / \mathrm{arRD}$ & 1 & 6 & {$[72,73]$} \\
\hline $\operatorname{TTC}^{\dagger}$ & NM_144596.2 & c. $115-2 A>G$ & p.(?) & $\operatorname{arRP}$ & 1 & 4 & [74] \\
\hline TULPI & NM_003322.3 & c. $1138 \mathrm{~A}>\mathrm{G}$ & p.(Thr380Ala) & $\operatorname{arRP}$ & 3 & 34 & {$[33,75,76]$} \\
\hline TULPI & NM_003322.3 & c. $1445 \mathrm{G}>\mathrm{A}$ & p. $(\operatorname{Arg} 482 \mathrm{Gln})$ & $\operatorname{arRP}$ & 1 & 8 & [75] \\
\hline TULPI & NM_003322.3 & c. $1466 \mathrm{~A}>\mathrm{G}$ & p.(Lys489Arg) & $\operatorname{arRP}$ & 4 & 19 & {$[41,76,77]$} \\
\hline ZNF513 & NM 144631.5 & c. $1015 \mathrm{~T}>\mathrm{C}$ & p.(Cys339Arg) & arRP & 1 & 4 & {$[78,79]$} \\
\hline
\end{tabular}

ACHM, achromatopsia; ad, autosomal dominant; ar, autosomal recessive; CSNB, congenital stationary night blindness; CRD, cone rod dystrophy; EORD, early onset retinal dystrophy; EORP, early onset RP; FA, fundus albipunctatus; LCA, Leber congenital amaurosis; RD, retinal dystrophy; RefSeq Id, reference sequence identifier; $\mathrm{RP}$, retinitis pigmentosa; xlRP, X-linked RP; ${ }^{\ddagger}$ novel gene identification; ${ }^{\dagger}$ novel phenotype association.

Table 2. Common variants reported as mutations in Pakistani patients with non-syndromic retinal dystrophies and their in silico pathogenicity prediction.

\begin{tabular}{|c|c|c|c|c|c|c|c|c|c|c|c|c|}
\hline Gene & RefSeq Id & $\begin{array}{c}\text { Nucleotide } \\
\text { variant }\end{array}$ & $\begin{array}{l}\text { Protein } \\
\text { variant }\end{array}$ & Phenotype & \# Families & \# Patients & Ref. & phyloP & $\begin{array}{c}\text { Grantham } \\
\text { distance }\end{array}$ & PolyPhen & SIFT & EVS \\
\hline$R P 1$ & NM_006269.1 & c. $1118 \mathrm{C}>\mathrm{T}$ & p.(Thr373Ile) & $\operatorname{arRP}$ & 2 & 11 & {$[64]$} & 0.61 & 89 & $\begin{array}{c}\text { Benign } \\
(0.01)\end{array}$ & $\begin{array}{c}\text { Tolerated } \\
\quad(0.50)\end{array}$ & $\begin{array}{c}\mathrm{T}=152 \\
\mathrm{C}=12,854 \\
(\mathrm{rs} 77775126)\end{array}$ \\
\hline RPGRIPI & NM_020366.3 & c. $1639 \mathrm{G}>\mathrm{T}$ & p.(Ala547Ser) & $\operatorname{arCRD}$ & 3 & 12 & [69] & 0.29 & 99 & $\begin{array}{c}\text { Probably } \\
\text { damaging } \\
(1.00)\end{array}$ & $\begin{array}{c}\text { Tolerated } \\
(0.49)\end{array}$ & $\begin{array}{c}\mathrm{T}=2,792 \\
\mathrm{G}=9,214 \\
(\mathrm{rs} 10151259)\end{array}$ \\
\hline$S E M A 4 A$ & NM_022367.3 & c. $2138 \mathrm{G}>\mathrm{A}$ & p. $(\operatorname{Arg} 713 \mathrm{Gln})$ & $\operatorname{adRP}$ & 1 & 4 & {$[27]$} & 1.25 & 43 & $\begin{array}{c}\text { Benign } \\
(0.23)\end{array}$ & $\begin{array}{c}\text { Tolerated } \\
(0.43)\end{array}$ & $\begin{array}{c}A=451 \\
G=12,555 \\
(r s 41265017)\end{array}$ \\
\hline
\end{tabular}

Ad, autosomal dominant; ar, autosomal recessive; CRD, cone-rod dystrophy; EVS, exome variant server; PolyPhen, polymorphism phenotyping; RefSeq Id, reference sequence identifier; RP, retinitis pigmentosa; SIFT, sorting tolerant from intolerant. 
Out of the 47 non-synonymous variants identified in Pakistani non-syndromic RD families (Table 1) three variants (SEMA4A, c.2138G $>\mathrm{A} ; R P 1$, c.1118C $>\mathrm{T} ; R P G R I P 1$, c.1639G $>\mathrm{T}$ ), are reported as single nucleotide polymorphisms (SNP) with high frequencies in the EVS (Table 2) $[27,64,69]$. In addition, SIFT also predicts these changes to be tolerated while except for the RPGRIP1 variant, the other two are considered to be benign by PolyPhen-2 (Table 2). Therefore, these variants could be segregating with the disease in the family by chance and the causative mutation may reside in another gene.

Figure 1. Distribution of non-syndromic Pakistani RD families according to their phenotypes. Ad, autosomal dominant; ar, autosomal recessive; CRD, cone-rod dystrophy; CSNB, congenital stationary night blindness; LCA, Leber congenital amaurosis; RP, retinitis pigmentosa; $\mathrm{xl}$, X-linked.

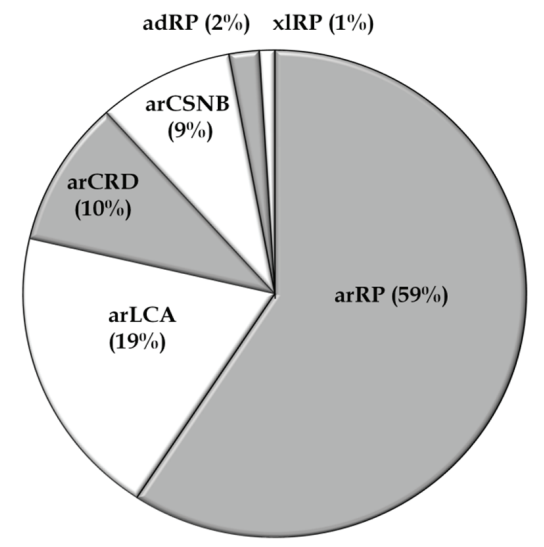

Figure 2. Occurrence of gene defects in non-syndromic RD families in Pakistan. Numbers of families with mutations in respective genes are indicated between parentheses.

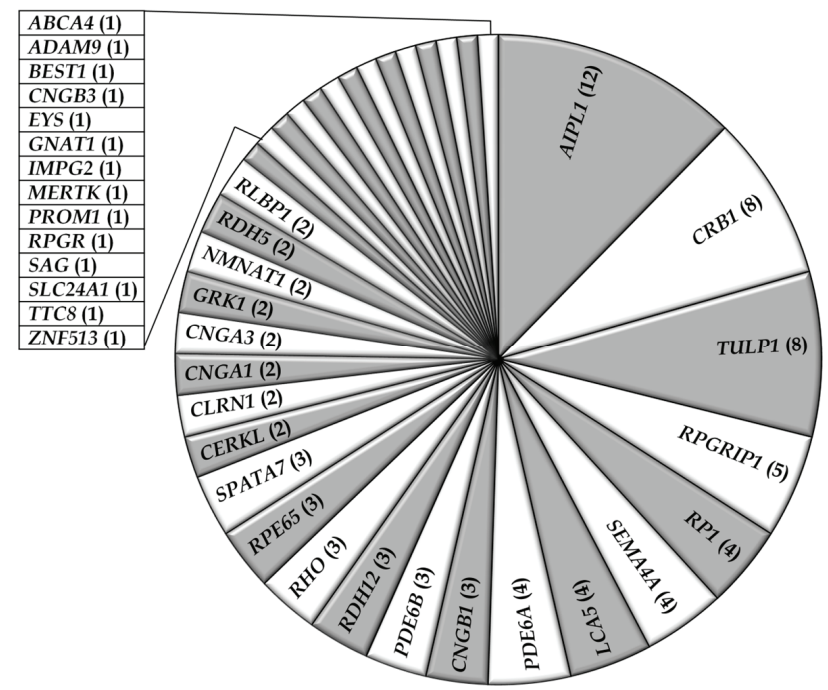




\subsection{Overview of Molecular Genetic Studies in Syndromic RDs in Pakistan}

In addition to the non-syndromic families, data of 52 syndromic RD families with a total of 139 affected individuals were collected from 22 studies. Usher syndrome represented about $36 \%$ of the families in this group, whereas BBS (33\%), MKS (13\%), JBTS (10\%), and SLSN (8\%), accounted for the other families (Table 3; Figure 3). The most commonly mutated gene associated with syndromic RD in the Pakistani population was cadherin 23 (CDH23; MIM\# 605516), which has been reported to be mutated in persons with Usher type 1, followed by TMEM67 (MIM\# 609884), the gene mutated in persons with autosomal recessive MKS (Table 3; Figure 4). As expected for the syndromic mutations, all the reported disease associated alleles are rare variants and in silico analysis predicted these variants to have a deleterious effect on protein function (Table S2).

\section{Discussion}

The Pakistani population is known for its high rate of consanguinity $(>60 \%)$, but it is still remarkable that $97 \%$ of the families with inherited RDs had an autosomal recessive mode of inheritance. It is, therefore, not surprising that Pakistani families have been instrumental in pinpointing a number of the underlying gene defects through homozygosity mapping [80,81]. Genetic studies of Pakistani families with RD have previously facilitated the identification of eleven novel RD genes, i.e., AIPL1 [34], BEST1 [35], CC2D2A (MIM\# 612013) [82], CDH23 (MIM\# 605516) [83], IMPG2 (MIM\# 607056) [51], LCA5 (MIM\# 611408) [53], NMNAT1 (MIM:608700) [55,56], ZNF513 (MIM\# 613598) [78], PCDH15 (MIM\# 605514) [84], SEMA4A [27], and SLC24A1 (MIM\# 603617) [71]. In addition, mutations in CLRN1 (MIM\# 606397) and TTC8 (MIM\# 608132), which had been previously implicated in the syndromic retinal phenotypes USH3 (MIM\# 276902), and BBS (MIM\# 209900), respectively, were found to cause non-syndromic arRP [39,74]. Mutations in $R P 1$, which had previously been shown to be involved in adRP, were found to segregate in a recessive manner in 3 Pakistani families [64]. In addition to the novel genes identified in the affected Pakistani families, five novel RD loci including three non-syndromic, i.e., CORD8 (MIM\# 605549), [85], RP29 (MIM\# 612165), [86], and RP32 [87], and two syndromic, i.e., USH1H (MIM\# 612632), [88], and USH1K [89], have also been identified in Pakistani families.

Table 3. Mutations identified in Pakistani patients with syndromic retinal dystrophies.

\begin{tabular}{|c|c|c|c|c|c|c|c|}
\hline Gene & RefSeq Id & Nucleotide variant & Protein variant & Phenotype & \# Families & \# Patients & References \\
\hline AHII & NM_017651.4 & c.2370dup & p. $\left(\right.$ Lys $\left.791^{*}\right)$ & $\operatorname{arJBTS}$ & 1 & 2 & [90] \\
\hline ARL6 & NM_032146.3 & c. $281 \mathrm{~T}>\mathrm{C}$ & p.(Ile94Thr) & arBBS & 1 & 5 & [91] \\
\hline ARL6 & NM_032146.3 & c. $123+1119 \mathrm{del}$ & p.(?) & $\operatorname{arBBS}$ & 1 & 1 & [92] \\
\hline$A R L 13 B$ & NM_182896.2 & c. $236 \mathrm{G}>\mathrm{A}$ & p. $(\operatorname{Arg} 79 \mathrm{Gln})$ & $\operatorname{arJBTS}$ & 1 & 3 & [93] \\
\hline BBSI & NM_02464.9.4 & c. $47+1 \mathrm{G}>\mathrm{T}$ & p.(?) & arBBS & 1 & 2 & [94] \\
\hline$B B S I$ & NM_02464.9.4 & c. $442 \mathrm{G}>\mathrm{A}$ & p.(Asp148Asn) & arBBS & 1 & 2 & [94] \\
\hline$B B S 2$ & NM_031885.3 & c. $1237 \mathrm{C}>\mathrm{T}$ & p. $\left(\operatorname{Arg} 413^{*}\right)$ & arBBS & 1 & 1 & [95] \\
\hline BBS5 & NM_152384.2 & c. $2 \mathrm{~T}>\mathrm{A}$ & p.(Met1Lys) & arBBS & 2 & 2 & [95] \\
\hline BBS10 & NM_024685.3 & c.271dup & p.(Cys91Leufs*5) & arBBS & 2 & 4 & [96] \\
\hline BBS10 & NM_024685.3 & c. $1075 \mathrm{C}>\mathrm{T}$ & p. $\left(\mathrm{G} \ln 359^{*}\right)$ & arBBS & 1 & 7 & [91] \\
\hline
\end{tabular}


Table 3. Cont.

\begin{tabular}{|c|c|c|c|c|c|c|c|}
\hline Gene & RefSeq Id & Nucleotide variant & Protein variant & Phenotype & \# Families & \# Patients & References \\
\hline BBSIO & NM_024685.3 & c.1091del & p.(Asn364Thrfs*5) & arBBS & 1 & 1 & [96] \\
\hline BBSIO & NM_024685.3 & c.1958_1967del & p.(Ser653Ilefs*4) & $\operatorname{arBBS}$ & 1 & 2 & [97] \\
\hline BBS10 & NM_024685.3 & c. $2121 \mathrm{dup}$ & p.(Lys708*) & $\operatorname{arBBS}$ & 1 & 1 & [96] \\
\hline BBS12 & NM_152618.2 & c. $1589 \mathrm{~T}>\mathrm{C}$ & p.(Leu530Pro) & $\operatorname{arBBS}$ & 2 & 2 & [95] \\
\hline BBS12 & NM_152618.2 & c. $2102 \mathrm{C}>\mathrm{A}$ & p. $(\operatorname{Ser} 701 *)$ & $\operatorname{arBBS}$ & 1 & 3 & [98] \\
\hline$C C 2 D 2 A^{\ddagger}$ & NM_001080522.2 & c. $2003+1 \mathrm{G}>\mathrm{C}$ & p.(?) & arJBTS & 1 & 5 & {$[82]$} \\
\hline $\mathrm{CDH} 23^{\ddagger}$ & NM_022124.5 & c. $1114 \mathrm{C}>\mathrm{T}$ & p. $(\mathrm{G} \ln 372 *)$ & arUSH1 & 1 & 3 & [83] \\
\hline $\mathrm{CDH} 23$ & NM_022124.5 & c. $2587+1 \mathrm{G}>\mathrm{A}$ & p.(?) & arUSH1 & 1 & 4 & [99] \\
\hline $\mathrm{CDH} 23$ & NI & NI & p. $\left(\operatorname{Arg} 1305^{*}\right)$ & arUSH1 & 1 & 4 & [99] \\
\hline $\mathrm{CDH} 23^{ \pm}$ & NM_022124.5 & c.3106_3106+11delinsTGGT & p.(Gly1036delinsTrpCys) & arUSH1 & 1 & 5 & [83] \\
\hline $\mathrm{CDH} 23^{*}$ & NM_022124.5 & c. $6050-9 \mathrm{G}>A$ & p.(?) & arUSH1 & 4 & 13 & [83] \\
\hline $\mathrm{CDH} 23^{*}$ & NM_022124.5 & c. $6050-1 \mathrm{G}>\mathrm{C}$ & p.(?) & arUSH1 & 1 & 6 & [83] \\
\hline $\mathrm{CDH} 23^{*}$ & NM_022124.5 & c.6054_6074del & p.(Val2019_Val2025del) & arUSH1 & 1 & 3 & [83] \\
\hline $\mathrm{CDH} 23^{4}$ & NM_022124.5 & c. $6845 \mathrm{del}$ & p.(Asn2282Thrfs*91) & arUSH1 & 1 & 3 & [83] \\
\hline $\mathrm{CDH} 23^{*}$ & NM_022124.5 & c. $7198 \mathrm{C}>\mathrm{T}$ & p.(Pro2400Ser) & arUSH1 & 1 & 4 & [83] \\
\hline $\mathrm{CDH} 23^{\ddagger}$ & NM_022124.5 & c. $8150 \mathrm{~A}>\mathrm{G}$ & p.(Asp2717Gly) & arUSH1 & 1 & 3 & {$[83]$} \\
\hline $\mathrm{CDH} 23^{*}$ & NM_022124.5 & c.8208_8209del & p.(Val2737Alafs*2) & arUSH1 & 2 & 11 & [83] \\
\hline CEP290 & NM_025114.3 & c. $.5668 \mathrm{G}>\mathrm{T}$ & p. $\left(\right.$ Gly $\left.1890^{*}\right)$ & arJBTS & 1 & 1 & {$[100,101]$} \\
\hline$I Q C B I$ & NM_001023570.2 & c. $488-1 \mathrm{G}>\mathrm{A}$ & p.(?) & $\operatorname{arSLSN}$ & 1 & 1 & {$[41,102]$} \\
\hline$I Q C B I$ & NM_001023570.2 & c. $1465 \mathrm{C}>\mathrm{T}$ & p. $\left(\operatorname{Arg} 489^{*}\right)$ & $\operatorname{arSLSN}$ & 1 & 1 & [102] \\
\hline$I Q C B I$ & NM_001023570.2 & c. $1796 \mathrm{~T}>\mathrm{G}$ & p. $(* 599$ Serext*2) & $\operatorname{arSLSN}$ & 1 & 1 & [102] \\
\hline NPHP4 & NM_015102.3 & c.3272dup & p.(Ser1092Valfs*11) & $\operatorname{arSLSN}$ & 1 & 1 & [102] \\
\hline $\mathrm{PCDH}^{\ddagger} 5^{\ddagger}$ & NM_001142763.1 & c. $7 \mathrm{C}>\mathrm{T}$ & p. $(\operatorname{Arg} 3 *)$ & arUSH1 & 1 & 5 & [84] \\
\hline $\mathrm{PCDHI}^{\ddagger}$ & NM_001142763.1 & c. $1927 \mathrm{C}>\mathrm{T}$ & p. $\left(\operatorname{Arg} 643^{*}\right)$ & arUSH1 & 1 & 3 & [103] \\
\hline PCDH15 & NM_001142763.1 & c. $3389-2 A>G$ & $\mathrm{p} .(?)$ & arUSH1 & 1 & 3 & [84] \\
\hline TCTN2 & NM_024809.3 & c. $1873 \mathrm{C}>\mathrm{T}$ & p. $\left(\mathrm{G} \ln 625^{*}\right)$ & arJBTS & 1 & 4 & [104] \\
\hline TMEM67 & NM_153704.5 & c.647del & p.(Val217Leufs*5) & arMKS & 1 & 2 & [105] \\
\hline TMEM67 & NM_153704.5 & c. $715-2 A>G$ & p.(?) & arMKS & 1 & 1 & [105] \\
\hline TMEM67 & NM_153704.5 & c. $1127 \mathrm{~A}>\mathrm{C}$ & p.(Gln376Pro) & arMKS & 2 & 2 & [105] \\
\hline TMEM67 & NM_153704.5 & c. $1575+1 \mathrm{G}>\mathrm{A}$ & p.(?) & arMKS & 3 & 5 & [105] \\
\hline TTC 8 & NM_144596.2 & c. $1049+2 \_1049+4 \mathrm{del}$ & p.(?) & $\operatorname{arBBS}$ & 1 & 3 & [106] \\
\hline USHIG & NM_173477.2 & c. $163 \_164+13 \mathrm{del}$ & p. $\left(\mathrm{Gly} 56^{*}\right)$ & arUSH1 & 1 & 4 & [107] \\
\hline
\end{tabular}

Ar, autosomal recessive; BBS, Bardet-Biedl syndrome; JBTS, Joubert syndrome; MKS, Meckel syndrome; NI, not indicated; RefSeq Id, reference sequence identifier; SLSN, Senior-Loken syndrome; USH1, Usher syndrome type 1; ${ }^{\ddagger}$ novel gene identification; ${ }^{\dagger}$ novel phenotype association.

In the 103 non-syndromic Pakistani RD families described so far, mutations were most frequently found in AIPL1, CRB1, TULP1, RPGRIP1, RP1, SEMA4A, LCA5, and PDE6A (Table 1; Figure 2). A direct comparison with other RD populations is difficult as comprehensive studies of this kind are rare. In a recent study of Abu-Safieh et al. (2012) comprising 150 Saudi Arabian RD families, similar results were observed as RP1, TULP1, RPGRIP1, and CRBI were found to be the most frequently mutated genes [108]. 
Figure 3. Prevalences of syndromic RD phenotypes. BBS, Bardet-Biedl syndrome; JBTS, Joubert syndrome; MKS, Meckel syndrome; SLS, Senior-Loken syndrome; USH, Usher syndrome.

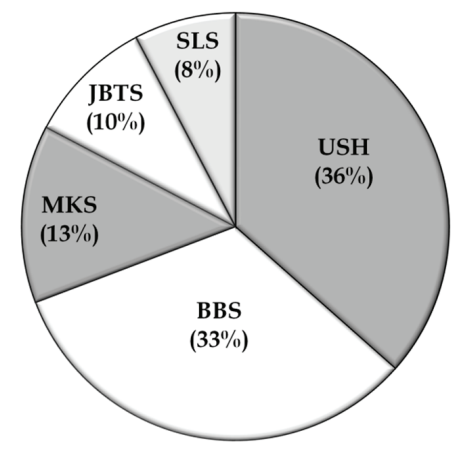

Figure 4. Occurrence of gene defects in syndromic RD families in Pakistan. Numbers of families with mutations in respective genes are indicated between parentheses.

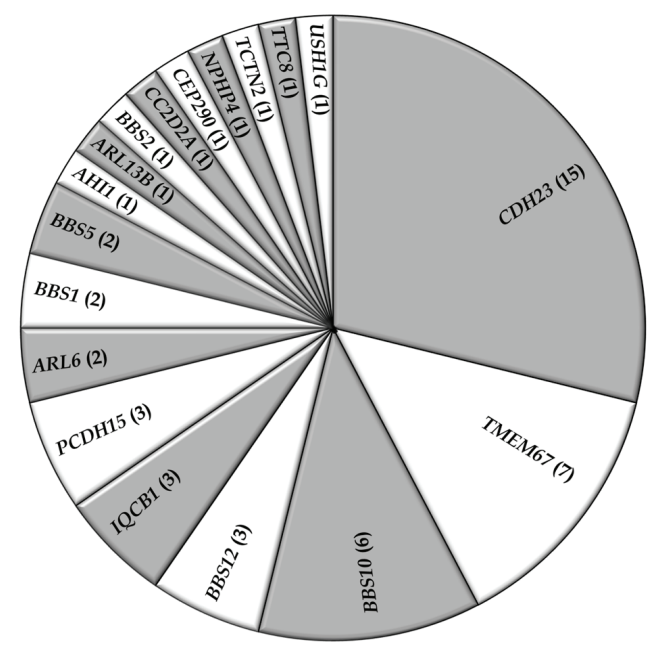

A worldwide general literature study revealed arRP-associated mutations distributed in $U S H 2 A$ (12\%; MIM\# 276901), ABCA4 (8\%), PDE6B (7\%; MIM\# 180072), CNGB1 (6\%), and PDE6A (5\%; MIM\# 180071) [109]. In a more recent study of 230 Dutch persons with isolated or arRP [110], the most frequently mutated genes were EYS (11\%; MIM\# 602772), and CRB1 (11\%) followed by USH $2 A(10 \%), A B C A 4$ (9\%), and PDE6B (7\%). As opposed to these studies the absence of USH $2 A$ variants in individuals of Pakistani origin is probably due to the fact that the most frequent arRP-associated variant, c.2299del;p.(E767fs), is almost invariably found in compound heterozygous states with second mutations that are considered to be mild [111], precluding their detection in a homozygosity mapping approach. Other differences can only be attributed to divergent genetic backgrounds of these populations [112,113]. 
Although 113/118 variants listed in Tables 1 and 3 have only been identified in Pakistani patients, seven variants (SEMA4A, p.(Asp345His) and p.(Phe350Cys); TULP1, p.(Thr380Ala); LCA5, p.(Pro384Glnfs*17); RPGRIP1, p.(Arg827Leu); TMEM67, c.1575+1G $>$ A and p.(Gln37Pro)), are more frequent than others, and therefore they seem to be population-specific. The six most frequent variants, p.(Trp278*) in AIPL1, p.(Lys489Arg) and p.(Thr380Ala) in TULP1, p.(Asp345His) and p.(Phe350Cys) in SEMA4A, p.(Pro384Glnfs*17) in LCA5 (Table 1), explain about 25\% of the non-syndromic Pakistani RD families. The p.Trp278* variant has been identified as the most frequent AIPL1 variant worldwide in many LCA studies [114,115], suggesting that this variant is relatively old. The six frequent variants mentioned above, together with five other variants in RDH12 (MIM\# 608830), p.(Arg169Gln); RHO, p.(Glu150Lys); RP1, p.(Glu488*), RPGRIP1, p.(Arg827Leu), and SPATA7, p.(Arg85*), account for approximately 34\% (35/103) of all non-syndromic RD families from Pakistan. A cost-effective initial genetic screening of Pakistani persons with RD therefore could be to analyze these variants using Sanger sequencing. For example, 10 amplicons covers the most frequent variants mentioned above. Alternatively, a larger subset of variants can be captured by arrayed primer extension (APEX) analysis or other allele-specific genotyping methods [116-119].

Three of the 47 missense mutations (RP1: c.1118C $>\mathrm{T}, R P G R I P 1:$ c.1639G $>\mathrm{T}, S E M A 4 A$ : c. $2138 \mathrm{G}>\mathrm{A}$ ) reported to be associated with $\mathrm{RD}$ in Pakistani families are found at higher frequencies in EVS. In silico analysis also predict them likely to be non-pathogenic, therefore they should be considered as non-causative (Table 2) [27,64,69]. As these variants on their own are not sufficient to explain the phenotype in these six families (two, three and one with RP1, RPGRIP1 and $S E M A 4 A$ mutations, respectively) they must still be considered genetically unresolved.

Of all the non-syndromic and syndromic arRD families $(n=146)$, which are genetically resolved, compound heterozygous mutations were identified in only four non-syndromic RD families $(4 / 146=2.7 \%)$. These compound heterozygous mutations were identified in SEMA4A. This finding on one hand favors the utility of homozygosity based gene identification strategies for Pakistani RD families. While on the other hand it also indicates that in a small but significant proportion of the families $(\sim 2 / 100)$, compound heterozygous mutations might be able to explain the phenotype. These mutations will certainly be overlooked if one only considers homozygosity mapping based approaches to pinpoint causative genetic defects.

\section{Conclusions}

This review provides a comprehensive overview of genetic causes of non-syndromic and syndromic retinal diseases in Pakistan, the results of which can be used to design a cost-effective screening platform for future genetic testing in Pakistan. For genetically unsolved non-syndromic $\mathrm{RD}$ cases, we propose a sequencing-based pre-screening genetic test in which 10 different amplicons capture the most frequent mutations described for Pakistani RD patients. In consanguineous families, homozygosity directed sequence analysis has demonstrated its potential to unravel genetic defect underlying recessive diseases. 


\section{Acknowledgments}

This work was supported by grant no. PAS/I-9/Project awarded (to R.Q. and M.A.), by the Pakistan Academy of Sciences and a core grant from the COMSATS Institute of Information Technology. This work was also financially supported by the Foundation Fighting Blindness, USA, the Stichting Nederlands Oogheelkundig Onderzoek, the Nelly Reef Foundation, the Stichting ter Verbetering van het Lot der Blinden (to F.P.M.C., R.W.J.C., and A.I.d.H.), the Gelderse Blinden Stichting (to F.P.M.C.), the Rotterdamse Stichting Blindenbelangen, the Stichting Blindenhulp, the Stichting A.F. Deutman Researchfonds Oogheelkunde, and the Stichting voor Ooglijders (to F.P.M.C. and M.I.K.). F.P.M.C. and M.I.K. were also supported by the following foundations: the Algemene Nederlandse Vereniging ter Voorkoming van Blindheid, the Landelijke Stichting voor Blinden en Slechtzienden, the Stichting Retina Nederland Fonds, and the Novartis fund, that contributed through UitZicht. The funding organizations had no role in the design or conduct of this research. They provided unrestricted grants.

\section{Author Contributions}

Conception and design: FPMC, MIK, MAz, RQ, RWJC, and AIH; collected the data: MIK, MAz, and MAj; wrote the manuscript: MIK, MAz, MAj, RWJC, AIH, FPMC, and RQ.

\section{Conflicts of Interest}

The authors declare no conflict of interest.

\section{References}

1. Berger, W.; Kloeckener-Gruissem, B.; Neidhardt, J. The molecular basis of human retinal and vitreoretinal diseases. Prog. Retin. Eye Res. 2010, 29, 335-375.

2. Moradi, P.; Moore, A.T. Molecular genetics of infantile-onset retinal dystrophies. Eye 2007, 21, 1344-1351.

3. Den Hollander, A.I.; Black, A.; Bennett, J.; Cremers, F.P.M. Lighting a candle in the dark: Advances in genetics and gene therapy of recessive retinal dystrophies. J. Clin. Invest. 2010, 120, 3042-3053.

4. Reiners, J.; Nagel-Wolfrum, K.; Jurgens, K.; Marker, T.; Wolfrum, U. Molecular basis of human usher syndrome: Deciphering the meshes of the usher protein network provides insights into the pathomechanisms of the usher disease. Exp. Eye Res. 2006, 83, 97-119.

5. Hildebrandt, F.; Zhou, W. Nephronophthisis-associated ciliopathies. J. Am. Soc. Nephrol. 2007, 18, 1855-1871.

6. Hildebrandt, F.; Benzing, T.; Katsanis, N. Ciliopathies. N. Engl. J. Med. 2011, 364, 1533-1543.

7. Robson, A.G.; Michaelides, M.; Saihan, Z.; Bird, A.C.; Webster, A.R.; Moore, A.T.; Fitzke, F.W.; Holder, G.E. Functional characteristics of patients with retinal dystrophy that manifest abnormal parafoveal annuli of high density fundus autofluorescence; a review and update. Doc. Ophthalmol. 2008, 116, 79-89. 
8. Jay, M. On the heredity of retinitis pigmentosa. Br. J. Ophthalmol. 1982, 66, 405-416.

9. Ayuso, C.; Millan, J.M. Retinitis pigmentosa and allied conditions today: A paradigm of translational research. Genome Med. 2010, 2, 34.

10. Adhi, M.I.; Ahmed, J. Frequency and clinical presentation of retinal dystrophies - A hospital based study. Pak. J. Ophthalmol. 2002, 18, 106-110.

11. Bittles, A.H. Endogamy, consanguinity and community disease profiles. Community Genet. 2005, 8, 17-20.

12. Bittles, A. Consanguinity and its relevance to clinical genetics. Clin. Genet. 2001, 60, 89-98.

13. Lander, E.S.; Botstein, D. Homozygosity mapping: A way to map human recessive traits with the DNA of inbred children. Science 1987, 236, 1567-1570.

14. Woods, C.G.; Cox, J.; Springell, K.; Hampshire, D.J.; Mohamed, M.D.; McKibbin, M.; Stern, R.; Raymond, F.L.; Sandford, R.; Malik Sharif, S.; et al. Quantification of homozygosity in consanguineous individuals with autosomal recessive disease. Am. J. Hum. Genet. 2006, 78, 889-896.

15. Retinal Information Network. Available online: https://sph.uth.edu/retnet/ (accessed on 2 August 2013).

16. Estrada-Cuzcano, A.; Roepman, R.; Cremers, F.P.M.; den Hollander, A.I.; Mans, D.A. Non-syndromic retinal ciliopathies: Translating gene discovery into therapy. Hum. Mol. Genet. 2012, 21, R111-R124.

17. Downs, K.; Zacks, D.N.; Caruso, R.; Karoukis, A.J.; Branham, K.; Yashar, B.M.; Haimann, M.H.; Trzupek, K.; Meltzer, M.; Blain, D.; et al. Molecular testing for hereditary retinal disease as part of clinical care. Arch. Ophthalmol. 2007, 125, 252-258.

18. Koenekoop, R.K.; Lopez, I.; den Hollander, A.I.; Allikmets, R.; Cremers, F.P.M. Genetic testing for retinal dystrophies and dysfunctions: Benefits, dilemmas and solutions. Clin. Exp. Ophthalmol. 2007, 35, 473-485.

19. Brooks, B.P.; Macdonald, I.M.; Tumminia, S.J.; Smaoui, N.; Blain, D.; Nezhuvingal, A.A.; Sieving, P.A. National Ophthalmic Disease Genotyping, N. Genomics in the era of molecular ophthalmology: Reflections on the national ophthalmic disease genotyping network (eyegene). Arch. Ophthalmol. 2008, 126, 424-425.

20. National Centre for Biotechnology information. Available online: http://www.ncbi.nlm. nih.gov/pubmed/ (accessed on 21 November 2013).

21. Online Mendelian Inheritance in Man. Available online: http://www.omim.org/ (accessed on 21 November 2013).

22. The Human Gene Mutation Database. Available online: http://www.hgmd.cf.ac.uk/ac/ index.php/ (accessed on 13 May 2013).

23. Adzhubei, I.A.; Schmidt, S.; Peshkin, L.; Ramensky, V.E.; Gerasimova, A.; Bork, P.; Kondrashov, A.S.; Sunyaev, S.R. A method and server for predicting damaging missense mutations. Nat. Methods 2010, 7, 248-249.

24. Kumar, P.; Henikoff, S.; Ng, P.C. Predicting the effects of coding non-synonymous variants on protein function using the sift algorithm. Nat. Protoc. 2009, 4, 1073-1081. 
25. NHLBI GO Exome Sequencing Project (ESP). Available online: http://evs.gs.washington.edu/ EVS/ (accessed on 1 July 2013).

26. Bessant, D.A.; Khaliq, S.; Hameed, A.; Anwar, K.; Payne, A.M.; Mehdi, S.Q.; Bhattacharya, S.S. Severe autosomal dominant retinitis pigmentosa caused by a novel rhodopsin mutation (Ter349Glu). Mutations in brief no. 208. Online. Hum. Mutat. 1999, 13, 83.

27. Abid, A.; Ismail, M.; Mehdi, S.Q.; Khaliq, S. Identification of novel mutations in the SEMA4A gene associated with retinal degenerative diseases. J. Med. Genet. 2006, 43, 378-381.

28. Maugeri, A.; Klevering, B.J.; Rohrschneider, K.; Blankenagel, A.; Brunner, H.G.; Deutman, A.F.; Hoyng, C.B.; Cremers, F.P.M. Mutations in the ABCA4 (ABCR) gene are the major cause of autosomal recessive cone-rod dystrophy. Am. J. Hum. Genet. 2000, 67, 960-966.

29. Khan, M.I.; Ajmal, M.; Micheal, S.; Azam, M.; Hussain, A.; Shahzad, A.; Venselaar, H.; Bokhari, H.; de Wijs, I.; Hoefsloot, L.; et al. Homozygosity mapping identifies genetic defects in four consanguineous families with retinal dystrophy from pakistan. Clin. Genet. 2013, 84, 290-293.

30. Parry, D.A.; Toomes, C.; Bida, L.; Danciger, M.; Towns, K.V.; McKibbin, M.; Jacobson, S.G.; Logan, C.V.; Ali, M.; Bond, J.; et al. Loss of the metalloprotease ADAM9 leads to cone-rod dystrophy in humans and retinal degeneration in mice. Am. J. Hum. Genet. 2009, 84, 683-691.

31. Khaliq, S.; Abid, A.; Hameed, A.; Anwar, K.; Mohyuddin, A.; Azmat, Z.; Shami, S.A.; Ismail, M.; Mehdi, S.Q. Mutation screening of Pakistani families with congenital eye disorders. Exp. Eye Res. 2003, 76, 343-348.

32. Damji, K.F.; Sohocki, M.M.; Khan, R.; Gupta, S.K.; Rahim, M.; Loyer, M.; Hussein, N.; Karim, N.; Ladak, S.S.; Jamal, A.; et al. Leber's congenital amaurosis with anterior keratoconus in pakistani families is caused by the Trp278X mutation in the AIPL1 gene on 17p. Can. J. Ophthalmol. 2001, 36, 252-259.

33. McKibbin, M.; Ali, M.; Mohamed, M.D.; Booth, A.P.; Bishop, F.; Pal, B.; Springell, K.; Raashid, Y.; Jafri, H.; Inglehearn, C.F. Genotype-phenotype correlation for leber congenital amaurosis in Northern Pakistan. Arch. Ophthalmol. 2010, 128, 107-113.

34. Sohocki, M.M.; Bowne, S.J.; Sullivan, L.S.; Blackshaw, S.; Cepko, C.L.; Payne, A.M.; Bhattacharya, S.S.; Khaliq, S.; Mehdi, S.Q.; Birch, D.G.; et al. Mutations in a new photoreceptor-pineal gene on 17p cause leber congenital amaurosis. Nat. Genet. 2000, 24, 79-83.

35. Davidson, A.E.; Millar, I.D.; Urquhart, J.E.; Burgess-Mullan, R.; Shweikh, Y.; Parry, N.; O’Sullivan, J.; Maher, G.J.; McKibbin, M.; Downes, S.M.; et al. Missense mutations in a retinal pigment epithelium protein, bestrophin-1, cause retinitis pigmentosa. Am. J. Hum. Genet. 2009, 85, 581-592.

36. Ali, M.; Ramprasad, V.L.; Soumittra, N.; Mohamed, M.D.; Jafri, H.; Rashid, Y.; Danciger, M.; McKibbin, M.; Kumaramanickavel, G.; Inglehearn, C.F. A missense mutation in the nuclear localization signal sequence of CERKL (p.R106S) causes autosomal recessive retinal degeneration. Mol. Vis. 2008, 14, 1960-1964. 
37. Littink, K.W.; Koenekoop, R.K.; van den Born, L.I.; Collin, R.W.J.; Moruz, L.; Veltman, J.A.; Roosing, S.; Zonneveld, M.N.; Omar, A.; Darvish, M.; et al. Homozygosity mapping in patients with cone-rod dystrophy: Novel mutations and clinical characterizations. Invest. Ophthalmol. Vis. Sci. 2010, 51, 5943-5951.

38. Avila-Fernandez, A.; Riveiro-Alvarez, R.; Vallespin, E.; Wilke, R.; Tapias, I.; Cantalapiedra, D.; Aguirre-Lamban, J.; Gimenez, A.; Trujillo-Tiebas, M.J.; Ayuso, C. CERKL mutations and associated phenotypes in seven spanish families with autosomal recessive retinitis pigmentosa. Invest. Ophthalmol. Vis. Sci. 2008, 49, 2709-2713.

39. Khan, M.I.; Kersten, F.F.; Azam, M.; Collin, R.W.J.; Hussain, A.; Shah, S.T.A.; Keunen, J.E.E.; Kremer, H.; Cremers, F.P.M.; Qamar, R.; et al. CLRN1 mutations cause nonsyndromic retinitis pigmentosa. Ophthalmology 2011, 118, 1444-1448.

40. Zhang, Q.; Zulfiqar, F.; Riazuddin, S.A.; Xiao, X.; Ahmad, Z.; Riazuddin, S.; Hejtmancik, J.F. Autosomal recessive retinitis pigmentosa in a Pakistani family mapped to CNGA1 with identification of a novel mutation. Mol. Vis. 2004, 10, 884-889.

41. Ajmal, M. Personal Communications. Department of biosciences, COMSATS Institute of Information Technology, Islamabad, Pakistan, and Department of Human Genetics, Radboud University Medical Center, Nijmegen, the Netherlands, 2014.

42. Azam, M.; Collin, R.W.J.; Shah, S.T.A.; Shah, A.A.; Khan, M.I.; Hussain, A.; Sadeque, A.; Strom, T.M.; Thiadens, A.A.H.J.; Roosing, S.; et al. Novel CNGA3 and CNGB3 mutations in two Pakistani families with achromatopsia. Mol. Vis. 2010, 16, 774-781.

43. Saqib, M.A.; Awan, B.M.; Sarfraz, M.; Khan, M.N.; Rashid, S.; Ansar, M. Genetic analysis of four Pakistani families with achromatopsia and a novel S4 motif mutation of CNGA3. Jpn. J. Ophthalmol. 2011, 55, 676-680.

44. Azam, M.; Collin, R.W.J.; Malik, A.; Khan, M.I.; Shah, S.T.A.; Shah, A.A.; Hussain, A.; Sadeque, A.; Arimadyo, K.; Ajmal, M.; et al. Identification of novel mutations in pakistani families with autosomal recessive retinitis pigmentosa. Arch. Ophthalmol. 2011, 129, 1377-1378.

45. Den Hollander, A.I.; ten Brink, J.B.; de Kok, Y.J.M.; van Soest, S.; van den Born, L.I.; van Driel, M.A.; van de Pol, T.J.R.; Payne, A.M.; Bhattacharya, S.S.; Kellner, U.; et al. Mutations in a human homologue of Drosophila crumbs cause retinitis pigmentosa (RP12). Nat. Genet. 1999, 23, 217-221.

46. Lotery, A.J.; Malik, A.; Shami, S.A.; Sindhi, M.; Chohan, B.; Maqbool, C.; Moore, P.A.; Denton, M.J.; Stone, E.M. CRB1 mutations may result in retinitis pigmentosa without para-arteriolar RPE preservation. Ophthalmic Genet. 2001, 22, 163-169.

47. Khan, M.I.; Collin, R.W.J.; Arimadyo, K.; Micheal, S.; Azam, M.; Qureshi, N.; Faradz, S.M.H.; den Hollander, A.I.; Qamar, R.; Cremers, F.P.M. Missense mutations at homologous positions in the fourth and fifth laminin A G-like domains of eyes shut homolog cause autosomal recessive retinitis pigmentosa. Mol. Vis. 2010, 16, 2753-2759.

48. Naeem, M.A.; Chavali, V.R.; Ali, S.; Iqbal, M.; Riazuddin, S.; Khan, S.N.; Husnain, T.; Sieving, P.A.; Ayyagari, R.; Hejtmancik, J.F.; et al. GNAT1 associated with autosomal recessive congenital stationary night blindness. Invest. Ophthalmol. Vis. Sci. 2012, 53, 1353-1361. 
49. Azam, M.; Collin, R.W.J.; Khan, M.I.; Shah, S.T.A.; Qureshi, N.; Ajmal, M.; den Hollander, A.I.; Qamar, R.; Cremers, F.P.M. A novel mutation in GRK1 causes oguchi disease in a consanguineous Pakistani family. Mol. Vis. 2009, 15, 1788-1793.

50. Zhang, Q.; Zulfiqar, F.; Riazuddin, S.A.; Xiao, X.; Yasmeen, A.; Rogan, P.K.; Caruso, R.; Sieving, P.A.; Riazuddin, S.; Hejtmancik, J.F. A variant form of Oguchi disease mapped to $13 q 34$ associated with partial deletion of GRK1 gene. Mol. Vis. 2005, 11, 977-985.

51. Bandah-Rozenfeld, D.; Collin, R.W.J.; Banin, E.; van den Born, L.I.; Coene, K.L.M.; Siemiatkowska, A.M.; Zelinger, L.; Khan, M.I.; Lefeber, D.J.; Erdinest, I.; et al. Mutations in IMPG2, encoding interphotoreceptor matrix proteoglycan 2, cause autosomal-recessive retinitis pigmentosa. Am. J. Hum. Genet. 2010, 87, 199-208.

52. Ahmad, A.; Daud, S.; Kakar, N.; Nurnberg, G.; Nurnberg, P.; Babar, M.E.; Thoenes, M.; Kubisch, C.; Ahmad, J.; Bolz, H.J. Identification of a novel LCA5 mutation in a Pakistani family with Leber congenital amaurosis and cataracts. Mol. Vis. 2011, 17, 1940-1945.

53. Den Hollander, A.I.; Koenekoop, R.K.; Mohamed, M.D.; Arts, H.H.; Boldt, K.; Towns, K.V.; Sedmak, T.; Beer, M.; Nagel-Wolfrum, K.; McKibbin, M.; et al. Mutations in LCA5, encoding the ciliary protein lebercilin, cause leber congenital amaurosis. Nat. Genet. 2007, 39, 889-895.

54. Shahzadi, A.; Riazuddin, S.A.; Ali, S.; Li, D.; Khan, S.N.; Husnain, T.; Akram, J.; Sieving, P.A.; Hejtmancik, J.F.; Riazuddin, S. Nonsense mutation in MERTK causes autosomal recessive retinitis pigmentosa in a consanguineous Pakistani family. Br. J. Ophthalmol. 2010, 94, 1094-1099.

55. Falk, M.J.; Zhang, Q.; Nakamaru-Ogiso, E.; Kannabiran, C.; Fonseca-Kelly, Z.; Chakarova, C.; Audo, I.; Mackay, D.S.; Zeitz, C.; Borman, A.D.; et al. NMNAT1 mutations cause leber congenital amaurosis. Nat. Genet. 2012, 44, 1040-1045.

56. Koenekoop, R.K.; Wang, H.; Majewski, J.; Wang, X.; Lopez, I.; Ren, H.; Chen, Y.; Li, Y.; Fishman, G.A.; Genead, M.; et al. Mutations in NMNAT1 cause leber congenital amaurosis and identify a new disease pathway for retinal degeneration. Nat. Genet. 2012, 44, 1035-1039.

57. Riazuddin, S.A.; Zulfiqar, F.; Zhang, Q.; Yao, W.; Li, S.; Jiao, X.; Shahzadi, A.; Amer, M.; Iqbal, M.; Hussnain, T.; et al. Mutations in the gene encoding the alpha-subunit of rod phosphodiesterase in consanguineous Pakistani families. Mol. Vis. 2006, 12, 1283-1291.

58. Ali, S.; Riazuddin, S.A.; Shahzadi, A.; Nasir, I.A.; Khan, S.N.; Husnain, T.; Akram, J.; Sieving, P.A.; Hejtmancik, J.F.; Riazuddin, S. Mutations in the beta-subunit of rod phosphodiesterase identified in consanguineous Pakistani families with autosomal recessive retinitis pigmentosa. Mol. Vis. 2011, 17, 1373-1380.

59. Zhang, Q.; Zulfiqar, F.; Xiao, X.; Riazuddin, S.A.; Ahmad, Z.; Caruso, R.; MacDonald, I.; Sieving, P.; Riazuddin, S.; Hejtmancik, J.F. Severe retinitis pigmentosa mapped to 4p15 and associated with a novel mutation in the PROM1 gene. Hum. Genet. 2007, 122, 293-299.

60. Mackay, D.S.; Dev Borman, A.; Moradi, P.; Henderson, R.H.; Li, Z.; Wright, G.A.; Waseem, N.; Gandra, M.; Thompson, D.A.; Bhattacharya, S.S.; et al. RDH12 retinopathy: Novel mutations and phenotypic description. Mol. Vis. 2011, 17, 2706-2716.

61. Ajmal, M.; Khan, M.I.; Neveling, K.; Khan, Y.M.; Ali, S.H.; Ahmed, W.; Iqbal, M.S.; Azam, M.; den Hollander, A.I.; Collin, R.W.J.; et al. Novel mutations in RDH5 cause fundus albipunctatus in two consanguineous Pakistani families. Mol. Vis. 2012, 18, 1558-1571. 
62. Azam, M.; Khan, M.I.; Gal, A.; Hussain, A.; Shah, S.T.A.; Khan, M.S.; Sadeque, A.; Bokhari, H.; Collin, R.W.J.; Orth, U.; et al. A homozygous p.Glu150Lys mutation in the opsin gene of two pakistani families with autosomal recessive retinitis pigmentosa. Mol. Vis. 2009, 15, 2526-2534.

63. Naz, S.; Ali, S.; Riazuddin, S.A.; Farooq, T.; Butt, N.H.; Zafar, A.U.; Khan, S.N.; Husnain, T.; Macdonald, I.M.; Sieving, P.A.; et al. Mutations in RLBP1 associated with fundus albipunctatus in consanguineous Pakistani families. Br. J. Ophthalmol. 2011, 95, 1019-1024.

64. Khaliq, S.; Abid, A.; Ismail, M.; Hameed, A.; Mohyuddin, A.; Lall, P.; Aziz, A.; Anwar, K.; Mehdi, S.Q. Novel association of RP1 gene mutations with autosomal recessive retinitis pigmentosa. J. Med. Genet. 2005, 42, 436-438.

65. Riazuddin, S.A.; Zulfiqar, F.; Zhang, Q.; Sergeev, Y.V.; Qazi, Z.A.; Husnain, T.; Caruso, R.; Riazuddin, S.; Sieving, P.A.; Hejtmancik, J.F. Autosomal recessive retinitis pigmentosa is associated with mutations in RP1 in three consanguineous Pakistani families. Invest. Ophthalmol. Vis. Sci. 2005, 46, 2264-2270.

66. Simovich, M.J.; Miller, B.; Ezzeldin, H.; Kirkland, B.T.; McLeod, G.; Fulmer, C.; Nathans, J.; Jacobson, S.G.; Pittler, S.J. Four novel mutations in the RPE65 gene in patients with Leber congenital amaurosis. Hum. Mutat. 2001, 18, 164.

67. Coppieters, F.; de Baere, E.; Leroy, B. Development of a next-generation sequencing platform for retinal dystrophies, with LCA and RP as proof of concept. Bull. Soc. Belg. Ophtalmol. 2011, 317, 59-60.

68. Vervoort, R.; Lennon, A.; Bird, A.C.; Tulloch, B.; Axton, R.; Miano, M.G.; Meindl, A.; Meitinger, T.; Ciccodicola, A.; Wright, A.F. Mutational hot spot within a new RPGR exon in $\mathrm{X}$-linked retinitis pigmentosa. Nat. Genet. 2000, 25, 462-466.

69. Hameed, A.; Abid, A.; Aziz, A.; Ismail, M.; Mehdi, S.Q.; Khaliq, S. Evidence of rpgrip1 gene mutations associated with recessive cone-rod dystrophy. J. Med. Genet. 2003, 40, 616-619.

70. Waheed, N.K.; Qavi, A.H.; Malik, S.N.; Maria, M.; Riaz, M.; Cremers, F.P.M.; Azam, M.; Qamar, R. A nonsense mutation in S-antigen (p.Glu306*) causes Oguchi disease. Mol. Vis. 2012, 18, 1253-1259.

71. Riazuddin, S.A.; Shahzadi, A.; Zeitz, C.; Ahmed, Z.M.; Ayyagari, R.; Chavali, V.R.; Ponferrada, V.G.; Audo, I.; Michiels, C.; Lancelot, M.E.; et al. A mutation in SLC24A1 implicated in autosomal-recessive congenital stationary night blindness. Am. J. Hum. Genet. 2010, $87,523-531$.

72. Mackay, D.S.; Ocaka, L.A.; Borman, A.D.; Sergouniotis, P.I.; Henderson, R.H.; Moradi, P.; Robson, A.G.; Thompson, D.A.; Webster, A.R.; Moore, A.T. Screening of SPATA7 in patients with Leber congenital amaurosis and severe childhood-onset retinal dystrophy reveals disease-causing mutations. Invest. Ophthalmol. Vis. Sci. 2011, 52, 3032-3038.

73. Wang, H.; den Hollander, A.I.; Moayedi, Y.; Abulimiti, A.; Li, Y.; Collin, R.W.J.; Hoyng, C.B.; Lopez, I.; Abboud, E.B.; Al-Rajhi, A.A.; et al. Mutations in SPATA7 cause Leber congenital amaurosis and juvenile retinitis pigmentosa. Am. J. Hum. Genet. 2009, 84, 380-387. 
74. Riazuddin, S.A.; Iqbal, M.; Wang, Y.; Masuda, T.; Chen, Y.; Bowne, S.; Sullivan, L.S.; Waseem, N.H.; Bhattacharya, S.; Daiger, S.P.; et al. A splice-site mutation in a retina-specific exon of BBS8 causes nonsyndromic retinitis pigmentosa. Am. J. Hum. Genet. 2010, 86, $805-812$.

75. Ajmal, M.; Khan, M.I.; Micheal, S.; Ahmed, W.; Shah, A.; Venselaar, H.; Bokhari, H.; Azam, A.; Waheed, N.K.; Collin, R.W.J.; et al. Identification of recurrent and novel mutations in TULP1 in Pakistani families with early-onset retinitis pigmentosa. Mol. Vis. 2012, 18, 1226-1237.

76. Iqbal, M.; Naeem, M.A.; Riazuddin, S.A.; Ali, S.; Farooq, T.; Qazi, Z.A.; Khan, S.N.; Husnain, T.; Riazuddin, S.; Sieving, P.A.; et al. Association of pathogenic mutations in TULP1 with retinitis pigmentosa in consanguineous Pakistani families. Arch. Ophthalmol. 2011, 129, 1351-1357.

77. Gu, S.; Lennon, A.; Li, Y.; Lorenz, B.; Fossarello, M.; North, M.; Gal, A.; Wright, A. Tubby-like protein-1 mutations in autosomal recessive retinitis pigmentosa. Lancet 1998, 351, 1103-1104.

78. Li, L.; Nakaya, N.; Chavali, V.R.; Ma, Z.; Jiao, X.; Sieving, P.A.; Riazuddin, S.; Tomarev, S.I.; Ayyagari, R.; Riazuddin, S.A.; et al. A mutation in ZNF513, a putative regulator of photoreceptor development, causes autosomal-recessive retinitis pigmentosa. Am. J. Hum. Genet. 2010, 87, 400-409.

79. Naz, S.; Riazuddin, S.A.; Li, L.; Shahid, M.; Kousar, S.; Sieving, P.A.; Hejtmancik, J.F.; Riazuddin, S. A novel locus for autosomal recessive retinitis pigmentosa in a consanguineous Pakistani family maps to chromosome 2p. Am. J. Ophthalmol. 2010, 149, 861-866.

80. Rafiq, M.A.; Ansar, M.; Marshall, C.R.; Noor, A.; Shaheen, N.; Mowjoodi, A.; Khan, M.A.; Ali, G.; Amin-ud-Din, M.; Feuk, L.; et al. Mapping of three novel loci for non-syndromic autosomal recessive mental retardation (NS-ARMR) in consanguineous families from pakistan. Clin. Genet. 2010, 78, 478-483.

81. Kakar, N.; Goebel, I.; Daud, S.; Nurnberg, G.; Agha, N.; Ahmad, A.; Nurnberg, P.; Kubisch, C.; Ahmad, J.; Borck, G. A homozygous splice site mutation in TRAPPC9 causes intellectual disability and microcephaly. Eur. J. Med. Genet. 2012, 55, 727-731.

82. Noor, A.; Windpassinger, C.; Patel, M.; Stachowiak, B.; Mikhailov, A.; Azam, M.; Irfan, M.; Siddiqui, Z.K.; Naeem, F.; Paterson, A.D.; et al. CC2D2A, encoding a coiled-coil and C2 domain protein, causes autosomal-recessive mental retardation with retinitis pigmentosa. Am. J. Hum. Genet. 2008, 82, 1011-1018.

83. Schultz, J.M.; Bhatti, R.; Madeo, A.C.; Turriff, A.; Muskett, J.A.; Zalewski, C.K.; King, K.A.; Ahmed, Z.M.; Riazuddin, S.; Ahmad, N.; et al. Allelic hierarchy of CDH23 mutations causing non-syndromic deafness DFNB12 or usher syndrome USH1D in compound heterozygotes. J. Med. Genet. 2011, 48, 767-775.

84. Ahmed, Z.M.; Riazuddin, S.; Bernstein, S.L.; Ahmed, Z.; Khan, S.; Griffith, A.J.; Morell, R.J.; Friedman, T.B.; Wilcox, E.R. Mutations of the protocadherin gene PCDH15 cause usher syndrome type 1f. Am. J. Hum. Genet. 2001, 69, 25-34. 
85. Ismail, M.; Abid, A.; Anwar, K.; Mehdi, S.Q.; Khaliq, S. Refinement of the locus for autosomal recessive cone-rod dystrophy (CORD8) linked to chromosome 1q23-q24 in a pakistani family and exclusion of candidate genes. J. Hum. Genet. 2006, 51, 827-831.

86. Hameed, A.; Khaliq, S.; Ismail, M.; Anwar, K.; Mehdi, S.Q.; Bessant, D.; Payne, A.M.; Bhattacharya, S.S. A new locus for autosomal recessive RP (RP29) mapping to chromosome 4q32-q34 in a pakistani family. Invest. Ophthalmol. Vis. Sci. 2001, 42, 1436-1438.

87. Zhang, Q.; Zulfiqar, F.; Xiao, X.; Riazuddin, S.A.; Ayyagari, R.; Sabar, F.; Caruso, R.; Sieving, P.A.; Riazuddin, S.; Hejtmancik, J.F. Severe autosomal recessive retinitis pigmentosa maps to chromosome 1p13.3-p21.2 between D1S2896 and D1S457 but outside ABCA4. Hum. Genet. 2005, 118, 356-365.

88. Ahmed, Z.M.; Riazuddin, S.; Khan, S.N.; Friedman, P.L.; Riazuddin, S.; Friedman, T.B. USH1H, a novel locus for type I Usher syndrome, maps to chromosome 15q22-23. Clin. Genet. 2009, 75, 86-91.

89. Jaworek, T.J.; Bhatti, R.; Latief, N.; Khan, S.N.; Riazuddin, S.; Ahmed, Z.M. USH1K, a novel locus for type I Usher syndrome, maps to chromosome 10p11.21-q21.1. J. Hum. Genet. 2012, 57, 633-637.

90. Utsch, B.; Sayer, J.A.; Attanasio, M.; Pereira, R.R.; Eccles, M.; Hennies, H.C.; Otto, E.A.; Hildebrandt, F. Identification of the first AHI1 gene mutations in nephronophthisis-associated Joubert syndrome. Pediatr. Nephrol. 2006, 21, 32-35.

91. Khan, S.; Ullah, I.; Irfanullah, I.; Touseef, M.; Basit, S.; Khan, M.N.; Ahmad, W. Novel homozygous mutations in the genes ARL6 and BBS10 underlying Bardet-Biedl syndrome. Gene 2013, 515, 84-88.

92. Chen, J.; Smaoui, N.; Hammer, M.B.; Jiao, X.; Riazuddin, S.A.; Harper, S.; Katsanis, N.; Riazuddin, S.; Chaabouni, H.; Berson, E.L.; et al. Molecular analysis of Bardet-Biedl syndrome families: Report of 21 novel mutations in 10 genes. Invest. Ophthalmol. Vis. Sci. 2011, 52, 5317-5324.

93. Cantagrel, V.; Silhavy, J.L.; Bielas, S.L.; Swistun, D.; Marsh, S.E.; Bertrand, J.Y.; Audollent, S.; Attie-Bitach, T.; Holden, K.R.; Dobyns, W.B.; et al. Mutations in the cilia gene ARL13B lead to the classical form of Joubert syndrome. Am. J. Hum. Genet. 2008, 83, 170-179.

94. Ajmal, M.; Khan, M.I.; Neveling, K.; Tayyab, A.; Jaffar, S.; Sadeque, A.; Ayub, H.; Abbasi, N.M.; Riaz, M.; Micheal, S.; et al. Exome sequencing identifies a novel and a recurrent BBS1 mutation in Pakistani families with Bardet-Biedl syndrome. Mol. Vis. 2013, 19, 644-653.

95. Harville, H.M.; Held, S.; Diaz-Font, A.; Davis, E.E.; Diplas, B.H.; Lewis, R.A.; Borochowitz, Z.U.; Zhou, W.; Chaki, M.; Macdonald, J.; et al. Identification of 11 novel mutations in eight BBS genes by high-resolution homozygosity mapping. J. Med. Genet. 2010, 47, 262-267. 
96. White, D.R.; Ganesh, A.; Nishimura, D.; Rattenberry, E.; Ahmed, S.; Smith, U.M.; Pasha, S.; Raeburn, S.; Trembath, R.C.; Rajab, A.; et al. Autozygosity mapping of Bardet-Biedl syndrome to 12q21.2 and confirmation of FLJ23560 as BBS10. Eur. J. Hum. Genet. 2007, $15,173-178$.

97. Agha, Z.; Iqbal, Z.; Azam, M.; Hoefsloot, L.H.; van Bokhoven, H.; Qamar, R. A novel homozygous 10 nucleotide deletion in BBS10 causes Bardet-Biedl syndrome in a Pakistani family. Gene 2013, 519, 177-181.

98. Pawlik, B.; Mir, A.; Iqbal, H.; Li, Y.; Nurnberg, G.; Becker, C.; Qamar, R.; Nurnberg, P.; Wollnik, B. A novel familial BBS12 mutation associated with a mild phenotype: Implications for clinical and molecular diagnostic strategies. Mol. Syndromol. 2010, 1, 27-34.

99. Bork, J.M.; Peters, L.M.; Riazuddin, S.; Bernstein, S.L.; Ahmed, Z.M.; Ness, S.L.; Polomeno, R.; Ramesh, A.; Schloss, M.; Srisailpathy, C.R.; et al. Usher syndrome 1D and nonsyndromic autosomal recessive deafness DFNB12 are caused by allelic mutations of the novel cadherin-like gene CDH23. Am. J. Hum. Genet. 2001, 68, 26-37.

100. Otto, E.A.; Ramaswami, G.; Janssen, S.; Chaki, M.; Allen, S.J.; Zhou, W.; Airik, R.; Hurd, T.W.; Ghosh, A.K.; Wolf, M.T.; et al. Mutation analysis of 18 nephronophthisis associated ciliopathy disease genes using a DNA pooling and next generation sequencing strategy. J. Med. Genet. 2011, 48, 105-116.

101. Sayer, J.A.; Otto, E.A.; O’Toole, J.F.; Nurnberg, G.; Kennedy, M.A.; Becker, C.; Hennies, H.C.; Helou, J.; Attanasio, M.; Fausett, B.V.; et al. The centrosomal protein nephrocystin- 6 is mutated in joubert syndrome and activates transcription factor ATF4. Nat. Genet. 2006, 38, 674-681.

102. Otto, E.A.; Helou, J.; Allen, S.J.; O’Toole, J.F.; Wise, E.L.; Ashraf, S.; Attanasio, M.; Zhou, W.; Wolf, M.T.F.; Hildebrandt, F. Mutation analysis in nephronophthisis using a combined approach of homozygosity mapping, CEL I endonuclease cleavage, and direct sequencing. Hum. Mutat. 2008, 29, 418-426.

103. Ahmed, Z.M.; Riazuddin, S.; Ahmad, J.; Bernstein, S.L.; Guo, Y.; Sabar, M.F.; Sieving, P.; Griffith, A.J.; Friedman, T.B.; Belyantseva, I.A.; et al. PCDH15 is expressed in the neurosensory epithelium of the eye and ear and mutant alleles are responsible for both USH1F and DFNB23. Hum. Mol. Genet. 2003, 12, 3215-3223.

104. Sang, L.; Miller, J.J.; Corbit, K.C.; Giles, R.H.; Brauer, M.J.; Otto, E.A.; Baye, L.M.; Wen, X.; Scales, S.J.; Kwong, M.; et al. Mapping the NPHP-JBTS-MKS protein network reveals ciliopathy disease genes and pathways. Cell 2011, 145, 513-528.

105. Smith, U.M.; Consugar, M.; Tee, L.J.; McKee, B.M.; Maina, E.N.; Whelan, S.; Morgan, N.V.; Goranson, E.; Gissen, P.; Lilliquist, S.; et al. The transmembrane protein meckelin (MKS3) is mutated in Meckel-Gruber syndrome and the wpk rat. Nat. Genet. 2006, 38, 191-196.

106. Ansley, S.J.; Badano, J.L.; Blacque, O.E.; Hill, J.; Hoskins, B.E.; Leitch, C.C.; Kim, J.C.; Ross, A.J.; Eichers, E.R.; Teslovich, T.M.; et al. Basal body dysfunction is a likely cause of pleiotropic Bardet-Biedl syndrome. Nature 2003, 425, 628-633.

107. Bashir, R.; Fatima, A.; Naz, S. A frameshift mutation in SANS results in atypical Usher syndrome. Clin. Genet. 2010, 78, 601-603. 
108. Abu-Safieh, L.; Alrashed, M.; Anazi, S.; Alkuraya, H.; Khan, A.O.; Al-Owain, M.; Al-Zahrani, J.; Al-Abdi, L.; Hashem, M.; Al-Tarimi, S.; et al. Autozygome-guided exome sequencing in retinal dystrophy patients reveals pathogenetic mutations and novel candidate disease genes. Genome Res. 2013, 23, 236-247.

109. Hartong, D.T.; Berson, E.L.; Dryja, T.P. Retinitis pigmentosa. Lancet 2006, 368, 1795-1809.

110. Neveling, K.; Collin, R.W.J.; Gilissen, C.; van Huet, R.A.; Visser, L.; Kwint, M.P.; Gijsen, S.J.; Zonneveld, M.N.; Wieskamp, N.; de Ligt, J.; et al. Next-generation genetic testing for retinitis pigmentosa. Hum. Mutat. 2012, 33, 963-972.

111. Seyedahmadi, B.J.; Rivolta, C.; Keene, J.A.; Berson, E.L.; Dryja, T.P. Comprehensive screening of the USH2A gene in usher syndrome type II and non-syndromic recessive retinitis pigmentosa. Exp. Eye Res. 2004, 79, 167-173.

112. Qamar, R.; Ayub, Q.; Mohyuddin, A.; Helgason, A.; Mazhar, K.; Mansoor, A.; Zerjal, T.; Tyler-Smith, C.; Mehdi, S.Q. Y-chromosomal DNA variation in pakistan. Am. J. Hum. Genet. 2002, 70, 1107-1124.

113. Collin, R.W.J.; van den Born, L.I.; Klevering, B.J.; de Castro-Miro, M.; Littink, K.W.; Arimadyo, K.; Azam, M.; Yazar, V.; Zonneveld, M.N.; Paun, C.C.; et al. High-resolution homozygosity mapping is a powerful tool to detect novel mutations causative of autosomal recessive RP in the dutch population. Invest. Ophthalmol. Vis. Sci. 2011, 52, 2227-2239.

114. Sohocki, M.M.; Perrault, I.; Leroy, B.P.; Payne, A.M.; Dharmaraj, S.; Bhattacharya, S.S.; Kaplan, J.; Maumenee, I.H.; Koenekoop, R.; Meire, F.M.; et al. Prevalence of AIPL1 mutations in inherited retinal degenerative disease. Mol. Genet. Metab. 2000, 70, 142-150.

115. Yzer, S.; Leroy, B.P.; de Baere, E.; de Ravel, T.J.; Zonneveld, M.N.; Voesenek, K.; Kellner, U.; Martinez Ciriano, J.P.; de Faber, J.T.H.N.; Rohrschneider, K.; et al. Microarray-based mutation detection and phenotypic characterization of patients with leber congenital amaurosis. Invest. Ophthalmol. Vis. Sci. 2006, 47, 1167-1176.

116. Muller, J.; Stoetzel, C.; Vincent, M.C.; Leitch, C.C.; Laurier, V.; Danse, J.M.; Helle, S.; Marion, V.; Bennouna-Greene, V.; Vicaire, S.; et al. Identification of 28 novel mutations in the Bardet-Biedl syndrome genes: The burden of private mutations in an extensively heterogeneous disease. Hum. Genet. 2010, 127, 583-593.

117. Kurg, A.; Tonisson, N.; Georgiou, I.; Shumaker, J.; Tollett, J.; Metspalu, A. Arrayed primer extension: Solid-phase four-color DNA resequencing and mutation detection technology. Genet. Test. 2000, 4, 1-7.

118. Jaakson, K.; Zernant, J.; Kulm, M.; Hutchinson, A.; Tonisson, N.; Hawlina, M.; Ravnic-Glavac, M.; Meltzer, M.; Caruso, R.; Testa, F.; et al. Genotyping microarray (gene chip) for the ABCR (ABCA4) gene. Hum. Mutat. 2003, 22, 395-403.

119. Avila-Fernandez, A.; Cantalapiedra, D.; Aller, E.; Vallespin, E.; guirre-Lamban, J.; Blanco-Kelly, F.; Corton, M.; Riveiro-Alvarez, R.; Allikmets, R.; Trujillo-Tiebas, M.J.; et al. Mutation analysis of 272 spanish families affected by autosomal recessive retinitis pigmentosa using a genotyping microarray. Mol. Vis. 2010, 16, 2550-2558. 


\section{Association Claims in the Sequencing Era}

\section{Sara L. Pulit, Maarten Leusink, Androniki Menelaou and Paul I. W. de Bakker}

Abstract: Since the completion of the Human Genome Project, the field of human genetics has been in great flux, largely due to technological advances in studying DNA sequence variation. Although community-wide adoption of statistical standards was key to the success of genome-wide association studies, similar standards have not yet been globally applied to the processing and interpretation of sequencing data. It has proven particularly challenging to pinpoint unequivocally disease variants in sequencing studies of polygenic traits. Here, we comment on a number of factors that may contribute to irreproducible claims of association in scientific literature and discuss possible steps that we can take towards cultural change.

Reprinted from Genes. Cite as: Pulit, S.L.; Leusink, M.; Menelaou, A.; de Bakker, P.I.W. Association Claims in the Sequencing Era. Genes 2014, 5, 196-213.

\section{The Evolution of Genetic Association Studies}

The Human Genome Project [1] remains the largest scientific endeavor in the biological sciences, spanning thirteen years, requiring hundreds of researchers around the globe, and costing $\$ 3$ billion. Called the "most important, most wondrous map ever produced by human kind" [2] by U.S. President Clinton upon its completion, the map of the genome catapulted the investigation of human disease into a new era. The study of complex traits and disease had previously been limited to genetic linkage studies, typically laborious efforts limited to constructing linkage maps in families and powered for discovering highly penetrant variants. Now, geneticists could identify single-base changes in the genome (single nucleotide polymorphisms, or SNPs) and, by measuring the frequencies of these changes in large groups of cases and controls, test SNPs for association with susceptibility to any number of diseases.

Upon the completion of the Human Genome Project, the field of genetics was faced with determining how best to hunt for such associations. The first widely used method was candidate gene studies, in which genes with suspected biological or functional relevance to the disease in question were selected for testing. These studies were applied to a variety of traits [3], but in the absence of community-wide standards for analysis, they were plagued with problems. Reviews of candidate gene studies found that small sample sizes, population stratification issues, weak effects, and a lack of statistical evidence for the claimed associations were common [3-6]. All of these problems contributed to the irreproducibility of initial findings; one review found that of 166 associations with more than two follow-up studies, only six (3.6\%) replicated [3].

Despite limited success in candidate gene studies, the number of replicated associations indicated that common variation (frequency $>5 \%$ ) indeed plays a role in genetic susceptibility to common disease (involving tens of genes and environmental factors) [7]. In 2005, the approach in genetics began shifting towards genome-wide association studies (GWAS) [8], which require no prior hypothesis about which genes are likely to influence disease. Instead, geneticists could test 
millions of common variants across the genome for association with the trait of interest. Systematic cataloguing of these variants [9] allowed for the design of genome-wide SNP arrays, ultimately allowing for low-cost capture of tens of thousands of variants in large samples.

The beginnings of GWAS were slow; initial studies produced few if any associated loci, and it quickly became clear that larger samples would be necessary for sufficient power to detect susceptibility variants [10]. With the formation of international consortia, collection of large samples, and assembly of imputation panels that allowed for testing of variants not present on SNP arrays came an explosion in discovered loci (Figure 1). Along with the rapid increase in the number of GWAS being performed came a large-scale effort to standardize the method. The community adopted the genome-wide significance $p$-value threshold of $5 \times 10^{-8}$, a $p$-value that reflects a Bonferroni correction for the approximately one million independent tests performed in a GWAS [11,12]. Methods for handling population stratification were developed [13], as were approaches for finding and removing poorly captured genotypes [14]. Replication of discovered loci also became a criterion for declaring a SNP to be associated with a disease $[15,16]$. With best practices in place, GWAS has become an efficient and robust method for discovering the contribution of common variation to susceptibility in common disease. The total number of SNPs associated with complex traits (at genome-wide significance) was only seven by the end of 2006, but by 2008, an additional 637 associations had been discovered [17]. Today, more than 6,000 disease-SNP associations have been reported.

Figure 1. Disease-susceptibility loci discovered to date in various complex traits, as reported in the National Human Genome Research Institute (NHGRI) genome-wide association studies (GWAS) catalog [17]. Early genome-wide association studies interrogated small samples and uncovered few, if any, loci associated with the trait of interest. However, collaborative efforts to assemble large-scale samples improved power and implicated tens and even hundreds of susceptibility loci, revealing a (roughly) linear relat

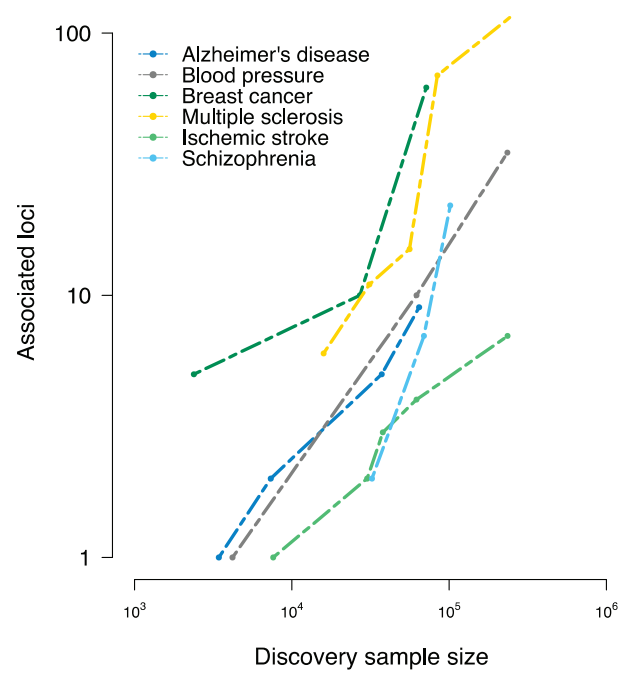


Because of established best practices and statistical thresholds, most GWAS findings are robust and reproducible. The method is, of course, not immune to error; for example, one GWAS looking for variants associated with longevity failed to correct for technical bias introduced by the use of two different SNP arrays, inducing spurious association [19]. The mistake went unnoticed until the results were published and several geneticists caught the error. The false report received attention from the genetics community and the media alike [20] and was later retracted [21].

More recently, scientists and the media have cast a critical eye towards the aspects of scientific culture that also give rise to false-positive findings in published work. The Economist recently published an article suggesting that science is not as self-correcting as many assume [22,23], and journals such as Nature and Science are publishing columns on scientific misconduct, peer-review, and other issues that contribute to the reporting of inaccurate results [24-28]. The last four years have also seen several published studies on the prevalence of false discoveries and misconduct, indicating that as many as " $1 \%$ of published papers are fraudulent" (about 20,000 papers each year) [25]. Given the increased attention from the public and scientists alike, and because genetics is in a transition phase as it moves from performing GWAS data to studying next-generation sequencing data, now is an ideal time to address some of these shortcomings.

\section{Sources of Error and Bias in Genetic Research}

A number of factors contribute to false-positive findings in published human genetic research. Technical artifact, such as mishandling of population stratification, poorly genotyped SNPs, and batch effects introduced by different SNP arrays or genotyping runs can cause spurious results, though a number of methods have been designed for detecting them [13,29-32]. Study design is also crucial in avoiding false positives. SNPs implicated in disease susceptibility typically have modest effect sizes (odds ratios ranging from 1.1-1.5) [18]; insufficient sample size to detect such effects can substantially reduce a study's power and increase the likelihood of discovering an artifact rather than a true association.

Although technical error can lead to false positives, a number of other forces in research culture also contribute to the number of published erroneous findings. In the span of just seven years, GWAS moved from small-scale efforts to studies of thousands of samples, leading to a heavy reliance on statistical methods to study large datasets. Yet, researchers' understanding of statistics has not kept pace with data generation, making them more likely to apply inappropriate statistical tests or perform tests they do not fully understand. The same holds true for the many programs written to analyze genome-wide datasets. Not all researchers using this software will fully understand the underlying methods, increasing the chance of false positives going unnoticed.

The peer-review process is also partially to blame for the introduction of false findings into scientific literature. Though researchers would seem ideal candidates for catching the mistakes of their peers, studies suggest that they often fail to catch errors (even when instructed that there are errors to find) [22]. Further, peer-reviewers are not provided all data underlying a paper and therefore cannot reproduce analyses to verify findings.

Studies also indicate that misconduct (which includes plagiarism, fraud, and duplicate publications) has been on the rise in recent years [33] and accounts for the majority of retracted 
papers in the life sciences [34]. A number of aspects of research culture likely contribute to such misconduct. Scientific research has become a field in which the number of articles a researcher produces is a primary measure of success. Career opportunities in the sciences also often hinge on the number of publications a scientist has produced. Conflating financial interests (whether in the form of employment or grants) and pressure to publish is a factor that potentially gives rise to false associations reported in scientific literature [35]. Anyone pressed to produce such a high volume of results, preferably at great speed, is more likely to miss mistakes in her own work.

Publication and funding bias are also problematic [36]. Although replicating a result is the backbone of establishing the veracity of a scientific claim, replication studies are less likely to be funded than discovery-focused experiments, and journals are less likely to publish them. Journals also typically do not publish negative studies, preferring to focus instead on novel results. The establishment of the impact factor system has further intensified journals' bias towards novelty. While selecting manuscripts for publication, journal editors consider not only the content of the manuscript, but also the potential that the paper will improve the journal's impact factor. The impact factor ranking system also heightens publishing competition for researchers. Researchers seek publication in only a very small set of highly selective journals in pursuit of many citations, widespread attention from the scientific community and popular media, and potential career advancement. In molecular biology and genetics, just six journals accounted for 85 of the 100 most-cited articles between 1998 and 2008 [37].

Figure 2. The growth of open access journals over time and around the world.

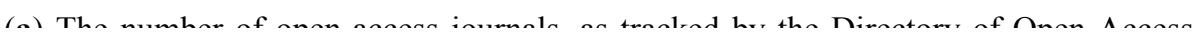

a.

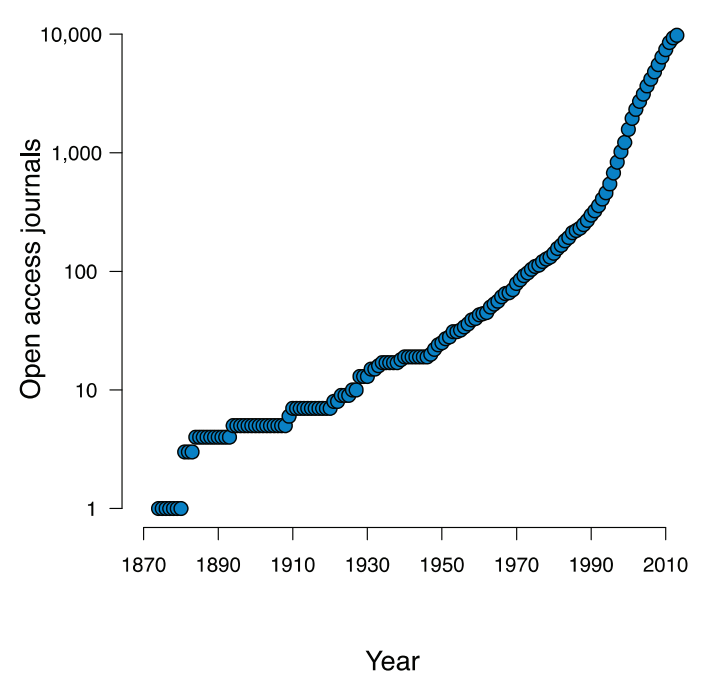

b.

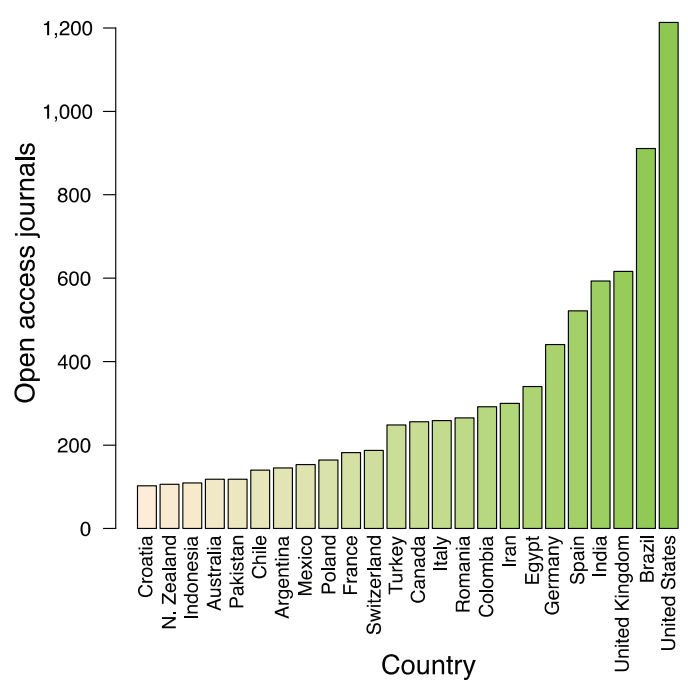


Increasing the prevalence of false positives are those journals that seek to turn a profit by preying off a research culture that keeps publishing at its center. The number of journals accepting manuscripts is large and rapidly growing. The Directory of Open Access Journals, which tracks open access journals and their credibility, has tracked the swift global expansion of the number of open access journals, including an addition of over 1000 journals in 2012 alone [38] (Figure 2). A "sting" conducted on open-access journals revealed that many of them are uninterested in scientific veracity; a paper concocted wholesale and containing glaring errors was accepted by more than $52 \%$ of the targeted journals [39]. The "sting" in part implicated a flawed peer-review system, as $40 \%$ of the submissions were reviewed and then accepted. The other $60 \%$ of submissions, however, were accepted without any indication of peer review, suggesting that many of the journals the "sting" targeted are focused more on profit rather than on scientific rigor, encouraging a culture that values a published finding over a robust result.

\section{Next-Generation Sequencing: New Technology, New Challenges}

The advent of next-generation sequencing (NGS) technology has ushered in a new wave of studies in human genetic research. Given that many complex traits involve tens and sometimes hundreds of loci [40,41], lower-frequency variants may also contribute to the architecture of human disease. However, these variants are weakly tagged by common SNPs and have therefore gone untested by GWAS efforts. Researchers using NGS data can test (nearly) the entire set of variants in a single genome, helping to complete the picture of the role of genetic variation in common disease. Yet, this new technology brings with it many challenges, giving rise to additional forces that may lead to false positives in scientific literature.

A number of technical errors can give rise to a false-positive association in a NGS disease study. Determining genotypes from sequencing reads is more challenging than determining genotypes from SNP array data $[42,43]$. Rather than measuring probe intensities, as is done with SNP arrays to determine genotypes, extracting genotypes from sequencing data involves multiple steps, including mapping sequencing reads to a reference, detecting bases that do not match the reference, and determining the genotypes of each individual at each base; errors can occur at any of these steps and are particularly likely in regions that are difficult to capture, such as those rich in GC content or that are highly repetitive. Further, determining inclusion and exclusion criteria for variants based on a host of sequencing metrics can be difficult, sometimes requiring manual review of each of the metrics to determine appropriate filters [44]. Even the most conservative variant calling and quality control (QC) cannot guarantee that "variants" that are actually artifacts will be removed from the dataset.

Study design flaws can also prompt false-positive results. Although methods for detecting population stratification have been developed and widely used in GWAS, our understanding of population stratification in rare variants is limited and may confound association results [45]. To reduce costs, an investigator may choose to sequence only cases and use external (previously sequenced) controls; this approach may introduce stratification because the controls may be sequenced using a different platform [46] or genotype-called using outdated software. Alternatively, an investigator may want to sequence only cases and then genotype the discovered 
variants in controls or have cases substantially outnumber the controls, but these approaches also inflate type I error [47].

So-called loss-of-function mutations are of particular interest in NGS studies since they truncate proteins and are thus good candidates for likely pathogenic mutations. However, determining the deleteriousness of a loss-of-function mutation can be challenging, and this class of variants is enriched for artifacts [48]. It may be tempting to relax statistical thresholds for loss-of-function mutations or produce functional results for them before assembling appropriate statistical evidence from the genetic data, but doing so can lead to error.

Figure 3. (a) The power to detect a genetic association is a function of sample size $(N)$, effect size $(\gamma)$, the frequency of the associated allele (p), and linkage disequilibrium (LD) between the tested and causal variants $\left(R^{2}\right)$, assuming an additive model [50]. (b) For sequencing studies, many thousands of samples will be necessary to detect single, low-frequency variants associated with disease risk at genome-wide significance (black

\section{a}

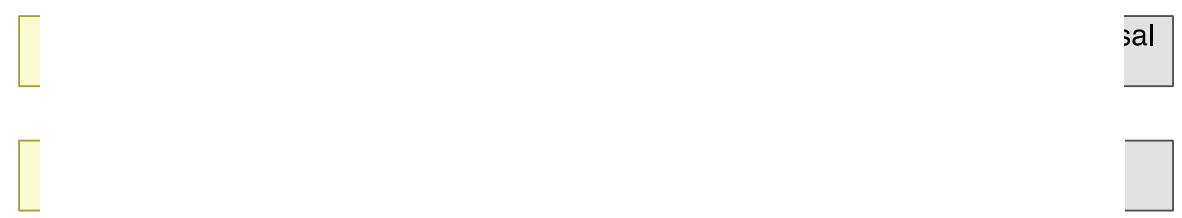

b

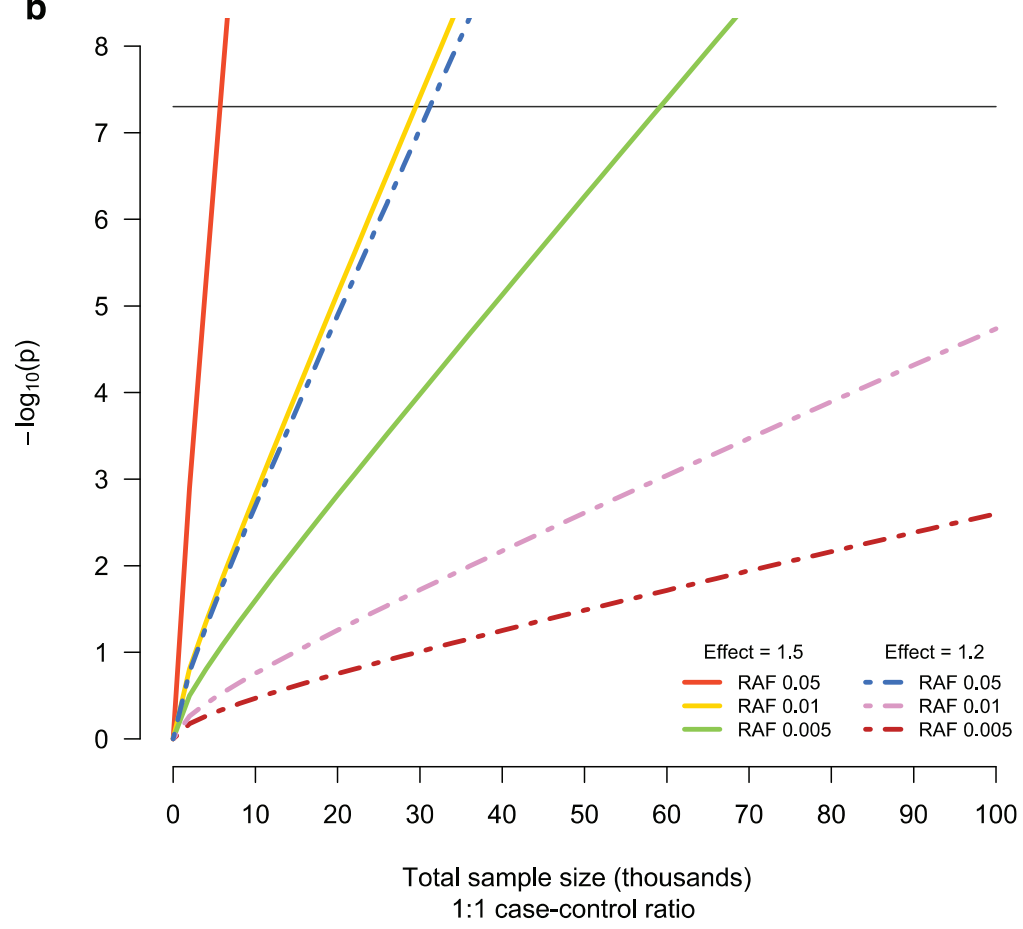


Though there are many challenges in performing an NGS study, the single largest problem plaguing sequencing disease studies to date is low statistical power to detect an association. The hypothesis that rare variants influencing susceptibility to common disease would have larger effect sizes than those discovered by GWAS has gone largely unsupported, and analyses indicate that NGS studies will require tens and even hundreds of thousands of samples to be well powered [46,49] (Figure 3). Consequently, assembling adequate sample sizes for NGS studies will take time, but researchers remain under pressure to publish. As a result, they will likely be anxious to push forward results that are not fully understood, lack statistical evidence, and may not even be real.

Finally, publishing bias will prove even more problematic in the sequencing age. The optimal choices for designing and performing a sequencing study-from phenotype ascertainment to selecting algorithms for calling and filtering variants and deciding which association tests to apply_remain difficult to discern. Sequencing studies that lack "positive" findings are highly informative but publishing bias will prevent such information from being widely disseminated. It will be more difficult to establish a standardized methodology for NGS studies, as was done for GWAS, because it is likely that "failed" experiments will not be given the same attention as studies reporting new and exciting results.

\section{Lacking Evidence: Examples from NGS Studies}

To publish disease-associated loci discovered through genome-wide association studies, it has become standard practice to meet basic criteria for discovery: appropriate sample and genotype cleaning, a SNP at genome-wide significance with a reasonable effect size and frequency, and replication in independent samples. Such standards do not yet exist for sequencing studies. Without criteria for claiming an association combined with publication bias and the pervasive pressure to "publish or perish," some NGS studies in complex traits have been published despite a paucity of statistical evidence.

A targeted sequencing project in anorexia nervosa (AN) patients claims an association between AN and the epoxide hydrolase 2 (EPHX2) gene, though the burden test $p$-value of the gene failed to meet exome-wide significance (discovery, $p=4 \times 10^{-4}$; replication, $p=6.2 \times 10^{-3}$ ) [51]. Because $E P H X 2$ has been linked to lipid traits and hypercholesterolemia is common in AN patients, the authors performed a variety of interaction tests between variants in EPHX2 and cholesterol and body mass index. They suggest that the results of the interaction tests of seven SNPs ( $p=0.004-0.045)$ are additional evidence for the role of EPHX2 in AN, but show no correction for multiple testing. A small sample size (1205 cases and 1719 controls), a large case-control ratio $(\sim 3: 1)$ in discovery, and mismatched ancestries between cases and controls (indicated by a principal component plot) may have also confounded the results. Although the authors acknowledge the need for additional replication, declaring the association between EPHX2 and AN "statistically compelling" seems premature.

Another study examined whole-exome sequencing (WES) from four samples with multiple sclerosis (MS) selected from a family with more than 15 affected individuals [52]. The group found one novel missense mutation in the tyrosine kinase 2 (TYK2) gene, an MS-susceptibility locus 
established through GWAS [53,54]. The authors performed genotyping of the variant in all remaining family members and report the percentage of affected and unaffected individuals carrying the variant $(10 / 14(71.4 \%)$ and $28 / 60(46.7 \%)$, respectively); the difference is not statistically significant ( $p=0.17$; not reported). Follow-up genotyping in an additional 2,104 cases and 1,543 controls revealed that the variant had a frequency of $0.8 \%$ in cases and $0.6 \%$ in controls, also not a statistically significant difference (which the authors state themselves). In fact, these frequencies are consistent with observed frequencies in several sets of healthy individuals [55]. Nonetheless, the authors conclude the variant has a modest effect on MS risk. Even though GWAS has established TYK2 as an MS-associated locus, the particular variant implicated by this study is severely lacking in statistical evidence and seems highly unlikely to confer disease susceptibility.

Some sequencing studies rely on functional follow-up of a variant or gene in the absence of statistically compelling genetic evidence. One recent paper examining a family with 22 members with early-onset myocardial infarction (EOMI) implicated a frameshift insertion in the guanyl cyclase 1, soluble alpha 3 (GUCY1A3) gene and a nonsynonymous single nucleotide variant (SNV) in the chaperonin containing TCP1 (CCT7) gene in susceptibility to disease [56]. The frequency of the variants in affected versus unaffected family members was not statistically significant, as the sample size was small. A search for other susceptibility variants in these two genes in 252 EOMI cases and 800 controls yielded counts that were also not statistically significant (Fisher's $p=0.023$ and $p=0.12$ for GUCY1A3 and CCT7, respectively). Functional work on both variants is provided as additional evidence, indicating that mutations in both GUCY1A3 and CCT7 in mice induce a protein deficiency that can accelerate the formation of clots that potentially cause infarction. Guanyl cyclase is a key gene in signal transduction involved in vasodilation, and it is possible that these two loci influence susceptibility to EOMI. However, the statistical evidence from the genetic data alone is not strong enough to claim association between the mutations and the trait. Moreover, it is not immediately obvious whether the conclusions of functional work in mouse models will be pertinent to humans or whether findings from such an extreme family will be extendable to EOMI in the general population [57].

A WES study in sporadic amyotrophic lateral sclerosis (ALS) trios also relied on functional work to bolster the finding in the absence of statistically compelling results [58]. The group identified de novo mutations in 47 ALS patients and discovered 25 de novo variants in 25 different genes, a distribution consistent with the (null) distribution in healthy individuals [59]. A pathway analysis indicated enrichment for chromatin regulator genes, such as the synovial sarcoma translocation (SS18L1 or CREST) gene, which contained a single de novo event in one sample. The authors found that variants in CREST inhibited neurite growth in animal models and claimed that de novo mutations in the gene confer ALS risk. However, a single de novo event from one individual, even if the variant is functional, is insufficient evidence to claim a role in disease susceptibility [60], and extensive replication efforts will be necessary to determine whether the ALS-CREST association is real.

A similar WES effort in Hirschsprung's disease sequenced two affected (related) samples and also used pathway analysis to investigate the role of the neuregulin 3 (NRG3) gene in disease susceptibility [61]. Three variants found in $N R G 3$ in the initial samples were followed up in 96 
cases and 110 controls; the variants were carried by a few cases and no controls (Fisher's $p=0.021, p=0.22, p=0.021$, for the three variants, respectively). The authors discuss the biological plausibility of the $N R G 3$ association based on the gene's role in the nervous system, but due to the small sample size, the genetic evidence for the association is lacking.

In each of these studies, the statistical evidence based on the provided data was insufficient. Although it is certainly possible that replication will show these findings to be real, their publication before gathering more persuasive statistical evidence from genetic data seems a symptom of a much larger problem. Researchers eager to publish novel results, peer reviewers not questioning the dearth of evidence, and journals enthusiastic to publish exciting stories that will garner both attention and citations all combine to allow findings that have not yet been demonstrated as statistically robust to enter scientific literature as such.

\section{Conclusions: Moving Towards Permanent Change}

A number of changes, addressing both technical challenges and cultural characteristics of research, can be implemented in order to improve the veracity of claims in published research. Though association testing approaches may differ between sequencing studies (such as selecting single-variant testing or gene-based testing), the universal application of particular thresholds will help to ensure the robustness of claimed associations. The statistical stringency of genome-wide significance at $5 \times 10^{-8}$ has served the genetics community well and has ensured the robustness of the majority of GWAS findings. Single-variant testing across whole-genome data should maintain this threshold, if not establish a more conservative one given the increased number of variants tested. Similarly, burden tests of genes should be held to an exome-wide significance level, estimated to be approximately $5 \times 10^{-7}$ [44]. Relaxing these thresholds, particularly tempting in the case of loss-of-function mutations or studies that analyze only a small set of genes, is unwise. A number of mechanisms make interpreting the true deleteriousness of a variant difficult; functional annotation alone does not guarantee a true loss-of-function mutation [44,48]. Findings from other association testing approaches, such as pathway analyses or polygenic modeling, should be evaluated based on appropriate Bonferroni correction for independent tests. Some may argue that it is overly conservative to hold a small set of genes or variants to an exome- or genome-wide threshold, but these smaller analyses are simply subsets of what will likely become exhaustive genome-wide searches. Contingent on the assembly of large datasets, testing every gene or variant is inevitable. False-positive signals can occur anywhere in the search space, and assuming these false positives will not occur in smaller, earlier searches is faulty logic [62].

Designing and performing a study correctly is also crucial to avoiding spurious associations. Large samples are of the utmost priority and will lead to additional discoveries, as demonstrated by a recent WES project in Alzheimer's that discovered rare variants in the phospholipase D family (Member 3) (PLD3) gene by whole-exome sequencing of 14 families and replicated the finding in a large-scale cohort (11,000 European-ancestry cases and controls, gene burden odds ratio $=2.75$, $p=1.44 \times 10^{-11} ; 302$ African-ancestry cases and controls, gene burden odds ratio $=5.48$, $p=1.4 \times 10^{-3}$ ) [63]. Leveraging population isolates [64] and assembling non-European samples [46] may also help in improving power, as was true in the GWAS era [65]. Because of 
varying study designs and technologies, standardizing NGS data processing and analysis is difficult and will likely take time as methods continue to develop. However, some best practices already exist $[42,43,55]$ and should be followed. Further, the methods sections of papers should be as clear and explicit as possible, reading as "how-to" guides to allow peer reviewers to catch technical mistakes and aid external replication efforts. Ideally, data should be made publicly available whenever possible. Each of these steps will improve the quality of NGS studies as they are increasingly used in the future to study complex traits (Figure 4a,b).

Designing a sequencing study not only involves consideration of the technical aspects of the study but also of the analysis team. Diversifying the team to include scientists with an array of expertise will improve the study's execution and increase the likelihood that the findings prove replicable. In addition to geneticists, computer scientists, statisticians, biologists, engineers, and clinicians can all contribute to different aspects of a sequencing study, from assembling the raw sequencing data to interpreting results. Methods for sequencing analysis are evolving rapidly, and multifaceted teams representing many scientific fields are optimal for keeping pace with this changing landscape.

Future scientific publications will also be improved by an open peer review system. Some journals, such as the journal of the European Molecular Biology Organization (EMBO), have already begun making peer review a public process to great success [66]. The exchange that occurs between scientists during peer review can be valuable not only for the authors, but also for other researchers. If reviews, responses and revisions are made public the scientific community will benefit as a whole by being able to design better studies and avoid errors that other researchers have made. Further, peer review is crucial to the scientific process, yet is rarely ever taught. Making peer review reports accessible to all can serve as a teaching tool, particularly for younger scientists who may be unfamiliar with the process [66].

Peer review is not the only aspect of publishing in scientific journals that should be open. Though some open-access journals were discredited in Science's recent "sting", several open-access journals have become highly reputable in the scientific community, and other journals are giving authors the option to make their article open-access. This trend towards open-access science should continue. Rather than hiding important discoveries behind exorbitantly priced subscriptions, journals should be making these findings easily accessible so that they may be discussed and retested by the scientific community and available to the public.

While changes such as statistical stringency, public peer review, and open-access articles are readily implementable, other changes in research culture will enhance the robustness of published findings, but will require a larger community effort. Journals should require researchers to increase transparency and meet certain criteria before submitting a manuscript for consideration. The Nature Publishing Group recently updated its editorial policies [67], requiring authors to fill out a checklist that accompanies their submitted manuscript. The checklist addresses areas of manuscripts, such as study design and analysis, which the editors have noticed are often not reported completely. The checklist also lends particular emphasis to justifying statistical analyses; the Nature Publishing Group will now consult with statisticians if there seem to be glaring analytic issues or if a referee suggests outside consultation. Other journals should adopt a similar strategy. 
Retractions of genome-wide association and next-generation sequencing studies have been limited and should remain as such. To further increase the responsibility of journals in helping prevent retractions, the genetics community could establish a retraction index (similar to impact factor) for each journal. Such an index would track retraction rate and thus encourage journal editors to rigorously review findings and pursue a thorough peer-review process. Of course, researchers must take on greater responsibility for retractions, as well. Currently, retraction notices can be limited in information or completely ambiguous [34]. Instead, authors should be required to provide retraction notices that detail the steps that led to the retraction. The notice should be published as a brief article for the journal's readership to see and appended to the original manuscript. Requiring a detailed notice will encourage authors to be particularly critical of their own work and may help deter future retractions (Figure 4c).

Journals should also work to rectify publication bias towards novel findings (Figure 4d). A study on de novo variation in Autism Spectrum Disorder (which did not discover any disease-susceptibility loci) extensively investigated population stratification in rare variants and the effects of different meta-analysis approaches on power [68]. A recent WES paper on Type 2 Diabetes also did not discover disease-associated genes, but performed a host of analyses to determine likely etiological architecture [69]. Such findings should be made accessible to the entire community, as they can clarify our understanding of the genetic architecture of complex traits and improve future studies.

Journals can also be used to educate the scientific community in areas where it is lacking, such as applications of statistics and data interpretation. Three papers published together in the Journal of the American Medical Association explain how to interpret genome-wide association studies [70-72]. This fall, Nature Methods began publishing the column Points of Significance [73-76], which addresses important statistical concepts such as $p$-values, significance, and the relationship between sample size and power. Nature also recently ran a column that discussed the role of bias, the inexact nature of measurements, and the important distinction between correlation and causation [77]. Such articles do a great community service and will remain invaluable teaching tools to the many genetics researchers without formal training in statistics, allowing them to evaluate claims of novelty, both in their work and the work of others.

Of course, in addition to all of these larger changes, the field will hugely benefit from changes on a smaller scale. Senior scientists should encourage younger members of the field to conduct rigorous experiments, remain vigilant to prevent error, and seek additional help when in doubt about an analysis or result. Mistakes or "negative" findings should be met with discussion about how to improve a scientific question or refine an experiment. Randy Schekman, who won the Nobel Prize for physiology or medicine in 2013, has recently sparked public debate by announcing that he and all members of his lab will no longer be publishing in Nature, Science, and Cell [78]. Schekman's announcement, while controversial because of its potentially detrimental effects on the careers of his younger lab members, has an important motivation: to publicly address the adverse effects that publication bias and lack of open-access have had on the field. Making younger scientists aware of the aspects of scientific culture that are damaging to the quality of published and public science and prompting them to push for change can only alter the field for the better. 
Figure 4. Trending topics on PubMed, 2000-2013. The cumulative number of times certain phrases appear per one million abstracts in PubMed. (a) Earlier genetic association studies (search terms: "candidate gene"; "linkage analysis" or "linkage study"; "genome-wide association study"). (b) Next-generation sequencing studies (search terms: "exome sequencing"; "whole-genome sequencing"). (c) Retraction notices (search terms: "retraction notice" or "notice of retraction"). (d) Claims of novelty and statistical significance (search terms: "novel"; "not statistically significant" or "not significant"; "statistically significant" removing "not statistically significant").

a

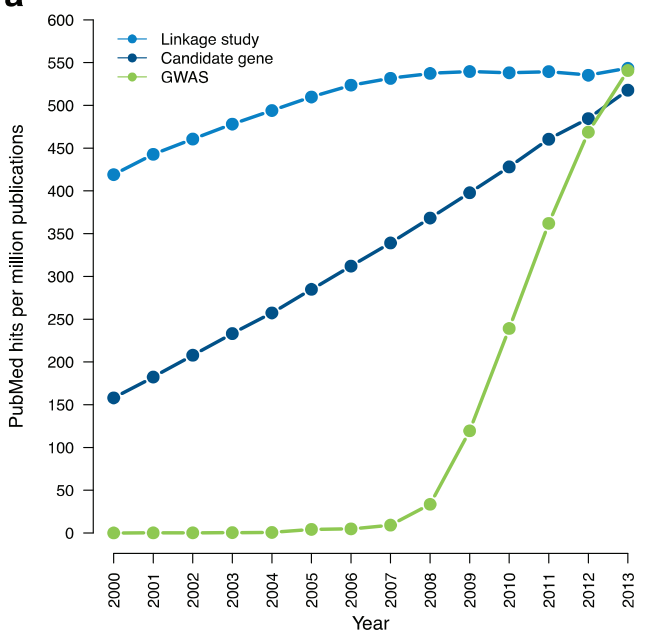

C



b



d

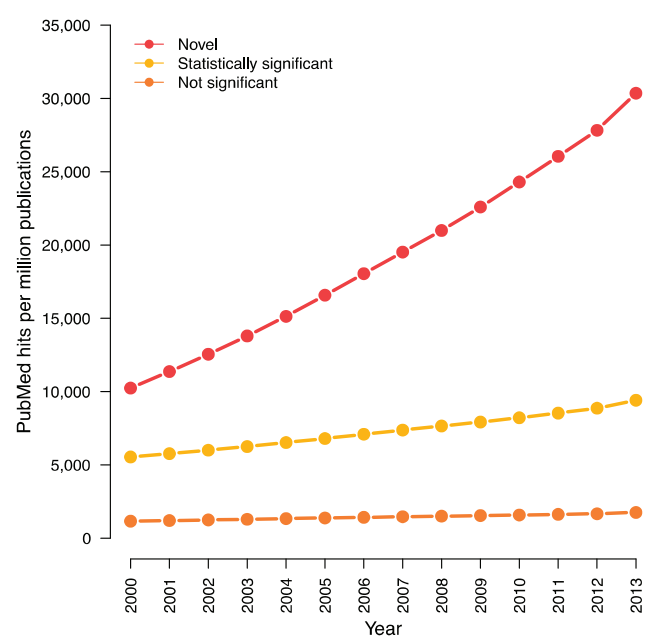

Fortunately, science in general and genetics in particular has proven to be a highly adaptive field. Peer-review is a relatively new process, becoming standard practice for most scientific journals in the second half of the 20th century [79]. In less than a decade, the foundation of the 
Public Library of Science (PLoS) has helped to revolutionize publishing in science, providing the scientific community with a family of open-access journals and encouraging other journals to follow suit in its pursuit of freely available scientific literature [80]. In genetics specifically, the community acknowledged that candidate gene studies were poorly performing and established standards for more robust genome-wide association studies. Again, the tide has begun to shift as communities to discuss pre-prints continue to grow [81,82], researchers increasingly use social media to discuss many of the issues addressed here, and journals begin altering their editorial and publishing processes. With a concerted effort to improve published work, the field of human genetics is capable of permanent change.

\section{Acknowledgements}

The authors would like to thank Ron Do and Jan Veldink for helpful conversations during the writing of this manuscript.

\section{Author Contributions}

Conceived and wrote the paper: Sara L. Pulit, Maarten Leusink, Androniki Menelaou and Paul I. W. de Bakker.

\section{Conflicts of Interest}

The authors declare no conflict of interest.

\section{References}

1. Lander, E.S.; Linton, L.M.; Birren, B.; Nusbaum, C.; Zody, M.C.; Baldwin, J.; Devon, K.; Dewar, K.; Doyle, M.; FitzHugh, W.; et al. Initial sequencing and analysis of the human genome. Nature 2001, 409, 860-921.

2. Clinton, W.J. Remarks made by the President on the Completion of the First Survey of the Entire Human Genome Project. The White House Office of the Press Secretary: Washington, DC, USA, 2014; pp. 1-7.

3. Hirschhorn, J.N.; Lohmueller, K.; Byrne, E.; Hirschhorn, K. A comprehensive review of genetic association studies. Genet. Med. 2002, 4, 45-61.

4. Hirschhorn, J.N.; Altshuler, D. Once and again-issues surrounding replication in genetic association studies. J. Clin. Endocrinol. Metab. 2002, 87, 4438-4441.

5. Ioannidis, J.P.; Ntzani, E.E.; Trikalinos, T.A.; Contopoulos-Ioannidis, D.G. Replication validity of genetic association studies. Nat. Genet. 2001, 29, 306-309.

6. Kathiresan, S.; Newton-Cheh, C.; Gerszten, R.E. On the interpretation of genetic association studies. Eur. Heart J. 2004, 25, 1378-1381.

7. Lohmueller, K.E.; Pearce, C.L.; Pike, M.; Lander, E.S.; Hirschhorn, J.N. Meta-analysis of genetic association studies supports a contribution of common variants to susceptibility to common disease. Nat. Genet. 2003, 33, 177-182. 
8. Hirschhorn, J.N.; Daly, M.J. Genome-wide association studies for common diseases and complex traits. Nat. Rev. Genet. 2005, 6, 95-108.

9. The International HapMap Consortium. A haplotype map of the human genome. Nature 2005, 437, 1299-1320.

10. Wang, W.Y.S.; Barratt, B.J.; Clayton, D.G.; Todd, J.A. Genome-wide association studies: Theoretical and practical concerns. Nat. Rev. Genet. 2005, 6, 109-118.

11. Dudbridge, F.; Gusnanto, A. Estimation of significance thresholds for genomewide association scans. Genet. Epidemiol. 2008, 32, 227-234.

12. Pe'er, I.; Yelensky, R.; Altshuler, D.; Daly, M.J. Estimation of the multiple testing burden for genomewide association studies of nearly all common variants. Genet. Epidemiol. 2008, 32, 381-385.

13. Price, A.L.; Patterson, N.J.; Plenge, R.M.; Weinblatt, M.E.; Shadick, N.A.; Reich, D. Principal components analysis corrects for stratification in genome-wide association studies. Nat. Genet. 2006, 38, 904-909.

14. Ioannidis, J.P.A.; Boffetta, P.; Little, J.; O’Brien, T.R.; Uitterlinden, A.G.; Vineis, P.; Balding, D.J.; Chokkalingam, A.; Dolan, S.M.; Flanders, W.D.; et al. Assessment of cumulative evidence on genetic associations: interim guidelines. Int. J. Epidemiol. 2008, 37, $120-132$.

15. Kraft, P.; Zeggini, E.; Ioannidis, J.P.A. Replication in genome-wide association studies. Stat. Sci. 2009, 24, 561-573.

16. Chanock, S.J.; Manolio, T.; Boehnke, M.; Boerwinkle, E.; Hunter, D.J.; Thomas, G.; Hirschhorn, J.N.; Abecasis, G.; Altshuler, D.; Bailey-Wilson, J.E.; et al. Replicating genotype-phenotype associations. Nature 2007, 447, 655-660.

17. Hindorff, L.A.; MacArthur, J.; Morales, J.; Junkins, H.A.; Hall, P.N.; Klemm, A.K.; Manolio, T.A. A Catalog of Published Genome-Wide Association Studies. Available online: www.genome.gov/gwastudies/ (accessed on 2 December 2013).

18. Visscher, P.M.; Brown, M.A.; McCarthy, M.I.; Yang, J. Five years of GWAS discovery. Am. J. Hum. Genet. 2012, 90, 7-24.

19. Sebastiani, P.; Solovieff, N.; Puca, A.; Hartley, S.W.; Melista, E.; Andersen, S.; Dworkis, D.A.; Wilk, J.B.; Myers, R.H.; Steinberg, M.H.; et al. Genetic signatures of exceptional longevity in humans. Science 2010, doi:10.1126/science.1190532.

20. Carmichael, M. The little flaw in the longevity-gene study that could be a big problem. Available online: http:/www.newsweek.com/little-flaw-longevity-gene-study-could-be-bigproblem-74703/ (accessed on 10 December 2013).

21. Sebastiani, P.; Solovieff, N.; Puca, A.; Hartley, S.W.; Melista, E.; Andersen, S.; Dworkis, D.A.; Wilk, J.B.; Myers, R.H.; Steinberg, M.H.; et al. Letters: Retraction. Science 2011, 333, 404.

22. Unreliable research: Trouble at the Lab. Economist 2013, 409, 27.

23. Problems with scientific reasearch: How science goes wrong. Economist 2013, 409, 12.

24. Neaves, W. The roots of research misconduct. Nature 2012, 488, 121-122.

25. Macilwain, C. The time is right to confront misconduct. Nature 2012, 488, 7. 
26. Corbyn, Z. Misconduct is the main cause of life-sciences retractions. Nature 2012, 490, 21.

27. Macilwain, C. Scientific misconduct: More cops, more robbers? Cell 2012, 149, 1417-1419.

28. Yong, E.; Ledford, H.; van Noorden, R. Research ethics: 3 ways to blow the whistle. Nature 2013, 503, 454-457.

29. The Wellcome Trust Case Control Consortium. Genome-wide association study of 14,000 cases of seven common diseases and 3000 shared controls. Nature 2007, 447, 661-678.

30. Zheng, G.; Freidlin, B.; Gastwirth, J.L. Robust genomic control for association studies. Am. J. Hum. Genet. 2006, 78, 350-356.

31. Clayton, D.G.; Walker, N.M.; Smyth, D.J.; Pask, R.; Cooper, J.D.; Maier, L.M.; Smink, L.J.; Lam, A.C.; Ovington, N.R.; Stevens, H.E.; et al. Population structure, differential bias and genomic control in a large-scale, case-control association study. Nat. Genet. 2005, 37, 1243-1246.

32. Plagnol, V.; Cooper, J.D.; Todd, J.A.; Clayton, D.G. A method to address differential bias in genotyping in large-scale association studies. PLoS Genet. 2007, 3, e74.

33. Steen, R.G. Retractions in the scientific literature: Is the incidence of research fraud increasing?

J. Med. Ethics 2011, 37, 249-253.

34. Fang, F.C.; Steen, R.G.; Casadevall, A. Misconduct accounts for the majority of retracted scientific publications. Proc. Natl. Acad. Sci. USA 2012, 109, 17028-17033.

35. Ioannidis, J.P.A. Why most published research findings are false. PLoS Med. 2005, 2, e124.

36. Young, N.S.; Ioannidis, J.P.A.; Al-Ubaydli, O. Why current publication practices may distort science. PLoS Med. 2008, 5, e201.

37. Ioannidis, J.P.A. Concentration of the most-cited papers in the scientific literature: Analysis of journal ecosystems. PLoS One 2006, 1, e5.

38. Bjornshauge, L.; Brage, S.; Mitchell, D.; Zeylon, R. Directory of Open Access Journals. Available online: www.doaj.org/ (accessed on 23 December 2013).

39. Bohannon, J. Who's afraid of peer review? Science 2013, 342, 60-65.

40. Stahl, E.A.; Wegmann, D.; Trynka, G.; Gutierrez-Achury, J.; Do, R.; Voight, B.F.; Kraft, P.; Chen, R.; Kallberg, H.J.; Kurreeman, F.A.S.; et al. Bayesian inference analyses of the polygenic architecture of rheumatoid arthritis. Nat. Genet. 2012, 44, 483-489.

41. Hemani, G.; Yang, J.; Vinkhuyzen, A.; Powell, J.E.; Willemsen, G.; Hottenga, J.-J.; Abdellaoui, A.; Mangino, M.; Valdes, A.M.; Medland, S.E.; et al. Inference of the genetic architecture underlying BMI and height with the use of 20,240 sibling pairs. Am. J. Hum. Genet. 2013, 93, 865-875.

42. DePristo, M.A.; Banks, E.; Poplin, R.; Garimella, K.V.; Maguire, J.R.; Hartl, C.; Philippakis, A.A.; del Angel, G.; Rivas, M.A.; Hanna, M.; et al. A framework for variation discovery and genotyping using next-generation DNA sequencing data. Nat. Genet. 2011, 43, 491-498. 
43. McKenna, A.; Hanna, M.; Banks, E.; Sivachenko, A.; Cibulskis, K.; Kernytsky, A.; Garimella, K.; Altshuler, D.; Gabriel, S.; Daly, M.; et al. The Genome Analysis Toolkit: A MapReduce framework for analyzing next-generation DNA sequencing data. Genome Res. 2010, 20, 1297-1303.

44. Do, R.; Kathiresan, S.; Abecasis, G.R. Exome sequencing and complex disease: Practical aspects of rare variant association studies. Hum. Mol. Genet. 2012, 21, R1-R9.

45. Mathieson, I.; McVean, G. Differential confounding of rare and common variants in spatially structured populations. Nat. Genet. 2012, 44, 243-246.

46. Kiezun, A.; Garimella, K.; Do, R.; Stitziel, N.O.; Neale, B.M.; McLaren, P.J.; Gupta, N.; Sklar, P.; Sullivan, P.F.; Moran, J.L.; et al. Exome sequencing and the genetic basis of complex traits. Nat. Genet. 2012, 44, 623-630.

47. Li, B.; Leal, S.M. Discovery of rare variants via sequencing: Implications for the design of complex trait association studies. PLoS Genet. 2009, 5, e1000481.

48. MacArthur, D.G.; Balasubramanian, S.; Frankish, A.; Huang, N.; Morris, J.; Walter, K.; Jostins, L.; Habegger, L.; Pickrell, J.K.; Montgomery, S.B.; et al. A systematic survey of loss-of-function variants in human protein-coding genes. Science 2012, 335, 823-828.

49. Kryukov, G.V.; Shpunt, A.; Stamatoyannopoulos, J.A.; Sunyaev, S.R. Power of deep, all-exon resequencing for discovery of human trait genes. Proc. Natl. Acad. Sci. USA 2009, 106, 3871-3876.

50. Chapman, J.M.; Cooper, J.D.; Todd, J.A.; Clayton, D.G. Detecting disease associations due to linkage disequilibrium using haplotype tags: A class of tests and the determinants of statistical power. Hum. Hered. 2003, 56, 18-31.

51. Scott-Van Zeeland, A.A.; Bloss, C.S.; Tewhey, R.; Bansal, V.; Torkamani, A.; Libiger, O.; Duvvuri, V.; Wineinger, N.; Galvez, L.; Darst, B.F.; et al. Evidence for the role of EPHX2 gene variants in anorexia nervosa. Mol. Psychiatry 2013, doi:10.1038/mp.2013.91.

52. Dyment, D.A.; Cader, M.Z.; Chao, M.J.; Lincoln, M.R.; Morrison, K.M.; Disanto, G.; Morahan, J.M.; de Luca, G.C.; Sadovnick, A.D.; Lepage, P.; et al. Exome sequencing identifies a novel multiple sclerosis susceptibility variant in the TYK2 gene. Neurology 2012, 79, 406-411.

53. Ban, M.; Goris, A.; Lorentzen, A.R.; Baker, A.; Mihalova, T.; Ingram, G.; Booth, D.R.; Heard, R.N.; Stewart, G.J.; Bogaert, E.; et al. Replication analysis identifies TYK2 as a multiple sclerosis susceptibility factor. Eur. J. Hum. Genet. 2009, 17, 1309-1313.

54. Australia and New Zealand Multiple Sclerosis Genetics Consortium. Genome-wide association study identifies new multiple sclerosis susceptibility loci on chromosomes 12 and 20. Nat. Genet. 2009, 41, 824-828.

55. Abecasis, G.R.; Auton, A.; Brooks, L.D.; DePristo, M.A.; Durbin, R.M.; Handsaker, R.E.; Kang, H.M.; Marth, G.T.; McVean, G.A. An integrated map of genetic variation from 1092 human genomes. Nature 2012, 491, 56-65.

56. Erdmann, J.; Stark, K.; Esslinger, U.B.; Rumpf, P.M.; Koesling, D.; de Wit, C.; Kaiser, F.J.; Braunholz, D.; Medack, A.; Fischer, M.; et al. Dysfunctional nitric oxide signalling increases risk of myocardial infarction. Nature 2013, 504, 432-436. 
57. Flannick, J.; Beer, N.L.; Bick, A.G.; Agarwala, V.; Molnes, J.; Gupta, N.; Burtt, N.P.; Florez, J.C.; Meigs, J.B.; Taylor, H.; et al. Assessing the phenotypic effects in the general population of rare variants in genes for a dominant Mendelian form of diabetes. Nat. Genet. 2013, 45, 1380-1385.

58. Chesi, A.; Staahl, B.T.; Jovičić, A.; Couthouis, J.; Fasolino, M.; Raphael, A.R.; Yamazaki, T.; Elias, L.; Polak, M.; Kelly, C.; et al. Exome sequencing to identify de novo mutations in sporadic ALS trios. Nat. Neurosci. 2013, 16, 851-855.

59. Neale, B.M.; Kou, Y.; Liu, L.; Ma'ayan, A.; Samocha, K.E.; Sabo, A.; Lin, C.-F.; Stevens, C.; Wang, L.-S.; Makarov, V.; et al. Patterns and rates of exonic de novo mutations in autism spectrum disorders. Nature 2012, 485, 242-245.

60. Gratten, J.; Visscher, P.M.; Mowry, B.J.; Wray, N.R. Interpreting the role of de novo protein-coding mutations in neuropsychiatric disease. Nat. Genet. 2013, 45, 234-238.

61. Yang, J.; Duan, S.; Zhong, R.; Yin, J.; Pu, J.; Ke, J.; Lu, X.; Zou, L.; Zhang, H.; Zhu, Z.; et al. Exome sequencing identified NRG3 as a novel susceptible gene of Hirschsprung's disease in a Chinese population. Mol. Neurobiol. 2013, 47, 957-966.

62. Lander, E.; Kruglyak, L. Genetic dissection of complex traits: Guidelines for interpreting and reporting linkage results. Nat. Genet. 1995, 11, 241-247.

63. Cruchaga, C.; Karch, C.M.; Jin, S.C.; Benitez, B.A.; Cai, Y.; Guerreiro, R.; Harari, O.; Norton, J.; Budde, J.; Bertelsen, S.; et al. Rare coding variants in the phospholipase D3 gene confer risk for Alzheimer's disease. Nature 2013, 505, 550-554.

64. Panoutsopoulou, K.; Tachmazidou, I.; Zeggini, E. In search of low-frequency and rare variants affecting complex traits. Hum. Mol. Genet. 2013, 22, R16-R21.

65. Pulit, S.L.; Voight, B.F.; de Bakker, P.I.W. Multiethnic genetic association studies improve power for locus discovery. PLoS One 2010, 5, e12600.

66. Pulverer, B. Transparency showcases strength of peer review. Nature 2010, 468, 29-31.

67. Nature Neuroscience Editors. Raising standards. Nat. Neurosci. 2013, 16, 517.

68. Liu, L.; Sabo, A.; Neale, B.M.; Nagaswamy, U.; Stevens, C.; Lim, E.; Bodea, C.A.; Muzny, D.; Reid, J.G.; Banks, E.; et al. Analysis of rare, exonic variation amongst subjects with autism spectrum disorders and population controls. PLoS Genet. 2013, 9, e1003443.

69. Lohmueller, K.E.; Sparsø, T.; Li, Q.; Andersson, E.; Korneliussen, T.; Albrechtsen, A.; Banasik, K.; Grarup, N.; Hallgrimsdottir, I.; Kiil, K.; et al. Whole-exome sequencing of 2000 danish individuals and the role of rare coding variants in type 2 diabetes. Am. J. Hum. Genet. 2013, 93, 1072-1086.

70. Attia, J.; Ioannidis, J.P.A.; Thakkinstian, A.; McEvoy, M.; Scott, R.J.; Minelli, C.; Thompson, J.; Infante-Rivard, C.; Guyatt, G. How to use an article about genetic association: A: Background concepts. JAMA 2009, 301, 74-81.

71. Attia, J.; Ioannidis, J.P.A.; Thakkinstian, A.; McEvoy, M.; Scott, R.J.; Minelli, C.; Thompson, J.; Infante-Rivard, C.; Guyatt, G. How to use an article about genetic association: B: Are the results of the study valid? JAMA 2009, 301, 191-197. 
72. Attia, J.; Ioannidis, J.P.A.; Thakkinstian, A.; McEvoy, M.; Scott, R.J.; Minelli, C.; Thompson, J.; Infante-Rivard, C.; Guyatt, G. How to use an article about genetic association: C: What are the results and will they help me in caring for my patients? JAMA 2009, 301, 304-308.

73. Krzywinski, M.; Altman, N. Points of significance: Importance of being uncertain. Nat. Methods 2013, 10, 809-810.

74. Krzywinski, M.; Altman, N. Points of significance: error bars. Nat. Methods 2013, 10, 921-922.

75. Krzywinski, M.; Altman, N. Points of significance: Significance, $\mathrm{P}$ values and t-tests. Nat. Methods 2013, 10, 1041-1042.

76. Krzywinski, M.; Altman, N. Points of significance: Power and sample size. Nat. Methods 2013, 10, 1139-1140.

77. Sutherland, W.J.; Spiegelhalter, D.; Burgman, M.A. Policy: Twenty tips for interpreting scientific claims. Nature 2013, 503, 335-337.

78. Schekman, R. "How journals like Nature, Cell and Science are damaging science." Available online: http://www.theguardian.com/commentisfree/2013/dec/09/how-journals-nature-sciencecell-damage-science/ (accessed on 20 December 2013).

79. Chapelle, F.H. The history and practice of peer review. Ground Water 2014, 52, 1.

80. Van Noorden, R. Open access: The true cost of science publishing. Nature 2013, 495, 426-429.

81. Coop, G.; Howie, B.; Pickrell, J. Haldane's Sieve. Available online: haldanessieve.org/ (accessed on 20 January 2014).

82. Cornell University Library. arXiv. Available online: www.arxiv.org/ (accessed on 20 January 2014). 


\section{Genetic Profiling for Risk Reduction in Human Cardiovascular Disease}

\section{Megan J. Puckelwartz and Elizabeth M. McNally}

Abstract: Cardiovascular disease is a major health concern affecting over 80,000,000 people in the U.S. alone. Heart failure, cardiomyopathy, heart rhythm disorders, atherosclerosis and aneurysm formation have significant heritable contribution. Supported by familial aggregation and twin studies, these cardiovascular diseases are influenced by genetic variation. Family-based linkage studies and population-based genome-wide association studies (GWAS) have each identified genes and variants important for the pathogenesis of cardiovascular disease. The advent of next generation sequencing has ushered in a new era in the genetic diagnosis of cardiovascular disease, and this is especially evident when considering cardiomyopathy, a leading cause of heart failure. Cardiomyopathy is a genetically heterogeneous disorder characterized by morphologically abnormal heart with abnormal function. Genetic testing for cardiomyopathy employs gene panels, and these panels assess more than 50 genes simultaneously. Despite the large size of these panels, the sensitivity for detecting the primary genetic defect is still only approximately $50 \%$. Recently, there has been a shift towards applying broader exome and/or genome sequencing to interrogate more of the genome to provide a genetic diagnosis for cardiomyopathy. Genetic mutations in cardiomyopathy offer the capacity to predict clinical outcome, including arrhythmia risk, and genetic diagnosis often provides an early window in which to institute therapy. This discussion is an overview as to how genomic data is shaping the current understanding and treatment of cardiovascular disease.

Reprinted from Genes. Cite as: Puckelwartz, M.J.; McNally, E.M. Genetic Profiling for Risk Reduction in Human Cardiovascular Disease. Genes 2014, 5, 214-234.

\section{Introduction}

Next generation sequencing has revolutionized the study of human genome variation and has the capacity to greatly influence health care decision making. The Human Genome Project, as conceived, was to sequence the first human genome in $\sim 15$ years at a cost of almost $\$ 3$ billion using traditional dideoxy chain termination sequencing. In under 10 years, massively parallel next generation sequencing has led to the routine sequencing of exomes and whole genomes. Now achieved in weeks and at a cost that is multiple orders of magnitude less than the first genome, personalized genetic information is now widely available. These rapid advances in sequencing technology require new ways of collecting, analyzing, and disseminating genomic data. Herein, we discuss the ways that genomic information is currently being applied and how that data is shaping the ability to understand and treat cardiovascular disease (CVD).

Genetic variation is considered a contributory component for nearly all disease, whether single gene familial disorders or more common, complex traits with multiple gene involvement. Single gene or "Mendelian" disorders can be attributed to one gene as both necessary and sufficient to cause a 
large component of the disease phenotype. With complex traits, the gene-gene and gene-environment interactions are multifactorial. CVD consists of both single gene-familial disorders and common, complex disease. CVD is a major health concern affecting over $80,000,000$ people in the U.S. alone [1]. CVD extends to heart failure and cardiomyopathy, heart rhythm disorders, atherosclerosis and thromboembolic events, aneurysm and others disorders. Familial aggregation and twin studies demonstrate that most, if not all, of CVD is heavily influenced by a genetic component [2-4].

\section{Genetic Variation in CVD}

Beginning in the 1980s, family-based linkage analysis was used to identify regions of the genome responsible for monogenic disease. The success of these methods required large families with penetrant phenotypes. Polymorphic genetic markers segregated with the phenotype of interest in large multi-generational families to identify chromosomal regions bearing the causal genes of interest [5]. Such familial linkage studies were highly successful in identifying genes for multiple forms of CVD. In 1989, linkage analysis defined the chromosomal location responsible for hypertrophic cardiomyopathy [6]. The next year, this data was used to identify mutations in the causative gene, MYH7, encoding $\beta$-myosin heavy chain [7]. Genetic determinants for Long QT syndrome, multiple cardiomyopathies, Marfan's disease, and forms of congenital heart disease were identified highlighting both extensive locus and allelic heterogeneity [8-14]. However, these methods remain limited by the need for large families, a feature often not available since CVD confers survival disadvantage. Furthermore, much of CVD is under the influence of multiple genetic loci, and therefore requires alternative statistical methods and larger phenotypically and genetically characterized cohorts [15]. The HapMap project annotated the location of millions of single-nucleotide polymorphisms (SNPs) and took advantage of the long haplotype structure of the human genome [16]. Concurrently, commercially available platforms such as SNP arrays were developed that allowed simultaneous sampling of hundreds of thousands of SNPs paving the path for genome-wide association studies (GWAS). GWAS, which correlates SNPs with disease phenotypes, does not require a specific mode of inheritance and takes advantage of the extensive linkage disequilibrium (LD) in the human genome. In order to have enough statistical power to detect correlation, these large-scale association studies typically assess thousands to millions of SNPs across the genome in hundreds to thousands of cases and controls. According to the National Human Genome Research Institute (NHGRI) Catalog of Published Genome-Wide Association Studies [17] over 2800 strong SNP associations have been identified $\left(p<1 \times 10^{-8}\right)$ to date, and many of these are CVD-associated traits.

\subsection{The Overlap between GWAS Hits and Monogenic Disease in CVD}

CVD related phenotypes are well suited for GWAS because many CVDs have readily quantifiable discernable traits. Intriguingly, many GWAS "hits" overlap considerably with the same genes already linked to the disease though familial linkage studies. For example, Newton-Cheh and colleagues conducted a meta-analysis of three GWAS from $\sim 14,000$ individuals to examine the 
duration of QT interval from surface electrocardiograms [18]. QT duration reflects electrical depolarization and repolarization of the cardiac ventricles. A long QT interval is a biomarker for arrhythmias and a risk factor for sudden death. Non-familial QT disorders are still highly heritable $\left(\mathrm{h}^{2} \approx 0.35\right)$, indicating a genetic component. GWAS identified 10 loci with significant $\left(p<5 \times 10^{-8}\right)$ association with QT interval. Five loci were those known to be involved in Mendelian long-QT syndromes, while the other five loci were genes that offer additional insights into variation at the QT interval. In total, the variation at these 10 loci accounted for $5.4 \%-6.5 \%$ of variation in the QT interval, which is quite high by GWAS standards. Genetic testing for the Mendelian form of long QT currently identifies mutations in $\sim 75 \%$ of probands, so the additional GWAS loci may represent new candidate genes for mutation screening in familial long QT disorders.

$B A G 3$ (B-cell lymphoma 2-associated athanogene 3) is another example of GWAS results informing rare, Mendelian disease. In 2011, Villard and colleagues performed GWAS to identify loci contributing to sporadic dilated cardiomyopathy. Dilated cardiomyopathy (DCM) is exemplified by left ventricular dilation and systolic dysfunction, and is a major cause of heart failure and the principle indicator for heart transplant [19]. DCM has a high heritable component with $20 \%-35 \%$ of DCM patients having an affected first-degree relative [20]. More than 50 genes have been implicated in familial monogenetic DCM [21-23]. GWAS was performed with DNA from 1179 sporadic (non-familial) DCM patients and 1108 controls using 500,000 SNPs [19]. The authors identified a DCM-associated non-synonymous SNP (p. C151R) in the coding region of $B A G 3$. BAG3 is a co-chaperone that regulates HSP70 [24]. Further analysis of $B A G 3$ non-synonymous SNPs found another higher frequency SNP also associated with DCM. The authors investigated $B A G 3$ variation in familial DCM based on both the apparent association of $B A G 3$ non-synonymous SNPs with sporadic DCM and previously reported linkage with familial DCM between markers on chromosome 10 that include the $B A G 3$ locus [25]. In a cohort of 168 cases from DCM families, the authors identified additional likely pathogenic mutations in $B A G 3$. Features of DCM were identified in 16 of 18 mutation carriers in the cohort [19]. In the same year, Norton and colleagues identified $B A G 3$ mutations in familial DCM [26]. The authors also created a $B A G 3$ knockdown zebrafish model that recapitulated the DCM and heart failure found in patients [26]. Together, the GWAS and familial data implicate BAG3 in DCM and indicate that genes can harbor common variation that influences risk of disease in sporadic cases and rare variation that accounts for familial disease.

\subsection{The Missing Heritability of GWAS}

Despite the overlap of GWAS findings with monogenic disease, GWAS associations often account for only a small proportion of genetic variation. Also, the vast majority of variants identified by GWAS does not explain the high heritability or reveal the causal mechanism for the cardiovascular phenotype in question [27-29]. Using GWAS, McPherson and colleagues identified an interval on chromosome 9q21 that consistently associated with coronary heart disease in more than 23,000 participants from 6 independent cohorts [30]. Homozygotes for the risk allele have a $30 \%-40 \%$ increased risk for coronary artery disease (CAD). This finding remains perplexing as this region on 9q21 has no annotated genes and is not associated with known CAD risk factors [30]. 
This same chromosome 9q21 region has also been associated with myocardial infarction in a GWAS with 4587 cases and 12,676 controls [31]. Homozygotes for the allele have 1.64 times increased risk for myocardial infarction compared to noncarriers. Despite the evidence that this region is important for CVD pathophysiology, no disease mechanism has yet been identified.

GWAS is ultimately based on the idea that common diseases are caused by common genetic variants, each with small effect. Through additive and interactive effects, in conjunction with the environment, GWAS variants explain disease [27,32,33]. Recently, the common disease common variant hypothesis has been called into question due to the observation of missing heritability $[34,35]$. Missing heritability refers to the proportion of genetic variance that is not explained by the effect of common variants identified by GWAS. Several explanations have been suggested to explain missing heritability. It is possible that the number of variants responsible for a trait has been significantly underestimated, and that many more yet identified variants with very small effect sizes must be discovered. Another possibility is the presence of rare variants with larger effect size. Such rare variants are undetectable using present day SNP arrays, which are biased towards SNPs with allele frequencies close to $50 \%$. There is also a possibility that missing heritability arises from variation caused by structural variants in the genome, also difficult to detect with SNP arrays. Lastly, there is also the possibility that gene-gene interactions and gene-environment interactions are of major importance, but are not appropriately modeled by current methods.

GWAS typically interrogate SNPs with a minor allele frequency (MAF) $>5 \%$ while largely ignoring variants with lower population-based frequencies (MAF: $0.5 \%-5 \%$ ) and those SNPs that are rare $(\mathrm{MAF}<0.5 \%)$. The rationale for ignoring these variants relates to the strong linkage disequilibrium (LD) in the human genome. The human genome has an estimated mutation rate of approximately $1.4 \times 10^{-8}$ or approximately 40 new mutations per generation. Projected over the current population of $\sim 7$ billion, the world currently has 300-400 million new mutations this generation [36]. Within the coding region alone, there are $\sim 13,000$ nonsynonymous variants per genome [37,38]. The National Heart Lung and Blood Institute sponsored exome sequencing project (NHLBI ESP) sequenced $\sim 15,000$ human-protein coding genes in $>2000$ individuals [39]. This study revealed an abundance of rare variants that were often population specific, potentially offering some support that rare variation explains some component of missing heritability. Alleles that confer high risk of disease are subject to negative selection pressure and would not reach high population frequencies. However, many different rare alleles in the same genes or gene pathways would induce the same phenotype across the population despite the "rare" genetic etiology. Johansen and colleagues recently performed GWAS and resequencing to determine mutational burden of rare variants in individuals with hypertriglyceridemia versus control subjects [40]. Hypertriglyceridemia is polygenic in nature and confers risk for cardiovascular disease. In general, GWAS variants explain $<10 \%$ of variation for lipid traits $[41,42]$. Loci associated with GWAS signals were assessed for rare variation by focusing on protein-coding sequences of four GWAS-associated genes [40]. The authors identified a significant number of rare variants in individuals with hypertriglyceridemia compared to controls. An additional more restricted study, which analyzed only rare variants unique to cases and removing all reported variants without functional deficits, also confirmed a greater mutational burden in cases compared to controls [40]. 
These data indicate that GWAS-identified genes may carry rare variation that contributes to the heritability of a complex trait, and rare SNPs with relatively large effects on common disease may not be identified by GWAS studies. The underlying assumption that genotypes can be inferred between common alleles in strong LD may be flawed. Inferring genotypes based on strong LD may misestimate variation across the genome. While haplotype structure may be maintained with several to many common SNPs still inherited together, rare variation may occur between these SNPs, reducing the predictive value of the common SNPs. This rare variation may be the result of a higher than expected mutation rate and/or population structure.

\subsection{Rare Variation as a Cause of CVD}

Next generation sequencing (NGS) provides a method to identify rare genetic variation. Massively parallel, array-based sequencing dramatically reduced cost and increased efficiency of DNA sequencing. Depending on the platform, sequencing reads range from $\sim 35$ base pairs to up to $\sim 1000$ base pairs. As the generation of sequence has become far more facile with NGS, large-scale alignment and interpretation has become the rate limiting step. Bioinformatics tools are available for alignment to the referent genome and calling variants (reviewed in [43,44]). A number of efforts are currently underway to catalog human genetic variation. The 1000 Genomes project has sequenced 1092 individuals from 14 populations using a combination of low coverage whole genome sequencing and exome sequencing [45]. It is estimated that this dataset captures $98 \%$ of SNPs at a frequency of $1 \%$, finding 38 million SNPs, $59 \%$ of which were novel [45]. The NHLBI ESP provided whole exome sequencing on $>6500$ individuals, including 180,000 exons in 23,000 genes. The ESP is derived from diverse, well-phenotyped populations, including the Atherosclerosis Risk in Communities (ARIC) study, the Coronary Artery Risk Development in Young Adults (CARDIA) study, the Cardiovascular Health Study, the Framingham Heart Study, the Jackson Heart Study, and the Multi-Ethnic Study of Atherosclerosis. The data from this project will examine the genetic contribution to early-onset myocardial infarction, low-density lipoprotein cholesterol, body mass index/type 2 Diabetes mellitus, blood pressure, and ischemic stroke.

These databases are not only providing a rich dataset to identify rare variation, but they are also providing better allele frequencies across diverse populations for alleles once thought to be rare and pathogenic. Jabbari and colleagues examined the NHLBI ESP database $(n=6503)$ for variants previously associated with Catecholaminergic Polymorphic Ventricular Tachycardia (CPVT) and compared the frequency of those variants with the expected prevalence of CPVT in the population [46]. CPVT is a rare, lethal, hereditary cardiac disease characterized by fatal ventricular arrhythmias in the absence of structural defects of the heart or abnormal electrocardiographic findings [47]. Eleven percent of variants previously associated with CPVT were found in the ESP population, corresponding to a 1:150 prevalence of CPVT in the population, much higher than the known 1:10,000 prevalence. The 1000 Genomes $(1 \mathrm{KG})$ database was evaluated for the presence of predicted and previously reported pathogenic variation in three genes associated with cardiomyopathy (MYH7, MYBPC3 and TTN) [48]. Nine percent of the population was identified as having a pathogenic variant $(9 \%)$, which exceeds population prevalence estimates for dilated and hypertrophic cardiomyopathy $(0.04 \%-0.2 \%$, respectively). Similar studies have been performed for 
other cardiovascular diseases including Brugada Syndrome, cardiac channelopathies and arrhythmogenic right ventricular cardiomyopathy [49-51]. Since in every case the predicted pathogenic variation vastly exceeds the known disease prevalence, it suggests that on their own, these variants are not sufficient to cause disease. Whether these variants represent "at risk" genotypes for developing milder forms of disease is not known and requires prospective studies.

\section{The Genetics of Dilated Cardiomyopathy}

Cardiomyopathy is marked by a morphologically abnormal ventricle and frequently is associated with heart failure. Cardiomyopathy is divided into four groups: dilated (DCM), hypertrophic (HCM), restrictive (RCM), and arrhythmogenic right ventricular (ARVC), and the most common mode of inheritance is autosomal dominant. Depending on screening methods, nonischemic DCM, defined as DCM not arising from myocardial infarct or ischemia, is familial in $25 \%-50 \%$ of cases [52]. Greater than 50 genes have been implicated in familial DCM, and the majority of mutations are phenocopies as there are no outward clinical signs that predict the specific gene mutation. Identification of the genetic cause of cardiomyopathy is clinically important because of the high incidence of sudden death and clinical progression, which can be medically managed. In 2007, genetic testing relied on commercially available panels that interrogated only 5 genes using traditional sequencing. In 2011, Meder and colleagues developed an array-based panel that enriched for coding regions of 29 known and 18 novel, potential cardiomyopathy genes, followed by next-generation sequencing [53]. Current panels now include $>50$ genes [54], and the estimated sensitivity in DCM for detecting a pathogenic mutation is under 50\% (Partners Healthcare [55]).

\subsection{Next Generation Sequencing Identifies TTN as a Major Contributor to DCM}

Next-generation sequencing facilitated the screening of TTN for DCM-causing variants. The TTN gene includes $>350$ exons and encodes the giant sarcomere protein titin, which ranges in size from $\sim 27,000$ to $\sim 33,000$ amino acids depending on isoform, making it the largest human protein [56]. Together, two titin molecules span the sarcomere, providing both passive and active contractile forces [57-60]. Previous work has linked TTN to dilated cardiomyopathy in families, but extensive screening was limited due to its large size [61-63]. Herman and colleagues developed an array to capture TTN exons and sequence TTN in patients with DCM $(n=312)$ [64]. TTN truncating mutations accounted for approximately $25 \%$ of familial DCM, but had a minimal contribution to hypertrophic cardiomyopathy ( $1 \%)$. Approximately $30 \%$ of TTN truncating variants identified were putative splice site disrupting mutations whose effect on function can be difficult to assess in silico.

\subsection{Beyond Panel Based Sequencing for Cardiomyopathy and Beyond}

Despite the inclusion of $T T N$, the sensitivity for detecting a DCM mutation remains at just under 50\%. There are several explanations for the missing variation. First, there are likely novel genes not yet associated with cardiomyopathy; second, certain genetic variation may not be readily 
detectable with NGS and SNP analysis. For example, nucleotide repeat expansions and structural variation is not commonly determined by NGS, as analytic methods are biased toward SNP analysis and small insertions and deletions; Third, pathogenic variation may arise from combinations of pathogenic variation, and analysis is generally biased towards finding a single pathogenic variant. This bias reflects that most families with inherited cardiomyopathy have autosomal dominant inheritance; Fourth, pathogenic variation may be non-coding and at this point, these regions are not captured by gene panels. To combat these problems and provide a more comprehensive variant profile, whole exome sequencing (WES) and whole genome sequencing (WGS) are being applied to identify disease-causing variation for many different diseases (Table 1). WES interrogates the coding portion of the genome; approximately $1 \%-2 \%$ of nuclear DNA, although at higher coverage than-comparably priced WGS [65]. Interrogating only a small portion of the genome, as in WES, is less expensive than WGS, and it is currently the most-readily interpreted, as approximately $85 \%$ of Mendelian-disease causing mutations cause changes in the coding sequence of the genome [66]. WES relies on commercially available sequence-capture arrays to enrich for the coding subset of genomic DNA, followed by massively parallel, next-generation sequencing of the enriched fragments. The choice of exome kit is an important consideration as the exome is approximately 30 megabases, and exome capture kits interrogate anywhere from 50 to 100 megabases, depending on the provider. Most of the additional sequences are untranslated regions (UTRs) that may be important for disease pathogenesis.

Table 1. Comparison of Panel, whole exome sequencing (WES) and whole genome sequencing (WGS).

\begin{tabular}{cccc}
\hline & Panel & WES & WGS \\
\hline Variation in Known Genes & yes & yes & yes \\
Novel Gene Identification & no & yes & yes \\
Structural Variation & no & limited & yes \\
Non-coding Variation & no & limited & yes \\
Repeat testing required if first pass negative & yes & yes & no \\
\hline
\end{tabular}

\subsection{Exome Sequencing of Multiple Family Members Improves Identification of}

\section{Pathogenic Variation}

Campbell and colleagues used exome sequencing on three members of a large multi-generational family with classical DCM. After sequencing, variants were filtered by frequency and protein prediction algorithms [67]. Eight potentially causative mutations were shared across the three family members, significantly reducing the potential variants to be considered. Variants were tested for segregation across the other family members and only one variant segregated with disease, TNNT2 R173W, a known cardiomyopathy gene. These data are particularly convincing as the variant segregates across all affected members of the family, including fourth-degree relatives [67]. The underlying assumption with this analysis is that a single variant accounts for disease in all family members, an assumption that may or may not hold true. In 2013, Wells and others used WES in a large, multi-generational family with DCM of unknown etiology [68]. The proband had 
undergone extensive unrevealing panel testing for DCM. The authors selected 3 distantly related affected family members for WES. Distantly related family members that are obligate carriers of the same mutation should share fewer variants by descent than closely related members, allowing for easier filtering of potentially causative variants. Variants were then filtered for rarity, functional significance, conservation and autosomal-dominant inheritance [68]. Variants were also prioritized using the VAAST tool, a probabilistic search tool that combines conservation, amino acid substitution chemistry, and frequency data to build a unified likelihood-framework to identify damaged genes and disease-causing variants [69]. Heterozygous, nonsense, nonsynonymous and splice site variants shared between the 3 affected candidates were filtered based on rare frequency in $1 \mathrm{KG}$ and ESP, leaving 26 candidates for analysis. Wells and colleagues then compared these variants to variants identified in $\sim 70$ exomes previously sequenced by their laboratory. Variants identified in multiple exomes were removed, leaving 2 putative variants. This comparison allowed for removal of false positives that may be inherent to some sequencing platforms and variant calling pipelines. An RBM20 variant was identified in an unrelated patient with familial-DCM, consistent with its role in causing disease. The authors confirmed segregation within the larger pedigree of the variant in $R B M 20$, a recently identified cardiomyopathy gene [70], providing statistical support for RBM20 causing DCM. It is interesting to note that this finding is largely based on frequency in both the general population and in a cohort already sequenced by the laboratory. The authors did perform filtering that considered evolutionary conservation (Genomic Evolutionary Rate Profiling) and functional effects (Polyphen2) but these tools did not reduce the list as extensively as population and cohort frequency combined (8 variants versus 2 variants, respectively $[69,71,72]$. These data indicate that exome sequencing followed by extensive filtering, in conjunction with segregation analysis can identify rare, DCM-causing variation in known cardiomyopathy genes.

\subsection{Identifying Cardiomyopathy Modifier Loci Using Broad Based Sequencing}

With large gene panels or WES/WGS additional, disease-modifying variation can be identified. Intra-familial variability including age of onset, severity and penetrance is a hallmark of DCM, but the loci that modify DCM phenotypes have not been well elucidated [73,74]. Roncarati and colleagues used WES to investigate clinical variability in an extended family with 14 subjects that included four family members with severe DCM that required heart transplant in early adulthood [75]. WES was performed on three severely affected and one unaffected family member. Variants were filtered for rarity, predicted pathogenicity and inheritance. The filtering process left a list of only 28 variants that where further filtered through the Human Phenotype Ontology project, which uses formal ontology to capture phenotypic information to identify relationships between different genes and phenotypes [76]. Through this analysis, the authors identified eight genes with variation associated with Mendelian disease [75], and two of eight LMNA and TTN, were known to cause DCM. A missense LMNA mutation, previously identified in an unrelated DCM patient, was confirmed in all affected family members [77]. A TTN variant was identified in five family members, four of whom were severely affected, while the fifth is likely too young to yet be symptomatic. Doubly heterozygous family members had a more severe clinical course than the $L M N A$-only family members, indicating that the TTN variant modifies the clinical progression [75]. 
This mutational stratification is expected to prove useful for assessing clinical risk and guiding treatment. Broad based sequencing through gene panels or WES/WGS is now positioned to outline the contribution of multiple variants to DCM development.

\subsection{WES/WGS Can Identify New Genes for Cardiomyopathy}

In 2011, Theis and colleagues used genome-wide mapping and exome sequencing in a consanguineous family with autosomal recessive DCM [78]. Genome-wide linkage analysis was performed in nineteen family members and a significant LOD score was identified on chromosome $7 q 21$, in a region containing $>250$ genes. Exome sequencing was performed on 2 affected siblings, and variants were called and filtered without taking into account the linkage peak on 7q21. Synonymous, intergenic and intronic variants were removed from further consideration. Variants were filtered based on presence in $1 \mathrm{KG}$, HapMap, and in the authors' collection of exome sequences. Heterozygous SNPs were excluded due to the autosomal recessive inheritance mode. This extensive filtering left only 3 homozygous missense variants and only 1 was not present in unaffected family members, a mutation in GATADl that maps to the already identified linkage region on 7q21. GATAD1 encodes the GATA zinc finger domain-containing protein 1 which is ubiquitously expressed and is thought to bind to a histone modification site that regulates gene expression [79]. Immunohistochemistry with an antibody to GATAD1 revealed an abnormal staining pattern in the proband heart compared to control heart [78]. These data implicate GATADI in the pathogenesis of DCM indicating that exome sequencing can be used to identify novel DCM genes.

\subsection{Limitations of WES}

While fruitful, exome sequencing does have limitations. There are inherent technical limitations associated with the method. WES requires a capture step, which is limited by design of capture oligonucleotides. Not all genes or exons are adequately annotated and therefore will not be properly included in the methods to capture exons. Furthermore, there can be inconsistencies in capture resulting in poorly covered exons and off-target sequencing. Capture efficiency only approximates $70 \%-80 \%$ in part due to the high GC content of exonic sequence. Probably the most notable limitation is that only $1 \%-2 \%$ of the entire genome is evaluated. Because approximately $85 \%$ of described Mendelian mutations occur in the coding regions of genes, it is assumed that Mendelian disease is more likely to be caused by mutations in protein coding exons than in non-coding sequences. However, over a third of Mendelian diseases reported in OMIM have no known molecular basis. It is reasonable to conclude that some of these missing mutations are either structural variants or that they occur in non-coding regions of the genome, exome sequencing is not suited to interrogate either of these possibilities.

Copy number variants (CNVs) are regions, $>50 \mathrm{bp}$ in length, that differ from the expected diploid status [80]. CNVs are an important component of genomic variation in humans and some contribute to disease including cardiovascular disease [81-83]. A recent study by Norton and colleagues identified a large deletion in $B A G 3$ in a large multi-generational family with DCM [26]. 
Whole exome sequencing was performed on 4 of the affected family members and comparative genomic hybridization was performed on the proband to detect copy number variations. The technical limitations of WES prevented identification of the large $B A G 3$ deletion $(>8.7 \mathrm{~kb})$. Algorithms are being created to aid in the use of exome sequencing for the detection of copy number variation $[84,85]$. However, these methods come with a variety of limitations and cannot detect other types of structural variation such as uniparental disomies or chromosomal rearrangements, both exceedingly important for disease pathogenesis. WGS, as opposed to WES, may offer a better method to detect some structural variants, but may require improved analytic tools for specificity and sensitivity of structural variant detection.

\section{WGS as a Tool to Investigate Non-Coding Variation for CVD}

Perhaps even more important to understanding disease etiology is the investigation of non-coding variation. Over $98 \%$ of the genome is non-coding, and WGS captures nearly 100 fold more of this information compared to WES. However, the interpretation of non-coding variation is currently far more challenging than the interpretation of coding variants. Often referred to as the "dark matter" of the genome, these regions can include microRNAs, long non-coding RNAs, splice variants and regulatory elements that can directly cause or modulate disease phenotypes [37]. miRNAs are important regulators of heart function and recent studies have revealed miRNA misexpression in human cardiac disease and animal models of heart failure [86-88]. For example, miR-208 is encoded by an intron within MYH6, which encodes a myosin heavy chain and is in close proximity to MYH7 [87,88]. mir-208 null mice do not hypertrophy in response to cardiac stress and null mice do not upregulate Myh7 [89,90]. Silencing of miR-208 reduces cardiac remodeling, deterioration of heart function, and improves survival in a rat model of heart failure, while overexpression of miR-208 in cardiomyocytes leads to cardiomyocyte hypertrophy [90,91]. Another miR, miR-1 is the most highly expressed miRNA in the murine heart $[88,92]$. miR-1 targets HAND2, a transcription factor important for expansion of ventricular cardiomyocytes. Deletion of miR-1 results in 50\% perinatal lethality due to ventricular septal defects [88]. The majority of surviving mice exhibit sudden death due to conduction defects. Overexpression of miR-1 in embryonic cardiomyocytes caused thin-walled ventricles leading to death at embryonic day 13.5 [86]. These data underscore the importance of miRNAs in cardiac phenotypes. Variation in these and other non-coding regions of the genome may play a vital role in the disease process. Annotation of the non-coding genome is currently underway to aid in the interpretation of these variants. The ENCODE project (Encyclopedia of DNA Elements) has assigned biochemical functions to $80 \%$ of the genome [93]. Only twelve percent of SNPs identified by GWAS as disease-associated are located in the vicinity of a protein-coding region even though SNPs in coding regions are over-represented on SNP arrays. However, over $60 \%$ of disease-associated SNPs identified by GWAS lie within functional, non-coding regions, especially in promoters and enhancers [94]. 


\subsection{WGS Has Greater Sensitivity than WES}

WGS is only now emerging as an alternative to WES since higher cost and more complex analysis limited uptake of WGS. Recently, WGS was used to identify a putative causative variant in a family with two children affected by a previously unreported disease defined by cardiomyopathy and progressive muscle weakness [95]. Wang and colleagues performed WES on one sibling and WGS on the other sibling. WES was performed to a depth of $118 \times$ with $>90 \%$ target regions covered by $\geq 10$ reads, while WGS was performed to a depth of $81 \times$. Variants were filtered using ANNOVAR, which relies on frequency and functional variation [96]. After validation with Sanger sequencing and transmission pattern testing, only two genes remained as candidates. One, TAF1L, is homologous to $\mathrm{TAF}(\mathrm{II}) 250$ and is specifically expressed in testis. The other candidate, $R B C K 1$, codes for an E3 ubiquitin-protein ligase. In this family, RBCK1 had two truncating mutations, each inherited from one parent [95]. $R B C K 1$ was considered a good candidate for both its rarity and the involvement of other ubiquitin-ligase proteins in muscle disease. The WES data set failed to reveal the RBCK1 variants despite good coverage over the targeted regions including the exons of $R B C K 1$. Upon reanalysis, coverage was very low ( 2 and 4 reads respectively) for the two mutations, with only one read containing a mutation [95]. Further investigation revealed a high GC content surrounding these mutations [97]. Previous studies have also noted that uneven exome coverage has resulted in filtering of disease genes [98]. This study serves as a proof of principle that whole genome sequencing can be used to identify rare Mendelian, cardiomyopathy phenotypes, and, in some instances, may be more sensitive than WES.

\subsection{Limitations of $W G S$}

Two of the major limitations of WGS are size and cost. To achieve average coverage $\sim 35-40 \times$ with WGS requires approximately $125 \mathrm{~Gb}$ of generated sequencing data. Figure 1A compares the amount of data generated from panel (blue), exome (red) and whole genome sequencing (green). WGS produces an order of magnitude more data than WES. All three technologies call variants proportionate to the amount of data generated with WGS calling $\sim 4$ million variants per genome, WES calling $\sim 90 \mathrm{~K}$, and gene panels calling far fewer (Figure 1B). At this time, clinical WGS is more expensive than either WES or panel sequencing at approximately $\$ 9000-\$ 9500$ for WGS, $\sim \$ 7000$ for WES and $\sim \$ 4000$ for a pan-cardiomyopathy panel. The price for both WES and WGS is considerably more than the cost of a panel. However, this only remains true if a pathogenic mutation is identified in the first panel. The value of each test can be thought of in terms of the cost per variant identified, and with this metric WGS is a better value (Figure 1D). Panel sequencing is $\sim \$ 1.70$, WES is $\sim \$ 0.08(8 \phi)$ and WGS is $\sim \$ 0.002(0.2 \phi)$ per variant detected. While only a handful of variants may be germane to identifying the cause of an individual's primary cardiomyopathy, the other sequence data remains available where it may provide useful guidance for life-style and medical decisions. 
Figure 1. Size and Cost Considerations of Next-Generation Sequencing. (A) The amount of data generated by a typical cardiomyopathy gene panel of $\sim 50$ genes (green), whole exome sequencing (red) and whole genome Sequencing (blue) is shown; (B) The approximate number of variants produced by each method is indicated; (C) The Clinical cost of each method ranges from $\sim \$ 4000$ (cardiomyopathy gene panel, green) to $\sim \$ 9500$ (whole genome sequencing, blue); (D) The cost per variant is greatly reduced for WGS $(\$ 0.002$, blue) versus WES $(\$ 0.08$, red) and gene panel-based sequencing (\$1.70, green). Boxes indicate parameters used to calculate values in A-D including coverage, base pairs interrogated and total output.

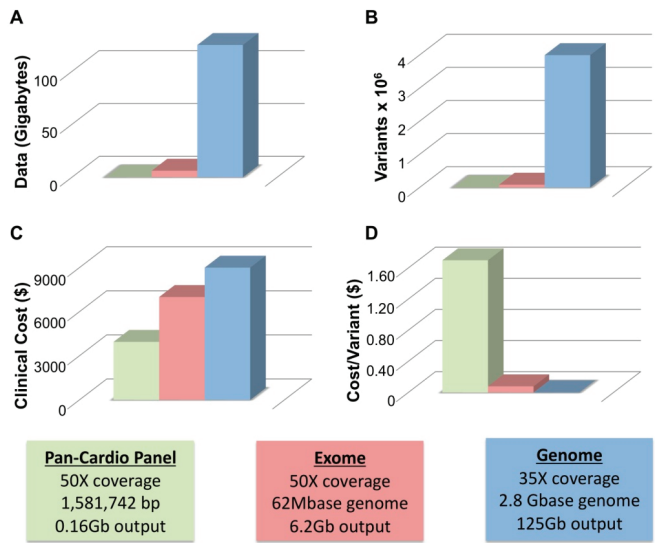

\subsection{Multi-Pass Filtering Methods Allow for More Efficient Variant Identification}

WGS produces vastly more data than either panels or WES, and this is the double-edged sword of broad based sequence analysis. To cope with this problem we (and others) have adopted stepwise, multi-pass filtering methods (Figure 2). In the first pass, candidate genes are analyzed for the phenotype of interest, in this case cardiomyopathy. Typically, exonic variants in candidate genes are filtered for frequency and for potential protein pathogenicity. A number of tools are freely available that predict the impact of amino acid changes on protein structure and function including PolyPhen-2, GERP, SIFT, PhastCons, Panther and Conseq [72,99-103]. MaxEnt can be used to score the strength of splice site variants [104]. If filtering candidate genes does not produce meaningful variation, the search can be expanded to less attractive candidates or to all rare protein coding variation and finally to non-coding variation. Non-coding variants can be filtered for frequency, however this is more challenging as many frequency databases are biased towards coding regions. Conservation of sequences across multiple species may provide clues about the selection acting on a sequence. The data generated by the ENCODE project will also provide information about variation in functional elements. The complexity of analysis increases with each pass. While this approach approximates panel and WES analysis, it is an improvement in several ways. With this model, WGS only needs to be performed once, while patients that are panel negative will need additional sequencing. In the case of WES, exome capture kits are often updated due to changing gene annotations and the inclusion of newly understood non-coding sequence, requiring retesting with 
new kits. WGS is less likely to need additional sequencing, and moreover, may provide additional data that over a lifetime can be used to inform not only the primary health concern for which data was collected, but medical choices throughout a patient's life.

Figure 2. Pipeline for WGS Variant Identification. WGS produces $\sim 4$ million variants per genome and requires extensive filtering to identify variants of interest. Shown here is a potential pipeline to identify variants. The first pass of the pipeline entails only reviewing variants in the coding regions of genes of interest and filtering by frequency, protein pathogenicity, and mode of inheritance (segregation in available family members). If no variant is identified, a second pass includes the same filtering steps, but on variants in all coding regions. The third pass includes analysis of non-coding variation using frequency, conservation and ENCODE annotation, along with mode of inheritance. The complexity of analysis increases with each pass.

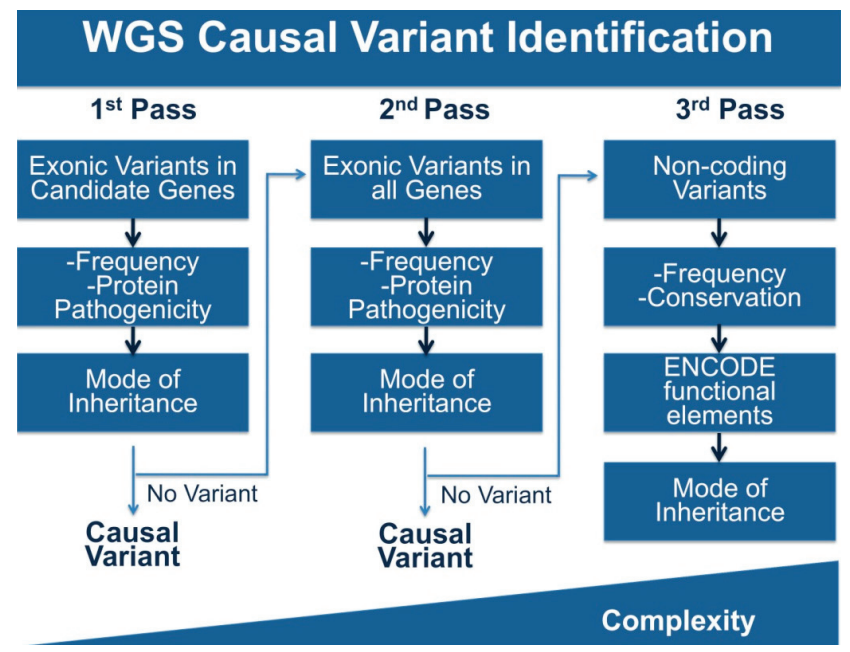

\section{Incidental Findings and Their Importance for CVD Related Phenotypes}

Incidental findings are a concern with all genetic assessment and especially so for WES and WGS. However, incidental findings are not unique to genetic testing and are part of medical decision making for any mode of testing, including imaging and blood tests. WGS may provide many more incidental findings than any other tests available, and this has led to new recommendations for delivering results of incidental findings from genetic research and testing. In 2006, an NHLBI working recommended reporting research results to study participants when the risk of disease is significant and has important health implications including sudden death or considerable risk of morbidity especially when therapeutic interventions are available [105]. In 2013, the American College of Medical Genetics and Genomics (ACMG) made recommendations for reporting incidental findings in exome and genome sequencing [106]. The ACMG recommended that laboratories performing sequencing should identify and report mutations in genes included on their minimal list. Notably, this list includes 24 phenotypes of which a third are 
cardiovascular disorders for which penetrance is high and clinical interventions are available [106]. Of the 57 genes for which recommendations were made to report incidental findings, more than half (34) were CVD-associated genes.

\section{Conclusions}

In the case of CVD genetic profiling, there are often medical management decisions that can reduce risk. This is the case whether the initial genetic profiling was done for assessing CVD risk or whether the genetic profiling was done to assess risk of other inherited diseases. For example, risks for cardiomyopathy and especially arrhythmias can be managed medically, with increased surveillance or even with device insertion. Risks for developing atherosclerosis or aneurysms can be mitigated through drug or even surgical intervention. Importantly, since CVD disorders can be associated with sudden cardiac death, the capacity to intervene based on genetic risk profiles is evident. With the improvement in genetic databases that are accompanied by robust phenotyping, it should be possible to more accurately predict risk for CVD.

\section{Acknowledgments}

This work was supported in part by National Institutes of Health NIH AR052646, NIH HL61322, NIH NS072027, and the Doris Duke Charitable Foundation.

\section{Author Contributions}

Megan J. Puckelwartz researched the topics and drafted the manuscript. Elizabeth M. McNally revised the manuscript.

\section{Conflicts of Interest}

The authors declare no conflict of interest.

\section{References}

1. Go, A.S.; Mozaffarian, D.; Roger, V.L.; Benjamin, E.J.; Berry, J.D.; Borden, W.B.; Bravata, D.M.; Dai, S.; Ford, E.S.; Fox, C.S.; et al. Executive summary: Heart disease and stroke statistics-2013 update: A report from the American Heart Association. Circulation 2013, 127, 143-152.

2. Marenberg, M.E.; Risch, N.; Berkman, L.F.; Floderus, B.; de Faire, U. Genetic susceptibility to death from coronary heart disease in a study of twins. N. Engl. J. Med. 1994, 330, 1041-1046.

3. Post, W.S.; Larson, M.G.; Myers, R.H.; Galderisi, M.; Levy, D. Heritability of left ventricular mass: The Framingham Heart Study. Hypertension 1997, 30, 1025-1028.

4. Adams, T.D.; Yanowitz, F.G.; Fisher, A.G.; Ridges, J.D.; Nelson, A.G.; Hagan, A.D.; Williams, R.R.; Hunt, S.C. Heritability of cardiac size: An echocardiographic and electrocardiographic study of monozygotic and dizygotic twins. Circulation 1985, 71, 39-44. 
5. Botstein, D.; White, R.L.; Skolnick, M.; Davis, R.W. Construction of a genetic linkage map in man using restriction fragment length polymorphisms. Am. J. Hum. Genet. 1980, 32, 314-331.

6. Jarcho, J.A.; McKenna, W.; Pare, J.A.; Solomon, S.D.; Holcombe, R.F.; Dickie, S.; Levi, T.; Donis-Keller, H.; Seidman, J.G.; Seidman, C.E. Mapping a gene for familial hypertrophic cardiomyopathy to chromosome 14q1. N. Engl. J. Med. 1989, 321, 1372-1378.

7. Geisterfer-Lowrance, A.A.; Kass, S.; Tanigawa, G.; Vosberg, H.P.; McKenna, W.; Seidman, C.E.; Seidman, J.G. A molecular basis for familial hypertrophic cardiomyopathy: A beta cardiac myosin heavy chain gene missense mutation. Cell 1990, 62, 999-1006.

8. Basson, C.T.; Bachinsky, D.R.; Lin, R.C.; Levi, T.; Elkins, J.A.; Soults, J.; Grayzel, D.; Kroumpouzou, E.; Traill, T.A.; Leblanc-Straceski, J.; et al. Mutations in human TBX5 [corrected] cause limb and cardiac malformation in Holt-Oram syndrome. Nat. Genet. 1997, $15,30-35$.

9. Curran, M.E.; Splawski, I.; Timothy, K.W.; Vincent, G.M.; Green, E.D.; Keating, M.T. A molecular basis for cardiac arrhythmia: HERG mutations cause long QT syndrome. Cell 1995, 80, 795-803.

10. Dietz, H.C.; Cutting, G.R.; Pyeritz, R.E.; Maslen, C.L.; Sakai, L.Y.; Corson, G.M.; Puffenberger, E.G.; Hamosh, A.; Nanthakumar, E.J.; Curristin, S.M.; et al. Marfan syndrome caused by a recurrent de novo missense mutation in the fibrillin gene. Nature 1991, 352, $337-339$.

11. Garg, V.; Kathiriya, I.S.; Barnes, R.; Schluterman, M.K.; King, I.N.; Butler, C.A.; Rothrock, C.R.; Eapen, R.S.; Hirayama-Yamada, K.; Joo, K.; et al. GATA4 mutations cause human congenital heart defects and reveal an interaction with TBX5. Nature 2003, 424, 443-447.

12. Garg, V.; Muth, A.N.; Ransom, J.F.; Schluterman, M.K.; Barnes, R.; King, I.N.; Grossfeld, P.D.; Srivastava, D. Mutations in NOTCH1 cause aortic valve disease. Nature 2005, 437, 270-274.

13. Schott, J.J.; Benson, D.W.; Basson, C.T.; Pease, W.; Silberbach, G.M.; Moak, J.P.; Maron, B.J.; Seidman, C.E.; Seidman, J.G. Congenital heart disease caused by mutations in the transcription factor NKX2-5. Science 1998, 281, 108-111.

14. Tartaglia, M.; Mehler, E.L.; Goldberg, R.; Zampino, G.; Brunner, H.G.; Kremer, H.; van der Burgt, I.; Crosby, A.H.; Ion, A.; Jeffery, S.; et al. Mutations in PTPN11, encoding the protein tyrosine phosphatase SHP-2, cause Noonan syndrome. Nat. Genet. 2001, 29, 465-468.

15. Lander, E.; Kruglyak, L. Genetic dissection of complex traits: Guidelines for interpreting and reporting linkage results. Nat. Genet. 1995, 11, 241-247.

16. International HapMap Consortium. The International HapMap Project. Nature 2003, 426, 789-796.

17. National Human Genome Research Institute (NHGRI) Catalog of Published Genome-Wide Association Studies. Available online: http://www.genome.gov/gwasstudies/ (accessed on 25 February 2014). 
18. Newton-Cheh, C.; Eijgelsheim, M.; Rice, K.M.; de Bakker, P.I.; Yin, X.; Estrada, K.; Bis, J.C.; Marciante, K.; Rivadeneira, F.; Noseworthy, P.A.; et al. Common variants at ten loci influence QT interval duration in the QTGEN Study. Nat. Genet. 2009, 41, 399-406.

19. Villard, E.; Perret, C.; Gary, F.; Proust, C.; Dilanian, G.; Hengstenberg, C.; Ruppert, V.; Arbustini, E.; Wichter, T.; Germain, M.; et al. A genome-wide association study identifies two loci associated with heart failure due to dilated cardiomyopathy. Eur. Heart J. 2011, 32, $1065-1076$.

20. Jefferies, J.L.; Towbin, J.A. Dilated cardiomyopathy. Lancet 2010, 375, 752-762.

21. Hershberger, R.E.; Norton, N.; Morales, A.; Li, D.; Siegfried, J.D.; Gonzalez-Quintana, J. Coding sequence rare variants identified in MYBPC3, MYH6, TPM1, TNNC1, and TNNI3 from 312 patients with familial or idiopathic dilated cardiomyopathy. Circ. Cardiovasc. Genet. 2010, 3, 155-161.

22. Rampersaud, E.; Kinnamon, D.D.; Hamilton, K.; Khuri, S.; Hershberger, R.E.; Martin, E.R. Common susceptibility variants examined for association with dilated cardiomyopathy. Ann. Hum. Genet. 2010, 74, 110-116.

23. Tiret, L.; Mallet, C.; Poirier, O.; Nicaud, V.; Millaire, A.; Bouhour, J.B.; Roizes, G.; Desnos, M.; Dorent, R.; Schwartz, K.; et al. Lack of association between polymorphisms of eight candidate genes and idiopathic dilated cardiomyopathy: The CARDIGENE study. J. Am. Coll. Cardiol. 2000, 35, 29-35.

24. Takayama, S.; Xie, Z.; Reed, J.C. An evolutionarily conserved family of Hsp70/Hsc70 molecular chaperone regulators. J. Biol. Chem. 1999, 274, 781-786.

25. Ellinor, P.T.; Sasse-Klaassen, S.; Probst, S.; Gerull, B.; Shin, J.T.; Toeppel, A.; Heuser, A.; Michely, B.; Yoerger, D.M.; Song, B.S.; et al. A novel locus for dilated cardiomyopathy, diffuse myocardial fibrosis, and sudden death on chromosome 10q25-26. J. Am. Coll. Cardiol. 2006, 48, 106-111.

26. Norton, N.; Li, D.; Rieder, M.J.; Siegfried, J.D.; Rampersaud, E.; Zuchner, S.; Mangos, S.; Gonzalez-Quintana, J.; Wang, L.; McGee, S.; et al. Genome-wide studies of copy number variation and exome sequencing identify rare variants in BAG3 as a cause of dilated cardiomyopathy. Am. J. Hum. Genet. 2011, 88, 273-282.

27. Reich, D.E.; Lander, E.S. On the allelic spectrum of human disease. Trends Genet. 2001, 17, 502-510.

28. Wang, W.Y.; Barratt, B.J.; Clayton, D.G.; Todd, J.A. Genome-wide association studies: Theoretical and practical concerns. Nat. Rev. Genet. 2005, 6, 109-118.

29. Manolio, T.A.; Collins, F.S.; Cox, N.J.; Goldstein, D.B.; Hindorff, L.A.; Hunter, D.J.; McCarthy, M.I.; Ramos, E.M.; Cardon, L.R.; Chakravarti, A.; et al. Finding the missing heritability of complex diseases. Nature 2009, 461, 747-753.

30. McPherson, R.; Pertsemlidis, A.; Kavaslar, N.; Stewart, A.; Roberts, R.; Cox, D.R.; Hinds, D.A.; Pennacchio, L.A.; Tybjaerg-Hansen, A.; Folsom, A.R.; et al. A common allele on chromosome 9 associated with coronary heart disease. Science 2007, 316, 1488-1491. 
31. Helgadottir, A.; Thorleifsson, G.; Manolescu, A.; Gretarsdottir, S.; Blondal, T.; Jonasdottir, A.; Sigurdsson, A.; Baker, A.; Palsson, A.; Masson, G.; et al. A common variant on chromosome 9p21 affects the risk of myocardial infarction. Science 2007, 316, 1491-1493.

32. Lander, E.S. The new genomics: Global views of biology. Science 1996, 274, 536-539.

33. Pritchard, J.K.; Cox, N.J. The allelic architecture of human disease genes: Common disease-common variant ... or not? Hum. Mol. Genet. 2002, 11, 2417-2423.

34. Gibson, G. Rare and common variants: Twenty arguments. Nat. Rev. Genet. 2011, 13, 135-145.

35. Manolio, T.A. Cohort studies and the genetics of complex disease. Nat. Genet. 2009, 41, 5-6.

36. Sun, J.X.; Helgason, A.; Masson, G.; Ebenesersdottir, S.S.; Li, H.; Mallick, S.; Gnerre, S.; Patterson, N.; Kong, A.; Reich, D.; et al. A direct characterization of human mutation based on microsatellites. Nat. Genet. 2012, 44, 1161-1165.

37. Marian, A.J.; Belmont, J. Strategic approaches to unraveling genetic causes of cardiovascular diseases. Circ. Res. 2011, 108, 1252-1269.

38. Ng, P.C.; Levy, S.; Huang, J.; Stockwell, T.B.; Walenz, B.P.; Li, K.; Axelrod, N.; Busam, D.A.; Strausberg, R.L.; Venter, J.C. Genetic variation in an individual human exome. PLoS Genet. 2008, 4, e1000160.

39. Tennessen, J.A.; Bigham, A.W.; O’Connor, T.D.; Fu, W.; Kenny, E.E.; Gravel, S.; McGee, S.; Do, R.; Liu, X.; Jun, G.; et al. Evolution and functional impact of rare coding variation from deep sequencing of human exomes. Science 2012, 337, 64-69.

40. Johansen, C.T.; Wang, J.; Lanktree, M.B.; Cao, H.; McIntyre, A.D.; Ban, M.R.; Martins, R.A.; Kennedy, B.A.; Hassell, R.G.; Visser, M.E.; et al. Excess of rare variants in genes identified by genome-wide association study of hypertriglyceridemia. Nat. Genet. 2010, 42, 684-687.

41. Aulchenko, Y.S.; Ripatti, S.; Lindqvist, I.; Boomsma, D.; Heid, I.M.; Pramstaller, P.P.; Penninx, B.W.; Janssens, A.C.; Wilson, J.F.; Spector, T.; et al. Loci influencing lipid levels and coronary heart disease risk in 16 European population cohorts. Nat. Genet. 2009, 41, 47-55.

42. Kathiresan, S.; Willer, C.J.; Peloso, G.M.; Demissie, S.; Musunuru, K.; Schadt, E.E.; Kaplan, L.; Bennett, D.; Li, Y.; Tanaka, T.; et al. Common variants at 30 loci contribute to polygenic dyslipidemia. Nat. Genet. 2009, 41, 56-65.

43. Li, H.; Homer, N. A survey of sequence alignment algorithms for next-generation sequencing. Brief. Bioinforma. 2010, 11, 473-483.

44. Nielsen, R.; Paul, J.S.; Albrechtsen, A.; Song, Y.S. Genotype and SNP calling from next-generation sequencing data. Nat. Rev. Genet. 2011, 12, 443-451.

45. Abecasis, G.R.; Auton, A.; Brooks, L.D.; DePristo, M.A.; Durbin, R.M.; Handsaker, R.E.; Kang, H.M.; Marth, G.T.; McVean, G.A. An integrated map of genetic variation from 1092 human genomes. Nature 2012, 491, 56-65.

46. Jabbari, J.; Jabbari, R.; Nielsen, M.W.; Holst, A.G.; Nielsen, J.B.; Haunso, S.; Tfelt-Hansen, J.; Svendsen, J.H.; Olesen, M.S. New exome data question the pathogenicity of genetic variants previously associated with catecholaminergic polymorphic ventricular tachycardia. Circ. Cardiovasc. Genet. 2013, 6, 481-489. 
47. Priori, S.G.; Napolitano, C.; Memmi, M.; Colombi, B.; Drago, F.; Gasparini, M.; DeSimone, L.; Coltorti, F.; Bloise, R.; Keegan, R.; et al. Clinical and molecular characterization of patients with catecholaminergic polymorphic ventricular tachycardia. Circulation 2002, 106, 69-74.

48. Golbus, J.R.; Puckelwartz, M.J.; Fahrenbach, J.P.; Dellefave-Castillo, L.M.; Wolfgeher, D.; McNally, E.M. Population-based variation in cardiomyopathy genes. Circ. Cardiovasc. Genet. 2012, 5, 391-399.

49. Risgaard, B.; Jabbari, R.; Refsgaard, L.; Holst, A.G.; Haunso, S.; Sadjadieh, A.; Winkel, B.G.; Olesen, M.S.; Tfelt-Hansen, J. High prevalence of genetic variants previously associated with Brugada syndrome in new exome data. Clin. Genet. 2013, 84, 489-495.

50. Kapplinger, J.D.; Landstrom, A.P.; Salisbury, B.A.; Callis, T.E.; Pollevick, G.D.; Tester, D.J.; Cox, M.G.; Bhuiyan, Z.; Bikker, H.; Wiesfeld, A.C.; et al. Distinguishing arrhythmogenic right ventricular cardiomyopathy/dysplasia-associated mutations from background genetic noise. J. Am. Coll. Cardiol. 2011, 57, 2317-2327.

51. Andreasen, C.; Refsgaard, L.; Nielsen, J.B.; Sajadieh, A.; Winkel, B.G.; Tfelt-Hansen, J.; Haunso, S.; Holst, A.G.; Svendsen, J.H.; Olesen, M.S. Mutations in genes encoding cardiac ion channels previously associated with sudden infant death syndrome (SIDS) are present with high frequency in new exome data. Can. J. Cardiol. 2013, 29, 1104-1109.

52. Petretta, M.; Pirozzi, F.; Sasso, L.; Paglia, A.; Bonaduce, D. Review and metaanalysis of the frequency of familial dilated cardiomyopathy. Am. J. Cardiol. 2011, 108, 1171-1176.

53. Meder, B.; Haas, J.; Keller, A.; Heid, C.; Just, S.; Borries, A.; Boisguerin, V.; Scharfenberger-Schmeer, M.; Stahler, P.; Beier, M.; et al. Targeted next-generation sequencing for the molecular genetic diagnostics of cardiomyopathies. Circ. Cardiovasc. Genet. 2011, 4, 110-122.

54. Zimmerman, R.S.; Cox, S.; Lakdawala, N.K.; Cirino, A.; Mancini-DiNardo, D.; Clark, E.; Leon, A.; Duffy, E.; White, E.; Baxter, S.; et al. A novel custom resequencing array for dilated cardiomyopathy. Genet. Med. 2010, 12, 268-278.

55. Partners Healthcare. Available online: http://pcpgm.partners.org/ (accessed on 25 February 2014).

56. Opitz, C.A.; Kulke, M.; Leake, M.C.; Neagoe, C.; Hinssen, H.; Hajjar, R.J.; Linke, W.A. Damped elastic recoil of the titin spring in myofibrils of human myocardium. Proc. Natl. Acad. Sci. USA 2003, 100, 12688-12693.

57. Granzier, H.L.; Irving, T.C. Passive tension in cardiac muscle: Contribution of collagen, titin, microtubules, and intermediate filaments. Biophys. J. 1995, 68, 1027-1044.

58. Horowits, R.; Kempner, E.S.; Bisher, M.E.; Podolsky, R.J. A physiological role for titin and nebulin in skeletal muscle. Nature 1986, 323, 160-164.

59. Muhle-Goll, C.; Habeck, M.; Cazorla, O.; Nilges, M.; Labeit, S.; Granzier, H. Structural and functional studies of titin's fn3 modules reveal conserved surface patterns and binding to myosin S1-A possible role in the Frank-Starling mechanism of the heart. J. Mol. Biol. 2001, $313,431-447$. 
60. Cazorla, O.; Wu, Y.; Irving, T.C.; Granzier, H. Titin-based modulation of calcium sensitivity of active tension in mouse skinned cardiac myocytes. Circ. Res. 2001, 88, 1028-1035.

61. Siu, B.L.; Niimura, H.; Osborne, J.A.; Fatkin, D.; MacRae, C.; Solomon, S.; Benson, D.W.; Seidman, J.G.; Seidman, C.E. Familial dilated cardiomyopathy locus maps to chromosome 2q31. Circulation 1999, 99, 1022-1026.

62. Gerull, B.; Atherton, J.; Geupel, A.; Sasse-Klaassen, S.; Heuser, A.; Frenneaux, M.; McNabb, M.; Granzier, H.; Labeit, S.; Thierfelder, L. Identification of a novel frameshift mutation in the giant muscle filament titin in a large Australian family with dilated cardiomyopathy. J. Mol. Med. 2006, 84, 478-483.

63. Gerull, B.; Gramlich, M.; Atherton, J.; McNabb, M.; Trombitas, K.; Sasse-Klaassen, S.; Seidman, J.G.; Seidman, C.; Granzier, H.; Labeit, S.; et al. Mutations of TTN, encoding the giant muscle filament titin, cause familial dilated cardiomyopathy. Nat. Genet. 2002, 30, 201-204.

64. Herman, D.S.; Lam, L.; Taylor, M.R.; Wang, L.; Teekakirikul, P.; Christodoulou, D.; Conner, L.; DePalma, S.R.; McDonough, B.; Sparks, E.; et al. Truncations of titin causing dilated cardiomyopathy. N. Engl. J. Med. 2012, 366, 619-628.

65. Ng, S.B.; Turner, E.H.; Robertson, P.D.; Flygare, S.D.; Bigham, A.W.; Lee, C.; Shaffer, T.; Wong, M.; Bhattacharjee, A.; Eichler, E.E.; et al. Targeted capture and massively parallel sequencing of 12 human exomes. Nature 2009, 461, 272-276.

66. Majewski, J.; Schwartzentruber, J.; Lalonde, E.; Montpetit, A.; Jabado, N. What can exome sequencing do for you? J. Med. Genet. 2011, 48, 580-589.

67. Campbell, N.; Sinagra, G.; Jones, K.L.; Slavov, D.; Gowan, K.; Merlo, M.; Carniel, E.; Fain, P.R.; Aragona, P.; di Lenarda, A.; et al. Whole exome sequencing identifies a troponin T mutation hot spot in familial dilated cardiomyopathy. PLoS One 2013, 8, e78104.

68. Wells, Q.S.; Becker, J.R.; Su, Y.R.; Mosley, J.D.; Weeke, P.; D’Aoust, L.; Ausborn, N.L.; Ramirez, A.H.; Pfotenhauer, J.P.; Naftilan, A.J.; et al. Whole exome sequencing identifies a causal RBM20 mutation in a large pedigree with familial dilated cardiomyopathy. Circ. Cardiovasc. Genet. 2013, 6, 317-326.

69. Yandell, M.; Huff, C.; Hu, H.; Singleton, M.; Moore, B.; Xing, J.; Jorde, L.B.; Reese, M.G. A probabilistic disease-gene finder for personal genomes. Genome Res. 2011, 21, 1529-1542.

70. Li, D.; Morales, A.; Gonzalez-Quintana, J.; Norton, N.; Siegfried, J.D.; Hofmeyer, M.; Hershberger, R.E. Identification of novel mutations in RBM20 in patients with dilated cardiomyopathy. Clin. Transl. Sci. 2010, 3, 90-97.

71. Davydov, E.V.; Goode, D.L.; Sirota, M.; Cooper, G.M.; Sidow, A.; Batzoglou, S. Identifying a high fraction of the human genome to be under selective constraint using GERP++. PLoS Comput. Biol. 2010, 6, e1001025.

72. Adzhubei, I.A.; Schmidt, S.; Peshkin, L.; Ramensky, V.E.; Gerasimova, A.; Bork, P.; Kondrashov, A.S.; Sunyaev, S.R. A method and server for predicting damaging missense mutations. Nat. Methods 2010, 7, 248-249. 
73. Millat, G.; Bouvagnet, P.; Chevalier, P.; Sebbag, L.; Dulac, A.; Dauphin, C.; Jouk, P.S.; Delrue, M.A.; Thambo, J.B.; Le Metayer, P.; et al. Clinical and mutational spectrum in a cohort of 105 unrelated patients with dilated cardiomyopathy. Eur. J. Med. Genet. 2011, 54, e570-e575.

74. Lakdawala, N.K.; Dellefave, L.; Redwood, C.S.; Sparks, E.; Cirino, A.L.; Depalma, S.; Colan, S.D.; Funke, B.; Zimmerman, R.S.; Robinson, P.; et al. Familial dilated cardiomyopathy caused by an alpha-tropomyosin mutation: The distinctive natural history of sarcomeric dilated cardiomyopathy. J. Am. Coll. Cardiol. 2010, 55, 320-329.

75. Roncarati, R.; Viviani Anselmi, C.; Krawitz, P.; Lattanzi, G.; von Kodolitsch, Y.; Perrot, A.; di Pasquale, E.; Papa, L.; Portararo, P.; Columbaro, M.; et al. Doubly heterozygous LMNA and TTN mutations revealed by exome sequencing in a severe form of dilated cardiomyopathy. Eur. J. Hum. Genet. 2013, 21, 1105-1111.

76. Robinson, P.N.; Kohler, S.; Bauer, S.; Seelow, D.; Horn, D.; Mundlos, S. The Human Phenotype Ontology: A tool for annotating and analyzing human hereditary disease. Am. J. Hum. Genet. 2008, 83, 610-615.

77. Perrot, A.; Hussein, S.; Ruppert, V.; Schmidt, H.H.; Wehnert, M.S.; Duong, N.T.; Posch, M.G.; Panek, A.; Dietz, R.; Kindermann, I.; et al. Identification of mutational hot spots in LMNA encoding lamin $\mathrm{A} / \mathrm{C}$ in patients with familial dilated cardiomyopathy. Basic Res. Cardiol. 2009, 104, 90-99.

78. Theis, J.L.; Sharpe, K.M.; Matsumoto, M.E.; Chai, H.S.; Nair, A.A.; Theis, J.D.; de Andrade, M.; Wieben, E.D.; Michels, V.V.; Olson, T.M. Homozygosity mapping and exome sequencing reveal GATAD1 mutation in autosomal recessive dilated cardiomyopathy. Circ. Cardiovasc. Genet. 2011, 4, 585-594.

79. Vermeulen, M.; Eberl, H.C.; Matarese, F.; Marks, H.; Denissov, S.; Butter, F.; Lee, K.K.; Olsen, J.V.; Hyman, A.A.; Stunnenberg, H.G.; et al. Quantitative interaction proteomics and genome-wide profiling of epigenetic histone marks and their readers. Cell 2010, 142, 967-980.

80. Alkan, C.; Coe, B.P.; Eichler, E.E. Genome structural variation discovery and genotyping. Nat. Rev. Genet. 2011, 12, 363-376.

81. Iafrate, A.J.; Feuk, L.; Rivera, M.N.; Listewnik, M.L.; Donahoe, P.K.; Qi, Y.; Scherer, S.W.; Lee, C. Detection of large-scale variation in the human genome. Nat. Genet. 2004, 36, 949-951.

82. Tuzun, E.; Sharp, A.J.; Bailey, J.A.; Kaul, R.; Morrison, V.A.; Pertz, L.M.; Haugen, E.; Hayden, H.; Albertson, D.; Pinkel, D.; et al. Fine-scale structural variation of the human genome. Nat. Genet. 2005, 37, 727-732.

83. Kidd, J.M.; Cooper, G.M.; Donahue, W.F.; Hayden, H.S.; Sampas, N.; Graves, T.; Hansen, N.; Teague, B.; Alkan, C.; Antonacci, F.; et al. Mapping and sequencing of structural variation from eight human genomes. Nature 2008, 453, 56-64.

84. Krumm, N.; Sudmant, P.H.; Ko, A.; O’Roak, B.J.; Malig, M.; Coe, B.P.; Quinlan, A.R.; Nickerson, D.A.; Eichler, E.E. Copy number variation detection and genotyping from exome sequence data. Genome Res. 2012, 22, 1525-1532. 
85. Magi, A.; Tattini, L.; Cifola, I.; D’Aurizio, R.; Benelli, M.; Mangano, E.; Battaglia, C.; Bonora, E.; Kurg, A.; Seri, M.; et al. EXCAVATOR: Detecting copy number variants from whole-exome sequencing data. Genome Biol. 2013, 14, R120.

86. Zhao, Y.; Samal, E.; Srivastava, D. Serum response factor regulates a muscle-specific microRNA that targets Hand2 during cardiogenesis. Nature 2005, 436, 214-220.

87. Thum, T.; Galuppo, P.; Wolf, C.; Fiedler, J.; Kneitz, S.; van Laake, L.W.; Doevendans, P.A.; Mummery, C.L.; Borlak, J.; Haverich, A.; et al. MicroRNAs in the human heart: A clue to fetal gene reprogramming in heart failure. Circulation 2007, 116, 258-267.

88. Zhao, Y.; Ransom, J.F.; Li, A.; Vedantham, V.; von Drehle, M.; Muth, A.N.; Tsuchihashi, T.; McManus, M.T.; Schwartz, R.J.; Srivastava, D. Dysregulation of cardiogenesis, cardiac conduction, and cell cycle in mice lacking miRNA-1-2. Cell 2007, 129, 303-317.

89. Van Rooij, E.; Sutherland, L.B.; Qi, X.; Richardson, J.A.; Hill, J.; Olson, E.N. Control of stress-dependent cardiac growth and gene expression by a microRNA. Science 2007, 316, 575-579.

90. Callis, T.E.; Pandya, K.; Seok, H.Y.; Tang, R.H.; Tatsuguchi, M.; Huang, Z.P.; Chen, J.F.; Deng, Z.; Gunn, B.; Shumate, J.; et al. MicroRNA-208a is a regulator of cardiac hypertrophy and conduction in mice. J. Clin. Invest. 2009, 119, 2772-2786.

91. Montgomery, R.L.; Hullinger, T.G.; Semus, H.M.; Dickinson, B.A.; Seto, A.G.; Lynch, J.M.; Stack, C.; Latimer, P.A.; Olson, E.N.; van Rooij, E. Therapeutic inhibition of miR-208a improves cardiac function and survival during heart failure. Circulation 2011, 124, 1537-1547.

92. Rao, P.K.; Toyama, Y.; Chiang, H.R.; Gupta, S.; Bauer, M.; Medvid, R.; Reinhardt, F.; Liao, R.; Krieger, M.; Jaenisch, R.; et al. Loss of cardiac microRNA-mediated regulation leads to dilated cardiomyopathy and heart failure. Circ. Res. 2009, 105, 585-594.

93. Bernstein, B.E.; Birney, E.; Dunham, I.; Green, E.D.; Gunter, C.; Snyder, M. An integrated encyclopedia of DNA elements in the human genome. Nature 2012, 489, 57-74.

94. Hindorff, L.A.; Sethupathy, P.; Junkins, H.A.; Ramos, E.M.; Mehta, J.P.; Collins, F.S.; Manolio, T.A. Potential etiologic and functional implications of genome-wide association loci for human diseases and traits. Proc. Natl. Acad. Sci. USA 2009, 106, 9362-9367.

95. Wang, K.; Kim, C.; Bradfield, J.; Guo, Y.; Toskala, E.; Otieno, F.G.; Hou, C.; Thomas, K.; Cardinale, C.; Lyon, G.J.; et al. Whole-genome DNA/RNA sequencing identifies truncating mutations in RBCK1 in a novel Mendelian disease with neuromuscular and cardiac involvement. Genome Med. 2013, 5, 67.

96. Wang, K.; Li, M.; Hakonarson, H. ANNOVAR: Functional annotation of genetic variants from high-throughput sequencing data. Nucleic Acids Res. 2010, 38, e164.

97. Benjamini, Y.; Speed, T.P. Summarizing and correcting the GC content bias in high-throughput sequencing. Nucleic Acids Res. 2012, 40, e72.

98. Sirmaci, A.; Edwards, Y.J.; Akay, H.; Tekin, M. Challenges in whole exome sequencing: An example from hereditary deafness. PLoS One 2012, 7, e32000.

99. Siepel, A.; Bejerano, G.; Pedersen, J.S.; Hinrichs, A.S.; Hou, M.; Rosenbloom, K.; Clawson, H.; Spieth, J.; Hillier, L.W.; Richards, S.; et al. Evolutionarily conserved elements in vertebrate, insect, worm, and yeast genomes. Genome Res. 2005, 15, 1034-1050. 
100. Ng, P.C.; Henikoff, S. SIFT: Predicting amino acid changes that affect protein function. Nucleic Acids Res. 2003, 31, 3812-3814.

101. Cooper, G.M.; Stone, E.A.; Asimenos, G.; Green, E.D.; Batzoglou, S.; Sidow, A. Distribution and intensity of constraint in mammalian genomic sequence. Genome Res. 2005, 15, 901-913.

102. Thomas, P.D.; Kejariwal, A.; Campbell, M.J.; Mi, H.; Diemer, K.; Guo, N.; Ladunga, I.; Ulitsky-Lazareva, B.; Muruganujan, A.; Rabkin, S.; et al. PANTHER: A browsable database of gene products organized by biological function, using curated protein family and subfamily classification. Nucleic Acids Res. 2003, 31, 334-341.

103. Ashkenazy, H.; Erez, E.; Martz, E.; Pupko, T.; Ben-Tal, N. ConSurf 2010: Calculating evolutionary conservation in sequence and structure of proteins and nucleic acids. Nucleic Acids Res. 2010, 38, W529-W533.

104. Yeo, G.; Burge, C.B. Maximum entropy modeling of short sequence motifs with applications to RNA splicing signals. J. Comput. Biol. 2004, 11, 377-394.

105. Bookman, E.B.; Langehorne, A.A.; Eckfeldt, J.H.; Glass, K.C.; Jarvik, G.P.; Klag, M.; Koski, G.; Motulsky, A.; Wilfond, B.; Manolio, T.A.; et al. Reporting genetic results in research studies: Summary and recommendations of an NHLBI working group. Am. J. Med. Genet. A 2006, 140, 1033-1040.

106. Green, R.C.; Berg, J.S.; Grody, W.W.; Kalia, S.S.; Korf, B.R.; Martin, C.L.; McGuire, A.L.; Nussbaum, R.L.; O’Daniel, J.M.; Ormond, K.E.; et al. ACMG recommendations for reporting of incidental findings in clinical exome and genome sequencing. Genet. Med. 2013, $15,565-574$. 


\title{
Illuminating the Transcriptome through the Genome
}

\author{
David J. Elliott
}

\begin{abstract}
Sequencing the human genome was a huge milestone in genetic research that revealed almost the total DNA sequence required to create a human being. However, in order to function, the DNA genome needs to be expressed as an RNA transcriptome. This article reviews how knowledge of genome sequence information has led to fundamental discoveries in how the transcriptome is processed, with a focus on new system-wide insights into how pre-mRNAs that are encoded by split genes in the genome are rearranged by splicing into functional mRNAs. These advances have been made possible by the development of new post-genome technologies to probe splicing patterns. Transcriptome-wide approaches have characterised a "splicing code" that is embedded within and has a significant role in deciphering the genome, and is deciphered by RNA binding proteins. These analyses have also found that most human genes encode multiple mRNA isoforms, and in some cases proteins, leading in turn to a re-assessment of what exactly a gene is. Analysis of the transcriptome has given insights into how the genome is packaged and transcribed, and is helping to explain important aspects of genome evolution.
\end{abstract}

Reprinted from Genes. Cite as: Elliott, D.J. Illuminating the Transcriptome through the Genome. Genes 2014, 5, 235-253.

\section{Introduction}

The completion of the human genome sequence [1,2] brought together key scientific and philosophical questions, including exactly what we are as a species and individuals. However, in order to function, the genome has to be expressed. The primary expression product of the genome is RNA, and the complete set of all RNA molecules made through copying the genome into RNA is called the transcriptome (Figure 1). After transcription in the nucleus, mRNAs are translated into protein in the cytoplasm to yield the proteome while other RNAs have noncoding functions [3].

A key feature of human (and other eukaryotic) genes is their split exon-intron structure [4,5]. Figure 2 shows the exon-intron structure of a typical human gene displayed on a genome browser [6]. The exons include the protein coding information of the gene while introns are the intervening sequences between them. The term exon refers to the fact that exon sequences are expressed in the mRNA made from the gene, as opposed to introns which are removed (intron refers to intragenic regions) [7]. The presence of introns within genes and the long intergenic sequences between genes mean that only a small fraction of the human genome is truly protein-encoding. To put some figures on this, human protein-encoding genes contribute $\sim 33.5 \%$ of the human genome sequence [1,2] but exons alone comprise $2.94 \%$ of the genome [8]. Protein coding exons make up a smaller proportion still $(1.2 \%)$ of the genome. This is because there are at least partially untranslated exons in every mRNA (some of which can have important regulatory roles), and some exons remain entirely untranslated (see below). 
Genes need to be transcribed over their full length (including both introns and exons) to generate precursor mRNAs (pre-mRNAs). Transcription of long genes represents a considerable energy investment by the cell. One of the longest genes in the human genome, DYSTROPHIN takes in the order of 16 hours to transcribe, yet produces a final mRNA of just $\sim 14 \mathrm{~kb}$ that would have just taken $\sim 7$ minutes to transcribe by itself, assuming an elongation rate of $2 \mathrm{~kb} /$ minute [9]. It is calculated that $\sim 95 \%$ of RNA does not leave the nucleus [10]. Nuclear-retained RNA includes intron sequences and some long ncRNAs that are also spliced but retained in the nucleus.

Figure 1. Information flow from the genome to the proteome. The genome represents an archive of information embedded in DNA. This information is transcribed as RNA to give the transcriptome, and then translated into protein to give the proteome.

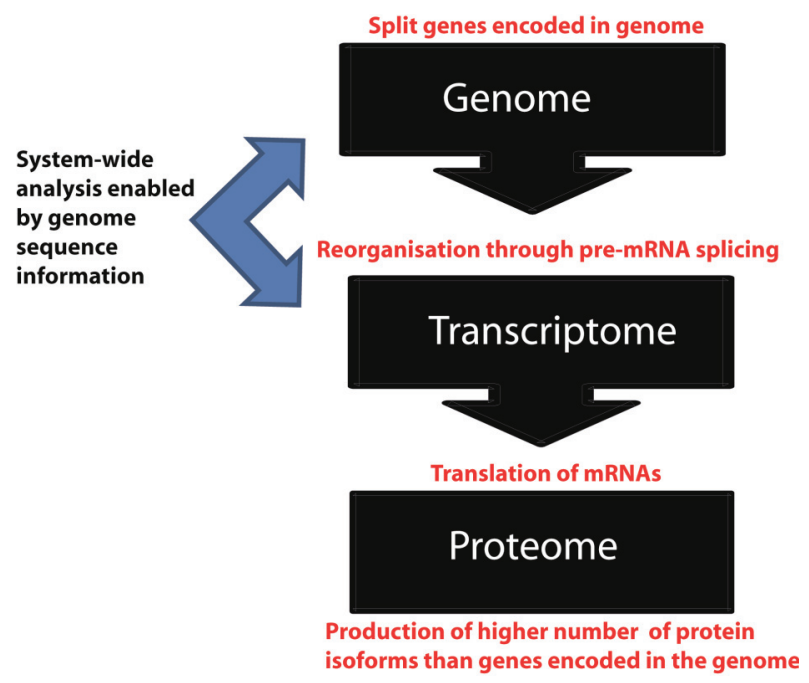

Figure 2. The intron-exon structure of a typical human gene displayed on the UCSC genome browser [6]. Introns are shown as lines (the "arrowheads" in the lines indicate the direction of transcription). Exons are shown as vertical bars. Coding exons are shown as thicker vertical bars than non-coding exon sequence. This example shows the NASP gene. The gene structures shown are "Refseq genes" that represent known human protein-coding and non-protein-coding genes taken from the NCBI RNA reference sequences collection. Notice that this single gene locus contains three distinct Refseq annotations containing different exon structures. Conserved sequences detected by comparative genomic information from 100 vertebrate genome sequences are shown at the bottom as a Phastcons plot- the higher values are most conserved, and often correspond to exons.

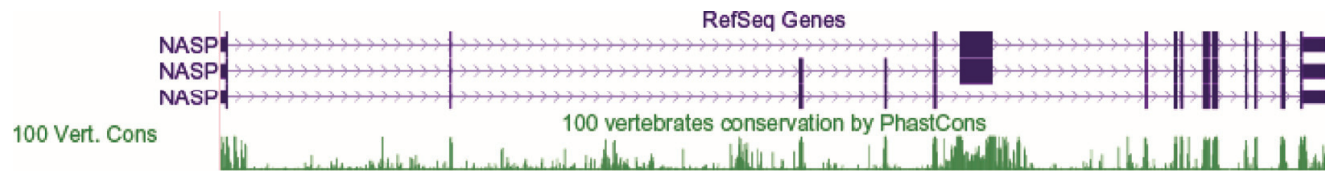


The splicing reaction is catalysed by the spliceosome. While introns are generally discarded after splicing, some introns can yield functional RNAs after splicing (e.g., miRNAs) [11,12]. Spliceosomes themselves are multi-component machines containing five snRNAs and at least 200 proteins, making them one of the most complicated assemblies in the cell $[13,14]$. Spliceosomes assemble de novo around each intron to be removed. A typical gene containing eight exons would require the assembly of eight spliceosomes to create a functional mRNA. Input of energy is required for spliceosomes to properly assemble through multiple ATP-dependent RNA helicases and other energy consuming proteins (including GTPases) [15].

Prior to completion of the human genome sequence, research into splicing typically looked at single genes and exons as models. However, while these detailed studies continue to be very important, the advances in genomics catalysed by genome sequencing projects have spawned parallel advances in transcriptomics, enabling a much broader system-wide dissection of RNA processing pathways. Here I review some of these new insights. While the focus here is on pre-mRNA splicing, transcriptome-wide analyses have also been directed at other aspects of genome expression, including RNA editing, RNA stability, expression of ncRNAs, polyadenylation and translation.

\section{The Human Genome Sequence Has Led to New Global Insights into the Control of Splicing}

In the 1980s examination of a limited number of genes led to the identification of short conserved sequences called 5' and 3' splice sites at exon-intron junctions [16]. The availability of the human and other genome sequences have enabled these studies to be extended genome-wide [17]. Most human exons are spliced together by a single kind of major spliceosome that recognises most $5^{\prime}$ and $3^{\prime}$ splice sites. In addition a second minor spliceosome exists in parallel that decodes a smaller subset of intron-exon junctions [18]. This minor spliceosome has a different but overlapping complement of snRNAs to the major spliceosome. Recent transcriptome-wide data show a key snRNA component in the minor spliceosome (called U6ATAC) is an important gene expression switch controlling patterns of splicing [19]. In the rest of this review the activities of the major and minor spliceosomes are not separately distinguished.

The conserved 5' and 3' splice site sequences encoded in the genome at exon-intron junctions are quite short. Furthermore, scattered within introns are short sequence elements called pseudoexons. Pseudoexons "look like" exons in that they have 5' and 3' splice sites, but are not selected as exons by the spliceosome. Estimates from model human genes suggest pre-mRNA splicing is remarkably accurate [20]. However, transcriptome-wide analyses of splicing patterns using RNAseq do detect some errors in splicing (at a rate of around $0.7 \%$ errors/intron) - these errors have been termed "noisy splicing", and might contribute to gene and protein evolution by enabling new mRNA isoforms to be made even at low frequencies [21].

The spliceosome uses several mechanisms to accurately decode exon/intron structure using information embedded in the transcriptome. Firstly, in humans and most vertebrates, spliceosomes recognise exons from introns through a process called exon definition [22-24]. The advantage of exon definition is that since exons are quite small they should be easier to identify as discrete units compared most (considerably longer) vertebrate introns. In exon definition, early spliceosome 
components bind to the pre-mRNA and "flag" exons to be spliced together. Following exon definition, pairs of exons are then joined together by splicing which removes the intervening intron sequences. Amongst the early binding components of the spliceosome involved in exon definition are the U1 snRNP RNA-protein complex that recognises the $5^{\prime}$ splice site, and a protein dimer which recognises $3^{\prime}$ splice sites called U2AF (U2AF65 and U2AF35 are the two proteins in the dimer).

Figure 3. Transcriptome-wide data can be used to predict the splicing code in specific genes. In this example sequences within a cassette exon in the mouse NASP gene have been analysed using genome and transcriptome wide datasets to pinpoint splicing control sequences. Firstly a Chasin Z-score plot was used that can identify sequences predictive of exonic splicing enhancers and silencers [32,34]: the four exonic splicing enhancer (ESE) sequences identified are shown as peaks in the plot above the sequence. In this example, these ESE sequences were individually mutated to test function in minigenes (the Chasin profiles of the mutants M1-M4 are shown compared to the wild type sequence: notice the change in the Z-score plot removes predicted ESE activity for each mutant). The positions of these ESEs mapped to binding sites for the splicing factor Tra2 $\beta$, both individually identified using cross linking immunoprecipitation (CLIP) in the mouse testis and predicted from the in vivo binding site generated from transcriptome-wide Tra2 $\beta$ binding data from the mouse testis. This figure is adapted from [32].

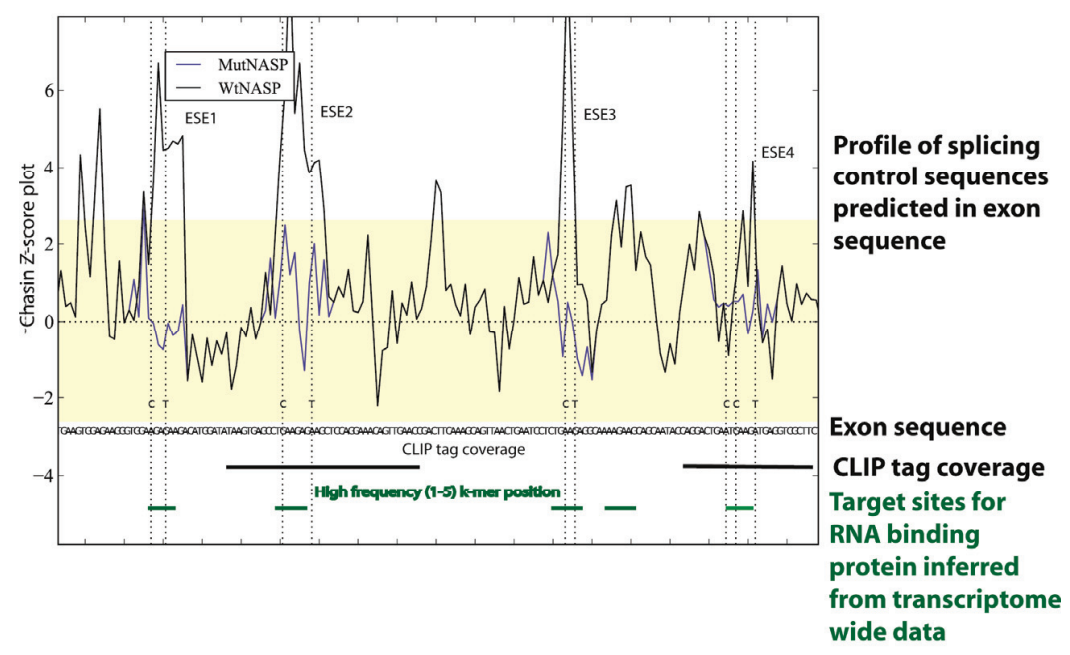

A second mechanism that facilitates accurate decoding of the genome is the presence of a splicing code that helps to differentiate between exons and introns in pre-mRNA. Before the sequencing of the human and other eukaryotic genomes, the important sequences controlling splicing of an exon were usually worked out on a gene by gene basis, using a finite number of model exons. The importance of exon sequences outside the splice junctions for splicing were first identified in pioneering experiments using model exons in the FIBRONECTIN and $\beta$ globin 
genes $[25,26]$. It is now known that pre-mRNAs each contain multiple short nucleotide sequences that can enhance or silence splicing of their associated exons [27-29]. Exon sequences can function as exonic splicing enhancers (abbreviated ESEs) that help the spliceosome to recognise exons, or exonic splicing silencers (abbreviated ESSs) that inhibit spliceosome recognition by the spliceosome. Similarly, intron associated sequences can function as intronic splicing enhancers (abbreviated ISE) or Intronic Splicing Silencers (abbreviated ISSs). Splicing enhancers are bound by proteins or complexes of proteins, including the SR proteins that contain domains enriched in serine and arginine residues, and splicing silencers are frequently bound by heterogeneous ribonucleoproteins (abbreviated hnRNPs).

The availability of genome sequences have allowed system-wide approaches to identify splicing enhancers and silencers that control splicing and led to significant insights into the "splicing code" [29]. These approaches have included machine learning approaches to utilize hundreds of features in pre-mRNAs including motifs bound by RNA binding proteins and RNA secondary structure predictions to predict in vivo splicing decisions [30]. Computer programmes have also been devised that can computationally predict positions of predicted splicing enhancers and silencers and the target sequences of RNA binding proteins in an input genomic sequence (e.g., Figure 3) [30-33]. The combination of these system-wide experimental and bioinformatic analyses show the splicing code is maintained as nucleotide information in the genome. The splicing code has similar importance to the genetic code that is deciphered to read amino acid sequences from mRNAs. However, the splicing code is much more complex than the genetic code. While the genetic code uses triplet codons to specify amino acids, in the splicing code multiple sequence elements act in combination to decipher the exon/intron structure of pre-mRNAs [30].

Because they are needed for exons to be spliced into mRNAs, ESE sequences have been maintained in exons as well as the codons that specify amino acid sequences [35]. The intronic sequences that flank exons are often also strongly conserved between species (Figure 4 shows as an example conserved nucleotide sequences flanking an exon in the mouse Neurexin3 gene downloaded from the UCSC genome browser). Comparison of the human and mouse genomes [1,36] which diverged 75 million years ago show that alternative exons are usually flanked by much longer stretches of conserved intron sequences than constitutive exons, consistent with more elaborate control mechanisms [37,38]. Conservation in these exon flanking intron sequences in some cases is much higher than in promoters, suggesting that one of the main functions of conserved noncoding sequences between mouse and human is the regulation of alternative splicing [37]. Even non-protein coding exons can be highly conserved in the genome. Noncoding exons include highly conserved "poison exons" (for example see Figure 5), that when included insert premature translational termination codons and lead to mRNA decay [32,39]. "Poison exons" are very important for auto-regulation of RNA binding proteins that control splicing $[39,40]$. Together these studies show the maintenance of splicing control sequences has had a significant impact in constraining genome evolution. 
Figure 4. A functional requirement to maintain splicing control sequences constrains evolution of the genome. This screenshot is downloaded from the UCSC genome browser [6] and shows conserved intron sequences flanking the alternatively spliced AS4 exon from the mouse Nrxn3 gene. The conserved sequences are shaded. At the top, the UCSC gene annotations show that this cassette exon is included in $3 / 4$ mRNA isoforms made from this gene. At the bottom the Phastcons plot shows that the flanking intron sequences are also highly conserved as well as the exon sequence (exons might be conserved because of their protein-coding content). Conservation of these intron sequences are likely important to control tissue-specific splicing of this exon by the spliceosome. Known alternative events are annotated on the UCSC track "Alt events", and can be shown alongside the gene structure (here the cassette exon is annotated in the alt events track, and is in purple).

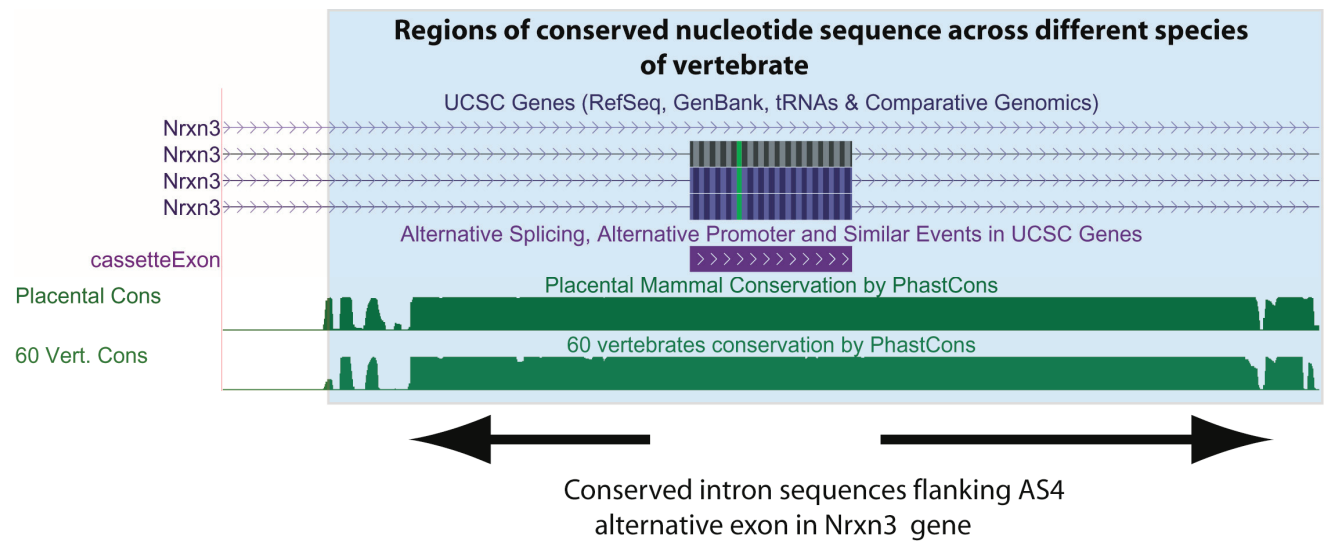

Genome sequences have been used to help develop technologies aimed to globally dissect RNA processing pathways [41]. These technologies can identify the target sites of RNA binding proteins transcriptome-wide. In cross linking immunoprecipitation (abbreviated CLIP) experiments RNA binding proteins are cross-linked in situ to their target RNAs within intact cells using ultra violet irradiation, followed by immunoprecipitation of the RNA protein complexes and amplification by PCR [42-44]. Once unique target sites are identified by next generation sequencing (these are called CLIP tags), these can be mapped onto genome sequences to reveal the initial binding sites in the transcriptome. Transcriptome-wide CLIP analyses have enabled maps to be drawn of the target sites of RNA binding proteins relative to regulated exons, and these maps can be used to predict mechanisms of splicing control [45]. For example, CLIP tags of the RNA binding protein Tra2 $\beta$ that is needed for splicing inclusion for a regulated cassette exon in the NASP gene are shown in Figure 3 [32]. 
Figure 5. Non protein coding exons are conserved in the genome. Some non-coding exons are highly conserved in the human genome and play important roles in controlling the expression levels of splicing regulator proteins. The TRA2A gene encodes the splicing regulator protein Tra $2 \alpha$ and contains a poison exon that contains multiple stop codons and is only inserted into some mRNAs. Despite not containing coding information, the TRA2A poison exon is highly conserved across species (indicated by the Phastcons score). Notice that the TRA2A gene encodes also alternative 5' splice sites and uses alternative promoters. This screenshot was downloaded from the UCSC genome browser [6].

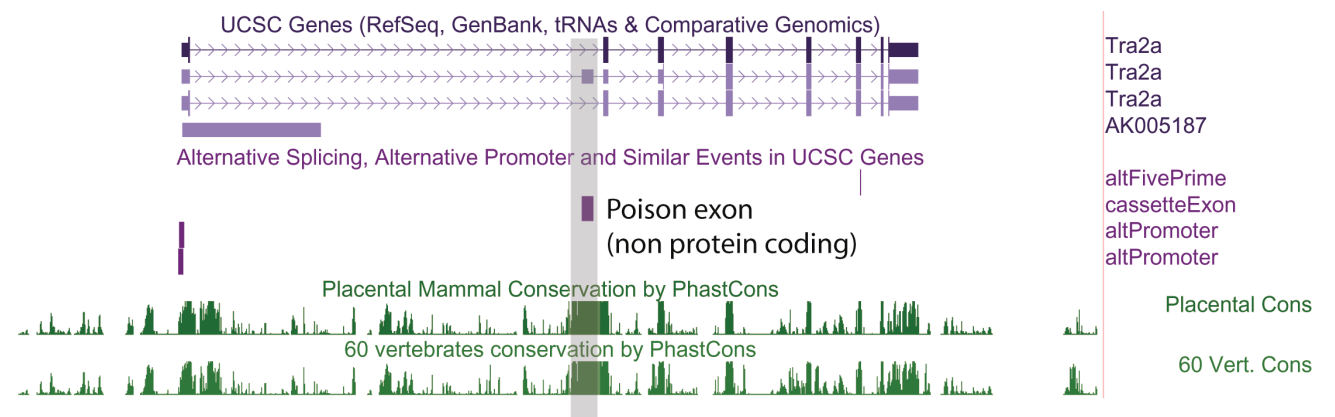

The completion of the human genome sequence enabled the development of comprehensive microarrays to interrogate gene expression. These global techniques include the development of splice-sensitive microarrays. These microarrays either detect specific exons in the transcriptome or the use of specific splice junctions and report splicing patterns in mRNA [46,47]. Transcriptome-wide patterns of alternative splicing can also be detected by RNAseq $[48,49]$. These technologies have been used to search for exons mis-spliced after depletion of particular RNA binding proteins from cells. By analysing thousands of exons in parallel the splicing events that are regulated by specific RNA binding proteins can be comprehensively identified. These experiments have shown some individual RNA binding proteins bind to and regulate similar mRNAs. For example, the NOVA protein regulates splicing of a functionally coherent set of genes involved in synapse function [50]. Other splicing regulators might similarly have coherent RNA functional targets, including T-STAR and SAM68 which regulate regional alternative splicing of the synaptic neurexin genes in the brain $[51,52]$.

Knowing which splicing events are regulated by what proteins at a global level has been used to derive general rules. For example, binding of the NOVA proteins upstream of exons tends to block splicing, while binding of the same proteins downstream of exons enhance splicing $[44,45]$. These rules governing the RNA motifs bound by NOVA and their role in activating or repressing associated exon inclusion are conserved between flies and mammals, although the actual target mRNAs are different [53]. Similar rules have also been uncovered for PTB and some other splicing regulator proteins $[45,54,55]$. Recent developments to understand the splicing code have compared binding maps for different RNA binding proteins, and show that some functionally collaborate with each other to generate tissue specific splicing patterns [56]. 
Transcriptome-wide insights have have also revealed the involvement of RNA binding proteins in other aspects of genome biology. Alu sequences are retro-transposable elements that frequently insert into introns, and have sequence similarities to exons [57]. There are over 15,000 Alu sequences in the human genome, many of which are inserted into introns. The RNA binding protein hnRNP $\mathrm{C}$ has an important role for in protecting the transcriptome from potential mis-splicing caused by the insertion of Alu retrotransposable elements into genomic introns [58]. Depletion of hnRNPC leads to aberrant inclusion of around 1000 Alu-derived exons into the transcriptome. Alu sequences contain polypyrimidine tracts that potentially bind U2AF65, leading to them being aberrantly included into mRNAs, but this splicing is blocked by hnRNPC.

\section{Analysis of the Human Transcriptome Led to the Realisation That Most Human Genes Encode Alternatively Spliced mRNAs}

Historically genes have been defined in different ways by different people at different times. Following the human genome sequencing project, the fundamental definition of what a gene actually is has been enriched, and to some extent clouded, by comparison of genome and transcriptome sequences $[59,60]$. Alternative mRNA isoforms can originate from the same genetic loci through use of alternative promoters and polyadenylation sites, and by the process of alternative splicing through which exons can be spliced into different combinations to give multiple mRNAs. Hence a "single gene" can encode multiple products. Alternative splicing fits into four different categories, depending on how variable splice junctions are utilised (Figure 6). In the simplest form of alternative splicing, called exon skipping, whole exons are either spliced into the mRNA or skipped (ignored by the spliceosome). Exon sequences can also be spliced into mRNA using different splice sites (alternative 5' splice site or 3' splice sites can be selected). Whole introns can also be left in the mRNA (this is called intron retention). Transcriptome-wide analyses show that in humans exon skipping is the most frequent form of alternative splicing, and intron retention the least frequent [61].

Figure 6. Types of alternative splicing events detected in the human transcriptome. Exons are shown as boxes, introns as lines, and splicing patterns as broken lines. mRNAs can be made from individual genes can using multiple alternative events, including different types of splicing, to build up complex patterns of alternative spliced mRNAs.




Evidence provided by comparative genome and transcriptome sequences has shown alternative splicing to be extremely frequent. Before the human genome was published, random sequencing of human mRNAs from their $3^{\prime}$ ends using oligo dT priming suggested $\sim 40 \%$ of human genes encoded alternatively spliced mRNAs [62,63]. During the human genome sequencing project, reconstruction of mRNAs from the gene rich chromosomes 19 and 22 upped this estimate to 59\% of genes encode alternative mRNAs, with 2-3 mRNA isoforms made/gene [1]. The use of microarrays to detect global patterns of alternative splicing indicated $73 \%-74 \%$ of human genes express alternatively spliced mRNA isoforms [46,64]. The most recently reported RNAseq analyses of the human transcriptome is consistent with $\sim 95 \%$ of multi-exonic genes expressing variant mRNA isoforms, with a plateaux of 10-11 isoforms/gene/cell line [65]. Usually one or two major mRNA isoforms predominate in a given cell line so many cell types will just express one major mRNA isoform $[60,65,66]$. $86 \%$ of genes have a minor isoform frequency of $15 \%$ or more, and more than $50 \%$ of alternative exons are tissue specific in expression [48,67]. Alternative events are now annotated on genome browsers like the UCSC genome browser (e.g., Figure 4) [6].

\section{To What Extent Can Human Complexity Be Ascribed to Alternative Splicing?}

Proteins make up large components of human bodies. Prior to the genome sequence estimates of human gene numbers went as high as 100,000. An initial surprise from the human genome sequence was a much lower protein coding gene number, initially counted at around 23,000 [1,2]. The most recent gene counts from the ENCODE consortia suggest only 20,687 human protein coding genes [8]. The number of proteins expressed in a human cell is in contrast estimated to be $\sim 100,000[68,69]$. This protein number represents an amplification factor of 5-fold compared to the counted number of genes. This total gene number in humans does not seem to be exceptionally higher than seemingly less sophisticated organisms. The genome of Haemophilus influenza contains 1743 predicted genes, the yeast Saccharomyces cerevisiae 6000 genes, Drosophila melanogaster $\sim 13,600$ genes, and nematode worms 18,425 genes [70-72].

This apparent failure of gene numbers to correlate with complexity has been called the gene number paradox, and counted as one of the major surprises from the human genome sequence. To what extent might alternative splicing and the resulting expansion in protein coding information help explain developmental and physiological complexity in humans (Figure 7)? Until recently this question has been difficult to address, since the higher number of mRNA and EST sequences available from humans made comparisons of alternative splicing frequency with other species biased. However, a recent modencode project based on RNAseq analysis to look at alternative splicing in C. elegans found $\sim 25 \%$ alternative splicing in 5000 genes, with around $30 \%$ of these being alternatively spliced between different developmental stages [73]. In depth experiments using RNAseq and tiling arrays do show a lower frequency $(60.7 \%)$ of genes in the fruitfly are alternatively spliced than in humans, often in a developmental or sex-specific fashion [74]. 
Figure 7. Alternative splicing amplifies genome information. Alternative splicing amplifies information in the human transcriptome relative to the genome.

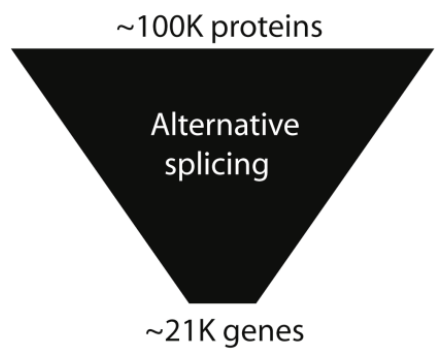

Hence amongst multicellular animals investigated in detail, humans do seem to exhibit higher levels of alternative splicing, which might lend credence to the idea that alternative splicing may be a factor contributing to human sophistication. If phylogenetic differences in alternative splicing frequency correlate with complexity, one might expect a decreased level in single celled organisms compared with multicellular organisms. On the one level less introns are found in the single celled baker's yeast Saccharomyces cerevisiae: only 5\% of genes contain introns in this yeast (290/6000 genes). However, these lower intron numbers are a bit misleading. The reason for a low overall intron number in this yeast is that many introns have been lost because of reverse transcriptase activity converting mRNAs into cDNAs, which then re-integrate into the genome through high levels of homologous recombination replacing originally intron containing genes [75]. While intron-containing genes generally are rare in yeast, alternative splicing of some of these introns are specifically utilised to control developmental timing of during meiosis that takes place under conditions of limiting nutrients [76,77] so alternative splicing is used to control a complex stage in the lifecycle of this single celled organism. Taken as a whole, it is difficult to draw general correlations between overall frequencies of alternative splicing and organism sophistication.

Alternative splicing patterns can also evolve rapidly and sometimes differ between closely related species. For example, despite almost $99 \%$ identity in protein coding information, comparative transcriptome analyses have shown that $6 \%-8 \%$ of alternative spliced exons have different inclusion patterns between humans and chimps. These observations are consistent with alternative splicing contributing to species-species differences, and transcriptomes being more distinct between species than protein coding information [78]. Although the major conclusion from evolutionary comparisons is that much alternative splicing is not conserved between species, comparative genomics show some alternative exons have been highly conserved during evolution [79-81]. These include the highly conserved "AS4" alternative exon in the Neurexin3 gene (abbreviated Nrxn3, Figure 4) that is conserved across the vertebrate lineage [51]. The Neurexin genes have been linked with autism and schizophrenia, and mice genetically engineered to be unable to regulate this AS4 alternative exon in the Nrxn3 gene have different synaptic activity in the brain [82].

Post-genome analyses have also addressed the question what alternative splicing does. Protein sequences encoded by alternatively spliced exons are frequently involved in protein-protein 
interaction networks and contain signalling domains $[83,84]$. Some alternatively spliced mRNAs encode proteins with clearly different functional activity. For example, different mRNA splice isoforms encoding the FOXP1 transcription factor are made between neural stem cells and differentiated cells, and these encode proteins that activate different promoters [85]. Alternative splicing regulators have been implicated in human cognitive diseases like autism [86]. Different groups of genes are regulated by alternative splicing from those regulated by transcription [87].

Alternative splicing pathways have been shown to have important roles in controlling development, and some human diseases are caused by defects in alternative splicing including the multi-system disorder myotonic dystrophy [87]. However, individual RNA binding proteins likely regulate coordinated splicing programmes of many target exons, and each of these individual exons might only have somewhat subtle biological contributions. Furthermore, post-genome technologies are also starting to introduce a note of caution in the interpretation of high levels of alternative splicing. Some lower abundance splice variants might be non-functional isoforms that occur as a result of low frequency events mistakes in the splicing process if they are neither evolutionarily conserved nor protein-coding [21,88]. Hence the frequency of functional alternative splicing is likely to be lower than the total frequency of all alternative splicing events.

\section{Human Genome Packaging into Chromatin Correlates with Its Intron/Exon Structure}

Post genome analyses have shown that the genome and transcriptome are intimately linked. In particular the exon/intron structure of genes correlates with how the genome is packaged. Within the nucleus the genome is wrapped around protein complexes called nucleosomes to form chromatin. Each nucleosome is itself made up of eight positively charged histone molecules that can be modified by the addition of small chemical groups (typically methyl and acetyl groups) [89]. Packaging of the genome within chromatin is important to enable storage of chromosomes within the comparatively small space afforded by the nuclear volume.

After experimental treatment of chromatin with the enzyme DNAse I, the DNA sequences wrapped around nucleosomes remain protected, while the DNA linkages joining nucleosomes together become degraded. Genome-wide analysis of sequences protected from DNAse I digestion in humans and other species indicate a 1.5 fold enrichment of exon sequences over nucleosomes compared to intron sequences [90,91]. This means that in chromatin exons are preferentially (but not exclusively) associated with nucleosomes. This is likely to have a biochemical explanation: exon sequences are GC-enriched, while their flanking intron sequences are AT-rich. Nucleosomes interact more strongly with GC-rich sequences, which likely help anchor exons to nucleosomes. The association of exons with nucleosomes has in turn had important implications for genome evolution. A length of $\sim 150$ nucleotides of DNA is needed to wrap around a nucleosome, and this is also the average size of an exon. Hence nucleosome wrapping has placed an evolutionary constraint on exon size, while in contrast introns have been able to expand in size to thousands of nucleotides. 


\section{Most Splicing Occurs Co-Transcriptionally}

Another important connection between the genome and the transcriptome is their physical proximity during important RNA processing steps. In several species including humans much pre-mRNA splicing has been shown to take place co-transcriptionally [92]. This means that "full length" pre-mRNA copies of genes are not made. Instead processing takes place as pre-mRNAs are produced on nascent pre-mRNAs still attached to RNA polymerases engaged in transcription. Deep sequencing of fractionated nascent RNA in human cell lines and total RNA in the brain show that exons located more upstream in genes are most likely to be spliced on nascent RNA [65,93,94]. Transcription of the genome also functionally depends on components more "traditionally" thought to be involved in splicing. Transcriptome-wide analysis of gene expression following depletion of U1 snRNP (a component of the spliceosome that recognises 5' splice sites) has shown that high nuclear concentrations of U1 snRNP are needed to prevent premature intragenic polyadenylation at sites upstream of the proper 3' boundary of genes [95].

The fact that splicing takes place on chromatin during ongoing transcription has important implications for alternative splicing patterns. Single molecule experiments have shown that nucleosomes slow down the progress of transcription [96,97]. This means that the time taken to traverse nucleosomes can provide pauses in RNA polymerase II elongation, allowing the spliceosome a window to assemble on nascent pre-mRNA. Nucleosomes have thus been described as "speed bumps" [98-101]. Interestingly only true exons, and not pseudoexons, are associated with nucleosomes [90,91]. Exon association with nucleosomes is likely to help in their recognition by the spliceosome. Pauses in transcriptional elongation on bona fide exons might facilitate spliceosome assembly before potentially competing splice sites in downstream pseudoexons can be transcribed. Changes in chromatin structure can locally speed up or slow down transcription within genes and be important for controlling alternative splicing [99]. Local transcriptional pauses would provide spliceosomes a longer "window" of time in which to assemble and sometimes even carry out splicing of an exon before a competing downstream splice site appears [102,103]. Faster elongating RNA polymerase II enzymes would give spliceosomes less time to assemble on nascent pre-mRNA before competing exons were transcribed, and so would favour exon skipping. In some cases changes in histone modification can recruit or stabilise RNA binding proteins which regulate splicing of the pre-mRNAs made from the gene [104]. The interactions between the different processes in gene expression have been recently reviewed in [105].

\section{Conclusions}

The human genome sequence has provided a catalyst for understanding the transcriptome. System-wide approaches of the transcriptome have led to a fuller appreciation of how genomes work including how the human genome operates with a finite gene number and providing a system wide view of RNA processing. 


\section{Acknowledgments}

I thank Julian Venables for comments on the manuscript. This work was supported by the Wellcome Trust (grant numbers WT080368MA and WT089225/Z/09/Z to DJE) and the BBSRC (grant numbers BB/D013917/1 and BB/I006923/1 to DJE).

\section{Author Contributions}

DJE wrote the manuscript.

\section{Conflicts of Interest}

The author declares no conflict of interest.

\section{References}

1. Lander, E.S.; Linton, L.M.; Birren, B.; Nusbaum, C.; Zody, M.C.; Baldwin, J.; Devon, K.; Dewar, K.; Doyle, M.; FitzHugh, W.; et al. Initial sequencing and analysis of the human genome. Nature 2001, 409, 860-921.

2. Venter, J.C.; Adams, M.D.; Myers, E.W.; Li, P.W.; Mural, R.J.; Sutton, G.G.; Smith, H.O.; Yandell, M.; Evans, C.A.; Holt, R.A.; et al. The sequence of the human genome. Science 2001, 291, 1304-1351.

3. Fatica, A.; Bozzoni, I. Long non-coding RNAs: New players in cell differentiation and development. Nat. Rev. Genet. 2014, 15, 7-21.

4. Berget, S.M.; Moore, C.; Sharp, P.A. Spliced segments at the 5 ' terminus of adenovirus 2 late mRNA. Proc. Natl. Acad. Sci. USA 1977, 74, 3171-3175.

5. Chow, L.T.; Gelinas, R.E.; Broker, T.R.; Roberts, R.J. An amazing sequence arrangement at the 5' ends of adenovirus 2 messenger RNA. Cell 1977, 12, 1-8.

6. Karolchik, D.; Barber, G.P.; Casper, J.; Clawson, H.; Cline, M.S.; Diekhans, M.; Dreszer, T.R.; Fujita, P.A.; Guruvadoo, L.; Haeussler, M.; et al. The UCSC Genome Browser database: 2014 update. Nucleic Acids Res. 2014, 42, D764-D770.

7. Gilbert, W. Why genes in pieces? Nature 1978, 271, 501.

8. Dunham, I.; Kundaje, A.; Aldred, S.F.; Collins, P.J.; Davis, C.; Doyle, F.; Epstein, C.B.; Frietze, S.; Harrow, J.; Kaul, R.; et al. An integrated encyclopedia of DNA elements in the human genome. Nature 2012, 489, 57-74.

9. Tennyson, C.N.; Klamut, H.J.; Worton, R.G. The human dystrophin gene requires 16 hours to be transcribed and is cotranscriptionally spliced. Nat. Genet. 1995, 9, 184-190.

10. Jackson, D.A.; Pombo, A.; Iborra, F. The balance sheet for transcription: An analysis of nuclear RNA metabolism in mammalian cells. FASEB J. 2000, 14, 242-254.

11. Okamura, K.; Hagen, J.W.; Duan, H.; Tyler, D.M.; Lai, E.C. The mirtron pathway generates microRNA-class regulatory RNAs in Drosophila. Cell 2007, 130, 89-100.

12. Ruby, J.G.; Jan, C.H.; Bartel, D.P. Intronic microRNA precursors that bypass Drosha processing. Nature 2007, 448, 83-86. 
13. Jurica, M.S.; Moore, M.J. Pre-mRNA splicing: Awash in a sea of proteins. Mol. Cell 2003, $12,5-14$.

14. Wahl, M.C.; Will, C.L.; Luhrmann, R. The spliceosome: Design principles of a dynamic RNP machine. Cell 2009, 136, 701-718.

15. Brow, D.A. Allosteric cascade of spliceosome activation. Annu. Rev. Genet. 2002, 36, 333-360.

16. Mount, S.M. A catalogue of splice junction sequences. Nucleic Acids Res. 1982, 10, 459-472.

17. Lim, L.P.; Burge, C.B. A computational analysis of sequence features involved in recognition of short introns. Proc. Natl. Acad. Sci. USA 2001, 98, 11193-11198.

18. Patel, A.A.; Steitz, J.A. Splicing double: Insights from the second spliceosome. Nat. Rev. Mol. Cell. Biol. 2003, 4, 960-970.

19. Younis, I.; Dittmar, K.; Wang, W.; Foley, S.W.; Berg, M.G.; Hu, K.Y.; Wei, Z.; Wan, L.; Dreyfuss, G. Minor introns are embedded molecular switches regulated by highly unstable U6atac snRNA. Elife 2013, 2, e00780.

20. Fox-Walsh, K.L.; Hertel, K.J. Splice-site pairing is an intrinsically high fidelity process. Proc. Natl. Acad. Sci. USA 2009, 106, 1766-1771.

21. Pickrell, J.K.; Pai, A.A.; Gilad, Y.; Pritchard, J.K. Noisy splicing drives mRNA isoform diversity in human cells. PLoS Genet. 2010, 6, e1001236.

22. Berget, S.M. Exon recognition in vertebrate splicing. J. Biol. Chem. 1995, 270, 2411-2414.

23. Black, D.L. Finding splice sites within a wilderness of RNA. RNA 1995, 1, 763-771.

24. Robberson, B.L.; Cote, G.J.; Berget, S.M. Exon definition may facilitate splice site selection in RNAs with multiple exons. Mol. Cell. Biol. 1990, 10, 84-94.

25. Mardon, H.J.; Sebastio, G.; Baralle, F.E. A role for exon sequences in alternative splicing of the human fibronectin gene. Nucleic Acids Res. 1987, 15, 7725-7733.

26. Reed, R.; Maniatis, T. A role for exon sequences and splice-site proximity in splice-site selection. Cell 1986, 46, 681-690.

27. Black, D.L. Protein diversity from alternative splicing: A challenge for bioinformatics and post-genome biology. Cell 2000, 103, 367-370.

28. Maniatis, T.; Tasic, B. Alternative pre-mRNA splicing and proteome expansion in metazoans. Nature 2002, 418, 236-243.

29. Wang, Z.; Burge, C.B. Splicing regulation: From a parts list of regulatory elements to an integrated splicing code. RNA 2008, 14, 802-813.

30. Barash, Y.; Calarco, J.A.; Gao, W.; Pan, Q.; Wang, X.; Shai, O.; Blencowe, B.J.; Frey, B.J. Deciphering the splicing code. Nature 2010, 465, 53-59.

31. Schwartz, S.; Hall, E.; Ast, G. SROOGLE: Webserver for integrative, user-friendly visualization of splicing signals. Nucleic Acids Res. 2009, 37, W189-W192.

32. Grellscheid, S.; Dalgliesh, C.; Storbeck, M.; Best, A.; Liu, Y.; Jakubik, M.; Mende, Y.; Ehrmann, I.; Curk, T.; Rossbach, K.; et al. Identification of evolutionarily conserved exons as regulated targets for the splicing activator tra2beta in development. PLoS Genet. 2011, 7, e1002390. 
33. Paz, I.; Akerman, M.; Dror, I.; Kosti, I.; Mandel-Gutfreund, Y. SFmap: A web server for motif analysis and prediction of splicing factor binding sites. Nucleic Acids Res. 2010, 38, W281-W285.

34. Zhang, X.H.; Chasin, L.A. Computational definition of sequence motifs governing constitutive exon splicing. Genes Dev. 2004, 18, 1241-1250.

35. Caceres, E.F.; Hurst, L.D. The evolution, impact and properties of exonic splice enhancers. Genome Biol. 2013, 14, R143.

36. Waterston, R.H.; Lindblad-Toh, K.; Birney, E.; Rogers, J.; Abril, J.F.; Agarwal, P.; Agarwala, R.; Ainscough, R.; Alexandersson, M.; An, P.; et al. Initial sequencing and comparative analysis of the mouse genome. Nature 2002, 420, 520-562.

37. Sorek, R.; Ast, G. Intronic sequences flanking alternatively spliced exons are conserved between human and mouse. Genome Res. 2003, 13, 1631-1637.

38. Sugnet, C.W.; Srinivasan, K.; Clark, T.A.; O'Brien, G.; Cline, M.S.; Wang, H.; Williams, A.; Kulp, D.; Blume, J.E.; Haussler, D.; et al. Unusual intron conservation near tissue-regulated exons found by splicing microarrays. PLoS Comput. Biol. 2006, 2, e4.

39. Lareau, L.F.; Inada, M.; Green, R.E.; Wengrod, J.C.; Brenner, S.E. Unproductive splicing of SR genes associated with highly conserved and ultraconserved DNA elements. Nature 2007, 446, 926-929.

40. Ni, J.Z.; Grate, L.; Donohue, J.P.; Preston, C.; Nobida, N.; O'Brien, G.; Shiue, L.; Clark, T.A.; Blume, J.E.; Ares, M., Jr. Ultraconserved elements are associated with homeostatic control of splicing regulators by alternative splicing and nonsense-mediated decay. Genes Dev. 2007, 21, 708-718.

41. Buratti, E.; Baralle, M.; Baralle, F.E. From single splicing events to thousands: The ambiguous step forward in splicing research. Brief. Funct. Genomics 2013, 12, 3-12.

42. Licatalosi, D.D.; Mele, A.; Fak, J.J.; Ule, J.; Kayikci, M.; Chi, S.W.; Clark, T.A.; Schweitzer, A.C.; Blume, J.E.; Wang, X.; Darnell, J.C.; Darnell, R.B. HITS-CLIP yields genome-wide insights into brain alternative RNA processing. Nature 2008, 456, 464-469.

43. Konig, J.; Zarnack, K.; Rot, G.; Curk, T.; Kayikci, M.; Zupan, B.; Turner, D.J.; Luscombe, N.M.; Ule, J. iCLIP-Transcriptome-wide mapping of protein-RNA interactions with individual nucleotide resolution. J. Vis. Exp. 2011, 50, 2638.

44. Anko, M.L.; Neugebauer, K.M. RNA-protein interactions in vivo: Global gets specific. Trends Biochem. Sci. 2012, 37, 255-262.

45. Witten, J.T.; Ule, J. Understanding splicing regulation through RNA splicing maps. Trends Genet. 2011, 27, 89-97.

46. Johnson, J.M.; Castle, J.; Garrett-Engele, P.; Kan, Z.; Loerch, P.M.; Armour, C.D.; Santos, R.; Schadt, E.E.; Stoughton, R.; Shoemaker, D.D. Genome-wide survey of human alternative pre-mRNA splicing with exon junction microarrays. Science 2003, 302, 2141-2144.

47. Pan, Q.; Shai, O.; Misquitta, C.; Zhang, W.; Saltzman, A.L.; Mohammad, N.; Babak, T.; Siu, H.; Hughes, T.R.; Morris, Q.D.; et al. Revealing global regulatory features of mammalian alternative splicing using a quantitative microarray platform. Mol. Cell 2004, 16, 929-941. 
48. Pan, Q.; Shai, O.; Lee, L.J.; Frey, B.J.; Blencowe, B.J. Deep surveying of alternative splicing complexity in the human transcriptome by high-throughput sequencing. Nat. Genet. 2008, 40, 1413-1415.

49. Sultan, M.; Schulz, M.H.; Richard, H.; Magen, A.; Klingenhoff, A.; Scherf, M.; Seifert, M.; Borodina, T.; Soldatov, A.; Parkhomchuk, D.; et al. A global view of gene activity and alternative splicing by deep sequencing of the human transcriptome. Science 2008, 321, 956-960.

50. Ule, J.; Ule, A.; Spencer, J.; Williams, A.; Hu, J.S.; Cline, M.; Wang, H.; Clark, T.; Fraser, C.; Ruggiu, M.; et al. Nova regulates brain-specific splicing to shape the synapse. Nat. Genet. 2005, 37, 844-852.

51. Ehrmann, I.; Dalgliesh, C.; Liu, Y.; Danilenko, M.; Crosier, M.; Overman, L.; Arthur, H.M.; Lindsay, S.; Clowry, G.J.; Venables, J.P.; et al. The tissue-specific RNA binding protein T-STAR controls regional splicing patterns of neurexin pre-mRNAs in the brain. PLoS Genet. 2013, 9, e1003474.

52. Iijima, T.; Wu, K.; Witte, H.; Hanno-Iijima, Y.; Glatter, T.; Richard, S.; Scheiffele, P. SAM68 regulates neuronal activity-dependent alternative splicing of neurexin-1. Cell 2011, 147, 1601-1614.

53. Brooks, A.N.; Yang, L.; Duff, M.O.; Hansen, K.D.; Park, J.W.; Dudoit, S.; Brenner, S.E.; Graveley, B.R. Conservation of an RNA regulatory map between Drosophila and mammals. Genome Res. 2011, 21, 193-202.

54. Llorian, M.; Schwartz, S.; Clark, T.A.; Hollander, D.; Tan, L.Y.; Spellman, R.; Gordon, A.; Schweitzer, A.C.; de la Grange, P.; Ast, G.; et al. Position-dependent alternative splicing activity revealed by global profiling of alternative splicing events regulated by PTB. Nat. Struct. Mol. Biol. 2010, 17, 1114-1123.

55. Xue, Y.; Zhou, Y.; Wu, T.; Zhu, T.; Ji, X.; Kwon, Y.S.; Zhang, C.; Yeo, G.; Black, D.L.; Sun, H.; et al. Genome-wide analysis of PTB-RNA interactions reveals a strategy used by the general splicing repressor to modulate exon inclusion or skipping. Mol. Cell 2009, 36, 996-1006.

56. Zhang, C.; Frias, M.A.; Mele, A.; Ruggiu, M.; Eom, T.; Marney, C.B.; Wang, H.; Licatalosi, D.D.; Fak, J.J.; Darnell, R.B. Integrative modeling defines the Nova splicing-regulatory network and its combinatorial controls. Science 2010, 329, 439-443.

57. Sorek, R.; Lev-Maor, G.; Reznik, M.; Dagan, T.; Belinky, F.; Graur, D.; Ast, G. Minimal conditions for exonization of intronic sequences: $5^{\prime}$ splice site formation in Alu exons. Mol. Cell 2004, 14, 221-231.

58. Zarnack, K.; Konig, J.; Tajnik, M.; Martincorena, I.; Eustermann, S.; Stevant, I.; Reyes, A.; Anders, S.; Luscombe, N.M.; Ule, J. Direct competition between hnRNP C and U2AF65 protects the transcriptome from the exonization of Alu elements. Cell 2013, 152, 453-466.

59. Gerstein, M.B.; Bruce, C.; Rozowsky, J.S.; Zheng, D.; Du, J.; Korbel, J.O.; Emanuelsson, O.; Zhang, Z.D.; Weissman, S.; Snyder, M. What is a gene, post-ENCODE? History and updated definition. Genome Res. 2007, 17, 669-681. 
60. Mudge, J.M.; Frankish, A.; Harrow, J. Functional transcriptomics in the post-ENCODE era. Genome Res. 2013, 12, 1961-1973.

61. Ast, G. How did alternative splicing evolve? Nat. Rev. Genet. 2004, 5, 773-782.

62. Brett, D.; Hanke, J.; Lehmann, G.; Haase, S.; Delbruck, S.; Krueger, S.; Reich, J.; Bork, P. EST comparison indicates $38 \%$ of human mRNAs contain possible alternative splice forms. FEBS Lett. 2000, 474, 83-86.

63. Mironov, A.A.; Fickett, J.W.; Gelfand, M.S. Frequent alternative splicing of human genes. Genome Res. 1999, 9, 1288-1293.

64. Clark, T.A.; Schweitzer, A.C.; Chen, T.X.; Staples, M.K.; Lu, G.; Wang, H.; Williams, A.; Blume, J.E. Discovery of tissue-specific exons using comprehensive human exon microarrays. Genome Biol. 2007, 8, R64.

65. Djebali, S.; Davis, C.A.; Merkel, A.; Dobin, A.; Lassmann, T.; Mortazavi, A.; Tanzer, A.; Lagarde, J.; Lin, W.; Schlesinger, F.; et al. Landscape of transcription in human cells. Nature 2012, 489, 101-108.

66. Gonzalez-Porta, M.; Frankish, A.; Rung, J.; Harrow, J.; Brazma, A. Transcriptome analysis of human tissues and cell lines reveals one dominant transcript per gene. Genome Biol. 2013, 14, R70.

67. Wang, E.T.; Sandberg, R.; Luo, S.; Khrebtukova, I.; Zhang, L.; Mayr, C.; Kingsmore, S.F.; Schroth, G.P.; Burge, C.B. Alternative isoform regulation in human tissue transcriptomes. Nature 2008, 456, 470-476.

68. Harrison, P.M.; Kumar, A.; Lang, N.; Snyder, M.; Gerstein, M. A question of size: The eukaryotic proteome and the problems in defining it. Nucleic Acids Res. 2002, 30, 10831090.

69. Stamm, S. Signals and their transduction pathways regulating alternative splicing: A new dimension of the human genome. Hum. Mol. Genet. 2002, 11, 2409-2416.

70. Adams, M.D.; Celniker, S.E.; Holt, R.A.; Evans, C.A.; Gocayne, J.D.; Amanatides, P.G.; Scherer, S.E.; Li, P.W.; Hoskins, R.A.; Galle, R.F.; et al. The genome sequence of Drosophila melanogaster. Science 2000, 287, 2185-2195.

71. Fleischmann, R.D.; Adams, M.D.; White, O.; Clayton, R.A.; Kirkness, E.F.; Kerlavage, A.R.; Bult, C.J.; Tomb, J.F.; Dougherty, B.A.; Merrick, J.M.; et al. Whole-genome random sequencing and assembly of Haemophilus influenzae Rd. Science 1995, 269, 496-512.

72. Genome sequence of the nematode C. elegans: A platform for investigating biology. Science 1998, 282, 2012-2018.

73. Ramani, A.K.; Calarco, J.A.; Pan, Q.; Mavandadi, S.; Wang, Y.; Nelson, A.C.; Lee, L.J.; Morris, Q.; Blencowe, B.J.; Zhen, M.; et al. Genome-wide analysis of alternative splicing in Caenorhabditis elegans. Genome Res. 2011, 21, 342-348.

74. Graveley, B.R.; Brooks, A.N.; Carlson, J.W.; Duff, M.O.; Landolin, J.M.; Yang, L.; Artieri, C.G.; van Baren, M.J.; Boley, N.; Booth, B.W.; et al. The developmental transcriptome of Drosophila melanogaster. Nature 2011, 471, 473-479.

75. Roy, S.W. Intron-rich ancestors. Trends Genet. 2006, 22, 468-471. 
76. Munding, E.M.; Igel, A.H.; Shiue, L.; Dorighi, K.M.; Trevino, L.R.; Ares, M., Jr. Integration of a splicing regulatory network within the meiotic gene expression program of Saccharomyces cerevisiae. Genes Dev. 2010, 24, 2693-2704.

77. Pleiss, J.A.; Whitworth, G.B.; Bergkessel, M.; Guthrie, C. Rapid, transcript-specific changes in splicing in response to environmental stress. Mol. Cell 2007, 27, 928-937.

78. Calarco, J.A.; Xing, Y.; Caceres, M.; Calarco, J.P.; Xiao, X.; Pan, Q.; Lee, C.; Preuss, T.M.; Blencowe, B.J. Global analysis of alternative splicing differences between humans and chimpanzees. Genes Dev. 2007, 21, 2963-2975.

79. Barbosa-Morais, N.L.; Irimia, M.; Pan, Q.; Xiong, H.Y.; Gueroussov, S.; Lee, L.J.; Slobodeniuc, V.; Kutter, C.; Watt, S.; Colak, R.; et al. The evolutionary landscape of alternative splicing in vertebrate species. Science 2012, 338, 1587-1593.

80. Venables, J.P.; Vignal, E.; Baghdiguian, S.; Fort, P.; Tazi, J. Tissue-specific alternative splicing of Tak1 is conserved in deuterostomes. Mol. Biol. Evol. 2012, 29, 261-269.

81. Merkin, J.; Russell, C.; Chen, P.; Burge, C.B. Evolutionary dynamics of gene and isoform regulation in Mammalian tissues. Science 2012, 338, 1593-1599.

82. Aoto, J.; Martinelli, D.C.; Malenka, R.C.; Tabuchi, K.; Sudhof, T.C. Presynaptic neurexin-3 alternative splicing trans-synaptically controls postsynaptic AMPA receptor trafficking. Cell 2013, 154, 75-88.

83. Colak, R.; Kim, T.; Michaut, M.; Sun, M.; Irimia, M.; Bellay, J.; Myers, C.L.; Blencowe, B.J.; Kim, P.M. Distinct types of disorder in the human proteome: Functional implications for alternative splicing. PLoS Comput. Biol. 2013, 9, e1003030.

84. Ellis, J.D.; Barrios-Rodiles, M.; Colak, R.; Irimia, M.; Kim, T.; Calarco, J.A.; Wang, X.; Pan, Q.; O'Hanlon, D.; Kim, P.M.; et al. Tissue-specific alternative splicing remodels protein-protein interaction networks. Mol. Cell 2012, 46, 884-892.

85. Gabut, M.; Samavarchi-Tehrani, P.; Wang, X.; Slobodeniuc, V.; O'Hanlon, D.; Sung, H.K.; Alvarez, M.; Talukder, S.; Pan, Q.; Mazzoni, E.O.; et al. An alternative splicing switch regulates embryonic stem cell pluripotency and reprogramming. Cell 2011, 147, 132-146.

86. Voineagu, I.; Wang, X.; Johnston, P.; Lowe, J.K.; Tian, Y.; Horvath, S.; Mill, J.; Cantor, R.M.; Blencowe, B.J.; Geschwind, D.H. Transcriptomic analysis of autistic brain reveals convergent molecular pathology. Nature 2011, 474, 380-384.

87. Kalsotra, A.; Cooper, T.A. Functional consequences of developmentally regulated alternative splicing. Nat. Rev. Genet. 2011, 12, 715-729.

88. Hsu, S.N.; Hertel, K.J. Spliceosomes walk the line: Splicing errors and their impact on cellular function. RNA Biol. 2009, 6, 526-530.

89. Luger, K.; Mader, A.W.; Richmond, R.K.; Sargent, D.F.; Richmond, T.J. Crystal structure of the nucleosome core particle at 2.8 A resolution. Nature 1997, 389, 251-260.

90. Schwartz, S.; Meshorer, E.; Ast, G. Chromatin organization marks exon-intron structure. Nat. Struct. Mol. Biol. 2009, 16, 990-995.

91. Tilgner, H.; Nikolaou, C.; Althammer, S.; Sammeth, M.; Beato, M.; Valcarcel, J.; Guigo, R. Nucleosome positioning as a determinant of exon recognition. Nat. Struct. Mol. Biol. 2009, 16, 996-1001. 
92. Brugiolo, M.; Herzel, L.; Neugebauer, K.M. Counting on co-transcriptional splicing. F1000Prime Rep. 2013, 5, 9.

93. Tilgner, H.; Knowles, D.G.; Johnson, R.; Davis, C.A.; Chakrabortty, S.; Djebali, S.; Curado, J.; Snyder, M.; Gingeras, T.R.; Guigo, R. Deep sequencing of subcellular RNA fractions shows splicing to be predominantly co-transcriptional in the human genome but inefficient for lncRNAs. Genome Res. 2012, 22, 1616-1625.

94. Ameur, A.; Zaghlool, A.; Halvardson, J.; Wetterbom, A.; Gyllensten, U.; Cavelier, L.; Feuk, L. Total RNA sequencing reveals nascent transcription and widespread co-transcriptional splicing in the human brain. Nat. Struct. Mol. Biol. 2011, 18, U1435-U1157.

95. Kaida, D.; Berg, M.G.; Younis, I.; Kasim, M.; Singh, L.N.; Wan, L.; Dreyfuss, G. U1 snRNP protects pre-mRNAs from premature cleavage and polyadenylation. Nature 2010, 468, 664-668.

96. Hodges, C.; Bintu, L.; Lubkowska, L.; Kashlev, M.; Bustamante, C. Nucleosomal fluctuations govern the transcription dynamics of RNA polymerase II. Science 2009, 325, 626-628.

97. Kornblihtt, A.R.; Schor, I.E.; Allo, M.; Blencowe, B.J. When chromatin meets splicing. Nat. Struct. Mol. Biol. 2009, 16, 902-903.

98. Schwartz, S.; Ast, G. Chromatin density and splicing destiny: On the cross-talk between chromatin structure and splicing. EMBO J. 2010, 29, 1629-1636.

99. Carrillo Oesterreich, F.; Bieberstein, N.; Neugebauer, K.M. Pause locally, splice globally. Trends Cell. Biol. 2011, 21, 328-335.

100. Schor, I.E.; Allo, M.; Kornblihtt, A.R. Intragenic chromatin modifications: A new layer in alternative splicing regulation. Epigenetics 2010, 5, 174-179.

101. Tilgner, H.; Guigo, R. From chromatin to splicing: RNA-processing as a total artwork. Epigenetics 2010, 5, 180-184.

102. De la Mata, M.; Alonso, C.R.; Kadener, S.; Fededa, J.P.; Blaustein, M.; Pelisch, F.; Cramer, P.; Bentley, D.; Kornblihtt, A.R. A slow RNA polymerase II affects alternative splicing in vivo. Mol. Cell 2003, 12, 525-532.

103. Schor, I.E.; Rascovan, N.; Pelisch, F.; Allo, M.; Kornblihtt, A.R. Neuronal cell depolarization induces intragenic chromatin modifications affecting NCAM alternative splicing. Proc. Natl. Acad. Sci. USA 2009, 106, 4325-4330.

104. Luco, R.F.; Pan, Q.; Tominaga, K.; Blencowe, B.J.; Pereira-Smith, O.M.; Misteli, T. Regulation of alternative splicing by histone modifications. Science 2010, 327, 996-1000.

105. Braunschweig, U.; Gueroussov, S.; Plocik, A.M.; Graveley, B.R.; Blencowe, B.J. Dynamic integration of splicing within gene regulatory pathways. Cell 2013, 152, 1252-1269. 


\title{
Architecture of Inherited Susceptibility to Colorectal Cancer: A Voyage of Discovery
}

\author{
Nicola Whiffin and Richard S. Houlston
}

\begin{abstract}
This review looks back at five decades of research into genetic susceptibility to colorectal cancer (CRC) and the insights these studies have provided. Initial evidence of a genetic basis of CRC stems from epidemiological studies in the 1950s and is further provided by the existence of multiple dominant predisposition syndromes. Genetic linkage and positional cloning studies identified the first high-penetrance genes for CRC in the 1980s and 1990s. More recent genome-wide association studies have identified common low-penetrance susceptibility loci and provide support for a polygenic model of disease susceptibility. These observations suggest a high proportion of CRC may arise in a group of susceptible individuals as a consequence of the combined effects of common low-penetrance risk alleles and rare variants conferring moderate CRC risks. Despite these advances, however, currently identified loci explain only a small fraction of the estimated heritability to CRC. It is hoped that a new generation of sequencing projects will help explain this missing heritability.
\end{abstract}

Reprinted from Genes. Cite as: Whiffin, N.; Houlston, R.S. Architecture of Inherited Susceptibility to Colorectal Cancer: A Voyage of Discovery. Genes 2014, 5, 270-284.

\section{Introduction}

Colorectal cancer (CRC) is the third most common cancer worldwide with half a million new individuals diagnosed annually [1]. In the UK CRC affects $\sim 40,000$ individuals and is responsible for $\sim 16,000$ deaths each year (Cancer Research UK, 2013) amounting to a life-time risk of 5\%-6\%. It is now an established fact that inherited susceptibility has an important role in predisposition to CRC. The earliest evidence for this came from epidemiological studies in the 1950s which showed a two- to three-fold increased risk of CRC in first degree relatives of patients [2]. Subsequent studies have identified a number of CRC susceptibility genes. These discoveries have greatly increased our understanding of the mechanisms underlying CRC biology and have provided promising targets for therapeutic intervention. Moreover, the ability to identify individuals at increased risk of CRC is of important clinical relevance.

\section{Early Models of Genetic Susceptibility}

Large families with multiple individuals affected by CRC have long been reported in the clinic. It was not until the late 1950s, however, that epidemiological studies attempted to quantify this familial clustering by comparing the incidence of CRC in first degree relatives (FDRs) of cases to control groups [3-5]. Recent analysis estimates an approximate two-fold increase in risk in FDRs [2]. This risk increases further to four-fold when the relative is diagnosed with early-onset CRC ( $<45$ years of age), indicative of colorectal tumours developing in genetically susceptibly individuals at an earlier age. 
In 1969 Ashley [6] proposed that colonic cancer development could be ascribed to a series of carcinogenic "hits" on normal intestinal mucosa cells. He further noted that the number of necessary "hits" was lower for genetically susceptible individuals with the Mendelian predisposition syndrome familial adenomatous polyposis (FAP). In the same year, DeMars [7] suggested that FAP, along with other apparently autosomal dominant syndromes, is autosomally recessive at the cellular level; individuals with a germline mutation in one allele of a tumour suppressor gene develop cancer as a result of subsequent somatic mutations in the other gene copy.

Anderson [8], in 1974, made the first argument for a polygenic mechanism to cancer susceptibility based on the increased risk in FDRs of cancer patients being limited to two- to three-fold. He stated these results were "not indicative of strong genetic effects" and rather suggested a mechanism involving many genes with small effects acting in concert with environmental factors with larger effects. Whilst there is growing evidence to suggest that his conclusion is, at least in part, correct, the reasoning behind this statement is flawed as the relative risks associated with FDRs are likely to be underestimated. This is because calculations of relative risks typically include both sporadic and genetically susceptible cases that are then compared to the general population which, to compound the problem, also contains individuals that are genetically susceptible to CRC.

\section{Identification of Rare High-Penetrance Susceptibility Alleles}

Evidence for Mendelian transmission of CRC was first provided by reports of large families with CRC segregating in a dominant fashion. Perhaps the most notable case report is "family G" first described in 1913 by Warthin and subsequently revisited by Lynch and Krush in 1971 [9]. This family of over 650 blood relatives provided scientists with one of the longest, most detailed cancer genealogies in the world and was instrumental in establishing the syndrome of hereditary non-polyposis colorectal cancer (HNPCC or Lynch syndrome).

Table 1. Colorectal cancer predisposition syndromes and associated high-penetrance mutations.

\begin{tabular}{ccccc}
\hline Gene(s) & Syndrome & Risk in mutation carriers & Mode of inheritance & References \\
\hline APC & FAP & $90 \%$ by age 45 & Dominant & {$[10-14]$} \\
Mismatch repair & HNPCC/Lynch syndrome & $40 \%-80 \%$ by age 75 & Dominant & {$[15-26,32,33]$} \\
(MLH1/MSH2/MSH6/PMS2) & JPS & $17 \%-68 \%$ by age 60 & Dominant & {$[26,27]$} \\
SMAD4/BMPR1A & PJS & $39 \%$ by age 70 & Dominant & {$[28]$} \\
STK11 & MYH-associated polyposis & $35 \%-53 \%$ & Recessive & {$[34,35]$} \\
MUTYH & Oligopolyposis & & Dominant & {$[36]$} \\
POLD1/POLE & & & &
\end{tabular}

Family based genetic linkage and positional cloning studies in the late 1980s and early 1990s led to the identification of numerous CRC susceptibility genes (Table 1). The APC gene on chromosome 5 was the first gene to be shown to be associated with CRC susceptibility when it was identified as mutated in FAP patients [10-14]. Subsequently, mutations in genes of the mismatch repair (MMR) pathway, particularly $M S H 2, M L H 1$ and MSH6, the TGF- $\beta$ signalling pathway genes, SMAD4 and BMPR1A, and the serine/threonine kinase gene STK11, were revealed as the causes of 
HNPCC [15-26], Juvenile Polyposis syndrome (JPS) [27,28] and Peutz-Jeghers syndrome (PJS) [29] respectively. Risk alleles in these genes are rare $(<0.1 \%)$ and confer a $>10$ fold increase in risk of CRC. These genes are tumour suppressors conforming to DeMars' "two-hit" model of cancer susceptibility through an apparently dominant mode of inheritance. The clinical utility of testing for high penetrance mutations in these genes has long been established and identification of individuals with such mutations has been shown reduce CRC incidence through prevention strategies and screening [30,31].

\section{More Recent Models of Genetic Susceptibility}

Studies examining the difference in CRC development between monozygotic and dizygotic twins estimated that $\sim 35 \%$ of CRC could be ascribed to a genetic predisposition [37]. However, $<10 \%$ of all CRC can be accounted for by germline mutations in APC and the MMR genes and crucially $\sim 70 \%$ of the familial risk of CRC remains unexplained [38].

Over the past 20 years, extensive efforts to identify additional, highly penetrant cancer susceptibility genes for CRC through conventional linkage scans have met with limited success $[39,40]$. This strongly implies that any additional high penetrance CRC gene will individually account for only a small proportion of the familial risk. Statistical modelling of the pattern of familial occurrence of CRC after exclusion of known high-risk genes suggests that much of the inherited susceptibility is likely to be polygenic with the co-inheritance of multiple genetic variants, each with a modest individual effect, causing a wide range of risk in the population (Figure 1).

Over the past two decades candidate gene studies have identified rare moderately-penetrant risk alleles (minor allele frequency (MAF) $<2 \%$; relative risks (RRs) $>2.0$ ) and more recent genome-wide association studies (GWAS) have identified common, low-penetrance alleles (MAF $>10 \%$; OR $<1.5$ ). In reality, these variants are likely to occur as a continuum and the separate classes of risk alleles merely reflect the subgroups detectable using current methodologies.

\section{Rare, Moderately-Penetrant Disease-Causing Variants}

The "rare-variant" hypothesis suggests that much of the remaining heritability could be due to the combined effect of rare, moderately-penetrant risk alleles [41]. These variants are hypothesised to act independently and to confer modest, but detectable, increases in risk.

Attempts to identify this class of disease allele have mainly been through resequencing candidate genes in affected families, the success of which has been hampered by our limited knowledge of tumour biology. The identification of the missense variant, APC I1307K, carried by $\sim 6 \%$ of Ashkenazi Jews and conferring around a two-fold increase in risk of CRC [42] and the more recent discovery of the functional promoter variant $-93 \mathrm{G}>\mathrm{A}$ of $\mathrm{MLH} 1$, shown to predispose to microsatellite unstable CRC [43], represent rare successes of this approach.

A priori rare disease-causing alleles are likely to act in a dominant fashion; however, functional variants in the base-excision repair gene $M U T Y H$ provide an example of a recessive model of inheritance [34]. Biallelic or compound heterozygosity of the G396D and Y179C mutations in 
MUTYH, which are carried by around $1 \%-2 \%$ of the UK population, confers a CRC risk comparable to that seen in carriers of germline MMR mutations [35].

Figure 1. Polygenic model of disease susceptibility. The distribution of risk alleles in both cases and controls follows a normal distribution. However, cases have a shift towards a higher number of high risk alleles.

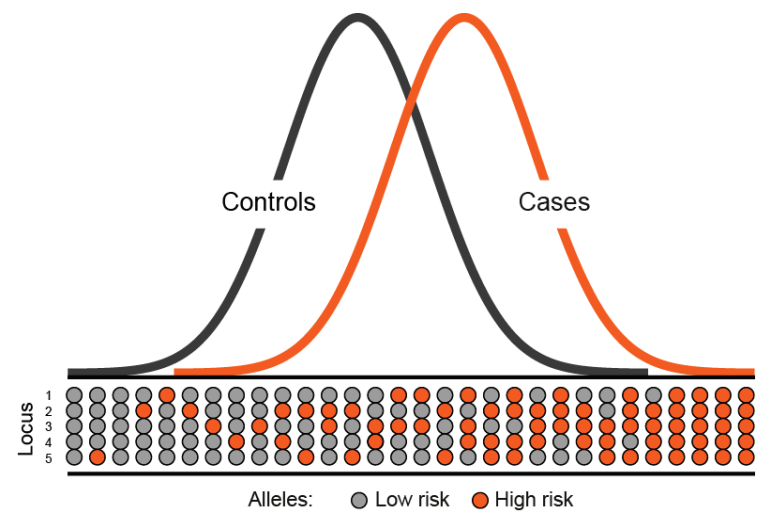

Mechanistically these variants are likely to be directly causal. For the variants in MUTYH, insights into biological basis of susceptibility came from the method in which they were discovered; a FAP family with no apparent $A P C$ mutation was found to possess a mutator phenotype reflective of defective base excision, resulting in somatic mutation of $A P C$ and other genes $[34,44]$. In contrast, the $A P C$ I1307K T $>$ A variant appears to increase replication errors in $A P C$ through generation of a run of eight adenines [42].

\section{Identification of Common Low-Penetrance Alleles}

The "common-variant, common-disease" hypothesis states that a substantial proportion of the remaining risk is likely to be accounted for by the summation of numerous low-penetrant genetic variants, each with a relatively high frequency in the population [45]. These variants have more subtle effects on gene regulation and predominantly reside within non-coding regions of the genome. Each individual variant is associated with only a modest increase in risk; however, collectively they may confer a substantial increase in disease susceptibility. These alleles rarely cause multiple cases in families and therefore cannot be detected through genetic linkage studies [46]. Initial attempts to identify this class of allele through candidate gene association studies were based on small case-control series and had little success; any proposed variants were not successfully validated in subsequent studies.

Genome-wide association studies (GWAS), typically based on genotyping of 300,000 to over 1 million SNPs, have proved to be a powerful approach in identifying common, low penetrance susceptibility loci for CRC without prior knowledge of location and function.

Since the first CRC GWAS in 2007, 18 CRC susceptibility loci have been identified in European populations (Table 2) [47-53]. While each individual risk allele confers only a small 
relative risk $(1.06<\mathrm{OR}<1.26)$, the $\mathrm{SNPs}$ are common $(\mathrm{MAF}>10 \%)$ and hence contribute significantly to the overall incidence of CRC. Moreover, by acting in concert they can impact significantly on an individual's risk of developing CRC (Figure 2) [54]. The design of association studies is advantageous as large numbers of unrelated case and control samples may be readily obtained, providing adequate power to detect loci with relatively small effects. This is in contrast to the difficulty in recruiting the extensive pedigrees required for linkage studies.

Table 2. Loci identified as associated with colorectal cancer through genome-wide association studies and meta-analyses.

\begin{tabular}{ccccccc}
\hline Locus & Nearest Gene(s) & GWAS tagSNP & Location & Risk Allele & Alt Allele & RAF \\
\hline $1 \mathrm{q} 41$ & DUSP10 & $\mathrm{rs} 6691170$ & $222,045,446$ & $\mathrm{~T}$ & $\mathrm{G}$ & 0.40 \\
$3 \mathrm{q} 26.2$ & TERC,MYNN & $\mathrm{rs} 10936599$ & $169,492,101$ & $\mathrm{C}$ & $\mathrm{T}$ & 0.75 \\
$6 \mathrm{p} 21.2$ & CDKN1A & $\mathrm{rs} 1321311$ & $36,622,900$ & $\mathrm{~T}$ & $\mathrm{G}$ & 0.21 \\
$8 \mathrm{q} 23.3$ & $E I F 3 H$ & $\mathrm{rs} 16892766$ & $117,630,683$ & $\mathrm{C}$ & $\mathrm{A}$ & 0.09 \\
$8 \mathrm{q} 24.21$ & MYC & $\mathrm{rs} 6983267$ & $128,413,305$ & $\mathrm{G}$ & $\mathrm{T}$ & 0.52 \\
$10 \mathrm{p} 14$ & GATA3 & $\mathrm{rs} 10795668$ & $8,701,219$ & $\mathrm{G}$ & $\mathrm{A}$ & 0.67 \\
$11 \mathrm{q} 13.4$ & POLD3 & $\mathrm{rs} 3824999$ & $74,345,550$ & $\mathrm{C}$ & $\mathrm{A}$ & 0.47 \\
$11 \mathrm{q} 23.1$ & FLJ45803 & $\mathrm{rs} 3802842$ & $111,171,709$ & $\mathrm{C}$ & $\mathrm{A}$ & 0.27 \\
$12 \mathrm{q} 13$ & DIP2B, ATF1 & $\mathrm{rs} 11169552$ & $51,155,663$ & $\mathrm{C}$ & $\mathrm{T}$ & 0.75 \\
$14 \mathrm{q} 22.2$ & BMP4 & $\mathrm{rs} 4444235$ & $54,410,919$ & $\mathrm{C}$ & $\mathrm{T}$ & 0.48 \\
$15 \mathrm{q} 13.3$ & SCG5, GREM1 & $\mathrm{rs} 4779584$ & $32,994,756$ & $\mathrm{~T}$ & $\mathrm{C}$ & 0.19 \\
$16 \mathrm{q} 22.1$ & CDH1 & $\mathrm{rs} 9929218$ & $68,820,946$ & $\mathrm{G}$ & $\mathrm{A}$ & 0.71 \\
$18 \mathrm{q} 21.2$ & SMAD7 & $\mathrm{rs} 4939827$ & $46,453,463$ & $\mathrm{~T}$ & $\mathrm{C}$ & 0.53 \\
$19 \mathrm{q} 13.11$ & RHPN2, GPATCH1 & $\mathrm{rs} 10411210$ & $33,532,300$ & $\mathrm{C}$ & $\mathrm{T}$ & 0.90 \\
20p12.3 & BMP2 & $\mathrm{rs} 961253$ & $6,404,281$ & $\mathrm{~A}$ & $\mathrm{C}$ & 0.37 \\
& & $\mathrm{rs} 4813802$ & $6,699,595$ & $\mathrm{G}$ & $\mathrm{T}$ & 0.34 \\
20q13.33 & LAMA5 & $\mathrm{rs} 4925386$ & $60,921,044$ & $\mathrm{C}$ & $\mathrm{T}$ & 0.68 \\
Xp22.2 & SHROOM2 & $\mathrm{rs5934683}$ & $9,751,474$ & $\mathrm{~T}$ & $\mathrm{C}$ & 0.56 \\
\hline
\end{tabular}

Figure 2. Plot showing the increase in odds ratio for colorectal cancer with an increasing number of risk alleles.

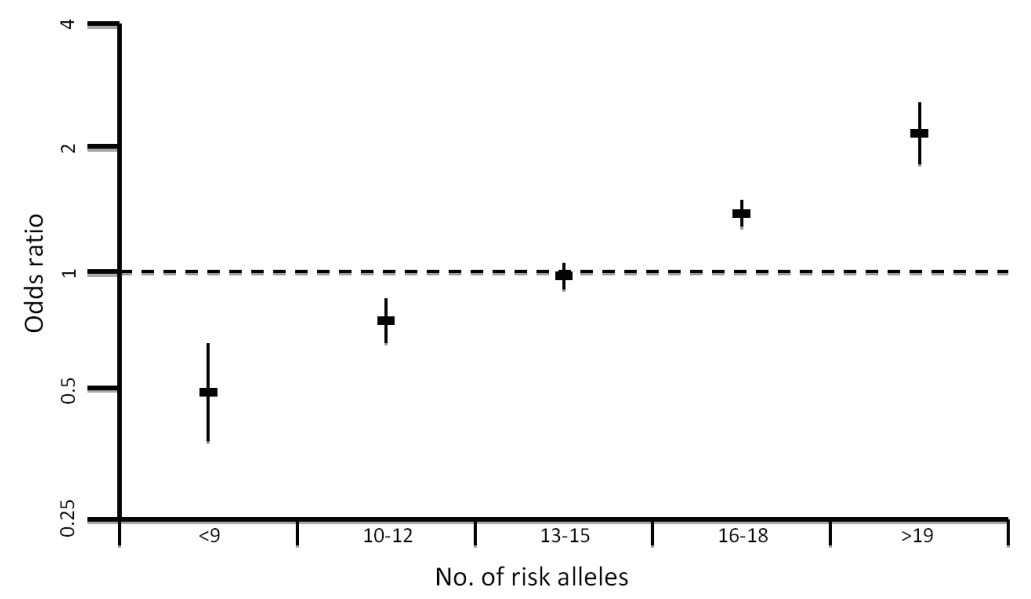


Importantly, few genes implicated in GWAS were previously evaluated in candidate gene studies, highlighting the importance of such an agnostic approach for gene discovery and understanding of CRC aetiology. None of the currently identified loci, for example, are involved in DNA repair, which is the principle pathway underscoring high-penetrance CRC susceptibility. Interestingly, five of the loci discovered to date are within or near to genes involved in the TGF- $\beta$ signalling pathway $[47,50]$. This pathway has already been implicated in pathogenesis of CRC, as the dominant CRC predisposition syndrome JPS is caused by high penetrance mutations in the TGF- $\beta$ family genes SMAD4 and BMPR1A $[13,14]$.

\section{Functional Effects of GWAS Loci}

Elucidating the basis of association at common low-penetrance loci represents a significant challenge. The tagging SNPs (tagSNPs) used in GWAS are not necessarily strong candidates for being causal and were instead chosen to capture variation across large genomic regions. Hence, establishing which of a set of highly correlated variants in linkage disequilibrium (LD) with the tagSNP is the true causal variant is a challenge. In addition, many GWAS loci map to non-coding regions or gene deserts, suggesting the true cause of the association at these regions is through subtle effects on gene expression rather than changes to protein coding sequence.

Fine mapping of CRC loci is still in its infancy with most studies attempting only to narrow down the location of a likely functional variant through imputation or re-sequencing [55-57]. These studies suggest candidate variants but very few functional studies have been carried out to test these assertions. To date, in only four regions has a SNP been proposed as the likely functional candidate and a mechanism of action suggested (8q23.3 [58], 8q24.21 [59,60], 11q23.1 [61], $18 \mathrm{q} 21.1$ [62]). The most intriguing of these regions is $8 \mathrm{q} 24.21$, which has pleiotropic effects on cancer susceptibility, also harbouring risk loci for breast [57], ovarian [63], bladder [64], CLL [65] and multiple independent loci for prostate cancer [66-69]. This is in contrast to most associations found to date which appear to be disease specific. The rs6983267 risk SNP is associated with both prostate and colorectal cancers and lies within an evolutionarily conserved region. The two alleles of rs6983267 show differential binding of the TCF4 transcription factor [59] to an enhancer element that has been shown to physically interact with the MYC gene promoter [60]. MYC is amplified or over-expressed in multiple cancer types leading to up-regulation of many genes controlling cell proliferation, so it is predicted that variation at this locus acts through subtle effects on MYC gene expression. The risk allele of rs6983267 has also been suggested as a marker of worse prognosis in CRC patients [70]. The strongest CRC GWAS association is at 18q21.1 $(\mathrm{RR}=1.26)$ within the $S M A D 7$ gene, which acts as an antagonist of the TGF- $\beta$ signalling pathway. Resequencing of the $17 \mathrm{~kb}$ region of linkage disequilibrium surrounding the GWAS tagSNP rs4939827 identified a novel variant, termed Novel1 (rs58920878), which was shown to affect SMAD7 expression [62]. 


\section{Impact of Common Variation on CRC Risk}

Collectively, the currently identified risk loci explain only $\sim 6 \%$ of the overall familial risk of CRC. This estimate is likely conservative as the effect of the causal variant at each locus is expected to be greater than the association detected through a GWAS tagSNP. In addition, as evinced by the 14q22 (BMP4) [52] association multiple risk variants may exist at each locus, including low-frequency variants with significantly larger effects. Moreover, epistatic interactions may exist between common risk loci, which could make the contribution of low-penetrance susceptibility alleles much higher. Such interactions remain difficult to detect due to substantial multiple testing penalties, and existing studies suggest the effects of most common low-penetrance alleles seem to be independent. In addition, interactions of these alleles with epigenetic regulation or environmental factors may lead to a greater increase in disease risk. For example the MLH1-93G $>$ A polymorphism has been shown to be associated with increased methylation of the MLH1 promoter [71]. Another important consideration is the possible modification of the effect of a low-penetrance allele by the presence of a high-penetrance mutation. The only evidence to support this assertion in CRC is a small study implicating the 8q23.3 and 11q23.1 CRC SNPs as modifiers of CRC risk in MMR mutation carriers [72]. Although there was initial hope for the use of low penetrance variants in the clinic, the small proportion of familial risk explained and the apparent lack of epistatic interactions between the variants leads to them being of low predictive power. The increased risk associated with having a high number of risk alleles (Figure 2) has the prospect of identifying individuals in the general population who might benefit from earlier screening [54].

\section{Identifying Novel CRC Susceptibility Loci}

It is unlikely that there are any common CRC risk loci with appreciable MAF (>10\%) and with relative risks $>1.1$ that remain to be uncovered given the size of existing GWAS studies. Small variant effect sizes combined with stringent thresholds for establishing statistical significance and financial constraints on the number of variants which can be followed up constrain GWAS study power. However, many GWAS have long tails of association with alleles of increasingly small effect, suggesting much of the remaining susceptibility may be embodied in a multitude of common risk alleles. Larger GWAS studies combining multiple phases and tens of thousands of cases may identify many more of these susceptibility loci, although the effect of these on CRC risk is likely to be minimal. Such studies have been conducted in both breast [73] and prostate [74] cancer identifying 41 and 23 novel risk loci respectively.

Commercial arrays used for GWAS capture a large proportion of common SNPs with minor allele frequencies $(\mathrm{MAF})>10 \%$. However, a much lower fraction of less common $(5 \%-10 \%$ MAF) and rare (MAF $<5 \%)$ SNPs are captured by these arrays. The power of GWAS to detect variants with MAF $<10 \%$ is therefore limited. Additionally, GWAS arrays are not optimally formatted to capture indels and copy number variants, both of which are likely to have roles in disease susceptibility. New "exome chips" have recently been released that aim to address some of these limitations. However the success of these new arrays remains to be evaluated. 
Since the completion of the human genome project in 2003, the utilisation of massively parallel sequencing technologies to identify variants has become feasible. Moreover, these methods can be used to detect small indels, substitutions and structural variants. Although in their infancy, such studies are beginning to identify additional variants. For example, highly-penetrant mutations have recently been identified in the proof reading domains of POLD1 (S478N) and POLE (L424V) in CRC families [36]. Another recent study identified 11 candidate CRC susceptibility genes with truncating mutations in two or more of 96 familial CRC cases [75]. To maximally utilise sequencing data, new bioinformatic techniques are required to remove sequencing errors and prioritise variants. Additionally, due to financial considerations such studies remain small and not powered to detect less common variants with moderate effects risk of disease. Using cases enriched for genetic susceptibility evidenced by a strong family history or early disease onset is a useful technique to increase the efficiency of these studies. In addition, utilising whole exome sequencing, as performed by Palles et al. [36], can dramatically reduce the costs associated with such projects. Coding variants are also much easier to interpret than those in non-coding regions. However, with every individual's genome harboring 250-300 putative loss of function variants [76] and many missense variants being of unknown effect, identification of the disease causing variant presents a significant challenge. Recent studies are working to interpret this class of variants [77] and algorithms such as SIFT [78], PolyPhen2 [79] and CONDEL [80] aim to guide researchers by predicting the functional effects of coding mutations. Work to develop similar methods to deal with non-coding regions is still in its infancy [81,82], however, recent evidence [83,84] suggests that these regions are also a priori likely to contain variants with a large effect on CRC risk.

\section{Conclusions}

Much has been achieved in the study of genetic susceptibility to CRC in the last five decades. The architecture underlying this susceptibility is now recognised to be defined by a spectrum of predisposition alleles with different effect sizes and frequencies in the population. GWAS has proved a successful approach for identification of novel low-penetrance CRC risk alleles, improving our understanding of disease aetiology and providing novel therapeutic targets. Determining the biological processes underlying the associations at these loci presents a significant challenge and will likely require large collaborations between genetic researchers and functional biologists. Despite these advances, a large proportion of the heritability to CRC remains unaccounted for. It is hoped that the new generation of sequencing projects will help to uncover this missing heritability.

\section{Acknowledgements}

Principle funding was provided to RSH by Cancer Research UK (C1298/A8362-Bobby Moore Fund for Cancer Research UK). NW is in receipt of a $\mathrm{PhD}$ studentship from the Institute of Cancer Research. 


\section{Author Contributions}

Manuscript was conceived and written by NW and RSH.

\section{Conflicts of Interest}

The authors declare no conflict of interest.

\section{References}

1. Jemal, A.; Siegel, R.; Ward, E.; Murray, T.; Xu, J.; Smigal, C.; Thun, M.J. Cancer statistics, 2006. CA Cancer J. Clin. 2006, 56, 106-130.

2. Johns, L.E.; Houlston, R.S. A systematic review and meta-analysis of familial colorectal cancer risk. Am. J. Gastroenterol. 2001, 96, 2992-3003.

3. Woolf, C.M. A genetic study of carcinoma of the large intestine. Am. J. Hum. Genet. 1958, 10, $42-47$.

4. Macklin, M.T. Inheritance of cancer of the stomach and large intestine in man. J. Natl. Canc. Inst. 1960, 24, 551-571.

5. Lovett, E. Familial factors in the etiology of carcinoma of the large bowel. Proc. R. Soc. Med. 1974, 67, 751-752.

6. Ashley, D.J. Colonic cancer arising in polyposis coli. J. Med. Genet. 1969, 6, 376-378.

7. DeMars, R. 23rd Annual Symposium on Fundamental Cancer Research; Williams and Wilkins: Baltimore, MD, USA, 1969; pp. 105-106.

8. Anderson, D.E. Genetic study of breast cancer: Identification of a high risk group. Cancer 1974, 34, 1090-1097.

9. Lynch, H.T.; Krush, A.J. Cancer family "G" Revisited: 1895-1970. Cancer 1971, 27, 1505-1511.

10. Bodmer, W.F.; Bailey, C.J.; Bodmer, J.; Bussey, H.J.; Ellis, A.; Gorman, P.; Lucibello, F.C.; Murday, V.A.; Rider, S.H.; Scambler, P.; et al. Localization of the gene for familial adenomatous polyposis on chromosome 5. Nature 1987, 328, 614-616.

11. Leppert, M.; Dobbs, M.; Scambler, P.; O’Connell, P.; Nakamura, Y.; Stauffer, D.; Woodward, S.; Burt, R.; Hughes, J.; Gardner, E.; et al. The gene for familial polyposis coli maps to the long arm of chromosome 5. Science 1987, 238, 1411-1413.

12. Herrera, L.; Kakati, S.; Gibas, L.; Pietrzak, E.; Sandberg, A.A. Gardner syndrome in a man with an interstitial deletion of 5q. Am. J. Med. Genet. 1986, 25, 473-476.

13. Groden, J.; Thliveris, A.; Samowitz, W.; Carlson, M.; Gelbert, L.; Albertsen, H.; Joslyn, G.; Stevens, J.; Spirio, L.; Robertson, M.; et al. Identification and characterization of the familial adenomatous polyposis coli gene. Cell 1991, 66, 589-600.

14. Kinzler, K.W.; Nilbert, M.C.; Su, L.K.; Vogelstein, B.; Bryan, T.M.; Levy, D.B.; Smith, K.J.; Preisinger, A.C.; Hedge, P.; McKechnie, D.; et al. Identification of fap locus genes from chromosome 5q21. Science 1991, 253, 661-665. 
15. Miyaki, M.; Konishi, M.; Tanaka, K.; Kikuchi-Yanoshita, R.; Muraoka, M.; Yasuno, M.; Igari, T.; Koike, M.; Chiba, M.; Mori, T. Germline mutation of msh6 as the cause of hereditary nonpolyposis colorectal cancer. Nat. Genet. 1997, 17, 271-272.

16. Papadopoulos, N.; Nicolaides, N.C.; Wei, Y.F.; Ruben, S.M.; Carter, K.C.; Rosen, C.A.; Haseltine, W.A.; Fleischmann, R.D.; Fraser, C.M.; Adams, M.D.; et al. Mutation of a mutl homolog in hereditary colon cancer. Science 1994, 263, 1625-1629.

17. Bronner, C.E.; Baker, S.M.; Morrison, P.T.; Warren, G.; Smith, L.G.; Lescoe, M.K.; Kane, M.; Earabino, C.; Lipford, J.; Lindblom, A.; et al. Mutation in the DNA mismatch repair gene homologue hmlh1 is associated with hereditary non-polyposis colon cancer. Nature 1994, 368, 258-261.

18. Strand, M.; Prolla, T.A.; Liskay, R.M.; Petes, T.D. Destabilization of tracts of simple repetitive DNA in yeast by mutations affecting DNA mismatch repair. Nature 1993, 365, 274-276.

19. Peltomaki, P.; Aaltonen, L.A.; Sistonen, P.; Pylkkanen, L.; Mecklin, J.P.; Jarvinen, H.; Green, J.S.; Jass, J.R.; Weber, J.L.; Leach, F.S.; et al. Genetic mapping of a locus predisposing to human colorectal cancer. Science 1993, 260, 810-812.

20. Aaltonen, L.A.; Peltomaki, P.; Leach, F.S.; Sistonen, P.; Pylkkanen, L.; Mecklin, J.P.; Jarvinen, H.; Powell, S.M.; Jen, J.; Hamilton, S.R.; et al. Clues to the pathogenesis of familial colorectal cancer. Science 1993, 260, 812-816.

21. Lindblom, A.; Tannergard, P.; Werelius, B.; Nordenskjold, M. Genetic mapping of a second locus predisposing to hereditary non-polyposis colon cancer. Nat. Genet. 1993, 5, 279-282.

22. Leach, F.S.; Nicolaides, N.C.; Papadopoulos, N.; Liu, B.; Jen, J.; Parsons, R.; Peltomaki, P.; Sistonen, P.; Aaltonen, L.A.; Nystrom-Lahti, M.; et al. Mutations of a muts homolog in hereditary nonpolyposis colorectal cancer. Cell 1993, 75, 1215-1225.

23. Drummond, J.T.; Li, G.M.; Longley, M.J.; Modrich, P. Isolation of an hmsh2-p160 heterodimer that restores DNA mismatch repair to tumor cells. Science 1995, 268, 1909-1912.

24. Palombo, F.; Gallinari, P.; Iaccarino, I.; Lettieri, T.; Hughes, M.; D’Arrigo, A.; Truong, O.; Hsuan, J.J.; Jiricny, J. Gtbp, a 160-kilodalton protein essential for mismatch-binding activity in human cells. Science 1995, 268, 1912-1914.

25. Akiyama, Y.; Sato, H.; Yamada, T.; Nagasaki, H.; Tsuchiya, A.; Abe, R.; Yuasa, Y. Germ-line mutation of the hmsh6/gtbp gene in an atypical hereditary nonpolyposis colorectal cancer kindred. Canc. Res. 1997, 57, 3920-3923.

26. Shibata, D.; Peinado, M.A.; Ionov, Y.; Malkhosyan, S.; Perucho, M. Genomic instability in repeated sequences is an early somatic event in colorectal tumorigenesis that persists after transformation. Nat. Genet. 1994, 6, 273-281.

27. Howe, J.R.; Roth, S.; Ringold, J.C.; Summers, R.W.; Jarvinen, H.J.; Sistonen, P.; Tomlinson, I.P.; Houlston, R.S.; Bevan, S.; Mitros, F.A.; et al. Mutations in the smad4/dpc4 gene in juvenile polyposis. Science 1998, 280, 1086-1088.

28. Howe, J.R.; Bair, J.L.; Sayed, M.G.; Anderson, M.E.; Mitros, F.A.; Petersen, G.M.; Velculescu, V.E.; Traverso, G.; Vogelstein, B. Germline mutations of the gene encoding bone morphogenetic protein receptor 1a in juvenile polyposis. Nat. Genet. 2001, 28, 184-187. 
29. Hemminki, A.; Markie, D.; Tomlinson, I.; Avizienyte, E.; Roth, S.; Loukola, A.; Bignell, G.; Warren, W.; Aminoff, M.; Hoglund, P.; et al. A serine/threonine kinase gene defective in peutz-jeghers syndrome. Nature 1998, 391, 184-187.

30. Jarvinen, H.J.; Aarnio, M.; Mustonen, H.; Aktan-Collan, K.; Aaltonen, L.A.; Peltomaki, P.; de La Chapelle, A.; Mecklin, J.P. Controlled 15-year trial on screening for colorectal cancer in families with hereditary nonpolyposis colorectal cancer. Gastroenterology 2000, 118, 829-834.

31. Burn, J.; Gerdes, A.M.; Macrae, F.; Mecklin, J.P.; Moeslein, G.; Olschwang, S.; Eccles, D.; Evans, D.G.; Maher, E.R.; Bertario, L.; et al. Long-term effect of aspirin on cancer risk in carriers of hereditary colorectal cancer: An analysis from the capp2 randomised controlled trial. Lancet 2011, 378, 2081-2087.

32. Nicolaides, N.C.; Papadopoulos, N.; Liu, B.; Wei, Y.F.; Carter, K.C.; Ruben, S.M.; Rosen, C.A.; Haseltine, W.A.; Fleischmann, R.D.; Fraser, C.M.; et al. Mutations of two pms homologues in hereditary nonpolyposis colon cancer. Nature 1994, 371, 75-80.

33. Hendriks, Y.M.; Jagmohan-Changur, S.; van der Klift, H.M.; Morreau, H.; van Puijenbroek, M.; Tops, C.; van Os, T.; Wagner, A.; Ausems, M.G.; Gomez, E.; et al. Heterozygous mutations in pms 2 cause hereditary nonpolyposis colorectal carcinoma (lynch syndrome). Gastroenterology 2006, 130, 312-322.

34. Al-Tassan, N.; Chmiel, N.H.; Maynard, J.; Fleming, N.; Livingston, A.L.; Williams, G.T.; Hodges, A.K.; Davies, D.R.; David, S.S.; Sampson, J.R.; et al. Inherited variants of myh associated with somatic g:C-->t:A mutations in colorectal tumors. Nat. Genet. 2002, 30, 227-232.

35. Lubbe, S.J.; di Bernardo, M.C.; Chandler, I.P.; Houlston, R.S. Clinical implications of the colorectal cancer risk associated with mutyh mutation. J. Clin. Oncol. 2009, 27, 3975-3980.

36. Palles, C.; Cazier, J.B.; Howarth, K.M.; Domingo, E.; Jones, A.M.; Broderick, P.; Kemp, Z.; Spain, S.L.; Guarino, E.; Salguero, I.; et al. Germline mutations affecting the proofreading domains of pole and pold1 predispose to colorectal adenomas and carcinomas. Nat. Genet. 2013, 45, 136-144.

37. Lichtenstein, P.; Holm, N.V.; Verkasalo, P.K.; Iliadou, A.; Kaprio, J.; Koskenvuo, M.; Pukkala, E.; Skytthe, A.; Hemminki, K. Environmental and heritable factors in the causation of cancer-Analyses of cohorts of twins from sweden, denmark, and finland. N. Engl. J. Med. 2000, 343, 78-85.

38. Lubbe, S.J.; Webb, E.L.; Chandler, I.P.; Houlston, R.S. Implications of familial colorectal cancer risk profiles and microsatellite instability status. J. Clin. Oncol. 2009, 27, 2238-2244.

39. Papaemmanuil, E.; Carvajal-Carmona, L.; Sellick, G.S.; Kemp, Z.; Webb, E.; Spain, S.; Sullivan, K.; Barclay, E.; Lubbe, S.; Jaeger, E.; et al. Deciphering the genetics of hereditary non-syndromic colorectal cancer. Eur. J. Hum. Genet. 2008, 16, 1477-1486.

40. Wiesner, G.L.; Daley, D.; Lewis, S.; Ticknor, C.; Platzer, P.; Lutterbaugh, J.; MacMillen, M.; Baliner, B.; Willis, J.; Elston, R.C.; et al. A subset of familial colorectal neoplasia kindreds linked to chromosome 9q22.2-31.2. Proc. Natl. Acad. Sci. USA 2003, 100, 12961-12965.

41. Bodmer, W.; Bonilla, C. Common and rare variants in multifactorial susceptibility to common diseases. Nat. Genet. 2008, 40, 695-701. 
42. Laken, S.J.; Petersen, G.M.; Gruber, S.B.; Oddoux, C.; Ostrer, H.; Giardiello, F.M.; Hamilton, S.R.; Hampel, H.; Markowitz, A.; Klimstra, D.; et al. Familial colorectal cancer in ashkenazim due to a hypermutable tract in apc. Nat. Genet. 1997, 17, 79-83.

43. Whiffin, N.; Broderick, P.; Lubbe, S.J.; Pittman, A.M.; Penegar, S.; Chandler, I.; Houlston, R.S. Mlh1-93G $>$ A is a risk factor for msi colorectal cancer. Carcinogenesis 2011, 32, 1157-1161.

44. Lipton, L.; Halford, S.E.; Johnson, V.; Novelli, M.R.; Jones, A.; Cummings, C.; Barclay, E.; Sieber, O.; Sadat, A.; Bisgaard, M.L.; et al. Carcinogenesis in myh-associated polyposis follows a distinct genetic pathway. Canc. Res. 2003, 63, 7595-7599.

45. Reich, D.E.; Lander, E.S. On the allelic spectrum of human disease. Trends Genet. 2001, 17, 502-510.

46. Risch, N.; Merikangas, K. The future of genetic studies of complex human diseases. Science 1996, 273, 1516-1517.

47. Broderick, P.; Carvajal-Carmona, L.; Pittman, A.M.; Webb, E.; Howarth, K.; Rowan, A.; Lubbe, S.; Spain, S.; Sullivan, K.; Fielding, S.; et al. A genome-wide association study shows that common alleles of smad7 influence colorectal cancer risk. Nat. Genet. 2007, 39, 1315-1317.

48. Dunlop, M.G.; Dobbins, S.E.; Farrington, S.M.; Jones, A.M.; Palles, C.; Whiffin, N.; Tenesa, A.; Spain, S.; Broderick, P.; Ooi, L.Y.; et al. Common variation near cdkn1a, pold3 and shroom 2 influences colorectal cancer risk. Nat. Genet. 2012, 44, 770-776.

49. Houlston, R.S.; Cheadle, J.; Dobbins, S.E.; Tenesa, A.; Jones, A.M.; Howarth, K.; Spain, S.L.; Broderick, P.; Domingo, E.; Farrington, S.; et al. Meta-analysis of three genome-wide association studies identifies susceptibility loci for colorectal cancer at 1q41, 3q26.2, 12q13.13 and 20q13.33. Nat. Genet. 2010, 42, 973-977.

50. Houlston, R.S.; Webb, E.; Broderick, P.; Pittman, A.M.; di Bernardo, M.C.; Lubbe, S.; Chandler, I.; Vijayakrishnan, J.; Sullivan, K.; Penegar, S.; et al. Meta-analysis of genome-wide association data identifies four new susceptibility loci for colorectal cancer. Nat. Genet. 2008, 40, 1426-1435.

51. Tenesa, A.; Farrington, S.M.; Prendergast, J.G.; Porteous, M.E.; Walker, M.; Haq, N.; Barnetson, R.A.; Theodoratou, E.; Cetnarskyj, R.; Cartwright, N.; et al. Genome-wide association scan identifies a colorectal cancer susceptibility locus on 11q23 and replicates risk loci at 8q24 and 18q21. Nat. Genet. 2008, 40, 631-637.

52. Tomlinson, I.P.; Carvajal-Carmona, L.G.; Dobbins, S.E.; Tenesa, A.; Jones, A.M.; Howarth, K.; Palles, C.; Broderick, P.; Jaeger, E.E.; Farrington, S.; et al. Multiple common susceptibility variants near bmp pathway loci grem1, bmp4, and bmp2 explain part of the missing heritability of colorectal cancer. PLoS Genet. 2011, 7, e1002105.

53. Tomlinson, I.P.; Webb, E.; Carvajal-Carmona, L.; Broderick, P.; Howarth, K.; Pittman, A.M.; Spain, S.; Lubbe, S.; Walther, A.; Sullivan, K.; et al. A genome-wide association study identifies colorectal cancer susceptibility loci on chromosomes 10p14 and 8q23.3. Nat. Genet. 2008, 40, 623-630. 
54. Lubbe, S.J.; di Bernardo, M.C.; Broderick, P.; Chandler, I.; Houlston, R.S. Comprehensive evaluation of the impact of 14 genetic variants on colorectal cancer phenotype and risk. Am. J. Epidemiol. 2012, 175, 1-10.

55. Whiffin, N.; Dobbins, S.E.; Hosking, F.J.; Palles, C.; Tenesa, A.; Wang, Y.; Farrington, S.M.; Jones, A.M.; Broderick, P.; Campbell, H.; et al. Deciphering the genetic architecture of low-penetrance susceptibility to colorectal cancer. Hum. Mol. Genet. 2013, 22, 5075-5082.

56. Carvajal-Carmona, L.G.; Cazier, J.B.; Jones, A.M.; Howarth, K.; Broderick, P.; Pittman, A.; Dobbins, S.; Tenesa, A.; Farrington, S.; Prendergast, J.; et al. Fine-mapping of colorectal cancer susceptibility loci at 8q23.3, 16q22.1 and 19q13.11: Refinement of association signals and use of in silico analysis to suggest functional variation and unexpected candidate target genes. Hum. Mol. Genet. 2011, 20, 2879-2888.

57. Easton, D.F.; Pooley, K.A.; Dunning, A.M.; Pharoah, P.D.; Thompson, D.; Ballinger, D.G.; Struewing, J.P.; Morrison, J.; Field, H.; Luben, R.; et al. Genome-wide association study identifies novel breast cancer susceptibility loci. Nature 2007, 447, 1087-1093.

58. Pittman, A.M.; Naranjo, S.; Jalava, S.E.; Twiss, P.; Ma, Y.; Olver, B.; Lloyd, A.; Vijayakrishnan, J.; Qureshi, M.; Broderick, P.; et al. Allelic variation at the 8q23.3 colorectal cancer risk locus functions as a cis-acting regulator of eif3h. PLoS Genet. 2010, 6, e1001126.

59. Tuupanen, S.; Turunen, M.; Lehtonen, R.; Hallikas, O.; Vanharanta, S.; Kivioja, T.; Bjorklund, M.; Wei, G.; Yan, J.; Niittymaki, I.; et al. The common colorectal cancer predisposition snp rs6983267 at chromosome 8q24 confers potential to enhanced wnt signaling. Nat. Genet. 2009, 41, 885-890.

60. Pomerantz, M.M.; Ahmadiyeh, N.; Jia, L.; Herman, P.; Verzi, M.P.; Doddapaneni, H.; Beckwith, C.A.; Chan, J.A.; Hills, A.; Davis, M.; et al. The 8q24 cancer risk variant rs6983267 shows long-range interaction with myc in colorectal cancer. Nat. Genet. 2009, 41, 882-884.

61. Pittman, A.M.; Webb, E.; Carvajal-Carmona, L.; Howarth, K.; di Bernardo, M.C.; Broderick, P.; Spain, S.; Walther, A.; Price, A.; Sullivan, K.; et al. Refinement of the basis and impact of common 11q23.1 variation to the risk of developing colorectal cancer. Hum. Mol. Genet. 2008, 17, 3720-3727.

62. Pittman, A.M.; Naranjo, S.; Webb, E.; Broderick, P.; Lips, E.H.; van Wezel, T.; Morreau, H.; Sullivan, K.; Fielding, S.; Twiss, P.; et al. The colorectal cancer risk at $18 \mathrm{q} 21$ is caused by a novel variant altering smad7 expression. Genome Res. 2009, 19, 987-993.

63. Ghoussaini, M.; Song, H.; Koessler, T.; Al Olama, A.A.; Kote-Jarai, Z.; Driver, K.E.; Pooley, K.A.; Ramus, S.J.; Kjaer, S.K.; Hogdall, E.; et al. Multiple loci with different cancer specificities within the 8q24 gene desert. J. Natl. Canc. Inst. 2008, 100, 962-966.

64. Kiemeney, L.A.; Thorlacius, S.; Sulem, P.; Geller, F.; Aben, K.K.; Stacey, S.N.; Gudmundsson, J.; Jakobsdottir, M.; Bergthorsson, J.T.; Sigurdsson, A.; et al. Sequence variant on 8q24 confers susceptibility to urinary bladder cancer. Nat. Genet. 2008, 40, 1307-1312. 
65. Crowther-Swanepoel, D.; Broderick, P.; di Bernardo, M.C.; Dobbins, S.E.; Torres, M.; Mansouri, M.; Ruiz-Ponte, C.; Enjuanes, A.; Rosenquist, R.; Carracedo, A.; et al. Common variants at 2q37.3, 8q24.21, 15q21.3 and 16q24.1 influence chronic lymphocytic leukemia risk. Nat. Genet. 2010, 42, 132-136.

66. Al Olama, A.A.; Kote-Jarai, Z.; Giles, G.G.; Guy, M.; Morrison, J.; Severi, G.; Leongamornlert, D.A.; Tymrakiewicz, M.; Jhavar, S.; Saunders, E.; et al. Multiple loci on 8q24 associated with prostate cancer susceptibility. Nat. Genet. 2009, 41, 1058-1060.

67. Amundadottir, L.T.; Sulem, P.; Gudmundsson, J.; Helgason, A.; Baker, A.; Agnarsson, B.A.; Sigurdsson, A.; Benediktsdottir, K.R.; Cazier, J.B.; Sainz, J.; et al. A common variant associated with prostate cancer in european and african populations. Nat. Genet. 2006, 38, 652-658.

68. Yeager, M.; Orr, N.; Hayes, R.B.; Jacobs, K.B.; Kraft, P.; Wacholder, S.; Minichiello, M.J.; Fearnhead, P.; Yu, K.; Chatterjee, N.; et al. Genome-wide association study of prostate cancer identifies a second risk locus at 8q24. Nat. Genet. 2007, 39, 645-649.

69. Gudmundsson, J.; Sulem, P.; Manolescu, A.; Amundadottir, L.T.; Gudbjartsson, D.; Helgason, A.; Rafnar, T.; Bergthorsson, J.T.; Agnarsson, B.A.; Baker, A.; et al. Genome-wide association study identifies a second prostate cancer susceptibility variant at 8q24. Nat. Genet. 2007, 39, 631-637.

70. Takatsuno, Y.; Mimori, K.; Yamamoto, K.; Sato, T.; Niida, A.; Inoue, H.; Imoto, S.; Kawano, S.; Yamaguchi, R.; Toh, H.; et al. The rs6983267 snp is associated with myc transcription efficiency, which promotes progression and worsens prognosis of colorectal cancer. Ann. Surg. Oncol. 2013, 20, 1395-1402.

71. Mrkonjic, M.; Roslin, N.M.; Greenwood, C.M.; Raptis, S.; Pollett, A.; Laird, P.W.; Pethe, V.V.; Chiang, T.; Daftary, D.; Dicks, E.; et al. Specific variants in the mlh1 gene region may drive DNA methylation, loss of protein expression, and msi-h colorectal cancer. PLoS One 2010, 5, e13314.

72. Wijnen, J.T.; Brohet, R.M.; van Eijk, R.; Jagmohan-Changur, S.; Middeldorp, A.; Tops, C.M.; van Puijenbroek, M.; Ausems, M.G.; Gomez Garcia, E.; Hes, F.J.; et al. Chromosome 8q23.3 and $11 \mathrm{q} 23.1$ variants modify colorectal cancer risk in lynch syndrome. Gastroenterology 2009, 136, 131-137.

73. Michailidou, K.; Hall, P.; Gonzalez-Neira, A.; Ghoussaini, M.; Dennis, J.; Milne, R.L.; Schmidt, M.K.; Chang-Claude, J.; Bojesen, S.E.; Bolla, M.K.; et al. Large-scale genotyping identifies 41 new loci associated with breast cancer risk. Nat. Genet. 2013, 45, 353-361, 361e1-361e2.

74. Eeles, R.A.; Olama, A.A.; Benlloch, S.; Saunders, E.J.; Leongamornlert, D.A.; Tymrakiewicz, M.; Ghoussaini, M.; Luccarini, C.; Dennis, J.; Jugurnauth-Little, S.; et al. Identification of 23 new prostate cancer susceptibility loci using the icogs custom genotyping array. Nat. Genet. 2013, 45, 385-391, 391e1-391e2.

75. Gylfe, A.E.; Katainen, R.; Kondelin, J.; Tanskanen, T.; Cajuso, T.; Hanninen, U.; Taipale, J.; Taipale, M.; Renkonen-Sinisalo, L.; Jarvinen, H.; et al. Eleven candidate susceptibility genes for common familial colorectal cancer. PLoS Genet. 2013, 9, e1003876. 
76. Abecasis, G.R.; Altshuler, D.; Auton, A.; Brooks, L.D.; Durbin, R.M.; Gibbs, R.A.; Hurles, M.E.; McVean, G.A. A map of human genome variation from population-scale sequencing. Nature 2010, 467, 1061-1073.

77. Thompson, B.A.; Spurdle, A.B.; Plazzer, J.-P.; Greenblatt, M.S.; Akagi, K.; Al-Mulla, F.; Bapat, B.; Bernstein, I.; Capellá, G.; den Dunnen, J.T.; et al. Application of a 5-tiered scheme for standardized classification of 2360 unique mismatch repair gene variants in the insight locus-specific database. Nat. Genet. 2014, 46, 107-115.

78. Kumar, P.; Henikoff, S.; Ng, P.C. Predicting the effects of coding non-synonymous variants on protein function using the sift algorithm. Nat. Protoc. 2009, 4, 1073-1081.

79. Adzhubei, I.A.; Schmidt, S.; Peshkin, L.; Ramensky, V.E.; Gerasimova, A.; Bork, P.; Kondrashov, A.S.; Sunyaev, S.R. A method and server for predicting damaging missense mutations. Nat. Methods 2010, 7, 248-249.

80. Gonzalez-Perez, A.; Lopez-Bigas, N. Improving the assessment of the outcome of nonsynonymous snvs with a consensus deleteriousness score, condel. Am. J. Hum. Genet. 2011, 88, 440-449.

81. Khurana, E.; Fu, Y.; Colonna, V.; Mu, X.J.; Kang, H.M.; Lappalainen, T.; Sboner, A.; Lochovsky, L.; Chen, J.; Harmanci, A.; et al. Integrative annotation of variants from 1092 humans: Application to cancer genomics. Science 2013, 342, 1235587.

82. Kircher, M.; Witten, D.M.; Jain, P.; O’Roak, B.J.; Cooper, G.M.; Shendure, J. A general framework for estimating the relative pathogenicity of human genetic variants. Nat. Genet. 2014, 46, 310-315.

83. Weedon, M.N.; Cebola, I.; Patch, A.M.; Flanagan, S.E.; de Franco, E.; Caswell, R.; Rodriguez-Segui, S.A.; Shaw-Smith, C.; Cho, C.H.; Lango Allen, H.; et al. Recessive mutations in a distal ptfla enhancer cause isolated pancreatic agenesis. Nat. Genet. 2014, 46, $61-64$.

84. Horn, S.; Figl, A.; Rachakonda, P.S.; Fischer, C.; Sucker, A.; Gast, A.; Kadel, S.; Moll, I.; Nagore, E.; Hemminki, K.; et al. Tert promoter mutations in familial and sporadic melanoma. Science 2013, 339, 959-961. 


\title{
Reading and Language Disorders: The Importance of Both Quantity and Quality
}

\author{
Dianne F. Newbury, Anthony P. Monaco and Silvia Paracchini
}

\begin{abstract}
Reading and language disorders are common childhood conditions that often co-occur with each other and with other neurodevelopmental impairments. There is strong evidence that disorders, such as dyslexia and Specific Language Impairment (SLI), have a genetic basis, but we expect the contributing genetic factors to be complex in nature. To date, only a few genes have been implicated in these traits. Their functional characterization has provided novel insight into the biology of neurodevelopmental disorders. However, the lack of biological markers and clear diagnostic criteria have prevented the collection of the large sample sizes required for well-powered genome-wide screens. One of the main challenges of the field will be to combine careful clinical assessment with high throughput genetic technologies within multidisciplinary collaborations.
\end{abstract}

Reprinted from Genes. Cite as: Newbury, D.F.; Monaco, A.P.; Paracchini, S. Reading and Language Disorders: The Importance of Both Quantity and Quality. Genes 2014, 5, 285-309.

\section{Introduction}

The disturbance of speech and language development is a common feature of many neurodevelopmental disorders [1]. Language impairment is often secondary to more pressing clinical features (e.g., in autistic disorders, epilepsy or periventricular heterotopia), but in some cases may represent the primary clinical concern ("specific language disorders", e.g., in Specific Language Impairment (SLI) and dyslexia) [1]. Specific language disorders typically occur in the absence of any gross neurodevelopmental difficulties, neurological or sensory impairments and with normal non-verbal intelligence. SLI is defined as a disturbance of oral language skills, whereas dyslexia is an impairment in the use and/or understanding of written language [2]. Both show a strong familial bias, and heritability estimates indicate that a high proportion of the phenotypic variation in each of these disorders can be attributed to genetic variation [3-5].

\section{Complex Traits, Complex Definitions}

Whilst the terms "dyslexia" and "SLI" are widely used in the clinical and research literature, both disorders lack clear diagnostic guidelines and are often defined chiefly in terms of exclusionary criteria [6]. DSM-5 (Diagnostic and Statistical Manual) classifies SLI as a language disorder, while dyslexia is categorized as a specific learning disorder. However, both diagnoses require that "the individual's difficulties must not be better explained by developmental, neurological, sensory (vision or hearing), or motor disorders and must significantly interfere with academic achievement, occupational performance, or activities of daily living" [7]. To complicate things further, deficits of language may vary considerably both between individuals and over the developmental trajectory in addition to the modality of language affected [6,8]. It is generally accepted that dyslexia primarily reflects a difficulty in the domain of phonological decoding 
(translating written letters into speech sounds) [9]. Nonetheless, it is very common to observe a phonological deficit in combination with other manifestations, like sensory or fine motor control problems $[10,11]$. SLI has been proposed to reflect a deficit in phonological short-term memory (the retention of verbal information for short periods of time) [12], auditory perception (the processing of brief and/or rapid auditory stimuli) [13] and/or the development and application of grammatical rules [14]. However, all of these theories are supported by varying amounts of evidence in the primary research literature.

\section{Changing and Heterogeneous Phenotypes}

In addition, the difficulties experienced by any given individual may cross linguistic and cognitive domains and often change as the child develops $[15,16]$. Such observations perhaps suggest that SLI and dyslexia cannot be treated as discrete clinical conditions. Instead, it is possible that these language disorders might represent complex end effects of the disruption of multiple cognitive development processes that overlap with, and are related to, the secondary language disorders mentioned above [17]. Under this model, the investigation of dimensions of impairment may be more relevant than the ascertainment of clinical cohorts. The observed co-occurrence of SLI and dyslexia ( $\sim 50 \%$, [18]) support such a hypothesis and has led researchers to suggest that both disorders may result from an impairment in phonological representation [17]. The clinical presentation of the deficit may represent the severity of the underlying impairment or the presence of additional language- or cognitive-related difficulties [17,19]. Thus, "specific" language disorders may be the exception, rather than the rule, since co-morbidities often extend outside of the linguistic domains. For example, weaknesses in motor skills and executive function and reduced functional brain laterality are commonly described in children with dyslexia or SLI [20-26]. Nonetheless, the causal relationships between symptoms and cognitive, linguistic and developmental markers have yet to be elucidated. Others maintain that SLI and dyslexia may still have separate etiologies, and the co-incidence may simply represent comorbidity, as seen with other neurodevelopmental disorders [19]. Identifying the genetic underpinning of these disorders is required to inform this debate and reach more definitive conclusions about the diagnosis. For example, are there shared or partially overlapping genetic factors that contribute to separate disorders? Can we talk about common etiologies? Or do the DSM-5 definitions correlate with distinct biological pathways? While it would be tempting to simplistically ask if candidate genes for a single disorder can also influence a disorder with a different diagnosis, we might be asking the wrong questions if the initial diagnoses are artificial clinical constructs and misleading with regards to etiology.

\section{Monogenic Conditions Back in the Picture}

For many language disorders that are associated with known syndromes, the genetic cause of the syndrome itself is known. These involve a wide range of genetic mutations, from point mutations (for example, $M E C P 2$ mutations in Rett syndrome [27]), to nucleotide expansions (for example, the expansion of the FMRI gene in Fragile X [16]) and deletion syndromes, such as velo-cardio-facial 
syndrome, which results from a 3-Mb deletion on chromosome 22q11.2 [28], or the duplication or deletion of an entire chromosome, such as Down syndrome and Turner syndrome [29-31]. Thus, it is likely that any population selected to have language impairment will harbor a subset of children with these recognized syndromes [32]. Single gene mutations have also been described for some specific language disorders. Mutations and disruptions of the FOXP2 gene lead to childhood apraxia of speech $[33,34]$, and point mutations in genes in the lysosomal pathway (GNPTAB, GNPTG and NAGPA) have been associated with persistent stuttering [35]. However, even in these severe and exceptional cases, there is often a high degree of heterogeneity between individuals. While some generalizations can be made, there is still considerable inter-individual variation. For example, individuals with FOXP2 disruptions invariably present with dyspraxic speech (the inability to make fine-tuned oromotor movements necessary for coherent speech), while others can present with both receptive and expressive language difficulties, only an expressive deficit, only with intellectual deficits or good performance on non-verbal intelligence tasks [36].

\section{Genetic Windows into Development}

One argument against the utility of understanding the genetic underpinnings of these rare syndromic language disorders has been to question their relevance to the biology of common forms of SLI and dyslexia. However, the identification of a specific candidate gene and mutations thereof can allow the development of targeted investigations in cellular or animal models, which, in turn, can point to mechanisms that might be relevant to more common forms of language-related conditions affecting thousands of children. An example of this is how the FOXP2 transcription factor regulates the expression of target genes, such as CNTNAP2, which has been shown to play a role in more common forms of language impairment [37], as well as other neurodevelopmental disorders [38-41] and normal language variation [42]. The increased resolution and power of genetic screening demonstrates that the boundary between monogenic and common traits may actually be less defined than that predicted previously. Recent large-scale studies have clearly shown that genes disrupted by highly penetrant mutations and leading to well-defined diseases can play a role in complex disorders, although this may only be relevant in a subset of cases [43]. Nonetheless, genetic contributions to the majority of specific language disorders are expected to be complex in nature and involve genetic variation in many genes, which combine to determine an overall risk of disorder [44].

\section{Classical Approaches}

Genetic contributions to neurodevelopmental disorders (both syndromic and specific forms) can be traced by linkage analyses. This approach can be applied to extended multi-generational families or large collections of small nuclear families and can involve the investigation of linkage-disequilibrium patterns [45]. Linkage analysis allows the identification of broad chromosome regions that co-segregate with a disorder in a given family unit. Since such studies consider segregation patterns rather than specific genetic variants, they can enable the identification of shared chromosome regions even when the pathological variants in each region differ between 
family units. This methodology is particularly powerful for the identification of genetic variants with high penetrance and expressivity and can be applied to more complex situations, such as that expected for both SLI and dyslexia.

\section{SLI and Dyslexia Linkage Loci}

Genome-wide screens or targeted analyses of dyslexic families have allowed the identification of linkage loci on chromosomes 15q21 (DYX1-OMIM\#127700) [46,47], 6p22.3-p21.3 (DYX2 - OMIM\#600202) [46,48-51], 2p16-p15 (DYX3-OMIM\#604254) [52,53], 3p12-q13 (DYX5-OMIM\#606896) [54,55], 18p11.2 (DYX6-OMIM\#606616) [56], 11 (DYX7-OMIM\#127700) [57], 1p36-p34 (DYX8-OMIM\#608995) [58,59] and Xq27.2-q28 (DYX9-OMIM\#300509) [60]. Subsequent fine-mapping efforts across linked loci, through the investigation of specific genetic variations or the characterization of individuals with chromosome imbalances, have led to the identification of putative risk variants in the $D C D C 2$ [61], KIAA0319 [62-64], DYX1C1 [65,66], C2orf3/MRPL19 [67], CYP19A1 [68] and ROBO1 [54] genes. Each of these candidate genes has a variable amount of support, ranging from observations limited to a single family to replication across multiple cohorts [69]. Nonetheless, functional analyses have led to an intriguing conversion upon pathways involving neuronal migration [70-76] and cilia motility [72,77-79], as discussed in later sections. Investigations of SLI are less advanced, but linkage studies have identified four loci of interest on chromosomes 7q35-q36 (SLI4 OMIM\#612514) [80], 13q21 (SLI3-OMIM\#607134) [81,82], 16q (SLI1-OMIM\#606711) [83-86] and 19q (SLI2-OMIM\#606712) [83-86], and subsequent studies have identified two candidate genes; CMIP and ATP2C2, both in SLI1 [87]. Microdeletions in the ZNF277 gene on chromosome 7 have also been implicated in SLI [88], as has the Human Leukocyte Antigen (HLA) locus [89].

Although the linkage loci described for SLI and dyslexia do not overlap, studies of other complex genetic disorders indicate that there may be hundreds of genetic variants contributing to any one phenotypic status. Since genetic analyses are likely to detect only the major gene effects within any given cohort (the so-called winners curse [90]), this observation may simply be a result of the number of studies performed rather than the reflection of separate pathologies per se.

\section{GWA Studies}

Advances in genetic technologies over the last decade have allowed enormous leaps in our characterization and understanding of both rare and common genetic variations at the sequence level. Projects, such as the HapMap ([91]) and 1000 Genomes ([92]), have provided us with catalogues of expected variations across multiple populations. Methodological advances, such as microarrays and high throughput genotyping and sequencing platforms, have allowed us to characterize known variants efficiently. Accordingly, gene identification shifted from linkage analysis to genome-wide association (GWA) studies [93]. GWA studies usually interrogate large cohorts of cases and controls (typical sample sizes range from 1000 up to 1,000,000), but can be extended to a regression analysis of variant frequency upon performance in phenotype-related quantitative tasks (quantitative GWA study). Since GWA studies consider the frequency of each 
variant independently, there is an underlying assumption that the causal variation (or a Single Nucleotide Polymorphism (SNP) in linkage disequilibrium with the causal variant) will be common across cases. As such, association studies do not allow for a high level of genetic heterogeneity between cases.

\section{GWA Studies of Speech and Language-Related Traits}

GWA studies of speech and language cohorts to date have not yielded consistent findings. Meaburn et al. (2008) applied a pooled genotyping method across two extreme samples selected from a twin cohort on the basis of reading ability (755 low reading ability cases and 747 high reading ability controls) using 100,000 SNPs, but did not identify any significant associations [94]. This may reflect the sparse density of the genotyping arrays employed at that time. Roeske et al. analyzed a discovery $(\mathrm{N}=200)$ and replication $(\mathrm{N}=186)$ cohort both selected for dyslexia and found association for a specific electrophysiological endophenotype of dyslexia ("mismatch negativity component" or MMN) $\left(p=5.14 \times \mathrm{e}^{-8}\right.$ in combined dataset) pointing to the SLC2A3 gene, which is implicated in glucose transport in the brain [95]. Field et al. 2013, performed a joint linkage and association study on 718 individuals from 101 dyslexia families with 100,000 SNPs. Again, they did not find any associations that met the threshold of genome-wide significance $\left(1 \times 10^{-8}\right.$ [96]) [97]. They did however, find suggestive association $\left(p=6.2 \times 10^{-7}\right)$ with an SNP 77 $\mathrm{Kb}$ downstream of the FGF18 gene, which has been implicated in lateralization [97]. Luciano et al. reported a meta-analysis of quantitative reading and language measures across two relatively large population-based samples (1177 individuals from 538 families and approximately 5000 cases) [98]. They found a suggestive level of association $\left(p=7.34 \times 10^{-8}\right)$ between variants in the $A B C C 13$ gene on chromosome 21 and non-word repetition (a marker of phonological short-term memory). However, they did not replicate the association to FGF18 [98]. Eicher et al. also used a population-based sample, but in their study, they selected low language and reading performers for their GWA study [99]. Their sample included 163 language impaired probands, 353 dyslexic probands and 174 comorbid probands (i.e., those with both language and reading impairment). They compared variant frequencies between these proband groups and the remainder of the population. They observed suggestive association with SNPs in ZNF385D $\left(p=5.45 \times 10^{-7}\right)$ and COL4A2 $\left(p=7.59 \times 10^{-7}\right)$ in the cases with the comorbid phenotype, and to SNPs in the NDST4 $\left(p=1.4 \times 10^{-7}\right)$ gene in language impaired probands [99].

Recently, it has been proposed that parent-of-origin effects could explain part of the missing heritability of complex traits, suggesting that the addition of these effects within GWA studies may be fruitful [100]. A recent study of 278 families with a language-impaired child, investigated child genotype and parent-of-origin effects [101]. They identified significant evidence for paternal parent-of-origin effects on chromosome $14 \mathrm{q} 12\left(p=3.74 \times 10^{-8}\right)$ and suggestive evidence for maternal parent-of-origin effects on chromosome $5 \mathrm{p} 13\left(p=1.16 \times 10^{-7}\right)$ [101]. The paternally-associated SNP on chromosome 14 yields a non-synonymous coding change within the NOP 9 gene, which has been reported to be significantly dysregulated in individuals with schizophrenia. 
Figure 1. Study design for quantitative phenotypes (a) genome-wide association (GWA) studies for speech and language-related traits typically use phenotypes across the entire distribution (population-based quantitative GWA studies). Others might apply a binary affection status under which low language-performing individuals are defined as "cases" and individuals within the "normal" language range (usually performance above the mean) as "controls". Under certain conditions, "super-controls" can provide more power, as they are selected to fall at the upper extreme of the distribution. If controls with phenotype data are not available, they may be derived from standard control populations under the knowledge that they might include a small proportion of cases. Quantitative GWA studies restricted to cases may be based on a phenotypic distribution restricted to the lower tail of the entire distribution or may be based on a phenotypic curve derived across cases samples, as denoted by the two normal distributions in (a) (note that in (a), the phenotype distribution may not necessarily be expected to be normal, although it is shown as such in the figure). (b) The pegboard test generates a quantitative measure for handedness (PegQ) that is normally distributed around a positive mean. PegQ strongly correlates with hand preference, so that individuals with positive scores are very likely to be right-handed (roughly $90 \%$ of the population), and individuals with negative scores are likely to be left handed. Typically genetic studies for handedness have used the categorical measures of hand-preference using a case-control (left vs. right) study design.

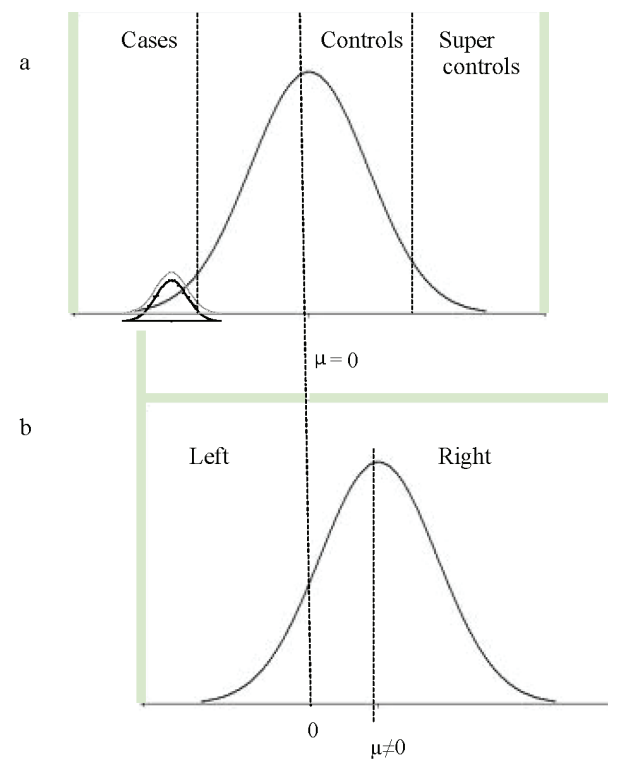

\section{GWA Study Design Factors}

The ability of GWA studies to identify risk variants depends upon several factors. These include the effect size of the variants, the frequency of the variants in the population under study and the 
population as a whole and, of course, sample size and study design [102]. The lack of replication described in the previous section is therefore perhaps not surprising: each started with a different hypothesis and definition of disorder and applied different selection procedures and association methodologies. Inconsistency across studies makes it hard to assess whether non-replication indicates the presence of false positives or is simply a function of study design or power to detect an association. In addition, the variations between independent studies means that it is not possible to simply combine existing cohorts to generate adequately powered meta-analyses.

As discussed above, some of the language-related GWA studies used population-based samples and chose to select low language-performing subjects as cases (i.e., the lower tail end of the distribution, marked as "cases" Figure 1a). Others studied speech and language-related traits across the entire distribution in a quantitative GWA approach (i.e., the entire normal distribution in Figure 1a). Such population-based approaches assume that genetic contributions to disorders of speech and language will be the same as those that contribute to speech and language-related traits across the entire distribution. This assumption is dependent upon the way in which disorders, such as SLI and dyslexia, are conceptualized: do they just represent the lower tail of the normal distribution with respect to speech and language (dimensional model) or is there a qualitative difference between dyslexic individuals and poor readers (categorical model)? (i.e., the difference between the "cases" and the lower normal distribution in Figure 1a).

\section{Dimensional and Categorical Models of Language Disorder}

The distinction between dimensional and categorical models of language disorders is still a matter of debate. Leonard argues that perhaps there is no tangible cause for SLI, and the mental representations of children with the label SLI are not distinct from other children [103]. Taxonomic and principal component analyses support this view, demonstrating that relationships between language-related measures do not differ between children affected by SLI and those with normal language development [104-106]. In addition, the ease of the acquisition of specific language features has also been shown to be consistent between children affected by SLI and those with normal language development [107]. Under this dimensional model, one can consider variation across the range when attempting to identify underlying genetic effects. Twin studies of SLI instead support a categorical distinction [4,108-111]. Such studies indicate that children who have speech and language difficulties that are severe enough to warrant clinical referral have a qualitatively different profile of impairment, which shows increased levels of heritability [110]. Thus, it is possible that some forms of speech and language impairment, at least, have different underlying pathology from those mechanisms that are important in normal language variation (i.e., the distinct lower normal distribution in Figure 1a). Under this hypothesis, one needs to specifically select children with SLI to identify these distinct underlying genetic effects. It is, of course, possible that both models contribute to some level: studies of the effects of specific genetic risk variants upon language development indicate that some genetic risk variants play a role across the entire distribution of ability [87,112-114], while others appear to play a role that is specific to impairment $[87,113]$. These data suggest that a variety of approaches will be required to delineate genetic effects underlying language impairment: GWA studies of individuals across a range of 
abilities will identify genes that contribute across the entire distribution, while studies of specific disorder subsets will be required to identify genes with more specific effects.

\section{Cross-Linguistic Difficulties of Speech and Language Disorders}

Given all these complexities, we extol the value of combining quantity with quality in the collection of larger cohorts carefully characterized at the phenotypic level. This is a major investment both in time and resources, but clearly represents the most promising way forward. The success of genetic mapping for complex traits has been largely facilitated by the collaboration of scientists and clinicians in large consortia, which have facilitated the collection of large sample sizes for genome mapping. This normally requires international collaborations, which pose additional complications for reading and language disorders. The psychometric tests used to assess reading and language skills cannot be separated from the language spoken in different countries and are not always directly comparable. In this context, the European NeuroDys project is working to define common guidelines for the collection and assessment of a large dyslexia cohort throughout research groups spread across Europe [115]. NeuroDys aims to exclude comorbidity and select severe dyslexic cases by selecting those more than 1.25 SD below grade level on a standardized word-reading test. Their strategy also includes the screening of control samples. Since dyslexia has a relatively high frequency, $5 \%-10 \%$ of population samples, routinely employed in GWA studies, will be expected to consist of individuals with dyslexia. GWA analysis of the NeuroDys cohort is currently underway. Analysis of dyslexia candidate genes in this cohort did not reveal any statistically significant association, highlighting many of the challenges covered in this review [116]. As discussed previously, universal inclusion/exclusion criteria are a good strategy to facilitate meta-analyses or cross-linguistic categorical GWA studies. Nonetheless, they do not entirely solve the challenge of obtaining large sample sizes for quantitative analyses. For example, single word reading tests tend to assess accuracy in English versus speed in transparent languages, like Italian or German. A composite score, including both accuracy and speed, as used by the NeuroDys study, can address this, but does not necessarily reflect the real reading difficulty in different populations. It is interesting to note that despite these differences, associations with candidate genes have been replicated in different languages; association with the $D C D C 2$ gene has been reported in English-speaking (Meng et al. 2005 [61], Scerri et al. 2011 [113]) and German-speaking (Schumacher et al. 2006 [117]) cohorts. Similarly, cross-linguistic studies find that features of the native language can act to modulate given aspects of the SLI phenotype. In general, those linguistic features that are considered "hardest" for a normally developing child to understand will represent areas of particular problem for children with SLI [118,119]. Within-child differences indicate that this generalization extends to bilingual children. Bilingual children with SLI encounter similar problems to monolingual children with SLI, and these problems appear to be language-specific [120]. These cross-linguistic variations represent an extra complication for meta-analyses and collaborative studies of speech and language impairments. If the features of the disorder vary across populations, the measurement of disability will be language-specific. Thus, multi-site efforts will not only need to consider the best way to overcome cross-ethnicity genetic differences, but also cross-ethnicity language differences. 


\section{Mega-, Meta- and Mixed-GWA Studies}

Many successful GWA studies in the literature now include hundreds of thousands of individuals (diabetes, body mass index, height) [121-125] compared to the hundreds employed in the studies described above. Such "mega-GWA studies" have been relatively successful in identifying significant and consistent loci that contribute to either disease status or continuous traits across the general population. However, it is clear from these large studies that each genetic risk variant is likely to explain only a small amount of the heritability and that several hundred risk loci are likely to underlie any given trait or disorder [126]. Besides an increased sample sizes, a possible route of future investigation may be provided by a model adopted in the study of psychiatric disorders. Much like SLI and dyslexia, there is much evidence for the existence of shared genetic effects between psychiatric disorders, such as bipolar disorder, depressive disorder and schizophrenia. "Mixed-GWA study" investigations of mixed cohorts across these disorders have recently identified risk factors that span these clinical boundaries, suggesting that there may be some common pathophysiologies across related disorders [127,128]. The above studies suggest that meta-analyses across the existing SLI and dyslexia cohorts would be a worthwhile effort, despite the challenges involved in the amalgamation of these highly heterogeneous samples.

\section{Quantitative vs. Qualitative, or Both}

Throughout this manuscript, we have clearly advocated the combined analyses of existing cohorts or the collection of new large homogeneous samples relevant to developmental disorders. However, is sample size all that matters? If that were the case, the field should probably shift towards questionnaire-based phenotypic assessments, which would be an efficient way to boost numbers. Nonetheless, there is an intrinsic value in investing in phenotypic assessments that are detailed and quantitative. Firstly, this strategy allows cohorts to be stratified in distinct subgroups according to distinct criteria, which might be required for different hypotheses. For example, it makes it possible to select for the severity of disorders or to change features along with revised diagnostic criteria (as in Figure 1b). In addition, association analyses that use quantitative measures are potentially more powerful, as they exploit the full range of variability of a given phenotype, allowing the direct investigation of dimensional and categorical models. A recent story describing a GWA study for handedness is emblematic of how small, detailed cohorts can provide power to detect relevant biological effects [129,130]. This GWA study was conducted in a small sample ( $\mathrm{N}=728$, well below the recommended standard) originally selected for a dyslexia diagnosis. In addition to language-related measures, this cohort was characterized with the pegboard test, which allows the derivation of a quantitative measure of relative hand skills (PegQ). This measure is normally distributed with a right-shifted mean and strongly correlates with hand preference (Figure 1b). Despite the small sample size, the screening led to a genome-wide significant result within the PCSK6 gene, known to regulate the NODAL protein pathway, essential for the left/right patterning in early embryonic development [131], and therefore represents an extremely interesting candidate for a lateralized phenotype. Although this association was replicated in two independent cohorts with developmental dyslexia, the risk variant appeared to confer an opposite effect 
(reduced relative laterality) in a general population cohort $(\mathrm{N}=2666)$. In support of these findings, subsequent gene enrichment analysis showed that genes controlling structural asymmetries were associated with the handedness measure in both cohorts regardless of a dyslexia diagnosis [130]. These findings demonstrate the importance of studying phenotypes within specific selected cohorts, as well as across the general population, as discussed above. It is possible that these effects are the result of risk variant interactions with different genotypic/biological backgrounds, as discussed below. A dyslexia-specific effect has also been observed for other traits. A variant in the MYO18B gene has also been implicated in mathematical ability specifically in children with dyslexia [132]. Hand preference (left $v s$. right) can readily be collected as a simple add-on question in any questionnaire battery. Thus, it would be very straightforward to re-analyze or meta-analyze existing GWA cohorts (even those collected for the investigation of different traits) using a case-control definition based on hand preference. A recent conference abstract describing a genome-wide analysis of hand preference data from more than 20,000 individuals did not find any genome-wide significant loci (Medland et al. ASHG, 2009 [133]). Similarly, a study of 4268 subjects from a population-based cohort, which included broad measures of laterality (hand or foot preference, ocular dominance or hand clasp), did not yield genome-wide significant associations [134]. These data suggest that the genetic effects at the population level are extremely low or, alternatively, that a categorical approach is not suitable for dissecting the genetics of this trait. It is likely that the biological regulation of hand preference involves complex and integrated processes that are not efficiently represented by the reductive phenotypes of left- versus right-handedness. If this can be said for a relatively transparent trait, like handedness, then we might expect these correlations to be even lower when considering a phenotype as complex as language. In addition, we must consider that the genetic effects might depend on sample stratification for a neurodevelopmental disorder definition.

\section{Filling the Gap}

Collecting large-scale GWA cohorts takes time, effort, funding and usually a concerted and collaborative effort between multiple research and clinical teams. Does this mean that we cannot make progress in this field until such statistical criteria are met? Perhaps the application of nextgeneration sequencing technologies to existing sample sets with detailed phenotypic information has the potential to fill this gap. While small individual family-based units do not provide enough power to map variants through linkage analyses alone, the application of this technique in combination with high-throughput sequencing can provide a powerful paradigm. The whole-exome or whole-genome sequencing of sporadic cases and their parents under the assumption of causative de novo mutations has proven successful in disorders, such as autism [135-138] and intellectual disability [139]. The sequencing of larger family units with multiple recurrent cases have allowed the identification of "ultra-rare" (often defined as $<0.01 \%$ ) or private (unique to the given family) mutations with high functional impact (i.e., the gain of a stop codon and frame shift mutations) that cosegregate with disorder [140,141]. A recent exome sequencing study of children with childhood apraxia of speech investigated known candidate genes for language development [142]. They 
reported potentially deleterious variants in FOXP1, CNTNAP2, ATP13A4, CNTNAP1, KIAA0319 and SETX, providing support for the role of these candidate genes [142].

\section{Difficulties of High-Throughput Sequencing Studies}

Although relatively simplistic in design, high throughput sequencing studies are far from straightforward. Rigorous quality control procedures and expert bioinformatics are needed, and the variation between platforms, capture assays and algorithms can be problematic. The main problem, though, is perhaps the proof of causality. The number of de novo mutations can be affected by environmental factors (e.g., paternal age [143]), but all being equal, the expected number of de novo mutations is approximately one per exome per generation. In contrast, the number of private mutations identified in an exome can be substantial, particularly as sequencing sensitivity and coverage increase. In addition, by design, these methods focus upon mutations that are likely to be private to the given individual and will not generalize between families. Studies of autistic disorder indicate that recurrent mutations in specific genes will be rare [135-137]. Furthermore, while the role of rare disruptive mutations is perhaps more tangible than that of common variation, these effects are still likely to function as part of a complex genetic model and represent risk variants rather than a causative mutation. Incomplete segregation, even within family units, is often observed for both rare mutations and copy number events, which can also prove a fruitful avenue of research in relatively small sample sizes. While large, disruptive events of genes that are known to be important can clearly be assigned some functionality, when a mutation or copy number variant is private, it can be very hard to distinguish between a functional effect that is subject to incomplete penetrance or modifier gene effects and a non-functional change. Such observations have led to the double-hit hypothesis in developmental disorders in which mutations and/or copy number events combine in an additive or epistatic manner to modulate the exact clinical presentation [136,144-148].

\section{Translational Relevance}

The pathway from association to functional evidence is a long, but necessary road if we are to truly elucidate the biological mechanisms underlying speech and language disorders. While a $p$-value $<0.5 \times 10^{-8}$ or the observation or private mutations in conserved motifs are certainly robust indicators of a genuine genetic susceptibility, these findings remain statistical predictions if they are not coupled by functional data. It is important to keep in mind that the risk variants identified by GWA studies are not necessarily functional and often represent proxies for the functional variant [102]. Functional investigations are being further progressed by projects, such as the Encyclopedia Of DNA Elements (ENCODE) and the Genetic European Variation in Health and Disease (GEUVADIS) project, which apply high-throughput methods to the study of gene expression and regulation to facilitate our understanding of the findings of GWA studies [149,150]. The functionality of rare coding mutations or copy number events is more tractable, but still not straightforward, especially when they are private. Changes to the coding sequence do not always result in protein dysfunction, and the interpretation of the severity of a given mutation often relies 
on in silico predictions [151]. Once the identity of the functional variant is established, its biological contribution to disorder needs to be clarified, a step which is usually achieved in animal models. The development of translational targets is not always clear, even for fully penetrant monogenic mutations with clear functional effects [152,153]. These limitations reflect the importance of considering genetic variants within relevant pathways and networks and are likely to be exacerbated for complex disorders.

\section{Biology beyond the $p$-Values}

The association of variants in the PCSK6 gene and relative hand skill introduced earlier acts to illustrate the importance of taking statistical associations forward with molecular investigations of the mechanism. As described above, the PCSK6 association with relative hand skill appears to confer specific effects in individuals with dyslexia. Given that the prevalence of left-handers is not higher among individuals with dyslexia compared to controls, at first glance, it appears difficult to explain this specificity. The very fact that this handedness measure was collected in individuals with dyslexia stems from a long-sought link between laterality and neurodevelopmental disorders [154]. Language is a lateralized behavior under the control of one specialized cerebral hemisphere (the left one, in most cases), and it has been suggested that language-related disorders might therefore be linked to handedness, which is the most obvious lateralized behavior. For many decades, researchers have looked with little success for a link between handedness and different psychiatric disorders, mainly by assessing the frequency of left-handers in patient cohorts [155]. Whilst largely inconclusive, an increased frequency of left-handers has been reported for schizophrenia [156]. Furthermore, imaging studies have shown that disorders, such as dyslexia and SLI, present with atypical and weaker cerebral lateralization [25]. Although, there is some controversy over whether these effects are causative or consequential [157].

\section{Converging Evidence towards Biological Pathways}

More direct evidence in support of the dyslexia-specific PCSK6 association comes from very recent functional studies. We have already eluded to the apparently connected functional role of dyslexia candidate genes. Functional studies and in vivo techniques have demonstrated that several of the dyslexia candidates described above (KIAA0319, DCDC2 and DYX1C1) are involved in early phases of fetal brain development and, in particular, in neuronal migration processes. In addition, $R O B O 1$ is a neuronal axon guidance receptor that is also important for cortex development. The importance of neuronal migration processes in dyslexia is thus now generally accepted in the literature [158]. However, it is not clear which exact mechanisms these candidate genes mediate. More recently, a role for cilia function and development has also been suggested as a common biological pathway between dyslexia candidate genes [159]. Cilia are essential in the establishment of left/right axis determination in the first weeks of embryogenesis by the activation of the NODAL pathway on the left side of the embryo. Mutations in genes controlling cilia function lead to a class of conditions often characterized by laterality defects (ciliopathies). DCDC2, DYX1C1 and KIAA0319 have recently been reported to form a novel co-expressed module 
in ciliated cells [79]. $D c d c 2$ has been implicated in the control of cilia length [72], and disruption of Dyxlc1 in mice and zebrafish [77,78] leads to laterality defects and impairments in cilia function, resembling that observed in primary ciliary dyskinesia (PCD). In humans, DYX1C1 mutations have been identified in 12 families with PCD [77]. KIAA0319 is characterized by five polycystic kidney disease (PKD) domains [160] typically found in proteins (e.g., PKD1 and PKD2) playing key roles in cilia function [161].

Cilial structures control many processes. In particular, they are important in neuronal migration, where they play a guiding role during cortical development. It has therefore been suggested that the roles of cilia in neuronal migration may be directly implicated in leading to cortical defects, which are at the basis of cognitive deficits in neurodevelopmental disorders [162]. Taken together, these observations support a possible interaction between biological pathways controlling the establishment of left/right structural asymmetries and neuronal migration early in development with genes implicated in dyslexia [159]. These interactions could be mediated by concomitant actions in controlling cilia function and could explain the PCSK6 association observed specifically in individuals with dyslexia. Furthermore, these data suggest for the first time that the mechanisms that control left/right body asymmetries maybe also be relevant in establishing functional brain asymmetries, contrary to previous evidence [163]. Incorrect reference order

\section{Conclusions}

We have discussed the various factors that can impact upon the relative success of genetic investigations in general and highlighted those factors that may be particularly pertinent to the investigation of speech and language disorders. While GWA studies have often been criticized for their high economic cost and little clinical benefits, they have contributed enormously to our understanding of human genome variation and appreciation of the complexity behind human biology and genetic disorders. Nonetheless, the application of these technologies to the study of genetic contributions to language and reading has not progressed as fast as other complex traits. One obvious limitation to the study of speech and language is the challenge of defining cases and ascertaining homogeneous cohorts with phenotypic measures that are universally relevant at the biological level. The translational application of genetic research to complex disorders will require the integration of large genomic datasets, functional genomic screenings and basic research projects aimed at studying the human brain. We know that genes and proteins do not act in isolation, and their functions differ between individuals, tissues, environments and over time; yet, we still consider risk variants as independent entities with fixed effects. Although we must accept that it is not currently possible to simultaneously consider all these effects within a single model, perhaps it is time to question the adherence to clinically defined strata in the genetic study of developmental disorders and, instead, promote the acceptance of cross-disorder studies that fully consider comorbidities across clinical symptoms and environmental factors, as well as regulatory effects and epigenetics. This reiterates the need for multidisciplinary collaborations to enable an increase in sample sizes, while maintaining detailed phenotypic assessments that ultimately will inform the definition of diagnostic criteria. In terms of future directions, it is difficult to establish whether resources would be better spent collecting large cohorts that meet a superficial categorical 
cutoff or, alternatively, in assembling smaller cohorts characterized across a range of detailed developmental phenotypes. What is clear is that the definition of universal guidelines will facilitate the coordinated collection of uniform and international cohorts, easing the burden of downstream analyses. Although larger collaborations are needed, a meta-analysis of existing resources would be a good starting point to establish such efforts. The reporting of complete data sets, even when events are considered non-significant or uninteresting in isolation, will also be crucial to the planning of future collections. Ultimately, functional evidence is necessary to definitely prove the downstream effects of genetic variants and to understand the biology of underlying disorders and neurodevelopment. Despite the lack of advances from genome-wide screening in speech and language disorders, the functional assessment of candidate genes has allowed considerable progress in the identification of specific biological processes that may be important in these phenotypes. In particular, neuronal migration and ciliogenesis have been highlighted as two, perhaps related, processes that may play a role in developmental dyslexia. These encouraging findings demonstrate the importance of the systematic integration of functional studies and genetic association or sequencing studies.

\section{Acknowledgments}

We would like to thank all the families, professionals and individuals who participated in our research. Dianne Newbury is a Medical Research Council (MRC) Career Development Fellow and a Junior Research Fellow at St. John's College, University of Oxford. The work of the Newbury lab is funded by the Medical Research Council (G1000569/1 and MR/J003719/1). Anthony P. Monaco is the President of Tufts University and a Visiting Professor at the Wellcome Trust Centre for Human Genetics. The work of the Monaco lab is funded by a program grant from the Wellcome Trust (092071). Silvia Paracchini is a Royal Society University Research Fellow. The work of the Wellcome Trust Centre in Oxford is supported by the Wellcome Trust (090532/Z/09/Z).

\section{Author Contributions}

All authors conceptualized and wrote this review.

\section{Conflicts of Interest}

The authors declare no conflict of interest.

\section{References}

1. Harel, S.; Greenstein, Y.; Kramer, U.; Yifat, R.; Samuel, E.; Nevo, Y.; Leitner, Y.; Kutai, M.; Fattal, A.; Shinnar, S. Clinical characteristics of children referred to a child development center for evaluation of speech, language, and communication disorders. Pediatr. Neurol. 1996, 15, 305-311.

2. American-Psychiatric-Association. Diagnostic and Statistical Manual of Mental Disorders, 4th ed. (dsm-iv); American Psychiatric Association: Washington, DC, USA, 1994. 
3. Bishop, D.V. The role of genes in the etiology of specific language impairment. J. Commun. Disord. 2002, 35, 311-328.

4. Hayiou-Thomas, M.E. Genetic and environmental influences on early speech, language and literacy development. J. Commun. Disord. 2008, 41, 397-408.

5. DeFries, J.C.; Fulker, D.W.; LaBuda, M.C. Evidence for a genetic aetiology in reading disability of twins. Nature 1987, 329, 537-539.

6. Bishop, D.V.M. Uncommon Understanding: Development and Disorders of Language Comprehension in Children; Psychology Press: Hove, UK, 1997.

7. American Psychiatric Association. Diagnostic and Statistical Manual of Mental Disorders: DSM-5, 5th ed.; American Psychiatric Publishing: Arlington, VA, USA, 2013.

8. Conti-Ramsden, G.; Botting, N. Characteristics of children attending language units in england: A national study of 7-year-olds. Int. J. Lang. Commun. Disord. 1999, 34, 359-366.

9. Snowling, M.J. Phonemic deficits in developmental dyslexia. Psychol. Res. 1981, 43, 219-234.

10. Ramus, F. Neurobiology of dyslexia: A reinterpretation of the data. Trends Neurosci. 2004, 27, 720-726.

11. Peterson, R.L.; Pennington, B.F. Developmental dyslexia. Lancet 2012, 379, 1997-2007.

12. Gathercole, S.E.; Baddeley, A.D. Phonological memory deficits in language disordered children: Is there a causal connection? J. Mem. Lang. 1990, 29, 336-360.

13. Tallal, P.; Piercy, M. Defects of non-verbal auditory perception in children with developmental aphasia. Nature 1973, 241, 468-469.

14. Gopnik, M. Feature-blind grammar and dysphagia. Nature 1990, 344, 715.

15. Bishop, D.V. The underlying nature of specific language impairment. J. Child Psychol. Psychiatry Allied Discip. 1992, 33, 3-66.

16. Abbeduto, L.; Brady, N.; Kover, S.T. Language development and fragile $\mathrm{x}$ syndrome: Profiles, syndrome-specificity, and within-syndrome differences. Ment. Retard. Dev. Disabil. Res. Rev. 2007, 13, 36-46.

17. Bishop, D.V.; Snowling, M.J. Developmental dyslexia and specific language impairment: Same or different? Psychol. Bull. 2004, 130, 858-886.

18. McArthur, G.M.; Hogben, J.H.; Edwards, V.T.; Heath, S.M.; Mengler, E.D. On the "specifics" of specific reading disability and specific language impairment. J. Child Psychol. Psychiatry Allied Discip. 2000, 41, 869-874.

19. Catts, H.W.; Adlof, S.M.; Hogan, T.P.; Weismer, S.E. Are specific language impairment and dyslexia distinct disorders? J. Speech Lang. Hear. Res. 2005, 48, 1378-1396.

20. Pieters, S.; de Block, K.; Scheiris, J.; Eyssen, M.; Desoete, A.; Deboutte, D.; van Waelvelde, H.; Roeyers, H. How common are motor problems in children with a developmental disorder: Rule or exception? Child Care Health Dev. 2012, 38, 139-145.

21. Flapper, B.C.; Schoemaker, M.M. Developmental coordination disorder in children with specific language impairment: Co-morbidity and impact on quality of life. Res. Dev. Disabil. 2013, 34, 756-763.

22. Gooch, D.; Hulme, C.; Nash, H.M.; Snowling, M.J. Comorbidities in preschool children at family risk of dyslexia. J. Child Psychol. Psychiatry Allied Discip. 2013, 55, 237-246. 
23. Bloom, J.S.; Garcia-Barrera, M.A.; Miller, C.J.; Miller, S.R.; Hynd, G.W. Planum temporale morphology in children with developmental dyslexia. Neuropsychologia 2013, 51, 1684-1692.

24. Johnson, B.W.; McArthur, G.; Hautus, M.; Reid, M.; Brock, J.; Castles, A.; Crain, S. Lateralized auditory brain function in children with normal reading ability and in children with dyslexia. Neuropsychologia 2013, 51, 633-641.

25. Badcock, N.A.; Bishop, D.V.; Hardiman, M.J.; Barry, J.G.; Watkins, K.E. Co-localisation of abnormal brain structure and function in specific language impairment. Brain Lang. 2012, 120, 310-320.

26. Stoodley, C.J.; Fawcett, A.J.; Nicolson, R.I.; Stein, J.F. Impaired balancing ability in dyslexic children. Exp. Brain Res. 2005, 167, 370-380.

27. Budden, S. Clinical variability in early speech-language development in females with rett syndrome. Dev. Med. Child Neurol. 2012, 54, 392-393.

28. Roizen, N.J.; Antshel, K.M.; Fremont, W.; AbdulSabur, N.; Higgins, A.M.; Shprintzen, R.J.; Kates, W.R. 22q11.2ds deletion syndrome: Developmental milestones in infants and toddlers. J. Dev. Behav. Pediatr. 2007, 28, 119-124.

29. Starke, M.; Albertsson Wikland, K.; Moller, A. Parents' descriptions of development and problems associated with infants with turner syndrome: A retrospective study. J. Paediatr. Child Health 2003, 39, 293-298.

30. Laws, G.; Bishop, D.V. Verbal deficits in down's syndrome and specific language impairment: A comparison. Int. J. Lang. Commun. Disord. 2004, 39, 423-451.

31. Laws, G.; Bishop, D.V. A comparison of language abilities in adolescents with down syndrome and children with specific language impairment. J. Speech Lang. Hear. Res. 2003, 46, 1324-1339.

32. Simpson, N.H.; Addis, L.; Brandler, W.M.; Slonims, V.; Clark, A.; Watson, J.; Scerri, T.S.; Hennessy, E.R.; Bolton, P.F.; Conti-Ramsden, G.; et al. Increased prevalence of sex chromosome aneuploidies in specific language impairment and dyslexia. Dev. Med. Child Neurol. 2013, doi:10.1111/dmcn.12294.

33. Lai, C.S.; Fisher, S.E.; Hurst, J.A.; Vargha-Khadem, F.; Monaco, A.P. A forkhead-domain gene is mutated in a severe speech and language disorder. Nature 2001, 413, 519-523.

34. Fisher, S.E.; Scharff, C. Foxp2 as a molecular window into speech and language. Trends Genet. 2009, 25, 166-177.

35. Kang, C.; Riazuddin, S.; Mundorff, J.; Krasnewich, D.; Friedman, P.; Mullikin, J.C.; Drayna, D. Mutations in the lysosomal enzyme-targeting pathway and persistent stuttering. N. Engl. J. Med. 2010, 362, 677-685.

36. Vernes, S.C.; Nicod, J.; Elahi, F.M.; Coventry, J.A.; Kenny, N.; Coupe, A.M.; Bird, L.E.; Davies, K.E.; Fisher, S.E. Functional genetic analysis of mutations implicated in a human speech and language disorder. Hum. Mol. Genet. 2006, 15, 3154-3167.

37. Vernes, S.C.; Newbury, D.F.; Abrahams, B.S.; Winchester, L.; Nicod, J.; Groszer, M.; Alarcon, M.; Oliver, P.L.; Davies, K.E.; Geschwind, D.H.; et al. A functional genetic link between distinct developmental language disorders. N. Engl. J. Med. 2008, 359, 2337-2345. 
38. Alarcon, M.; Abrahams, B.S.; Stone, J.L.; Duvall, J.A.; Perederiy, J.V.; Bomar, J.M.; Sebat, J.; Wigler, M.; Martin, C.L.; Ledbetter, D.H.; et al. Linkage, association, and gene-expression analyses identify cntnap2 as an autism-susceptibility gene. Am. J. Hum. Genet. 2008, 82, 150-159.

39. Arking, D.E.; Cutler, D.J.; Brune, C.W.; Teslovich, T.M.; West, K.; Ikeda, M.; Rea, A.; Guy, M.; Lin, S.; Cook, E.H.; et al. A common genetic variant in the neurexin superfamily member cntnap2 increases familial risk of autism. Am. J. Hum. Genet. 2008, 82, 160-164.

40. Friedman, J.I.; Vrijenhoek, T.; Markx, S.; Janssen, I.M.; van der Vliet, W.A.; Faas, B.H.; Knoers, N.V.; Cahn, W.; Kahn, R.S.; Edelmann, L.; et al. Cntnap2 gene dosage variation is associated with schizophrenia and epilepsy. Mol. Psychiatry 2008, 13, 261-266.

41. Allison, D.B.; Thiel, B.; St Jean, P.; Elston, R.C.; Infante, M.C.; Schork, N.J. Multiple phenotype modeling in gene-mapping studies of quantitative traits: Power advantages. Am. J. Hum. Genet. 1998, 63, 1190-1201.

42. Whitehouse, A.J.; Bishop, D.V.; Ang, Q.W.; Pennell, C.E.; Fisher, S.E. Cntnap2 variants affect early language development in the general population. Genes Brain Behav. 2011, 10, 451-456.

43. Blair, D.R.; Lyttle, C.S.; Mortensen, J.M.; Bearden, C.F.; Jensen, A.B.; Khiabanian, H.; Melamed, R.; Rabadan, R.; Bernstam, E.V.; Brunak, S.; et al. A nondegenerate code of deleterious variants in mendelian loci contributes to complex disease risk. Cell 2013, 155, 70-80.

44. Tallal, P.; Ross, R.; Curtiss, S. Familial aggregation in specific language impairment. J. Speech Hear. Disord. 1989, 54, 167-173.

45. Almasy, L.; Blangero, J. Human qtl linkage mapping. Genetica 2009, 136, 333-340.

46. Grigorenko, E.L.; Wood, F.B.; Meyer, M.S.; Hart, L.A.; Speed, W.C.; Shuster, A.; Pauls, D.L. Susceptibility loci for distinct components of developmental dyslexia on chromosomes 6 and 15. Am. J. Hum. Genet. 1997, 60, 27-39.

47. Schulte-Korne, G.; Grimm, T.; Nothen, M.M.; Muller-Myhsok, B.; Cichon, S.; Vogt, I.R.; Propping, P.; Remschmidt, H. Evidence for linkage of spelling disability to chromosome 15. Am. J. Hum. Genet. 1998, 63, 279-282.

48. Cardon, L.R.; Smith, S.D.; Fulker, D.W.; Kimberling, W.J.; Pennington, B.F.; DeFries, J.C. Quantitative trait locus for reading disability on chromosome 6. Science 1994, 266, 276-279.

49. Fisher, S.E.; Marlow, A.J.; Lamb, J.; Maestrini, E.; Williams, D.F.; Richardson, A.J.; Weeks, D.E.; Stein, J.F.; Monaco, A.P. A quantitative-trait locus on chromosome 6p influences different aspects of developmental dyslexia. Am. J. Hum. Genet. 1999, 64, 146-156.

50. Gayan, J.; Smith, S.D.; Cherny, S.S.; Cardon, L.R.; Fulker, D.W.; Brower, A.M.; Olson, R.K.; Pennington, B.F.; DeFries, J.C. Quantitative-trait locus for specific language and reading deficits on chromosome 6p. Am. J. Hum. Genet. 1999, 64, 157-164.

51. Kaplan, D.E.; Gayan, J.; Ahn, J.; Won, T.W.; Pauls, D.; Olson, R.K.; DeFries, J.C.; Wood, F.; Pennington, B.F.; Page, G.P.; et al. Evidence for linkage and association with reading disability on 6p21.3-22. Am. J. Hum. Genet. 2002, 70, 1287-1298. 
52. Fagerheim, T.; Raeymaekers, P.; Tonnessen, F.E.; Pedersen, M.; Tranebjaerg, L.; Lubs, H.A. A new gene $(\operatorname{dyx} 3)$ for dyslexia is located on chromosome 2. J. Med. Genet. 1999, 36, 664-669.

53. Francks, C.; Fisher, S.E.; Olson, R.K.; Pennington, B.F.; Smith, S.D.; DeFries, J.C.; Monaco, A.P. Fine mapping of the chromosome 2p12-16 dyslexia susceptibility locus: Quantitative association analysis and positional candidate genes sema4f and otx1. Psychiatr. Genet. 2002, 12, 35-41.

54. Hannula-Jouppi, K.; Kaminen-Ahola, N.; Taipale, M.; Eklund, R.; Nopola-Hemmi, J.; Kaariainen, H.; Kere, J. The axon guidance receptor gene robol is a candidate gene for developmental dyslexia. PLoS Genet. 2005, 1, e50.

55. Nopola-Hemmi, J.; Myllyluoma, B.; Haltia, T.; Taipale, M.; Ollikainen, V.; Ahonen, T.; Voutilainen, A.; Kere, J.; Widen, E. A dominant gene for developmental dyslexia on chromosome 3. J. Med. Genet. 2001, 38, 658-664.

56. Fisher, S.E.; Francks, C.; Marlow, A.J.; MacPhie, I.L.; Newbury, D.F.; Cardon, L.R.; Ishikawa-Brush, Y.; Richardson, A.J.; Talcott, J.B.; Gayan, J.; et al. Independent genome-wide scans identify a chromosome 18 quantitative-trait locus influencing dyslexia. Nat. Genet. 2002, 30, 86-91.

57. Hsiung, G.Y.; Kaplan, B.J.; Petryshen, T.L.; Lu, S.; Field, L.L. A dyslexia susceptibility locus (dyx7) linked to dopamine d4 receptor (drd4) region on chromosome 11p15.5. Am. J. Med. Genet. Part B Neuropsychiatr. Genet. 2004, 125B, 112-119.

58. Grigorenko, E.L.; Wood, F.B.; Meyer, M.S.; Pauls, J.E.; Hart, L.A.; Pauls, D.L. Linkage studies suggest a possible locus for developmental dyslexia on chromosome 1p. Am. J. Med. Genet. 2001, 105, 120-129.

59. Rabin, M.; Wen, X.L.; Hepburn, M.; Lubs, H.A.; Feldman, E.; Duara, R. Suggestive linkage of developmental dyslexia to chromosome 1p34-p36. Lancet 1993, 342, 178.

60. De Kovel, C.G.; Franke, B.; Hol, F.A.; Lebrec, J.J.; Maassen, B.; Brunner, H.; Padberg, G.W.; Platko, J.; Pauls, D. Confirmation of dyslexia susceptibility loci on chromosomes $1 \mathrm{p}$ and $2 \mathrm{p}$, but not $6 \mathrm{p}$ in a dutch sib-pair collection. Am. J. Med. Genet. Part B Neuropsychiatr. Genet. 2008, 147, 294-300.

61. Meng, H.; Smith, S.D.; Hager, K.; Held, M.; Liu, J.; Olson, R.K.; Pennington, B.F.; DeFries, J.C.; Gelernter, J.; O'Reilly-Pol, T.; et al. Dcdc2 is associated with reading disability and modulates neuronal development in the brain. Proc. Natl. Acad. Sci. USA 2005, 102, 17053-17058.

62. Francks, C.; Paracchini, S.; Smith, S.D.; Richardson, A.J.; Scerri, T.S.; Cardon, L.R.; Marlow, A.J.; MacPhie, I.L.; Walter, J.; Pennington, B.F.; et al. A 77-kilobase region of chromosome 6p22.2 is associated with dyslexia in families from the united kingdom and from the united states. Am. J. Hum. Genet. 2004, 75, 1046-1058.

63. Cope, N.; Harold, D.; Hill, G.; Moskvina, V.; Stevenson, J.; Holmans, P.; Owen, M.J.; O’Donovan, M.C.; Williams, J. Strong evidence that kiaa0319 on chromosome 6p is a susceptibility gene for developmental dyslexia. Am. J. Hum. Genet. 2005, 76, 581-591. 
64. Paracchini, S.; Thomas, A.; Castro, S.; Lai, C.; Paramasivam, M.; Wang, Y.; Keating, B.J.; Taylor, J.M.; Hacking, D.F.; Scerri, T.; et al. The chromosome $6 \mathrm{p} 22$ haplotype associated with dyslexia reduces the expression of kiaa0319, a novel gene involved in neuronal migration. Hum. Mol. Genet. 2006, 15, 1659-1666.

65. Taipale, M.; Kaminen, N.; Nopola-Hemmi, J.; Haltia, T.; Myllyluoma, B.; Lyytinen, H.; Muller, K.; Kaaranen, M.; Lindsberg, P.J.; Hannula-Jouppi, K.; et al. A candidate gene for developmental dyslexia encodes a nuclear tetratricopeptide repeat domain protein dynamically regulated in brain. Proc. Natl. Acad. Sci. USA 2003, 100, 11553-11558.

66. Bates, T.C.; Lind, P.A.; Luciano, M.; Montgomery, G.W.; Martin, N.G.; Wright, M.J. Dyslexia and dyx1c1: Deficits in reading and spelling associated with a missense mutation. Mol. Psychiatry 2009, 15, 1190-1196.

67. Anthoni, H.; Zucchelli, M.; Matsson, H.; Muller-Myhsok, B.; Fransson, I.; Schumacher, J.; Massinen, S.; Onkamo, P.; Warnke, A.; Griesemann, H.; et al. A locus on 2p12 containing the co-regulated mrpl19 and c2orf3 genes is associated to dyslexia. Hum. Mol. Genet. 2007, 16, 667-677.

68. Anthoni, H.; Sucheston, L.E.; Lewis, B.A.; Tapia-Paez, I.; Fan, X.; Zucchelli, M.; Taipale, M.; Stein, C.M.; Hokkanen, M.E.; Castren, E.; et al. The aromatase gene cyp19a1: Several genetic and functional lines of evidence supporting a role in reading, speech and language. Behav. Genet. 2012, 42, 509-527.

69. Scerri, T.S.; Schulte-Korne, G. Genetics of developmental dyslexia. Eur. Child Adolesc. Psychiatry 2010, 19, 179-197.

70. Currier, T.A.; Etchegaray, M.A.; Haight, J.L.; Galaburda, A.M.; Rosen, G.D. The effects of embryonic knockdown of the candidate dyslexia susceptibility gene homologue dyx1c1 on the distribution of gabaergic neurons in the cerebral cortex. Neuroscience 2011, 172, $535-546$.

71. Tammimies, K.; Vitezic, M.; Matsson, H.; Le Guyader, S.; Burglin, T.R.; Ohman, T.; Stromblad, S.; Daub, C.O.; Nyman, T.A.; Kere, J.; et al. Molecular networks of dyx1c1 gene show connection to neuronal migration genes and cytoskeletal proteins. Biol. Psychiatry 2013, 73, 583-590.

72. Massinen, S.; Hokkanen, M.E.; Matsson, H.; Tammimies, K.; Tapia-Paez, I.; Dahlstrom-Heuser, V.; Kuja-Panula, J.; Burghoorn, J.; Jeppsson, K.E.; Swoboda, P.; et al. Increased expression of the dyslexia candidate gene dcdc2 affects length and signaling of primary cilia in neurons. PLoS One 2011, 6, e20580.

73. Adler, W.T.; Platt, M.P.; Mehlhorn, A.J.; Haight, J.L.; Currier, T.A.; Etchegaray, M.A.; Galaburda, A.M.; Rosen, G.D. Position of neocortical neurons transfected at different gestational ages with shrna targeted against candidate dyslexia susceptibility genes. PLoS One 2013, 8, e65179.

74. Platt, M.P.; Adler, W.T.; Mehlhorn, A.J.; Johnson, G.C.; Wright, K.A.; Choi, R.T.; Tsang, W.H.; Poon, M.W.; Yeung, S.Y.; Waye, M.M.; et al. Embryonic disruption of the candidate dyslexia susceptibility gene homolog kiaa0319-like results in neuronal migration disorders. Neuroscience 2013, 248C, 585-593. 
75. Wang, Y.; Yin, X.; Rosen, G.; Gabel, L.; Guadiana, S.M.; Sarkisian, M.R.; Galaburda, A.M.; Loturco, J.J. Dcdc2 knockout mice display exacerbated developmental disruptions following knockdown of doublecortin. Neuroscience 2011, 190, 398-408.

76. Szalkowski, C.E.; Fiondella, C.G.; Galaburda, A.M.; Rosen, G.D.; Loturco, J.J.; Fitch, R.H. Neocortical disruption and behavioral impairments in rats following in utero rnai of candidate dyslexia risk gene kiaa0319. Int. J. Dev. Neurosci. 2012, 30, 293-302.

77. Tarkar, A.; Loges, N.T.; Slagle, C.E.; Francis, R.; Dougherty, G.W.; Tamayo, J.V.; Shook, B.; Cantino, M.; Schwartz, D.; Jahnke, C.; et al. Dyx1c1 is required for axonemal dynein assembly and ciliary motility. Nat. Genet. 2013, 45, 995-1003.

78. Chandrasekar, G.; Vesterlund, L.; Hultenby, K.; Tapia-Paez, I.; Kere, J. The zebrafish orthologue of the dyslexia candidate gene dyx1c1 is essential for cilia growth and function. PLoS One 2013, 8, e63123.

79. Ivliev, A.E.; 't Hoen, P.A.C.; van Roon-Mom, W.M.; Peters, D.J.M.; Sergeeva, M.G. Exploring the transcriptome of ciliated cells using in silico dissection of human tissues. PLoS One 2012, 7, e35618.

80. Villanueva, P.; Newbury, D.F.; Jara, L.; de Barbieri, Z.; Mirza, G.; Palomino, H.M.; Fernandez, M.A.; Cazier, J.B.; Monaco, A.P.; Palomino, H. Genome-wide analysis of genetic susceptibility to language impairment in an isolated chilean population. Eur. J. Hum. Genet. 2011, 19, 687-695.

81. Bartlett, C.W.; Flax, J.F.; Logue, M.W.; Smith, B.J.; Vieland, V.J.; Tallal, P.; Brzustowicz, L.M. Examination of potential overlap in autism and language loci on chromosomes 2, 7, and 13 in two independent samples ascertained for specific language impairment. Hum. Hered. 2004, 57, 10-20.

82. Bartlett, C.W.; Flax, J.F.; Logue, M.W.; Vieland, V.J.; Bassett, A.S.; Tallal, P.; Brzustowicz, L.M. A major susceptibility locus for specific language impairment is located on 13q21. Am.J. Hum. Genet. 2002, 71, 45-55.

83. SLI Consortium (SLIC). Highly significant linkage to the sli1 locus in an expanded sample of individuals affected by specific language impairment. Am. J. Hum. Genet. 2004, 74, 1225-1238.

84. SLI Consortium. A genomewide scan identifies two novel loci involved in specific language impairment. Am. J. Hum. Genet. 2002, 70, 384-398.

85. Monaco, A.P. Multivariate linkage analysis of specific language impairment (sli). Ann. Hum. Genet. 2007, 71, 660-673.

86. Falcaro, M.; Pickles, A.; Newbury, D.F.; Addis, L.; Banfield, E.; Fisher, S.E.; Monaco, A.P.; Simkin, Z.; Conti-Ramsden, G. Genetic and phenotypic effects of phonological short-term memory and grammatical morphology in specific language impairment. Genes Brain Behav. 2008, 7, 393-402.

87. Newbury, D.F.; Winchester, L.; Addis, L.; Paracchini, S.; Buckingham, L.L.; Clark, A.; Cohen, W.; Cowie, H.; Dworzynski, K.; Everitt, A.; et al. Cmip and atp2c2 modulate phonological short-term memory in language impairment. Am. J. Hum. Genet. 2009, 85, 264-272. 
88. Ceroni, F.; Simpson, N.H.; Francks, C.; Baird, G.; Conti-Ramsden, G.; Clark, A.; Bolton, P.F.; Hennessy, E.R.; Donnelly, P.; Bentley, D.R.; et al. Homozygous microdeletion of exon 5 in znf277 in a girl with specific language impairment. Eur. J. Hum. Genet. 2014, doi:10.1038/ejhg.2014.4.

89. Nudel, R.; Simpson, N.H.; Baird, G.; O’Hare, A.; Conti-Ramsden, G.; Bolton, P.F.; Hennessy, E.R.; Consortium, S.L.I.; Monaco, A.P.; Knight, J.C.; et al. Associations of hla alleles with specific language impairment. J. Neurodev. Disord. 2014, doi:10.1186/1866-1955-6-1.

90. Zollner, S.; Pritchard, J.K. Overcoming the winner's curse: Estimating penetrance parameters from case-control data. Am. J. Hum. Genet. 2007, 80, 605-615.

91. The International Hapmap Project. Available online: http://www.hapmap.org/ (accessed on 31 March 2014).

92. 1000 Genomes: A Deep Catalogue of Human Genetic Variation. Available online: http://www.1000genomes.org/ (accessed on 31 March 2014).

93. Visscher, P.M.; Brown, M.A.; McCarthy, M.I.; Yang, J. Five years of GWAS discovery. Am. J. Hum. Genet. 2012, 90, 7-24.

94. Meaburn, E.L.; Harlaar, N.; Craig, I.W.; Schalkwyk, L.C.; Plomin, R. Quantitative trait locus association scan of early reading disability and ability using pooled DNA and 100k snp microarrays in a sample of 5760 children. Mol. Psychiatry 2008, 13, 729-740.

95. Roeske, D.; Ludwig, K.U.; Neuhoff, N.; Becker, J.; Bartling, J.; Bruder, J.; Brockschmidt, F.F.; Warnke, A.; Remschmidt, H.; Hoffmann, P.; et al. First genome-wide association scan on neurophysiological endophenotypes points to trans-regulation effects on slc2a3 in dyslexic children. Mol. Psychiatry 2011, 16, 97-107.

96. Risch, N.; Merikangas, K. The future of genetic studies of complex human diseases. Science 1996, 273, 1516-1517.

97. Field, L.L.; Shumansky, K.; Ryan, J.; Truong, D.; Swiergala, E.; Kaplan, B.J. Dense-map genome scan for dyslexia supports loci at 4q13, 16p12, 17q22; suggests novel locus at 7q36. Genes Brain Behav. 2013, 12, 56-69.

98. Luciano, M.; Evans, D.M.; Hansell, N.K.; Medland, S.E.; Montgomery, G.W.; Martin, N.G.; Wright, M.J.; Bates, T.C. A genome-wide association study for reading and language abilities in two population cohorts. Genes Brain Behav. 2013, 12, 645-652.

99. Eicher, J.D.; Powers, N.R.; Miller, L.L.; Akshoomoff, N.; Amaral, D.G.; Bloss, C.S.; Libiger, O.; Schork, N.J.; Darst, B.F.; Casey, B.J.; et al. Genome-wide association study of shared components of reading disability and language impairment. Genes Brain Behav. 2013, 12, 792-801.

100. Mott, R.; Yuan, W.; Kaisaki, P.; Gan, X.; Cleak, J.; Edwards, A.; Baud, A.; Flint, J. The architecture of parent-of-origin effects in mice. Cell 2014, 156, 332-342.

101. Nudel, R.; Simpson, N.H.; Baird, G.; O’Hare, A.; Conti-Ramsden, G.; Bolton, P.F.; Hennessy, E.R.; SLIC; Ring, S.M.; Davey Smith, G.; et al. Genome-wide association analyses of child genotype effects and parent-of-origin effects in specific language impairment (sli). Genes Brain Behav. 2014, doi:10.1111/gbb.12127.

102. McCarthy, M.I.; Hirschhorn, J.N. Genome-wide association studies: Potential next steps on a genetic journey. Hum. Mol. Genet. 2008, 17, R156-R165. 
103. Leonard, L. Is specific language impairment a useful construct? In Cambridge Monographs and Texts in Applied Psycholinguistics; Rosenberg, S., Ed.; Cambridge University Press: Cambridge, UK, 1987; pp. 1-39.

104. Rescorla, L.; Ratner, N.B. Phonetic profiles of toddlers with specific expressive language impairment (sli-e). J. Speech Hear. Res. 1996, 39, 153-165.

105. Dollaghan, C.A. Taxometric analyses of specific language impairment in 3- and 4-year-old children. J. Speech Lang. Hear. Res. 2004, 47, 464-475.

106. Dollaghan, C.A. Taxometric analyses of specific language impairment in 6-year-old children. J. Speech Lang. Hear. Res. 2011, 54, 1361-1371.

107. Leonard, L.B. Specific langauage impairment across languages. Child Dev. Perspect. 2014, 8, 1-5.

108. Bishop, D.V. Is specific language impairment a valid diagnostic category? Genetic and psycholinguistic evidence. Philos. Trans. R. Soc. Lond. Ser. B Biol. Sci. 1994, 346, 105-111.

109. Hayiou-Thomas, M.E.; Oliver, B.; Plomin, R. Genetic influences on specific versus nonspecific language impairment in 4-year-old twins. J. Learn. Disabil. 2005, 38, 222-232.

110. Bishop, D.V.; Hayiou-Thomas, M.E. Heritability of specific language impairment depends on diagnostic criteria. Genes Brain Behav. 2008, 7, 365-372.

111. Eley, T.C.; Bishop, D.V.; Dale, P.S.; Oliver, B.; Petrill, S.A.; Price, T.S.; Purcell, S.; Saudino, K.J.; Simonoff, E.; Stevenson, J.; et al. Genetic and environmental origins of verbal and performance components of cognitive delay in 2-year-olds. Dev. Psychol. 1999, 35, 1122-1131.

112. Paracchini, S.; Steer, C.D.; Buckingham, L.L.; Morris, A.P.; Ring, S.; Scerri, T.; Stein, J.; Pembrey, M.E.; Ragoussis, J.; Golding, J.; et al. Association of the kiaa0319 dyslexia susceptibility gene with reading skills in the general population. Am. J. Psychiatry 2008, 165, 1576-1584.

113. Scerri, T.S.; Morris, A.P.; Buckingham, L.L.; Newbury, D.F.; Miller, L.L.; Monaco, A.P.; Bishop, D.V.; Paracchini, S. Dcdc2, kiaa0319 and cmip are associated with reading-related traits. Biol. Psychiatry 2011, 70, 237-245.

114. Newbury, D.F.; Paracchini, S.; Scerri, T.S.; Winchester, L.; Addis, L.; Richardson, A.J.; Walter, J.; Stein, J.F.; Talcott, J.B.; Monaco, A.P. Investigation of dyslexia and sli risk variants in reading- and language-impaired subjects. Behav. Genet. 2011, 41, 90-104.

115. Landerl, K.; Ramus, F.; Moll, K.; Lyytinen, H.; Leppanen, P.H.; Lohvansuu, K.; O’Donovan, M.; Williams, J.; Bartling, J.; Bruder, J.; et al. Predictors of developmental dyslexia in european orthographies with varying complexity. J. Child Psychol. Psychiatry Allied Discip. 2013, 54, 686-694.

116. Becker, J.; Czamara, D.; Scerri, T.S.; Ramus, F.; Csepe, V.; Talcott, J.B.; Stein, J.; Morris, A.; Ludwig, K.U.; Hoffmann, P.; et al. Genetic analysis of dyslexia candidate genes in the european cross-linguistic neurodys cohort. Eur. J. Hum. Genet. 2013, doi:10.1038/ejhg.2013.199.

117. Schumacher, J.; Anthoni, H.; Dahdouh, F.; Konig, I.R.; Hillmer, A.M.; Kluck, N.; Manthey, M.; Plume, E.; Warnke, A.; Remschmidt, H.; et al. Strong genetic evidence of dedc2 as a susceptibility gene for dyslexia. Am. J. Hum. Genet. 2006, 78, 52-62. 
118. Bedore, L.M.; Leonard, L.B. Grammatical morphology deficits in spanish-speaking children with specific language impairment. J. Speech Lang. Hear. Res. 2001, 44, 905-924.

119. Dispaldro, M.; Leonard, L.B.; Deevy, P. Clinical markers in italian-speaking children with and without specific language impairment: A study of non-word and real word repetition as predictors of grammatical ability. Int. J. Lang. Commun. Disord. 2013, 48, 554-564.

120. Paradis, J.; Crago, M.; Genesee, F.; Rice, M. French-english bilingual children with sli: How do they compare with their monolingual peers? J. Speech Lang. Hear. Res. 2003, 46, $113-127$.

121. Do, R.; Willer, C.J.; Schmidt, E.M.; Sengupta, S.; Gao, C.; Peloso, G.M.; Gustafsson, S.; Kanoni, S.; Ganna, A.; Chen, J.; et al. Common variants associated with plasma triglycerides and risk for coronary artery disease. Nat. Genet. 2013, 45, 1345-1352.

122. Global Lipids Genetics, C.; Willer, C.J.; Schmidt, E.M.; Sengupta, S.; Peloso, G.M.; Gustafsson, S.; Kanoni, S.; Ganna, A.; Chen, J.; Buchkovich, M.L.; et al. Discovery and refinement of loci associated with lipid levels. Nat. Genet. 2013, 45, 1274-1283.

123. Magi, R.; Manning, S.; Yousseif, A.; Pucci, A.; Santini, F.; Karra, E.; Querci, G.; Pelosini, C.; McCarthy, M.I.; Lindgren, C.M.; et al. Contribution of 32 GWAS-identified common variants to severe obesity in european adults referred for bariatric surgery. PLoS One 2013, 8, e70735.

124. Berndt, S.I.; Gustafsson, S.; Magi, R.; Ganna, A.; Wheeler, E.; Feitosa, M.F.; Justice, A.E.; Monda, K.L.; Croteau-Chonka, D.C.; Day, F.R.; et al. Genome-wide meta-analysis identifies 11 new loci for anthropometric traits and provides insights into genetic architecture. Nat. Genet. 2013, 45, 501-512.

125. Yang, J.; Loos, R.J.; Powell, J.E.; Medland, S.E.; Speliotes, E.K.; Chasman, D.I.; Rose, L.M.; Thorleifsson, G.; Steinthorsdottir, V.; Magi, R.; et al. Fto genotype is associated with phenotypic variability of body mass index. Nature 2012, 490, 267-272.

126. Lango, H.; Consortium, U.K.T.D.G.; Palmer, C.N.; Morris, A.D.; Zeggini, E.; Hattersley, A.T.; McCarthy, M.I.; Frayling, T.M.; Weedon, M.N. Assessing the combined impact of 18 common genetic variants of modest effect sizes on type 2 diabetes risk. Diabetes 2008, 57, 3129-3135.

127. Cross-Disorder Group of the Psychiatric Genomics Consortium; Genetic Risk Outcome of Psychosis (GROUP) Consortium. Identification of risk loci with shared effects on five major psychiatric disorders: A genome-wide analysis. Lancet 2013, 381, 1371-1379.

128. Cross-Disorder Group of the Psychiatric Genomics Consortium; Lee, S.H.; Ripke, S.; Neale, B.M.; Faraone, S.V.; Purcell, S.M.; Perlis, R.H.; Mowry, B.J.; Thapar, A.; Goddard, M.E.; et al. Genetic relationship between five psychiatric disorders estimated from genome-wide snps. Nat. Genet. 2013, 45, 984-994.

129. Scerri, T.S.; Brandler, W.M.; Paracchini, S.; Morris, A.P.; Ring, S.M.; Talcott, J.B.; Stein, J.; Monaco, A.P. Pcsk6 is associated with handedness in individuals with dyslexia. Hum. Mol. Genet. 2011, 20, 608-614. 
130. Brandler, W.M.; Morris, A.P.; Evans, D.M.; Scerri, T.S.; Kemp, J.P.; Timpson, N.J.; St Pourcain, B.; Smith, G.D.; Ring, S.M.; Stein, J.; et al. Common variants in left/right asymmetry genes and pathways are associated with relative hand skill. PLoS Genet. 2013, 9, e1003751.

131. Hamada, H.; Meno, C.; Watanabe, D.; Saijoh, Y. Establishment of vertebrate left-right asymmetry. Nat. Rev. Genet. 2002, 3, 103-113.

132. Ludwig, K.U.; Samann, P.; Alexander, M.; Becker, J.; Bruder, J.; Moll, K.; Spieler, D.; Czisch, M.; Warnke, A.; Docherty, S.J.; et al. A common variant in myosin-18b contributes to mathematical abilities in children with dyslexia and intraparietal sulcus variability in adults. Transl. Psychiatry 2013, 3, e229.

133. Medland, S.E.; Lindgren, C.M.; Magi, R.; Neale, B.M.; Albrecht, E.; Esko, T.; Evans, D.M.; Hottenga, J.J.; Ikram, M.A.; Mangino, M.; et al. Meta-Analysis of GWAS for Handedness: Results from the ENGAGE consortium. Available online: http://www.ashg.org/2009meeting/ abstracts/fulltext/f21141.htm/ (accessed on 31 March 2014).

134. Eriksson, N.; Macpherson, J.M.; Tung, J.Y.; Hon, L.S.; Naughton, B.; Saxonov, S.; Avey, L.; Wojcicki, A.; Pe'er, I.; Mountain, J. Web-based, participant-driven studies yield novel genetic associations for common traits. PLoS Genet. 2010, 6, e1000993.

135. Iossifov, I.; Ronemus, M.; Levy, D.; Wang, Z.; Hakker, I.; Rosenbaum, J.; Yamrom, B.; Lee, Y.H.; Narzisi, G.; Leotta, A.; et al. De novo gene disruptions in children on the autistic spectrum. Neuron 2012, 74, 285-299.

136. O’Roak, B.J.; Deriziotis, P.; Lee, C.; Vives, L.; Schwartz, J.J.; Girirajan, S.; Karakoc, E.; Mackenzie, A.P.; Ng, S.B.; Baker, C.; et al. Exome sequencing in sporadic autism spectrum disorders identifies severe de novo mutations. Nat. Genet. 2011, 43, 585-589.

137. Neale, B.M.; Kou, Y.; Liu, L.; Ma'ayan, A.; Samocha, K.E.; Sabo, A.; Lin, C.F.; Stevens, C.; Wang, L.S.; Makarov, V.; et al. Patterns and rates of exonic de novo mutations in asd. Nature 2012, 485, 242-245.

138. O’Roak, B.J.; Vives, L.; Girirajan, S.; Karakoc, E.; Krumm, N.; Coe, B.P.; Levy, R.; Ko, A.; Lee, C.; Smith, J.D.; et al. Sporadic autism exomes reveal a highly interconnected protein network of de novo mutations. Nature 2012, 485, 246-250.

139. Vissers, L.E.; de Ligt, J.; Gilissen, C.; Janssen, I.; Steehouwer, M.; de Vries, P.; van Lier, B.; Arts, P.; Wieskamp, N.; del Rosario, M.; et al. A de novo paradigm for mental retardation. Nat. Genet. 2011, 42, 1109-1112.

140. Toma, C.; Torrico, B.; Hervas, A.; Valdes-Mas, R.; Tristan-Noguero, A.; Padillo, V.; Maristany, M.; Salgado, M.; Arenas, C.; Puente, X.S.; et al. Exome sequencing in multiplex autism families suggests a major role for heterozygous truncating mutations. Mol. Psychiatry 2013, doi:10.1038/mp.2013.106.

141. Yu, T.W.; Chahrour, M.H.; Coulter, M.E.; Jiralerspong, S.; Okamura-Ikeda, K.; Ataman, B.; Schmitz-Abe, K.; Harmin, D.A.; Adli, M.; Malik, A.N.; et al. Using whole-exome sequencing to identify inherited causes of autism. Neuron 2013, 77, 259-273.

142. Worthey, E.A.; Raca, G.; Laffin, J.J.; Wilk, B.M.; Harris, J.M.; Jakielski, K.J.; Dimmock, D.P.; Strand, E.A.; Shriberg, L.D. Whole-exome sequencing supports genetic heterogeneity in childhood apraxia of speech. J. Neurodev. Disord. 2013, 5, 29. 
143. Kong, A.; Frigge, M.L.; Masson, G.; Besenbacher, S.; Sulem, P.; Magnusson, G.; Gudjonsson, S.A.; Sigurdsson, A.; Jonasdottir, A.; Jonasdottir, A.; et al. Rate of de novo mutations and the importance of father's age to disease risk. Nature 2012, 488, 471-475.

144. Girirajan, S.; Rosenfeld, J.A.; Coe, B.P.; Parikh, S.; Friedman, N.; Goldstein, A.; Filipink, R.A.; McConnell, J.S.; Angle, B.; Meschino, W.S.; et al. Phenotypic heterogeneity of genomic disorders and rare copy-number variants. N. Engl. J. Med. 2012, 367, 1321-1331.

145. Girirajan, S.; Rosenfeld, J.A.; Cooper, G.M.; Antonacci, F.; Siswara, P.; Itsara, A.; Vives, L.; Walsh, T.; McCarthy, S.E.; Baker, C.; et al. A recurrent 16p12.1 microdeletion supports a two-hit model for severe developmental delay. Nat. Genet. 2010, 42, 203-209.

146. Cooper, G.M.; Coe, B.P.; Girirajan, S.; Rosenfeld, J.A.; Vu, T.H.; Baker, C.; Williams, C.; Stalker, H.; Hamid, R.; Hannig, V.; et al. A copy number variation morbidity map of developmental delay. Nat. Genet. 2011, 43, 838-846.

147. Leblond, C.S.; Jutta, H.; Delorme, R.; Proepper, C.; Betancur, C.; Huguet, G.; Konyukh, M.; Chaste, P.; Ey, E.; Rastam, M.; et al. Genetic and functional analyses of shank2 mutations suggest a multiple hit model of autism spectrum disorders. PLoS Genet. 2012, 8, e1002521.

148. Newbury, D.F.; Mari, F.; Sadighi Akha, E.; Macdermot, K.D.; Canitano, R.; Monaco, A.P.; Taylor, J.C.; Renieri, A.; Fisher, S.E.; Knight, S.J. Dual copy number variants involving $16 \mathrm{p} 11$ and $6 \mathrm{q} 22$ in a case of childhood apraxia of speech and pervasive developmental disorder. Eur. J. Hum. Genet. 2013, 21, 361-365.

149. Consortium, E.P.; Birney, E.; Stamatoyannopoulos, J.A.; Dutta, A.; Guigo, R.; Gingeras, T.R.; Margulies, E.H.; Weng, Z.; Snyder, M.; Dermitzakis, E.T.; et al. Identification and analysis of functional elements in $1 \%$ of the human genome by the encode pilot project. Nature 2007, 447, 799-816.

150. Lappalainen, T.; Sammeth, M.; Friedlander, M.R.; 't Hoen, P.A.; Monlong, J.; Rivas, M.A.; Gonzalez-Porta, M.; Kurbatova, N.; Griebel, T.; Ferreira, P.G.; et al. Transcriptome and genome sequencing uncovers functional variation in humans. Nature 2013, 501, 506-511.

151. Gratten, J.; Visscher, P.M.; Mowry, B.J.; Wray, N.R. Interpreting the role of de novo protein-coding mutations in neuropsychiatric disease. Nat. Genet. 2013, 45, 234-238.

152. Laederich, M.B.; Horton, W.A. Fgfr3 targeting strategies for achondroplasia. Expert Rev. Mol. Med. 2012, 14, e11.

153. Foldynova-Trantirkova, S.; Wilcox, W.R.; Krejci, P. Sixteen years and counting: The current understanding of fibroblast growth factor receptor 3 (fgfr3) signaling in skeletal dysplasias. Hum. Mutat. 2012, 33, 29-41.

154. Eglinton, E.; Annett, M. Handedness and dyslexia: A meta-analysis. Percept. Mot. Skills 1994, 79, 1611-1616.

155. Bishop, D.V. How to increase your chances of obtaining a significant association between handedness and disorder. J. Clin. Exp. Neuropsychol. 1990, 12, 812-816.

156. Dragovic, M.; Hammond, G. Handedness in schizophrenia: A quantitative review of evidence. Acta Psychiatr. Scand. 2005, 111, 410-419.

157. Bishop, D.V. Cerebral asymmetry and language development: Cause, correlate, or consequence? Science 2013, doi:10.1126/science.1230531. 
158. Paracchini, S.; Scerri, T.; Monaco, A.P. The genetic lexicon of dyslexia. Annu. Rev. Genomics Hum. Genet. 2007, 8, 57-79.

159. Brandler, W.M.; Paracchini, S. The genetic relationship between handedness and neurodevelopmental disorders. Trends Mol. Med. 2014, 20, 83-90.

160. Velayos-Baeza, A.; Toma, C.; da Roza, S.; Paracchini, S.; Monaco, A.P. Alternative splicing in the dyslexia-associated gene kiaa0319. Mamm. Genome 2007, 18, 627-634.

161. Field, S.; Riley, K.L.; Grimes, D.T.; Hilton, H.; Simon, M.; Powles-Glover, N.; Siggers, P.; Bogani, D.; Greenfield, A.; Norris, D.P. Pkd111 establishes left-right asymmetry and physically interacts with pkd2. Development 2011, 138, 1131-1142.

162. Lee, J.E.; Gleeson, J.G. Cilia in the nervous system: Linking cilia function and neurodevelopmental disorders. Curr. Opin. Neurol. 2011, 24, 98-105.

163. Sun, T.; Walsh, C.A. Molecular approaches to brain asymmetry and handedness. Nat. Rev. Neurosci. 2006, 7, 655-662. 
MDPI AG

Klybeckstrasse 64

4057 Basel, Switzerland

Tel. +41 616837734

Fax +41 613028918

http://www.mdpi.com/

Genes Editorial Office

E-mail: genes@mdpi.com

http://www.mdpi.com/journal/genes 




ISBN 978-3-03842-170-2

www.mdpi.com 